\title{
INTEGRATED VERIFICATION EXPERIMENT DATA COLLECTED AS PART OF THE LOS ALAMOS NATIONAL LABORATORY SOURCE REGION PROGRAM
}

\author{
APPENDIX B \\ SURFACE GROUND MOTION \\ Thomas A. Weaver \\ Diane F. Baker \\ C. L. Edwards \\ Susan H. Freeman \\ Geophysics Group, EES-3 \\ Los Alamos National Laboratory \\ Los Alamos, New Mexico 87545
}

October 1993

\begin{abstract}
Surface ground motion was recorded for many of the integratea Verification Experiments using standard 10-, 25- and 100-g accelerometers, force-balanced accelerometers and, for some events, using golf balls and $0.39-\mathrm{cm}$ steel balls as surface inertial gauges (SIGs). This report contairis the semi-ore ressed acceleration, velocity, and displacement data for the acceleron eters fielcied and the individual observations for the SIG experiments. Most acceleration, velocity, anc displacement records have had calibrations applied and have been deramped, offset corrected, and deglitched but are otherwise unfiltered or processed from their original records. Digital data for all of these records are stored at Los Alamos National Laboratory.
\end{abstract}




\section{Table of Contents}

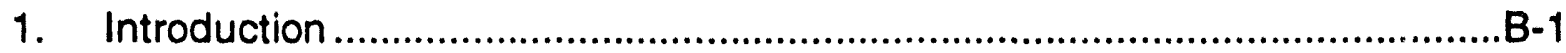

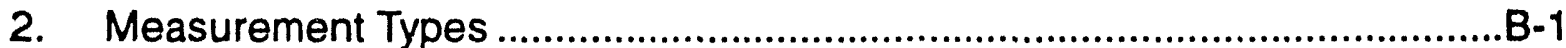

2.1 J-8 Surface Accelerometer Measurements .......................................

2.2 Extended Surface Accelerometer Measurements ............................... B-2

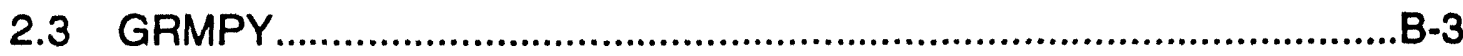

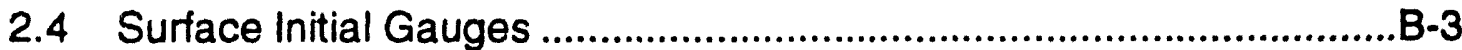

3. Surface Motion Data for the IVEs ...........................................................

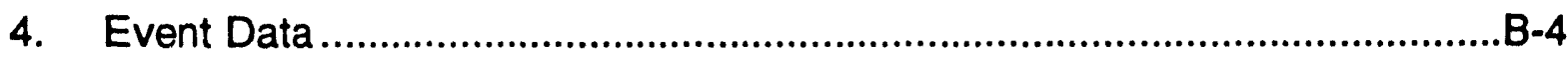

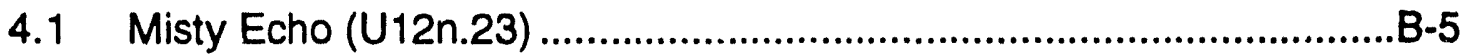

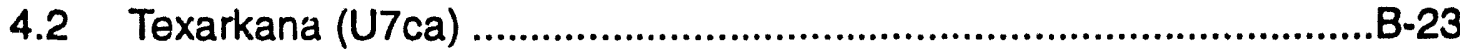

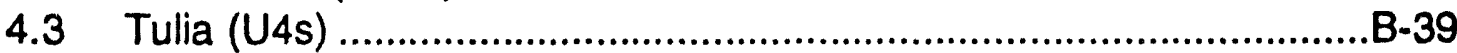

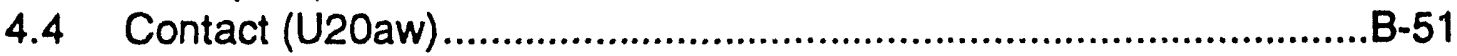

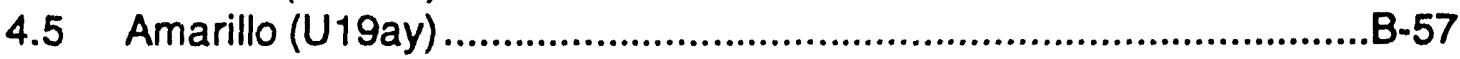

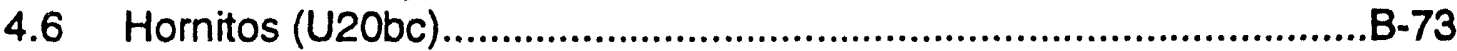

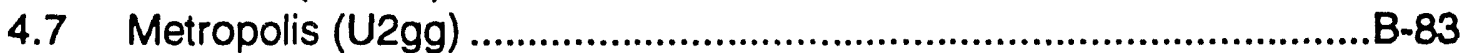

4.8 Mineral Quarry (U12n.22) .........................................................

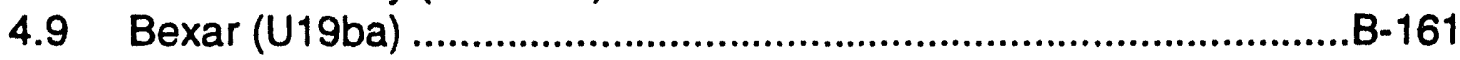

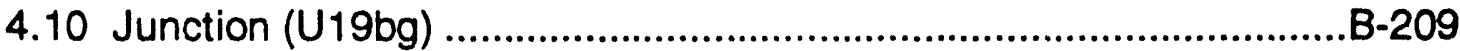

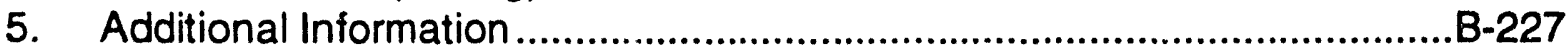

\section{List of Figures}

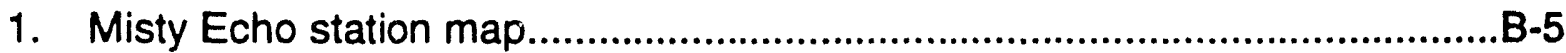

2. Misty Echo station data traces for the accelerometers .................................. B-7

3. Ground-motion-measurement array for Texarkana .......................................

4. Texarkana station data traces for the accelerometers ................................... B-25

5. Ground-motion-measurement array for Tulia..................................................39

6. Tulia station data traces for the accelerometers..........................................41

7. Contact/Amarillo experiment map..............................................................52

8. Ground-motion stations for Amarillo ........................................................59

9. Amarillo station data traces for the accelerometers...................................6-65

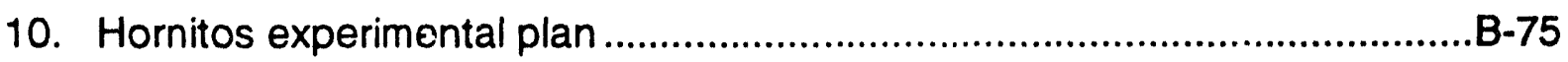

11. Metropolis deployment locations............................................................8-85

12. Results of the SIG experiment on Metropolis. ..........................................8-88

13. Spall limit on Metropolis as determined from the extended

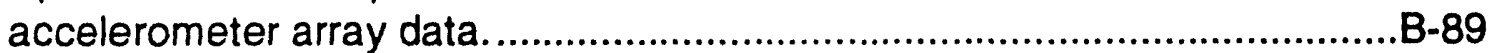

14. Metropolis station data traces for the accelerometers ..................................

15. Mineral Quarry station map.............................................................

16. Mineral Quarry station data traces for the accelerometers.......................B-131

17. Bexar station map...............................................................................162

18. Bexar station data traces for the accelerometers .................................... 165 
19. Junction SIG experiment plan

B-210

20. Junction station map

21. Junction data traces for the accelerometers

1. Misty Echo Ground-Motion Station Data ....................................................6

2. Texarkana Ground-Motion Station Data ....................................................24

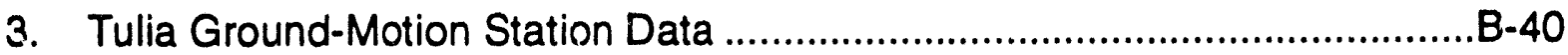

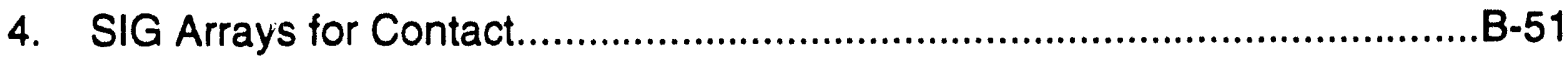

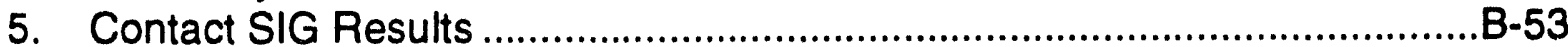

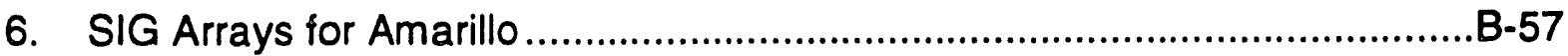

7. Amarillo Ground-Motion Station Data ........................................................

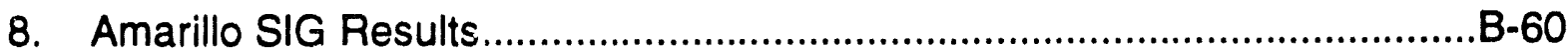

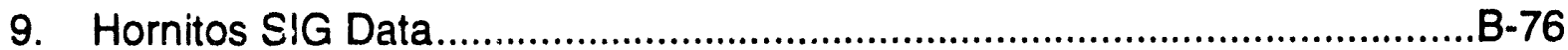

10. Metropolis Ground Motion Station Data......................................................8-86

11. Metropolis SIG Results .........................................................................90

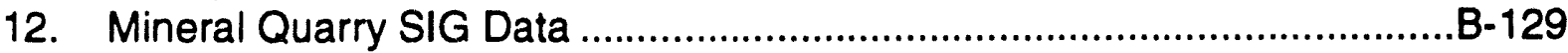

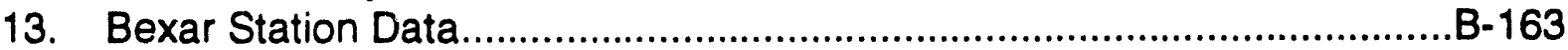

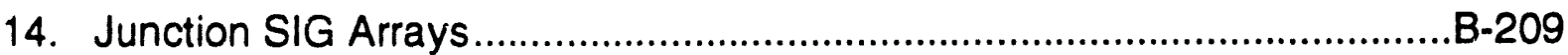

15. Junction Ground-Motion Station Data.....................................................

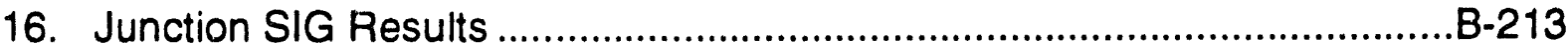




\section{Introduction}

Ground-motion analysis has been a key part of the Los Alamos National Laboratory (LANL) Source Region Program (SRP) for several reasons: near-source ground motion represents the up-going energy from an underground explosion, and that information is required to assess the total energy budget for an explosion; these data can be used to benchmark the numerical modeling of the nonlinear zone; and last, but not least, later time phenomena, such as spall closure, are believed to play significant roles in seismic and other signals used for detection, discrimination, and characterization of underground explosions. As a result, characterization of the close-in ground motions has been a major goal of the SRP.

One of the topics being addressed by the SRP is the three-dimensional characterization of the spall volume as a function of time (size, shape, volume, mass, ground motions, surface expression, and degree of damage). Surface observations of acceleration, velocity, and displacement provide constraints for spall and, when combined with the limited amount of subsurface ground-motion data (not presented in this report), allow a three-dimensional model to be generated.

Presented herein are the data from a variety of close-in ground-motion experiments conducted by LANL and its collaborators, Southern Methodist University (SMU) and the Defense Nuclear Agency, Albuquerque (DNA/A), on the 12 Integrated Verification Experiments (IVEs) of the SRP. Only 10 of the IVEs had ground-motion experiments performed; Ingot (U2gg) and Bullion (20bd) did not.

These data are the semi-processed acceleration, velocity, and displacement records for the accelerometers fielded and the individual observations for the surface inertial gauge (SIG) experiments. Most acceleration, velocity, and displacement records have had calibrations applied and have been deramped, offset corrected, and deglitched but are otherwise unfiltered or processed from their original records.

\section{Measurement Types}

Four types of ground-motion measurements were performed for the IVEs: (1) the standard J-8 surface accelerometer installation for the Nuclear Test Containment Program, deployed only on LANL-sponsored events; (2) extended surface accelerometer (ESA) measurements to define and characterize the spall zone; (3) GRMPY stations installed at and around surface ground zero (SGZ) for some events; and (4) arrays of SIGs to fill in those areas where we could not afford to place instrumentation. Not all types were used on each IVE; type 1 was deployed only on LANL-sponsored events; types 2 through 4 were added as needed to observe the ground motions at and around SGZ, through the spall zone, and beyond. 
2.1 J-8 Surface Accelerometer Measurements. LANL Group J-8 has instrumented the SGZ area on LANL-sponsored events with accelerometers (among other types of sensors) for the Test Director (and later, the Containment Program) since the first underground nuclear tests. In the latter half of this effort, the measurement array for these instruments has been standardized to include: one to three stations at $S G Z$, two stations at the trailer park (normally located near the perimeter fence); three or four stations at the perimeter fence, normally at azimuths $90^{\circ}$ to $120^{\circ}$ apart, and three or four stations at about one hole depth, $90^{\circ}$ to $120^{\circ}$ apart. Each station typically has three components of motion: vertical, radial, and tangential, though in some cases, only verticals have been employed. The accelerometers used for these stations are Endevco 10-, 25-, or 100-g instruments (Model 2262), depending on the anticipated motions. The plus directions on the data plots presented herein are: vertical, up; radial, toward SGZ; and tangential, clockwise around SGZ.

The data are recorded by the remote instrumentation digital system (RIDS). The input analog signals are low-pass-filtered to $100 \mathrm{~Hz}$ and digitized at $640 \mathrm{~Hz}$. J-8 has preprocessed the data, which may include corrections for timing, amplitude calibration, baseline offsets, integration ramps, noise, etc. EG\&G and EES-3 may also have further corrected these signals for residual integration ramps and baseline offsets.

Note: Offset or integral ramp corrections may not be obvious in the records presented because for clarity only the first $2 \mathrm{~s}$ of record are displayed in this report.

2.2 Extended Surface Accelerometer (ESA) Measurements. For some events, we have acquired ground-motion data that straddled the edge of predicted spall (Misty Echo, Metropolis, Mineral Quarry, and Bexar), and for one event, Bexar, daty out to at least three times the predicted spall radius were collected. To acquire these measurements, we installed portable stations consisting of accelerometers (and in some cases, seismometers), portable power (batteries), and portable digital recorders.

The accelerometers deployed were of two main types: Model SA-111 force-balanced accelerometers, built by Terra Technology; and 10- and 25-g Model 2262A piezoelectric accelerometers by Endevco. The Endevcos were borrowed from J-8 for these experiments.

Three types of portable digital recorders were used. For the two tunnel experiments, SMU furnished 16-bit, 6-channel REFTEK data acquisition system (DAS), made by Refraction Technology, and DNA/A furnished 12-bit, 3-channel PDAS recorders by Terra Technology. For Metropolis, only SMU REFTEKs were used; for Bexar, SMU and LANL REFTEKs were used together with several of both 3- and 6-channel portable digital recorders (PDR-2s), made by Kinemetrics, furnished by LANL. Data were 
recorded at several speeds, dependent upon recorders used and the nature of the experiment, ranging from 100 samples/s to 1000 samples/s.

The clocks on all of the portable digital recorders used for the four events with ESA arrays (Misty Echo, Metropolis, Mineral Quarry, and Bexar) were not the modern GPS (Global Positioning Satellite) clocks. As a result, timing for the extended accelerometer measurements on those events is suspect and has been corrected by average velocity travel times to each station. Do not attempt to draw significance from any time-ofarrival (TOA) data for these measurements.

EG\&G and EES-3 have corrected these signals for timing, amplitude calibration, integration ramps, and baseline offsets.

Note: Offset or integral ramp corrections may not be obvious in the records presented because for clarity only the first $2 \mathrm{~s}$ of record are displayed in this report.

2.3 GRMPY. GRMPY (GRound Motion Predicts Yield) was an experimental system being developed and tested by LANL for on-site verification of the Threshold Test Ban Treaty (TTBT). ${ }^{1}$ GRMPY employed a small array of accelerometers that were fielded in the vicinity of SGZ for an underground nuclear test and a recording van. Due to the nature of the system the locations for those stations are approximate because the stations did not require accurate surveys. Because the system was experimental, the configuration and location of the accelerometer stations, the recording system, and even the sensors could change from event to event. EG\&G and EES-3 may have corrected these signals for polarity, integration ramps, and baseline offsets.

Note: Offset or integral ramp corrections may not be obvious in the records presented because for clarity only $2 \mathrm{~s}$ of record are displayed in this report.

2.4 Surface Inertial Gauges (SIGs). One of the major topics to be addressed by the SRP is the three-dimensional characterization of the spall volume as a function of time: size, shape, volume, mass, ground motions, surface expression, and degree of damage. Observations made on earlier events have indicated that rocks, ranging in size from small pebbles to large boulders, can be ballistically launched during the spall period. Hundreds of ground-motion gauges would be needed to map ground motion in the spall zone, assuming the complexity indicated by the postshot crack maps of many events, but an experiment of that magnitude involving electronic gauges is prohibitively expensive. Using golf balls as a type of inertial gauge seemed to be a way of getting, at least in a relative sense, fairly inexpensive information about the spall zone: size, shape, and character (uniformity, intensity, impulsiveness) of the ground motions.

'Deupree, R. G. et. al., MISTY ECHO Proof Test of the GRMPY System, LA-11660-MS, 43 p., January 1990 


\section{Surface Motion Data for the IVEs}

The accelerometer data presented herein are the vertical components of surface acceleration and the derived velocity and displacement. At most ground-motion stations discussed in this report, three-component data (mainly vertical, radial, and tangential) were collected. The radial and tangential data are in the database and available should anyone require them. The data are reported as acceleration, in units

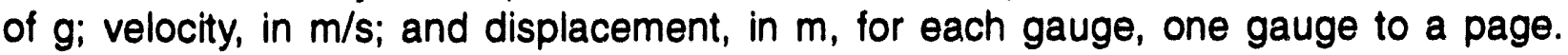
Only those gauges with valid records are included in this report. Later experiments indicated that any ground-motion data of the type and density reported herein are spatially aliased and probably do not represent more than a snapshot of outgoing wave generation (report in preparation). However, taken collectively, these data can be used to generate predictive curves for the average ground motion as a function of scaled range for contained underground explosions.

Accelerometer stations are named with a code as follows: three letters for the first three letters of the event name; followed by two letters to indicate the type of measurement (GM for J-8 stations; GR for GRMPY stations; and SG for extendedsurface-accelerometer stations); followed by a number for the station, followed by a letter, normally " $A$," representing a single station. The letters $B, C$, or D were used if there were multiple sensors at the same location, or, if there were multiple stations on a single portable DAS (e.g., Misty Echo, Metropolis, Mineral Quarry, and Bexar). In the last case, refer to the data tables for the event to find the location of each station. The gauge-data plots included in this report have two additional letters immediately following the station name, $Z$ for the vertical component and $A, V$, or $D$ for acceleration, velocity, and displacement, respectively. Therefore, MISGR1AZA would denote the vertical acceleration from station 1A of the GRMPY array on Misty Echo.

Two seconds of record are displayed for each station; the start time of the display for each station may have been shifted so that the signal onset is in the first third of the record. All times presented are from explosion zero time, in seconds.

The SIG data are presented as tables with location number, azimuth, and distance from $S G Z$, azimuth and distance moved, and any observational comments. For Metropolis we present one of many ways that these data may be portrayed for analysis. Azimuths have been corrected to true north.

\section{Event Data}

The following pages contain a brief description of the experiment setups and data for ten events: Misty Echo, Texarkana, Tulia. Contact, Amarillo, Hornitos, Metropolis, Mineral Quarry, Bexar, and Junction. 


\subsection{Misty Echo (U12n.23)}

Experiment design. The Area 12 tunnel event Misty Echo had an array of 18 surface ground motion stations: 8 GRMPY near and around SGZ, all inside the predicted spall zone, and 10 extended ground-motion stations (from just inside to well beyond the edge of predicted spall), fielded by SMU and DNAA (Fig. 1 and Table 1). Figure 2 (16 pages) contains the data traces for the accelerometer data.

GRMPY results. All GRMPY stations recorded spall signals. Two of the ten ESA stations failed to record good data ( $3 A$ and $9 A)$. Of the remaining eight, four recorded spall signals (2A, $5 \mathrm{~A}, 7 \mathrm{~A}$, and $13 \mathrm{~A})$.

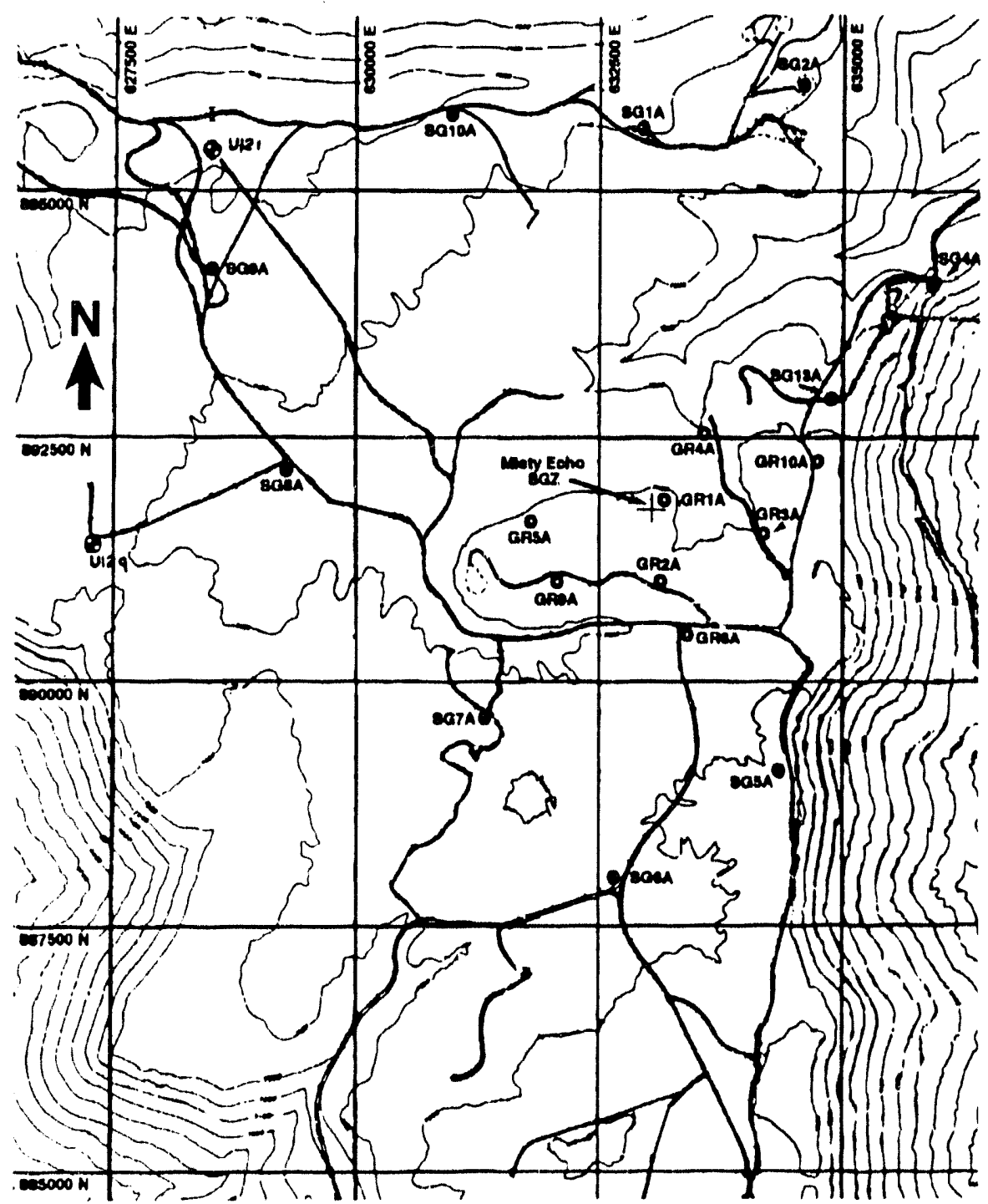

Fig. 1. Misty Echo station map. 
Table 1. Misty Echo Ground-Motion Station Data






\section{Misty Echo Surface Ground Motion}

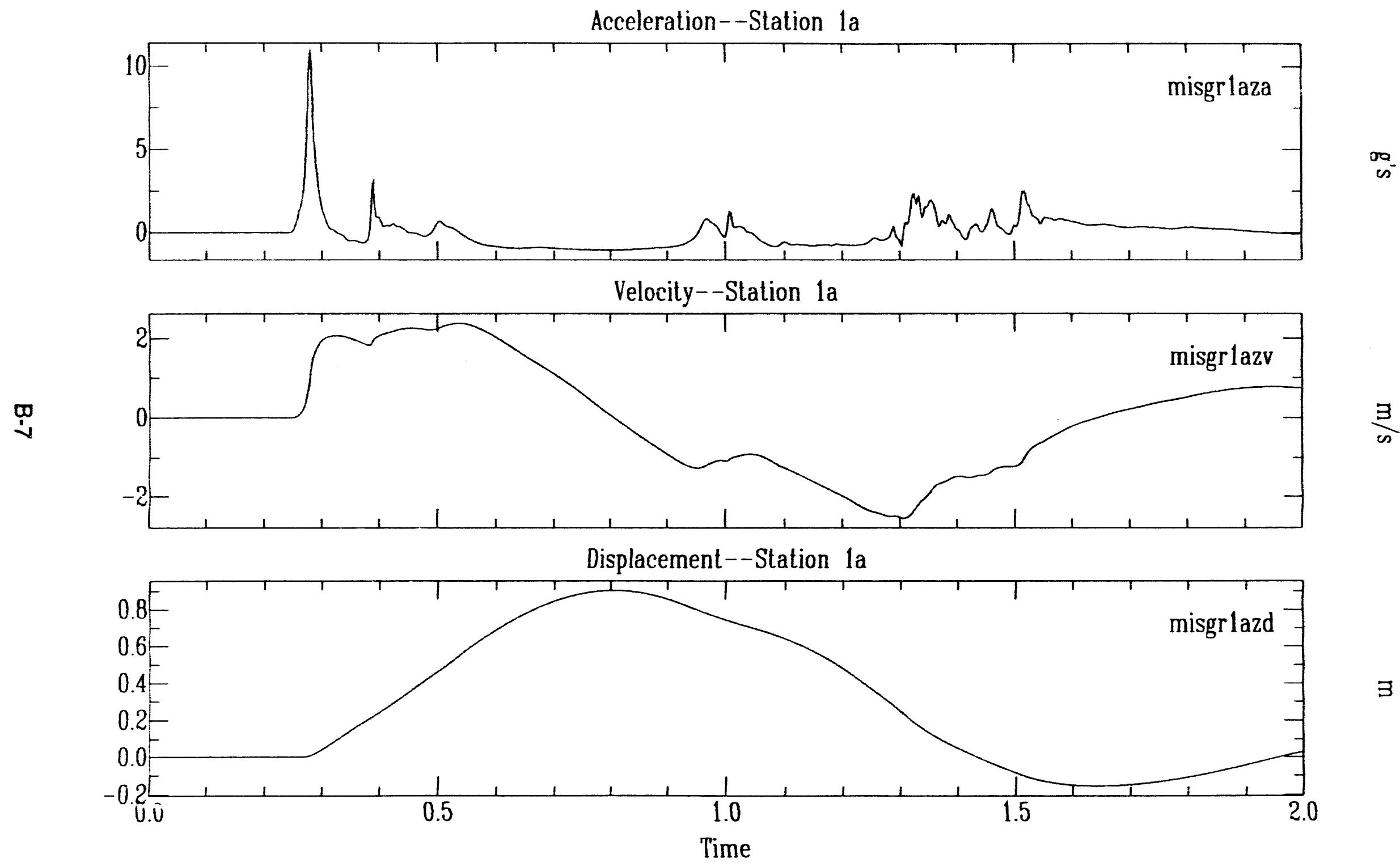


Misty Echo Surface Ground Motion

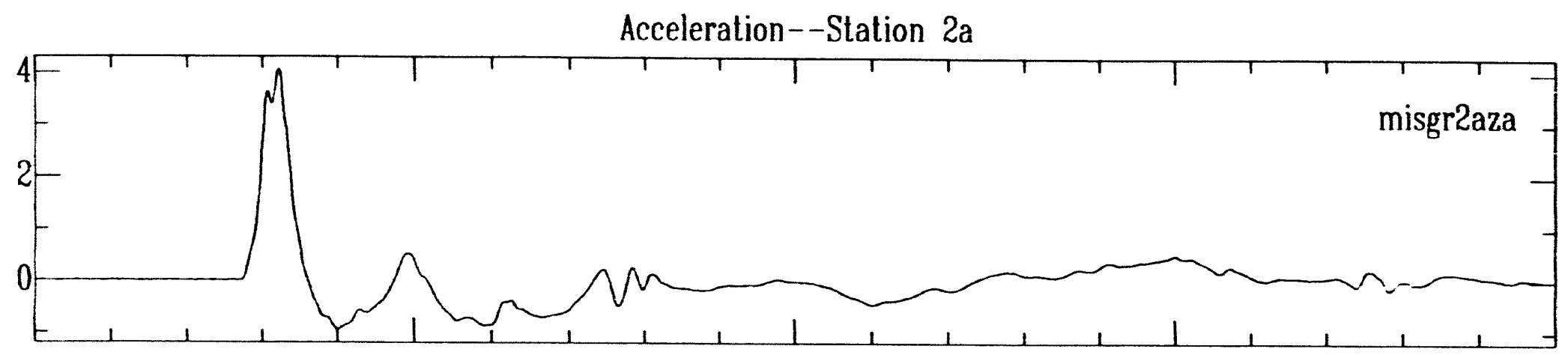

Velocity--Station 2a

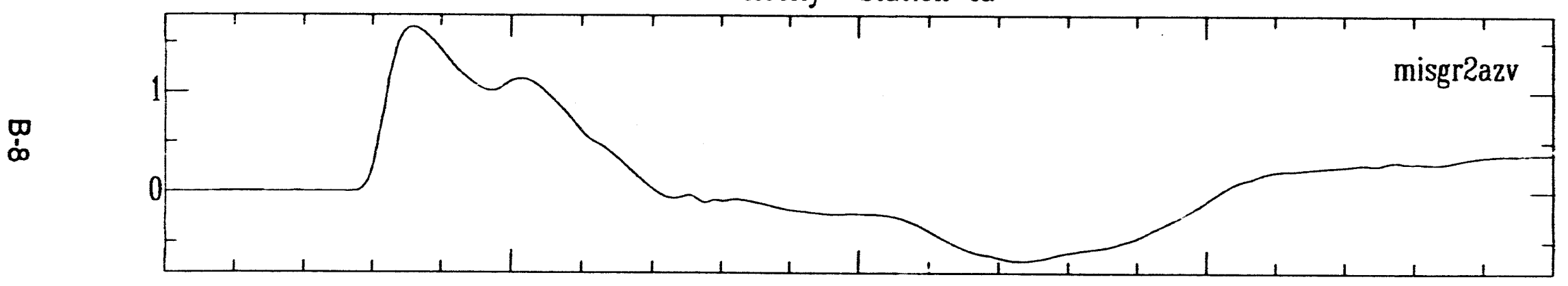

Displacement--Station 2a

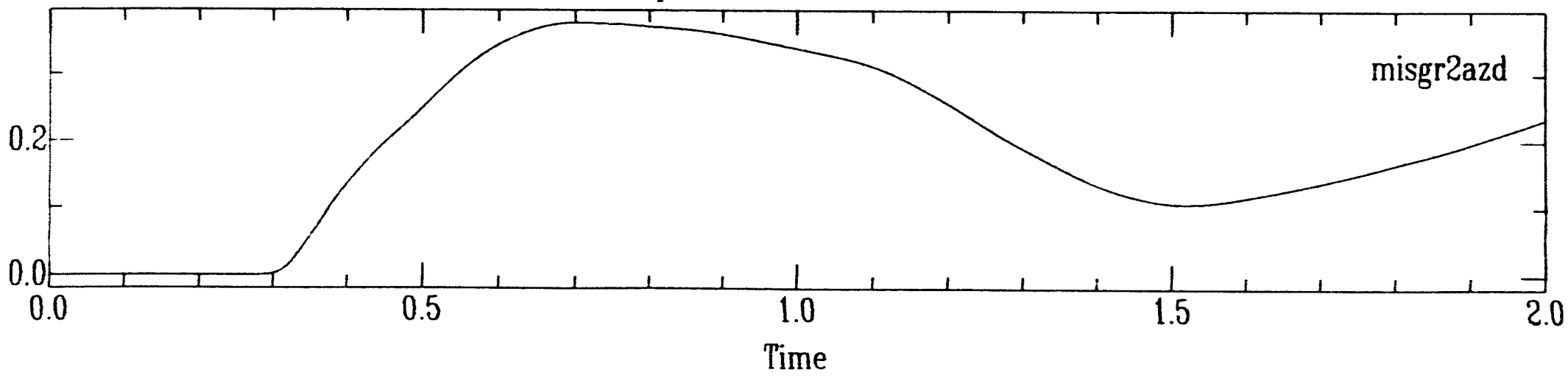




\section{Misty Echo Surface Ground Motion}

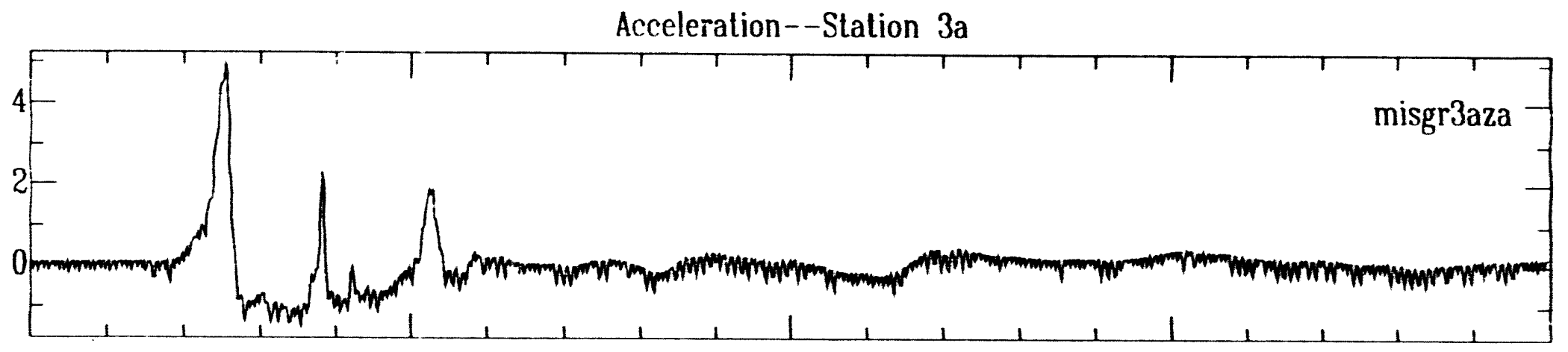

Velocity--Statiün 3a

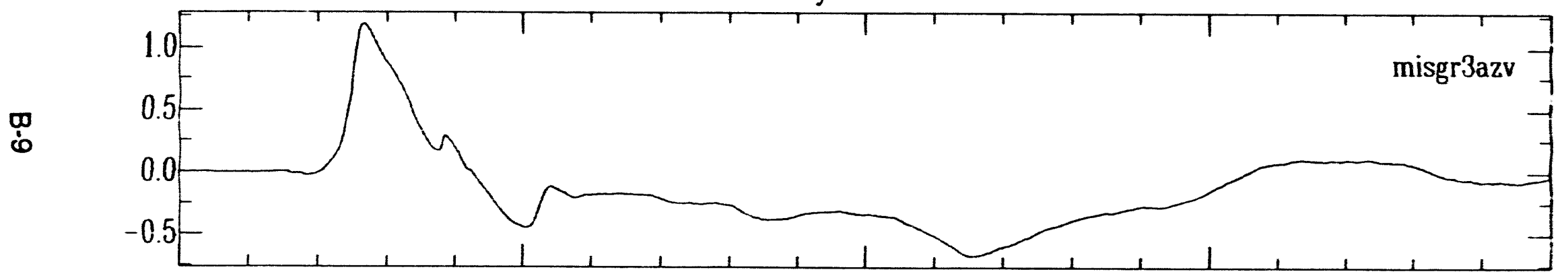

Displacement--Station 3a

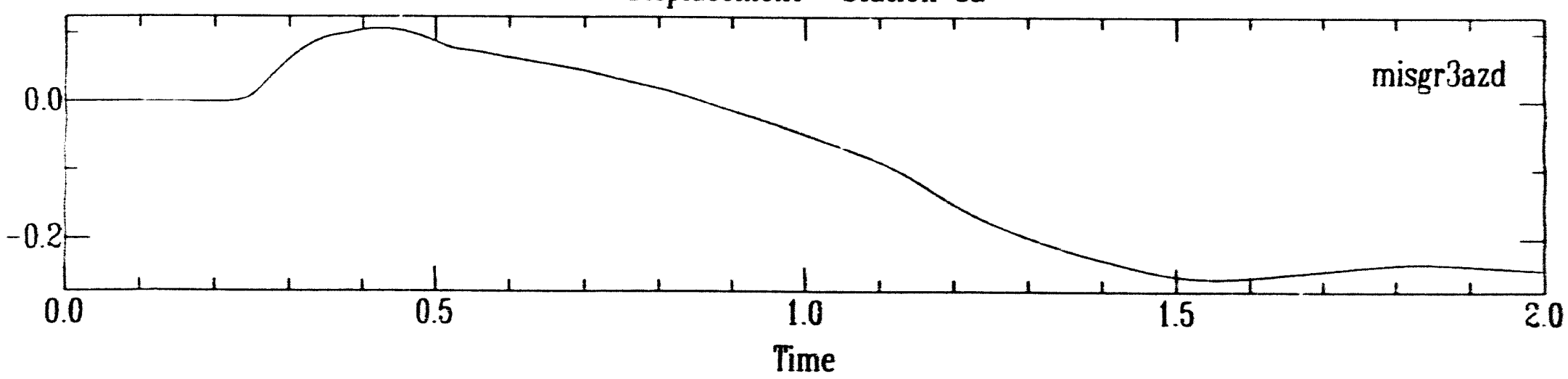




\section{Misty Echo Surface Ground Motion}

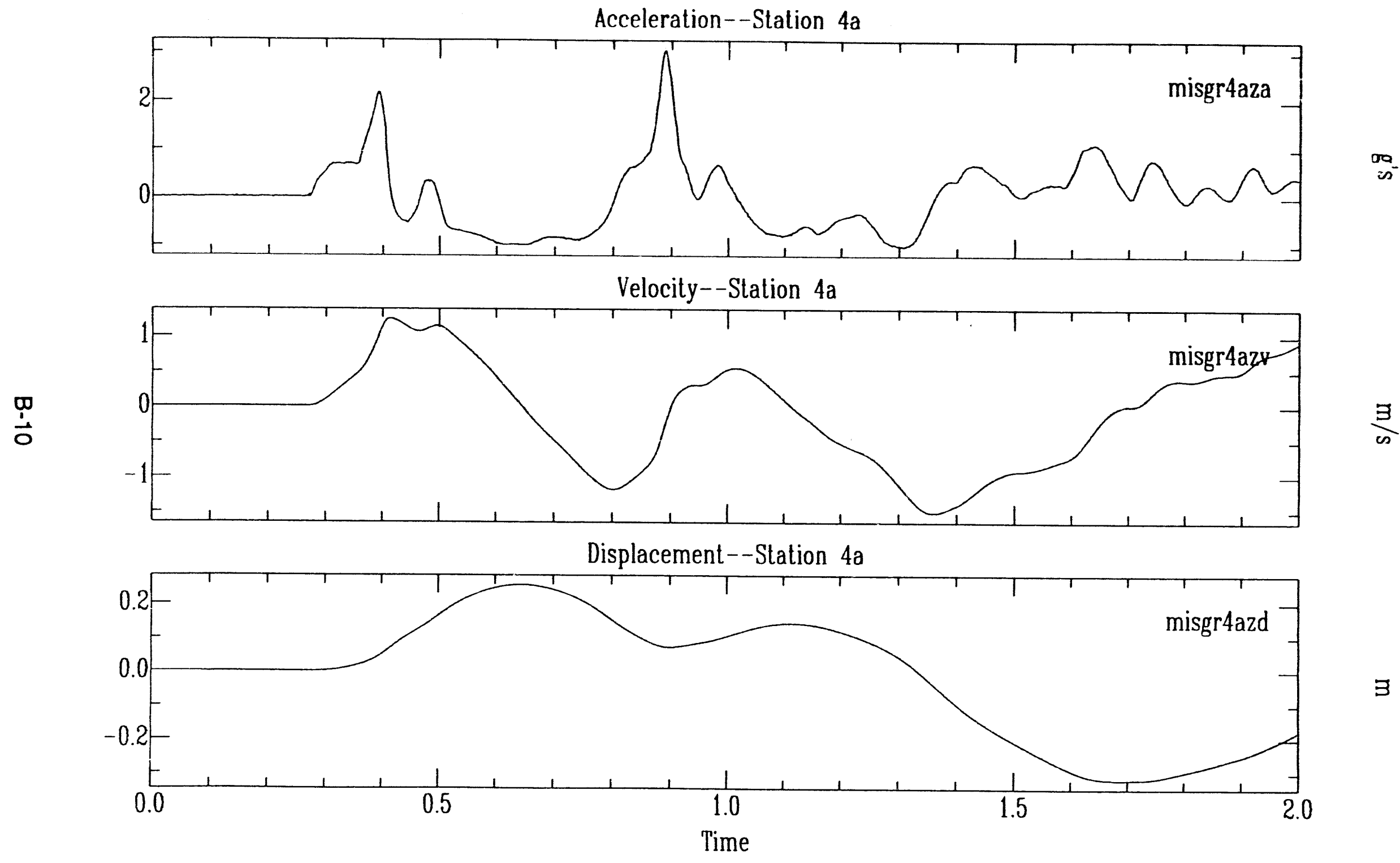




\section{Misty Echo Surface Ground Motion}

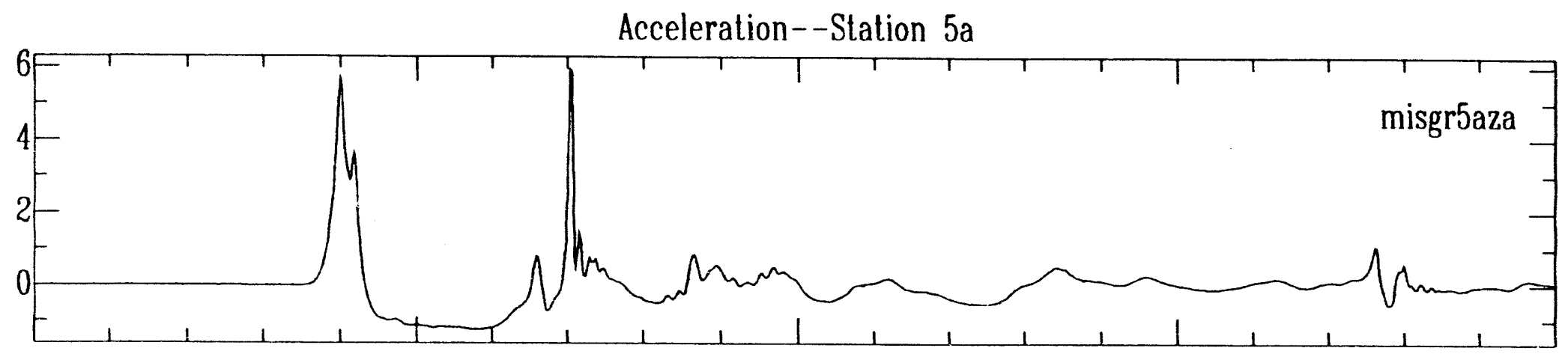

Velocity--Station 5a

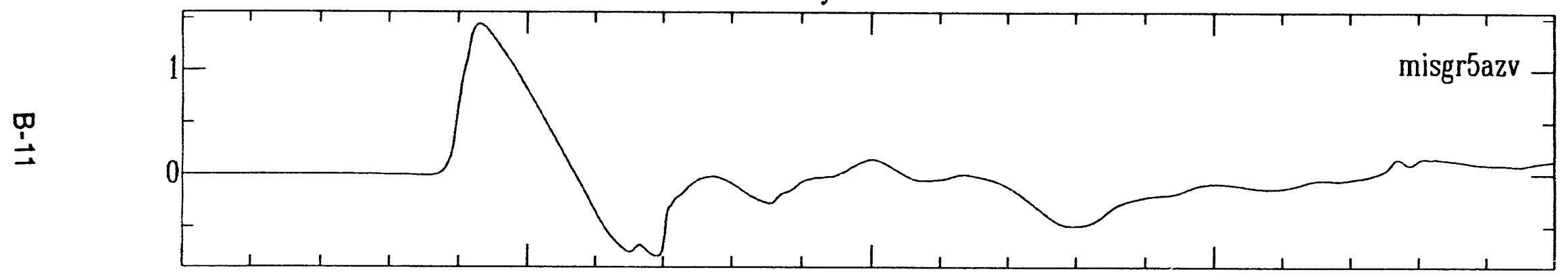

$\frac{3}{\infty}$

Displacement--Station 5a

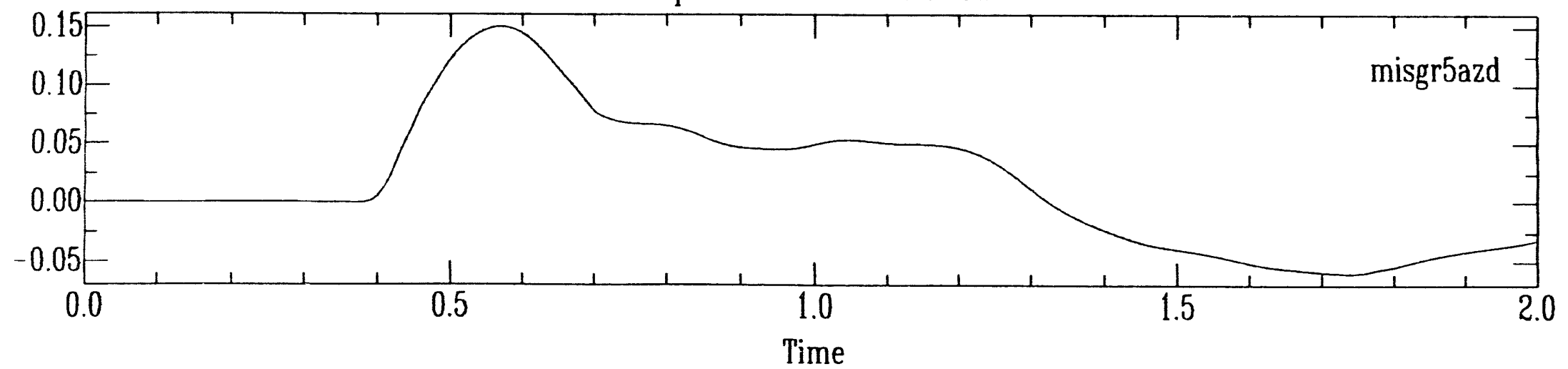




\section{Misty Echo Surface Ground Motion}

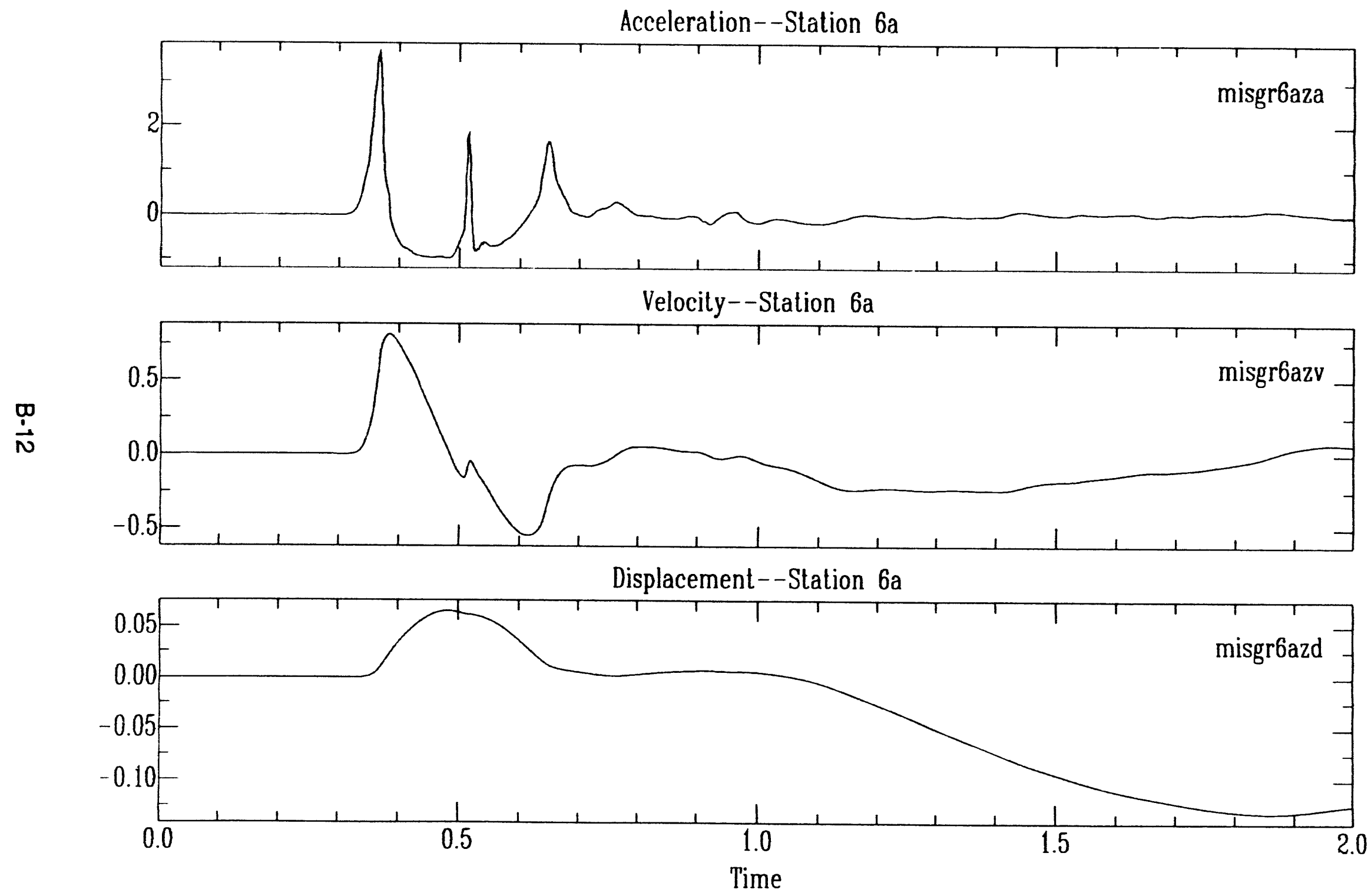


Misty Echo Surface Ground Motion

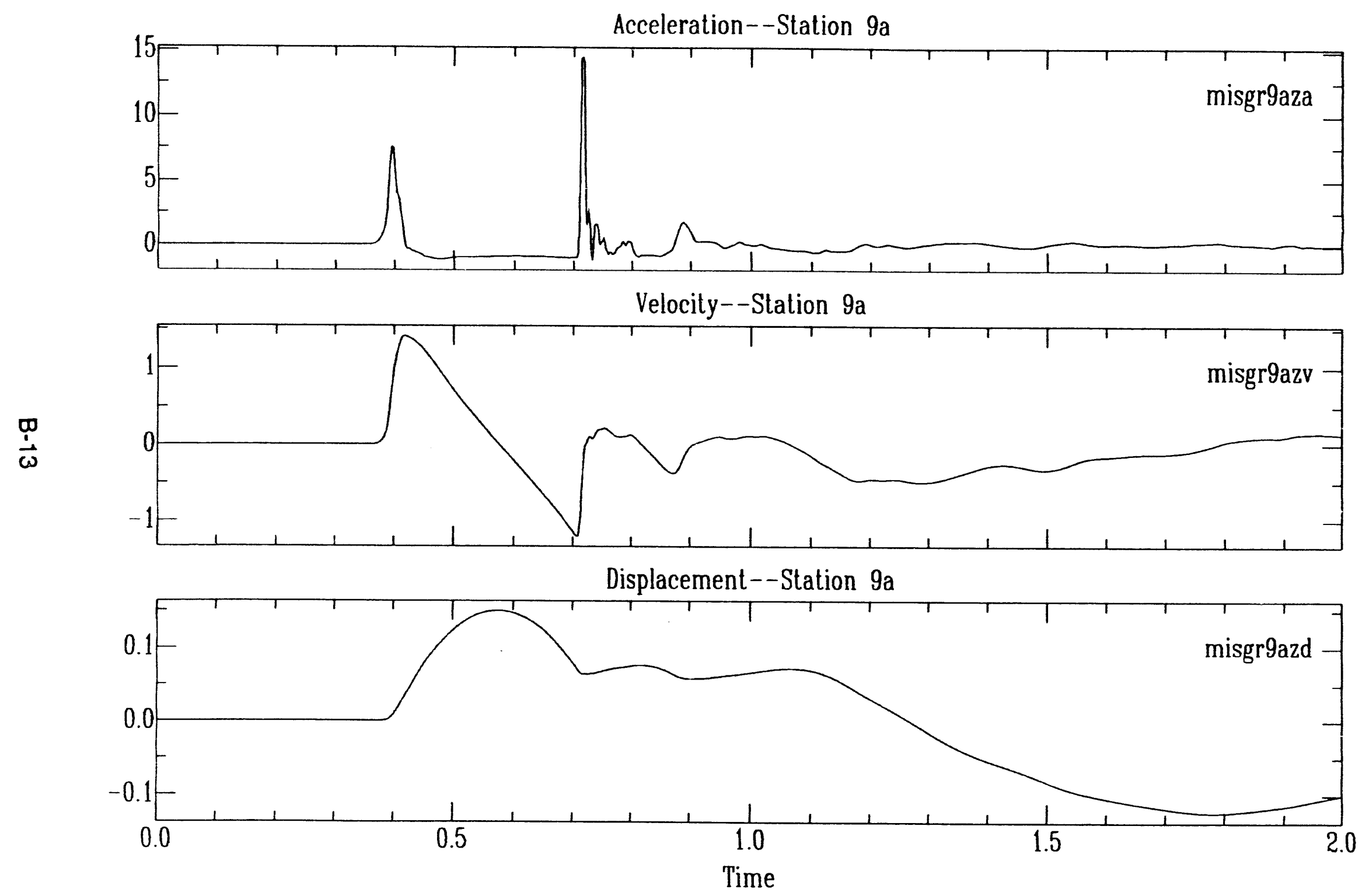


Misty Echo Surface Ground Motion

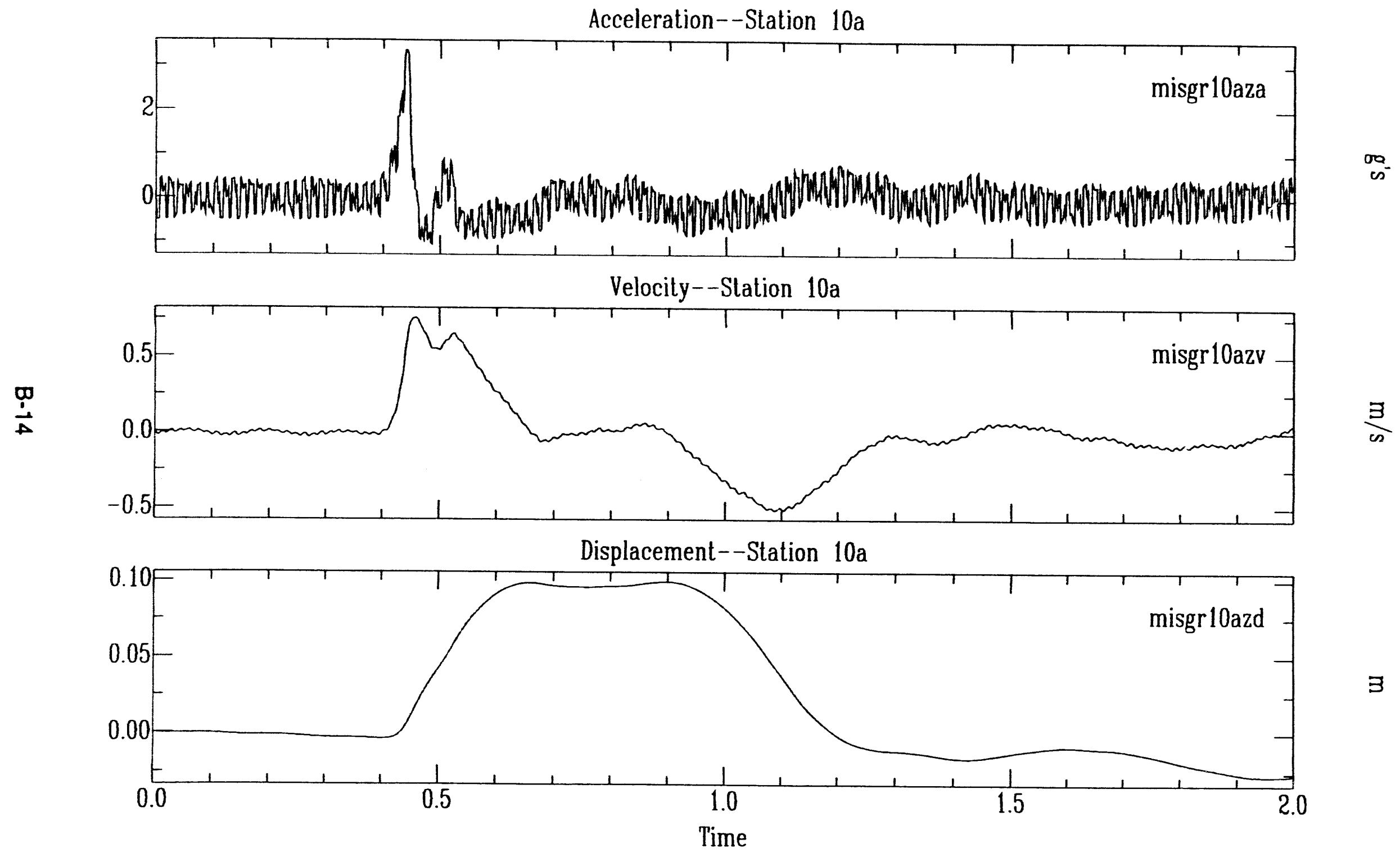




\section{Misty Echo Surface Ground Motion}

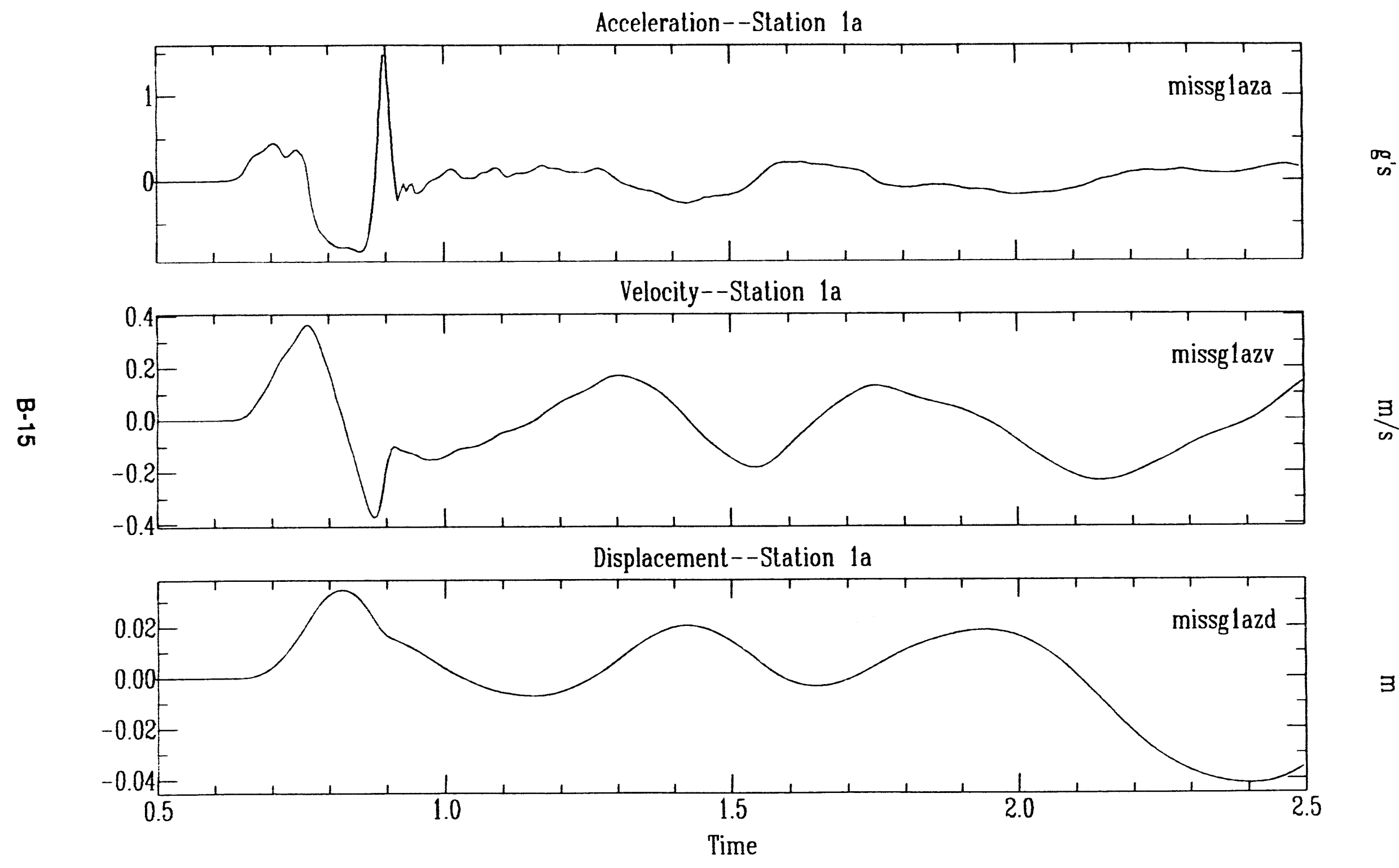


Misty Echo Surface Ground Motion

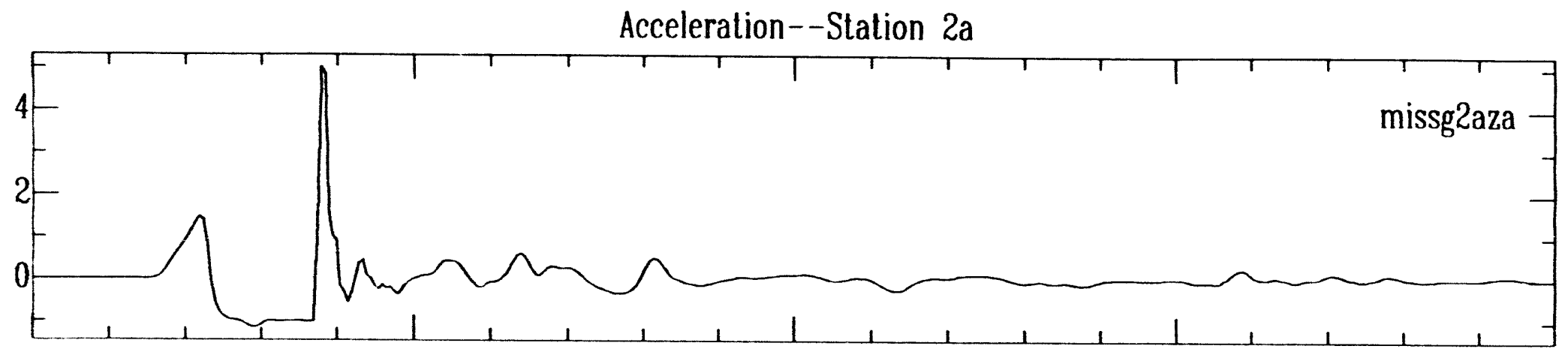

Velocity--Station 2a

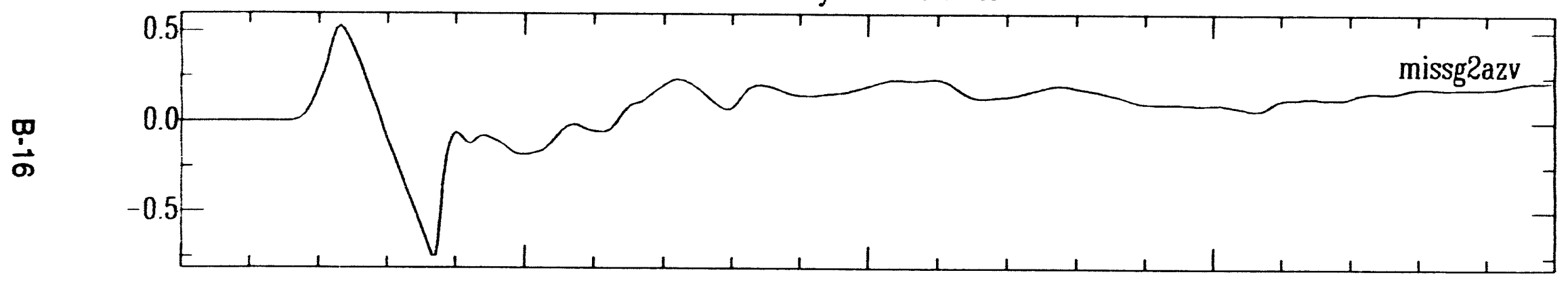

Displacement--Station 2a

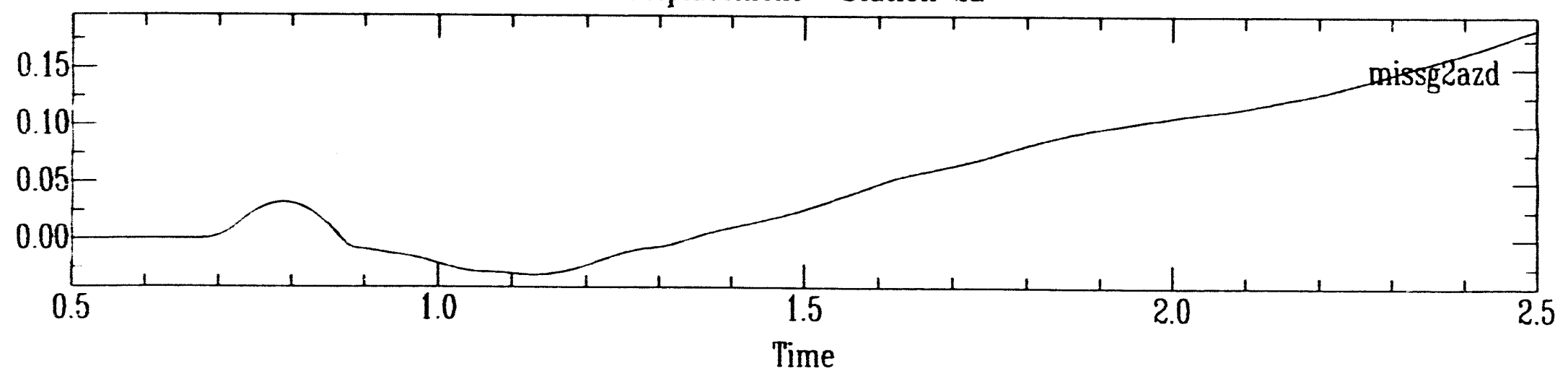


Misty Echo Surface Ground Motion

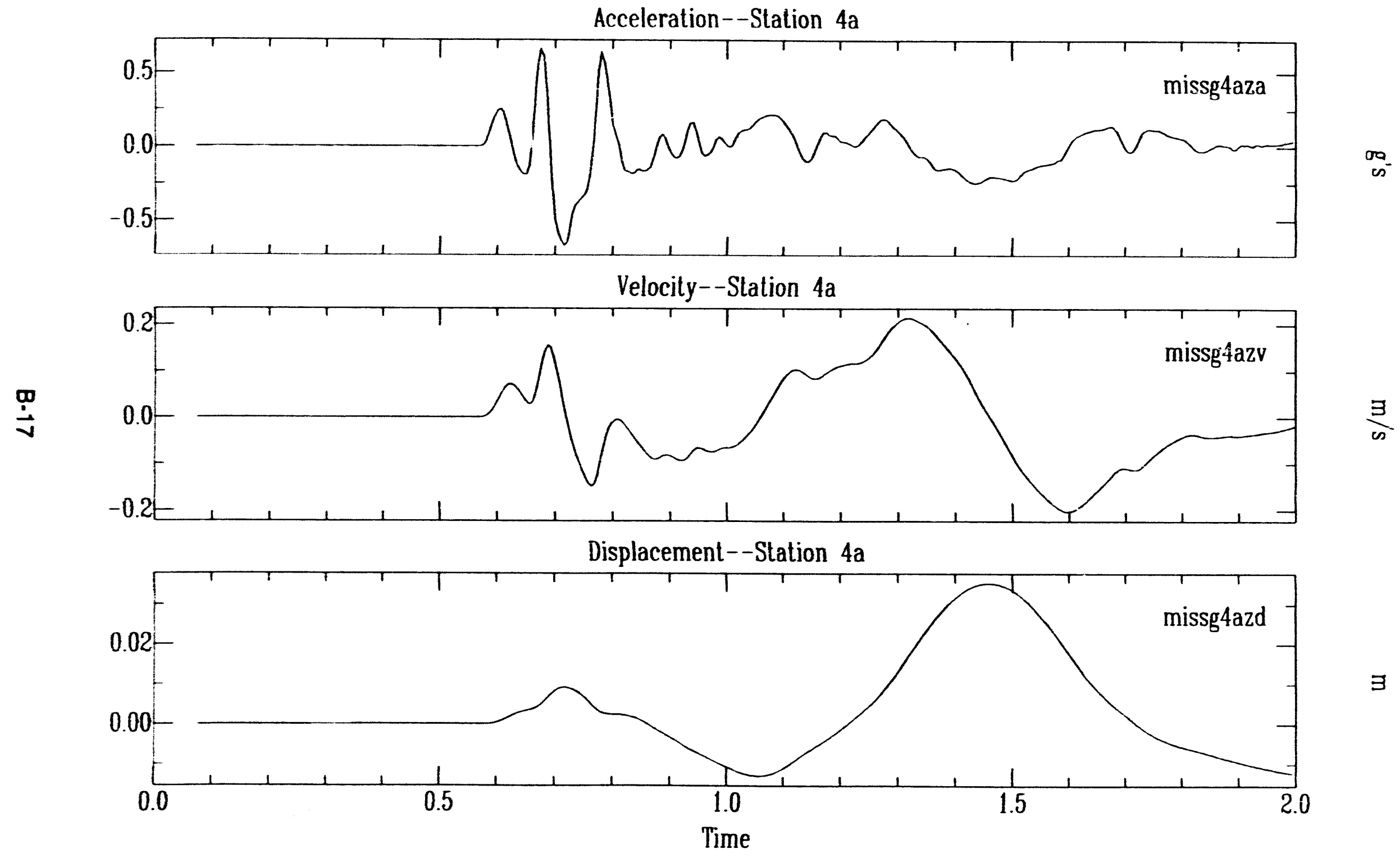




\section{Misty Echo Surface Ground Motion}

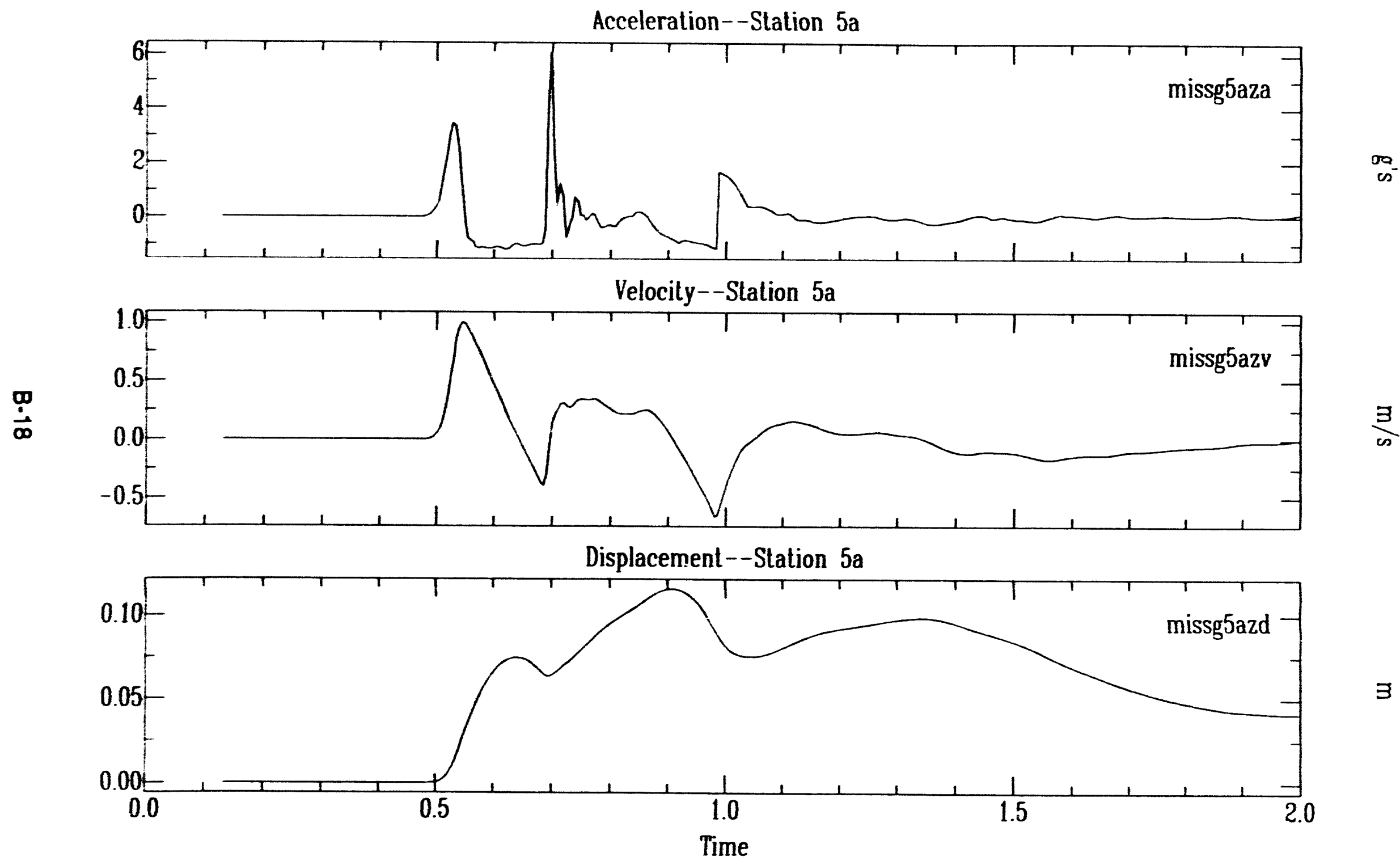




\section{Misty Echo Surface Ground Motion}

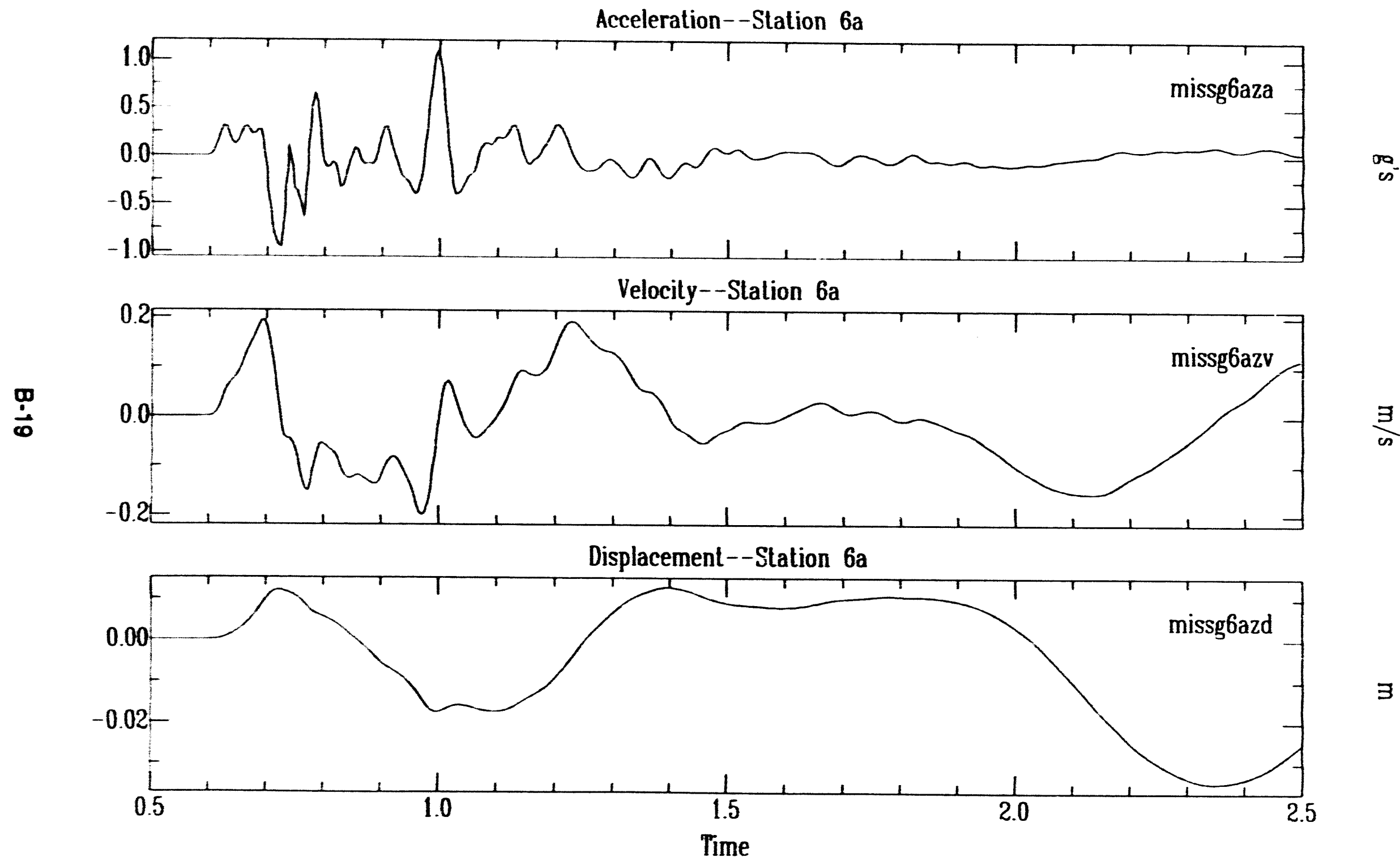


Misty Echo Surface Ground Motion

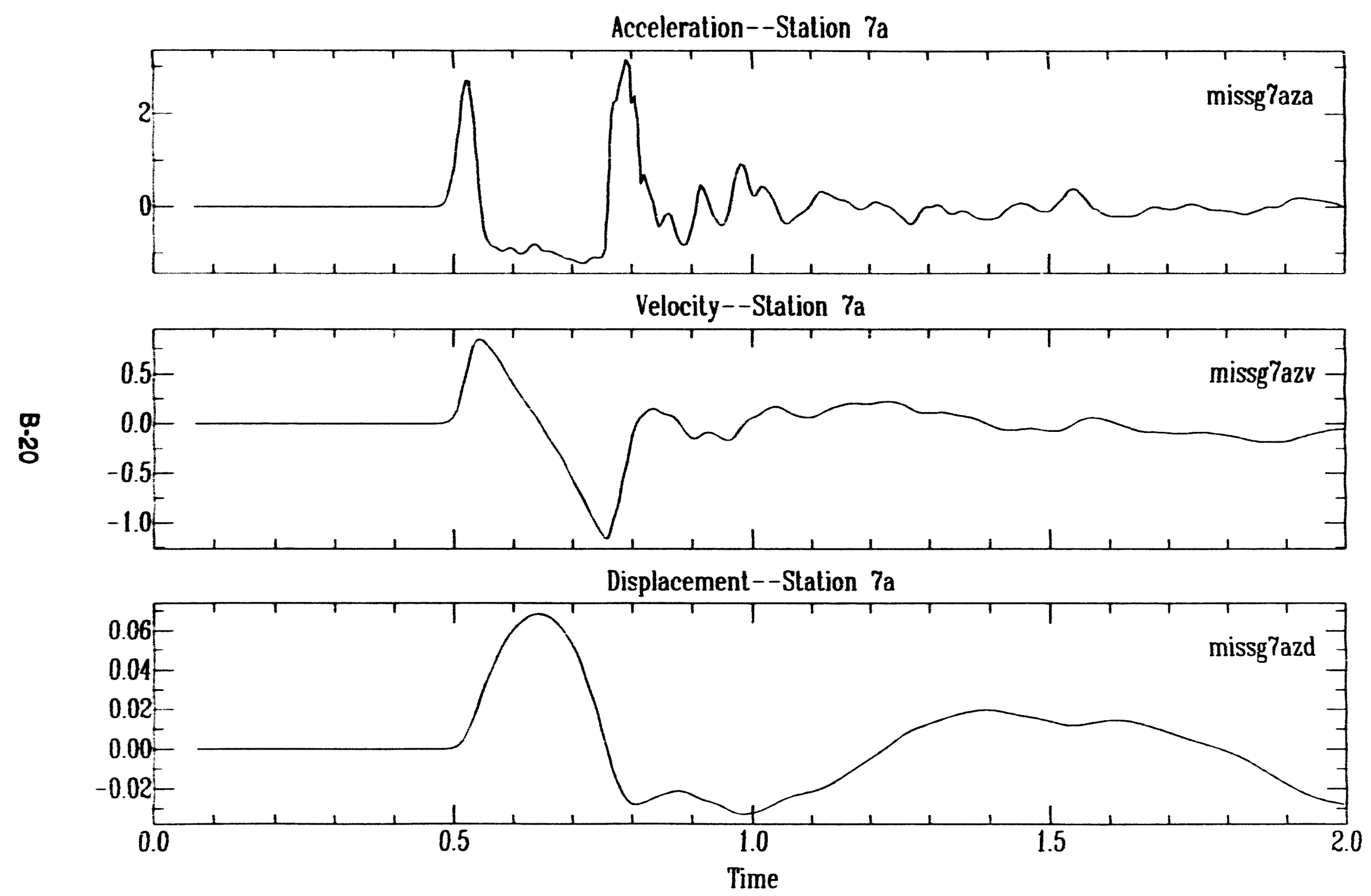


Misty Echo Surface Ground Motion

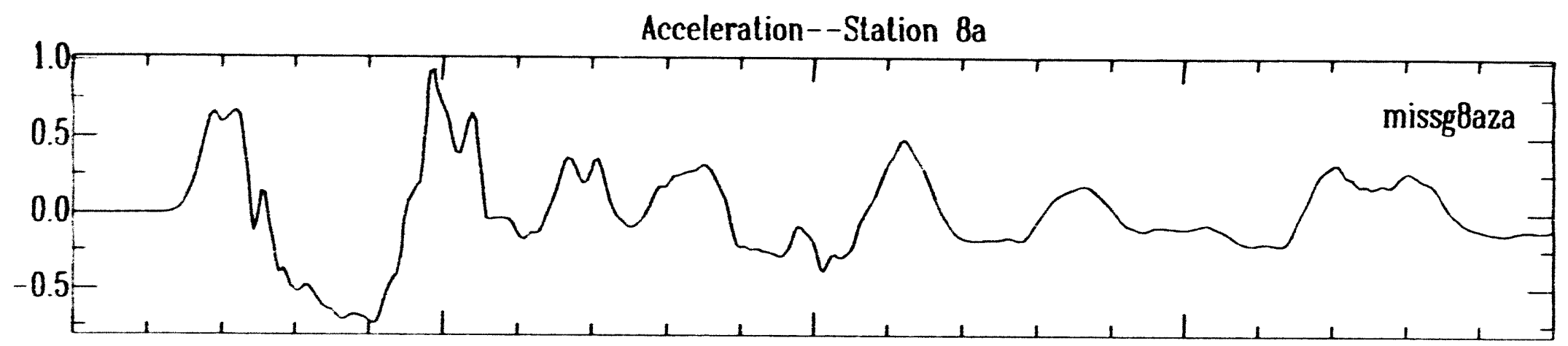

Velocity--Station Ba

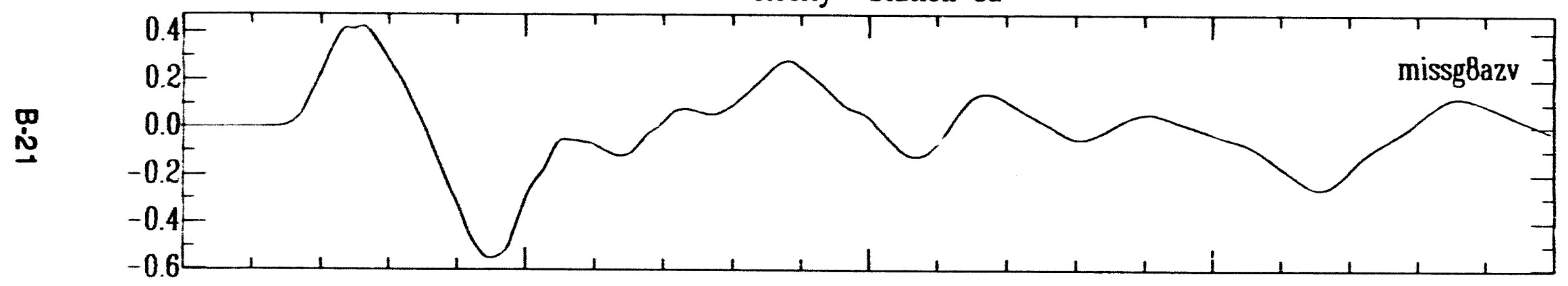

$\frac{3}{\infty}$

Displacement--Station 8a

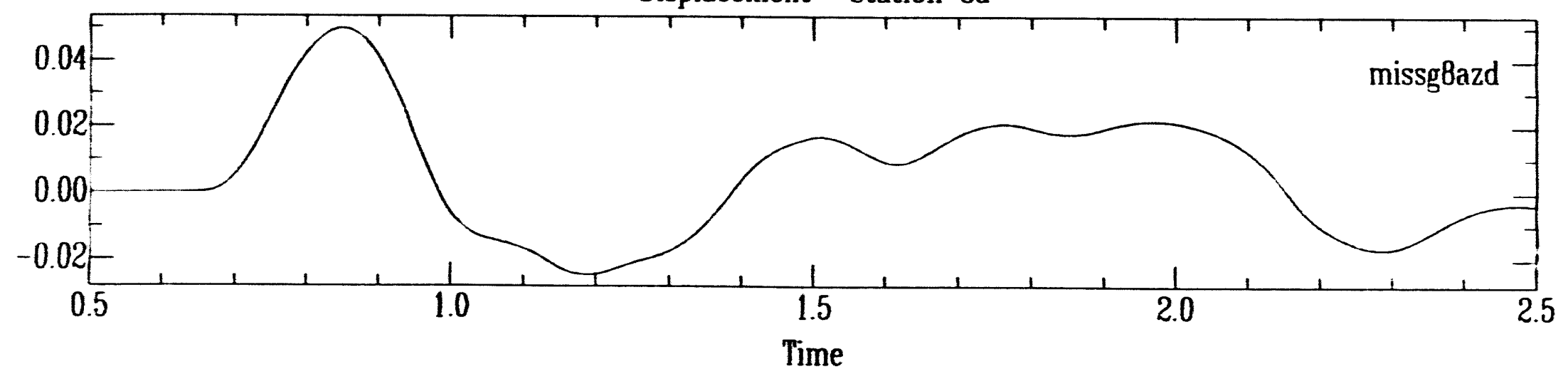




\section{Misty Echo Surface Ground Motion}

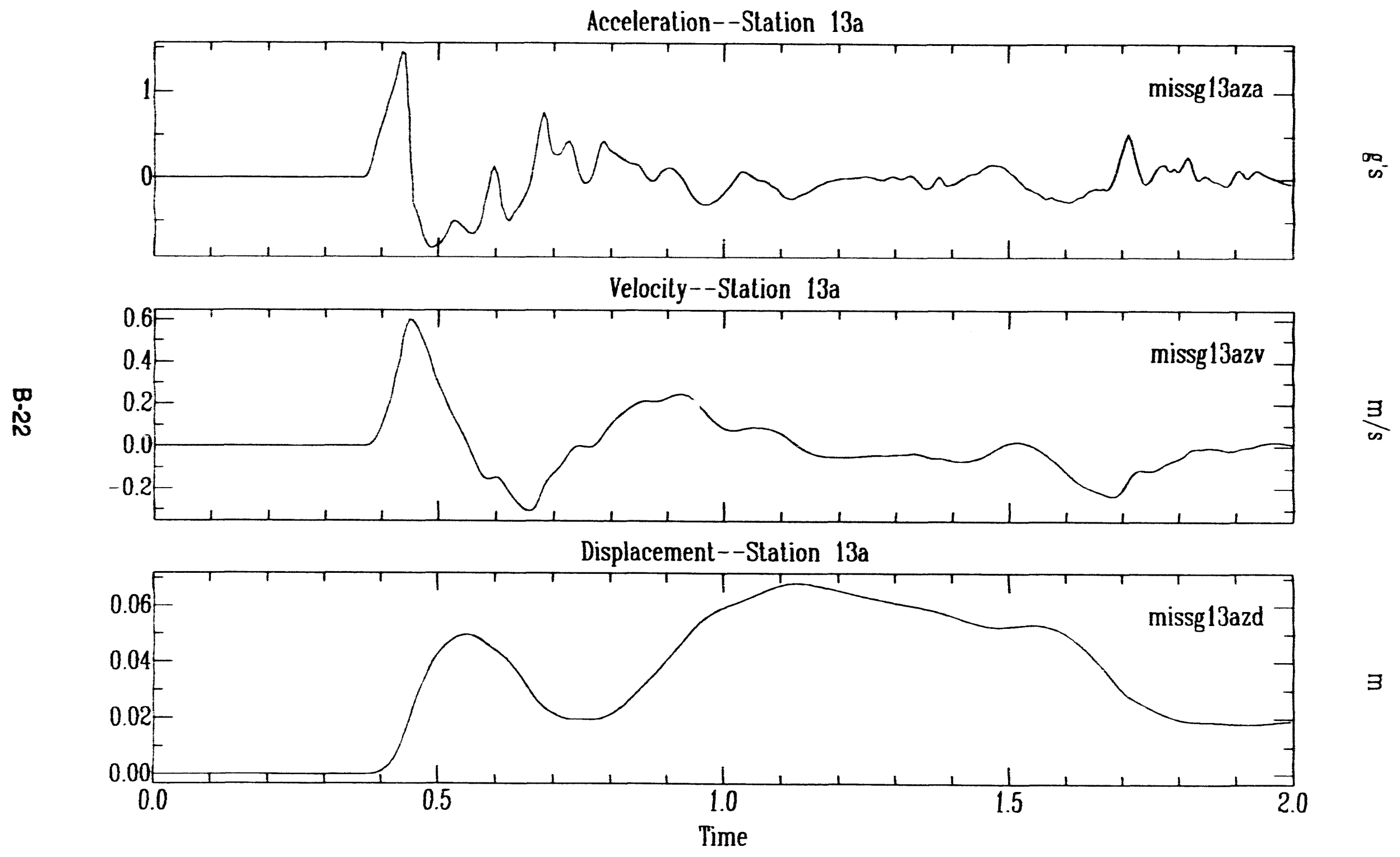




\subsection{Texarkana (U7ca).}

Experimental design. The Texarkana event had a surface array of $10 \mathrm{~J}-8$ stations (including three near SGZ and two at the trailer park) and four GRMPY stations, all of which were within the predicted spall zone (Fig. 3 and Table 2).

Results: The J-8 data were originally reported out in March 1989 (Memo J-8-89-58, R. S. Fitzhugh to F. N. App). All stations worked properly and all recorded spall signals.

Figure 4 (14 pages) contains the station data traces for the accelerometer data.

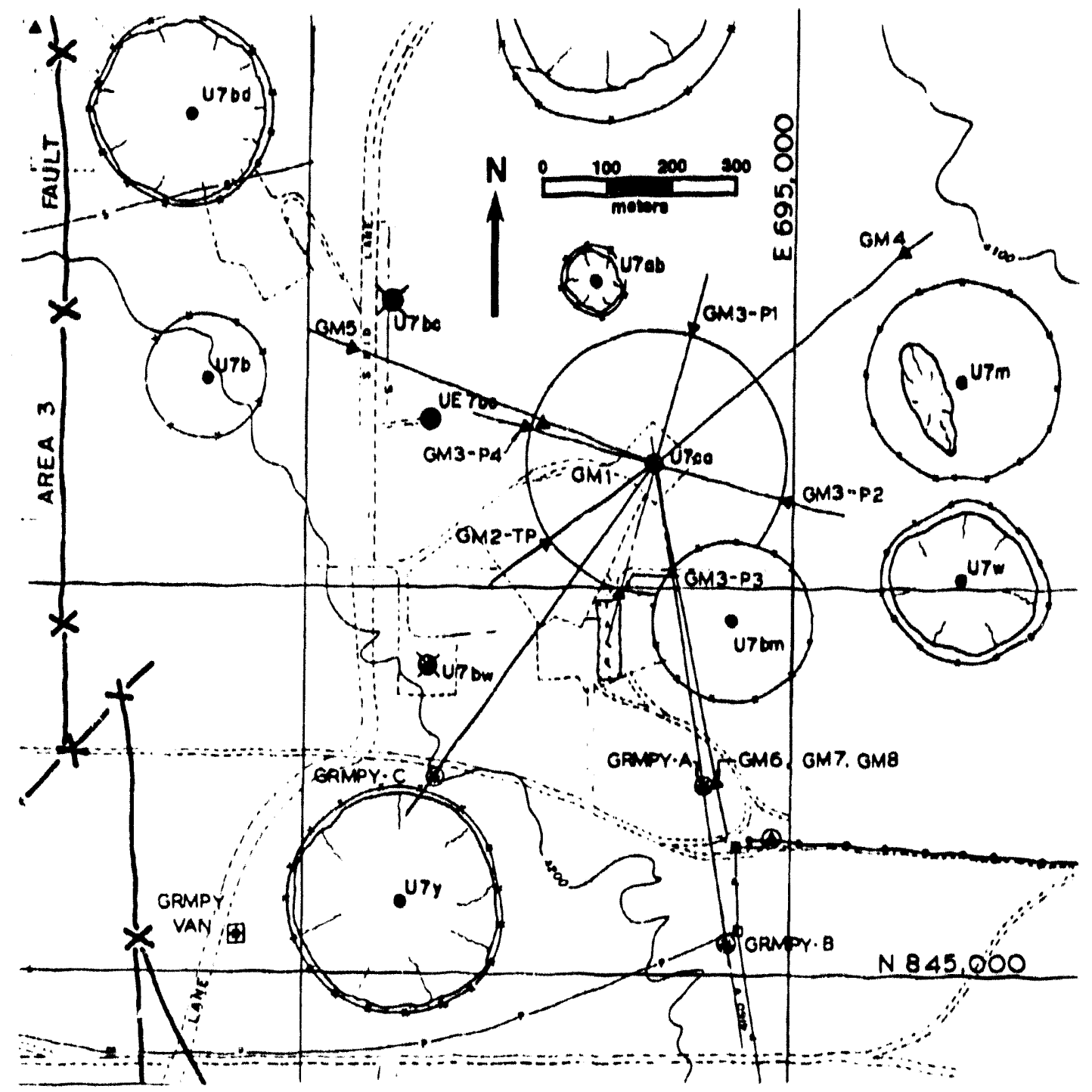

Fig. 3. Ground-motion-measurement array for Texarkana (U7ca). 
Table 2. Texarkana Ground-Motion Station Data

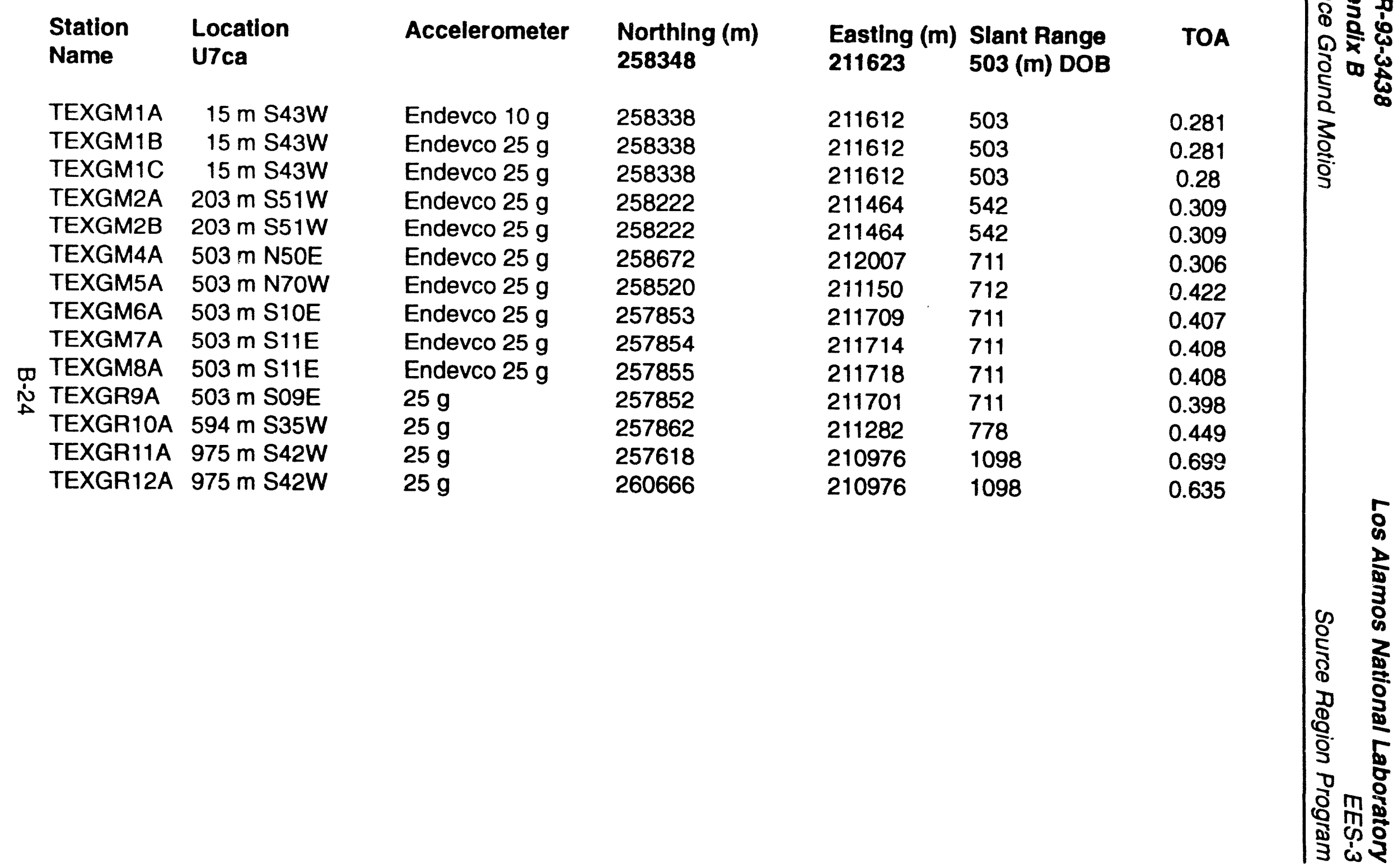




\section{Texarkana Surface Ground Motion}

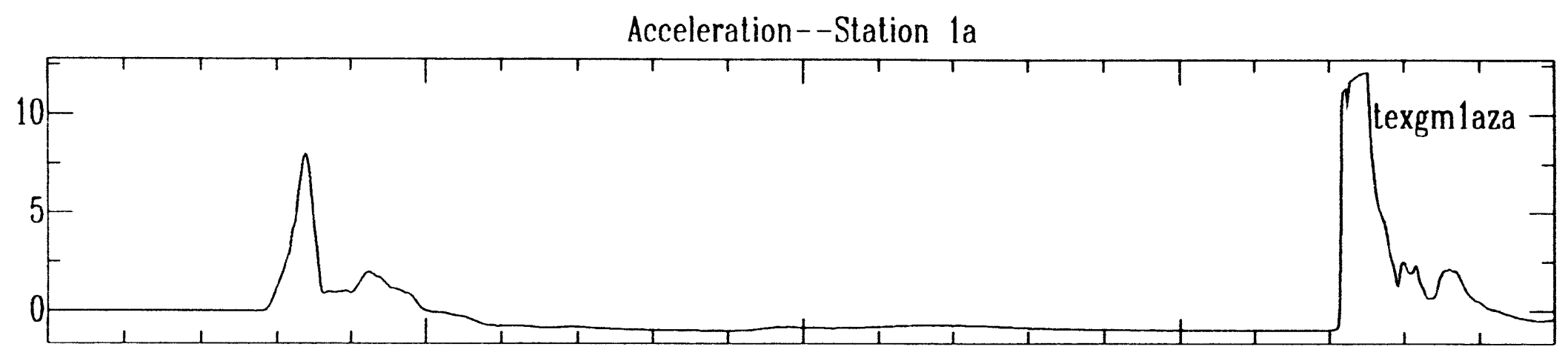

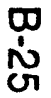

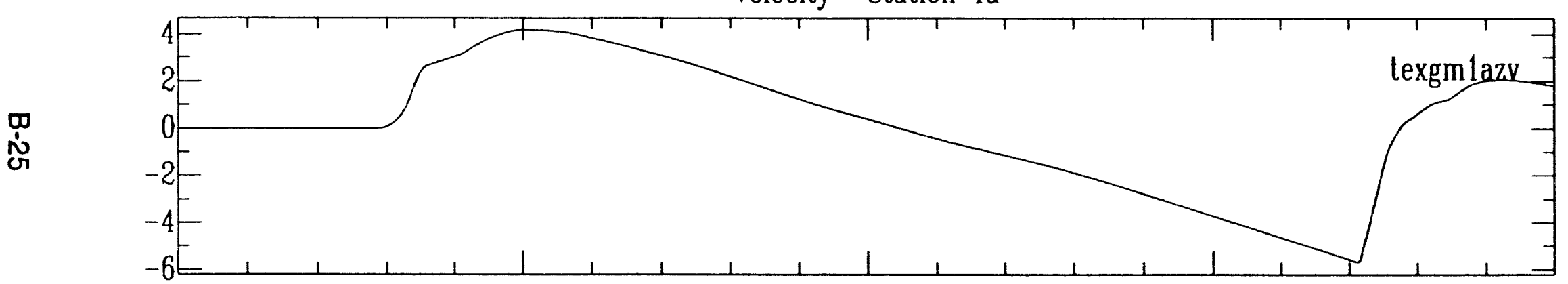

Displacement--Station 1a

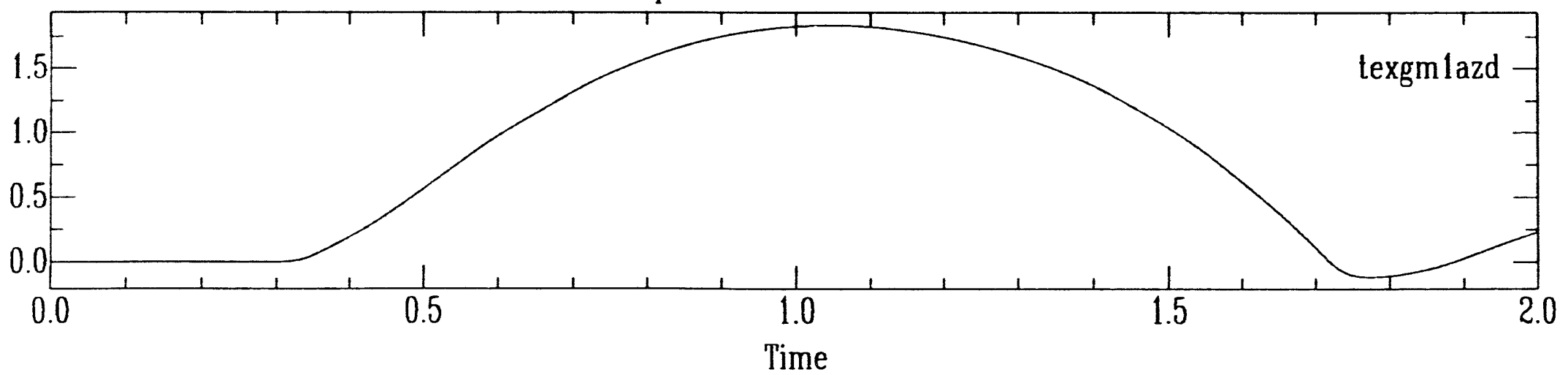




\section{Texarkana Surface Ground Motion}

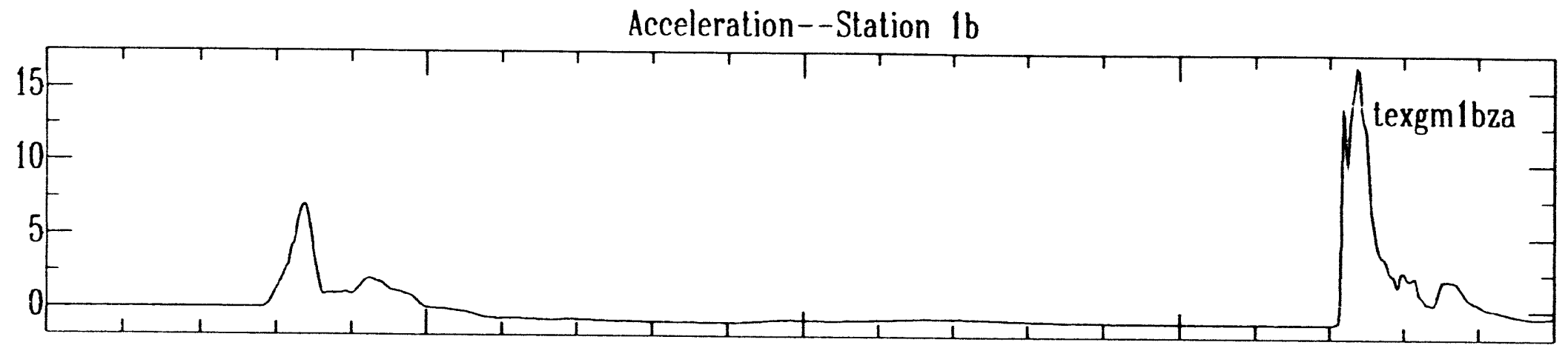

Velocity--Station 1b

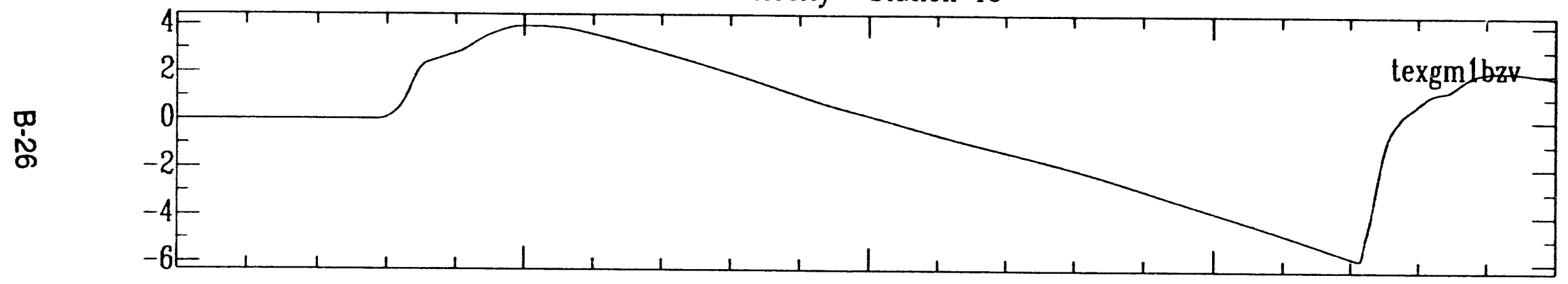

อ

Displacement--Station $1 \mathrm{~b}$

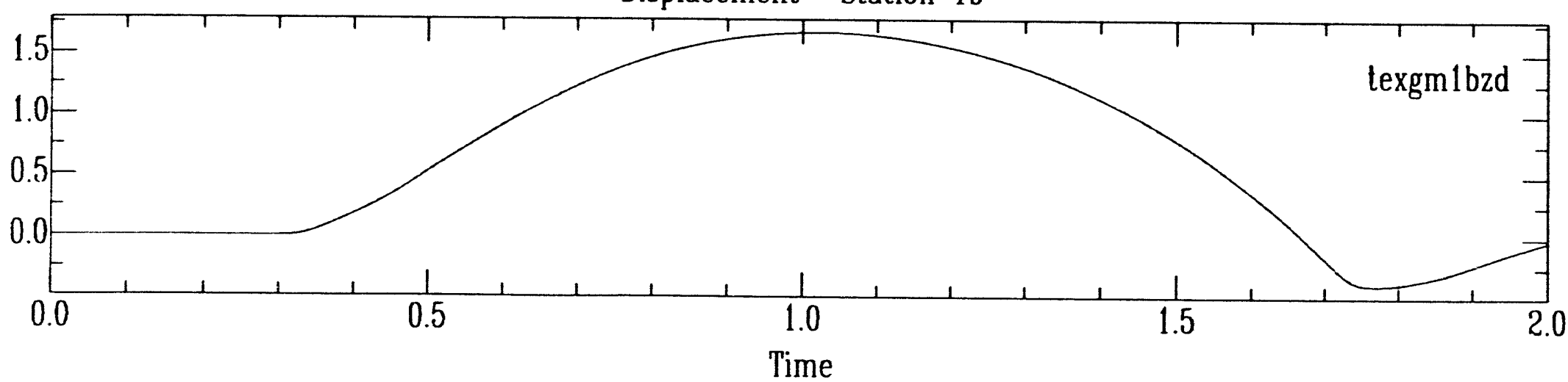




\section{Texarkana Surface Ground Motion}

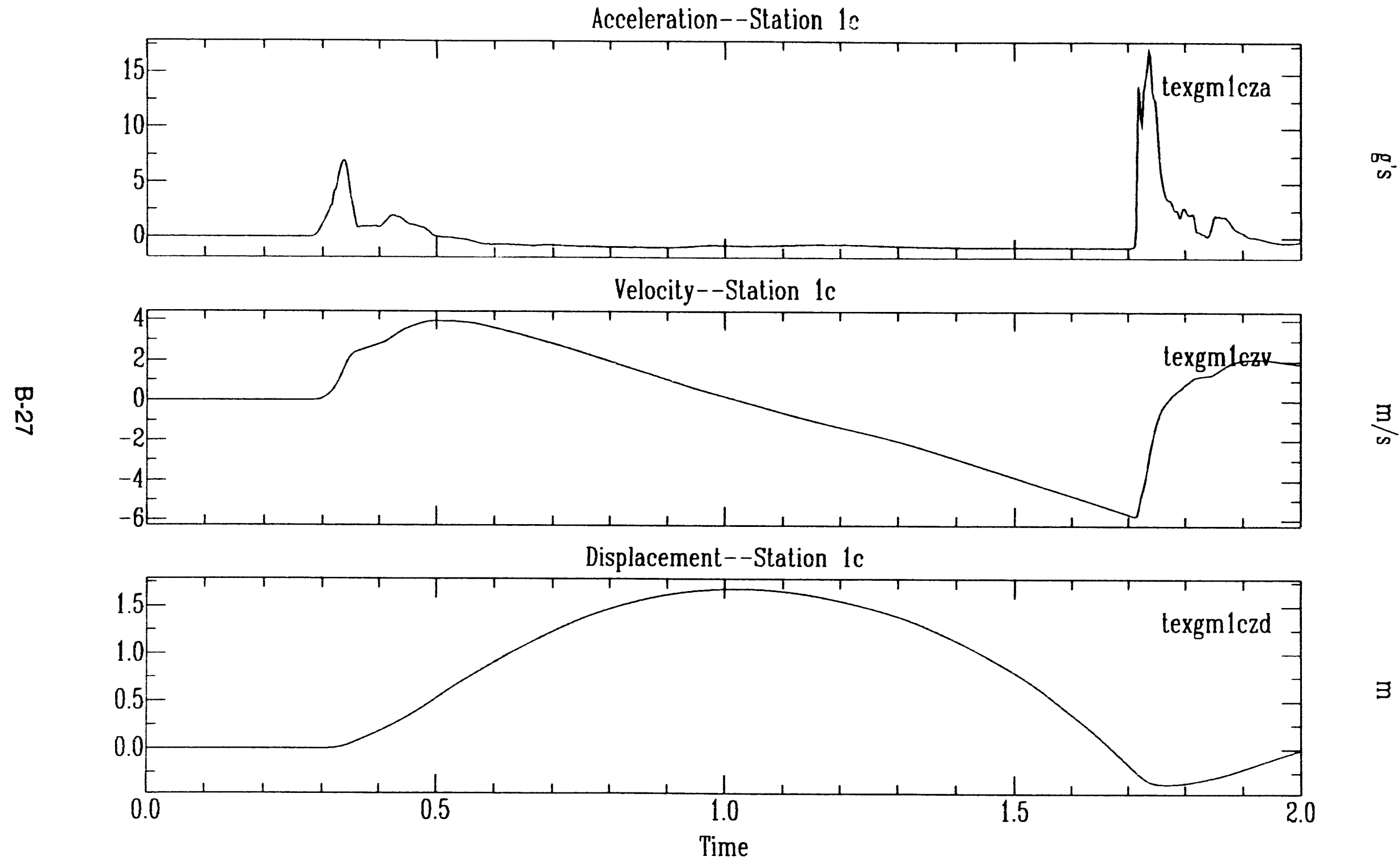




\section{Texarkana Surface Ground Motion}

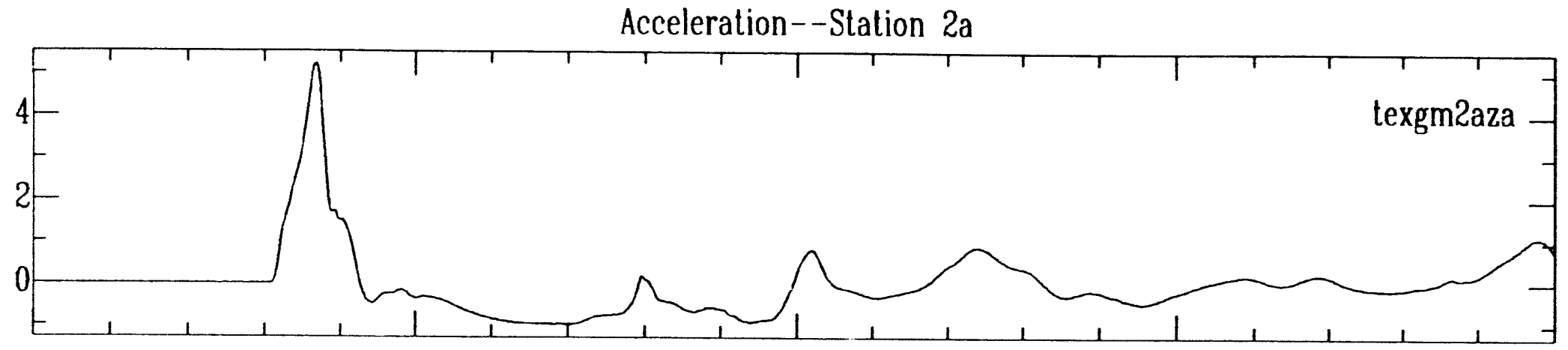

Velocity--Station 2a

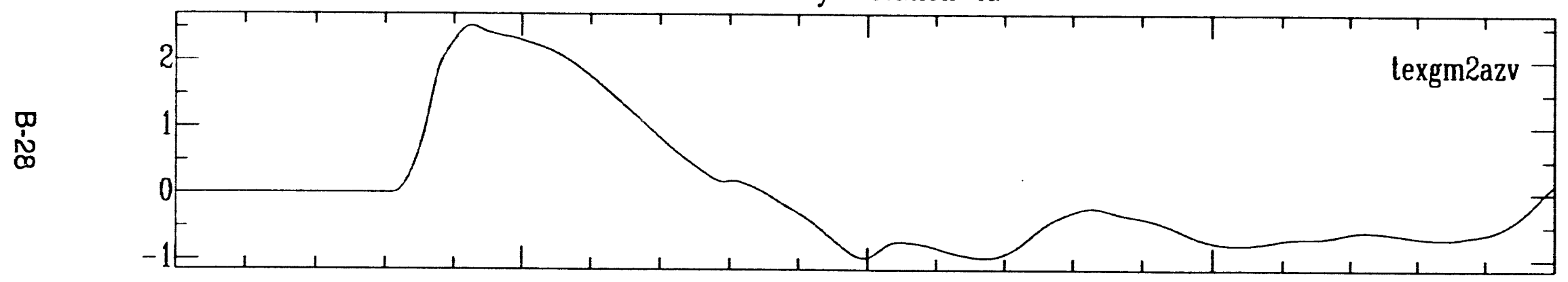

Displacement--Station 2a

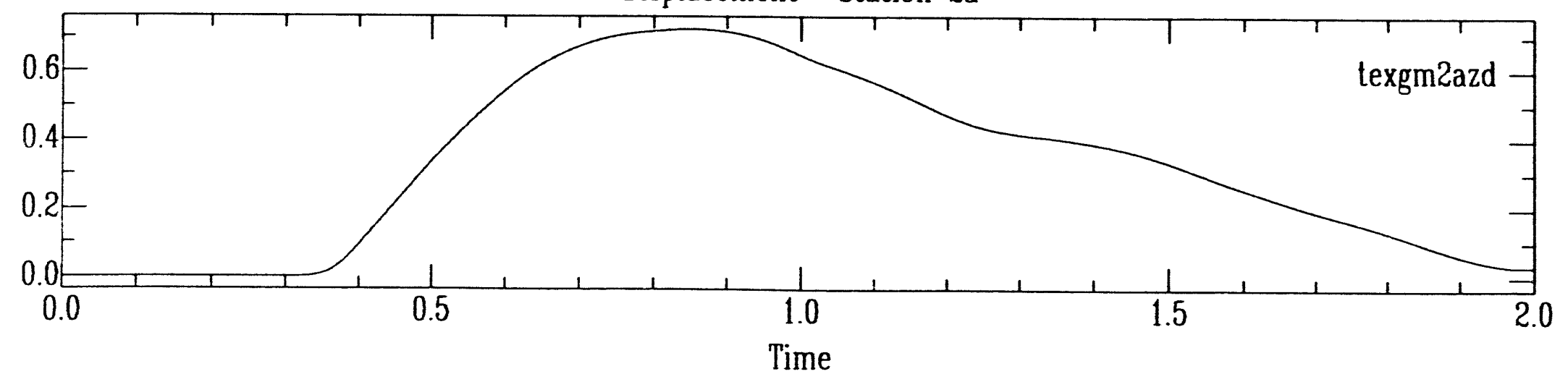




\section{Texarkana Surface Ground Motion}

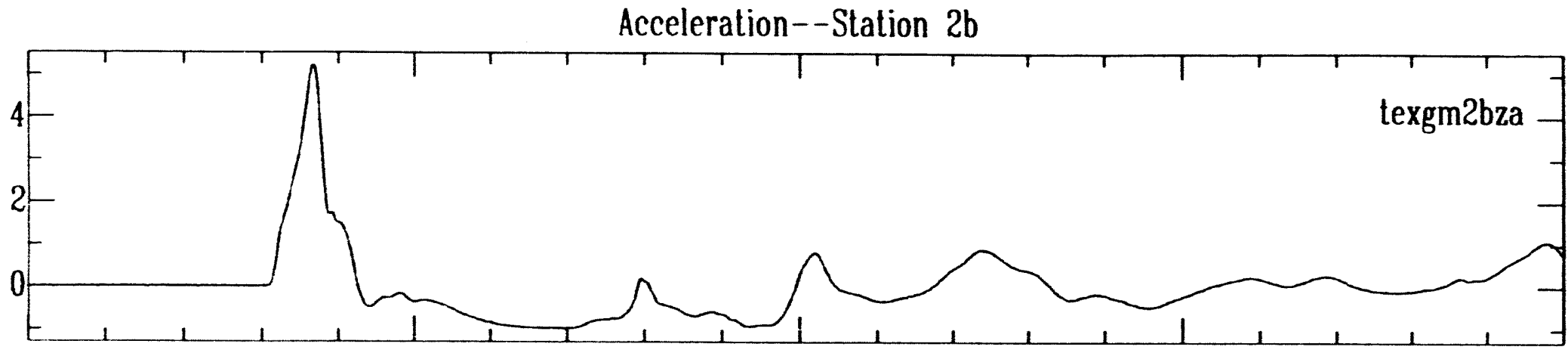

Velocity--Station $\mathbf{2 b}$

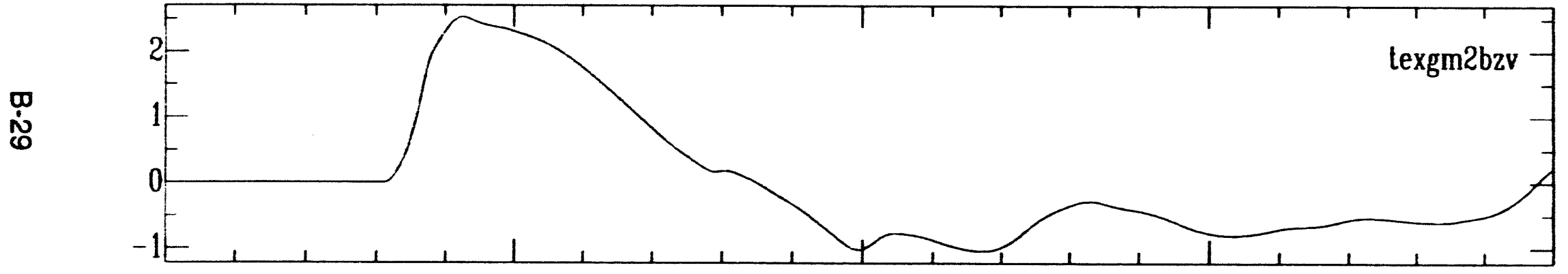

Displacement--Station 2b

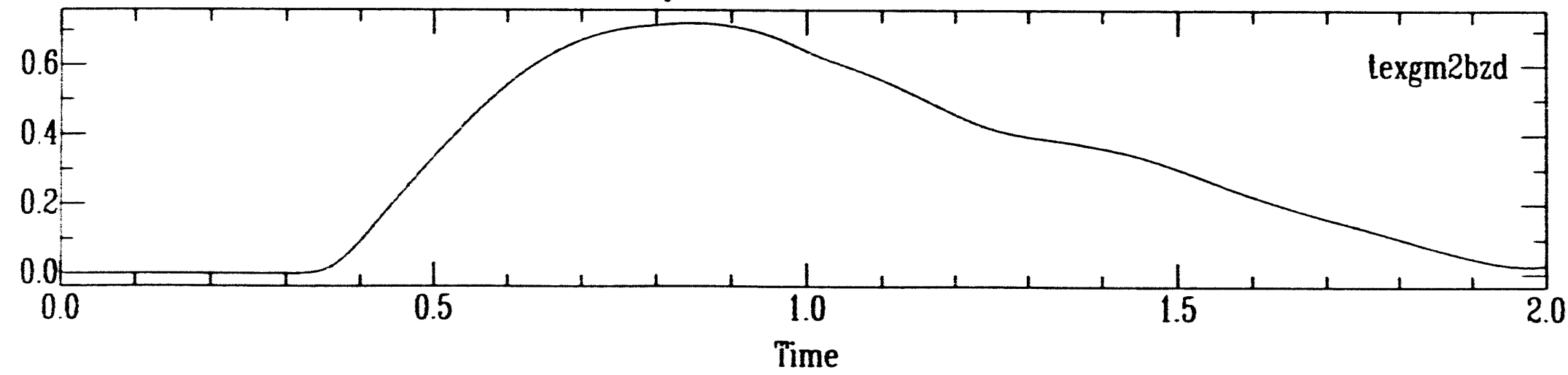



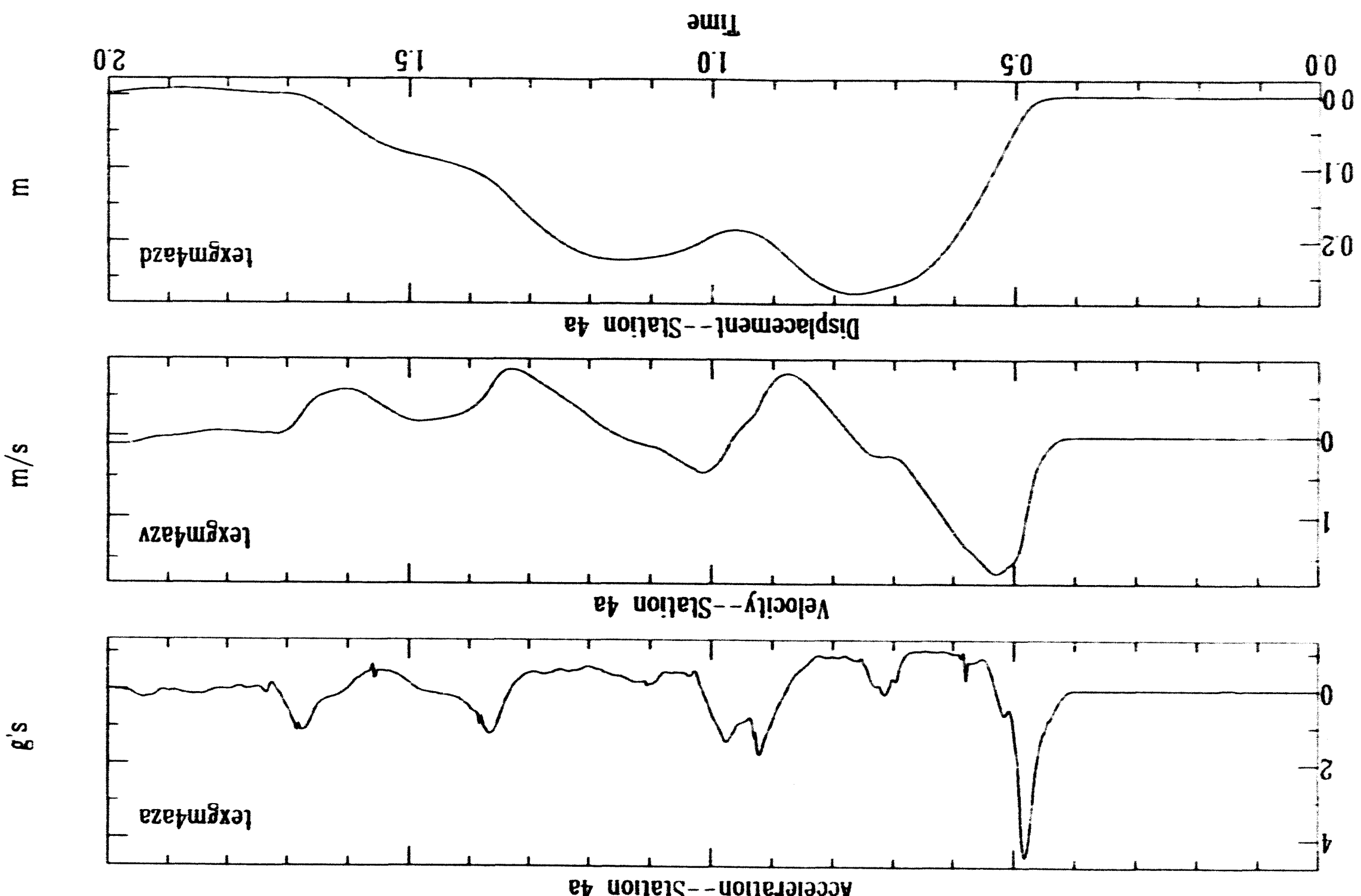

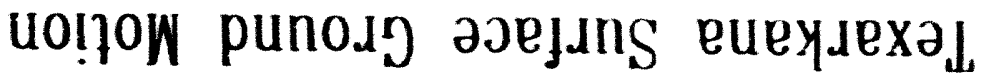




\section{Texarkana Surface Ground Motion}

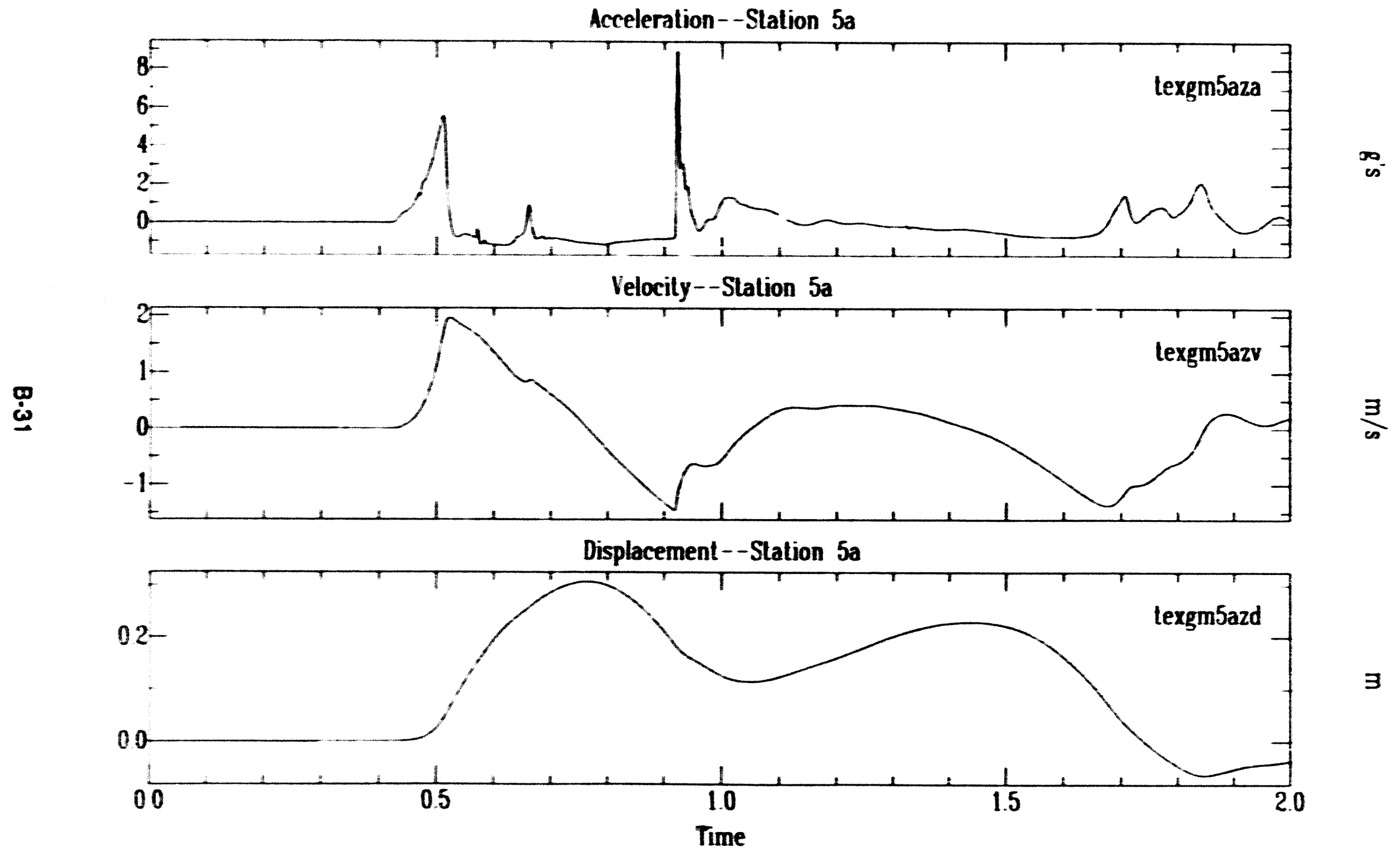




\section{Texarkana Surface Ground Motion}

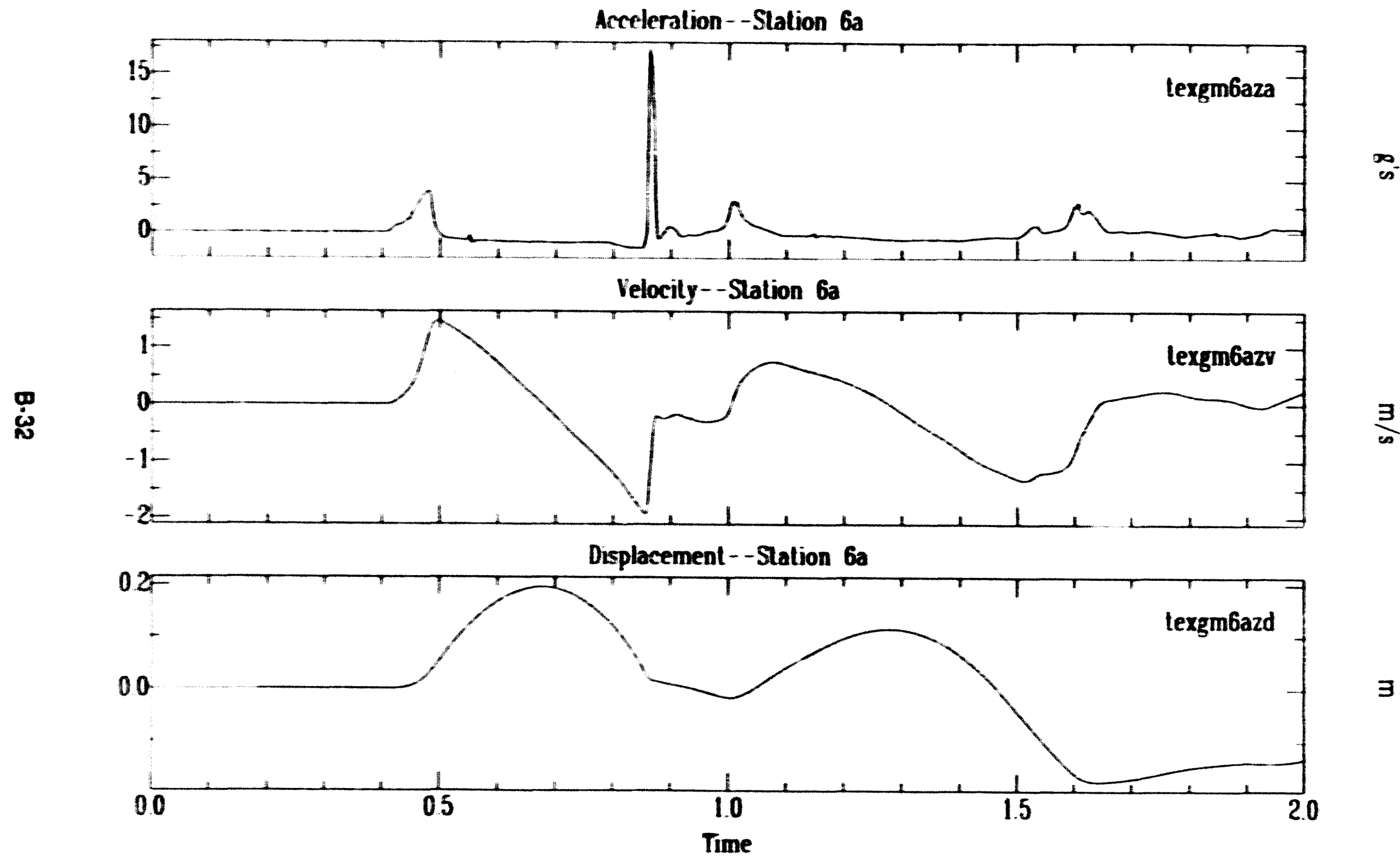




\section{Texarkana Surface Ground Motion}

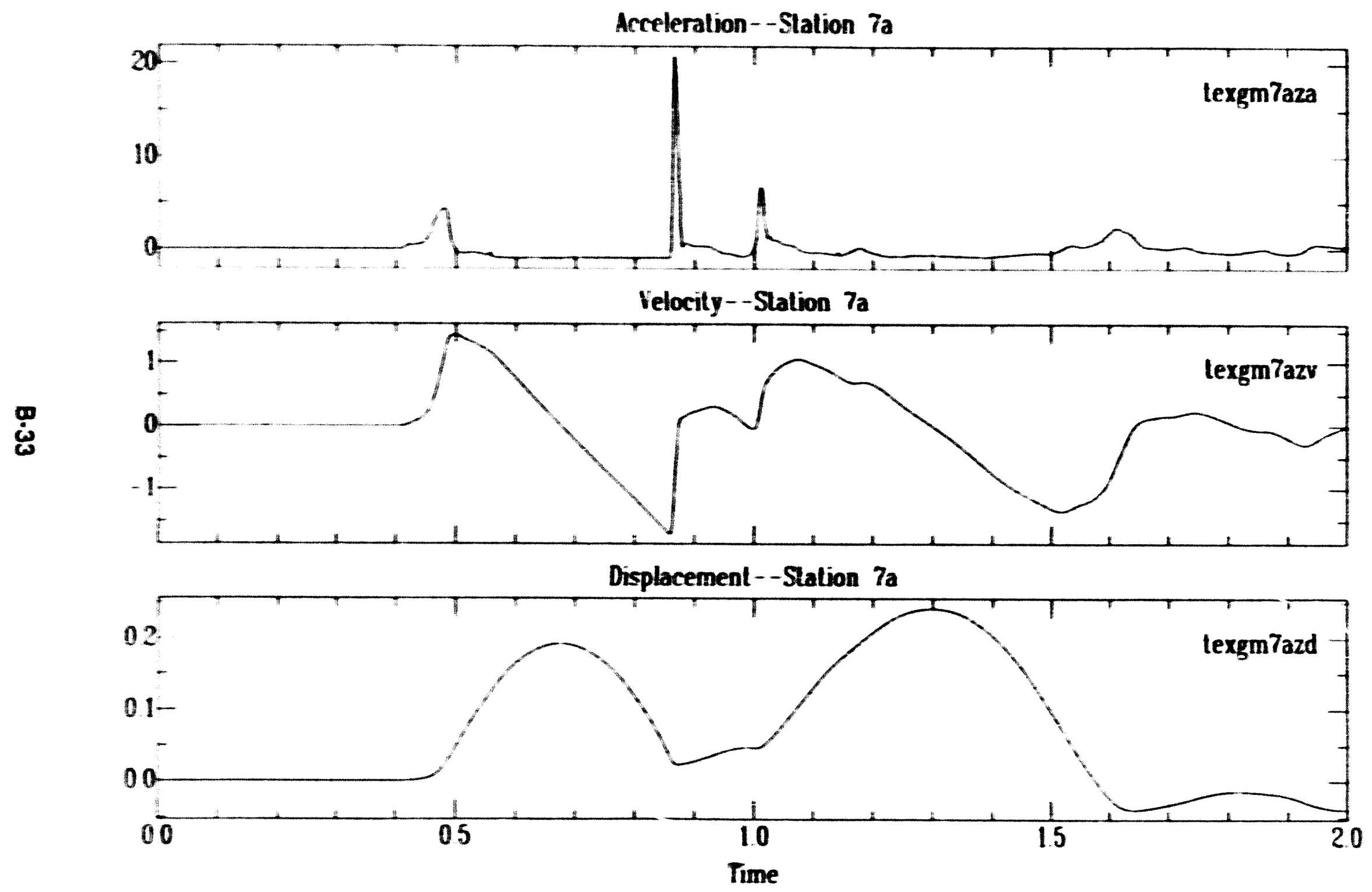




\section{Texarkana Surface Ground Motion}
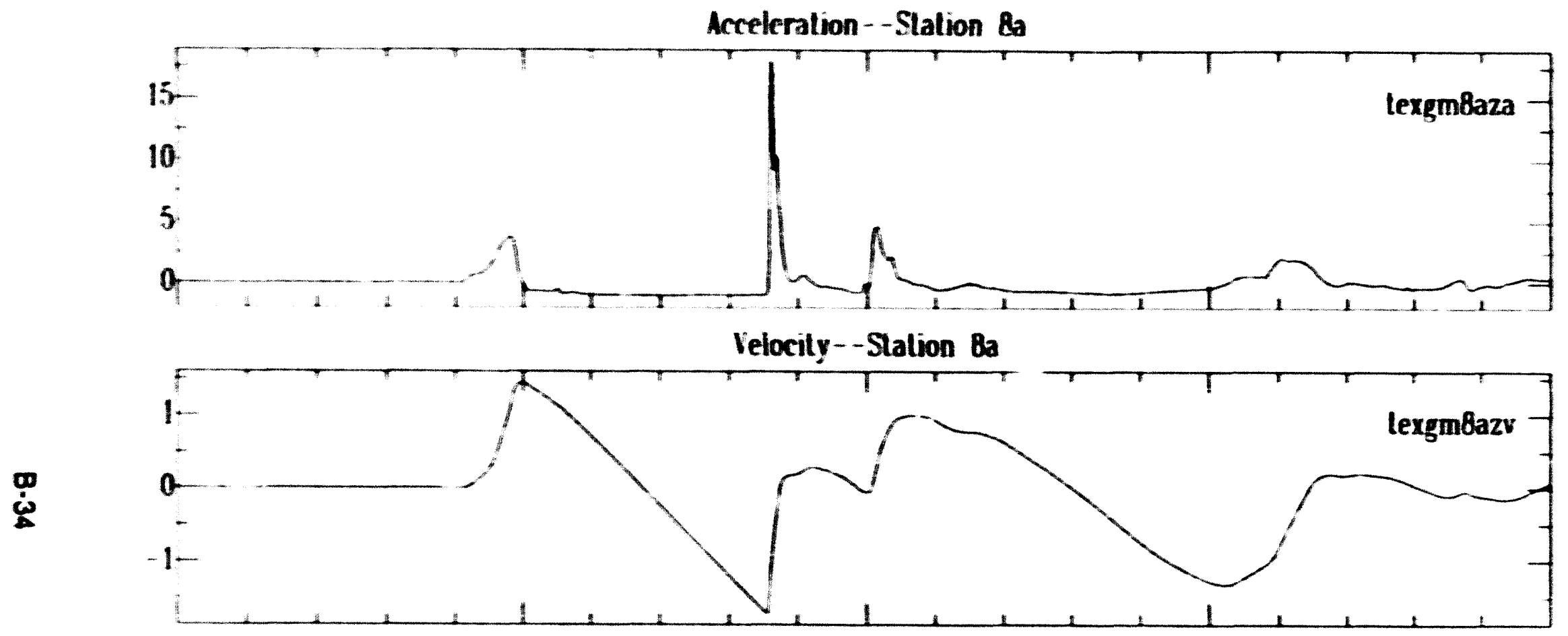

$\sum_{-\infty}^{3}$

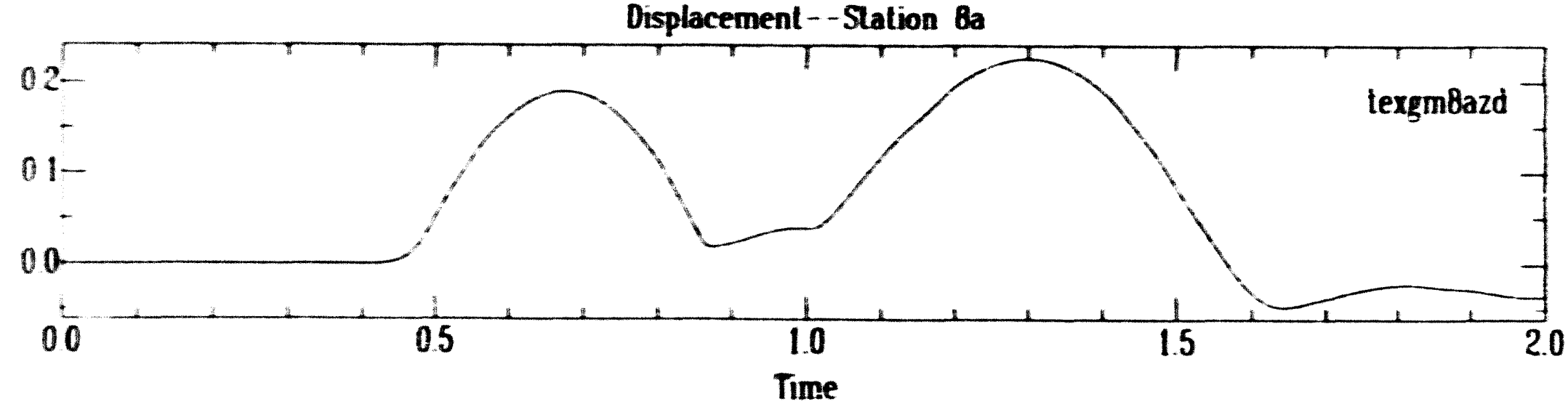



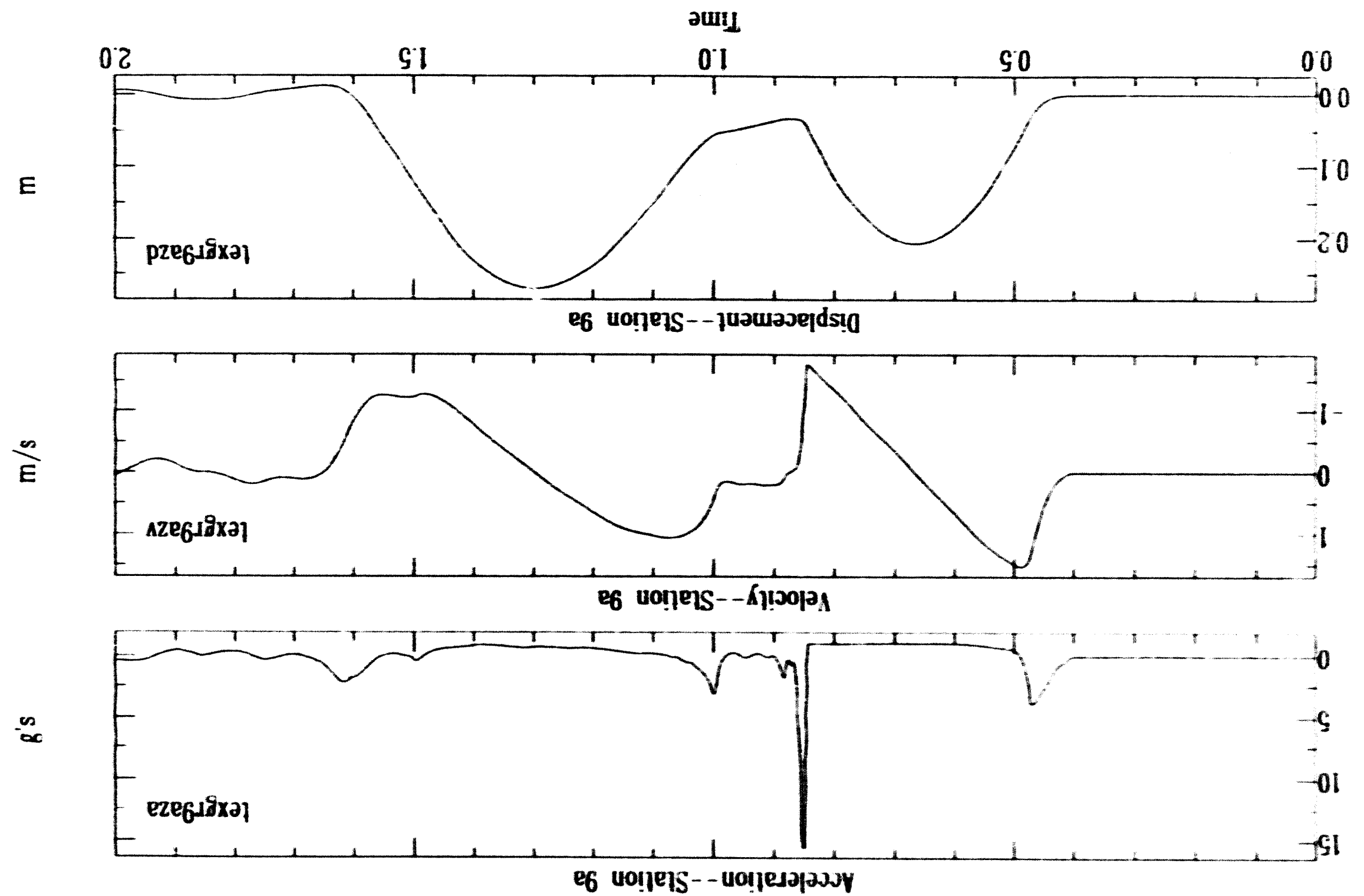

นо!̣on punodg әоеjans eueydexəd 


\section{Texarkana Surface Ground Motion}

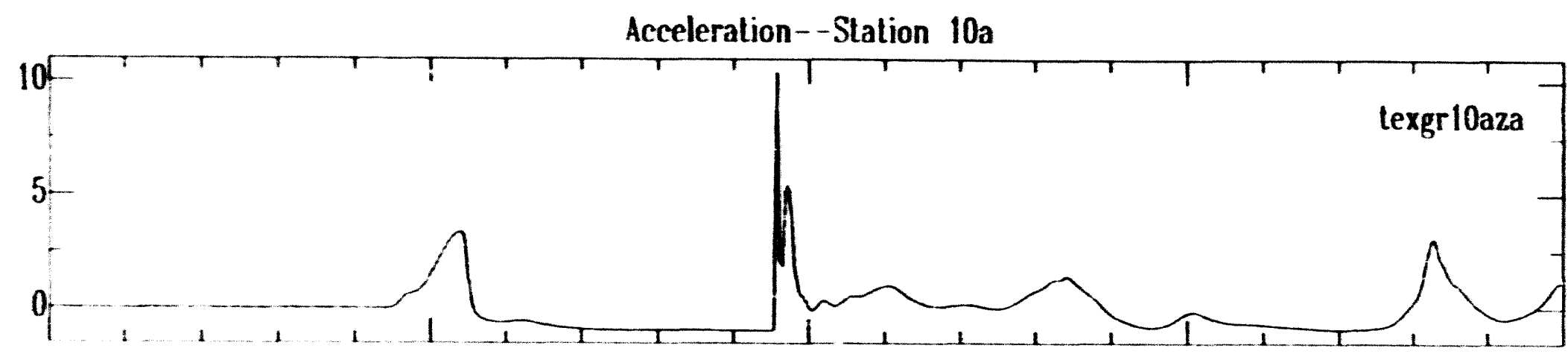

$\infty$

Velocily--Station 10a

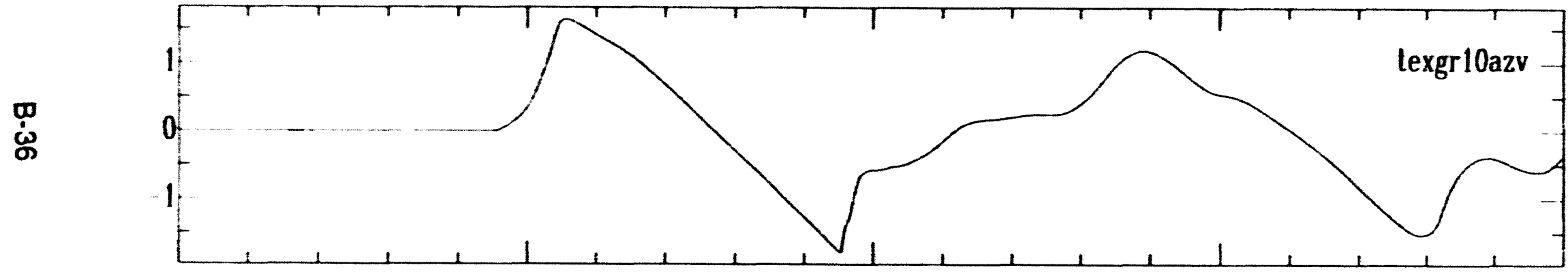

콩

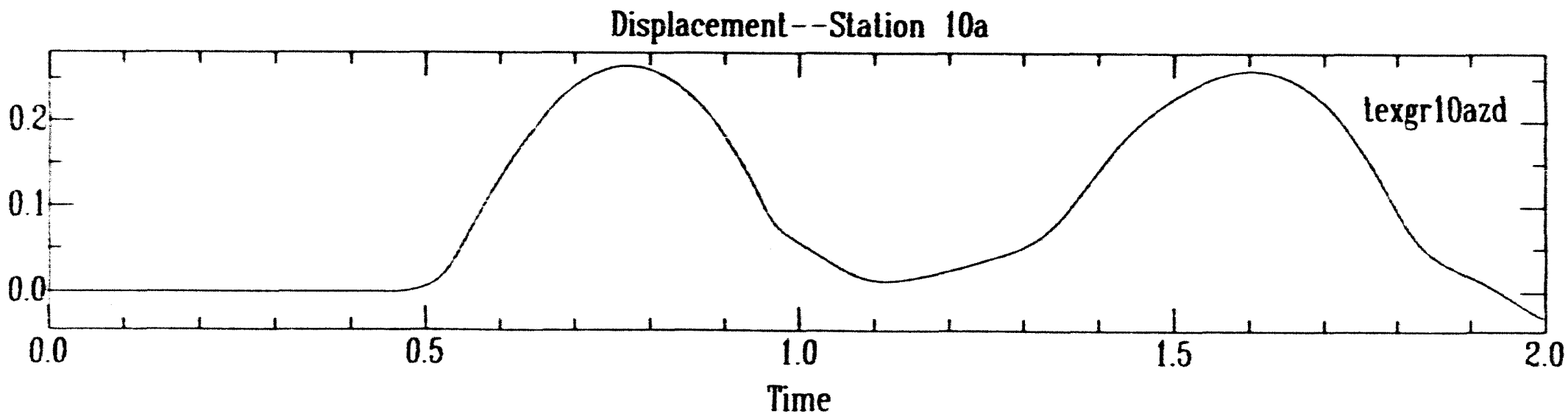



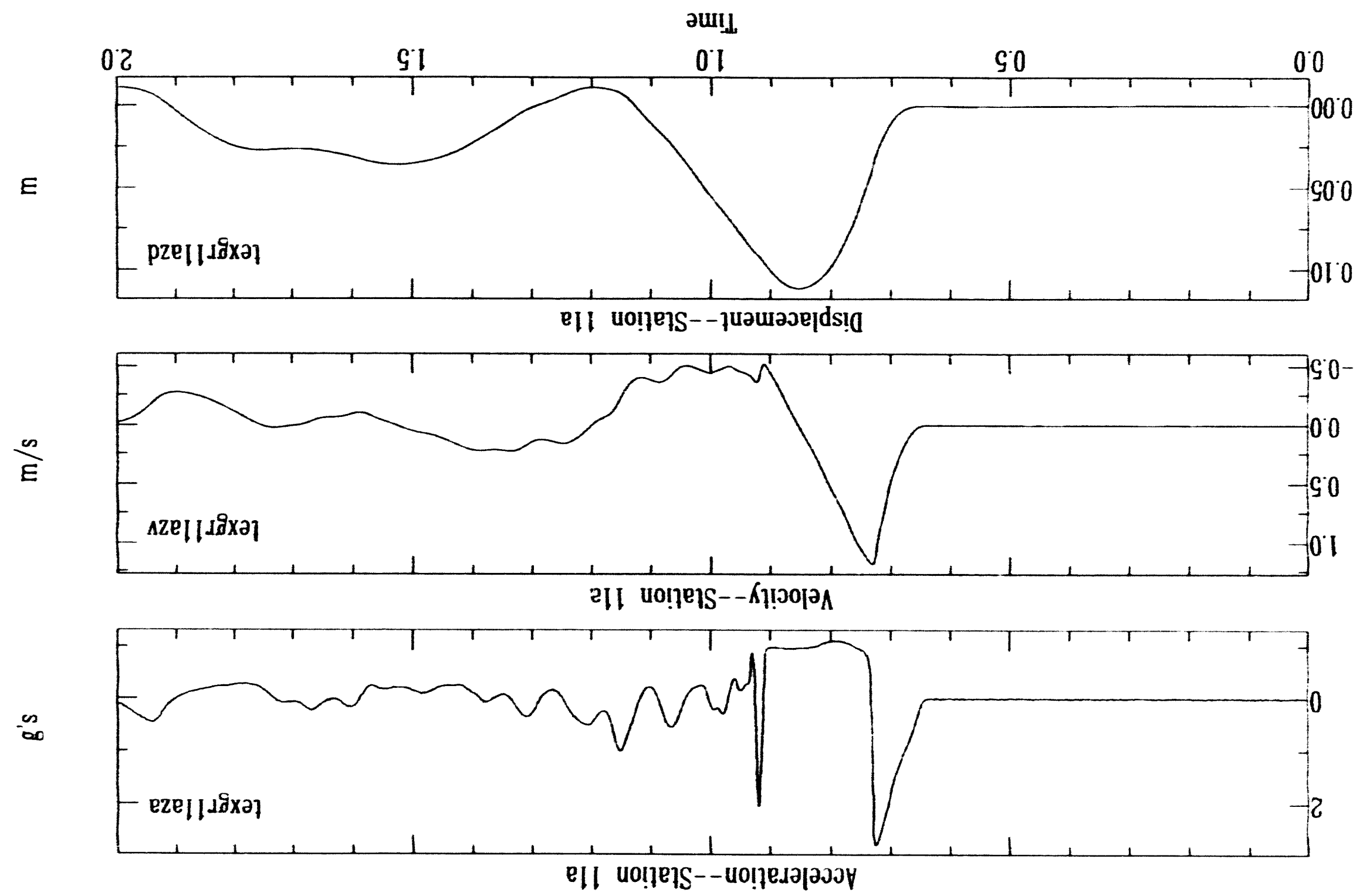

uo!now punods วоejuns eueysexad 


\section{Texarkana Surface Ground Motion}

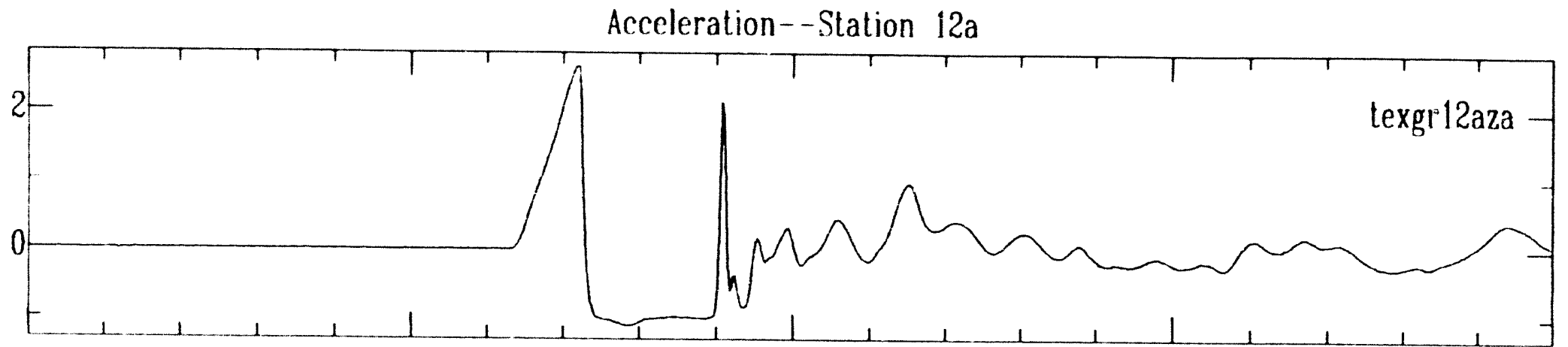

Velocity--Station 12a

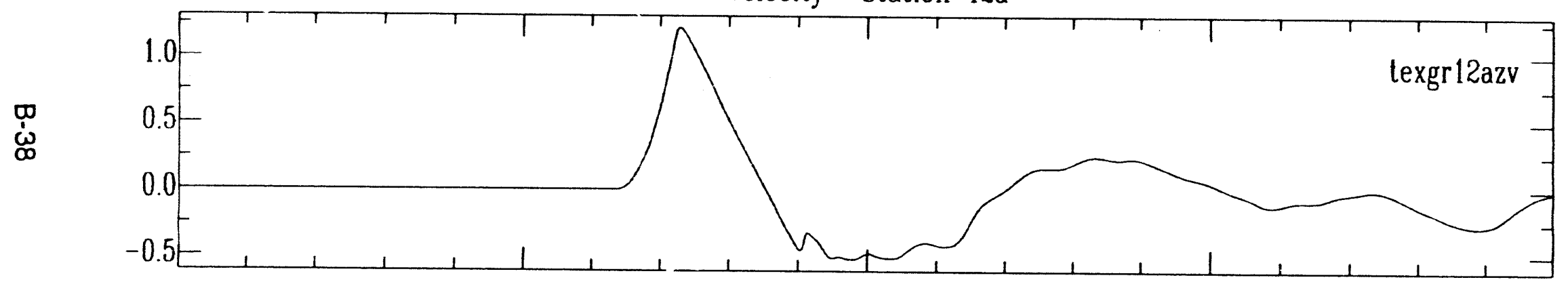

Displacement--Station $12 \mathrm{a}$

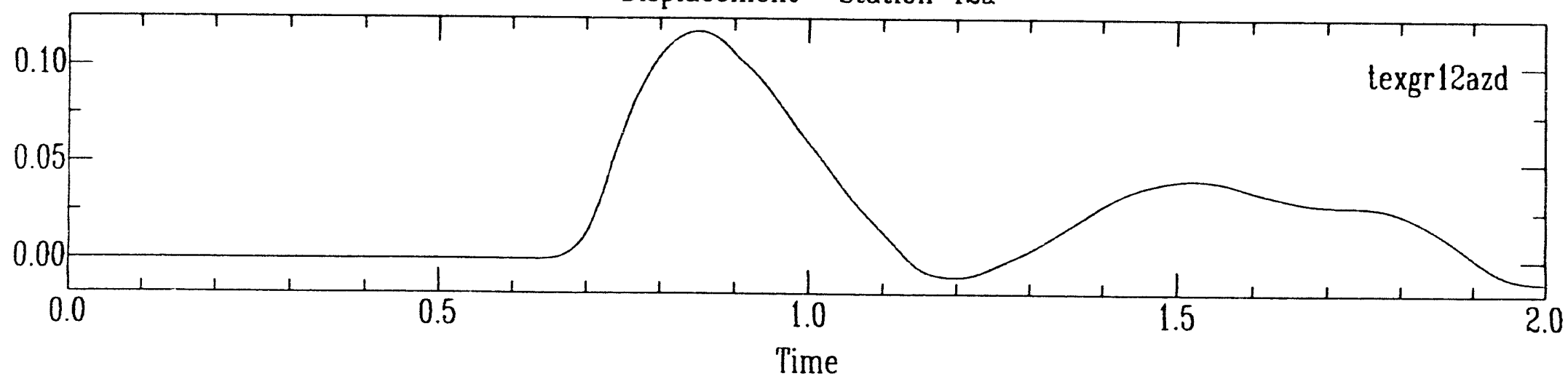




\subsection{Tulia (U4s)}

Experiment design. The only ground-motion measurements on Tulia were from the nine-station J-8 accelerometer array for containment, including the three stations near SGZ (Fig. 5 and Table 3).

Results. The J-8 data were originally reported out in June 1989 (Memo J-8-89-142, R. S. Fitzhugh to F. N. App). All stations functioned properly, and spall was recorded on stations $1 A, B$ and $C$, and $2 A$ and $B$. All other stations recorded signals that did not contain spall characteristics. Figure 6 ( 9 pages) contains the station data traces for the accelerometer data.

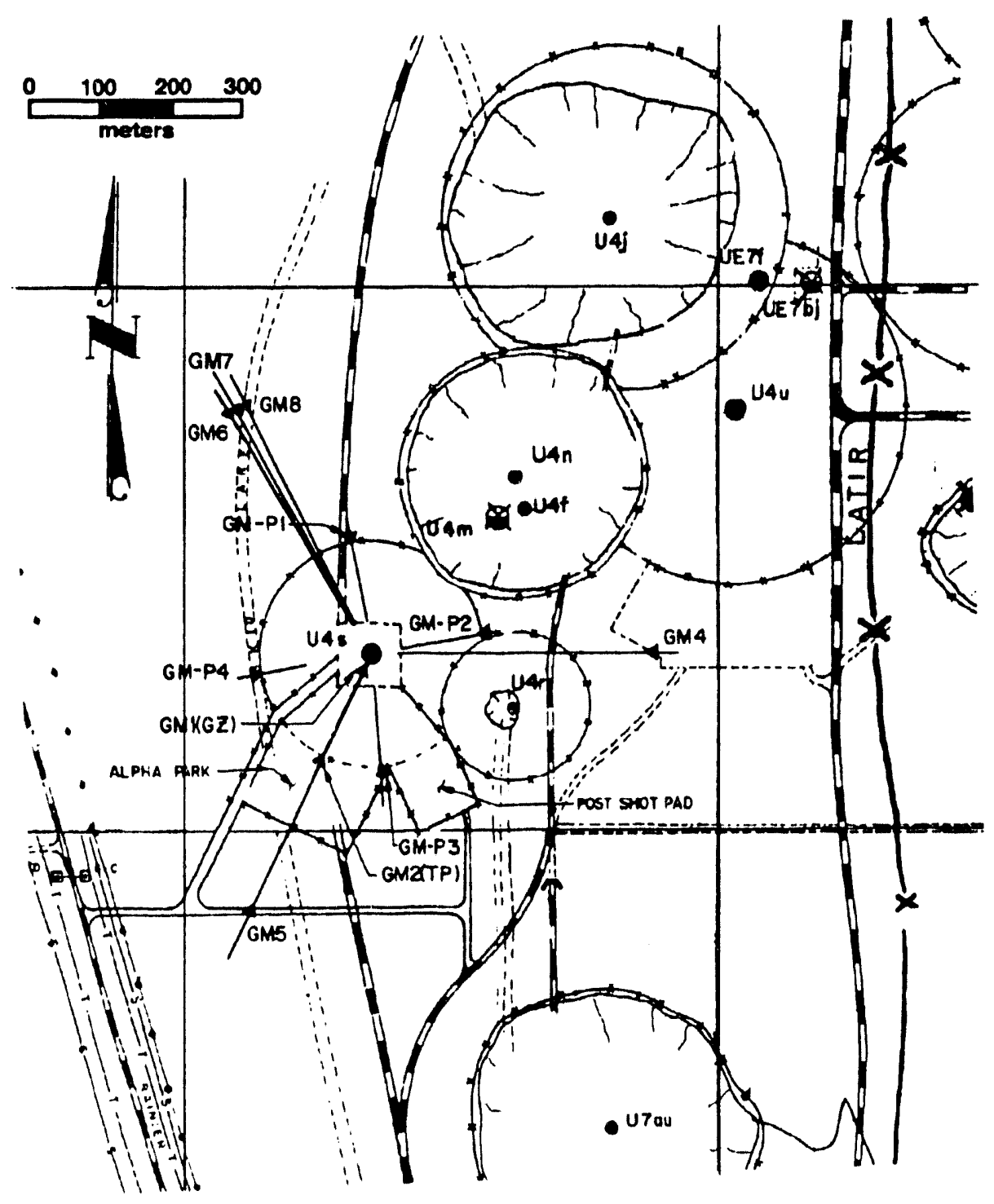

Fig. 5. Ground-motion-measurement array for Tulia. 
Table 3. Tulla Ground-Motion Station Data

\begin{tabular}{|c|c|c|c|c|c|c|}
\hline $\begin{array}{l}\text { Station } \\
\text { Name }\end{array}$ & $\begin{array}{l}\text { Station Loc } \\
\text { U4s }\end{array}$ & Accelerometer & $\begin{array}{l}\text { Northing (m) } \\
259324\end{array}$ & $\begin{array}{l}\text { Easting (m) } \\
206769\end{array}$ & $\begin{array}{l}\text { Slant Range } \\
396(\mathrm{~m}) \text { DOB }\end{array}$ & TOA \\
\hline TULGM1A & $15 \mathrm{~m} \mathrm{~S} 22 \mathrm{~W}$ & Endevco $10 \mathrm{~g}$ & 259310 & 206763 & 396 & 0.258 \\
\hline TULGM1B & $15 \mathrm{~m} \mathrm{~S} 22 \mathrm{~W}$ & Endevco $25 \mathrm{~g}$ & 259310 & 206763 & 396 & 0.259 \\
\hline TULGM1C & 15 m S22W & Endevco $25 \mathrm{~g}$ & 259310 & 206763 & 396 & 0.258 \\
\hline TULGM2A & $160 \mathrm{~m} \mathrm{~S} 23 \mathrm{~W}$ & Endevco $25 \mathrm{~g}$ & 259177 & 206707 & 427 & 0.28 \\
\hline TULGM2B & $160 \mathrm{~m} \mathrm{~S} 23 \mathrm{~W}$ & Endevco $25 \mathrm{~g}$ & 259177 & 206707 & 427 & 0.28 \\
\hline TULGM4A & $396 \mathrm{~m} \mathrm{E}$ & Endevco $10 \mathrm{~g}$ & 259324 & 207165 & 560 & 0.365 \\
\hline TULGM5A & $396 \mathrm{~m} \mathrm{~S} 23 W$ & Endevco $10 \mathrm{~g}$ & 258959 & 206615 & 560 & 0.353 \\
\hline TULGM6A & $396 \mathrm{~m} \mathrm{~N} 30 \mathrm{~W}$ & Endevco $10 \mathrm{~g}$ & 259667 & 206571 & 560 & 0.365 \\
\hline TULGM7A & 396 m N30W & Endevco $10 \mathrm{~g}$ & 259669 & 206574 & 560 & \\
\hline
\end{tabular}




\section{Tulia Surface Ground Motion}

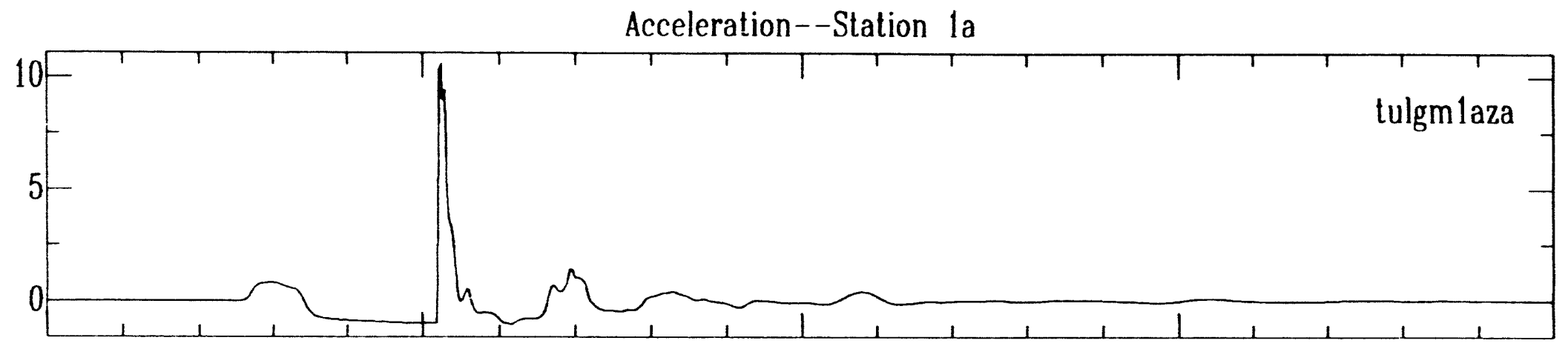

Velocity--Station la

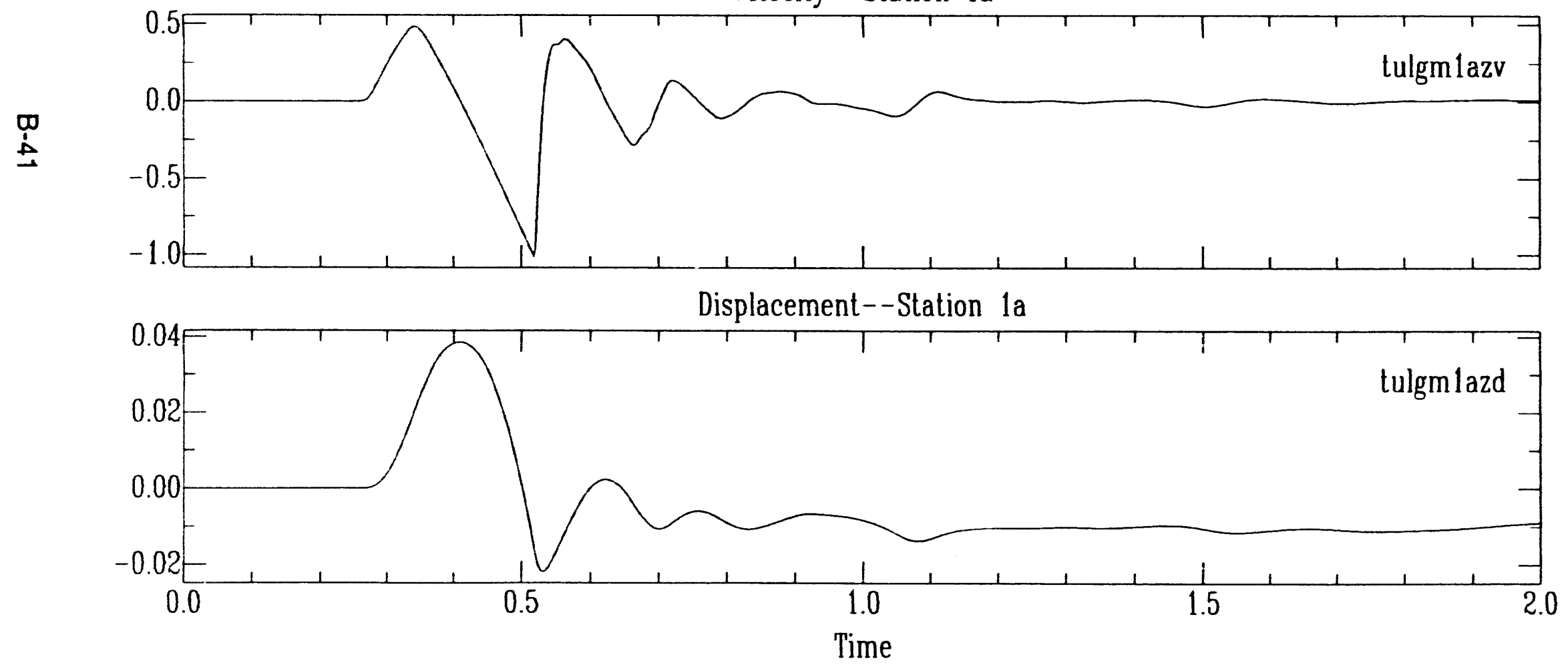




\section{Tulia Surface Ground Motion}

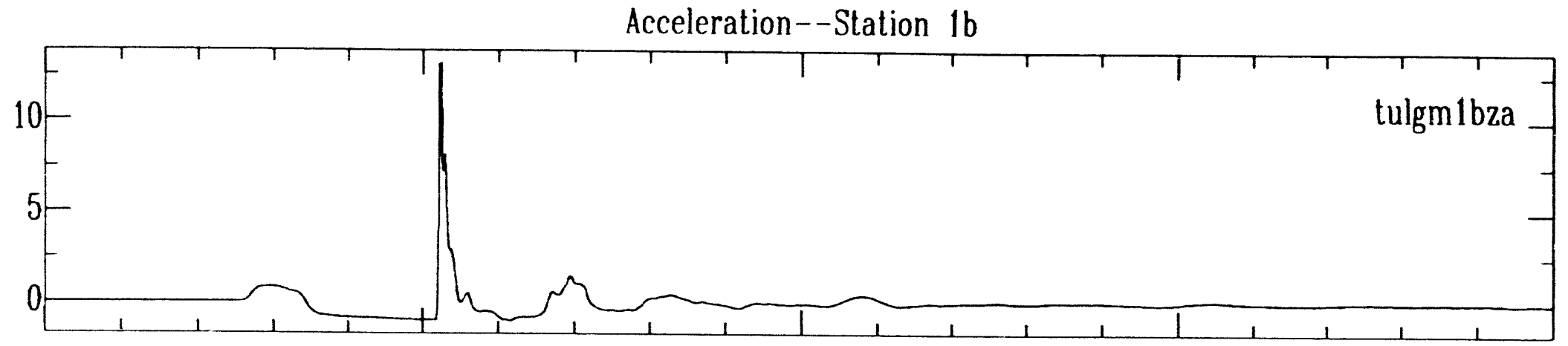

Velocity--Station $1 \mathrm{~b}$

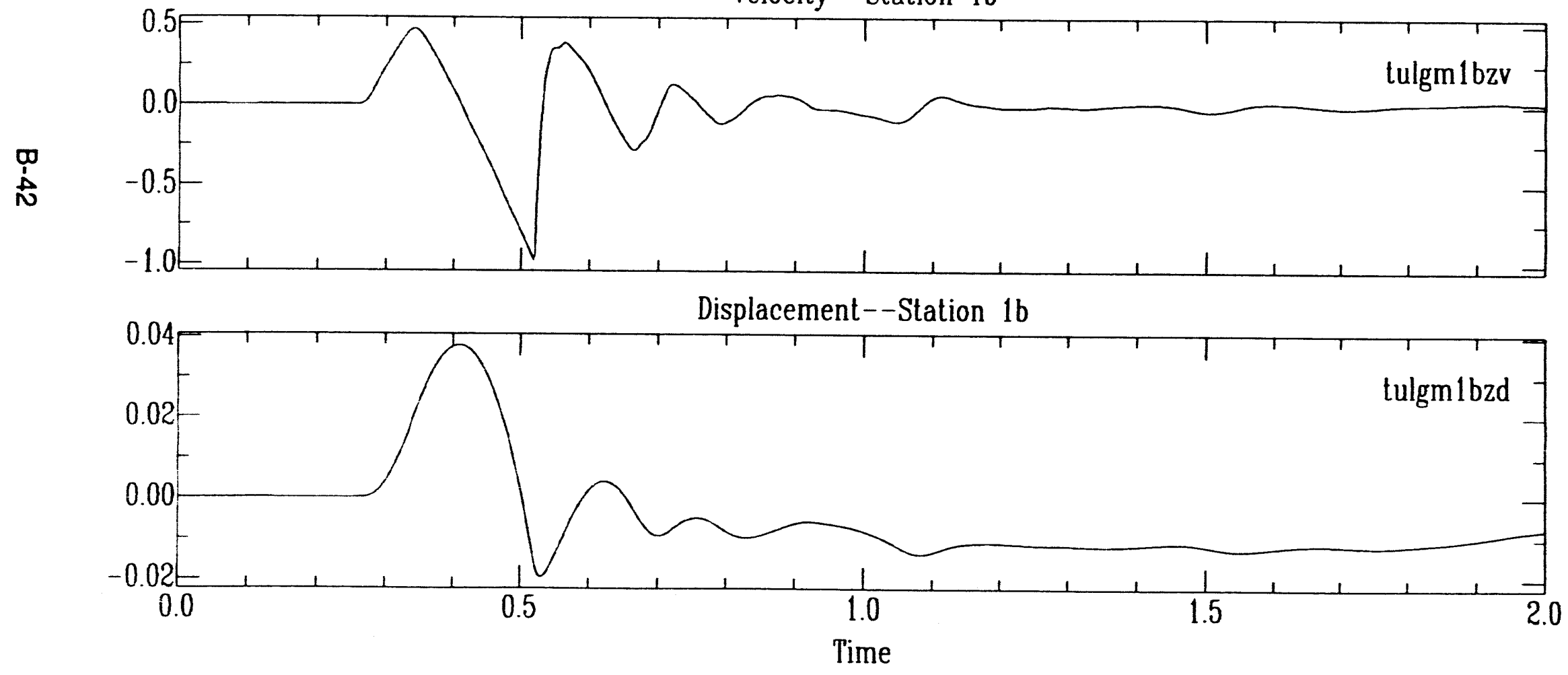




\section{Tulia Surface Ground Motion}

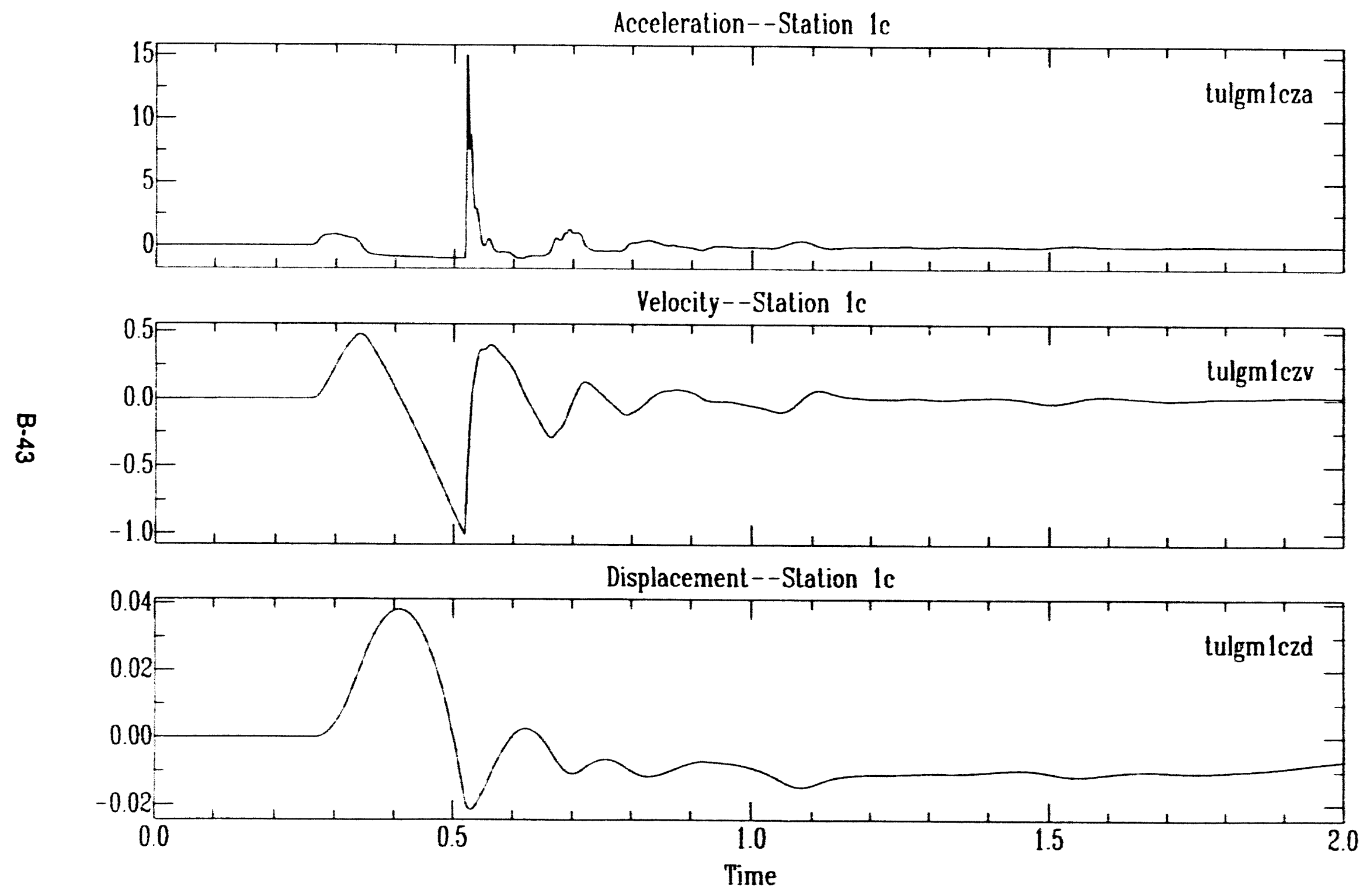




\section{Tulia Surface Ground Motion}

Acceleration--Station 2a

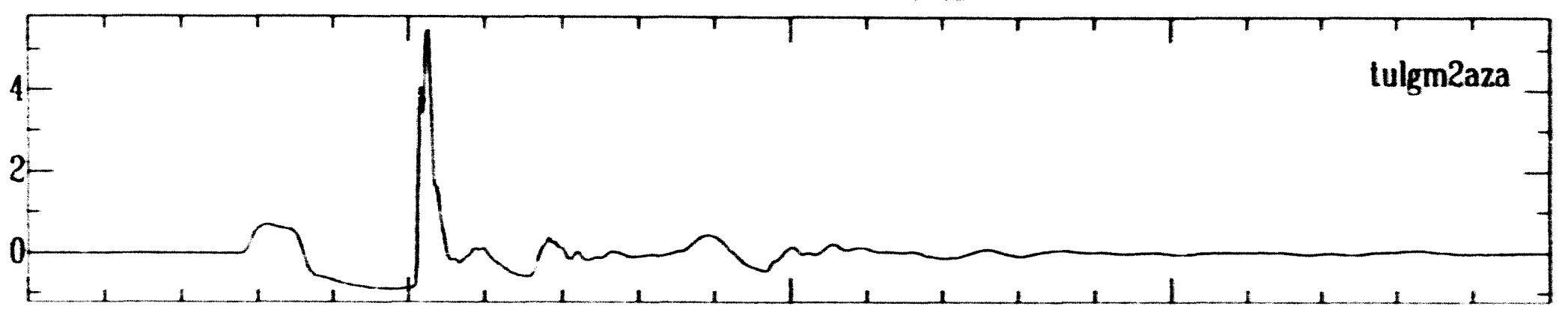

Velocity--Station $2 \mathrm{a}$

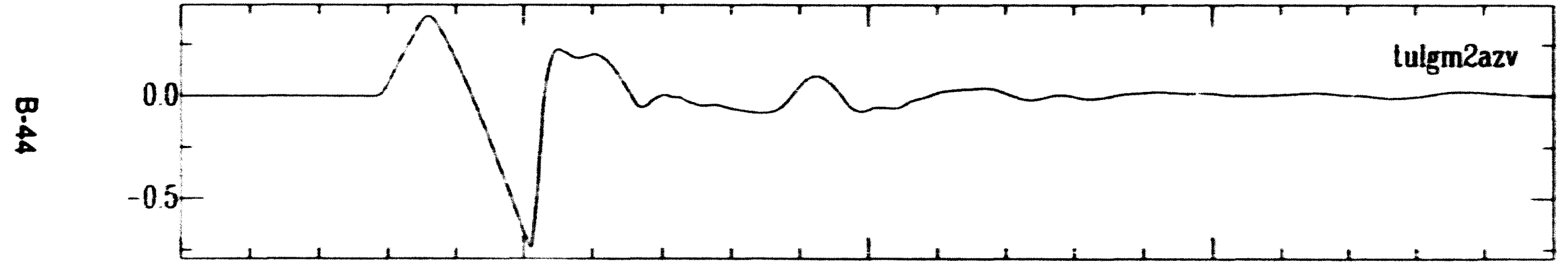

Displacement --Station 2a

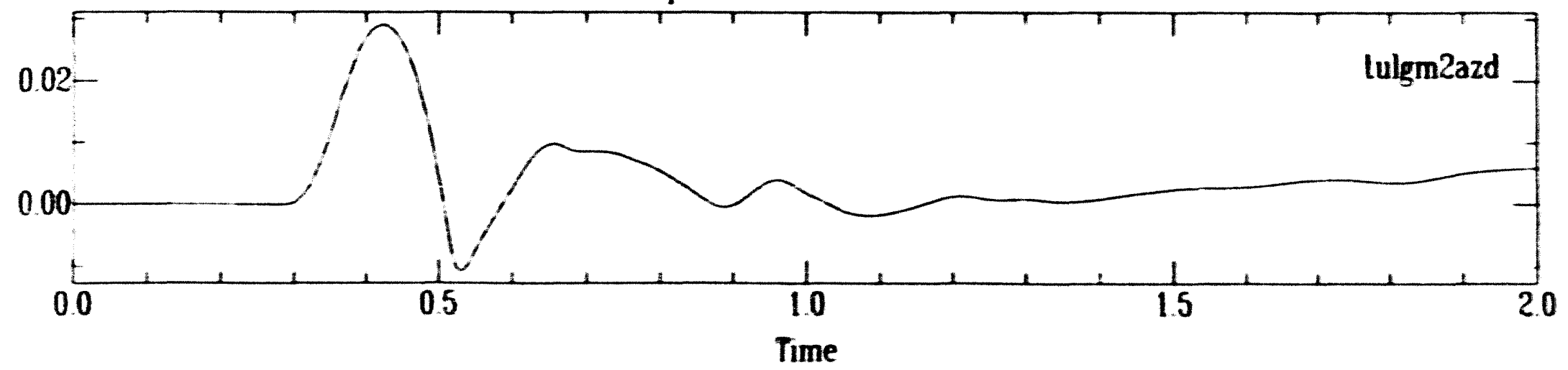


Tulia Surface Ground Motion

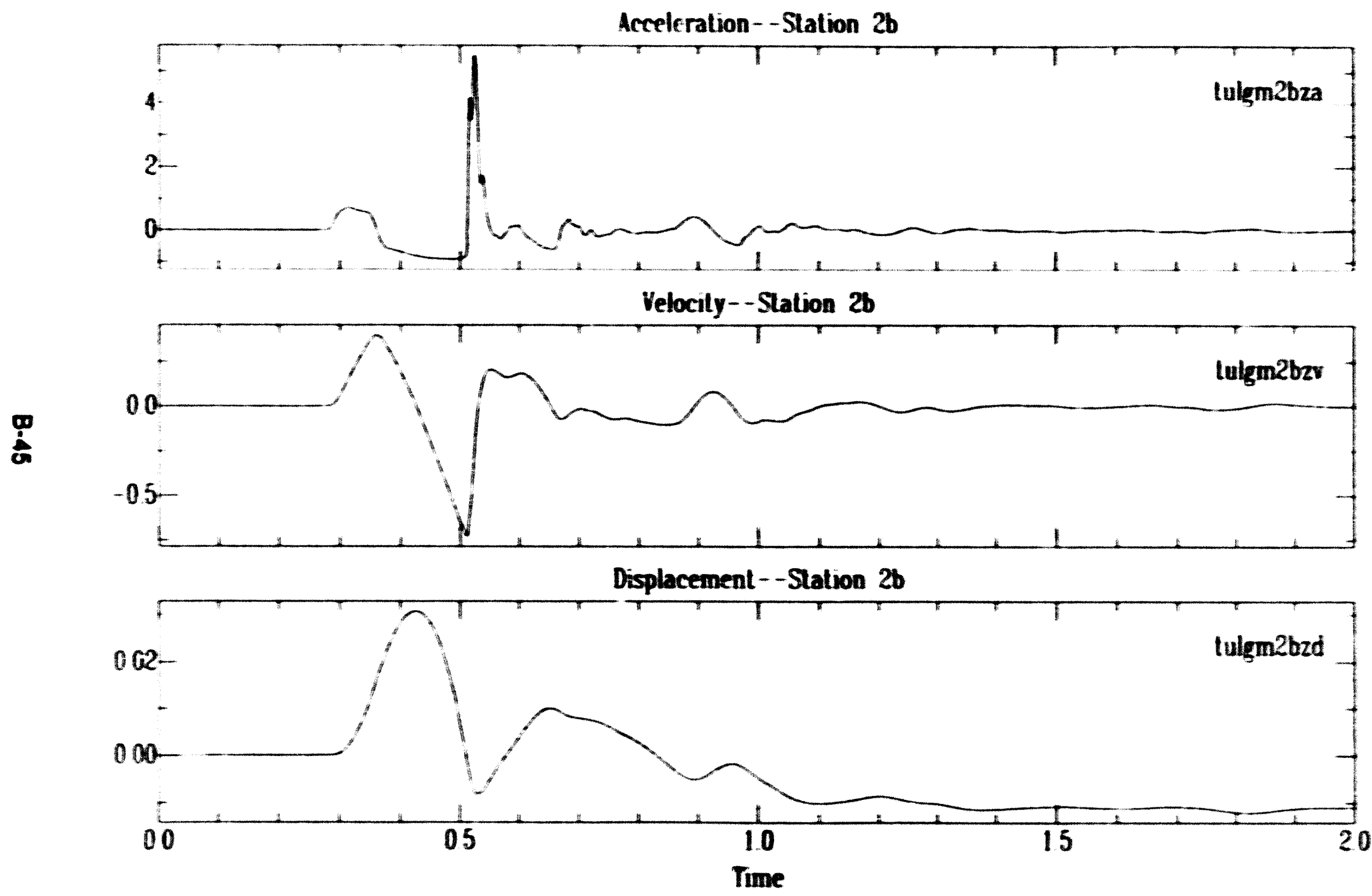


Tulia Surface Ground Molion

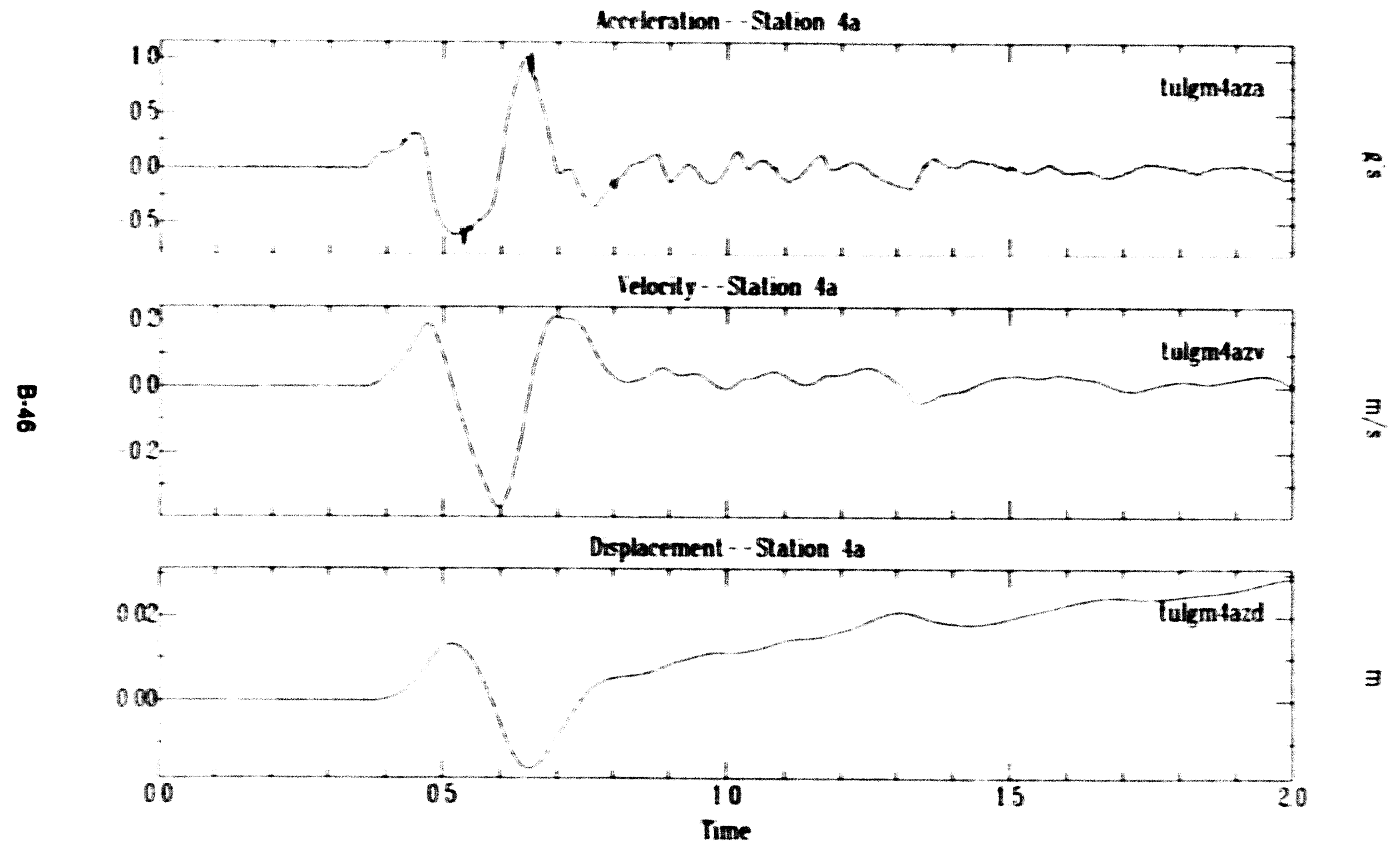




\section{Tulia Surface Ground Motion}

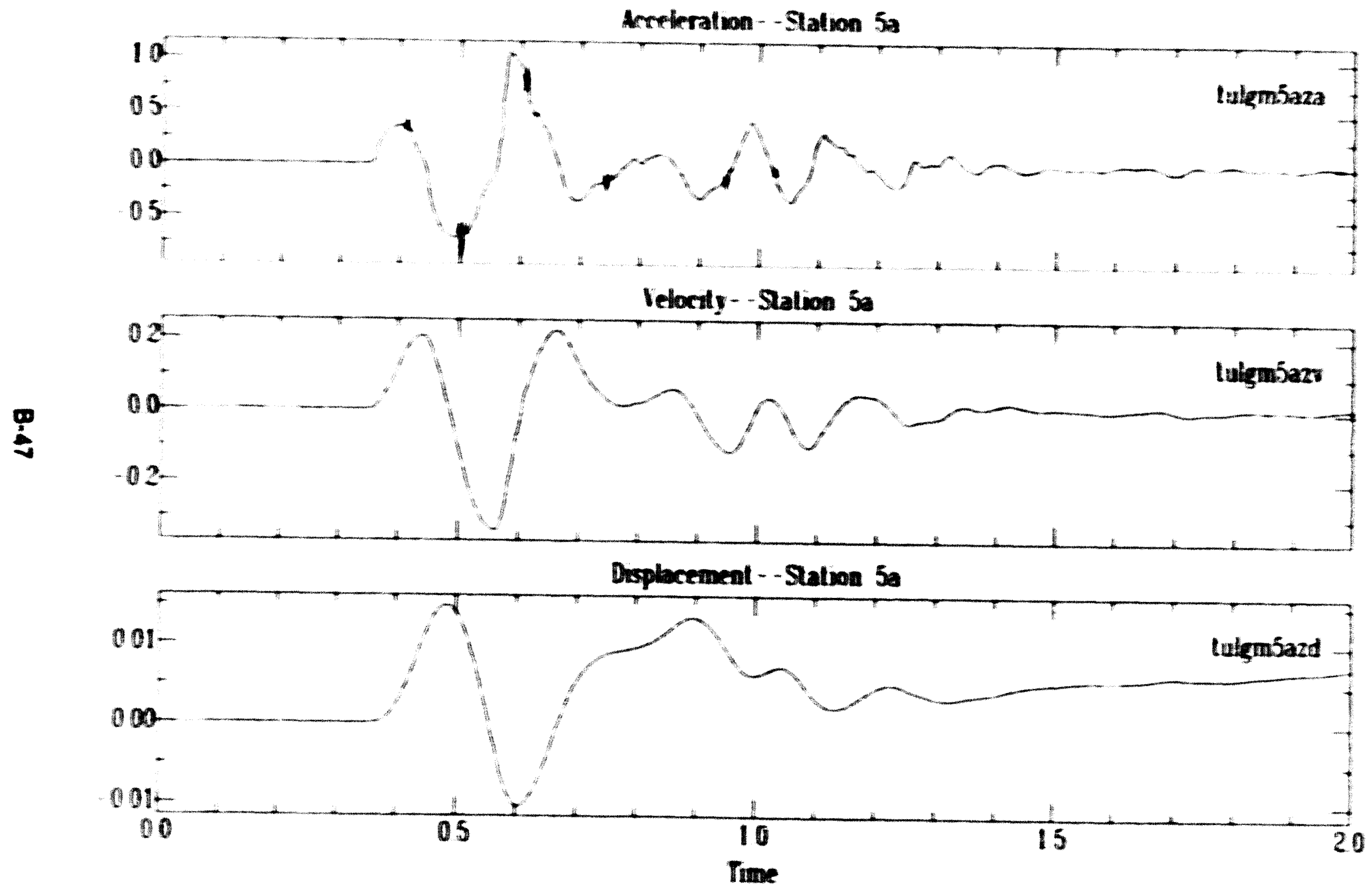




\section{Tulia Surface Ground Motion}

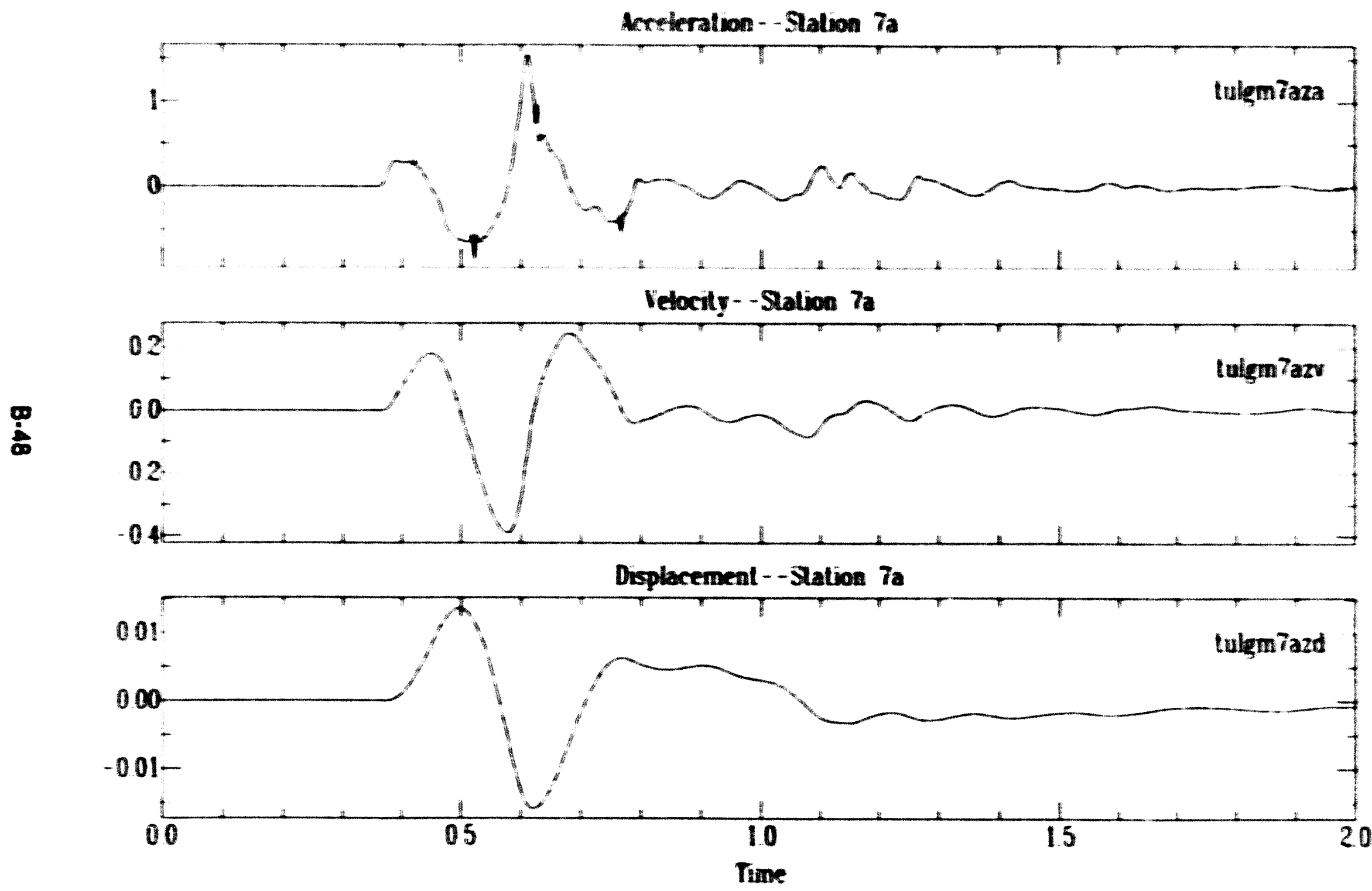




\section{Tulia Surface Ground Motion}

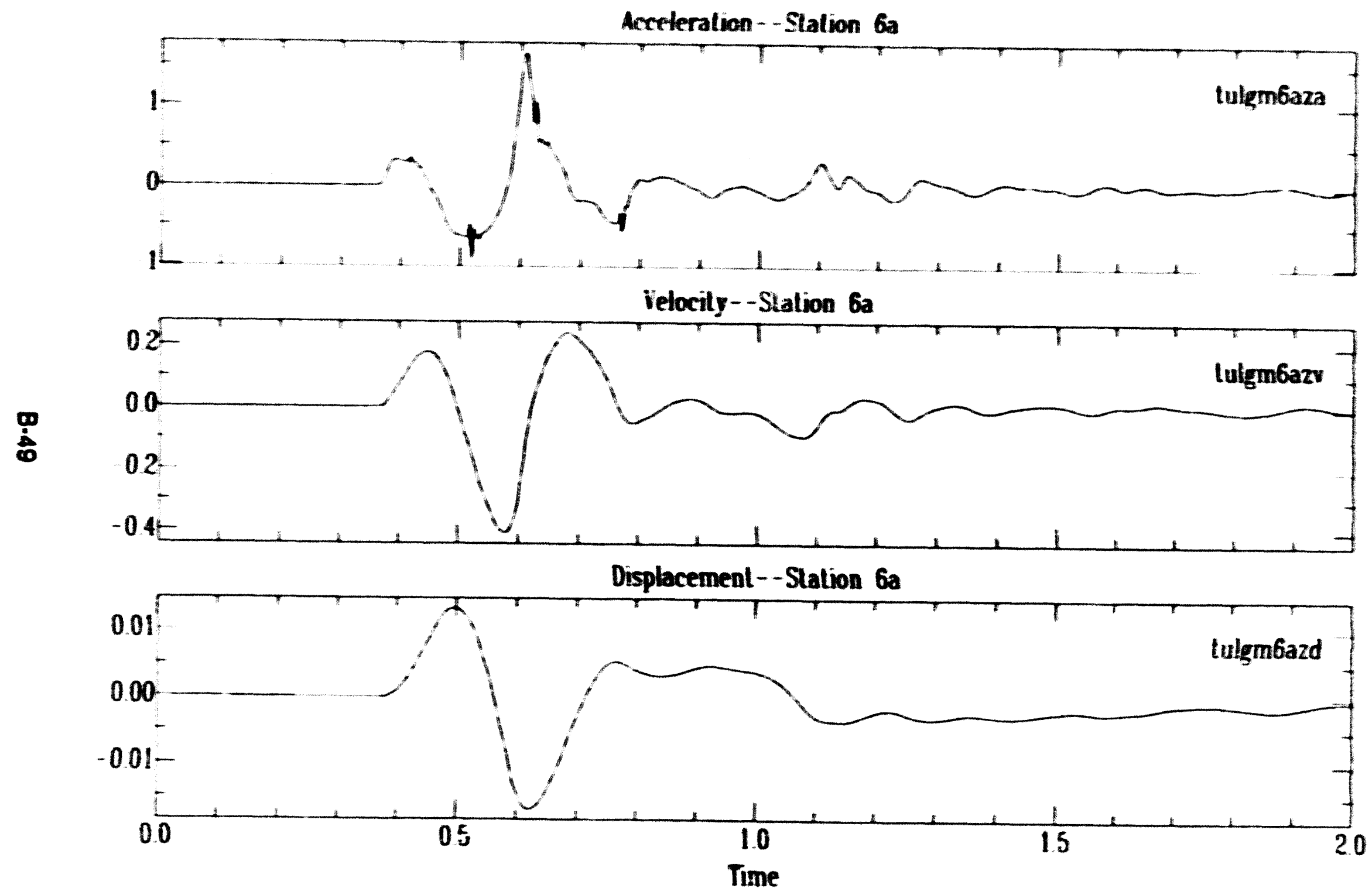




\subsection{Contact (U20aw)}

Experiment design. The only ground-motion measurement for Contact was the SIG experiment. Eleven arrays of golf balls ( 150 golf balls total) were installed around both the Contact and Amarillo emplacements. Table 4 is summary data about the arrays: number of balls, the approximate distance from the center of the array to Contact SGZ, and how each array was configured. The same configuration was used for both events (Fig. 7). Installation consisted of placing a golf ball directly on the ground (no dish), or into a plastic dish $(15.4-\mathrm{cm}$ diameter and $4-\mathrm{cm}$ lip) that was partially filled with soil and placed onto the ground surface.

Eight of the arrays (four around each emplacement) were placed across a 600-m zone that spanned the predicted edges of spall. The other three arrays were placed approximately halfway between the two events.

Table 4. SIG Arrays for Contact

(see also Fig. 7)

$\begin{array}{cccc}\begin{array}{c}\text { Array } \\ 1\end{array} & \begin{array}{l}\text { Ball } \\ \text { Total } \\ 15\end{array} & \begin{array}{c}\text { Ball Nos. } \\ 1.15\end{array} & \begin{array}{l}\text { Dist.(m) } \\ 3050\end{array} \\ 2 & 15 & 16.30 & 2600 \\ & & & \\ 3 & 14 & 31.44 & 1680 \\ 4 & 10 & 45.54 & 1525 \\ 5 & 8 & 55.62 & 1525 \\ 6 & 10 & 63.72 & 1525 \\ 7 & 24 & 73.96 & 3660 \\ 8 & 8 & 97.104 & 5200 \\ 9 & 10 & 105.114 & 7000 \\ 10 & 10 & 115.124 & 6700 \\ 11 & 26 & 125.150 & 6550\end{array}$

\begin{abstract}
Conflguration
$3 \times 5$ rectangular array, about 50.m spacing between balls

$3 \times 5$ rectangular array, about 50.m spacing between balls

2 parallel lines, bells 100-m spacing, lines 20-m spacing 2 parallel lines, balls $100 \cdot \mathrm{m}$ spacing, lines $20 \cdot \mathrm{m}$ spacing 2 parallel lines, balls 100-m spacing, lines $20-\mathrm{m}$ spacing 2 parallel lines, balls $100-\mathrm{m}$ spacing, lines $20-\mathrm{m}$ spacing $4 \times 6$ rectangular array, $50-\mathrm{m}$ spacing 1 line, 50-m spacing 2 parallel lines, balls 100-m spacing, lines 20-m spacing 2 parallel lines, balls $50 \cdot \mathrm{m}$ spacing, lines $10 \cdot \mathrm{m}$ spacing 1 line, 2 balls at each location, 50-m spacing
\end{abstract}

SIG results. Results of the SIG experiment can be found in Table 5. Three of the golf balls moved a significant distance: (1) Golf ball 22 (GB22), located in the east-central part of Array 2, jumped $6.4 \mathrm{~m}$ at $95^{\circ}$ azimuth; (2) GB32 jumped almost $3 \mathrm{~m}$ at $10^{\circ}$, and the dish it was in moved about $0.3 \mathrm{~m}$; and (3) GB41, located $10 \mathrm{~m} \mathrm{~N}$ of GB32, moved about $8.8 \mathrm{~m}$ at $40^{\circ}$. Both $\mathrm{GB3} 2$ and GB41 were located about $50 \mathrm{~m}$ east of the U2Oau pad. All three of these balls appear to have been placed in close proximity to fault systems.

The rest of the balls moved very little. Six other balls moved more than $10 \mathrm{~cm}$. None of the balls that moved showed any indication of more than one direction of movement, i.e., rolling backward and forward. 
टS-g

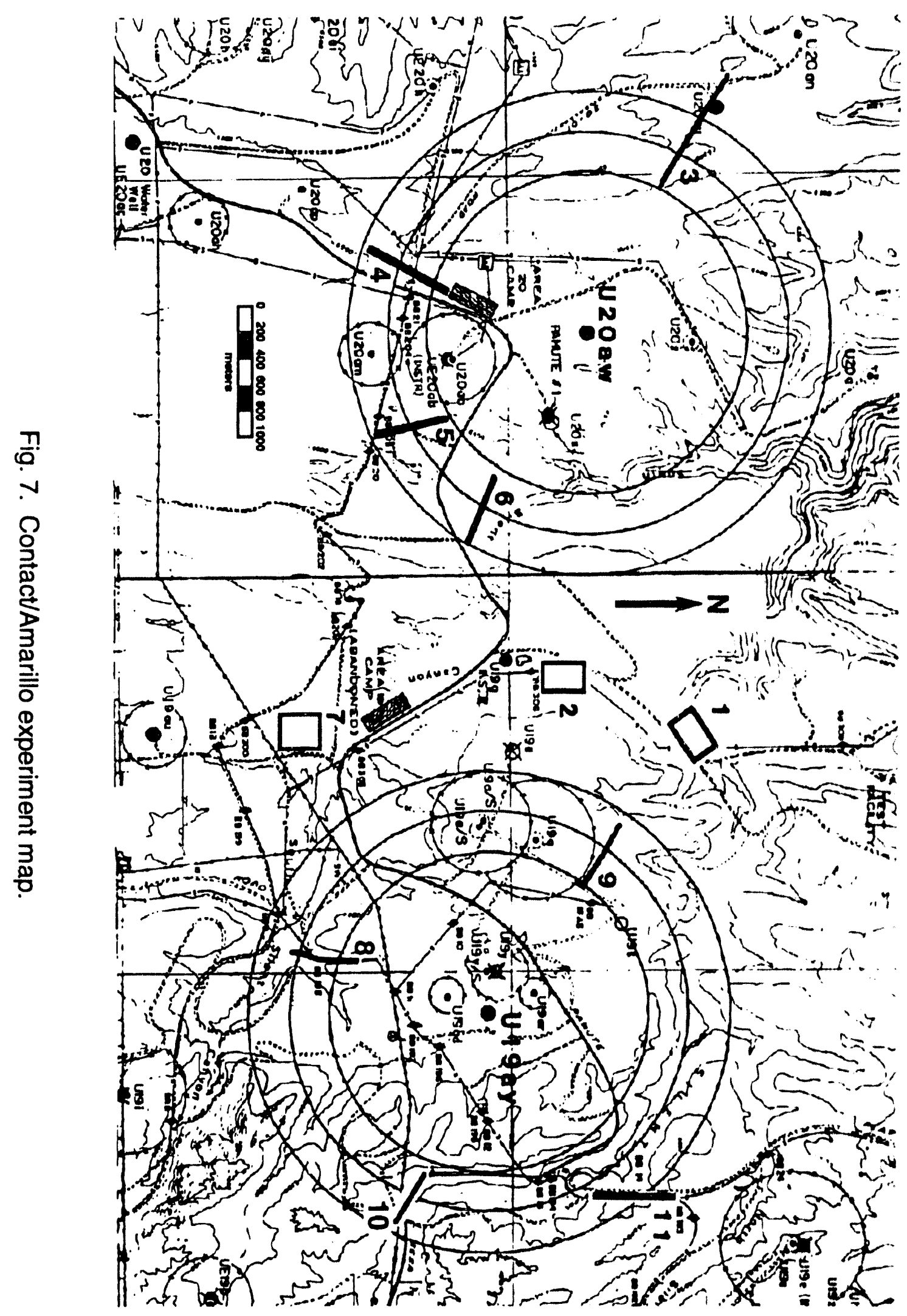


Table 5. Contact SIG Results

\begin{tabular}{|c|c|c|c|c|c|}
\hline Ball & Array & $\begin{array}{l}\text { Movement } \\
\text { Dist } \\
\text { (cm) }\end{array}$ & $A z$ & Placoment & Comments \\
\hline 1 & 1 & 0 & 0 & ground & \\
\hline 2 & 1 & 0 & 0 & ground & \\
\hline 3 & 1 & 0 & 0 & ground & \\
\hline 4 & 1 & 0 & 0 & ground & \\
\hline 5 & 1 & 0 & 0 & ground & \\
\hline 6 & 1 & 2.5 & 255 & ground & \\
\hline 7 & 1 & 0 & 0 & ground & \\
\hline 8 & 1 & 0 & 0 & ground & \\
\hline 9 & 1 & 0 & 0 & ground & \\
\hline 10 & 1 & 0 & 0 & ground & \\
\hline 11 & 1 & 8.9 & 70 & ground & Downslope \\
\hline 12 & 1 & 0 & 0 & ground & \\
\hline 13 & 1 & 0 & 0 & ground & \\
\hline 14 & 1 & 0 & 0 & ground & \\
\hline 15 & 1 & 5.1 & 310 & ground & Crack through clearing \\
\hline 16 & 2 & 7.6 & 15 & ground & Downslope \\
\hline 17 & 2 & 10.2 & 340 & ground & \\
\hline 18 & 2 & 19.1 & 240 & ground & Downslope \\
\hline 19 & 2 & 0 & 0 & ground & \\
\hline 20 & 2 & 10.2 & 255 & ground & \\
\hline 21 & 2 & 0 & 0 & ground & \\
\hline 22 & 2 & 640.1 & 95 & ground & \\
\hline 23 & 2 & 0 & 0 & ground & Rolled but stopped at origin \\
\hline 24 & 2 & 0 & 0 & ground & \\
\hline 25 & 2 & 0 & 0 & ground & \\
\hline 26 & 2 & 2.5 & 285 & ground & \\
\hline 27 & 2 & 10.2 & 345 & ground & \\
\hline 28 & 2 & 34.3 & 315 & ground & Downslope \\
\hline 29 & 2 & 0 & 0 & ground & \\
\hline 30 & 2 & 0 & 0 & ground & \\
\hline 31 & 3 & 0 & 0 & dish & \\
\hline 32 & 3 & 289.6 & 20 & dish & Pan moved $=30.5 \mathrm{~cm}$ north \\
\hline 33 & 3 & 0 & 0 & dish & \\
\hline 34 & 3 & 0 & 0 & dish & \\
\hline 35 & 3 & 0 & 0 & dish & \\
\hline 36 & 3 & 0 & 0 & dish & \\
\hline 37 & 3 & 0 & 0 & dish & \\
\hline 38 & 3 & 0 & 0 & dish & \\
\hline 39 & 3 & 0 & 0 & dish & \\
\hline 40 & 3 & 0 & 0 & dish & \\
\hline 41 & 3 & 887.7 & 55 & dish & \\
\hline 42 & 3 & 0 & 0 & dish & \\
\hline 43 & 3 & 0 & 0 & dish & \\
\hline 44 & 3 & 0 & 0 & dish & \\
\hline 45 & 4 & 0 & 0 & dish & \\
\hline
\end{tabular}


Table 5. Contact SIG Results (Continued)

\begin{tabular}{|c|c|c|c|c|c|}
\hline Ball & Array & $\begin{array}{l}\text { Move } \\
\text { Dist } \\
\text { (cm) }\end{array}$ & $\begin{array}{l}\text { ient } \\
\text { Az }\end{array}$ & Placoment & Comments \\
\hline 46 & 4 & 7.6 & 45 & dish & \\
\hline 47 & 4 & 7.6 & 135 & dish & \\
\hline 48 & 4 & 7.6 & 235 & dish & \\
\hline 49 & 4 & 0 & 0 & dish & \\
\hline 50 & 4 & 0 & 0 & dish & \\
\hline 51 & 4 & 2.5 & 15 & dish & Deer track next to dish \\
\hline 52 & 4 & 0 & 0 & dish & \\
\hline 53 & 4 & 7.6 & 75 & dish & E-W hairline crack next to dish \\
\hline 54 & 4 & 0 & 0 & dish & \\
\hline 55 & 5 & 0 & 0 & dish & \\
\hline 56 & 5 & 7.6 & 250 & dish & Dish slid $13-15 \mathrm{~cm}$ \\
\hline 57 & 5 & 0 & 0 & dish & \\
\hline 58 & 5 & 7.6 & 225 & dish & \\
\hline 59 & 5 & 0 & 0 & dish & \\
\hline 60 & 5 & 0 & 0 & dish & \\
\hline 61 & 5 & 0 & 0 & dish & \\
\hline 62 & 5 & 0 & 0 & dish & \\
\hline 63 & 6 & 0 & 0 & dish & \\
\hline 64 & 6 & 0 & 0 & dish & \\
\hline 65 & 6 & 7.6 & 105 & dish & Downslope \\
\hline 66 & 6 & 0 & 0 & dish & \\
\hline 67 & 6 & 0 & 0 & dish & \\
\hline 68 & 6 & 0 & 0 & dish & Gully bottom \\
\hline 69 & 6 & 5.1 & 60 & dish & \\
\hline 70 & 6 & 3.8 & 60 & dish & \\
\hline 71 & 6 & 7.6 & 75 & dish & \\
\hline 72 & 6 & 7.6 & 60 & dish & \\
\hline 73 & 7 & 0 & 0 & dish & \\
\hline 74 & 7 & 0 & 0 & dish & \\
\hline 75 & 7 & 0 & 0 & dish & \\
\hline 76 & 7 & 0 & 0 & dish & \\
\hline 77 & 7 & 0 & 0 & dish & \\
\hline 78 & 7 & 2.5 & & dish & Rolled into a depression \\
\hline 79 & 7 & 2.5 & 195 & dish & \\
\hline 80 & 7 & 2.5 & 195 & dish & \\
\hline 81 & 7 & 0 & 0 & dish & \\
\hline 82 & 7 & 2.5 & 15 & dish & \\
\hline 83 & 7 & 2.5 & 105 & dish & \\
\hline 84 & 7 & 2.5 & 195 & dish & \\
\hline 85 & 7 & 2.5 & 150 & dish & \\
\hline 86 & 7 & 2.5 & 195 & dish & \\
\hline 87 & 7 & 0 & 0 & dish & \\
\hline 88 & 7 & 0 & 0 & dish & \\
\hline 89 & 7 & 0 & 0 & dish & \\
\hline
\end{tabular}


Table 5. Contact SIG Results (Continued)

\begin{tabular}{|c|c|c|c|c|c|}
\hline Ball & Array & $\begin{array}{l}\text { Move } \\
\text { Dist } \\
\text { (cm) }\end{array}$ & $\begin{array}{l}\text { ient } \\
\text { Az }\end{array}$ & Placement & Comments \\
\hline 90 & 7 & 2.5 & 195 & dish & Animal tracks near dish \\
\hline 91 & 7 & 0 & 0 & dish & \\
\hline 92 & 7 & 0 & 0 & dish & \\
\hline 93 & 7 & 2.5 & 195 & dish & \\
\hline 94 & 7 & 0 & 0 & dish & \\
\hline 95 & 7 & 2.5 & 340 & dish & \\
\hline 96 & 7 & 15.2 & 150 & dish & Animal tracks near dish \\
\hline 97 & 8 & 0 & 0 & dish & \\
\hline 98 & 8 & 0 & 0 & dish & \\
\hline 99 & 8 & 0 & 0 & dish & \\
\hline 100 & 8 & 0 & 0 & dish & \\
\hline 101 & 8 & 0 & 0 & dish & \\
\hline 102 & 8 & 0 & 0 & dish & \\
\hline 103 & 8 & 0 & 0 & dish & \\
\hline 104 & 8 & 2.5 & 285 & dish & \\
\hline 105 & 9 & 0 & 0 & dish & \\
\hline 106 & 9 & 0 & 0 & dish & \\
\hline 107 & 9 & 0 & 0 & dish & \\
\hline 108 & 9 & 7.6 & 285 & dish & \\
\hline 109 & 9 & 0 & 0 & dish & \\
\hline 110 & 9 & 0 & 0 & dish & \\
\hline 111 & 9 & 0 & 0 & dish & \\
\hline 112 & 9 & 0 & 0 & dish & \\
\hline 113 & 9 & 0 & 0 & dish & \\
\hline 114 & 9 & 0 & 0 & dish & \\
\hline 115 & 10 & 0 & 0 & dish & \\
\hline 116 & 10 & 0 & 0 & dish & \\
\hline 117 & 10 & 0 & 0 & dish & \\
\hline 118 & 10 & 0 & 0 & dish & \\
\hline 119 & 10 & 0 & 0 & dish & \\
\hline 120 & 10 & 0 & 0 & dish & \\
\hline 121 & 10 & 0 & 0 & dish & \\
\hline 122 & 10 & 0 & 0 & dish & \\
\hline 123 & 10 & 0 & 0 & dish & \\
\hline 124 & 10 & 0 & 0 & dish & \\
\hline 125 & 11 & 0 & 0 & dish & North of Silent Canyon \\
\hline 126 & 11 & 0 & 0 & dish & \\
\hline 127 & 1 & 0 & 0 & dish & Same dish as Ball \#150 \\
\hline 128 & 11 & 0 & 0 & dish & Same dish as Ball \#149 \\
\hline 129 & 11 & 0 & 0 & dish & Same dish as Ball \#148 \\
\hline 130 & 11 & 0 & 0 & dish & Same dish as Ball \#147 \\
\hline 131 & 11 & 0 & 0 & dish & Same dish as Ball \#146 \\
\hline 132 & 11 & 0 & 0 & dish & Same dish as Ball \#145 \\
\hline 133 & 11 & 0 & 0 & dish & Dish adjacent to Ball \#144 \\
\hline 134 & 11 & 0 & 0 & dish & Dish adjacent to Ball \#143 \\
\hline 135 & 11 & 0 & 0 & dish & Dish adjacent to Ball \#142 \\
\hline
\end{tabular}


Table 5. Contact SIG Results (Concluded)

\begin{tabular}{|c|c|c|c|c|c|}
\hline Ball & Array & $\begin{array}{l}\text { Movement } \\
\text { Dist } \\
\text { (cm) }\end{array}$ & Az & Placement & Comments \\
\hline 136 & 11 & 0 & 0 & dish & Dish adjacent to Ball $\# 141$ \\
\hline 137 & 11 & 0 & 0 & dish & Dish adjacent to Ball \#140 \\
\hline 138 & 11 & 0 & 0 & dish & Dish adjacent to Ball \#139 \\
\hline 139 & 11 & 0 & 0 & dish & Dish adjacent to Ball \#140 \\
\hline 140 & 11 & 0 & 0 & dish & Dish adjacent to Ball \#137 \\
\hline 141 & 11 & 0 & 0 & dish & Dish adjacent to Ball \#136 \\
\hline 142 & 11 & 0 & 0 & dish & Dish adjacent to Ball \#135 \\
\hline 143 & 11 & 0 & 0 & dish & Dish adjacent to Ball \#134 \\
\hline 144 & 11 & 0 & 0 & dish & Dish adjacent to Ball \#133 \\
\hline 145 & 11 & 0 & 0 & dish & Same dish as Ball \#132 \\
\hline 146 & 11 & 0 & 0 & dish & Same dish as Ball \#131 \\
\hline 147 & 11 & 0 & 0 & dish & Same dish as Ball \#130 \\
\hline 148 & 11 & 0 & 0 & dish & Same dish as Ball \#129 \\
\hline 149 & 11 & 0 & 0 & dish & Same dish as Ball \#128 \\
\hline 150 & 11 & 0 & 0 & dish & Same dish as Ball \#127 \\
\hline
\end{tabular}




\subsection{Amarlllo (U19ay)}

Experiment design. The identical SIG experiment conducted for Contact a week earlier was reset and repeated for Amarillo (see Fig. 7 and Table 6). Mapping and retrieval of the SIGs began within a few hours of event detonation. Amarillo also had a standard J-8 6-station accelerometer array (including 3 stations near SGZ) (see Fig. 8 and Table 7). All of these stations were within the predicted spall zone.

SIG results. Recovery of the SIGs and fracture mapping began within $4 \mathrm{hrs}$ of event detonation. The weather prior to the event was hot and dry, with occasional wind gusts. This same weather pattem persisted for several days after the event. Postshot, very few of the SIGs had moved from their preshot positions (Table 7). Those few that did showed evidence of animal or bird tracks in the immediate vicinity or may have been moved as a result of the high wind gusts that were experienced the evening before the event (see comments in Table 7). There are no SIG movements that can uniquely be attributed to the ground motion from Amarillo.

Table 6. SIG Arrays for Amarillo (see also Fig. 7)

$\begin{array}{llll}\begin{array}{l}\text { Array } \\ 1\end{array} & \begin{array}{l}\text { Ball } \\ \text { Total } \\ 15\end{array} & \begin{array}{l}\text { Ball Nos. } \\ 1-15\end{array} & \begin{array}{l}\text { Dist.(m) } \\ 2620\end{array} \\ 2 & 15 & 16-30 & 2620 \\ 3 & 14 & 31-44 & 6700 \\ 4 & 10 & 45-54 & 5790 \\ 5 & 8 & 55-62 & 4570 \\ 6 & 10 & 63-72 & 3960 \\ 7 & 24 & 73-96 & 2620 \\ 8 & 8 & 97-104 & 1340 \\ 9 & 10 & 105-114 & 1520 \\ 0 & 10 & 115-124 & 1520 \\ 11 & 26 & 125-150 & 1830\end{array}$
Configuration
$3 \times 5$ rectangular array, about $50-\mathrm{m}$ spacing between balls
$3 \times 5$ rectangular array, about $50-\mathrm{m}$ spacing between balls
2 parallel lines, balls 100-m spacing, lines 20-m spacing 2 parallel lines, balls $100-\mathrm{m}$ spacing, lines $20-\mathrm{m}$ spacing 2 parallel lines, balls $100-\mathrm{m}$ spacing, lines $20-\mathrm{m}$ spacing 2 parallel lines, balls $100-\mathrm{m}$ spacing, lines $20-\mathrm{m}$ spacing $4 \times 6$ rectangular array, $50-\mathrm{m}$ spacing 1 line, 50-m spacing
2 parallel lines, balls $100-\mathrm{m}$ spacing, lines $20-\mathrm{m}$ spacing 2 parallel lines, balls $50-\mathrm{m}$ spacing, lines $10-\mathrm{m}$ spacing 1 line, 2 balls at each location, 50-m spacing

Accelerometer results. The J-8 data were originally reported out in July 1989 (Memo $\mathrm{J}-8-89-165$, R. S. Fitzhugh to F. N. App). All but one of the J-8 accelerometers (AMAGM6A) recorded spall signals. Station $6 \mathrm{~A}$ looks similar to a spall record, but the first maximum acceleration was just less the 1-g upward, and the dwell between first peak and slapdown never exceeded $-0.4 \mathrm{~g}$, whereas $-1.0 \mathrm{~g}$ would normally indicate spall. Figure 9 (continued over 8 pages) contains the data traces for the accelerometer data. 
Table 7. Amarillo J-8 Ground-Alotion Station Data

\begin{tabular}{|c|c|c|c|c|c|}
\hline $\begin{array}{l}\text { Station } \\
\text { Name }\end{array}$ & $\begin{array}{l}\text { Station Loc } \\
\text { u19ay }\end{array}$ & Accelerometer & $\begin{array}{l}\text { Northing (m) } \\
280233\end{array}$ & $\begin{array}{l}\text { Easting (m) } \\
180167\end{array}$ & $\begin{array}{l}\text { Stant Range } \\
640(m) 00 B\end{array}$ \\
\hline AMAGM1A & $15 \mathrm{~m} \mathrm{~N} 38 \mathrm{E}$ & Endevco $10 \mathrm{~g}$ & 2802451 & 80177 & 640 \\
\hline AMAGM1B & $15 \mathrm{~m} \mathrm{~N} 38 \mathrm{E}$ & Endevco $25 \mathrm{~g}$ & 280245 & 18017 & 640 \\
\hline AMAGM1C & $15 \mathrm{~m}$ N38E & Endevco $25 \mathrm{~g}$ & 280245 & 180177 & 640 \\
\hline AMAGM2A & $258 \mathrm{~m}$ N38E & Endevco $25 \mathrm{~g}$ & 280436 & 180325 & 690 \\
\hline AMAGM2B & $258 \mathrm{~m}$ N38E & Endevco $25 \mathrm{~g}$ & 280436 & 180325 & 690 \\
\hline AMAGM4A & $640 \mathrm{~m}$ N2OE & Endevco $20 \mathrm{~g}$ & 280834 & 180386 & 905 \\
\hline AMAGM5A & $644 \mathrm{~m}$ S39E & Endevco $20 \mathrm{~g}$ & 279731 & 180570 & 908 \\
\hline AMAGM6A & $640 \mathrm{~m}$ S80W & Endevco $20 \mathrm{~g}$ & 280122 & 179537 & 905 \\
\hline
\end{tabular}




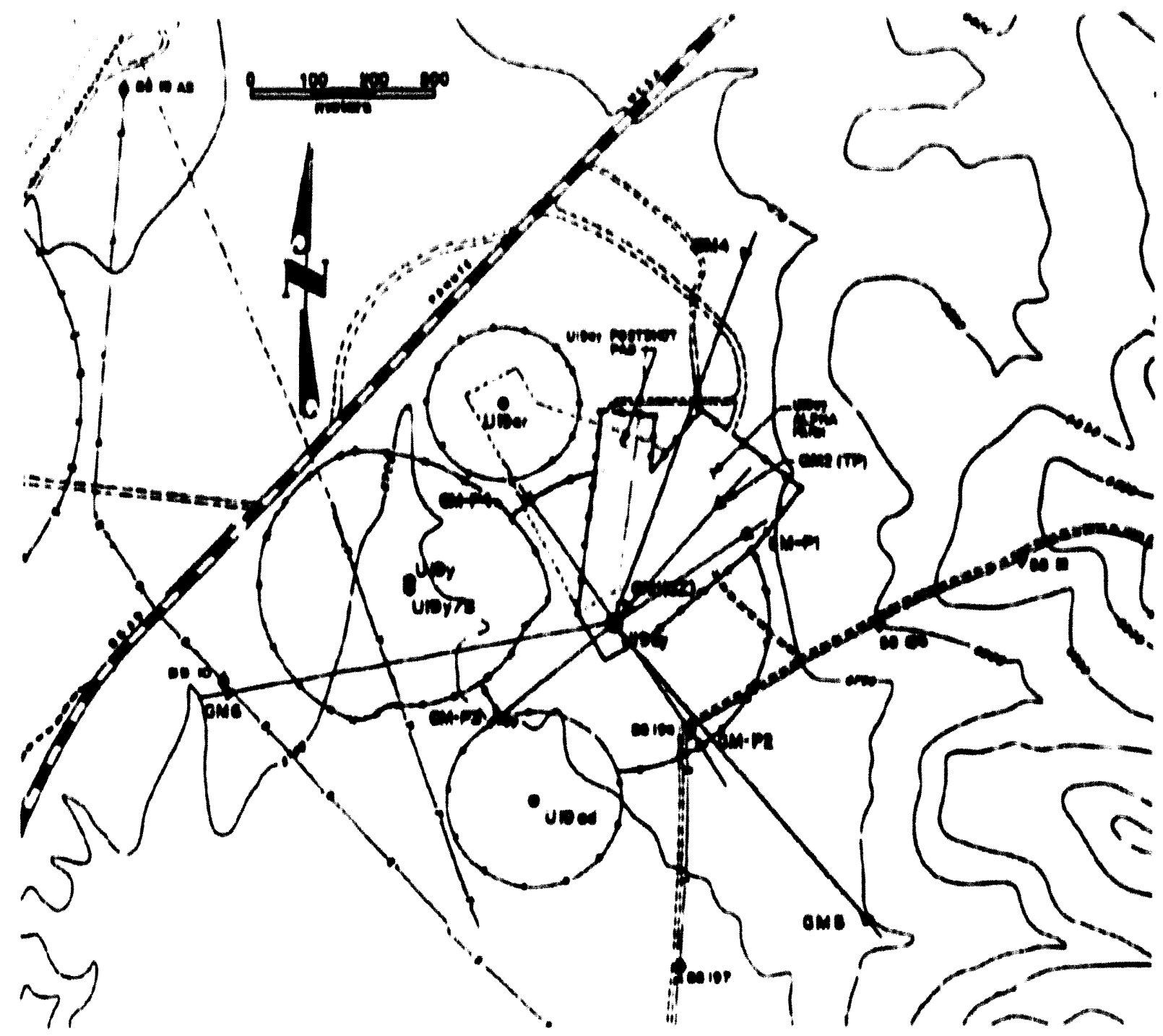

Fig. 8. Ground-motion stations for Amarillo. 
Toble A. Amarllo sto Roauhs

\begin{tabular}{|c|c|c|c|c|c|}
\hline Dall & Arroy & $\begin{array}{l}\text { Movemont } \\
\text { Didt } \\
\text { (em) }\end{array}$ & $\mathbf{A}$ & Plecomint & Commont \\
\hline 1 & 1 & 26 & 198 & ground & Wind? \\
\hline 2 & 1 & 0 & 0 & ground & \\
\hline 3 & 1 & 0 & 0 & ground & \\
\hline 4 & 1 & 0 & 0 & ground & \\
\hline 6 & 1 & 0 & 0 & oround & \\
\hline$\theta$ & 1 & 0 & 0 & ground & \\
\hline 7 & 1 & 0 & 0 & ground & \\
\hline$\theta$ & 1 & 0 & 0 & ground & \\
\hline$?$ & $!$ & 0 & 0 & ground & \\
\hline 10 & $?$ & 0 & 0 & ground & \\
\hline 11 & 1 & 0 & 0 & ground & \\
\hline $\begin{array}{l}12 \\
13\end{array}$ & $\begin{array}{l}1 \\
1\end{array}$ & 0 & 0 & ground & \\
\hline $\begin{array}{l}13 \\
14\end{array}$ & 1 & $\begin{array}{l}0 \\
0\end{array}$ & $\begin{array}{l}0 \\
0\end{array}$ & Ground & \\
\hline 16 & 1 & 0 & 0 & ground & \\
\hline 10 & 2 & 81 & 26 & ground & \\
\hline 17 & 2 & 0 & 0 & ground & \\
\hline 18 & 2 & 0 & 0 & ground & \\
\hline $\begin{array}{l}10 \\
20\end{array}$ & 2 & 0 & 0 & ground & \\
\hline $\begin{array}{l}20 \\
21\end{array}$ & 2 & 0 & 0 & ground & \\
\hline 22 & 2 & $\begin{array}{l}0 \\
0\end{array}$ & 0 & ground & \\
\hline 23 & 2 & 0 & $\begin{array}{l}0 \\
0\end{array}$ & $\begin{array}{l}\text { ground } \\
\text { ground }\end{array}$ & \\
\hline 24 & 2 & 0 & 0 & ground & \\
\hline 28 & 2 & 0 & 0 & ground & \\
\hline 20 & 2 & 0 & 0 & ground & \\
\hline 27 & 2 & 0 & 0 & ground & \\
\hline 28 & 2 & 0 & 0 & ground & \\
\hline 29 & 2 & 0 & 0 & ground & \\
\hline 30 & 2 & 0 & 0 & ground & \\
\hline 31 & 3 & 70 & 16 & dich & Wind? \\
\hline 34 & 3 & 0 & 0 & dich & \\
\hline 36 & 3 & 0 & 0 & dich & \\
\hline 30 & 3 & 0 & 0 & dich & \\
\hline 37 & 3 & 0 & 0 & dich & \\
\hline 38 & 3 & 0 & 0 & dich & \\
\hline 39 & 3 & 0 & 0 & dich & \\
\hline 10 & 3 & 0 & 0 & dich & \\
\hline 41 & 3 & 0 & 0 & dich & \\
\hline 12 & 3 & 0 & 0 & dich & \\
\hline 43 & 3 & 0 & 0 & dich & \\
\hline 44 & 3 & 0 & 0 & dich & \\
\hline 45 & 4 & 0 & 0 & dich & \\
\hline 46 & 4 & 0 & 0 & dich & \\
\hline 47 & 4 & 0 & 0 & dish & \\
\hline
\end{tabular}


Toble 0 Amerllle 210 hosulte (Continued)

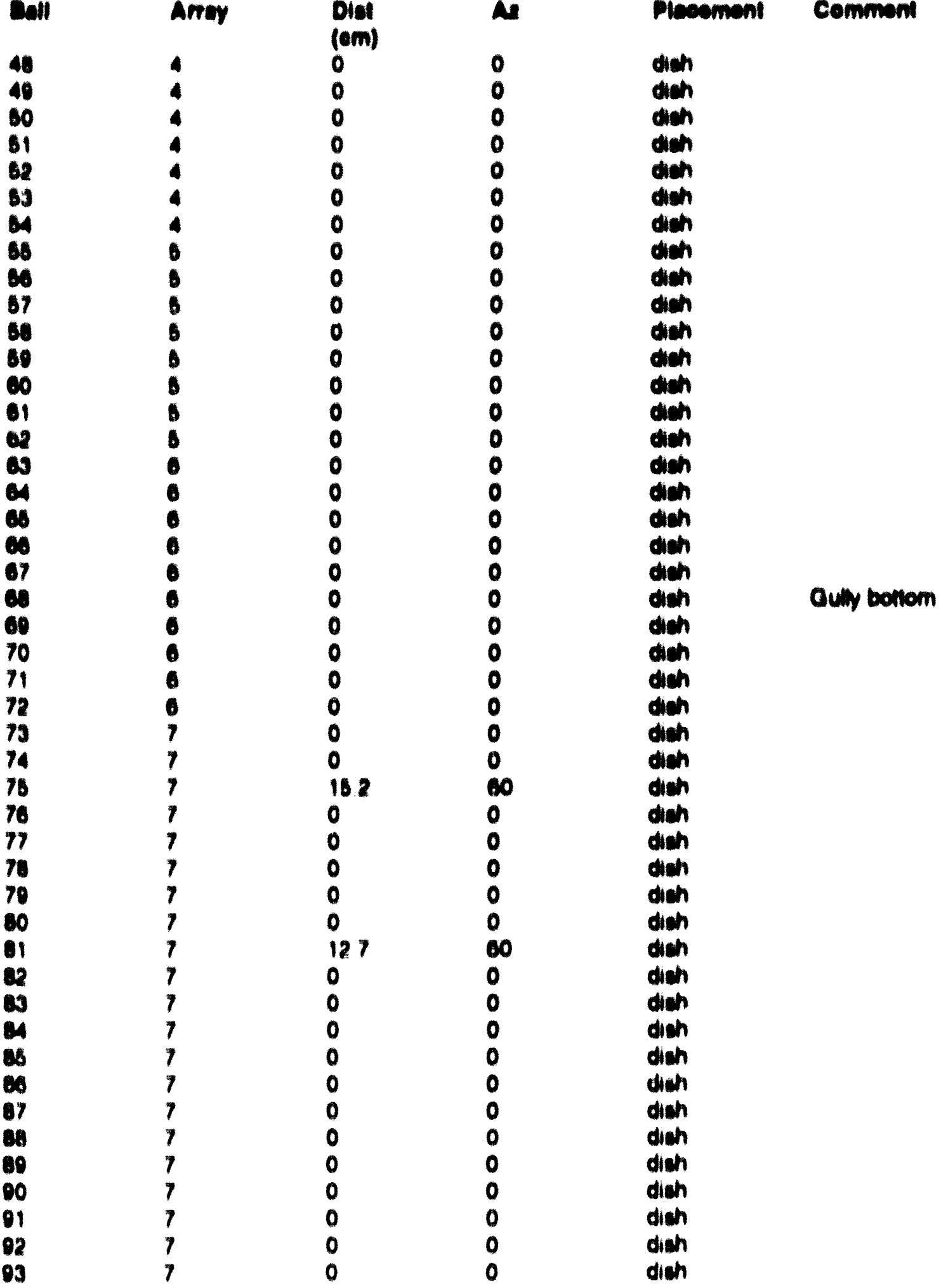


Toble t. Amarlle 210 Results (Continued)

\begin{tabular}{|c|c|c|c|c|c|}
\hline Call & Array & $\begin{array}{l}\text { Movemant } \\
\text { Diot } \\
\text { (om) }\end{array}$ & $\mathbf{M}$ & Pleoomont & Commont \\
\hline 84 & 1 & 0 & 0 & dich & \\
\hline 6 & 1 & 0 & 0 & dich & \\
\hline $\boldsymbol{0}$ & 7 & 0 & 0 & dich & \\
\hline 97 & 8 & 0 & 0 & $\operatorname{dish}$ & \\
\hline e8 & 8 & 70 & 226 & dich & \\
\hline 9 & 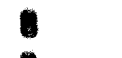 & 0 & 0 & dich & \\
\hline 100 & A & 0 & 0 & dich & \\
\hline 101 & - & 76 & 198 & dish & \\
\hline 102 & $\theta$ & 0 & 0 & dich & \\
\hline 103 & 8 & 0 & 0 & dich & \\
\hline 105 & ? & 0 & 0 & dich & \\
\hline 100 & $?$ & 0 & 0 & dich & \\
\hline 107 & 9 & 0 & 0 & dich & \\
\hline 100 & $?$ & 0 & 0 & dich & \\
\hline $\begin{array}{l}100 \\
110\end{array}$ & 9 & $\begin{array}{l}0 \\
0\end{array}$ & 0 & $\begin{array}{l}\text { dich } \\
\text { dich }\end{array}$ & \\
\hline $\begin{array}{l}110 \\
111\end{array}$ & 0 & $\begin{array}{l}0 \\
0\end{array}$ & 0 & dinen & \\
\hline 112 & 0 & 0 & 0 & dich & \\
\hline 113 & 9 & 0 & 0 & dien & \\
\hline 114 & 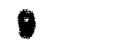 & 0 & 0 & dich & \\
\hline 116 & 10 & 0 & 0 & dich & \\
\hline 110 & 10 & 0 & 0 & dich & \\
\hline 117 & 10 & 0 & 0 & dich & \\
\hline 118 & 10 & 0 & 0 & dich & \\
\hline 110 & 10 & 0 & 0 & dien & \\
\hline 120 & 10 & 0 & 0 & dich & \\
\hline 121 & 10 & 0 & 0 & dich & \\
\hline 122 & 0 & 76 & 316 & dich & \\
\hline 123 & 10 & 76 & 46 & dich & \\
\hline 124 & 10 & 0 & 0 & dich & \\
\hline 126 & 11 & 0 & 0 & dich & North of Sllent Camyon \\
\hline 126 & 1 & 0 & 0 & dich & \\
\hline 127 & 11 & 0 & 0 & dich & Same diah as Ball 1160 \\
\hline 120 & 11 & 0 & 0 & dich & Same diah as Ball 1148 \\
\hline 120 & 11 & 0 & 0 & dich & Bame diah as Ball 148 \\
\hline 130 & 11 & 0 & 0 & dich & Bamo diah as Ball 1147 \\
\hline 131 & 11 & 0 & 0 & dich & Same diah os Ball 6140 \\
\hline 132 & 11 & 0 & 0 & dich & Same dich as Ball 146 \\
\hline 133 & 11 & 0 & 0 & dich & Dibh edjecont to Ball 1144 \\
\hline 134 & 11 & 0 & 0 & dich & Dish adjecent to Ball 143 \\
\hline 136 & 11 & 0 & 0 & dich & Dich adjacent to Ball "142 \\
\hline 130 & 11 & 0 & 0 & dieh & Dich adjecent to Ball 1141 \\
\hline 137 & 11 & 51 & 150 & dich & Dish edjecent to Ball 1140 \\
\hline 138 & 11 & 0 & 0 & dien & Diah adjacent to Ball 1130 \\
\hline 130 & 11 & 0 & 0 & dich & Dish adjacent to Ball $\| 138$ \\
\hline
\end{tabular}


Tablo 0. Amarillo sla Resulte (Conoluded)

\begin{tabular}{|c|c|c|c|c|}
\hline Arroy & $\begin{array}{l}\text { Movemant } \\
\text { Didt }\end{array}$ & A & Piccoment & Commont \\
\hline 140 & 0 & 0 & dich & Dish adjacent to Ball 137 \\
\hline 141 & 2.5 & 285 & dich & Dieh edjecent to Ball wi30 \\
\hline 11 & 0 & 0 & dich & Diah adjacent to Ball 136 \\
\hline 11 & 0 & 0 & dich & Diah adjacent to Ball 134 \\
\hline 11 & 0 & 0 & dich & Didi adjacent to Ball 133 \\
\hline 11 & 0 & $\mathbf{0}$ & dich & Same dich as Ball 132 \\
\hline 11 & $\mathbf{0}$ & $\mathbf{0}$ & dich & Same dich as Ball 131 \\
\hline 11 & $\mathbf{0}$ & $\mathbf{0}$ & dich & Same dich as Ball "130 \\
\hline 11 & $\mathbf{0}$ & $\mathbf{0}$ & dich & same dwh as Ball 120 \\
\hline 11 & 0 & 0 & dish & h as Ball 1128 \\
\hline
\end{tabular}




\section{Amarillo Surface Ground Motion}

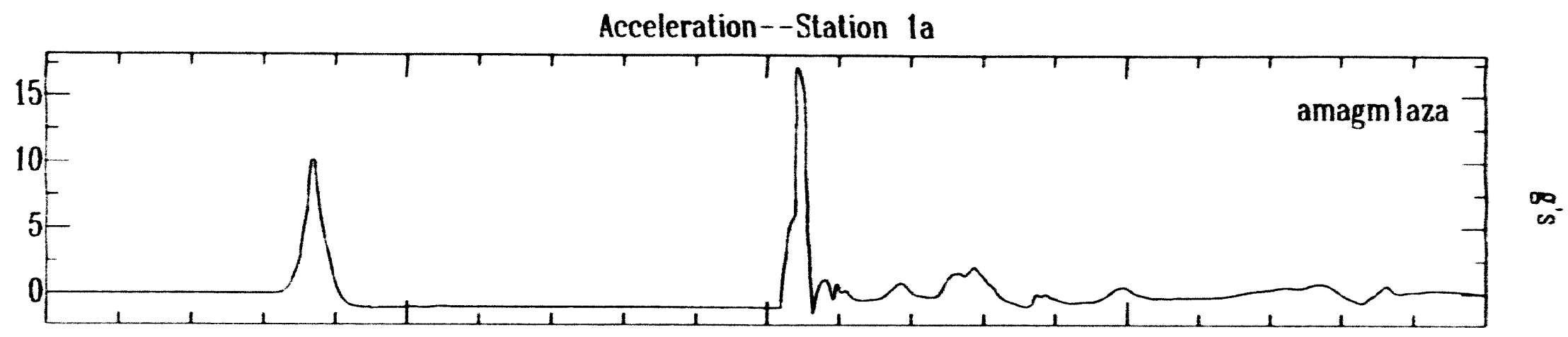

Velocily--Station la

品

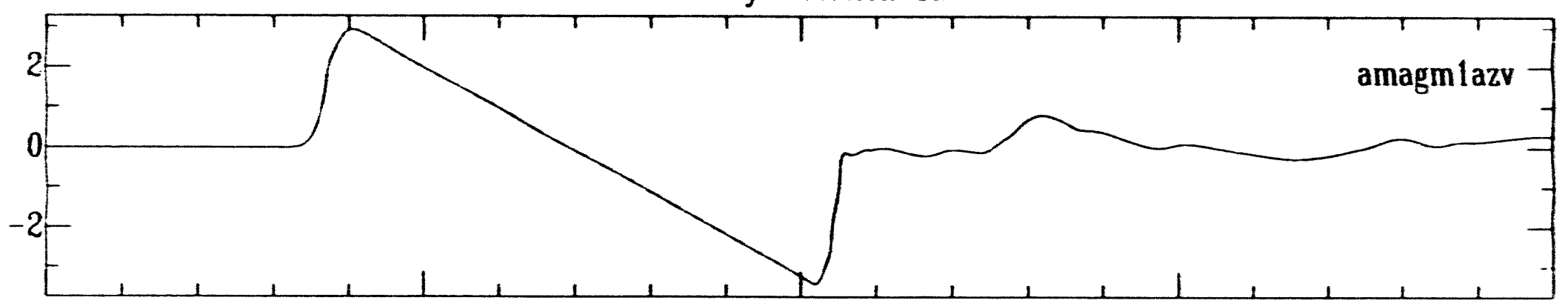

Displacement--Station la

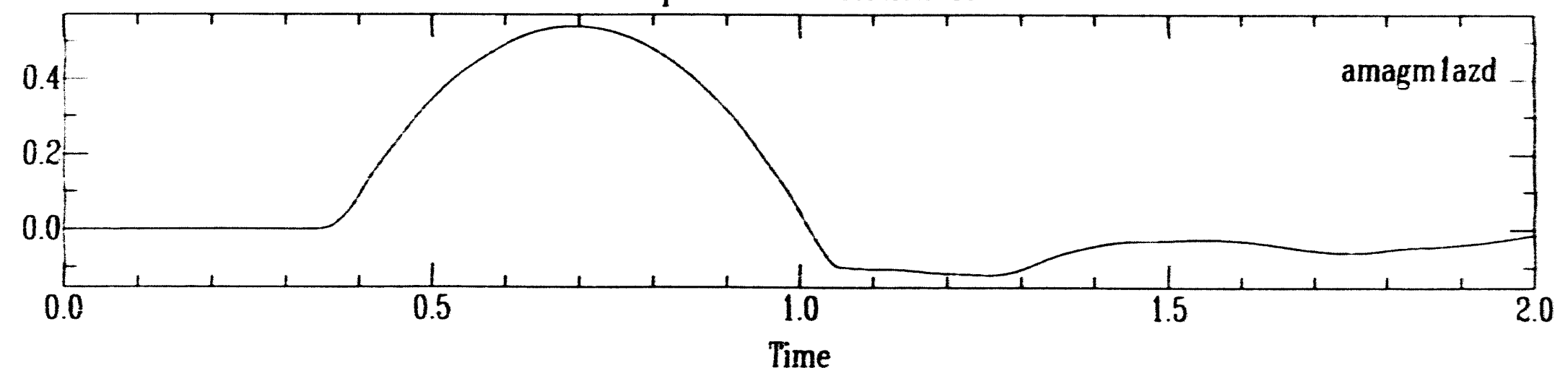




\section{Amarillo Surface Ground Motion}

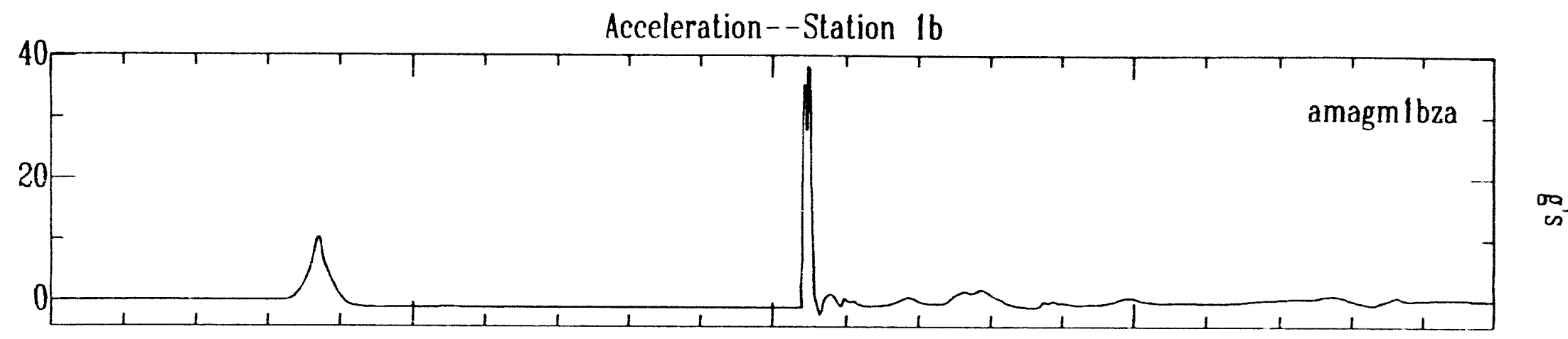

Velocity--Station 1b

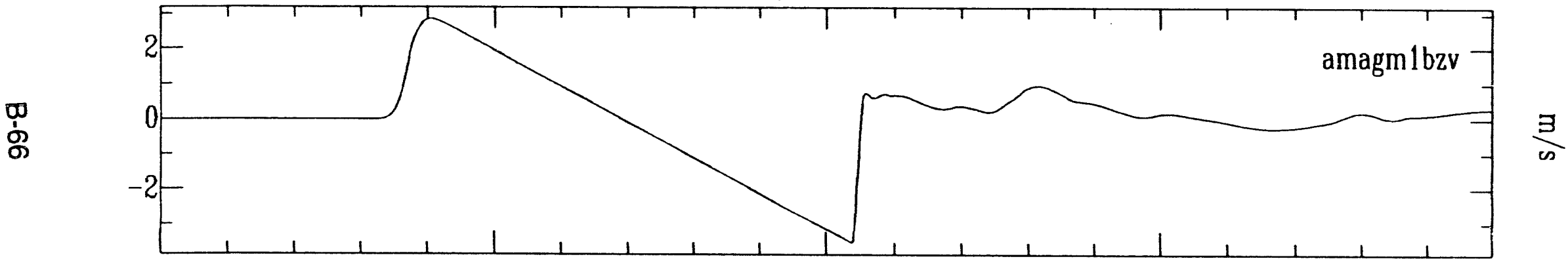

Displacement--Station 1b

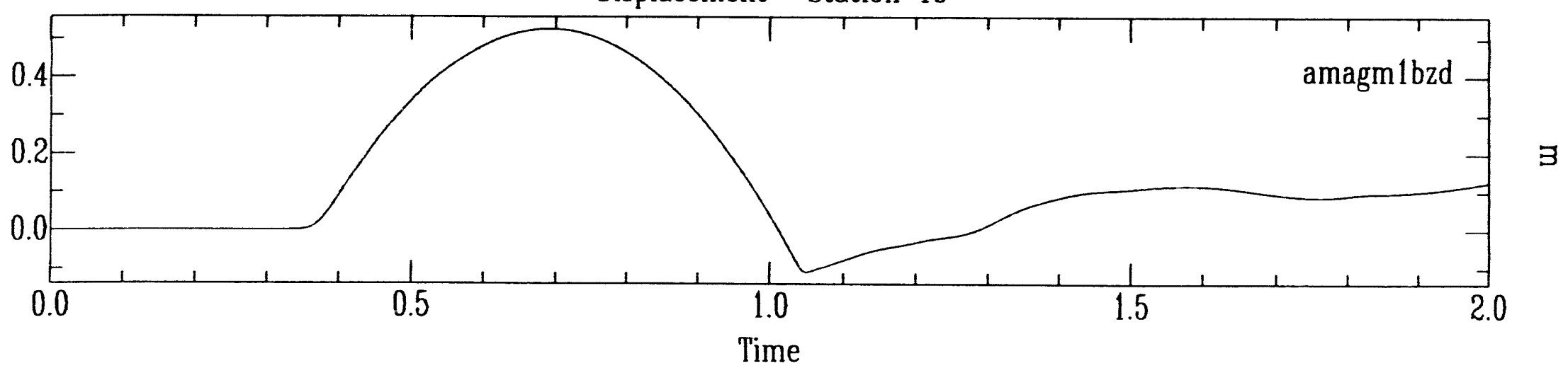




\section{Amarillo Surface Ground Motion}

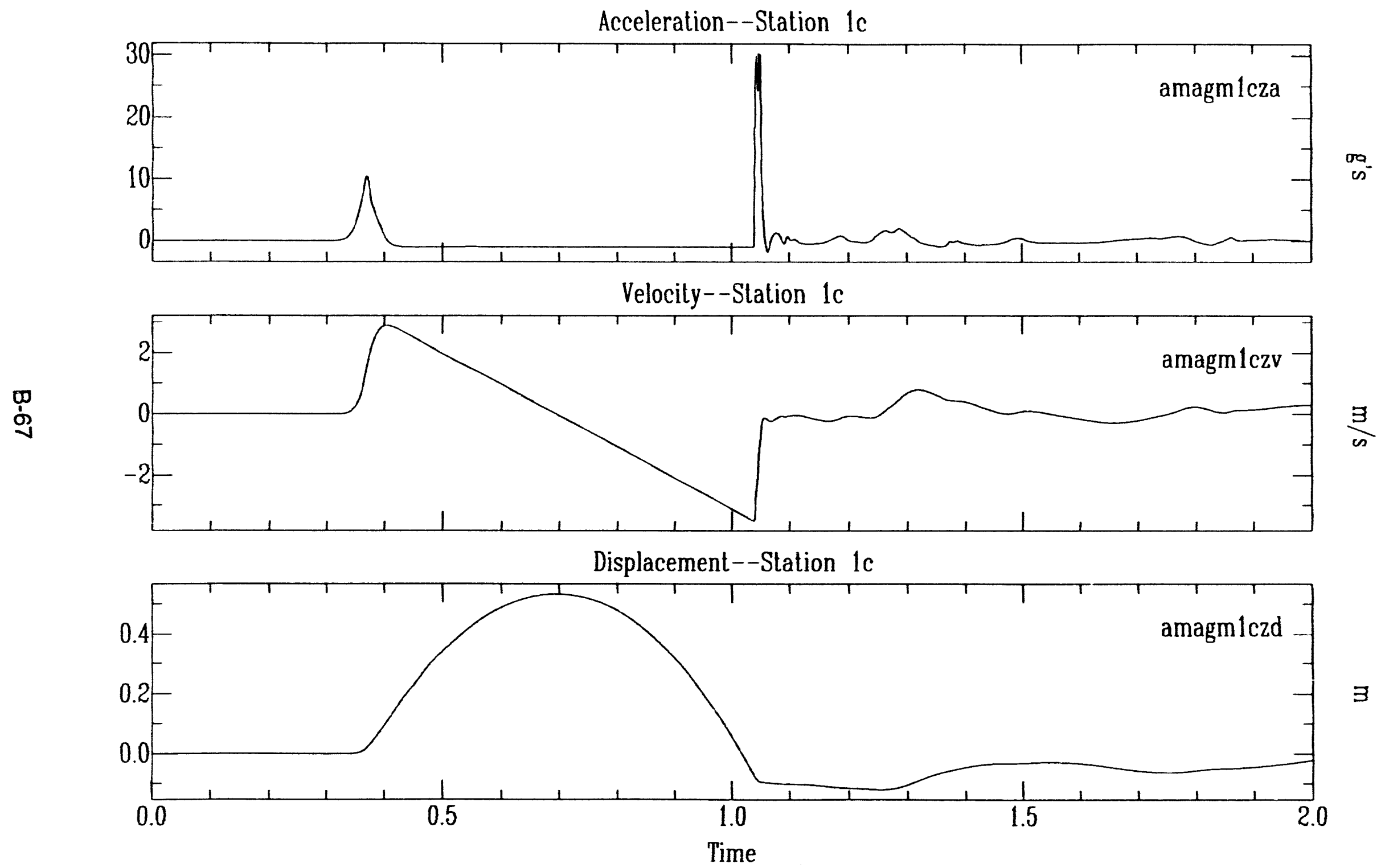




\section{Amarillo Surface Ground Motion}

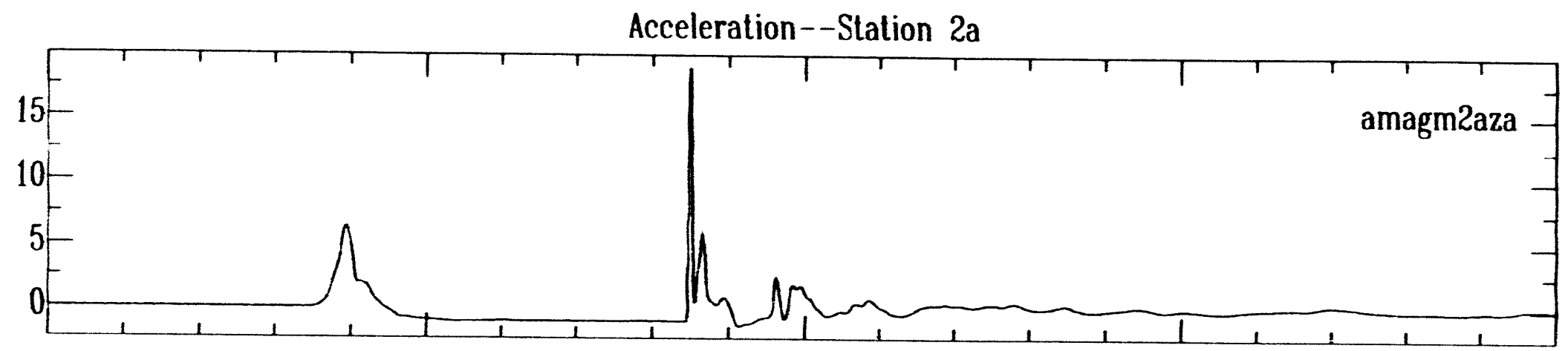

ஷு

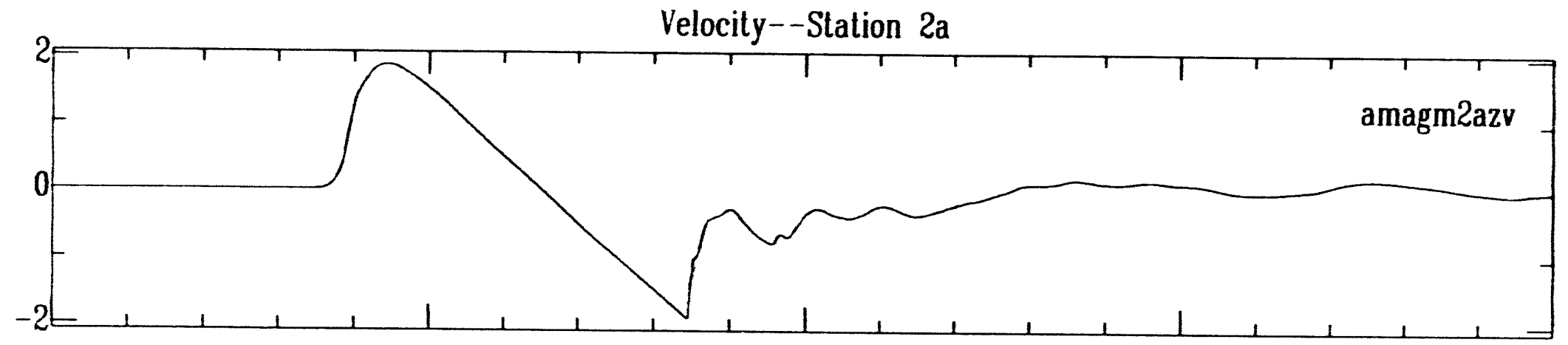

Displacement--Station 2a

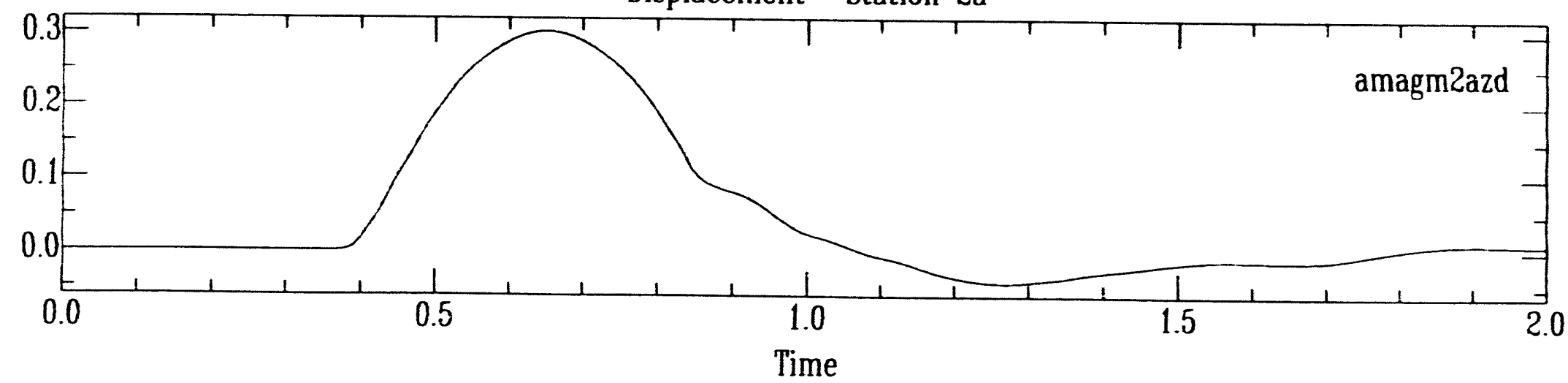




\section{Amarillo Surface Ground Motion}

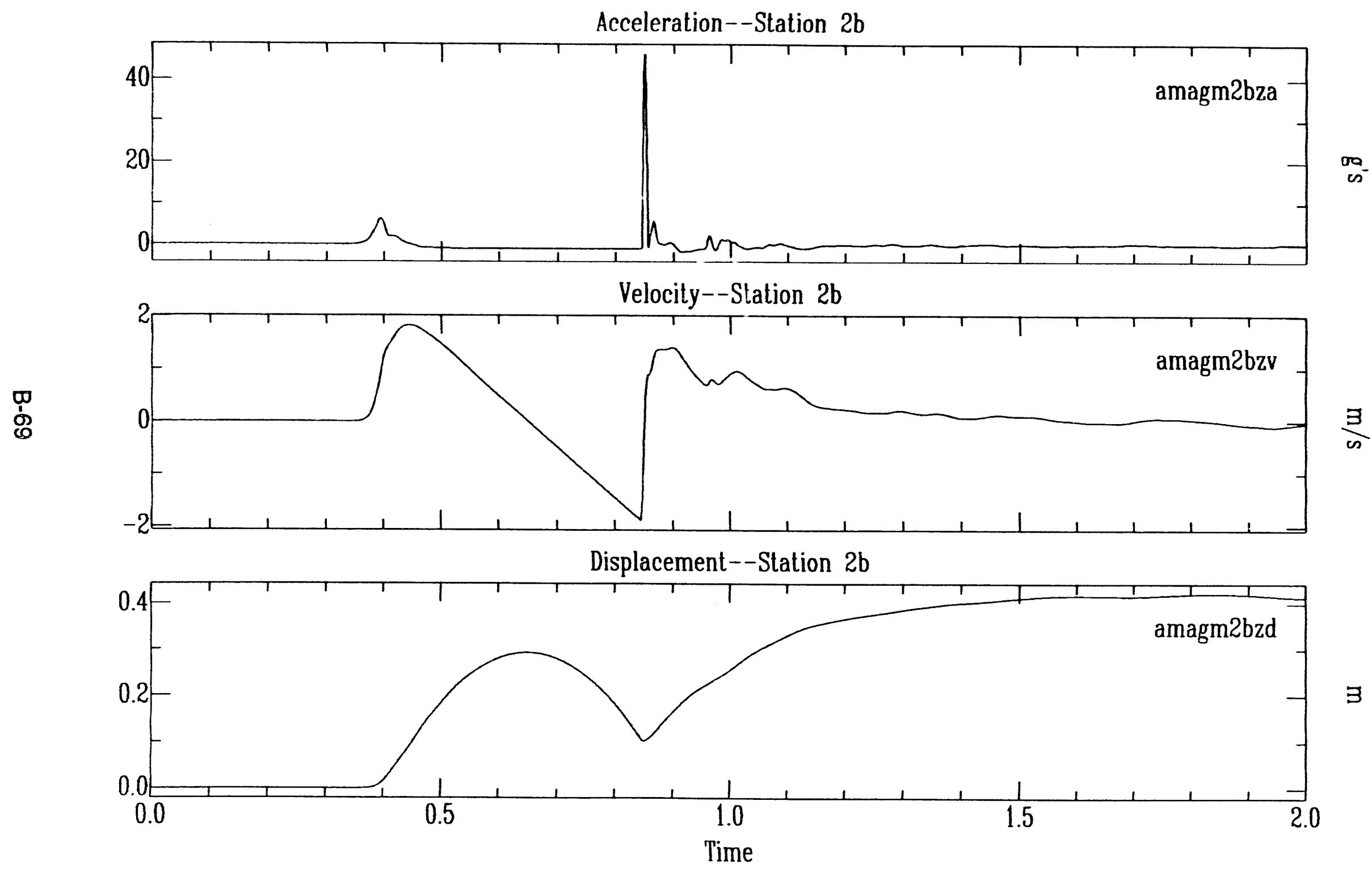




\section{Amarillo Surface Ground Motion}
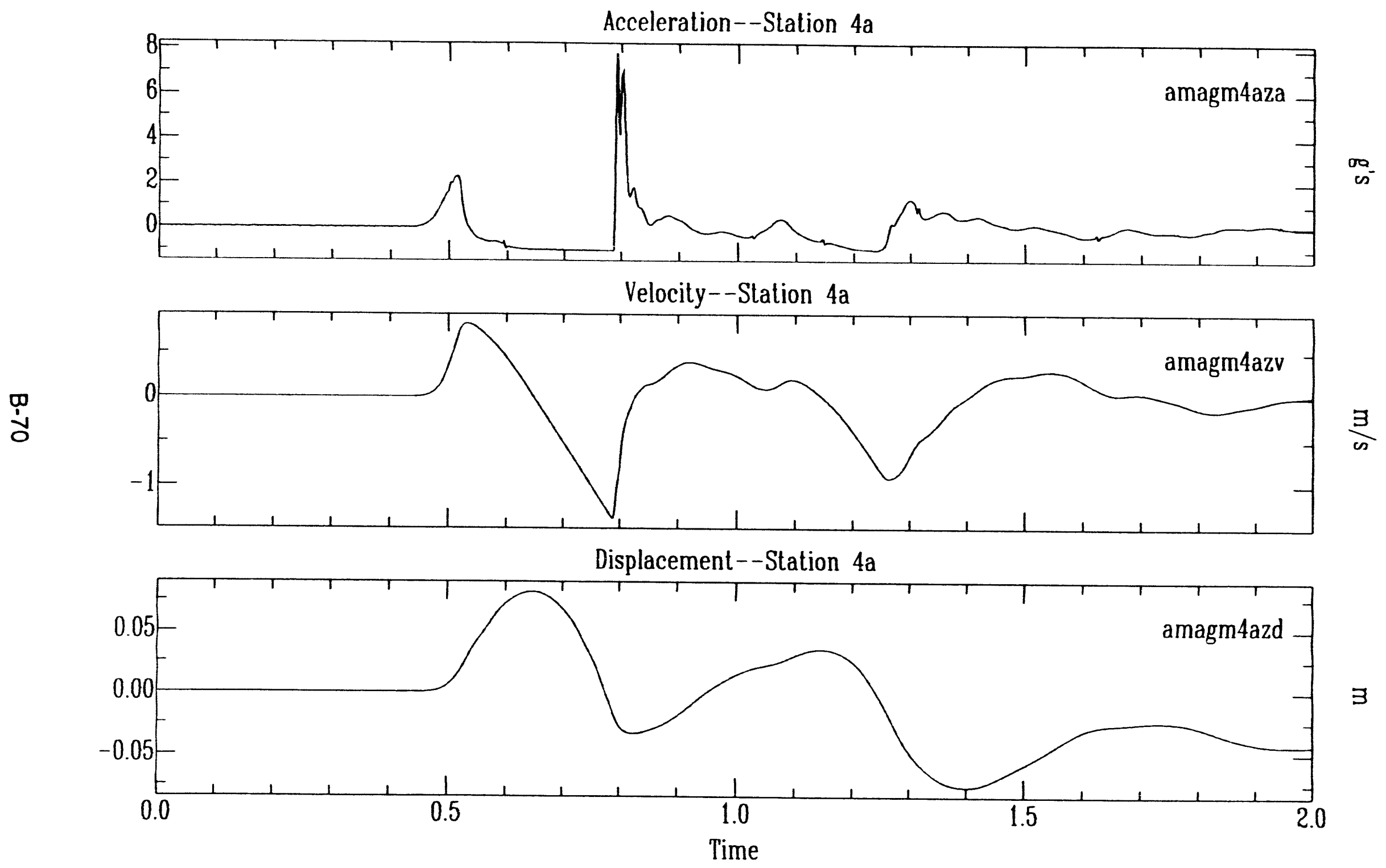


\section{Amarillo Surface Ground Motion}

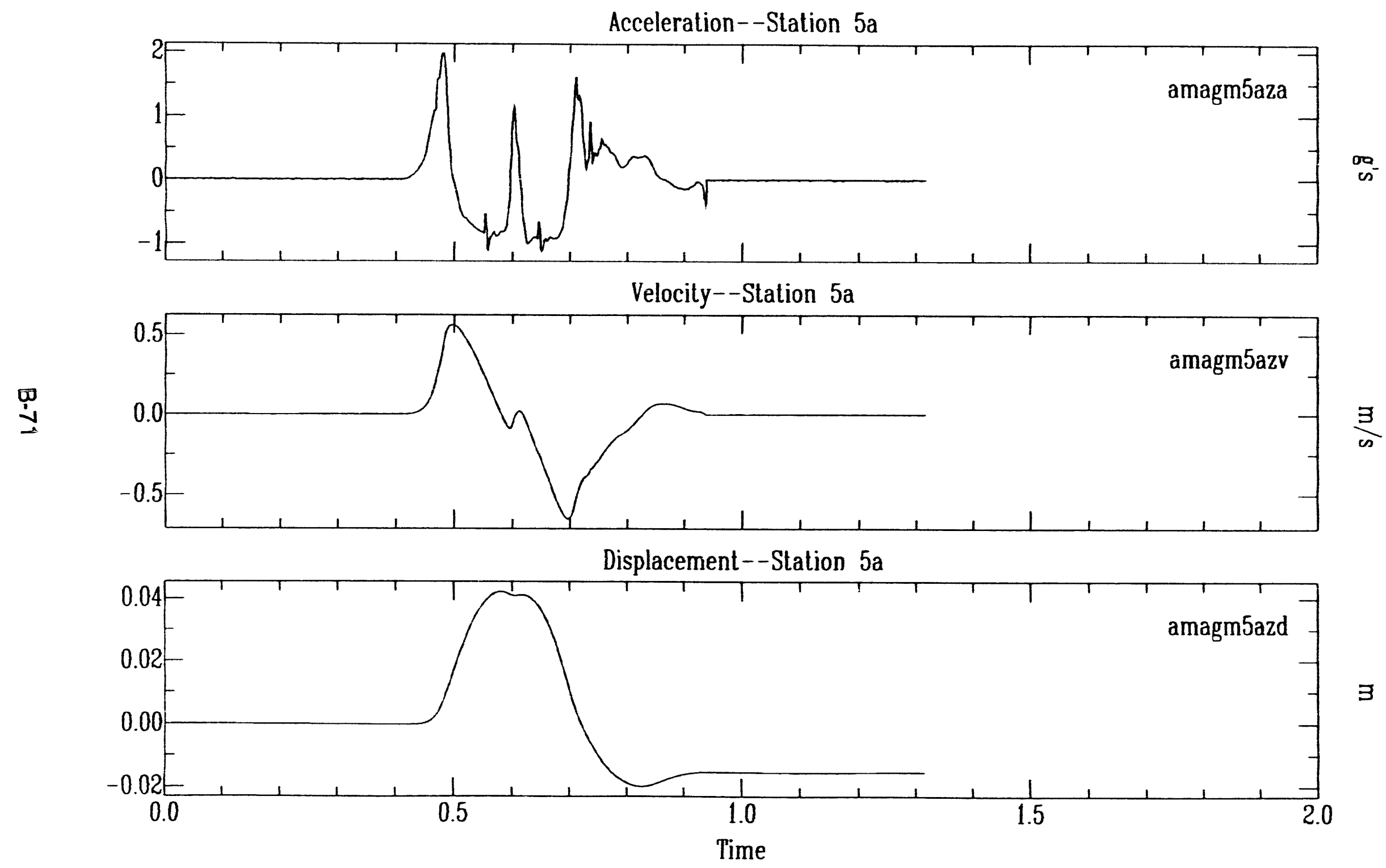




\section{Amarillo Surface Ground Motion}

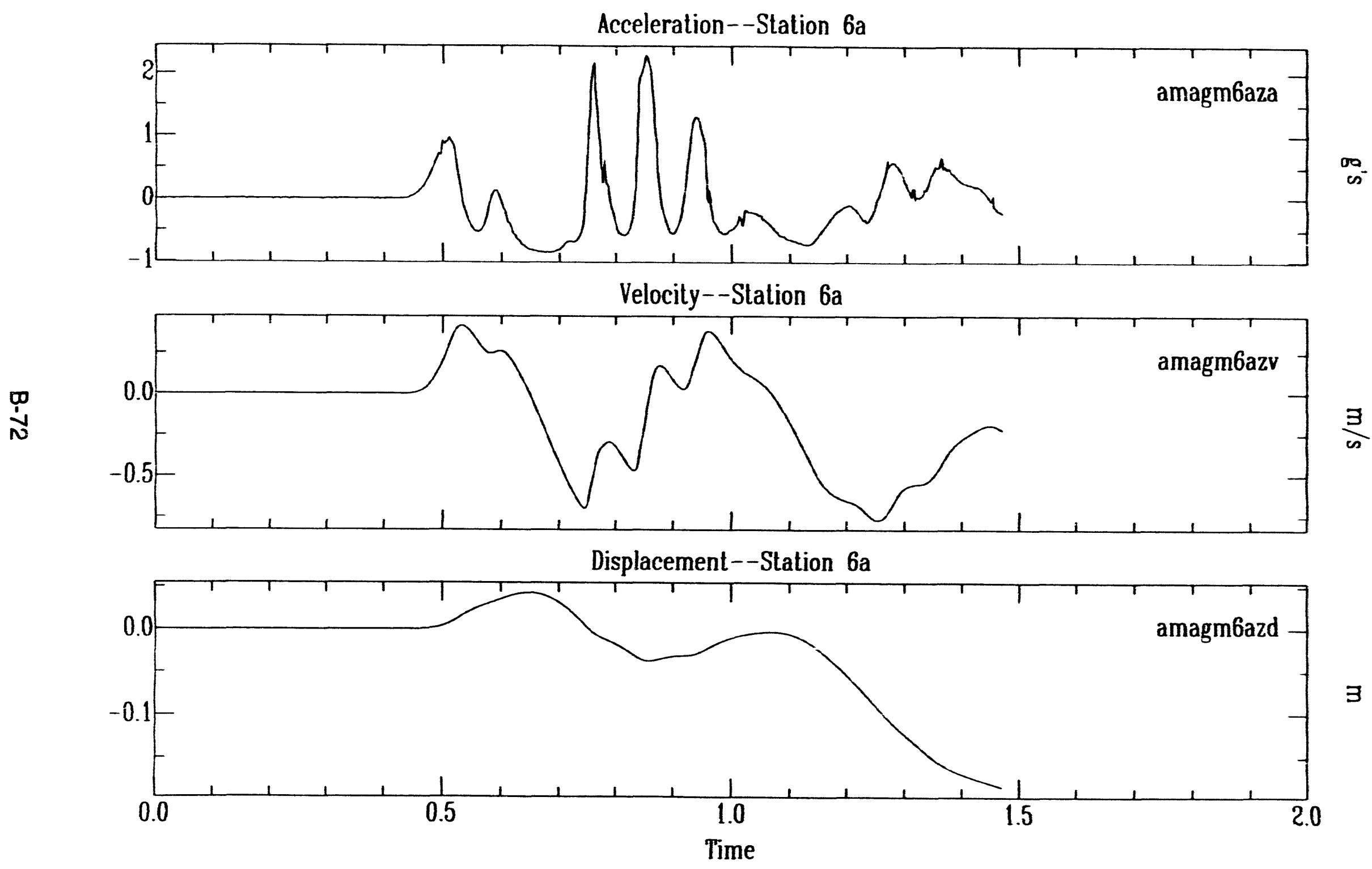




\subsection{Hornltos (U20bc)}

Experiment design. The only ground-motion measurement made on Homitos was the SIG experiment. SIGs were deployed at 147 sites on four azimuths. The azimuths and approximate distances are listed in Table 9, which also lists the postshot observations for each SIG. (For easy reference purposes, these four azimuths are called 'north.' 'east,' 'south.' and 'west.') For this experiment, a golf ball was used as the SIG. At each site, a $15.2-\mathrm{cm}$ diameter plastic dish filled with $-2 \mathrm{~cm}$ of soll was placed on a level cleared area. The golf ball was centered in the dish. Spacing between sites was approximately $30-40 \mathrm{~m}$ (Flg. 10).

SIG results. SIG retrieval was started the atternoon of the event and concluded 2 November 1989. The data from the SIGs are more complex than anticlpated. In many cases, just measuring the azimuth and distance the ball moved would ignore much of the data. The soil in the dishes held the traces of the ball rolling. For that reason, the azimuth and distance values in the table usually indicate the total distance the ball rolled on a single azimuth. In addition, the azimuth and distance on which the initial motion occurred are the ones listed in the table, when that could be determined.

In several instances, the ball rolled forward (forward is defined as the direction toward $S G Z$ ), then rolled backward (backward is away from SGZ), on the same azimuth. The distance is the length of the track, and the azimuth is the direction from the ball origin to its final position. However, at some time, the ball had to roll the opposite direction, $180^{\circ}$ off.

The following is a summary of observations on the SIG movements:

1. Based on SIG data, SGM within the spall zone was not concentric with SGZ. There was no SIG movement west of the Purse fault on the west azimuth (beyond the predicted edge of spall). On the other three azimuths, there was SIG motion at the furthest locations, which were well beyond the predicted edge of spall.

2. SIG movements were significantly different on the northern azimuth than on the other three. Several locations on the north azimuth indicated complex roll patterns, roughly a north-south motion and then an orthogonal east-west motion. On the other three azimuths, rolling was generally on a single azimuth.

3. On the east azimuth SIGs were deployed closer to SGZ than on the other three azimuths. At these closer locations, the SIGs were thrown from their dishes and, at the closest locations, the dishes had moved. None of the dishes were overturned, just moved horizontally. They were probably lofted, as there weren't traces in the dirt of the dishes sliding.

4. All but two of the SIGs at the first 17 locations on the east azimuth were thrown from their dishes. Some factors that may help explain this are: (1) closer 
proximlty to SGZ; (2) much of this part of the traverse is on a hill between two drainages; and (3) locations E.12 through E.17 are in the vicinity of the second drainage where the preexisting tractures were reactivated. Three of the lour SIGs that were thrown out of dishes on the north azimuth are also in tairly close proximity to SGZ. In addition, the north azimuth lollows a drainage that is very Ilkely structurally controlled. However, we have no explanation for the SIGe that were thrown out of dishes on the south azimuth. These locations were on a gentle, north-facing slope. We found very tew cracks or any type of surtace disruption on this slope. In addition, the SIC that moved the most was the furthest location on the south azimuth to be thrown out. If was close to the top of the slope.

5. Initial motion of the SIGs, in general, seems to have been toward SGZ. 


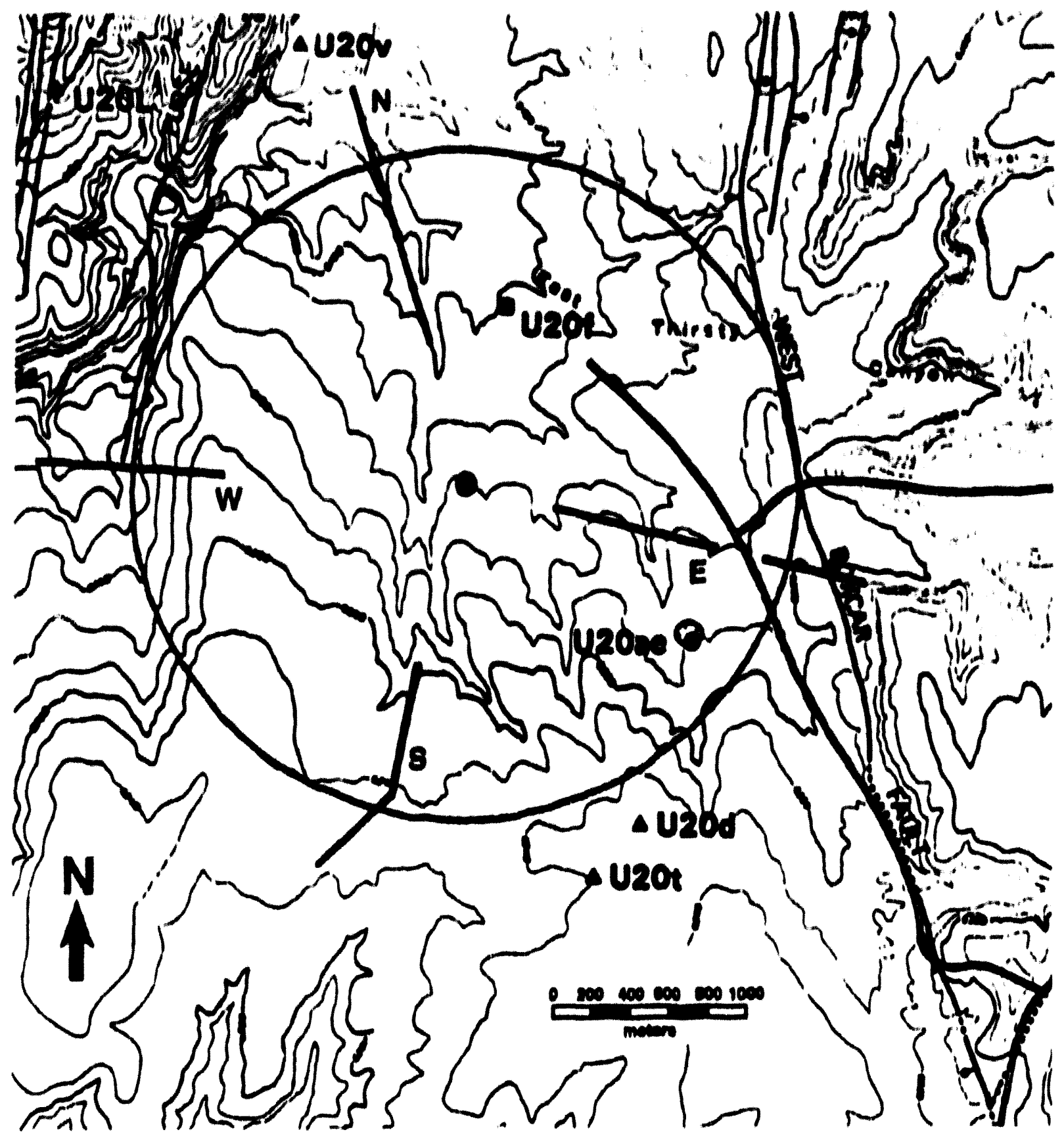

Fig. 10. Hornitos experimental plan. 


\begin{tabular}{|c|c|c|c|c|c|}
\hline Locar & Ard & $\begin{array}{l}\text { Dist } \\
\text { ser } \\
\text { inil }\end{array}$ & Dist & Ad & 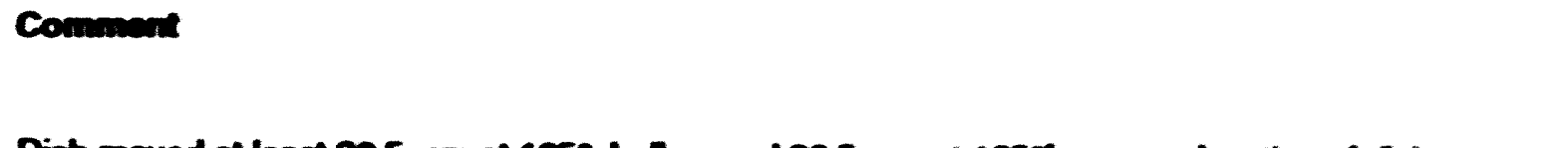 \\
\hline E-1 & 105 & 457 & 55.9 & 145 & Dish moved at least $30.5 \mathrm{~cm}$ al 165\%. bel moved $30.5 \mathrm{~cm}$ at $125 \%$ thom new bection of dish \\
\hline E-2 & 105 & 495 & 53.3 & 105 & Dish moved $-5.1 \mathrm{~cm}$ at 105" bel moved $48.3 \mathrm{~cm}$ from new boction of dish. \\
\hline E-3 & 105 & 533 & 45.7 & 135 & Dish moved $45.7 \mathrm{~cm}$ a $105^{\circ}$. bell moved $229 \mathrm{~cm}$ a $195^{\circ}$ som dish \\
\hline E-4 & 105 & 572 & 15.2 & 310 & Thrown hom dish. \\
\hline E-5 & 105 & 610 & 43.2 & 65 & Dish moved $12.7 \mathrm{~cm}$ at $105^{\circ}$, bell moved $38.1 \mathrm{~cm}$ a $75^{\circ}$ thom dish \\
\hline E-6 & 105 & 648 & 20.3 & 115 & Dish moved $20.3 \mathrm{~cm}$ at 115". ball stayed in dish. \\
\hline E-7 & 105 & 686 & 30.5 & 120 & Thrown from dish \\
\hline E-8 & 105 & 724 & 20.3 & 135 & Thrown tran dish. Crack $0.6 \mathrm{~m}$ west of ofh heeding $40^{\circ}-220^{\circ}$. \\
\hline E-9 & 105 & 762 & 33.0 & 150 & Thrown from dish \\
\hline E-10 & 105 & 800 & 20.3 & 135 & Thrown from dish, oift in dish stined b 135". \\
\hline E-11 & 105 & 838 & 10.6 & 75 & Thrown from dish. \\
\hline E-12 & 105 & 876 & 38.1 & 110 & 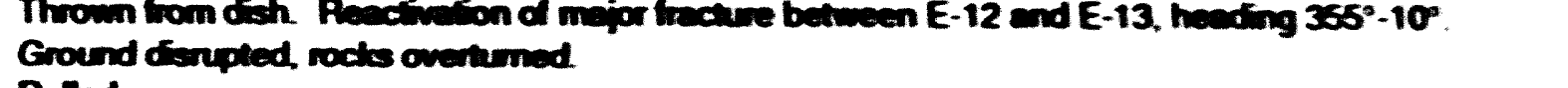 \\
\hline E-13 & 105 & 914 & 7.6 & 110 & Pollod \\
\hline E-14 & 105 & 953 & 0 & $\mathbf{0}$ & \\
\hline $\begin{array}{l}E-15 \\
E-16\end{array}$ & 100 & 991 & 11.8 & So & Thrown from dish. Reactivaled cracts between E-15 and E-16. heading 15'-25". \\
\hline E-17 & 105 & 1067 & 20.3 & 165 & 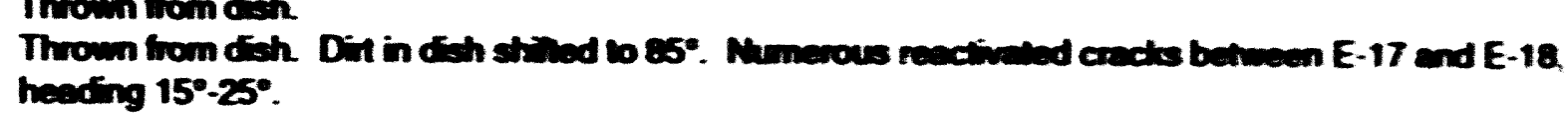 \\
\hline E-18 & 105 & 1105 & $\mathbf{0}$ & $\mathbf{0}$ & Fiolled in dish, ball was shilied, but ended up at origin. \\
\hline E-19 & 105 & 1143 & 5.1 & 285 & Rolled in dish, bell wes shilled, but ended up at origin. \\
\hline E-20 & 105 & 1181 & 7.6 & 265 & Rolled. \\
\hline E-21 & 105 & 1219 & 5.1 & 290 & Rolled \\
\hline $\begin{array}{l}E-22 \\
E-23\end{array}$ & $\begin{array}{l}105 \\
105\end{array}$ & $\begin{array}{l}1257 \\
1524\end{array}$ & $\begin{array}{l}3.8 \\
0\end{array}$ & $\begin{array}{l}245 \\
0\end{array}$ & Rolled. \\
\hline E-24 & 105 & 1562 & 25 & 275 & Polled. \\
\hline E-25 & 105 & 1600 & $\mathbf{0}$ & $\mathbf{0}$ & \\
\hline E-26 & 105 & 1638 & 5.1 & 275 & Movement questionable, rebbit tracks in vicinify. \\
\hline E-27 & 105 & 1676 & 7.6 & 110 & Pollod. \\
\hline E-28 & 105 & 1715 & 7.6 & 245 & \\
\hline E-29 & 105 & 1753 & 7.6 & 255 & Polled beck and forth \\
\hline$E-30$ & 100 & 1791 & 2.5 & 245 & Rolled \\
\hline E- & 100 & 100 & 10.2 & 100 & 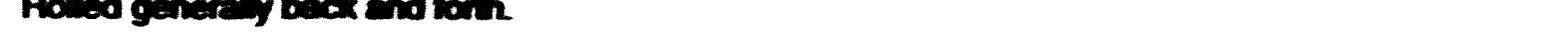 \\
\hline
\end{tabular}


8

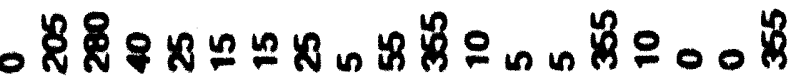

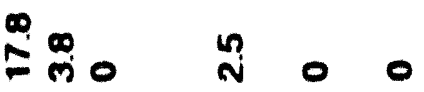

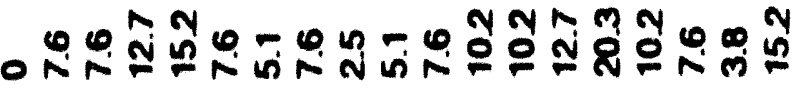

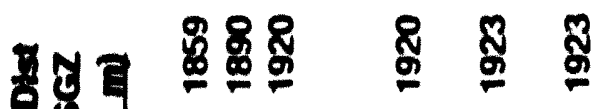

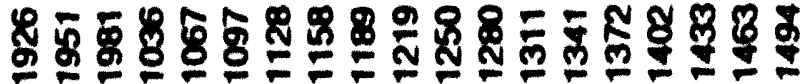

可重数

888 \& 8

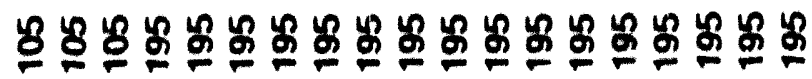

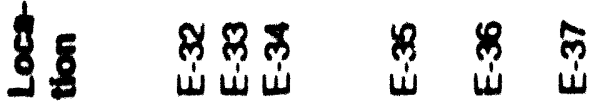

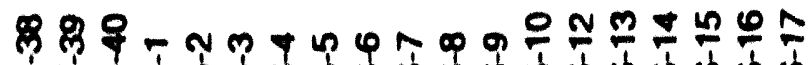

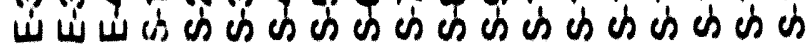




\begin{tabular}{|c|c|c|c|c|c|}
\hline $\begin{array}{l}\text { Loca- } \\
\text { tion }\end{array}$ & $\begin{array}{l}\text { Ad } \\
\text { from } \\
\text { (dea) }\end{array}$ & $\begin{array}{c}\text { Dist } \\
\text { SGZ } \\
m \text { II }\end{array}$ & $\begin{array}{c}\text { Dist } \\
\text { Wow } \\
\text { (cmi) }\end{array}$ & $\begin{array}{l}\text { Axd } \\
\text { (d)ep) }\end{array}$ & Commont \\
\hline S-18 & 195 & 1524 & 10.2 & 15 & Rolled back and forth. \\
\hline S-19 & 195 & 1554 & 15.2 & 20 & Rolled back and forth. \\
\hline S-20 & 195 & 1585 & 162.6 & 45 & Thrown out of dish. \\
\hline S-21 & 195 & 1615 & 15.2 & 5 & Rolled back and forth. \\
\hline S-22 & 195 & 1646 & 152 & 5 & Rolled back and forth. \\
\hline S-23 & 196 & 1676 & 6.4 & 15 & Rolled back and forth. \\
\hline $\begin{array}{l}\text { S-24 } \\
\text { S-25 }\end{array}$ & $\begin{array}{l}196 \\
197\end{array}$ & $\begin{array}{l}1707 \\
1737\end{array}$ & $\begin{array}{l}3.8 \\
3.8\end{array}$ & 15 & Rolled forward. \\
\hline $\begin{array}{l}\text { S-25 } \\
\text { S-26 }\end{array}$ & $\begin{array}{l}197 \\
197\end{array}$ & $\begin{array}{l}1737 \\
1768\end{array}$ & $\begin{array}{l}3.8 \\
6.4\end{array}$ & $\begin{array}{l}15 \\
355\end{array}$ & $\begin{array}{l}\text { Rolled forwand. } \\
\text { Rolled beck and forth }\end{array}$ \\
\hline S-27 & 198 & $\begin{array}{l}1768 \\
1798\end{array}$ & $\begin{array}{l}6.4 \\
7.6\end{array}$ & 350 & $\begin{array}{l}\text { Rolled back and forth. } \\
\text { Rolled beck and forth. }\end{array}$ \\
\hline S-28 & 198 & 1829 & 10.2 & 355 & Rolled beck and forth. \\
\hline S-29 & 199 & 1859 & 8.9 & 5 & Rolled beck and forth. \\
\hline o S-30 & 199 & 1890 & 5.1 & 30 & Rolled. \\
\hline S-31 & 200 & 1920 & 7.6 & $\mathbf{3 0}$ & Rolled. \\
\hline$\infty$ s-32 & 200 & 1951 & 12.7 & 10 & Rolled back and forth. \\
\hline S-33 & 200 & 1981 & 12.7 & 20 & Rolled beck and forth. \\
\hline S-34 & 201 & 2012 & 10.2 & 5 & Rolled beck and forth. \\
\hline S-35 & 201 & 2042 & 10.2 & 5 & Rolled back and forth. \\
\hline$W-1$ & 275 & 1250 & 12.7 & 135 & Rolled back and forth. \\
\hline W-2 & 275 & 1280 & 7.6 & 100 & Rolled back and forth. \\
\hline $\mathbf{W}-3$ & 275 & 1311 & 12.7 & 100 & $\begin{array}{l}\text { Polled back and forth; minor cracking } 3 \mathrm{~m} \text { east of dish, heading } 15^{\circ} \text {; crack } 6 \mathrm{~m} \text { west of dish. } \\
\text { heading } 15^{\circ}-25^{\circ} \text {; reectivaled crack } 3 \mathrm{~m} \text { west of dish, heading } \mathrm{N} \text {. }\end{array}$ \\
\hline S.W-4 & 275 & 1341 & 12.7 & 115 & Rolled back and forth. \\
\hline W-5 & 275 & 1372 & $\mathbf{0}$ & $\mathbf{0}$ & \\
\hline W-6 & 275 & 1402 & 3.8 & 100 & Rolled. \\
\hline $\begin{array}{ll}W-7 \\
w-0\end{array}$ & 275 & 1433 & 0 & 0 & \\
\hline $\begin{array}{l}W-8 \\
W-9\end{array}$ & $\begin{array}{l}275 \\
275\end{array}$ & $\begin{array}{l}1463 \\
1494\end{array}$ & $\begin{array}{l}3.8 \\
0\end{array}$ & $\begin{array}{l}285 \\
0\end{array}$ & Rolled backward. \\
\hline $\mathbf{W}-10$ & 275 & 1524 & 0 & 0 & \\
\hline W-11 & 275 & 1554 & $\mathbf{0}$ & $\mathbf{0}$ & \\
\hline W-12 & 275 & 1585 & 3.8 & 110 & Rolled. \\
\hline $\mathbf{W}-13$ & 275 & 1615 & 12.7 & 115 & Rolled beck and forth. \\
\hline
\end{tabular}


Table 9. Hornilos SIG Data (Continuad)

\begin{tabular}{|c|c|c|c|c|}
\hline $\begin{array}{l}\text { Loca- } \\
\text { tion }\end{array}$ & $\begin{array}{l}\text { Ad } \\
\text { from } \\
\text { (deal }\end{array}$ & $\begin{array}{l}\text { Dist } \\
\text { SGZ } \\
\text { mI }\end{array}$ & $\begin{array}{l}\text { Dist } \\
\text { Mov } \\
\text { (cm) }\end{array}$ & $\begin{array}{l}\text { Axd } \\
\text { ed } \\
\text { (dege }\end{array}$ \\
\hline W-14 & 275 & 1646 & 1.3 & 140 \\
\hline W-15 & 275 & 1676 & 0 & 0 \\
\hline W-19 & 275 & 1737 & 0 & 0 \\
\hline$W-16$ & 275 & 1707 & 0 & 0 \\
\hline W-17 & 275 & 1707 & 0 & 0 \\
\hline$W-18$ & 275 & 1710 & 0 & 0 \\
\hline$W-20$ & 275 & 1768 & 0 & 0 \\
\hline W-21 & 275 & 1798 & 0 & 0 \\
\hline W-22 & 275 & 1829 & 0 & 0 \\
\hline W-23 & 275 & 1859 & 0 & 0 \\
\hline W-24 & 275 & 1890 & 0 & 0 \\
\hline$W-25$ & 275 & 1920 & 0 & 0 \\
\hline W-26 & 275 & 1951 & 0 & 0 \\
\hline W-27 & 275 & 1981 & 0 & 0 \\
\hline W-28 & 275 & 2012 & 0 & 0 \\
\hline W-29 & 275 & 2042 & 0 & 0 \\
\hline$w-30$ & 275 & 2073 & 0 & 0 \\
\hline W-31 & 275 & 2103 & 0 & 0 \\
\hline w-32 & 275 & 2134 & 0 & 0 \\
\hline W-33 & 275 & 2164 & 0 & 0 \\
\hline W-34 & 275 & 2195 & 0 & 0 \\
\hline W-35 & 275 & 2225 & 0 & 0 \\
\hline$N-1$ & 345 & 762 & 12.7 & 325 \\
\hline $\mathrm{N}-2$ & 345 & 800 & 0 & 0 \\
\hline $\mathrm{N}-3$ & 345 & 838 & 12.7 & 280 \\
\hline$N-4$ & 345 & 876 & 2.5 & 140 \\
\hline $\mathrm{N}-5$ & 345 & 914 & 5.1 & 355 \\
\hline$N-6$ & 345 & 953 & 2.5 & 310 \\
\hline N-7 & 345 & 991 & 10.2 & 350 \\
\hline $\mathrm{N}-8$ & 345 & 1029 & 10.2 & 350 \\
\hline
\end{tabular}

Commont

Rolled. Reactivation of old fracture $9 \mathrm{~m}$ east of dish, heading $0^{\circ}$.

Location: $1.5 \mathrm{~m}$ south of $W-16$, east side of Purse fault.

Location: $6 \mathrm{~m}$ west of $W-16$, west side of Purse faul.

$20 \mathrm{~m}$ west of $W$-20-hairline crack on old pressure ridge, but animal burrow $3 \mathrm{~m}$ north not affected.

Thrown out of dish.

Major fracture $4.5 \mathrm{~m}$ north of dish not reactivated $\left(300^{\circ}\right)$.

Thrown out of dish. Minor reactivation of major fracture $2.5 \mathrm{~m}$ south of dish.

Rolled.

Dirt shifted in dish toward $280^{\circ}$.

Rolled.

Thrown out of dish; dirt in dish shifled.

Rolled back and forth on $350^{\circ}$ azimuth, then rolled heading $235^{\circ}$, and stopped at edge. Crack $3 \mathrm{~m}$ north of dish into gully bottom and up the other side, heading $75^{\circ}-125^{\circ}$. Lost crack in the rocks. 
Table 9. Hornitos SIG Data (Continued)

\begin{tabular}{|c|c|c|c|c|}
\hline $\begin{array}{l}\text { Loca- } \\
\text { tion }\end{array}$ & $\begin{array}{l}\text { Azl } \\
\text { from } \\
\text { (deg) }\end{array}$ & $\begin{array}{c}\text { Dist } \\
\text { sGZ } \\
\text { ml }\end{array}$ & $\begin{array}{l}\text { Dist } \\
\text { Move } \\
(\mathrm{cm})\end{array}$ & $\begin{array}{l}\text { Azl } \\
\text { ed } \\
\text { (deg) }\end{array}$ \\
\hline $\mathrm{N}-9$ & 345 & 1067 & 7.6 & 165 \\
\hline $\mathrm{N}-10$ & 345 & 1105 & 7.6 & 0 \\
\hline $\mathrm{N}-11$ & 345 & 1143 & 10.2 & 280 \\
\hline $\mathrm{N}-12$ & 345 & 1181 & 7.6 & 115 \\
\hline $\mathrm{N}-13$ & 345 & 1219 & 7.6 & 160 \\
\hline $\mathrm{N}-14$ & 345 & 1257 & 2.5 & 180 \\
\hline $\mathrm{N}-15$ & 345 & 1295 & 15.2 & 205 \\
\hline $\mathrm{N}-16$ & 345 & 1334 & 7.6 & 225 \\
\hline $\mathrm{N}-17$ & 345 & 1372 & 15.2 & 170 \\
\hline $\mathrm{N}-18$ & 345 & 1410 & 3.8 & 45 \\
\hline$N-19$ & 345 & 1448 & 10.2 & 165 \\
\hline $\mathrm{N}-20$ & 345 & 1486 & 12.7 & 350 \\
\hline $\mathrm{N}-21$ & 345 & 1524 & 5.1 & 160 \\
\hline $\mathrm{N}-22$ & 345 & 1524 & 7.6 & 330 \\
\hline $\mathrm{N}-23$ & 345 & 1562 & 7.6 & 325 \\
\hline $\mathrm{N}-24$ & 345 & 1562 & 7.6 & 150 \\
\hline $\mathrm{N}-25$ & 345 & 1600 & 2.5 & 160 \\
\hline $\mathrm{N}-26$ & 345 & 1638 & 7.6 & 295 \\
\hline $\mathrm{N}-27$ & 345 & 1676 & 2.5 & 180 \\
\hline $\mathrm{N}-28$ & 345 & 1715 & 2.5 & 335 \\
\hline $\mathrm{N}-29$ & 345 & 1753 & 0 & 0 \\
\hline $\mathrm{N}-30$ & 345 & 1791 & 5.1 & 235 \\
\hline
\end{tabular}

Comment

Rolled backward (downslope). Rock slide $6 \mathrm{~m}$ north of dish.

Thrown out of dish.

Rolled forward to edge of dish, then rolled along edge of dish both $E$ and $W$.

Rolled forward to edge of dish, then rolled along edge of dish both $E$ and W. Crack halfwe; between.

$\mathrm{N}-13$ and $\mathrm{N}-14$, at $215^{\circ}$.

Rolled forward, then side to side. Crack $3 \mathrm{~m}$ north of dish, discontinuous, general heading $355^{\circ}$.

Rolled back and forth on heading $205^{\circ}$, then to the southeast. Final offset $7.6 \mathrm{~cm}$ at $140^{\circ}$.

Roll pattern in dirt disrupted some. Initial motion may be on $165^{\circ}-345^{\circ}$ azimuth.

Rolled back and forth full $15.2 \mathrm{~cm}$. Final offset $6.4 \mathrm{~cm}$ at $170^{\circ}$. Crack halfway between $\mathrm{N}-16$ and $\mathrm{N}$ 17.

$3 \mathrm{~m}$ from edge of gully.

Rolled. Location: in the bottom of a gully.

Rolled back and forth. Some orthogonal motion toward the NE.

Rolled forward then in an arc toward the east to the back edge of dish. Final offset $7.6 \mathrm{~cm}$ at $350^{\circ}$.

Rolled forward then back to center. Location: 4.5 m east $\left(85^{\circ}\right)$ of $\mathrm{N}-22$.

Rolled back.

Rolled backward $7.6 \mathrm{~cm}$ at $325^{\circ}$, then along edge of dish to $235^{\circ}$. Located $9 \mathrm{~m}$ east of $\mathrm{N}-24$, on hard.

rock outcrop.

Rolled back and forth.

Rolled.

Roll pattern partially disrupted; some rolling on azimuth $150^{\circ}-330^{\circ}$. Cracks $3 \mathrm{~m}$ north of dish, general.

heading $50^{\circ}$ along gully bottom; crack spacing $4.5 \mathrm{~m}$ from each other.

Unknown roll pattern.

Rolled backward.

Strange roll pattern: segment $2.5 \mathrm{~cm}$ at $150^{\circ}$, at each end of this segment, a $5.1 \mathrm{~cm}$ arc orthogonal toward the west. Final offset $5.1 \mathrm{~cm}$ at $235^{\circ}$. 


\section{Table 9. Hornitos SIG Data (Concluded)}

\begin{tabular}{|c|c|c|c|c|c|}
\hline $\begin{array}{l}\text { Loca- } \\
\text { tion }\end{array}$ & $\begin{array}{l}\text { Azl } \\
\text { from } \\
\text { (deg) }\end{array}$ & $\begin{array}{l}\text { Dist } \\
\text { SGZ } \\
\text { m) }\end{array}$ & $\begin{array}{c}\text { Dist } \\
\text { Move } \\
\text { (cm) }\end{array}$ & $\begin{array}{l}\text { Azi } \\
\text { ed } \\
\text { (deg) }\end{array}$ & Comment \\
\hline N-31 & 345 & 1829 & 0 & 0 & Very minor hairline cracks on major fractures located $1.5 \mathrm{~m}$ northwest of dish, heading $350^{\circ}$. \\
\hline N-32 & 345 & 1867 & 7.6 & 160 & $\begin{array}{l}\text { Two roll segments: } 7.6 \mathrm{~cm} \text { at } 160^{\circ} \text {, then orthogonal set } 7.6 \mathrm{~cm} \text { at } 225^{\circ} \text { (final position). Roll tracks } \\
\text { cross. Crack heading } 340^{\circ} \text { close to dish. }\end{array}$ \\
\hline $\begin{array}{l}N-33 \\
N-34\end{array}$ & $\begin{array}{l}345 \\
345\end{array}$ & $\begin{array}{l}1905 \\
1943\end{array}$ & $\begin{array}{l}10.2 \\
0\end{array}$ & $\begin{array}{l}220 \\
0\end{array}$ & Strange roll pattern: $10.2 \mathrm{~cm}$ at $220^{\circ}, 5.1 \mathrm{~cm}$ at $325^{\circ}$, and $3.8 \mathrm{~cm}$ at $285^{\circ}$ (final position). \\
\hline $\mathrm{N}-35$ & 345 & 1981 & 0 & 0 & Small crack heading $45^{\circ}, 0.3 \mathrm{~m}$ west of dish. \\
\hline N-36 & 345 & 2019 & 12.7 & 180 & Rolled back and forth. \\
\hline $\mathrm{N}-37$ & 345 & 2057 & 10.2 & 195 & Rolled back and forth. \\
\hline
\end{tabular}

0
$\dot{1}$
0 
EES-3 


\subsection{Metropolis (U2gg)}

Experiment design. To better define a spall zone and its motions, two separate spallzone experiments were performed on Metropolis. A polar grid, centered on SGZ, was drawn to serve as a starting point for locations of the two experiments. The actual deployment locations are drawn on Figure 11. Instrumentation lines are approximately every $30^{\circ}$ of azimuth, and the distance between circle nodes is approximately $150 \mathrm{~m}$.

Of a possible 108 nodes, 71 were accessible to install arrays (see Table 10). Arrays were also installed at each of the accelerometer locations, even those not at nodes, to provide a method of comparing the movements of the balls to the quantitative measurements taken by the accelerometers.

SIG Experiment. For one experiment, a SIG array of $1-\mathrm{in}$. steel balls and golf balls was placed at each node of the array that was accessible. Although ball arrays cannot give quantitative data on SGM, they can give a more spatially complete characterization of ground motions throughout the spall zone. Analysis of ball motions (sometimes limited to their final resting places) can give some qualitative measure of how the surface reacted to the ground shock: (1) Is the shock impulsive enough to 'launch' the balls? (2) If the balls do not leave the ground, do they show preferential movement, e.g., away from SGZ? (3) Are the surface motions, as evidenced by ball displacements, at a given distance from SGZ of the same magnitude?

A SIG array consisted of 5 steel balls and 2 golf balls, placed in a cross (one crossbar was oriented along the azimuth to SGZ and the other perpendicular). One steel ball and the golf balls were placed at the intersection of the two crossbars, and a steel ball was placed at the end of each $10-\mathrm{m}$ arm. An $8-\mathrm{cm}$ nail was placed under each ball to mark its starting position. In the middle, the steel ball was set on a $25-\mathrm{cm}$ spike, one golf ball was set on a nail, and the other was placed in a $15-\mathrm{cm}$ plastic dish, partially filled with soil, and attached to the ground by a nail. The steel balls were color-coded in order to be able to track their movement: white in the middle, red toward SGZ, blue away from SGZ, orange to the left (when facing SGZ), and green to the right.

Accelerometer Experiment. The secona spall zone experiment involved placing 19 triaxial, force-balanced accelerometer packages about $150 \mathrm{~m}$ inside of and $150 \mathrm{~m}$ outside of the predicted edge of spall (ESA measurements). These quantitative results on the edge of spall were to be compared directly with the results of the SIG experiment and with the predictions of the limit of spall.

These experiments were designed to map and characterize ground motion in the spall $z o n e$ and determine its limits. Some of the questions that these experiments were to address are: (1) Is the spall zone circular? (2) Is the energy in the spall zone equally partitioned? (3) Does the transition from spall to no spall have any distinctive characteristics? (4) Does a collapse chimney in close proximity to an explosion 
capture/attenuate the shock wave? Which signals are affected: surface effects, local ground motion, regional seismic? (5) Does a fault, like the Yucca system, capture/reflect/attenuate the shock wave, and which signals are affected?

In addition to the spall-zone definition experiments, GRMPY fielded 14 stations inside the predicted spall zone.

In Figure 11, stations with solid circles had SIG arrays only; stations denoted by an "F" had a SIG array and an FBA package; stations denoted by a "G" had only a GRMPY station.

SIG Results. Observations of the SIG arrays began about 3 hours after zero time. The ball arrays at 9-8, 9-7, 8-8, 10-8, 11-8, $12-8$ were recorded Saturday, the day of the event. High winds and blowing dust caused fieldwork to be stopped early. Fieldwork was not possible Sunday because of the extremely poor weather conditions. During the following two days, all of the ball arrays were visited and the movements noted. The order in which each location was visited is usually considered very important. However, because most of the subtle effects were significantly altered and obliterated by the weather (i.e., the tracks of the balls rolling on the ground), the chronology is not a factor when analyzing the surface effects data.

In most cases, the balls apparently did not get enough of an impulse from the ground shock to be "launched," i.e., the balls apparently just rolled around on the surface of the ground. At a couple locations, the ground around the array was extremely disrupted. Figure 12 attempts to graphically depict the motions observed. These data are listed in Table 11.

Accelerometer Results. As noted in the section describing ESA measurements, the arrival times for these measurements are not accurate due to the drift in the internal clocks of the digital recorders. Nineteen stations were fielded with portable digital recorders (REFTEKs); GRMPY fielded 14 stations of which seven appear to have been collocated. All of the GRMPY stations appear to have spalled, but only nine of nineteen ESA stations appear to have spalled (METSG3A, 3B, 4A, 4B, 7A, 7B, 8A, 9A and 10A) and of these, four appear to have been right at the edge of spall (4A, 4B, 7A and 8A). The edge of spall as defined by the $1 \mathrm{~g} g$ vertical acceleration limit, from the accelerometer records, is depicted graphically in Figure 13. Note that the spall zone for Metropolis is not circular, and appears to extend outside the predicted zone along the Yucca fault and contract inside the prediction south and northwest of SGZ. Figure 14 (29 pages) contains the data traces for the accelerometer data. 


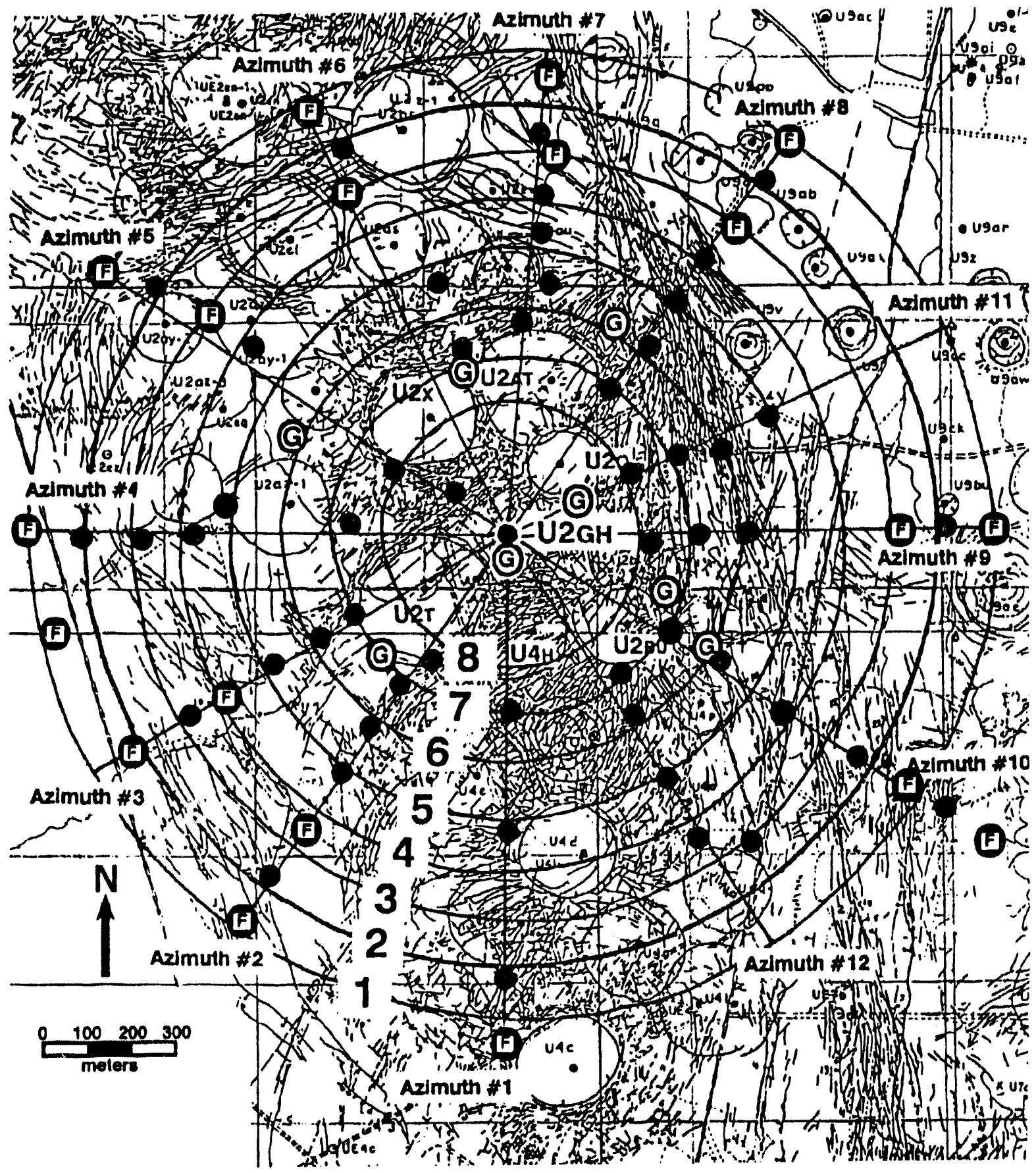

Fig. 11. Metropolis deployment locations. 
Table 10. Metropolis Ground Motion Station Data

\begin{tabular}{|c|c|c|c|c|c|c|}
\hline $\begin{array}{l}\text { Station } \\
\text { Name }\end{array}$ & $\begin{array}{l}\text { Station Loc } \\
\text { U2gh }\end{array}$ & Accelerometer & $\begin{array}{l}\text { Northing (m) } \\
262279\end{array}$ & $\begin{array}{l}\text { Easting }(m) \\
206746\end{array}$ & $\begin{array}{l}\text { Slant Range } \\
469(\mathrm{~m}) \mathrm{DOB}\end{array}$ & TOA \\
\hline METGR!A & $15 \mathrm{~m} \mathrm{~S}$ & Endevco $25 \mathrm{~g}$ & 262266 & 206745 & 469 & \\
\hline METGR2A & $230 \mathrm{~m} \mathrm{~N} 61 \mathrm{E}$ & Endevco $25 \mathrm{~g}$ & 262388 & 206948 & 522 & 0.285 \\
\hline METGR3A & $686 \mathrm{~m}$ N27E & Endevco $25 \mathrm{~g}$ & 262890 & 207056 & 830 & 0.466 \\
\hline METGR4A & $457 \mathrm{~m} \mathrm{S78E}$ & Endevco $25 \mathrm{~g}$ & 262184 & 207192 & 654 & 0.359 \\
\hline METGR5A & $474 \mathrm{~m} \mathrm{N17W}$ & Endevco $25 \mathrm{~g}$ & 262734 & 206610 & 667 & 0.393 \\
\hline METGR6A & $476 \mathrm{~m} \mathrm{~S} 47 \mathrm{~W}$ & Endevco $25 \mathrm{~g}$ & 261953 & 206398 & 669 & 0.387 \\
\hline METGR7A & 686 m S59E & Endevco $25 \mathrm{~g}$ & 261930 & 207335 & 830 & 0.458 \\
\hline METGR8A & $681 \mathrm{~m} \mathrm{N64W}$ & Eridevco $25 \mathrm{~g}$ & 262578 & 206133 & 827 & 0.471 \\
\hline METGR9A & $686 \mathrm{~m} \mathrm{N27E}$ & Endevco $25 \mathrm{~g}$ & 262890 & 207056 & 830 & 0.467 \\
\hline METGR9B & $686 \mathrm{~m}$ N27E & Endevco $25 \mathrm{~g}$ & 262890 & 207056 & 830 & 0.47 \\
\hline METGR9C & $686 \mathrm{~m}$ N27E & Endevco $25 \mathrm{~g}$ & 262890 & 207056 & 830 & 0.466 \\
\hline METGR10A & $686 \mathrm{~m}$ N27E & Endevco $25 \mathrm{~g}$ & 262890 & 207056 & 830 & 0.468 \\
\hline METGR10B & $686 \mathrm{~m} \mathrm{N27E}$ & Endevco $25 \mathrm{~g}$ & 262890 & 207056 & 830 & 0.465 \\
\hline METGR1OC & $686 \mathrm{~m}$ N27E & Endevco $25 \mathrm{~g}$ & 262890 & 207056 & 830 & 0.46 \\
\hline METSG1B & $1160 \mathrm{~m} \mathrm{~S}$ & Terra Tec $5.28 \mathrm{~g}$ & 261119 & 206746 & 1251 & 2.52 \\
\hline METSG2A & 810 m \&35W & Terra Tec $3.7 \mathrm{~g}$ & 261615 & 206281 & 937 & 2.536 \\
\hline METSG2B & $1070 \mathrm{~m} \mathrm{S35W}$ & Terra Tec $5.28 \mathrm{~g}$ & 261403 & 206132 & 1168 & 2.587 \\
\hline METSG3A & 745 m S60W & Terra Tec $3.7 \mathrm{~g}$ & 261569 & 206519 & 881 & 2.512 \\
\hline METSG3B & 994 m S60W & Terra Tec $5.28 \mathrm{~g}$ & 261781 & 205884 & 1100 & 2.553 \\
\hline METSG4A & $1100 \mathrm{~m} \mathrm{S89W}$ & Terra Tec $3.7 \mathrm{~g}$ & 262260 & 205646 & 1196 & 2.552 \\
\hline METSG4B & $1060 \mathrm{~m} \mathrm{N78W}$ & Terra Tec $5.28 \mathrm{~g}$ & 262059 & 205709 & 1159 & 2.578 \\
\hline METSG5A & 845 m N53W & Terra Tec $3.7 \mathrm{~g}$ & 262788 & 206071 & 967 & 2.401 \\
\hline METSG5B & $1100 \mathrm{~m} \mathrm{N56W}$ & Terra Tec $5.28 \mathrm{~g}$ & 262894 & 205834 & 1196 & 2.456 \\
\hline METSG6A & $860 \mathrm{~m} \mathrm{N25W}$ & Terra Tec $3.7 \mathrm{~g}$ & 263058 & 206383 & 979 & 2.526 \\
\hline METSG6B & 1175 m N25W & Terra Tec $5.28 \mathrm{~g}$ & 263344 & 206249 & 1265 & $\begin{array}{l}2.579 \\
2468\end{array}$ \\
\hline METSG7A & $880 \mathrm{~m} \mathrm{N7E}$ & Terra Tec $3.7 \mathrm{~g}$ & 263152 & 206853 & 997 & $\begin{array}{l}2.468 \\
251 ?\end{array}$ \\
\hline METSG7B & $1055 \mathrm{~m}$ N5E & Terra Tec $5.28 \mathrm{~g}$ & 263330 & 206838 & 1155 & 2.512 \\
\hline METSG8A & 875 m N37E & Terra Tec $3.7 \mathrm{~g}$ & 262978 & 207273 & 993 & 2.519 \\
\hline METSG8B & $1105 \mathrm{~m} \mathrm{~N} 36 \mathrm{E}$ & Terra Tec $5.28 \mathrm{~g}$ & 263173 & 207396 & 1201 & 2.583 \\
\hline METSGQA & $890 \mathrm{mE}$ & Terra Tec $3.7 \mathrm{~g}$ & 262279 & 207636 & 1006 & 2.465 \\
\hline METSG9A & $890 \mathrm{mE}$ & Terra Tec $3.7 \mathrm{~g}$ & 262279 & 207636 & 1006 & 2.465 \\
\hline
\end{tabular}


Table 10. Metropolis Ground Motion Station Data (Concluded)

\begin{tabular}{|c|c|c|c|c|c|c|}
\hline $\begin{array}{l}\text { Station } \\
\text { Name }\end{array}$ & $\begin{array}{l}\text { Station Loc } \\
\text { U2gh }\end{array}$ & Accelerometer & $\begin{array}{l}\text { Northing (m) } \\
262279\end{array}$ & $\begin{array}{l}\text { Easting (m) } \\
206746\end{array}$ & $\begin{array}{l}\text { Slant Range } \\
469 \text { (m) DOB }\end{array}$ & TOA \\
\hline $\begin{array}{l}\text { METSG9B } \\
\text { METSG10A }\end{array}$ & $\begin{array}{l}1095 m \mathrm{~m} \mathrm{E} \\
1085 \mathrm{~m} \mathrm{~S} 57 \mathrm{E}\end{array}$ & $\begin{array}{l}\text { Terra Tec } 5.28 \mathrm{~g} \\
\text { Terra Tec } 3.7 \mathrm{~g}\end{array}$ & $\begin{array}{l}262279 \\
262870\end{array}$ & $\begin{array}{l}207841 \\
207656\end{array}$ & $\begin{array}{l}1191 \\
1182\end{array}$ & $\begin{array}{l}2.525 \\
2.486\end{array}$ \\
\hline
\end{tabular}




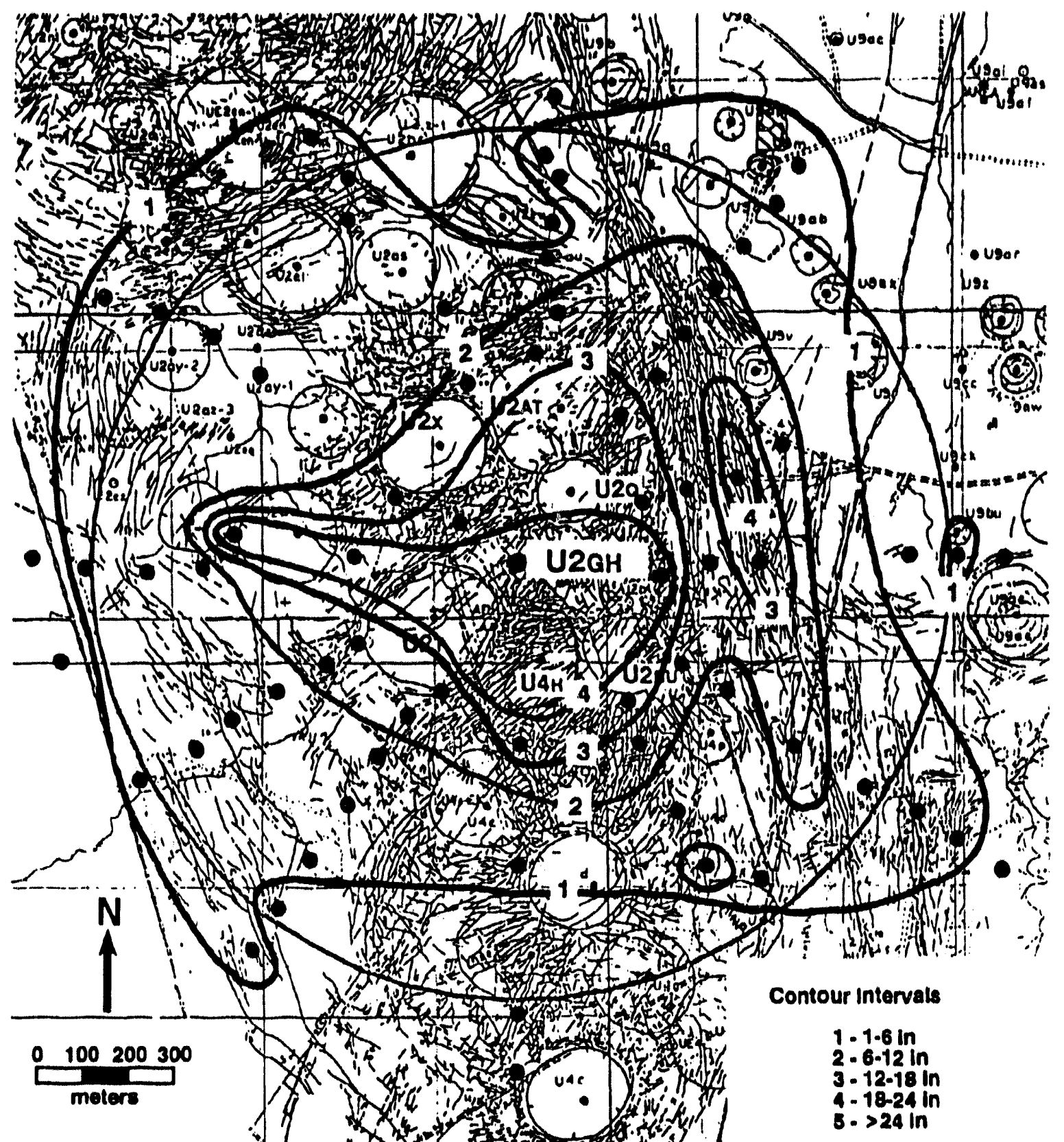

(Minimum of 2 balts in array moved at least the distance in an interval.)

Fig. 12. Results of the SIG experiment on Metropolis. Note that the motion patterns are not concentric around SGZ but appear to be influenced hy the Yucca Fault. 


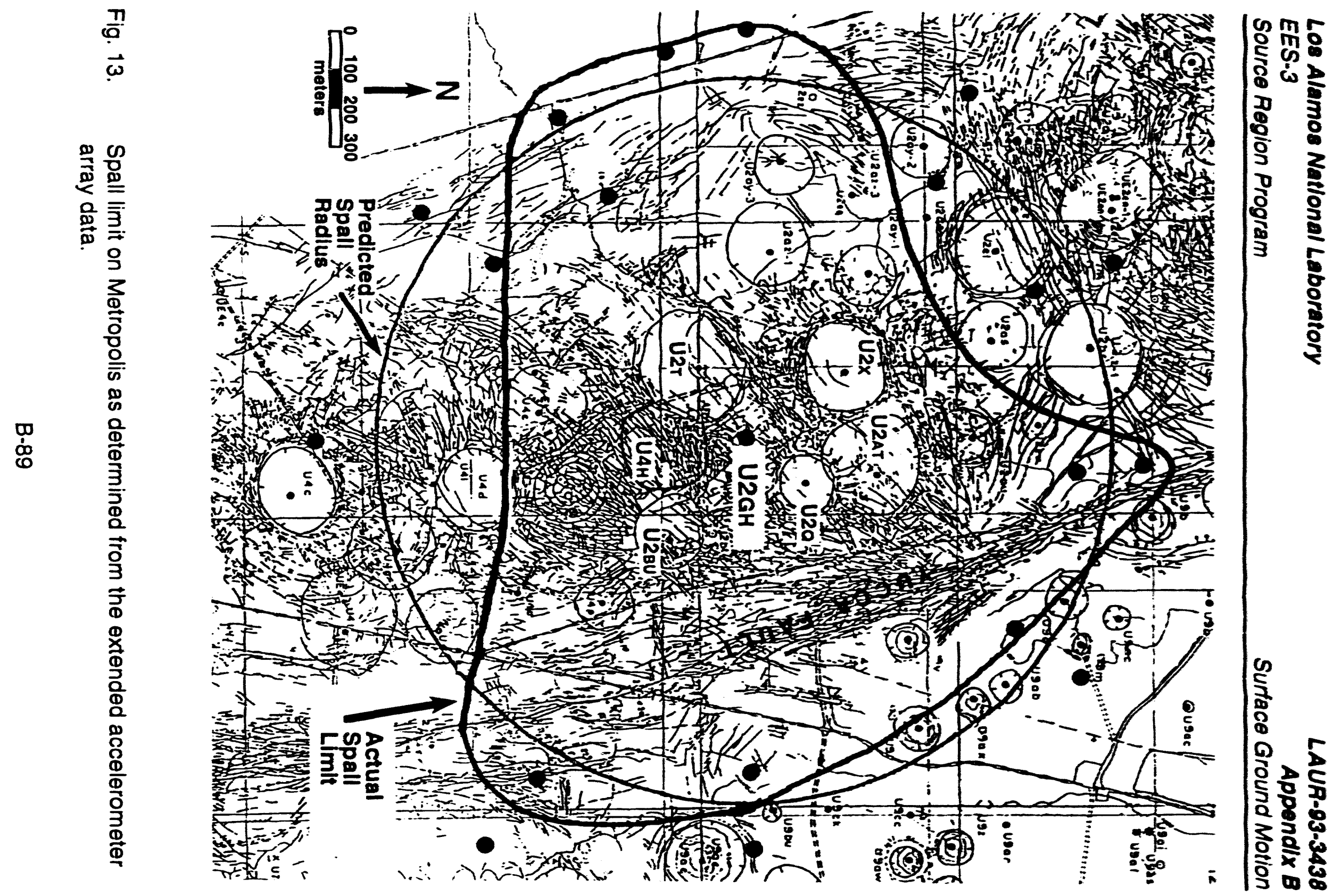


Table 11. Metropolls SiG Results

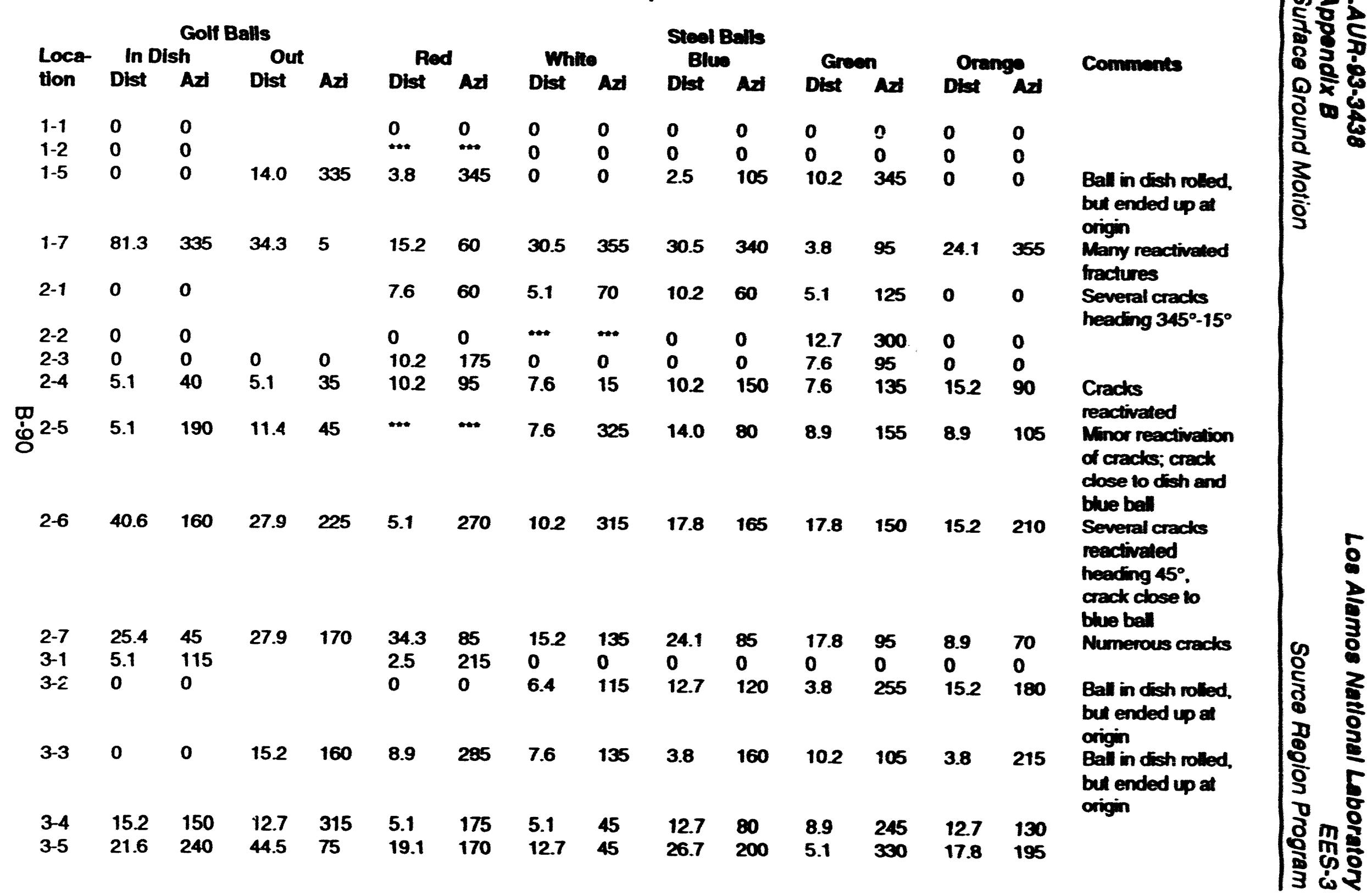




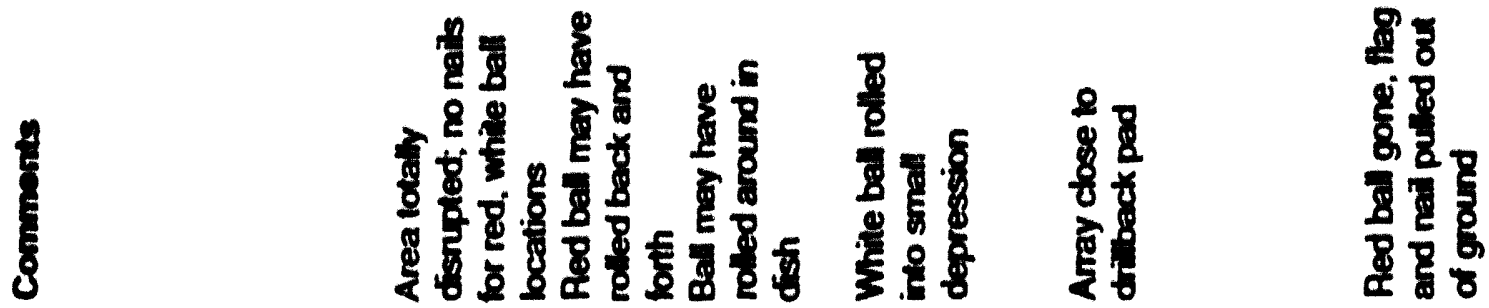

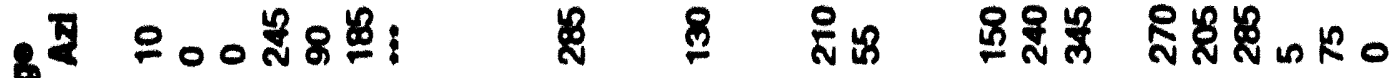

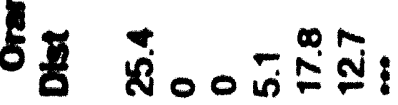

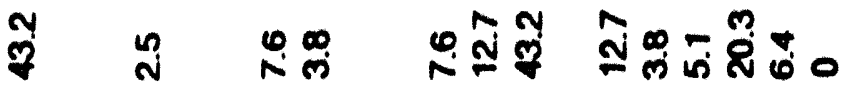

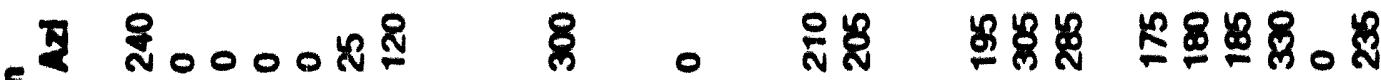
हु

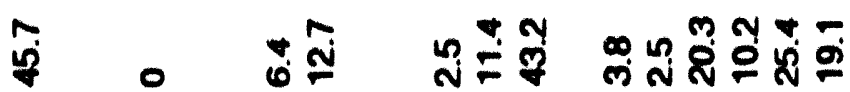

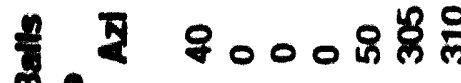

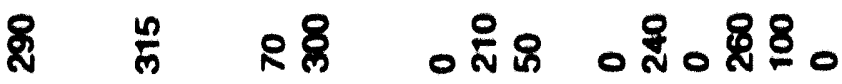

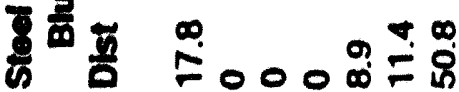

$\ddot{8}$

की -

꿍 뽕

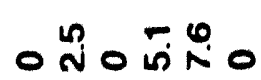

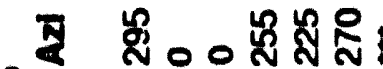

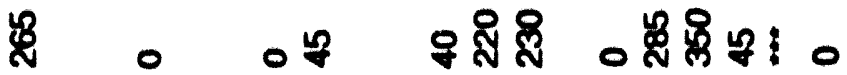

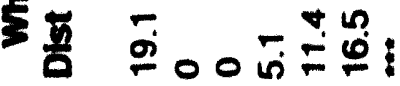

品 $\quad 0 \quad 0$ i்

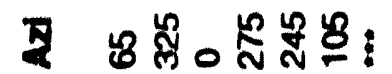

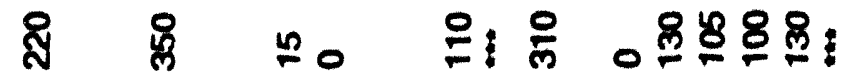

8

$\frac{1}{2}$

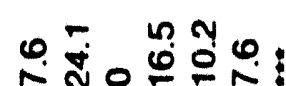

$\stackrel{\infty}{m}$ N

क:

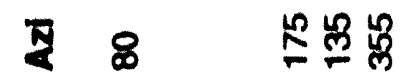

$\stackrel{8}{:}$

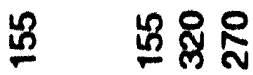

过点 के

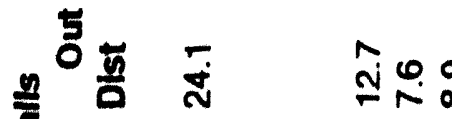

$\stackrel{\varphi}{Q}$

の

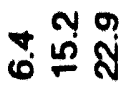

홍

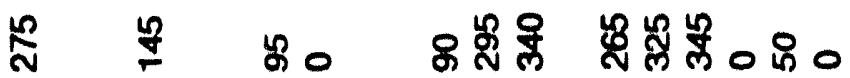

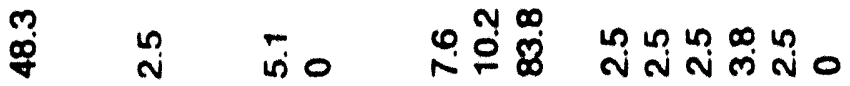

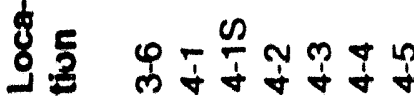

广

พn

$\forall \uparrow \infty$

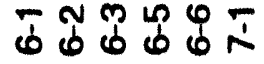


Table 11 Motropolis SIG Results (Continued)

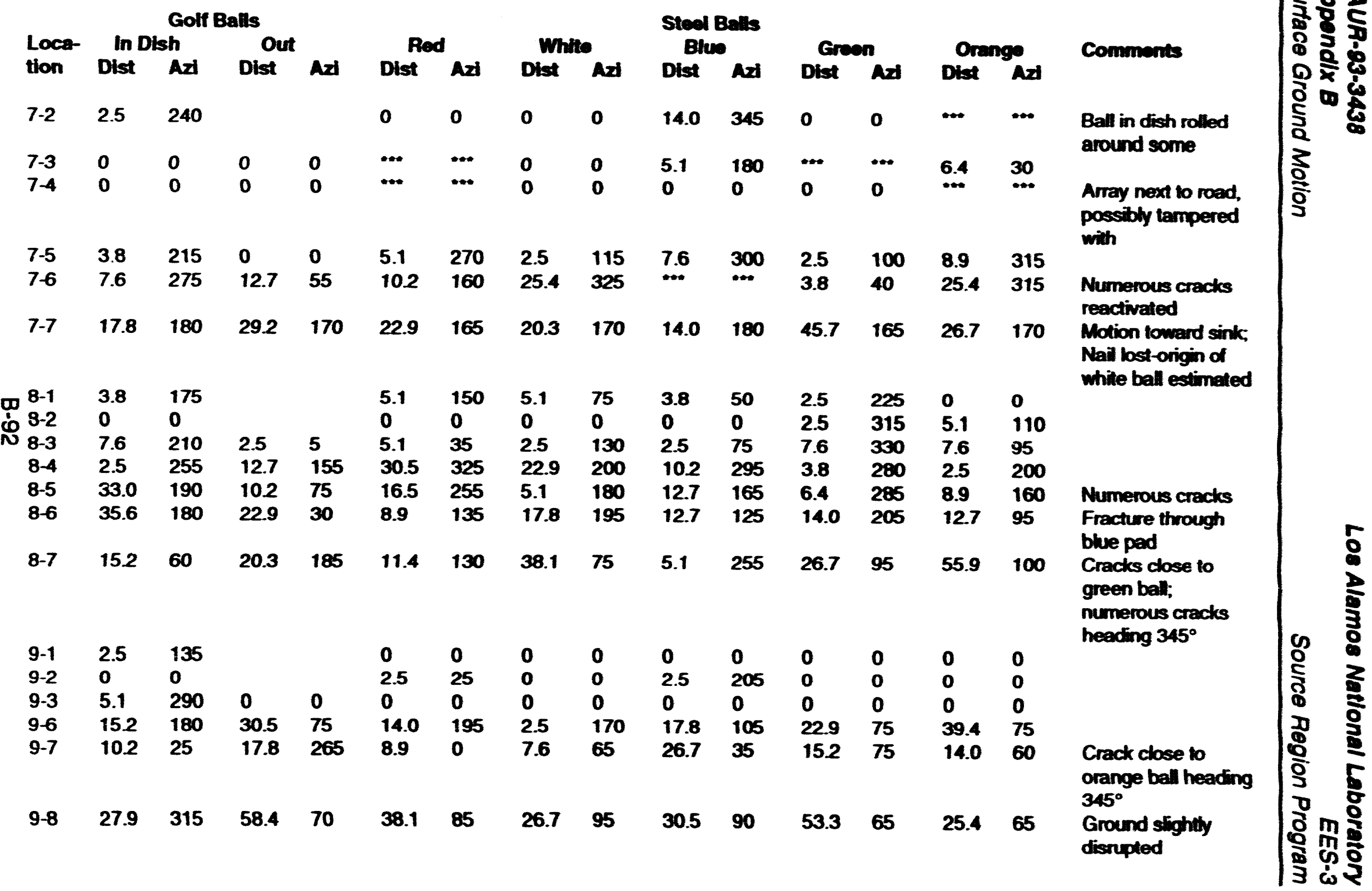


Tablo 11. Metropolls SIG Rasults (Conchuded)

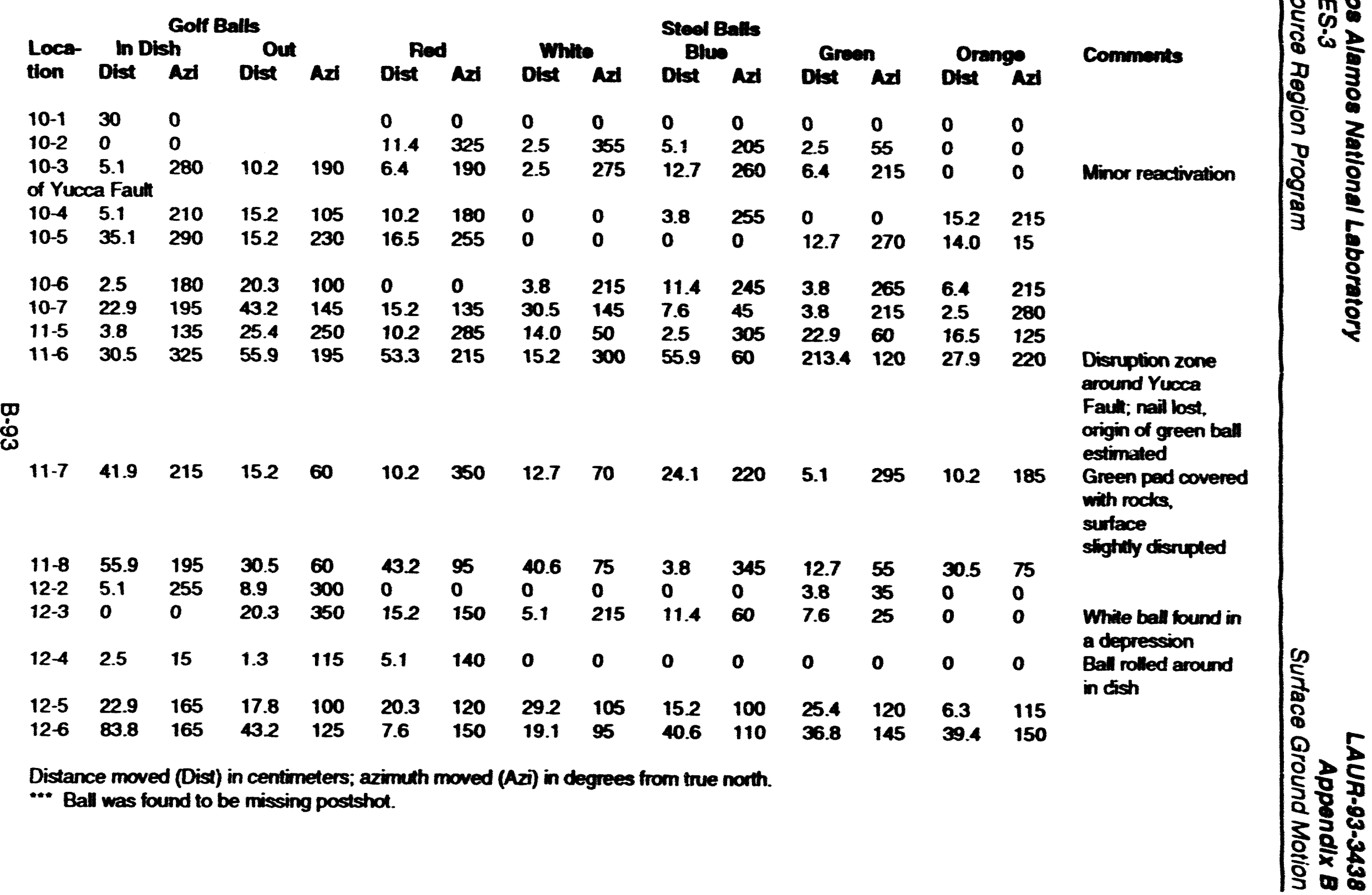




\section{Metropolis Surface Ground Motion}

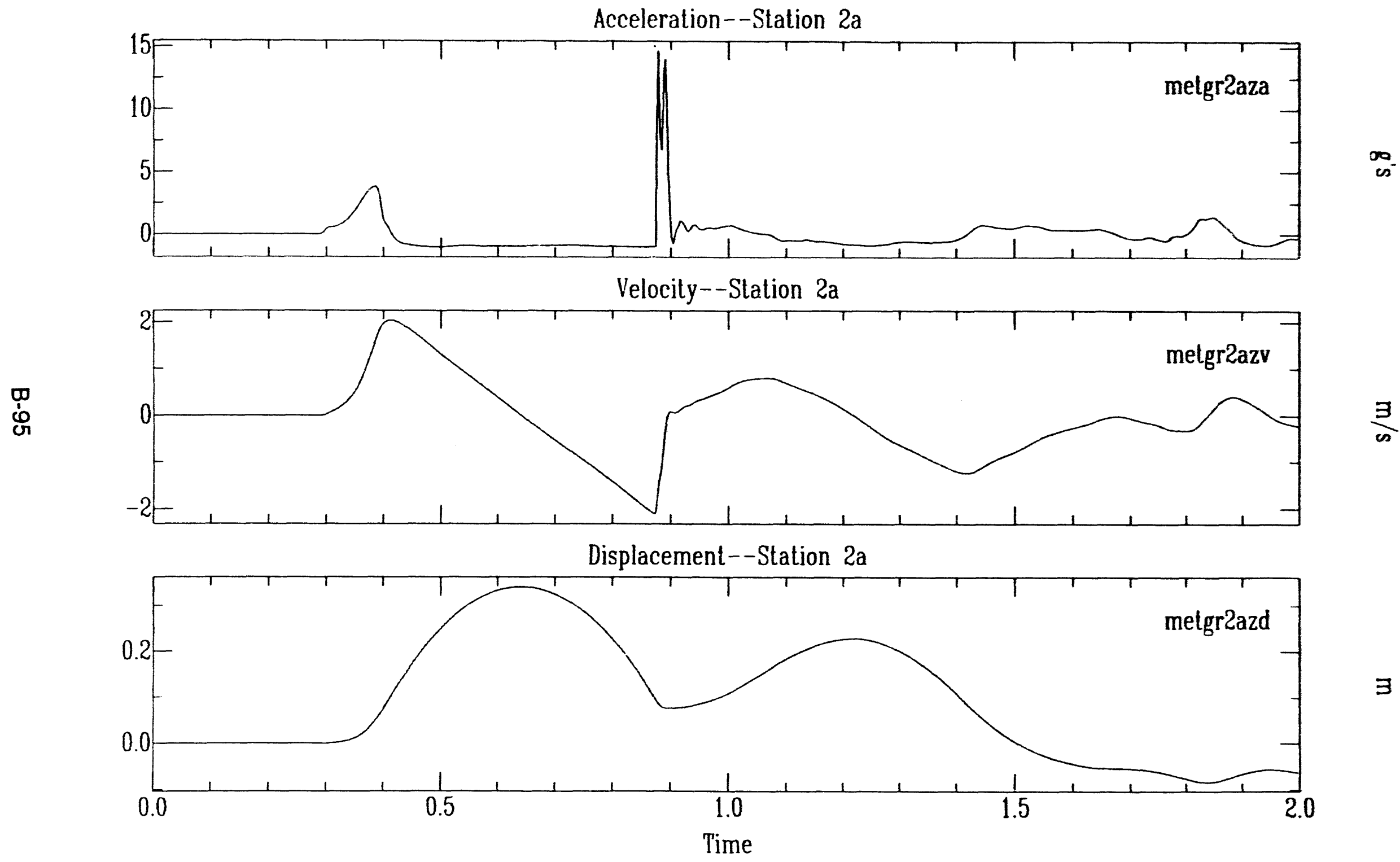




\section{Metropolis Surface Ground Motion}
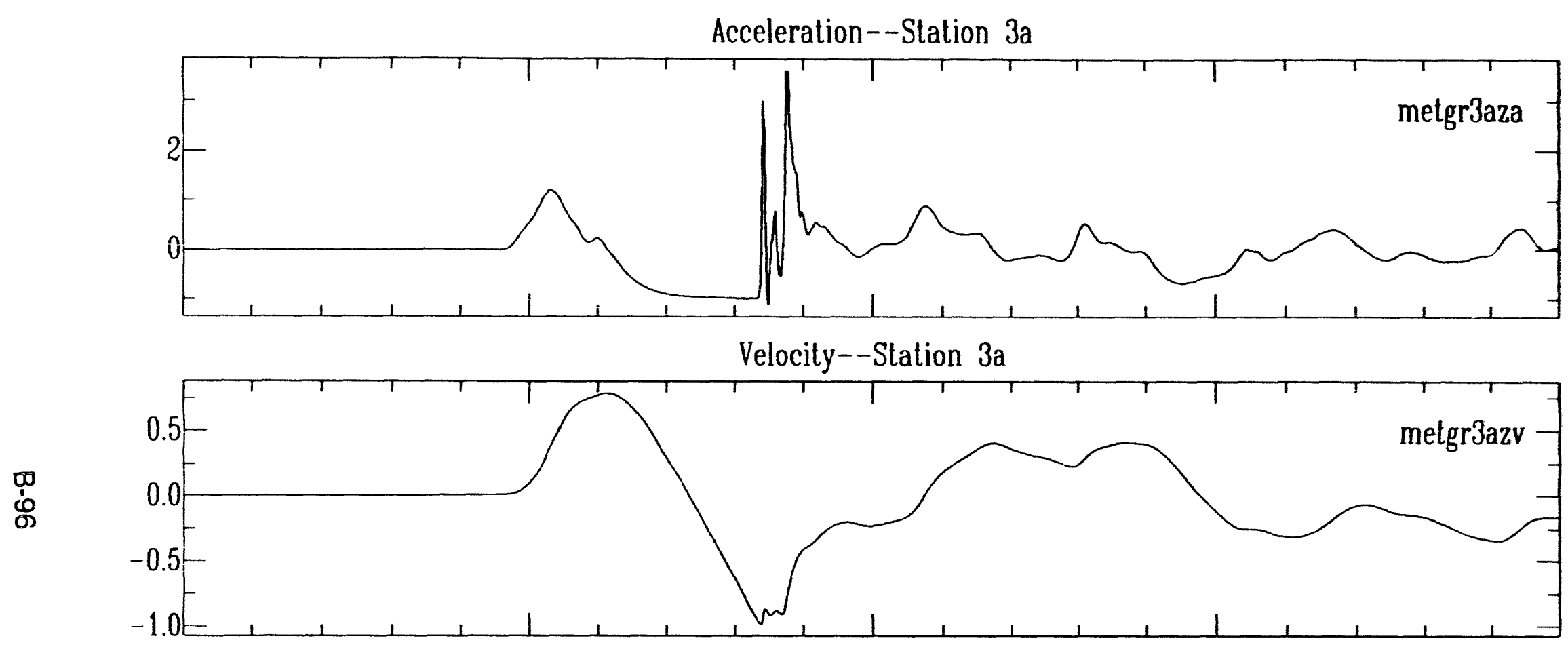

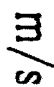

Displacement--Station 3a

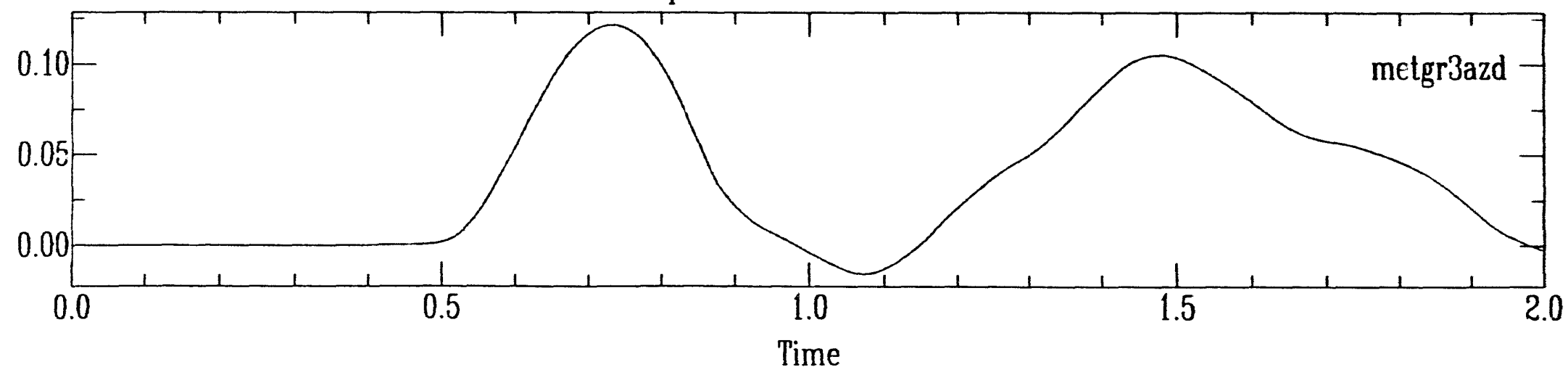

$\exists$ 
Metropolis Surface Ground Motion

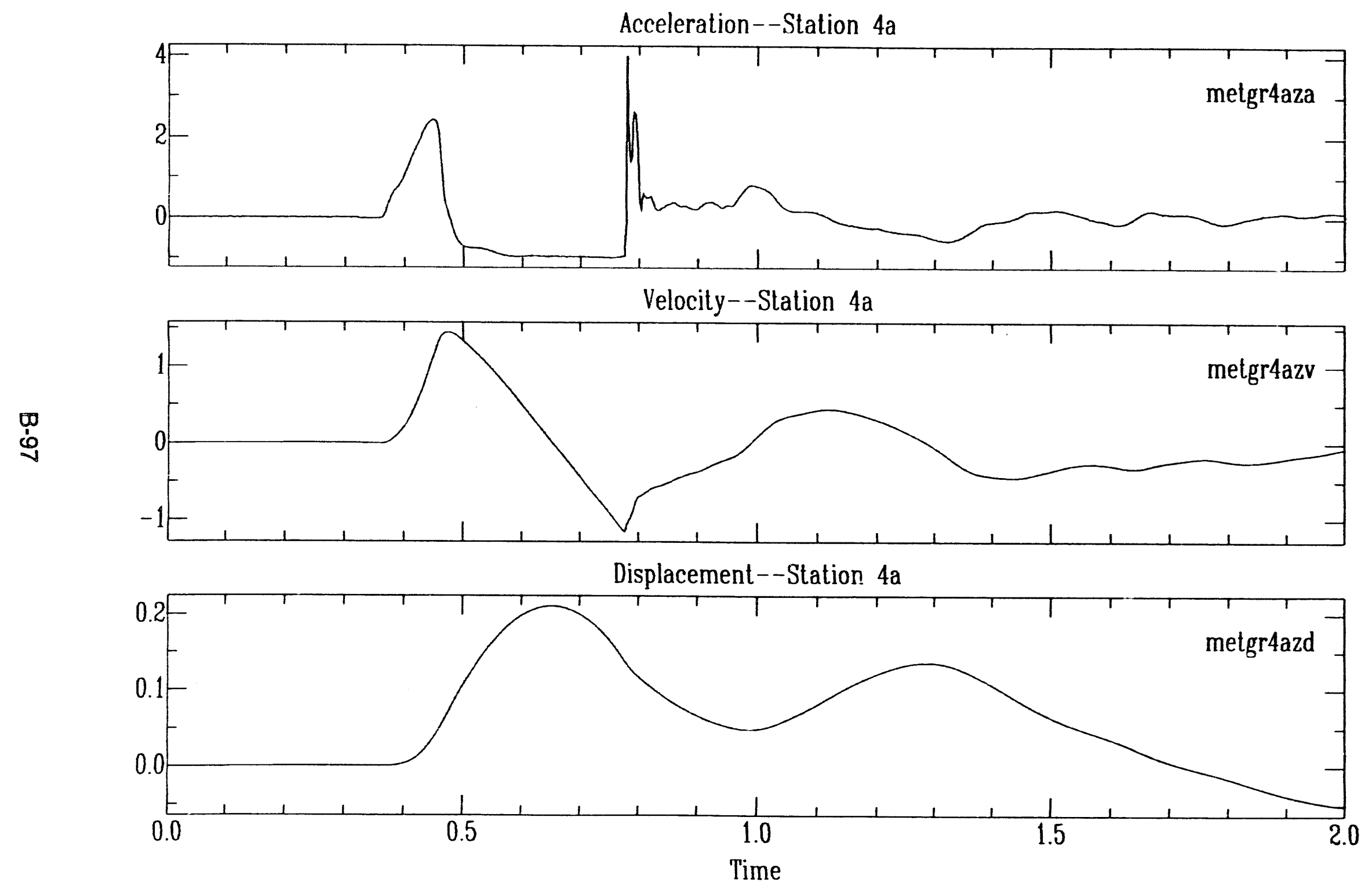




\section{Metropolis Surface Ground Motion}

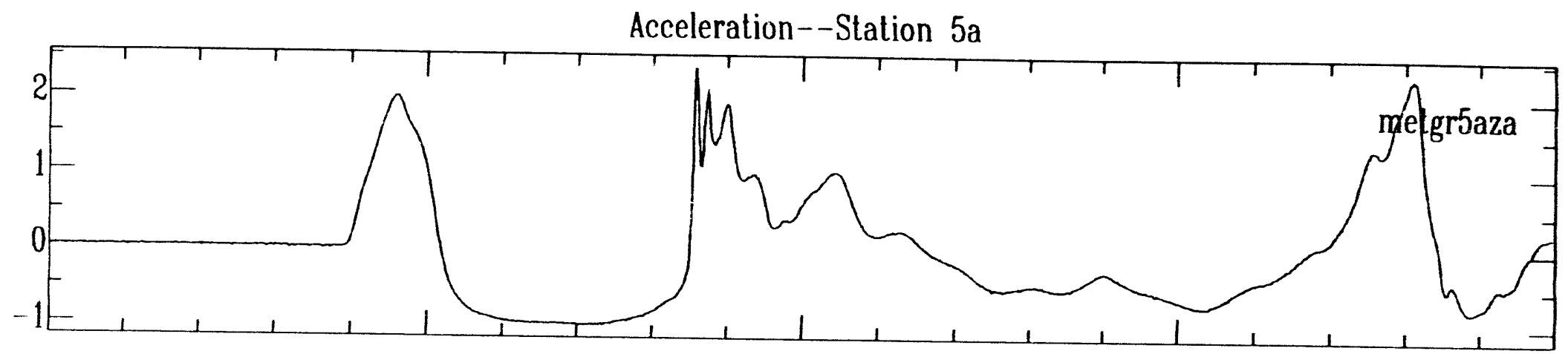

Velocity--Station $5 \mathrm{a}$

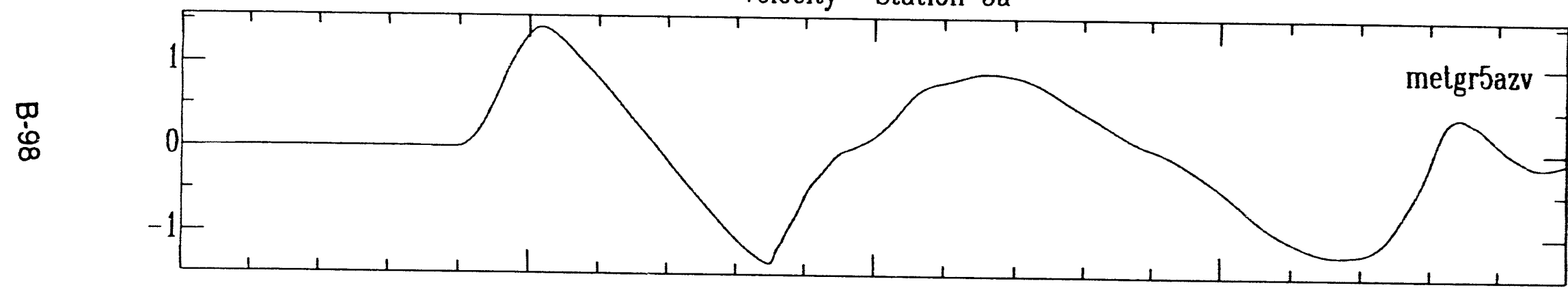

Displacement--Station 5a

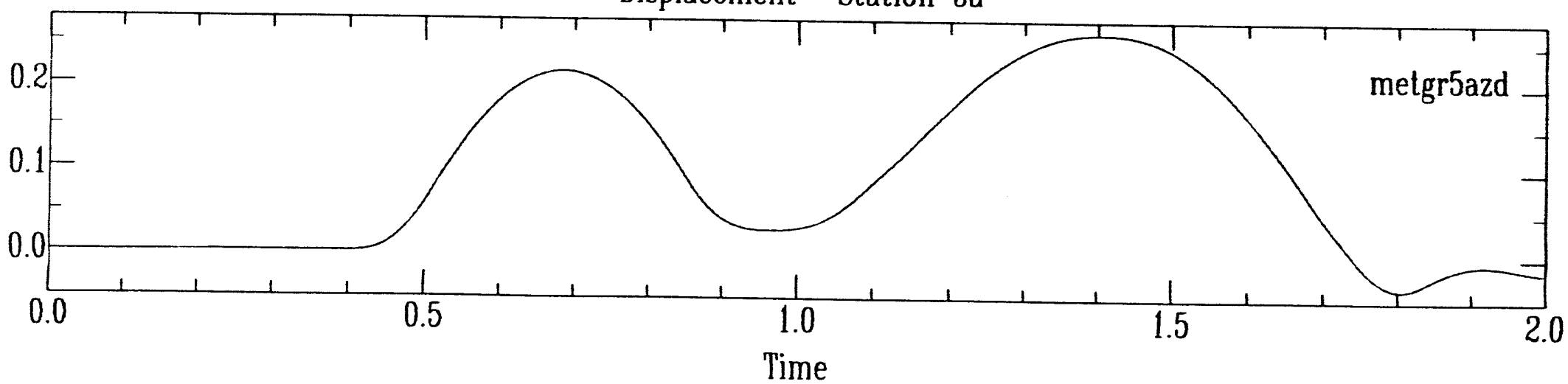


Metropolis Surface Ground Motion

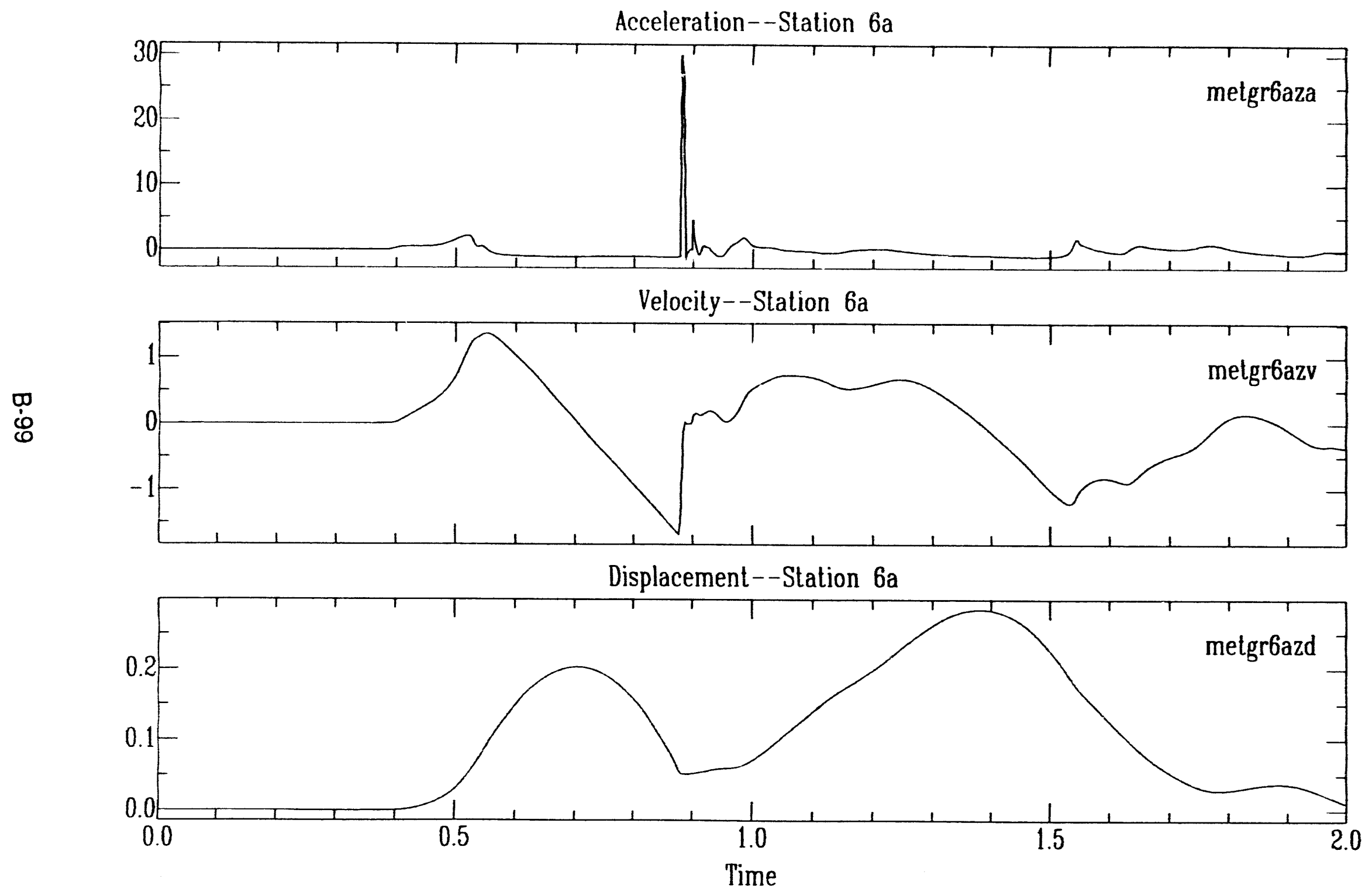




\section{Metropolis Surface Ground Motion}

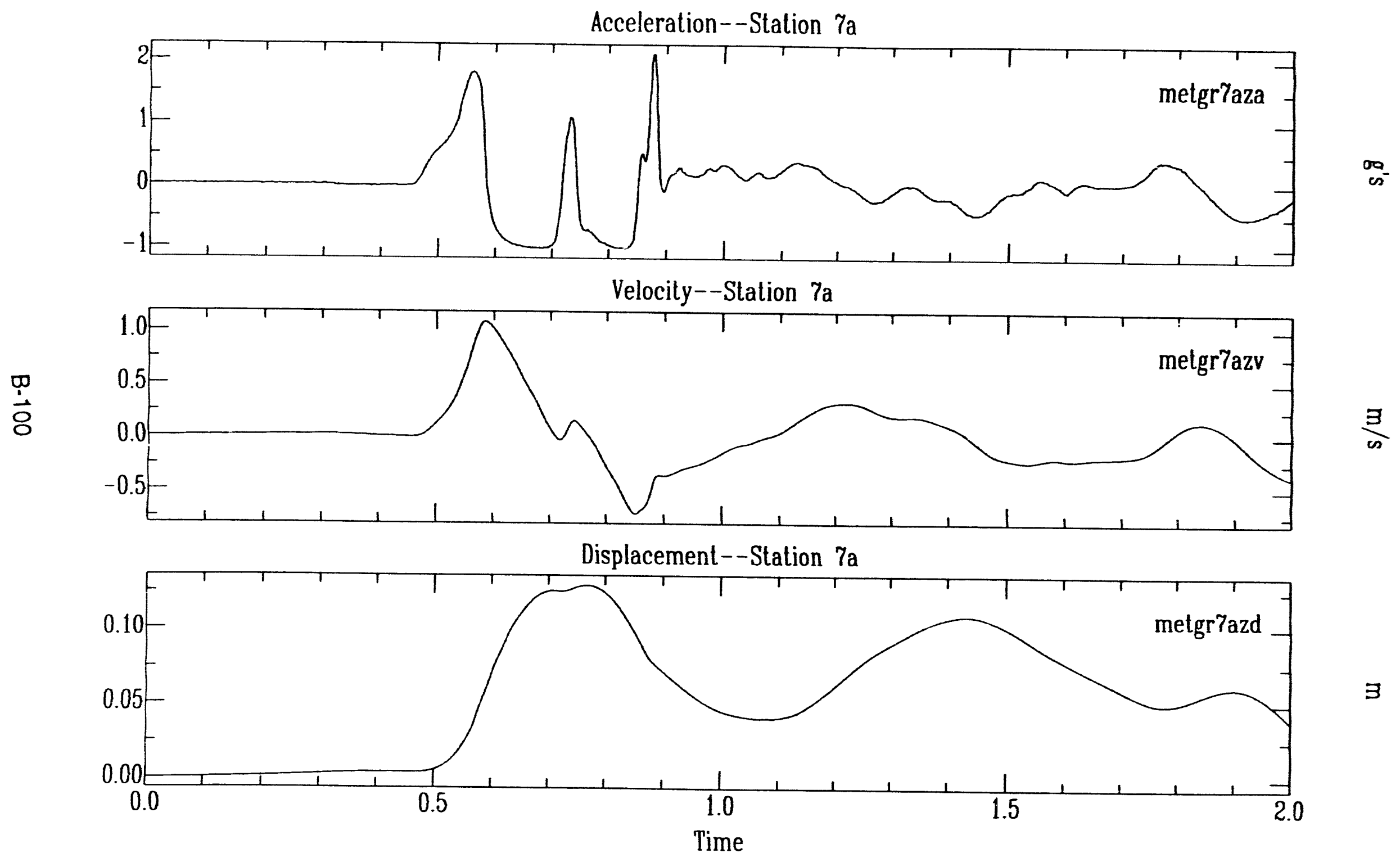


Metropolis Surface Ground Motion

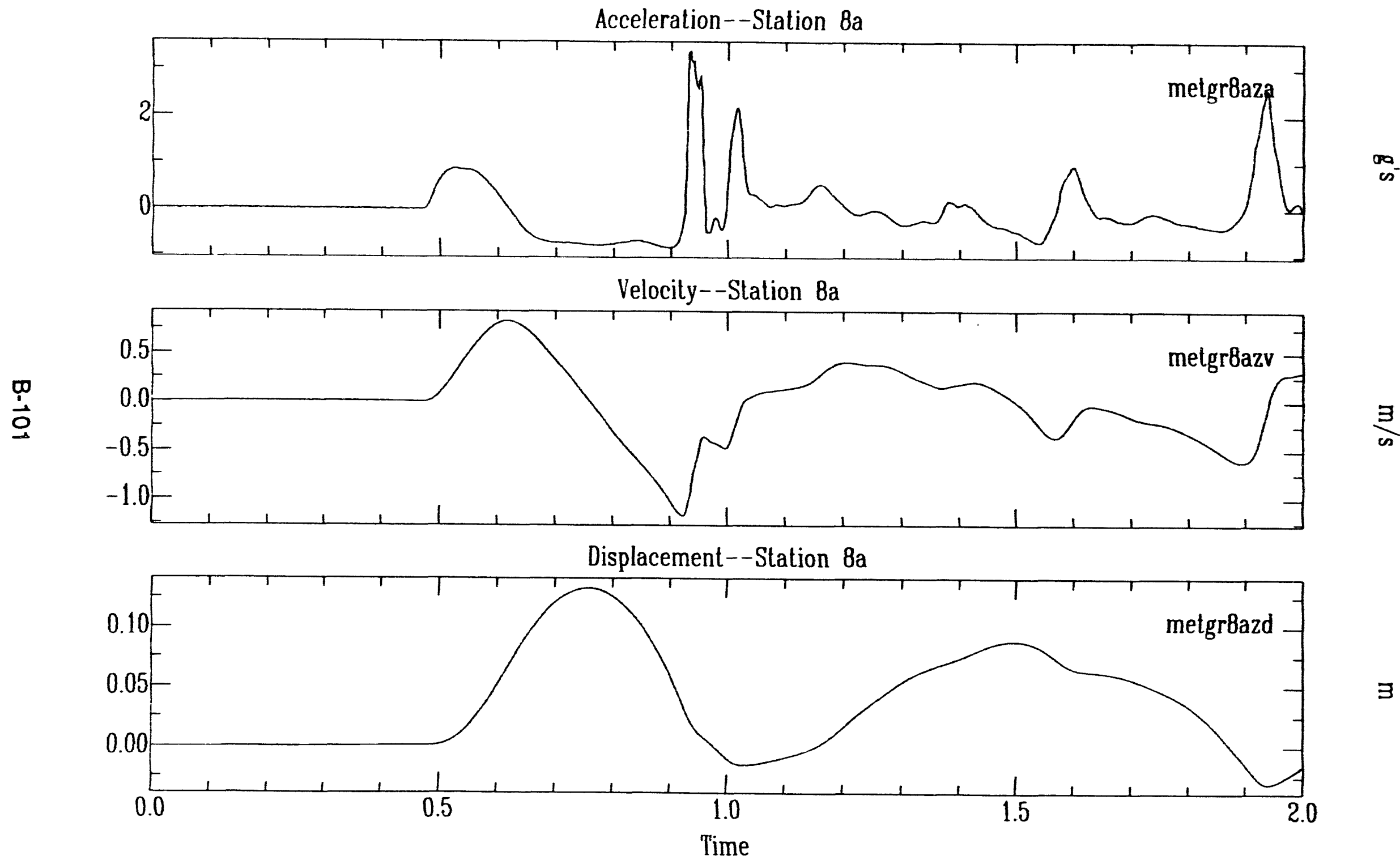




\section{Metropolis Surface Ground Motion}

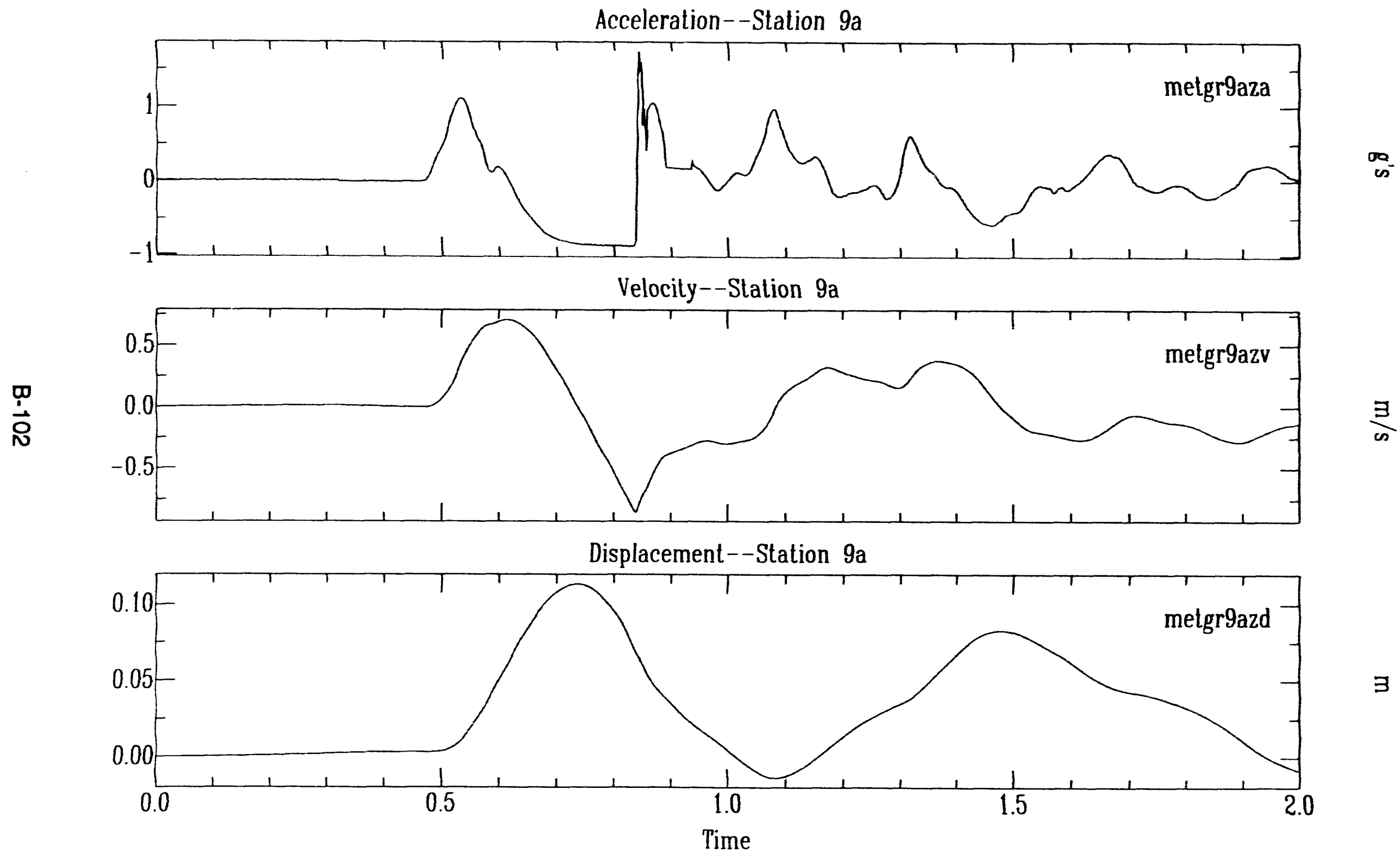




\section{Metropolis Surface Ground Motion}

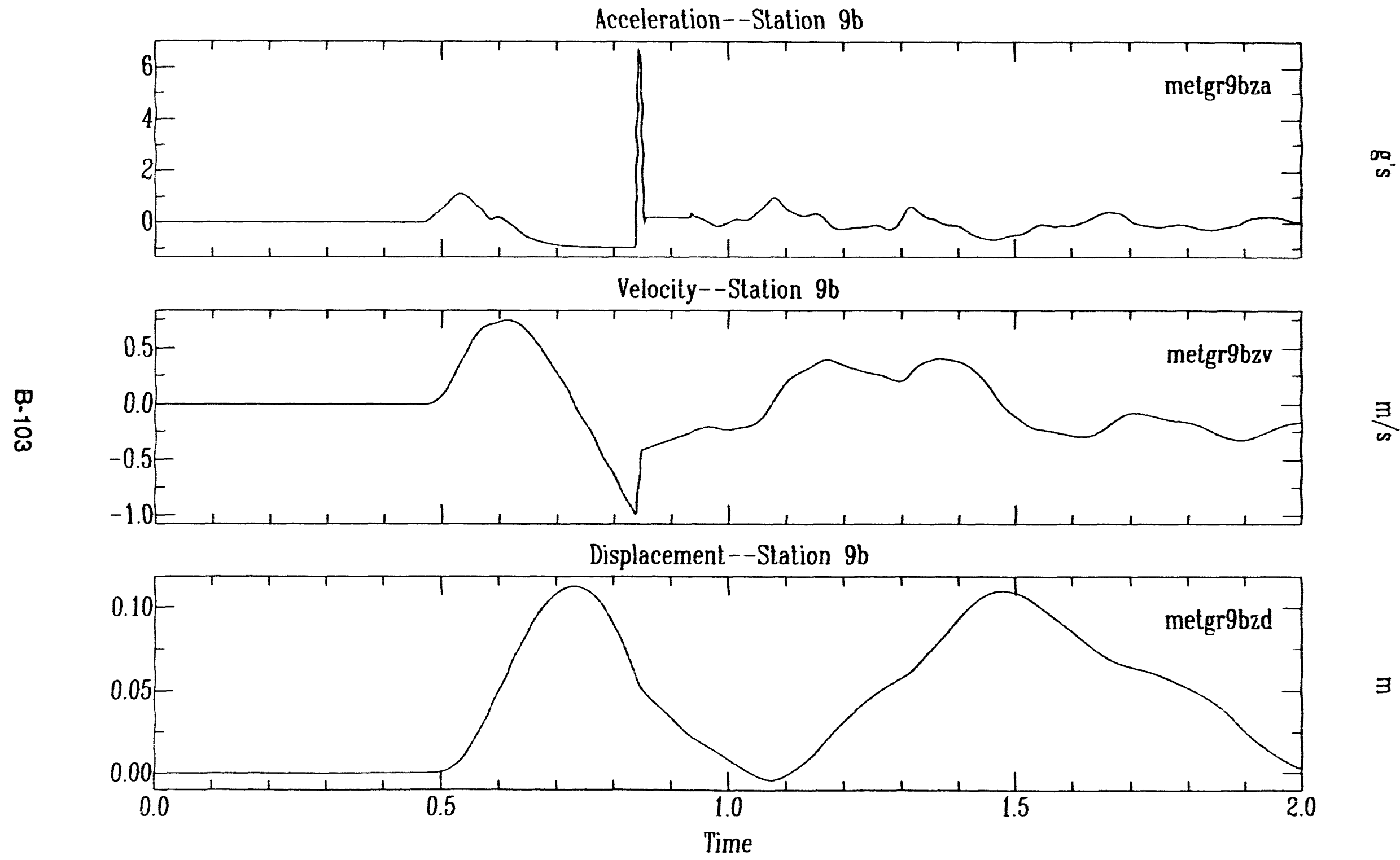




\section{Metropolis Surface Ground Motion}

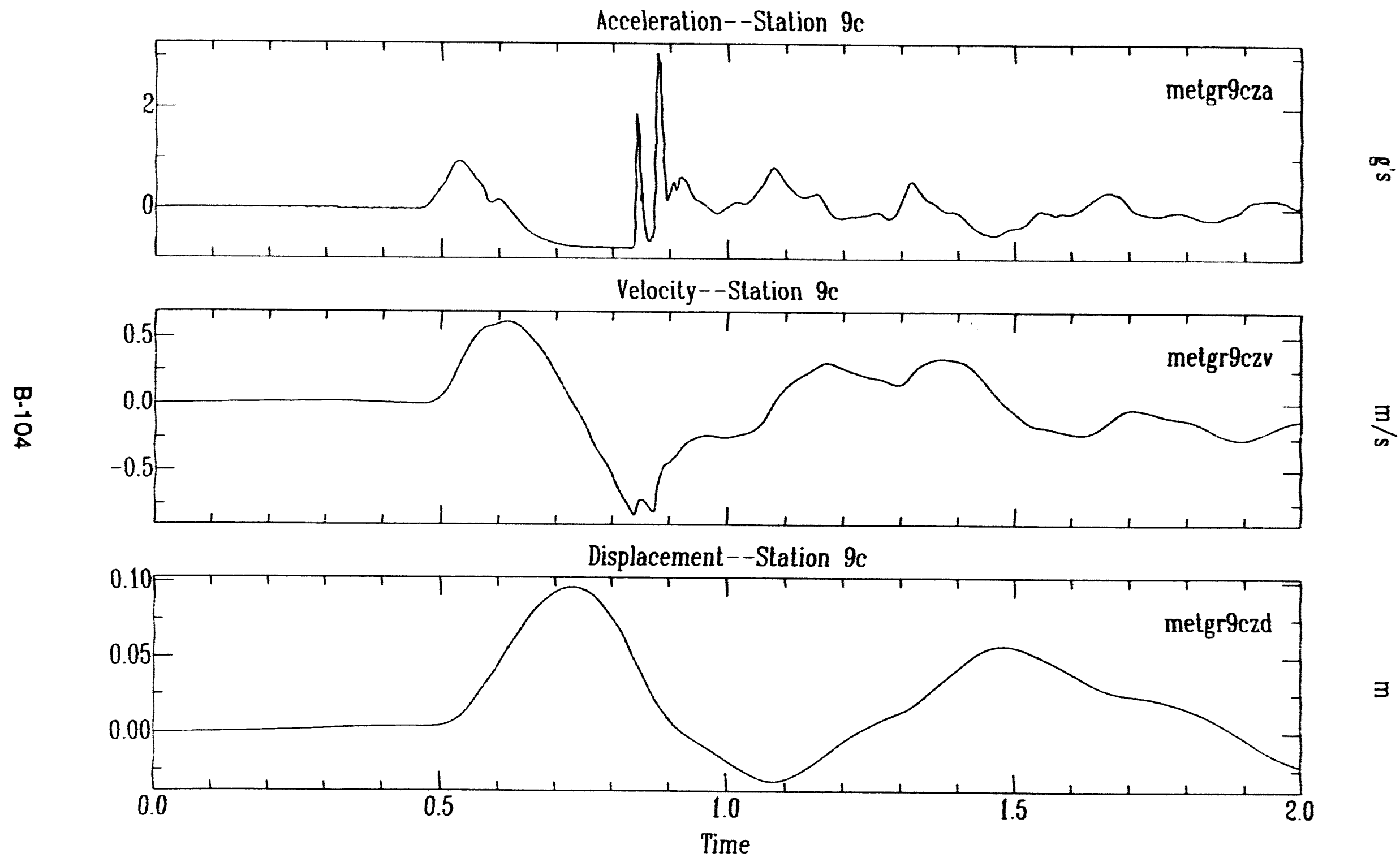



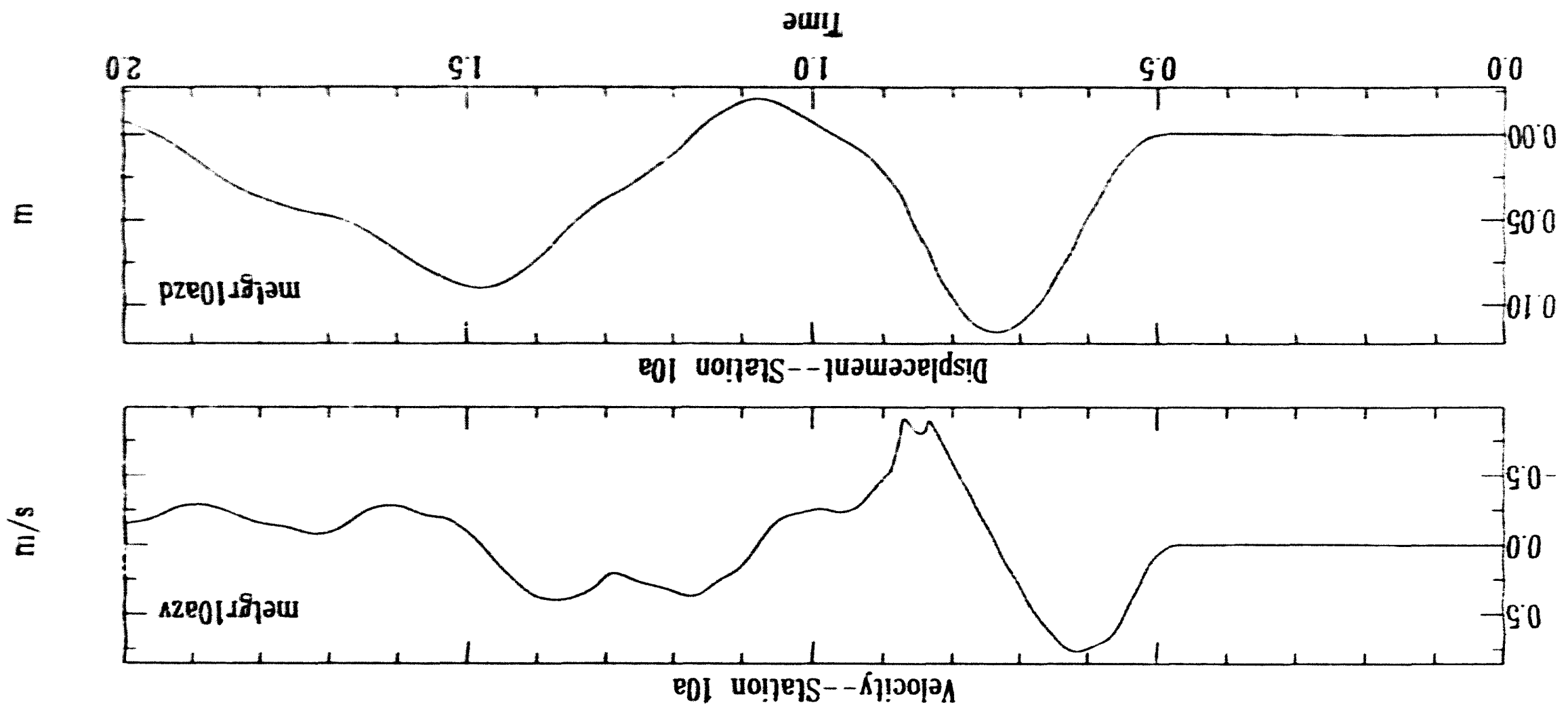

$\stackrel{\text { ต }}{\text { ஸ் }}$

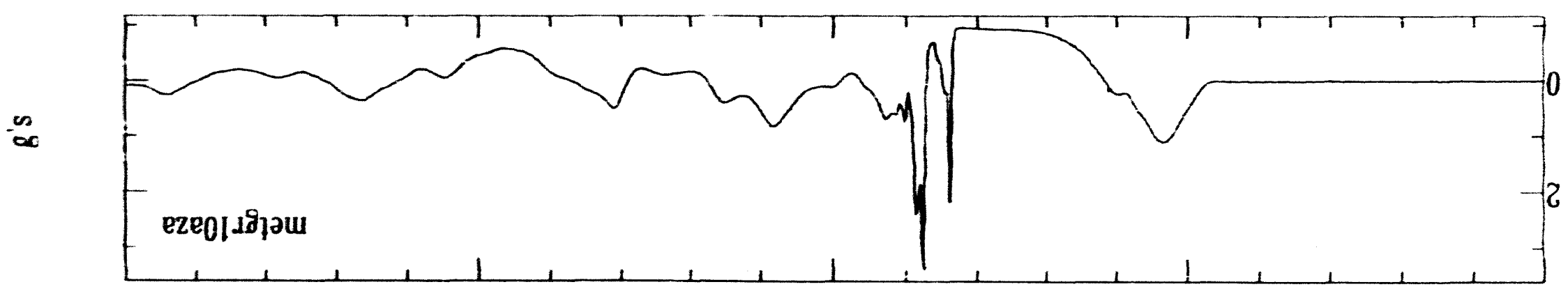

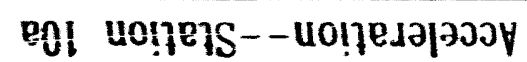

uo!jow punody วכejuns s!jododəw 


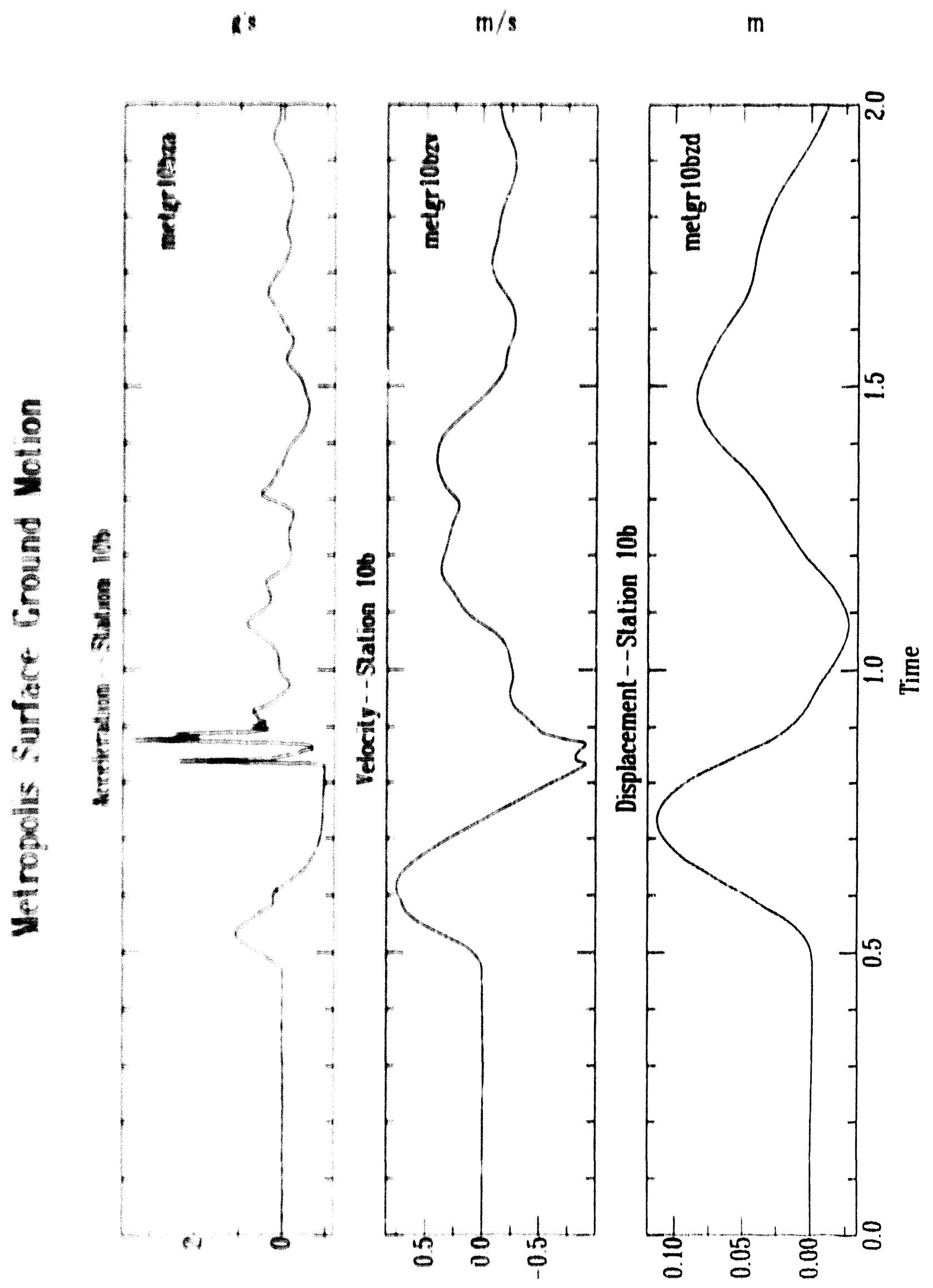

B. 106 


\section{Metropolis Surface Ground Motion}

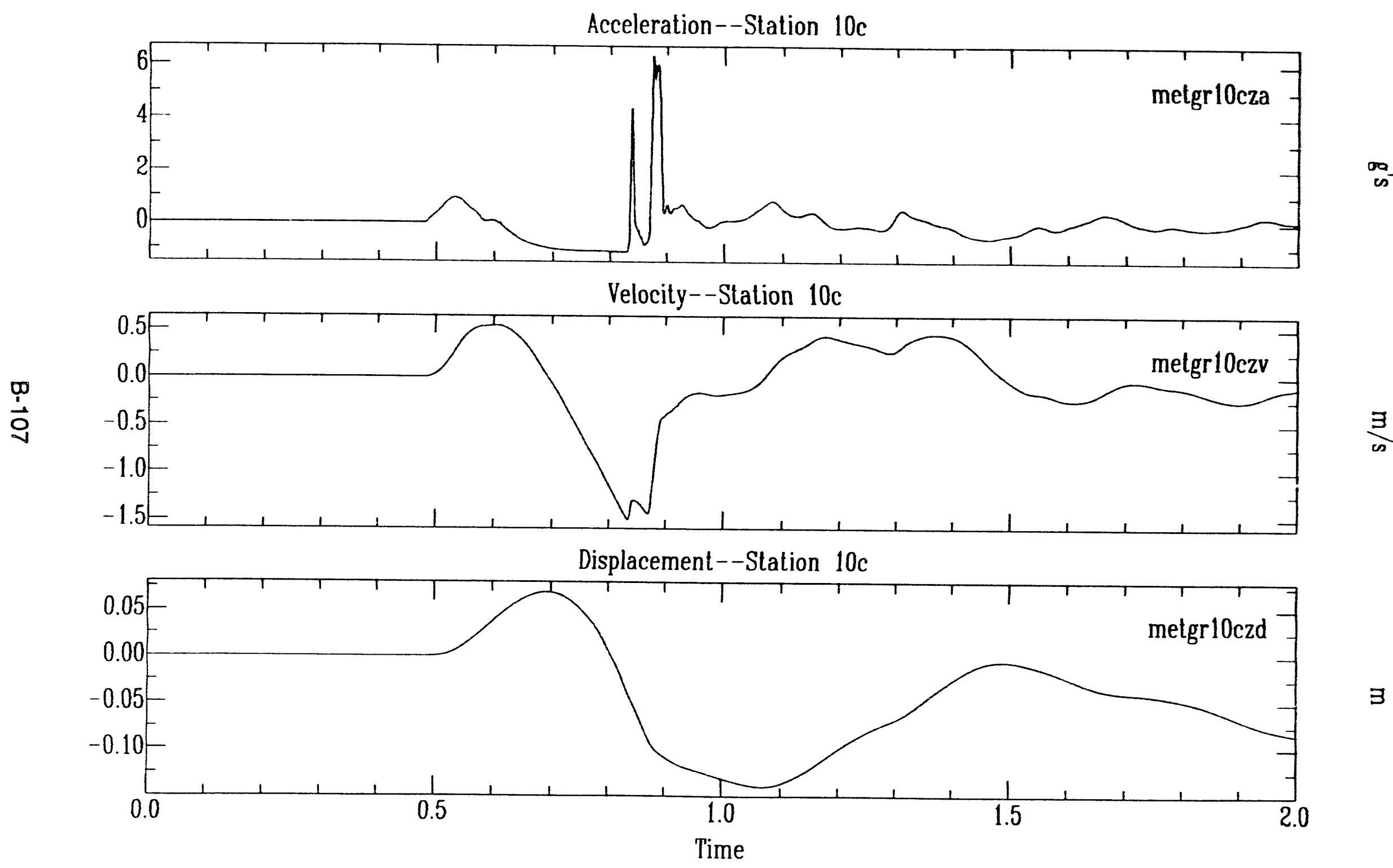




\section{Metropolis Surface Ground Motion}

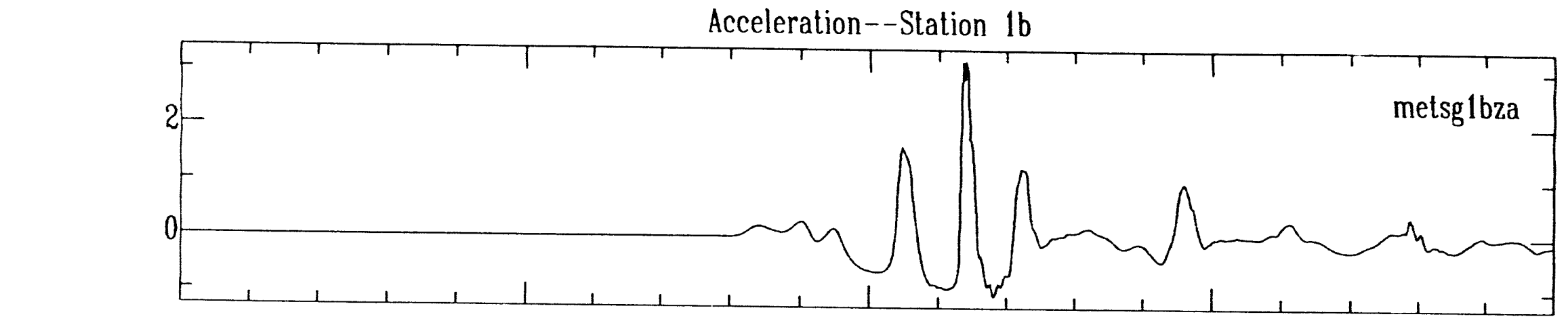

Velocity--Station $1 \mathrm{~b}$

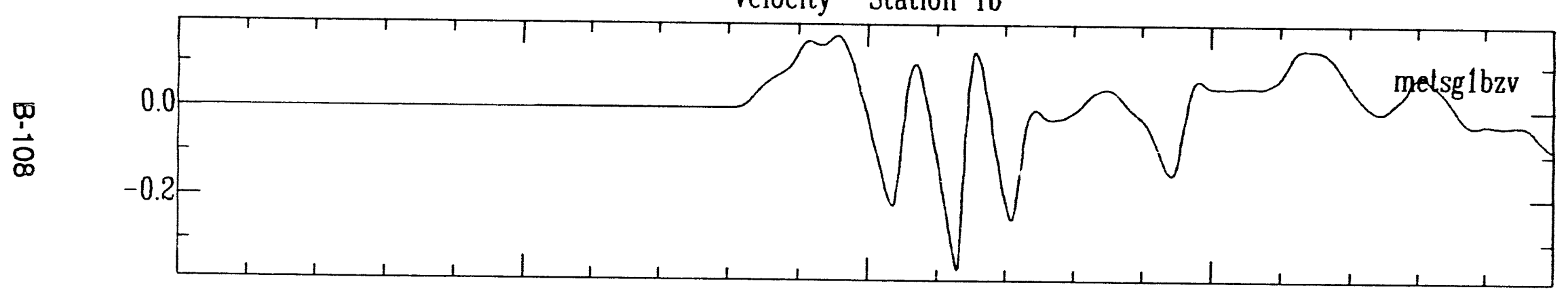

Displacement--Station $1 \mathrm{~b}$

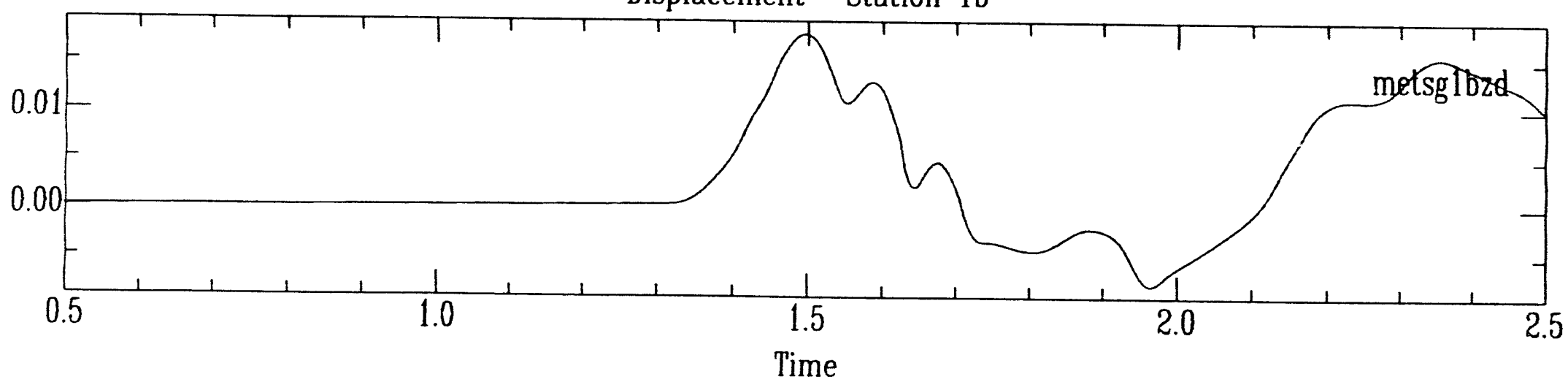




\section{Metropolis Surface Ground Motion}

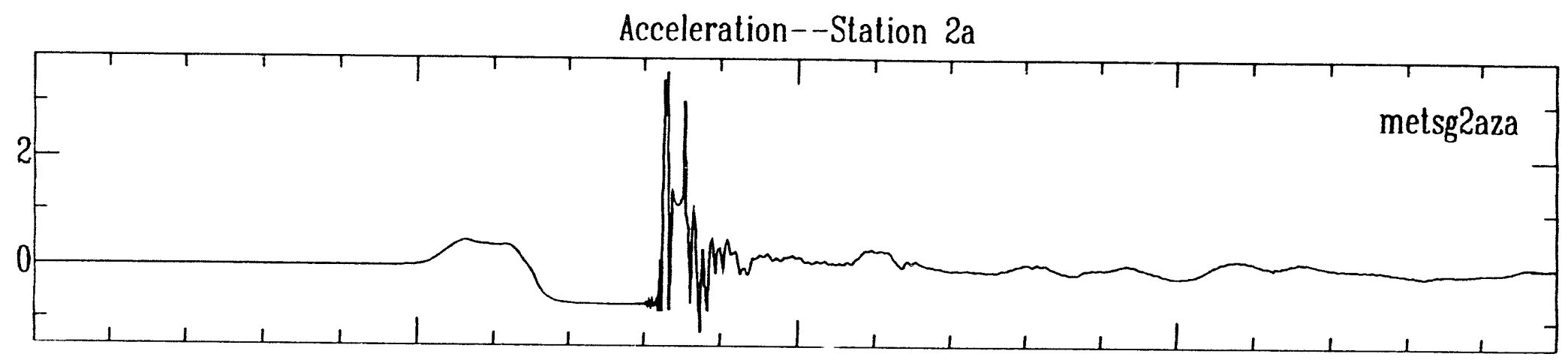

Velocity--Station 2a
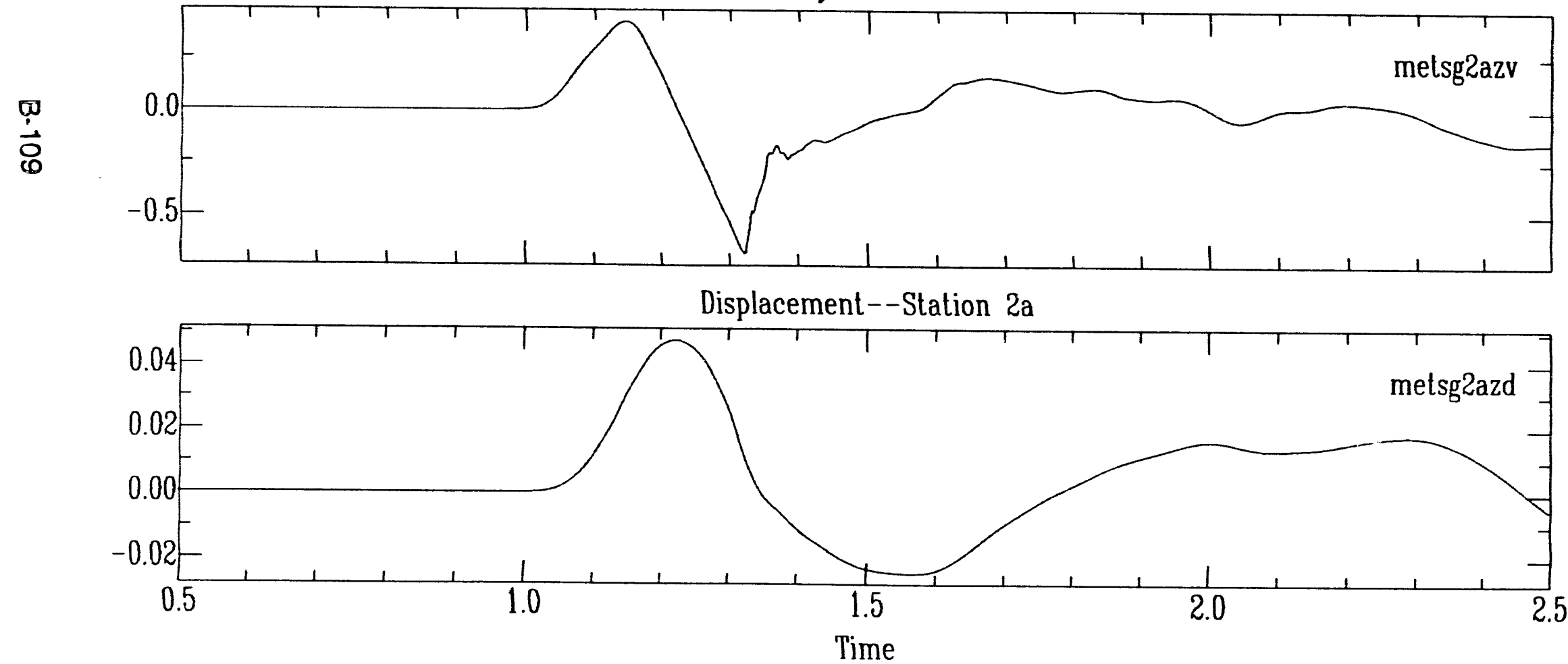
Metropolis Surface Ground Motion

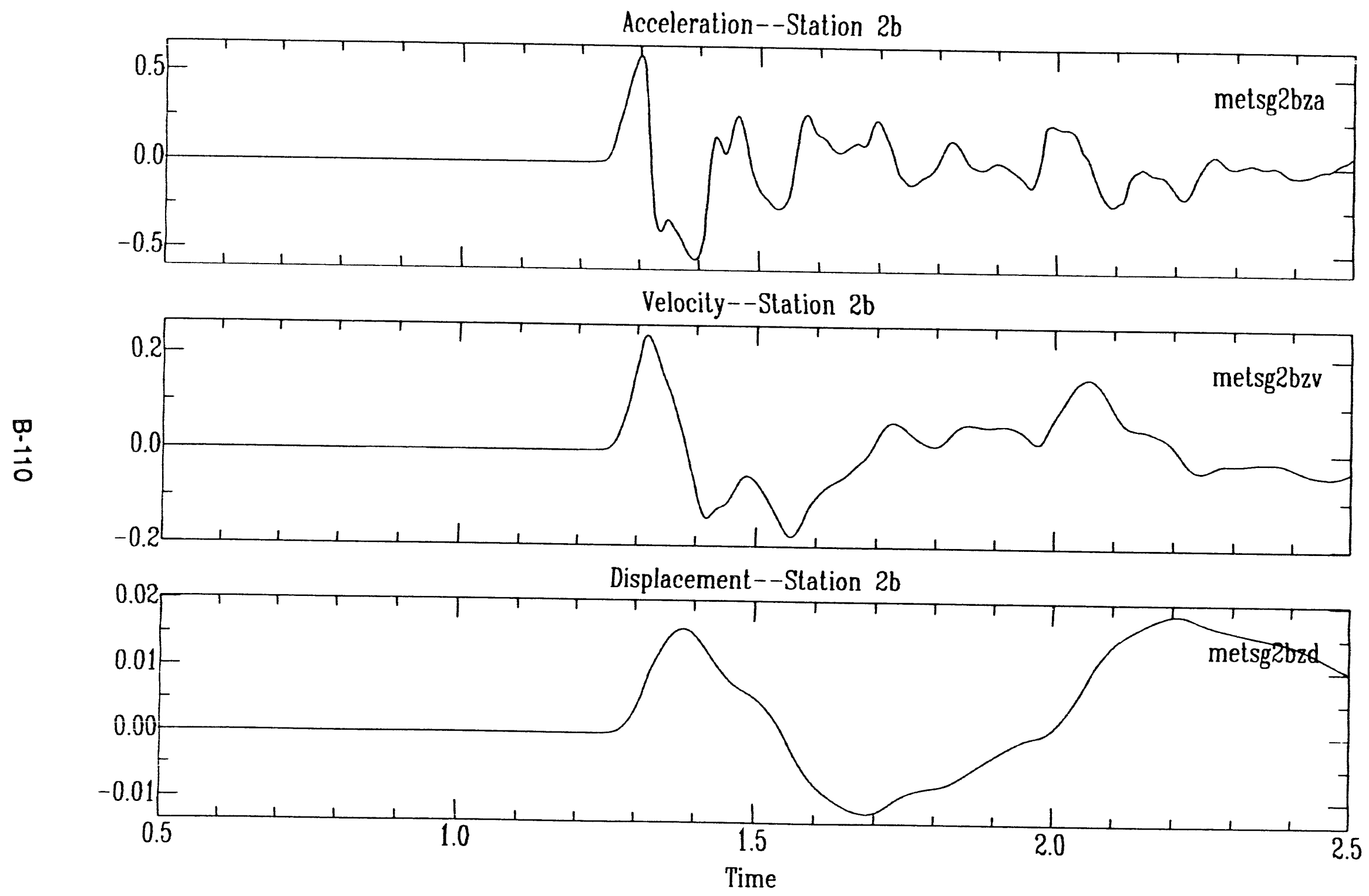


Metropolis Surface Ground Motion

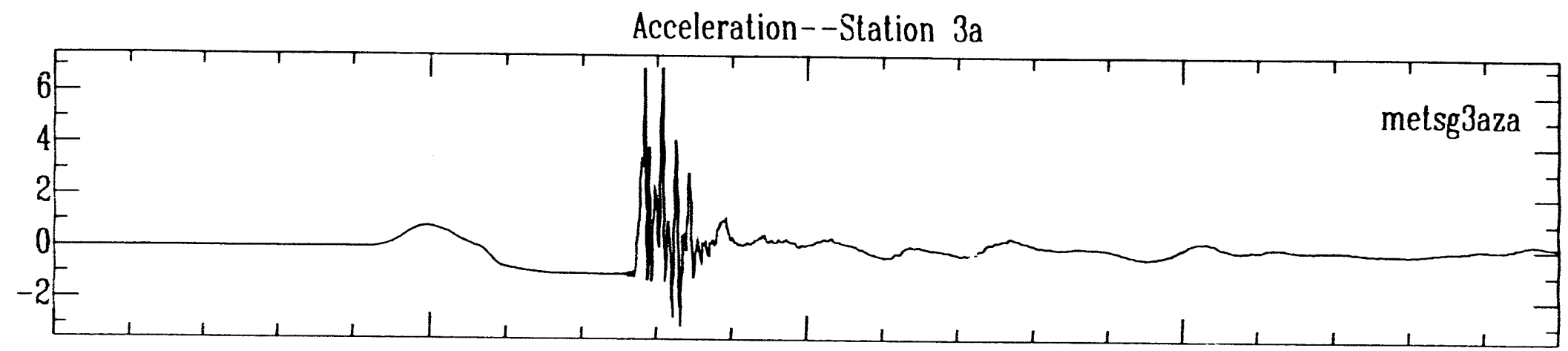

Velocity--Station 3a

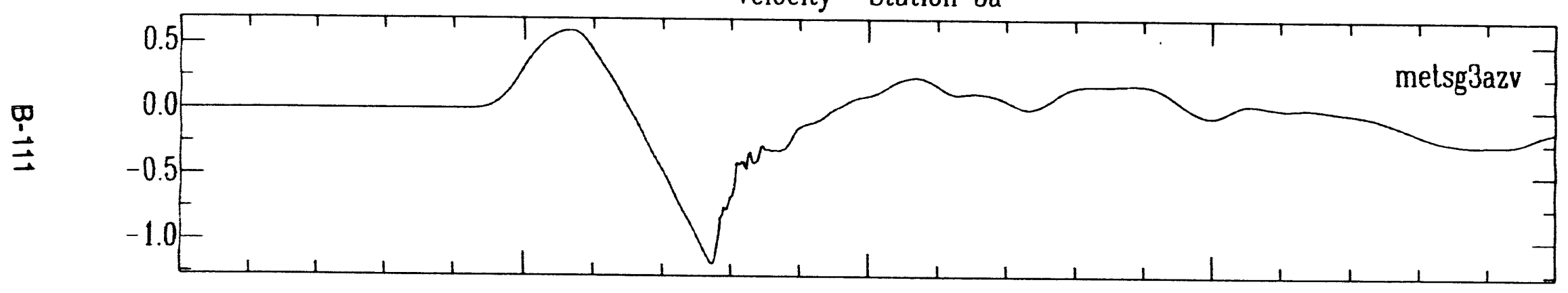

Displacement--Station $3 \mathrm{a}$

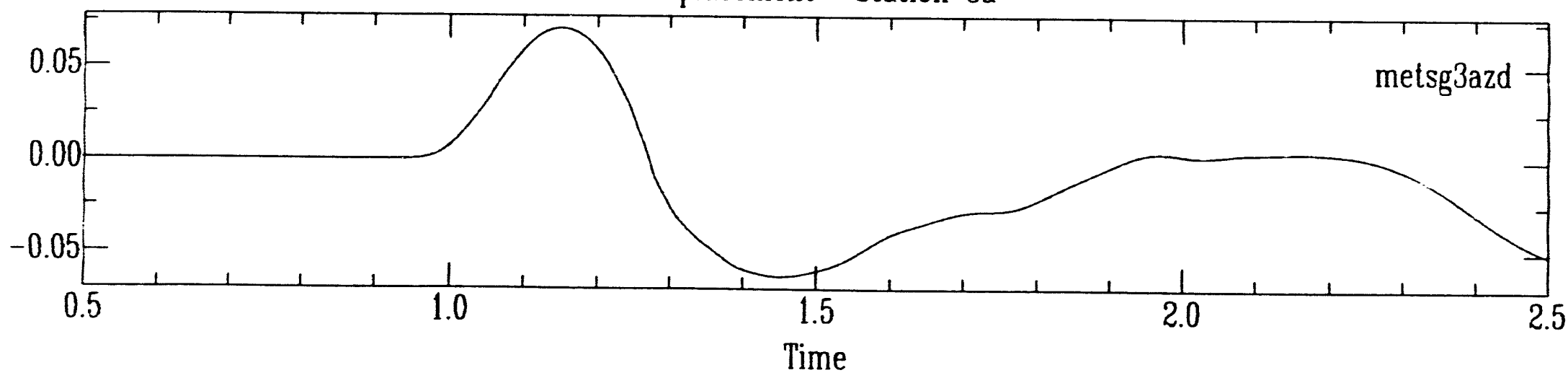


Metropolis Surface Ground Motion

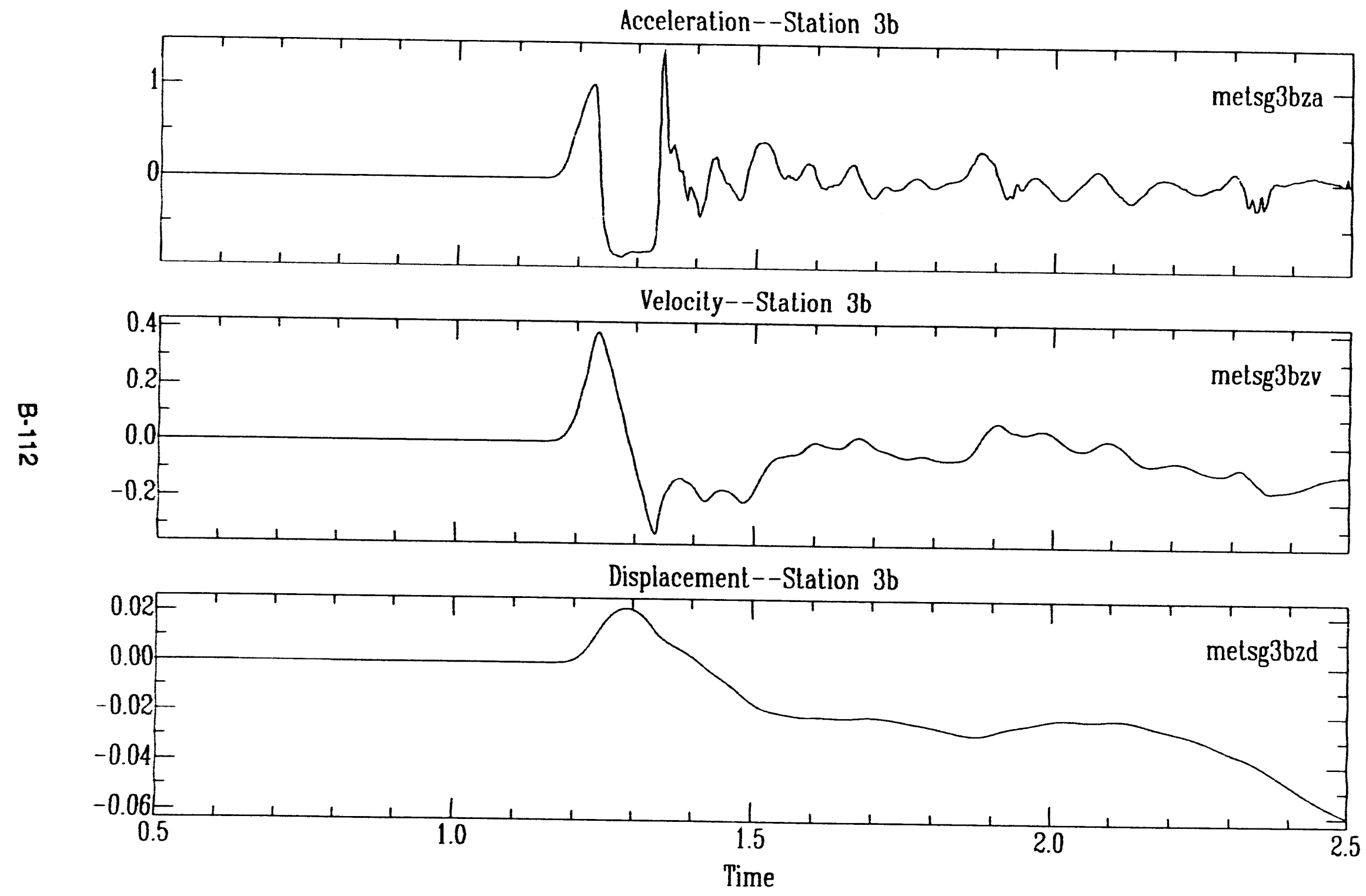




\section{Metropolis Surface Ground Motion}

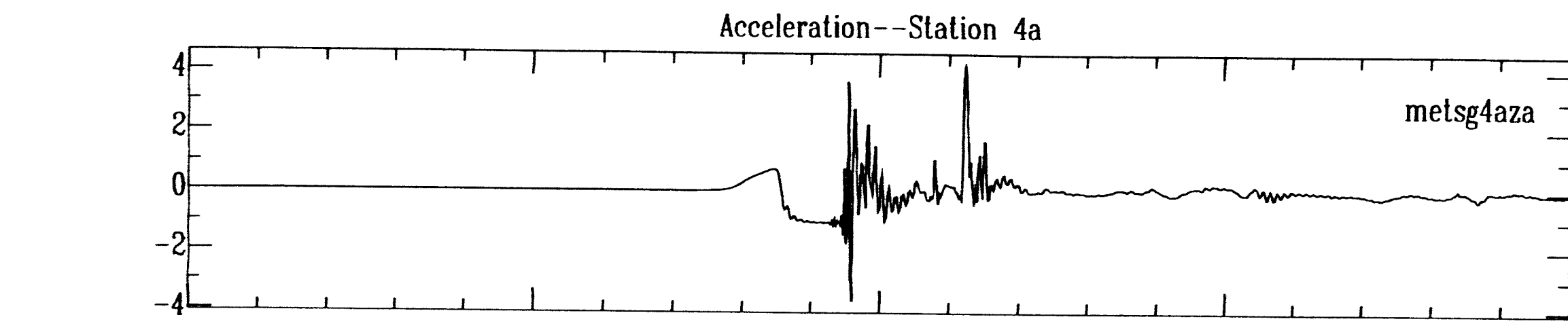

Velocity--Station 4a

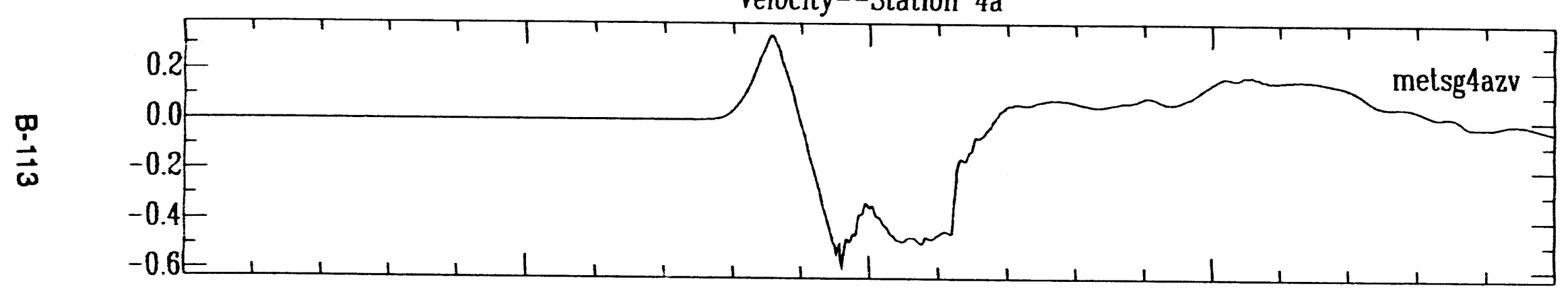

Displacement--Station 4a

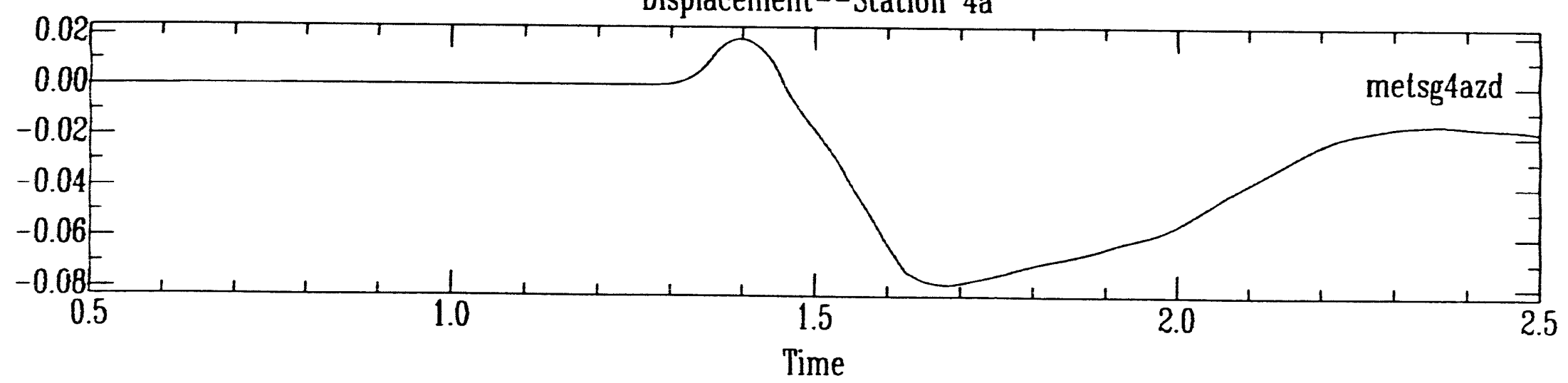




\section{Metropolis Surface Ground Motion}

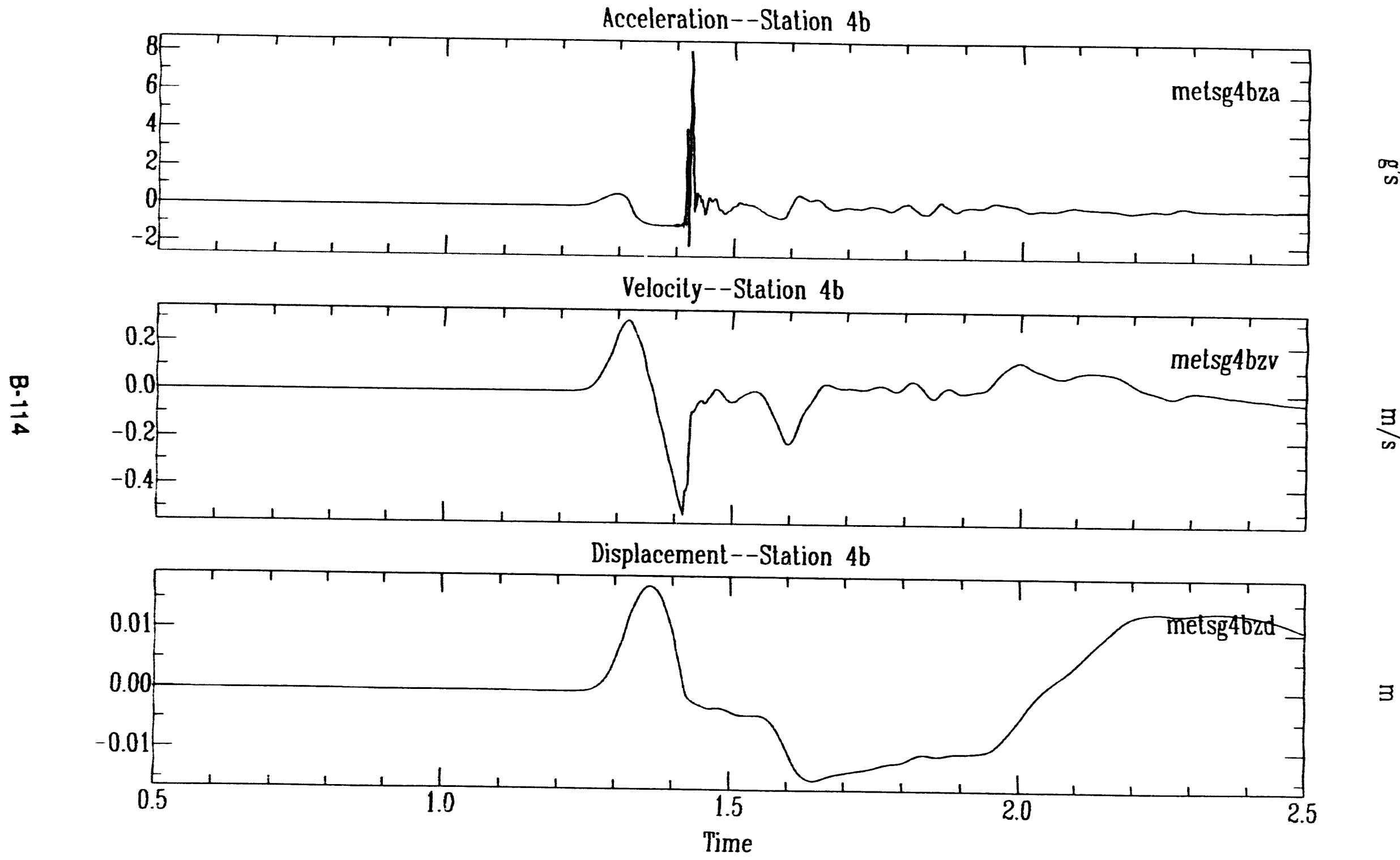




\section{Metropolis Surface Ground Motion}

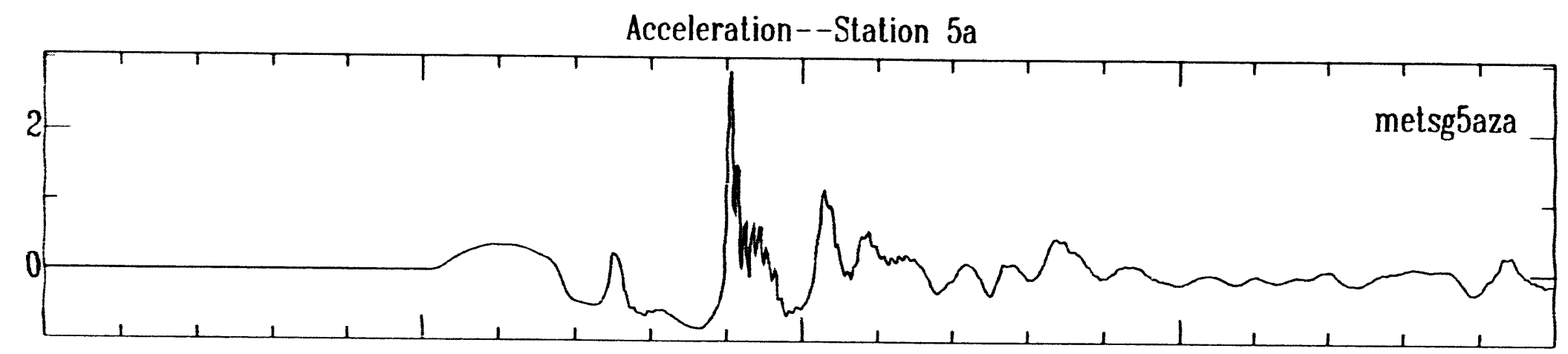

Velocity--Station 5a
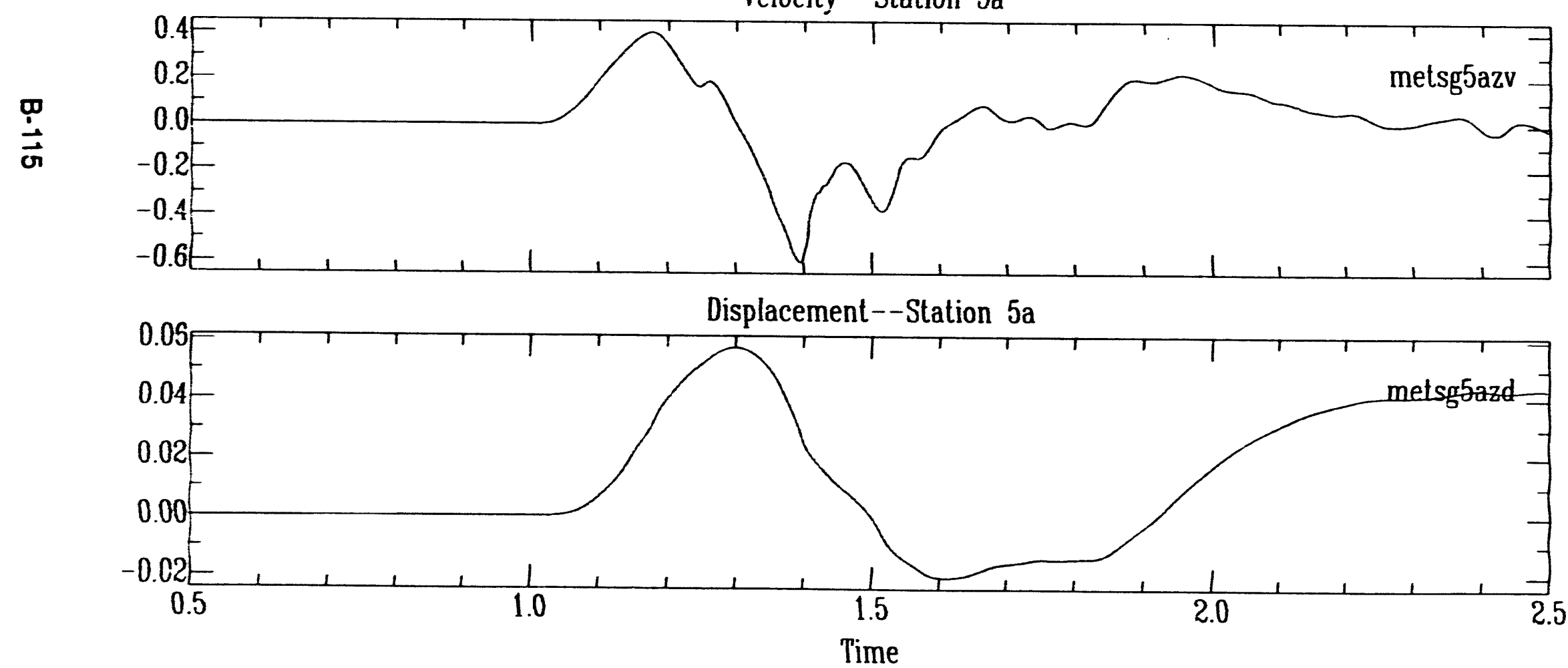


\section{Metropolis Surface Ground Motion}

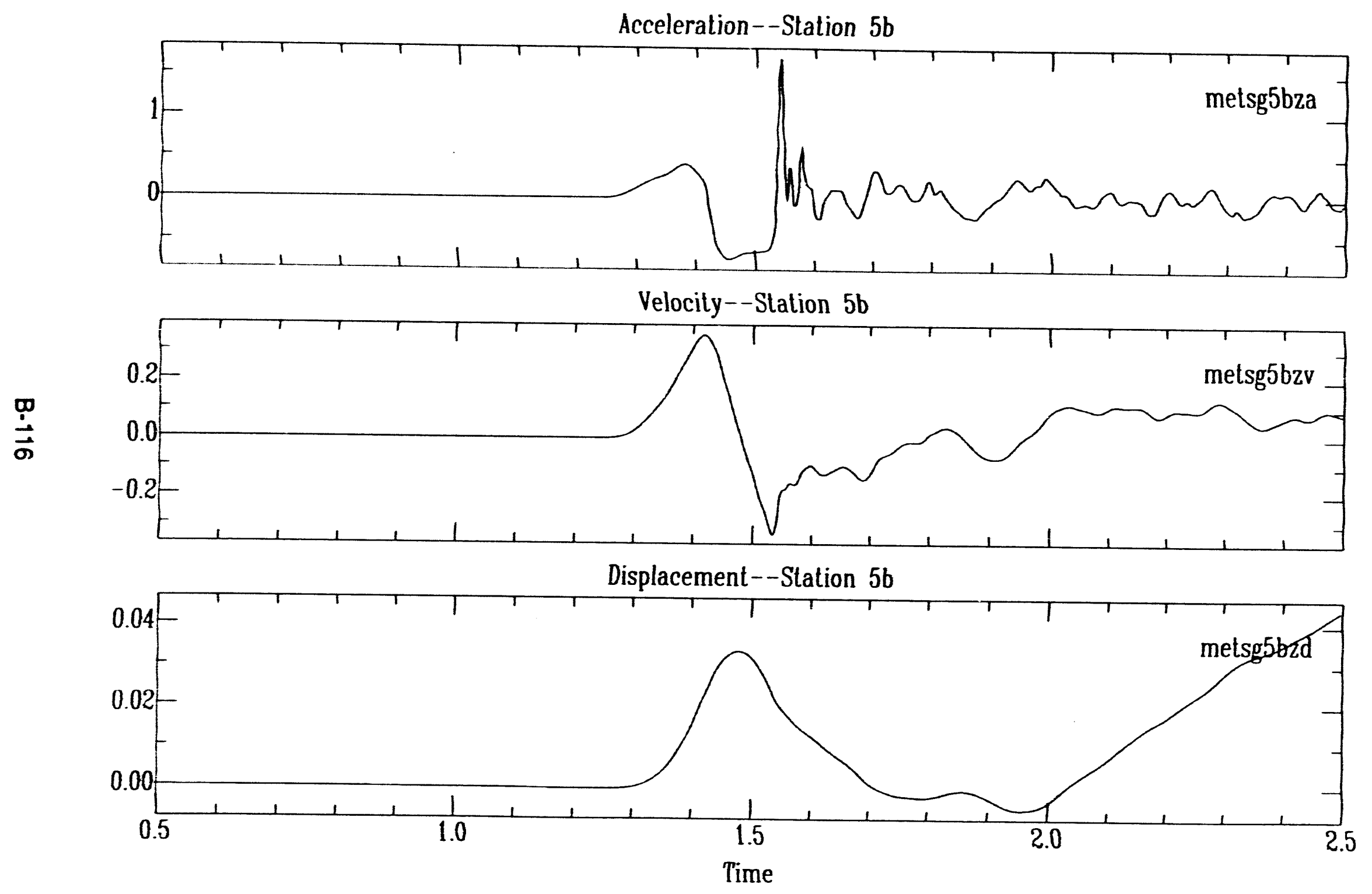

is 


\section{Metropolis Surface Ground Motion}

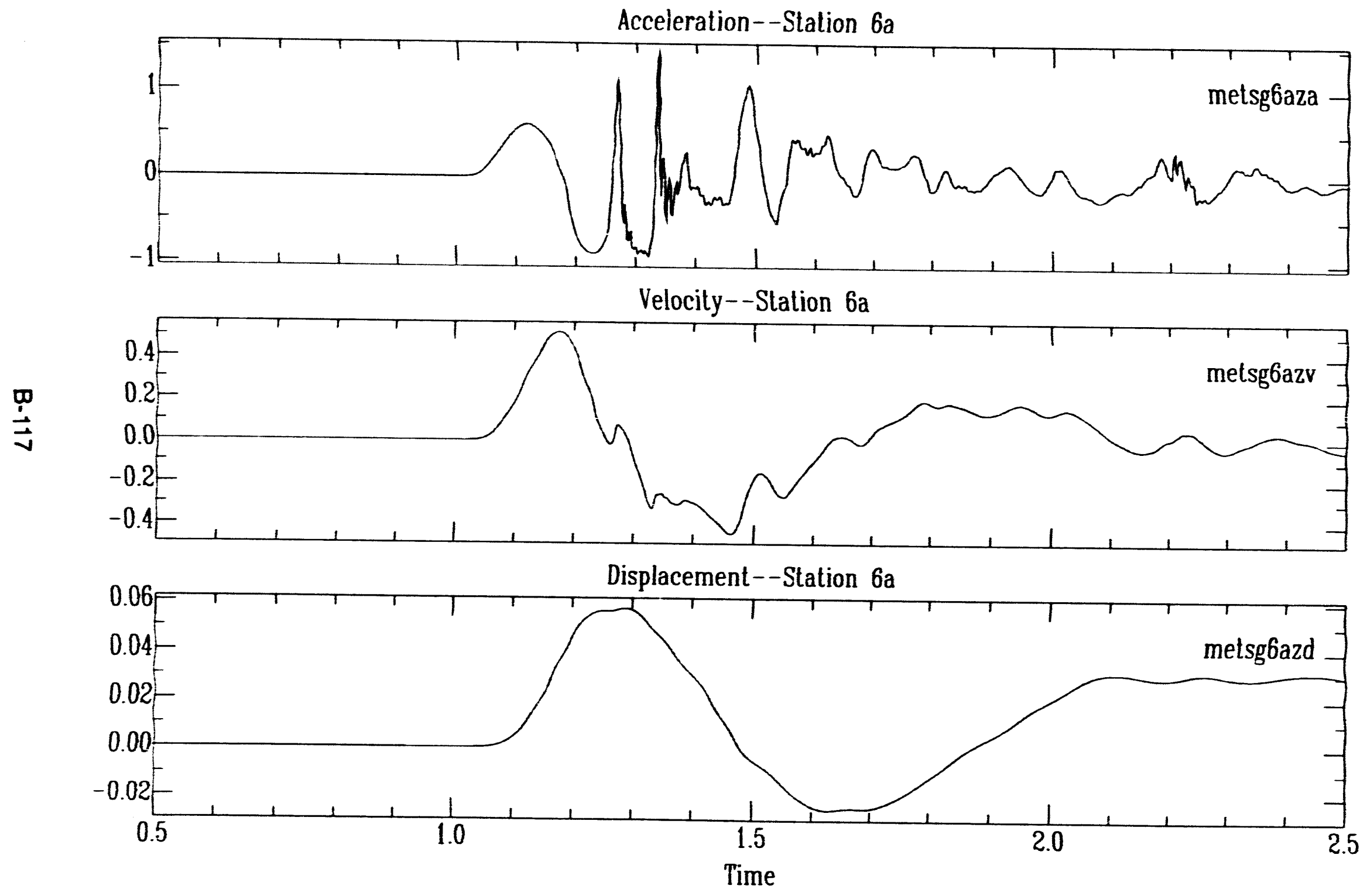




\section{Metropolis Surface Ground Motion}

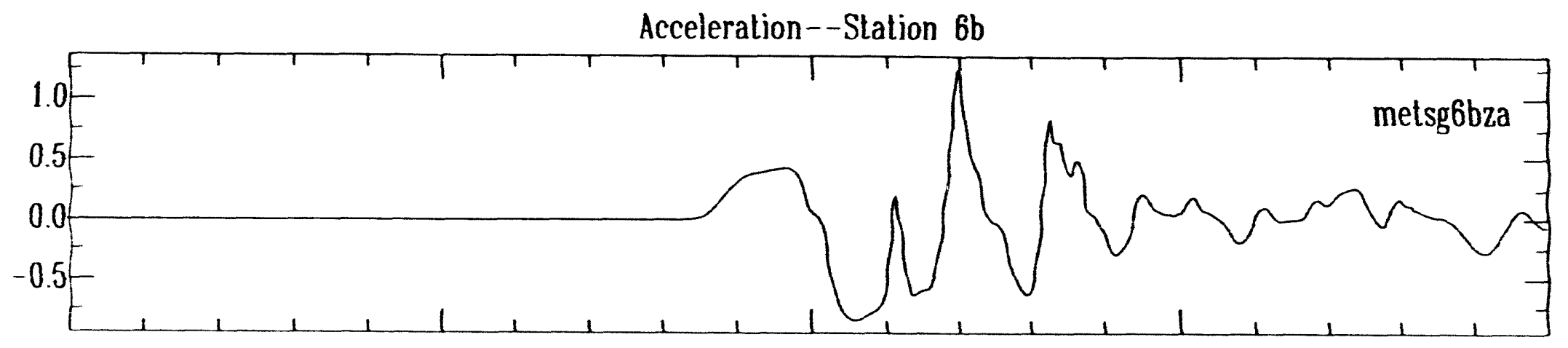

Velocity--Station 6b

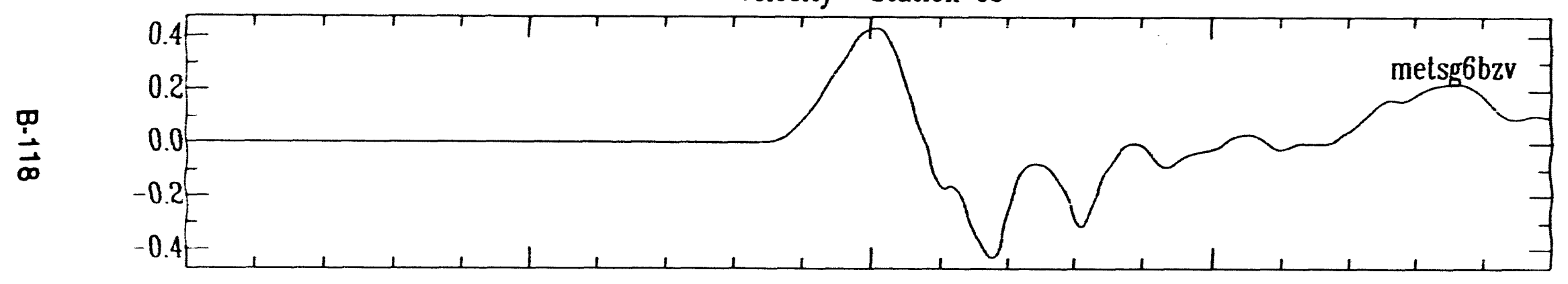

3

Displacement--Station $6 \mathrm{~b}$

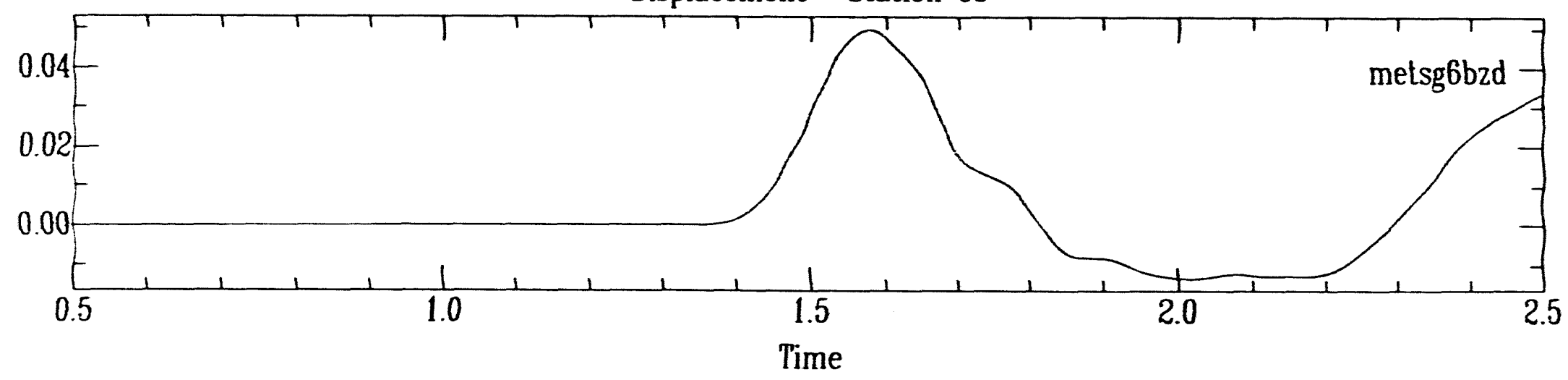




\section{Metropolis Surface Ground Motion}

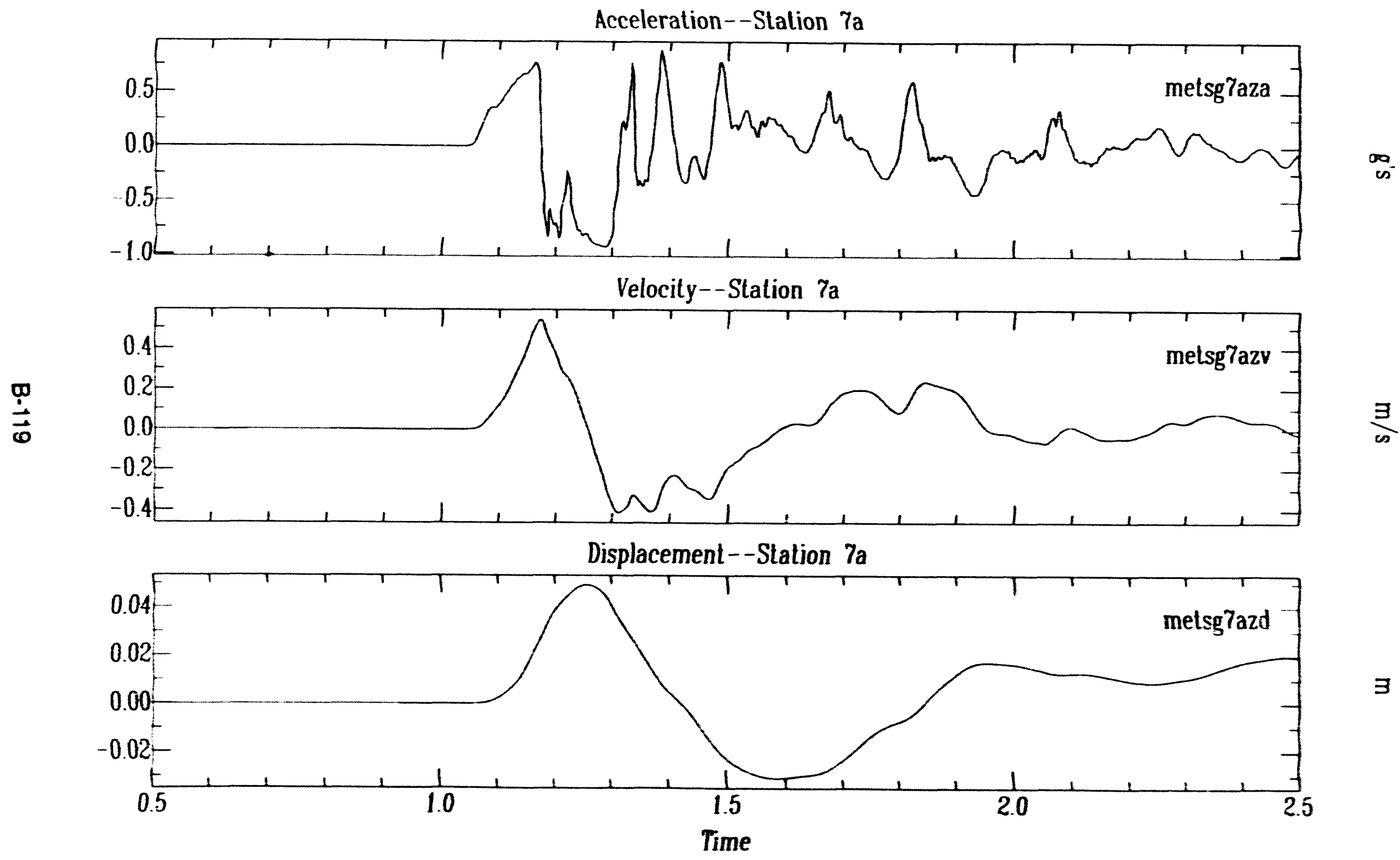




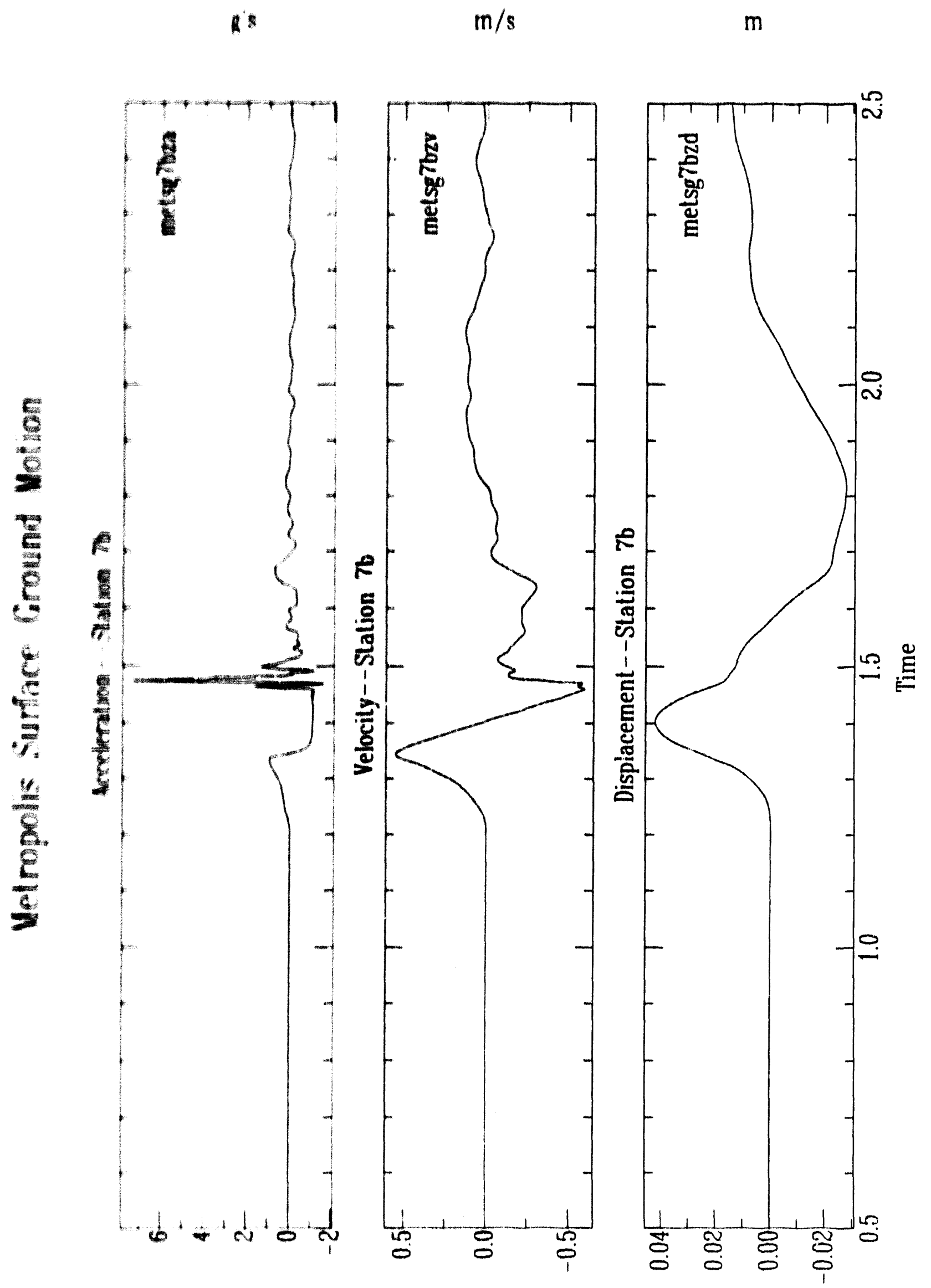

B. 120 
Metropolis Surface Ground Motion

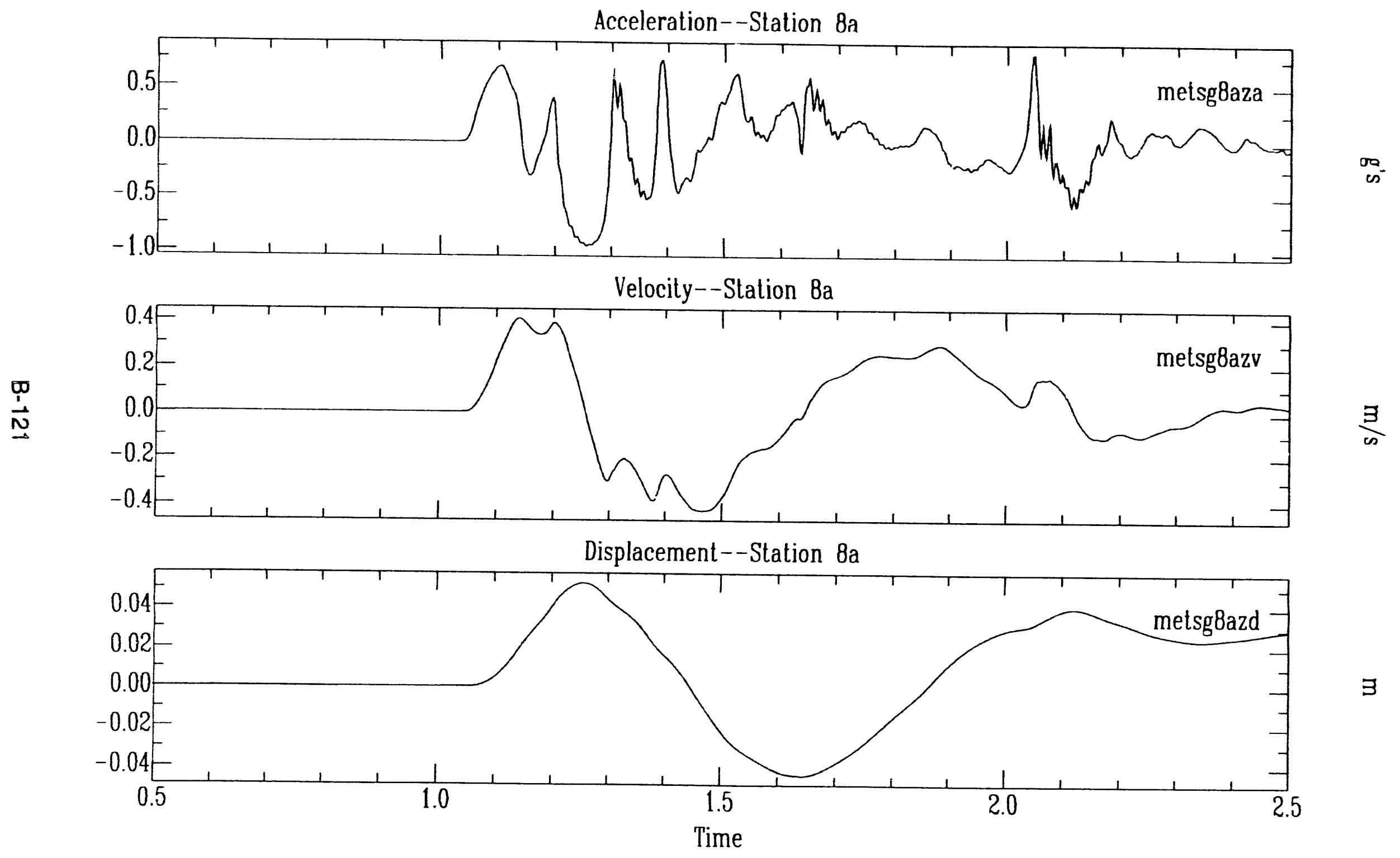




\section{Metropolis Surface Ground Motion}

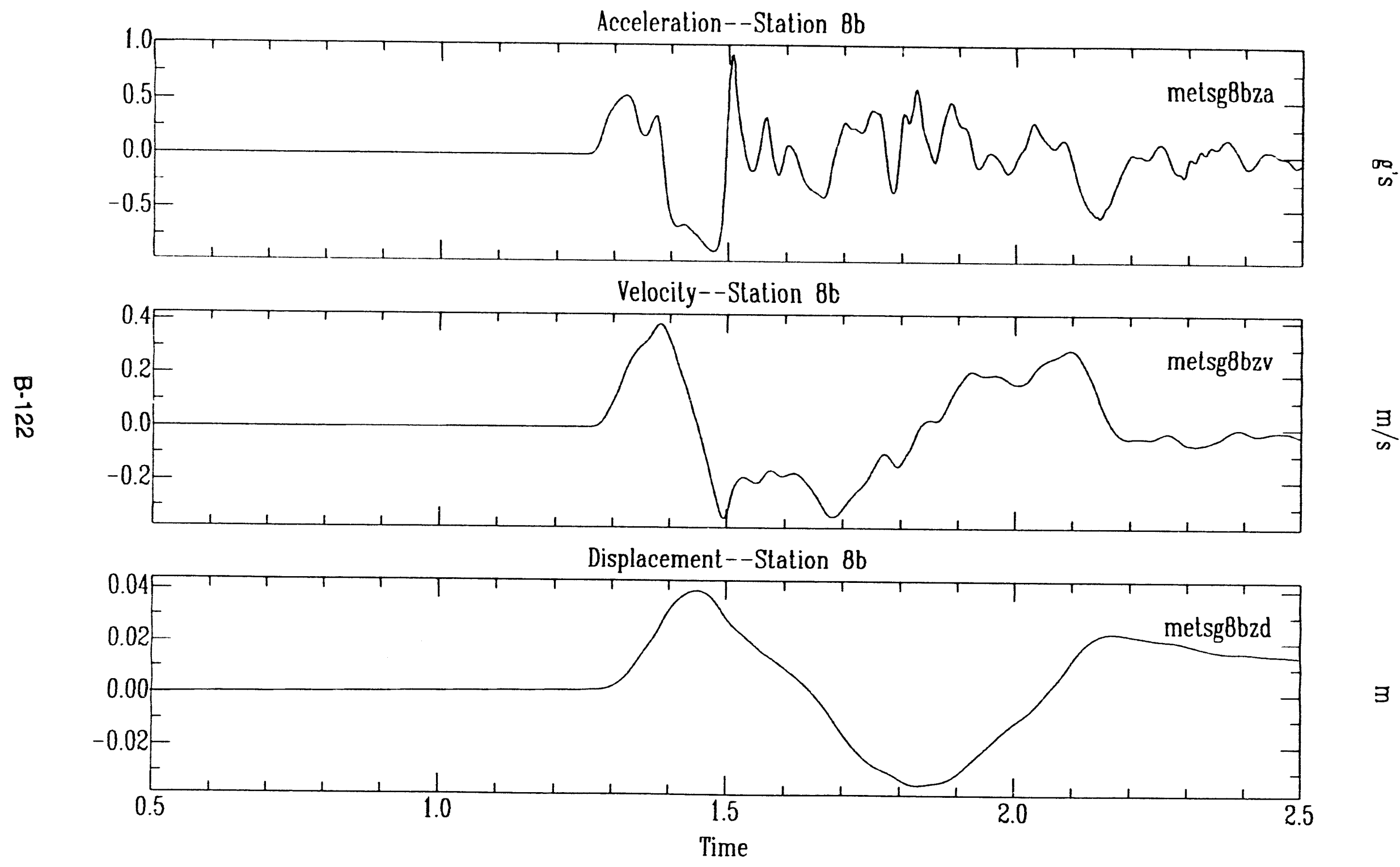




\section{Metropolis Surface Ground Motion}

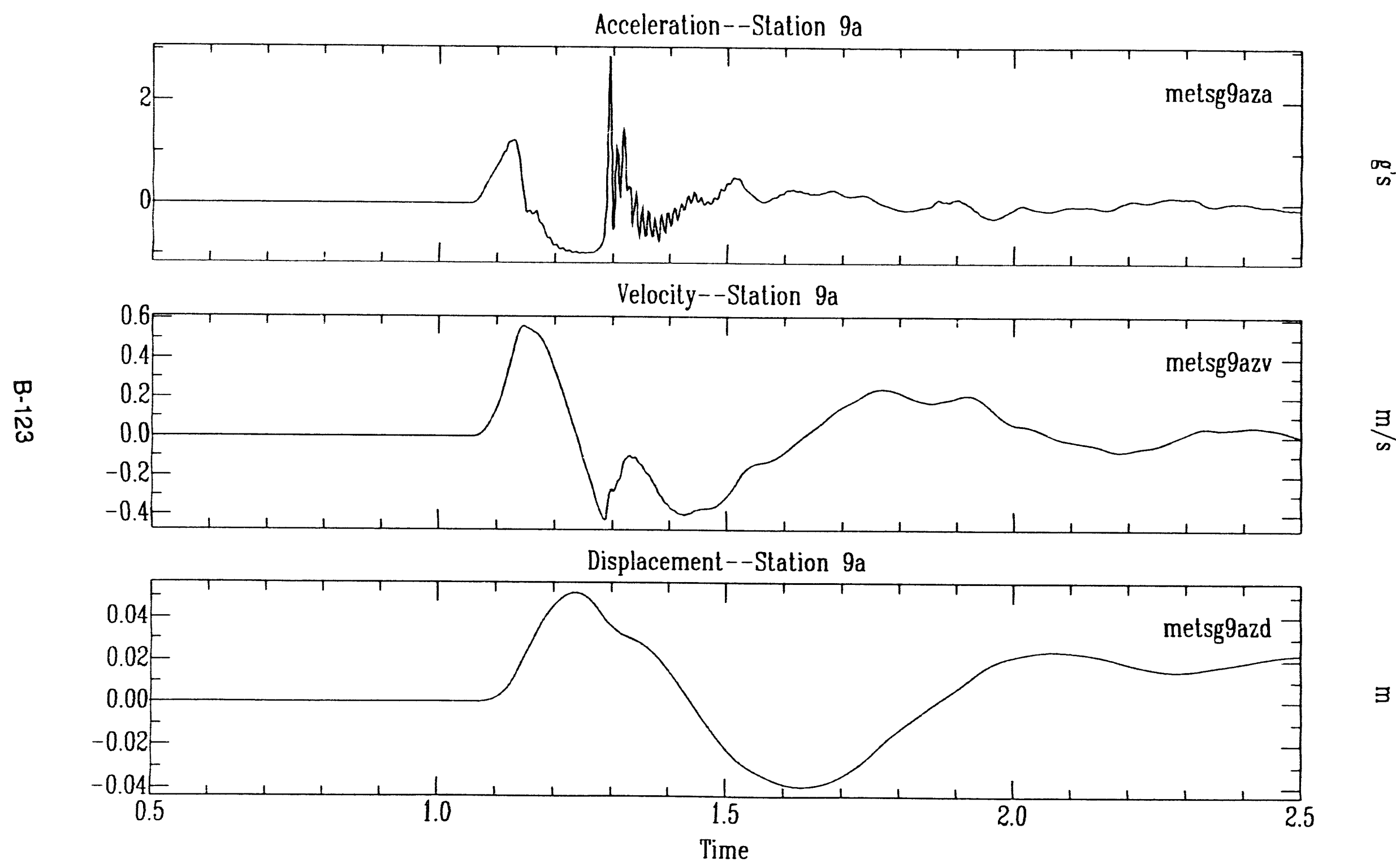


Metropolis Surface Ground Motion

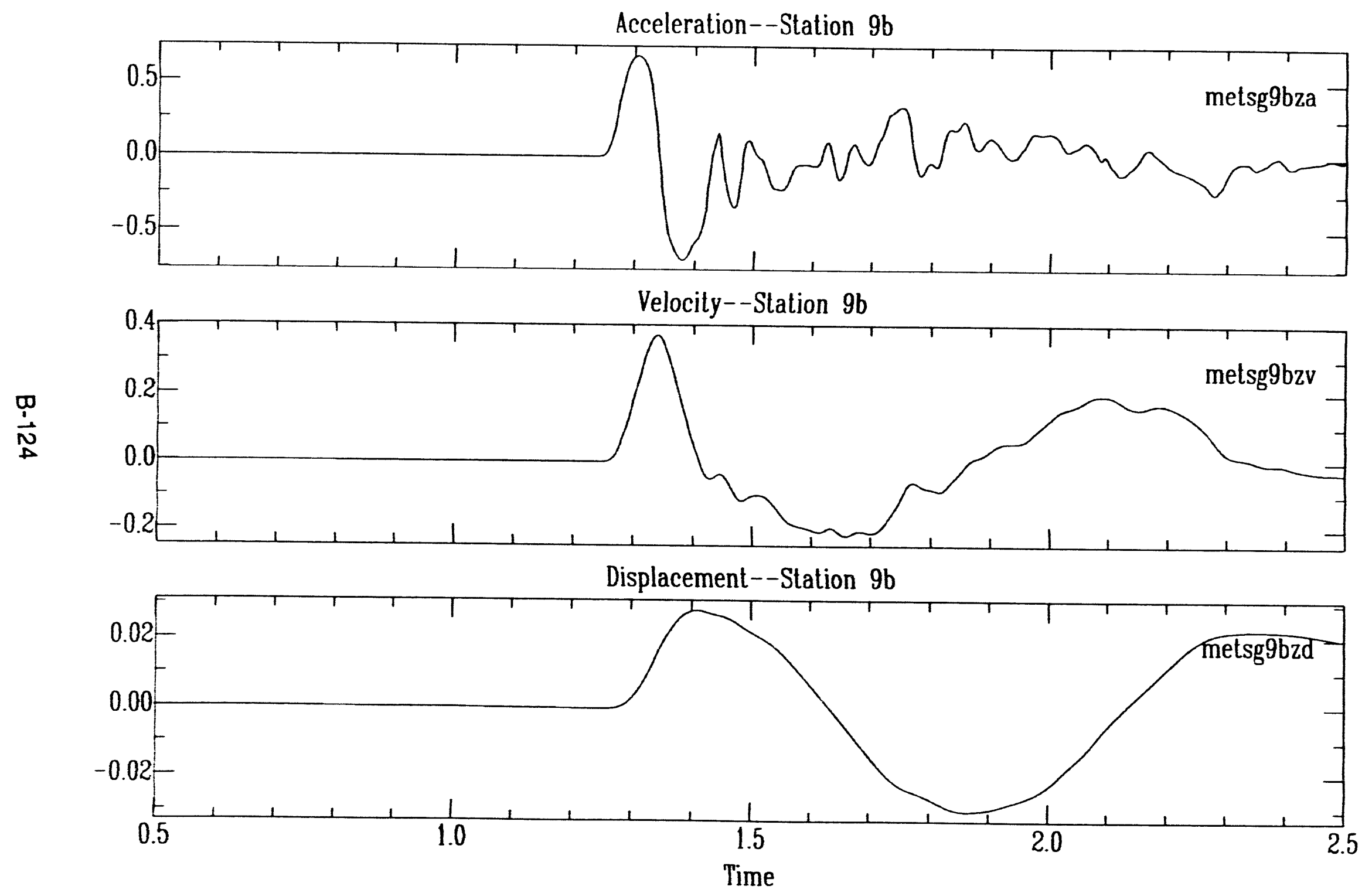




\section{Metropolis Surface Ground Motion}

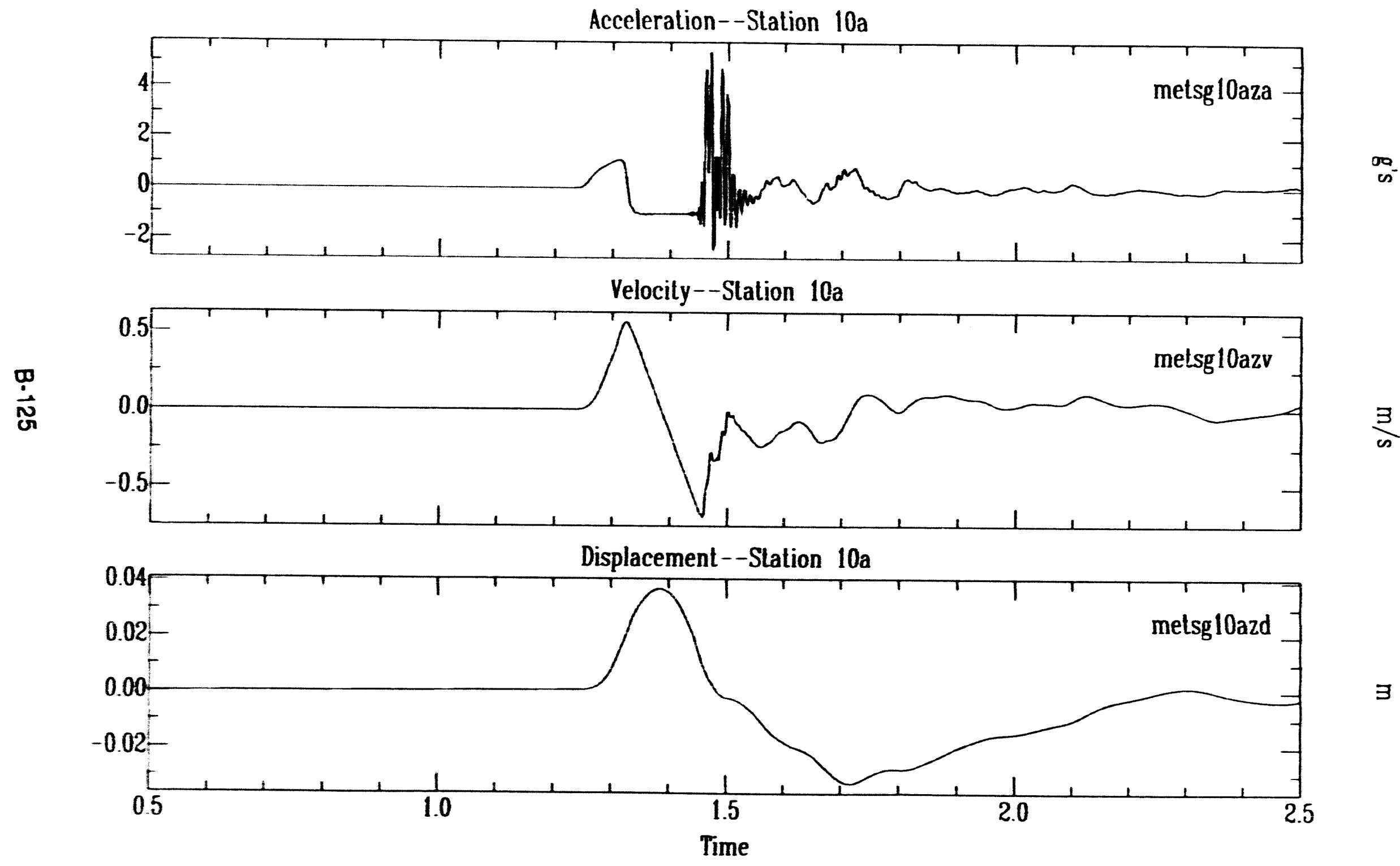




\section{Metropolis Surface Ground Motion}

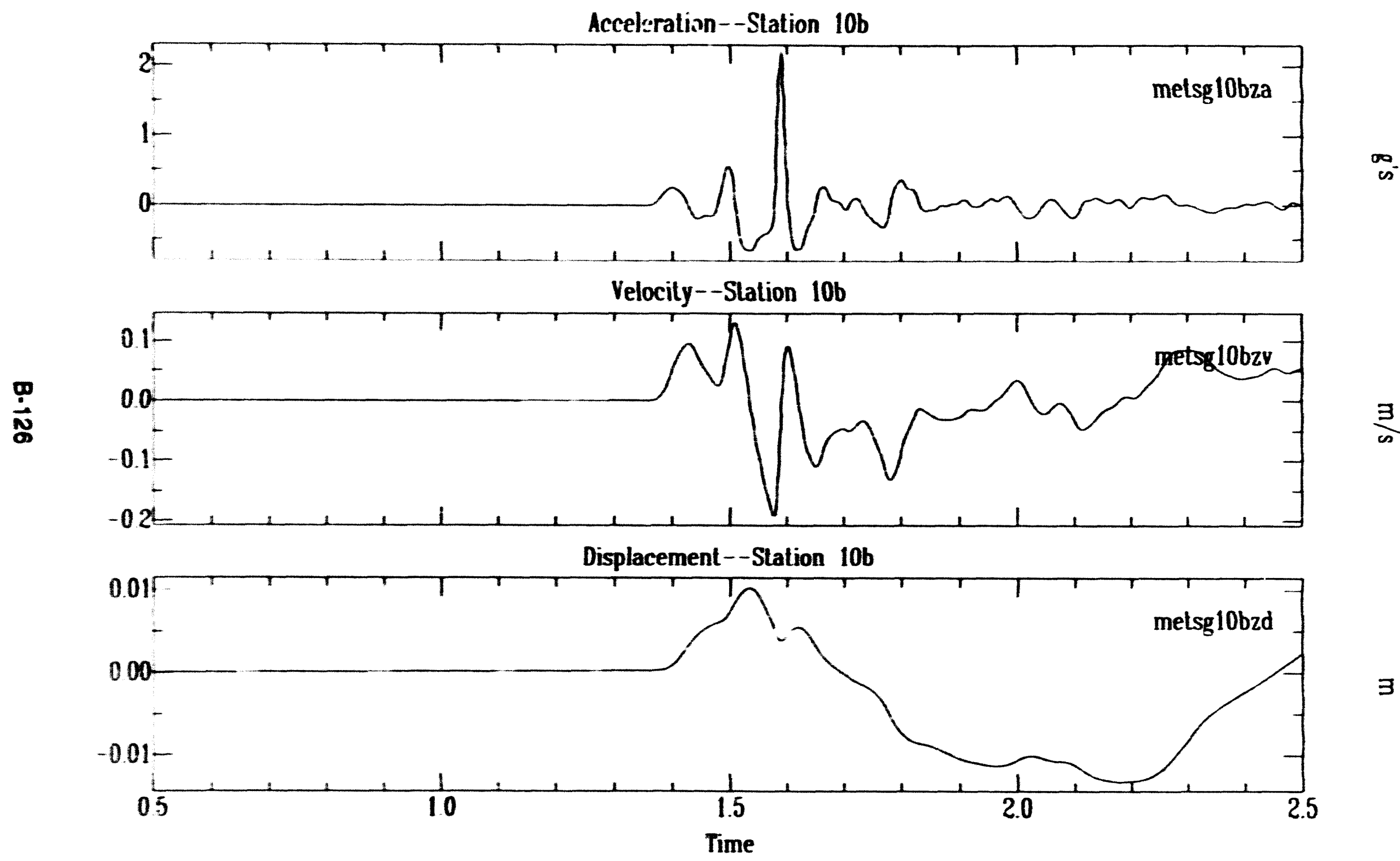




\subsection{Minoral Quarry (U12n.22)}

Experiment dosign. The Area 12 tunnel event, Mineral Quarry, had an array of 34 surface ground motion stations: 9 GRMPY (around SGZ, inside the predicted spall zone) and 25 ESA stations (from just outside to well beyond the edge of predicted 8pall), fielded by SMU and DNAA (80e Table 12 and Fig. 15).

Reeults. The records for the accelerometer stations fielded on Mineral Quarry are given in Fig. 16 (32 pages). Of the nine GRMPY stations fielded, seven recorded good spall signals and two. MINGR7A and $8 A$, recorded signals that indicated that the stations were just at the edge of spall. Of the 25 planned ESA stations, five (including MINSG3A, 5A, 14A, 15A and the 9-element array) were recorded on DNA PDASs, the data from which were not avallable as this report went to press. None of the ESA stations recorded signals that indicated spall. 


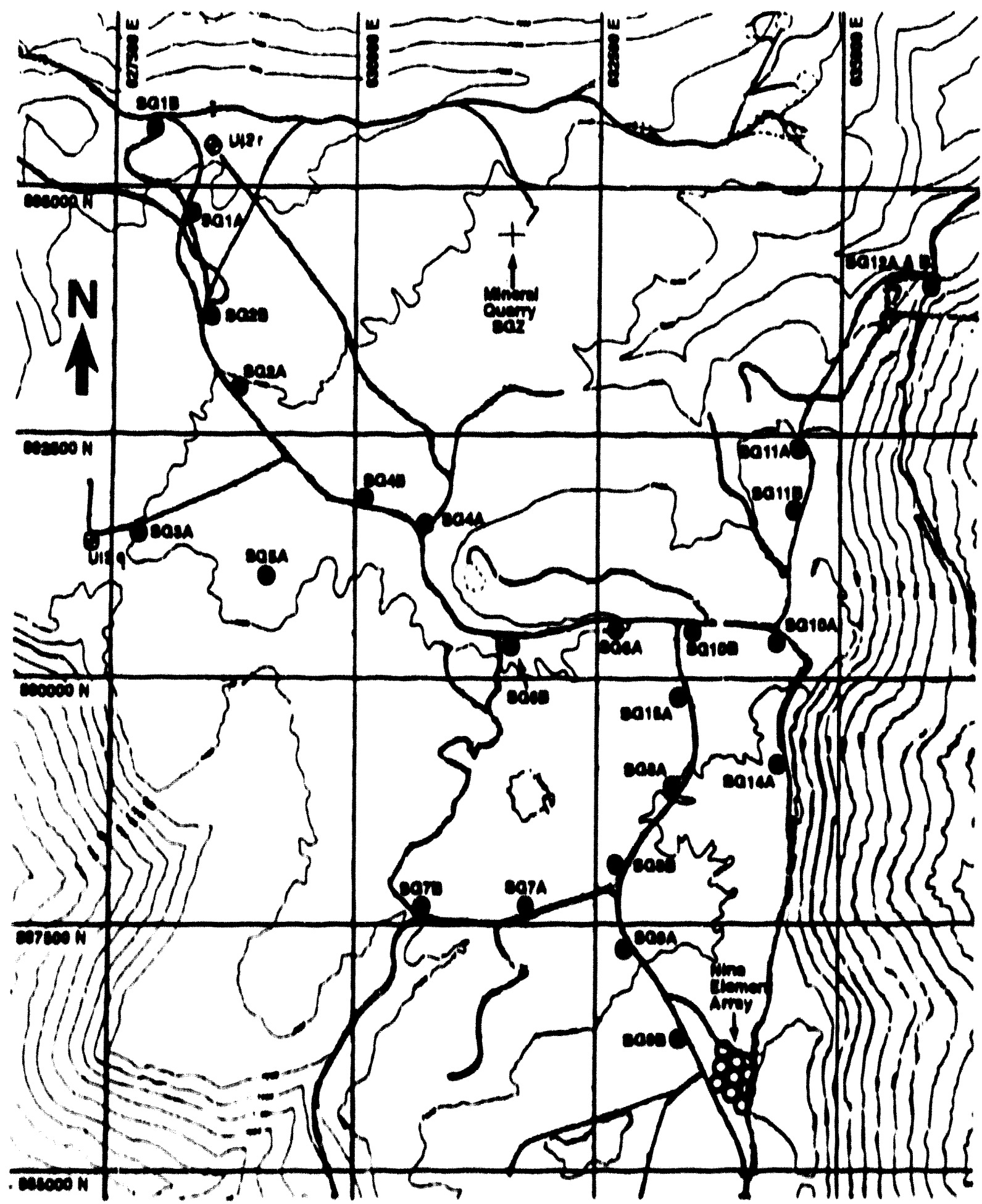

Fig. 15. Mineral Quarry station map. 


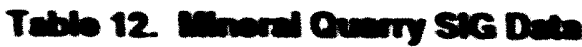

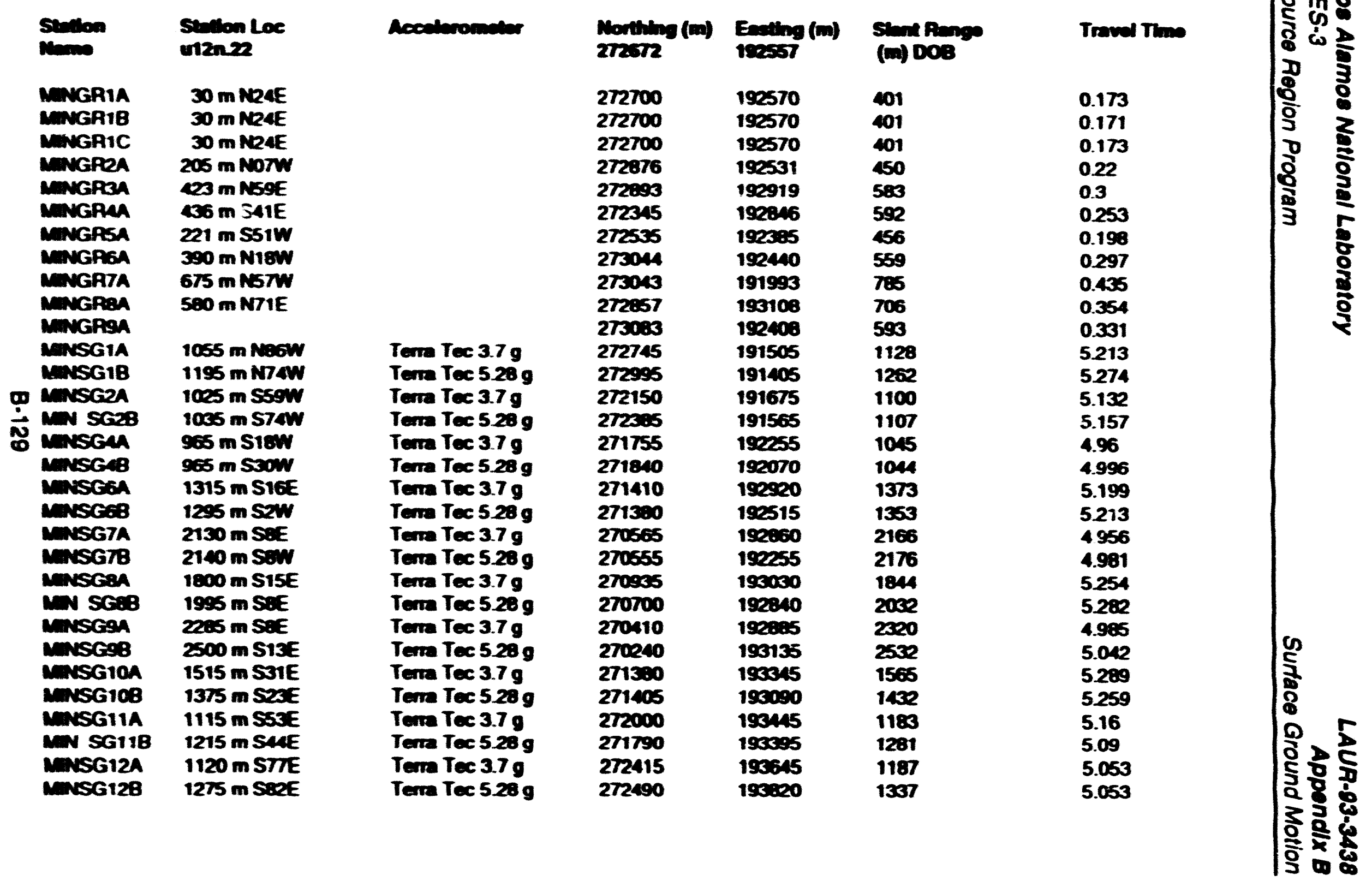




\section{Mineral Quarry Surface Ground Motion}

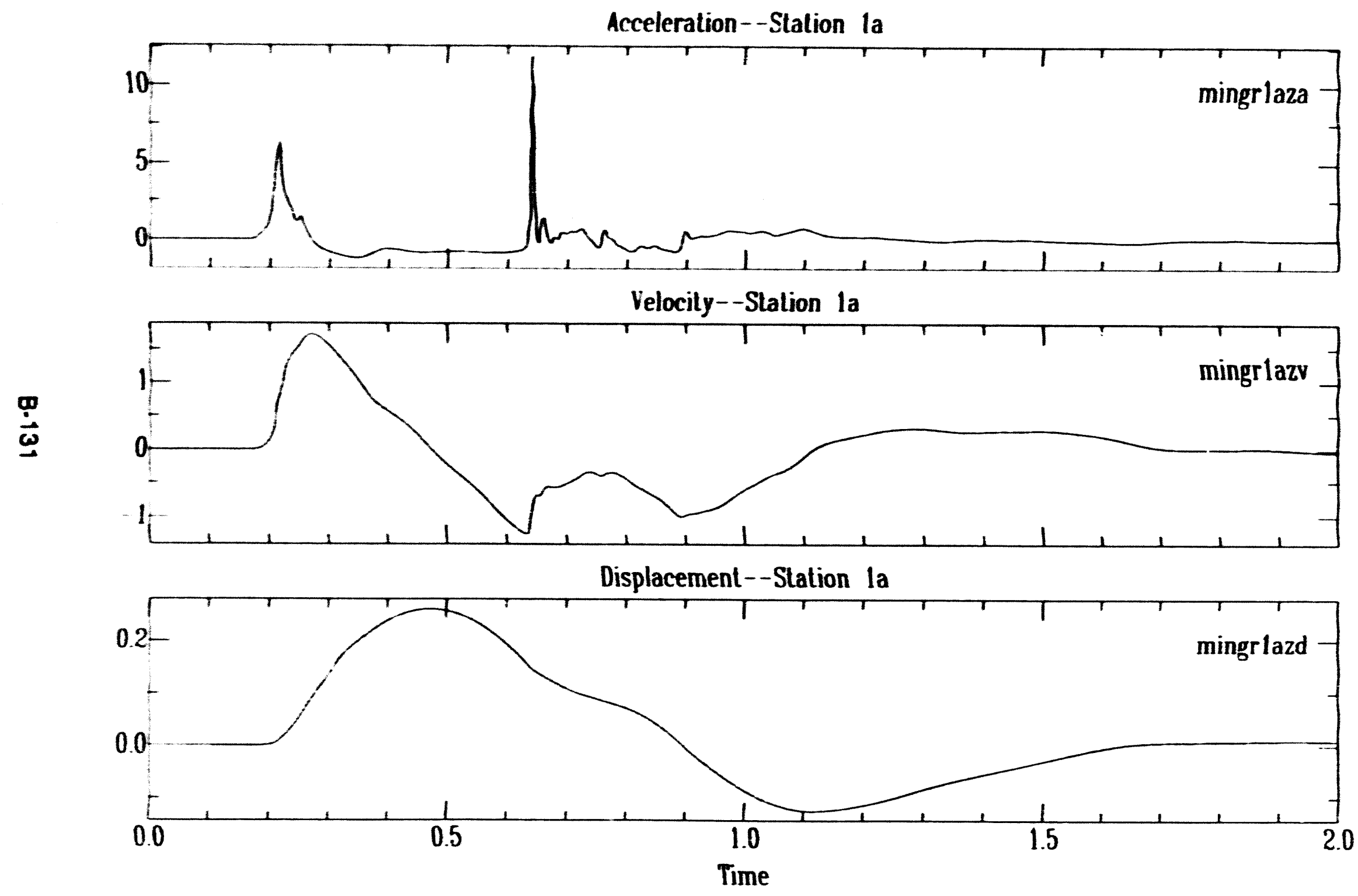




\section{Mineral Quarry Surface Ground Motion}

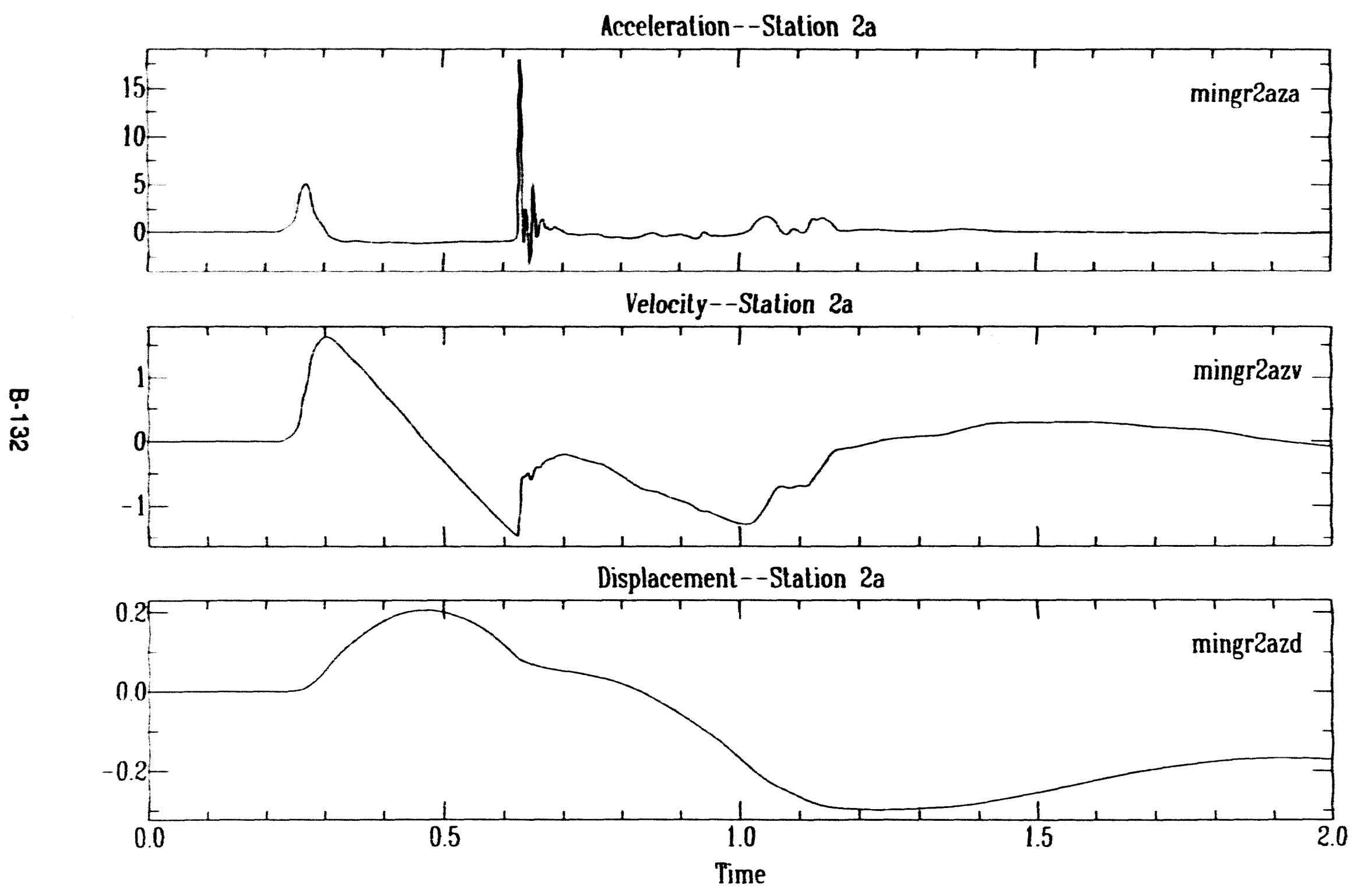




\section{Mineral Quarry Surface Ground Motion}

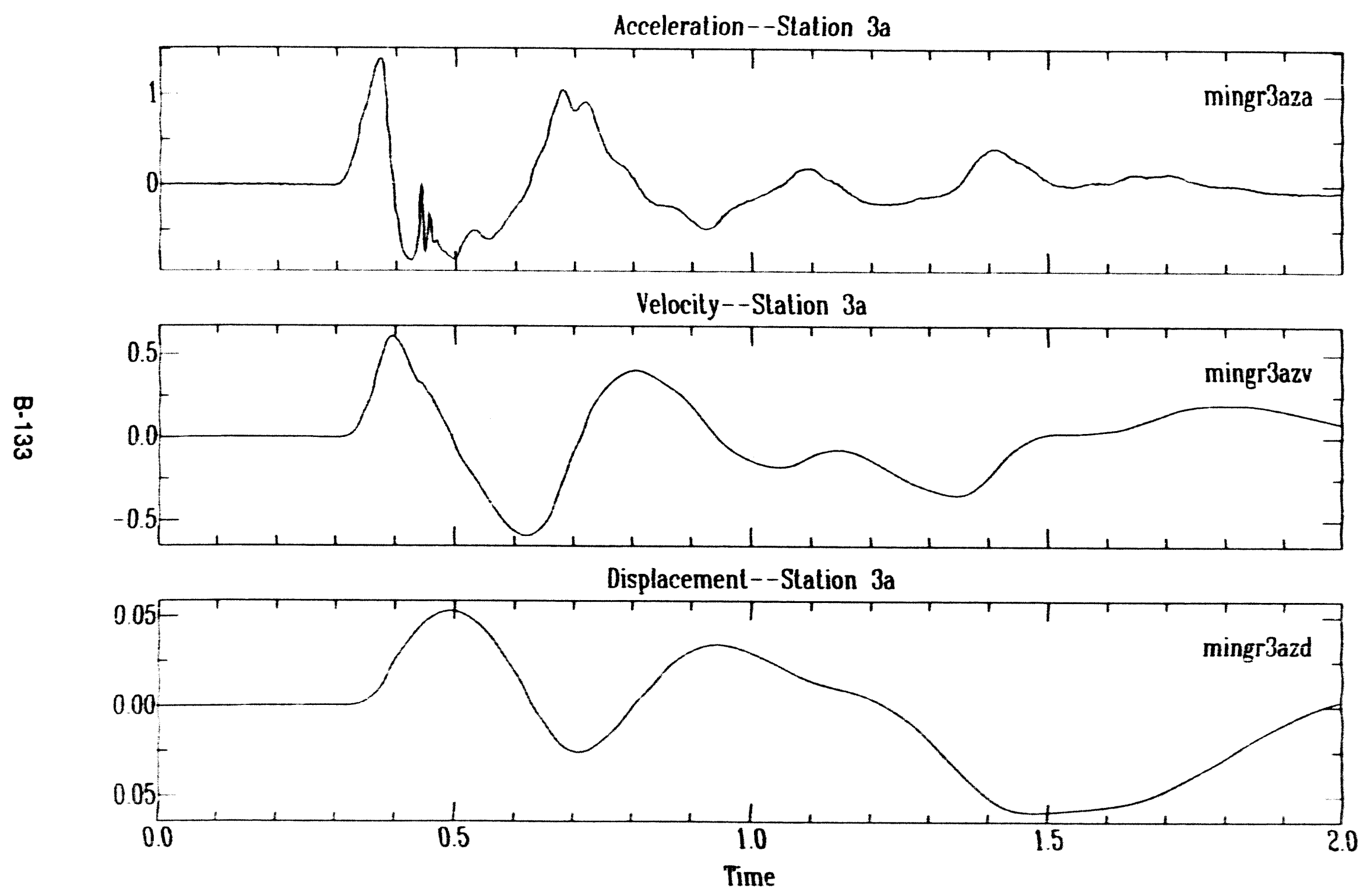




\section{Mineral Quarry Surface Ground Motion}

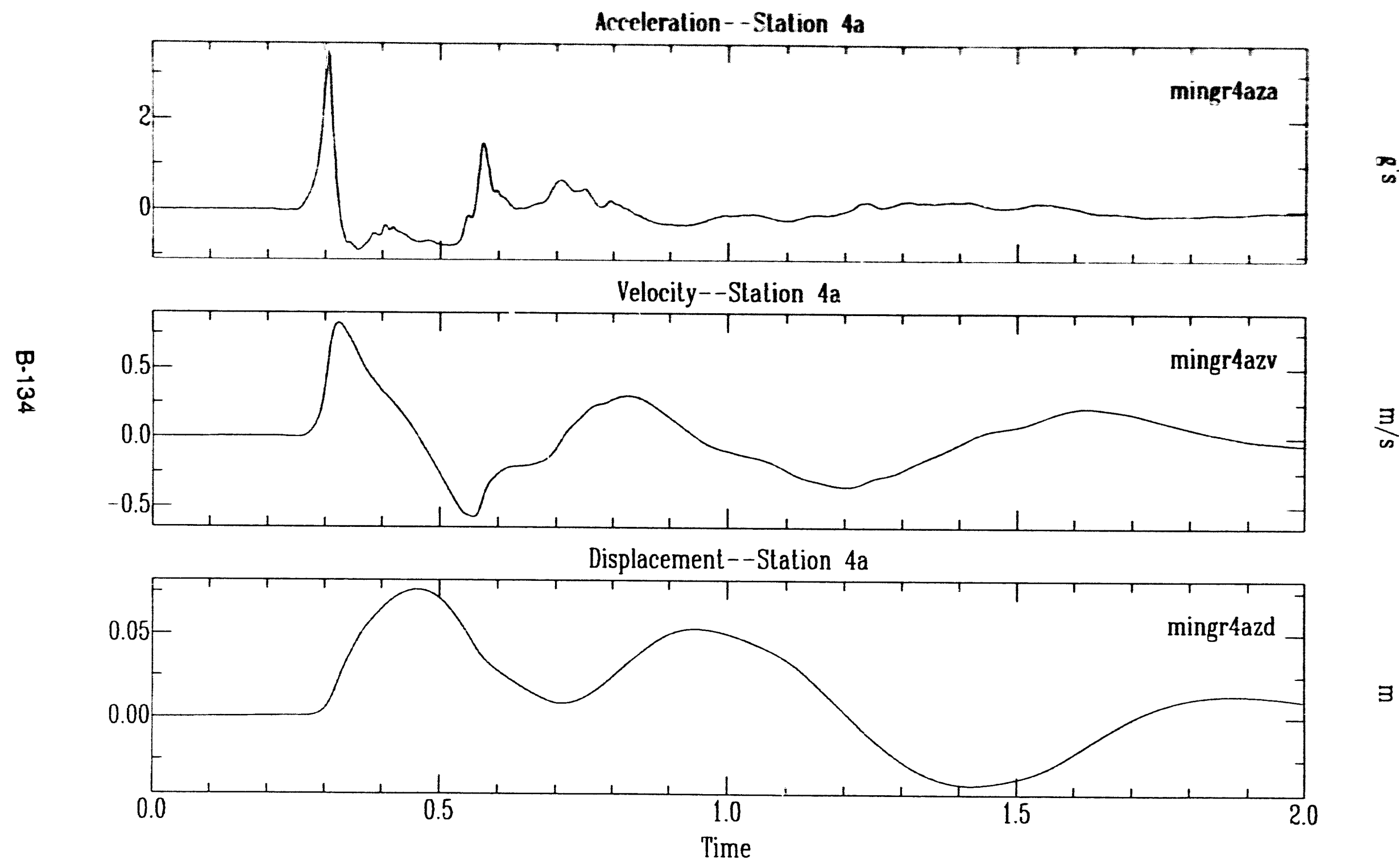




\section{Mineral Quarry Surface Ground Motion}

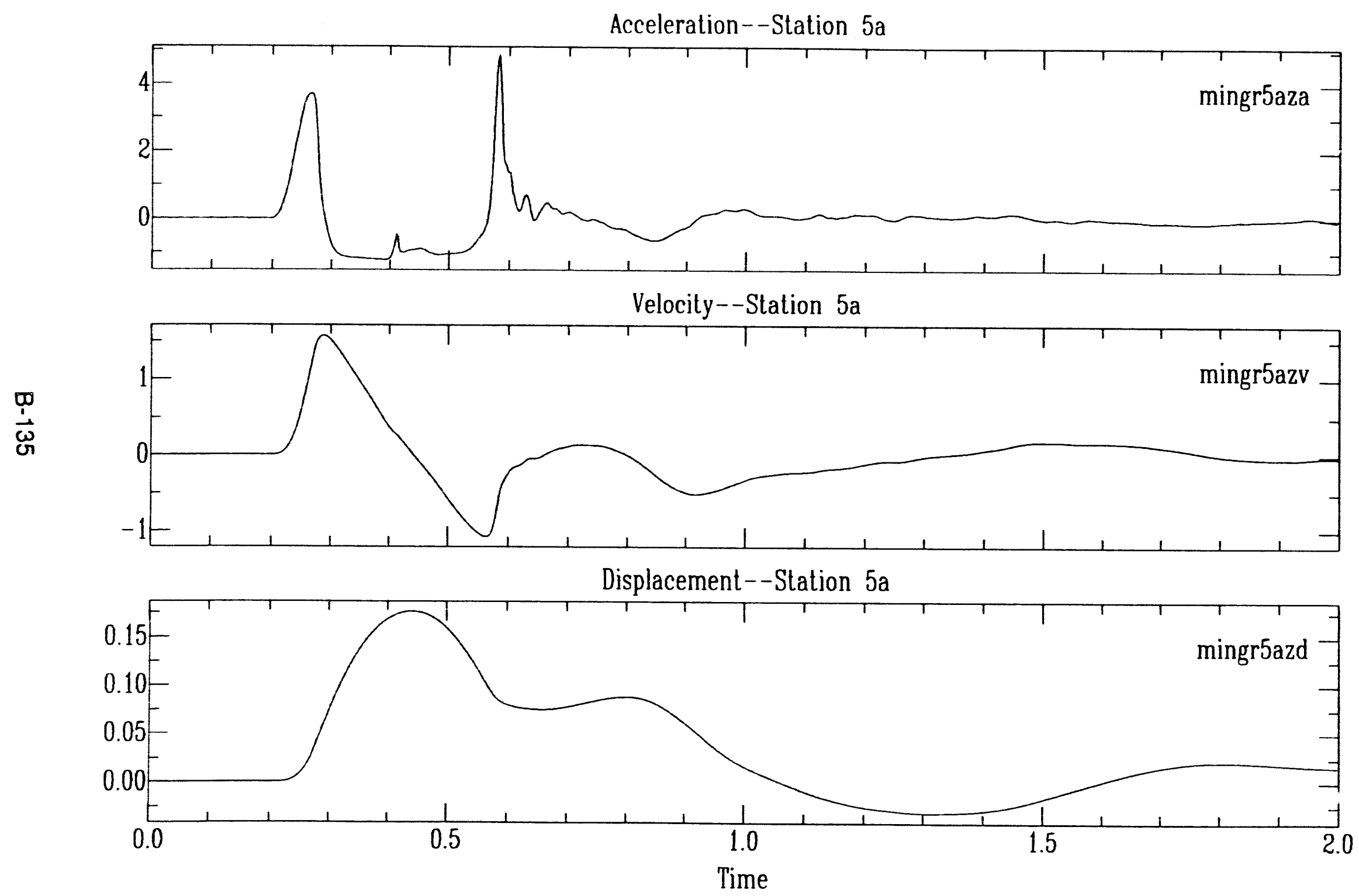




\section{Mineral Quarry Surface Ground Motion}

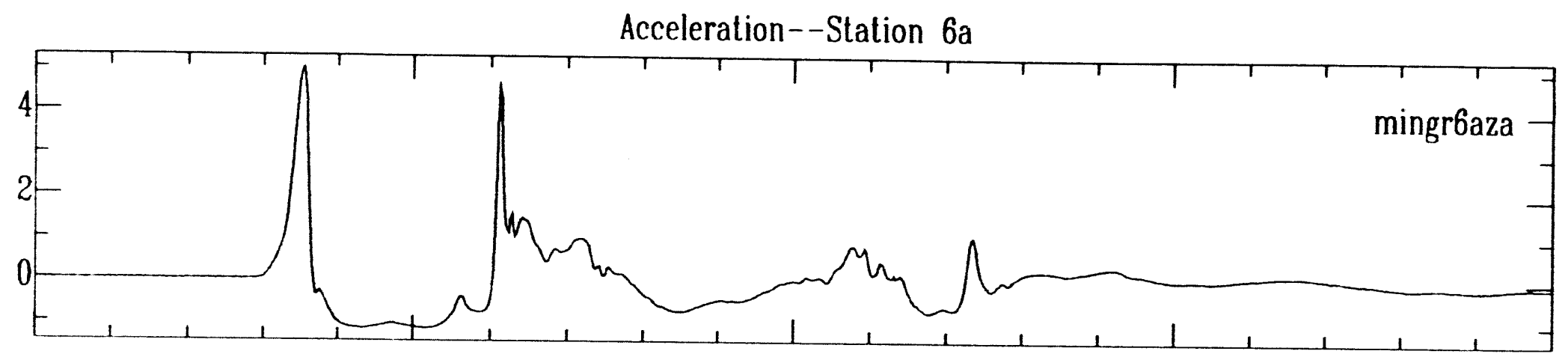

Velocity--Station $6 \mathrm{a}$

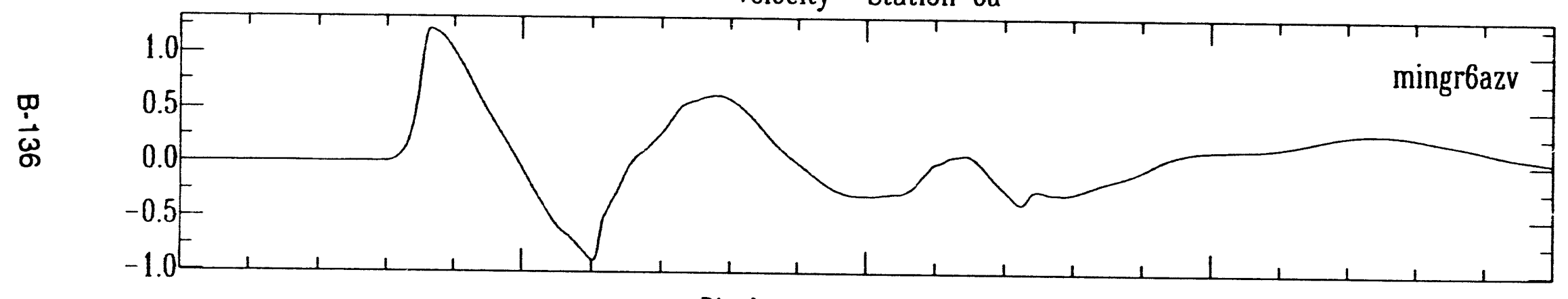

承

Displacement--Station 6a

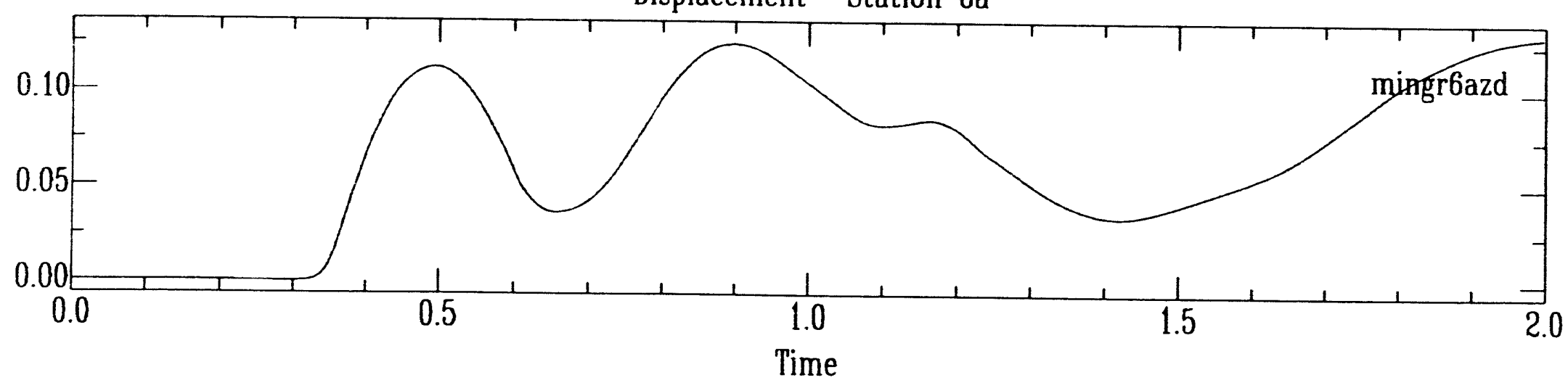




\section{Mineral Quarry Surface Ground Motion}

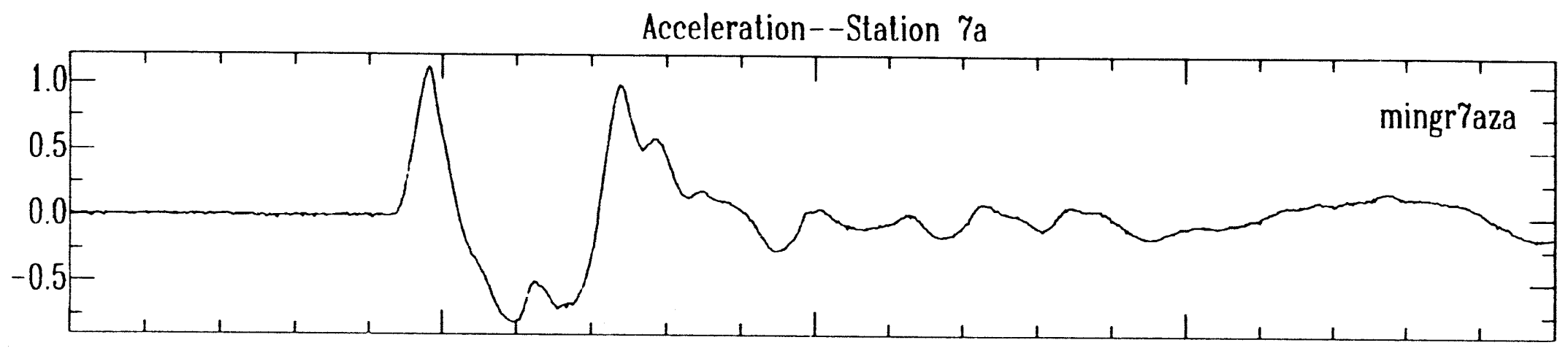

Velocity--Station 7a

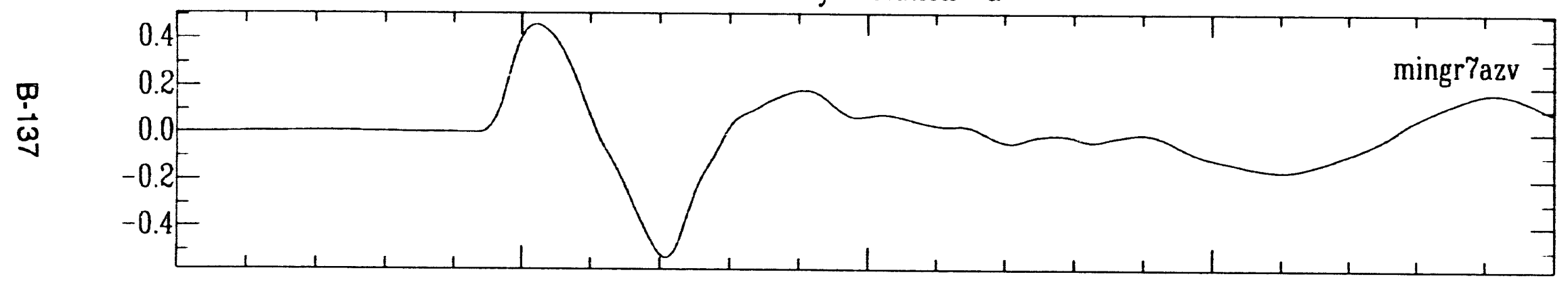

Displacement--Station 7a

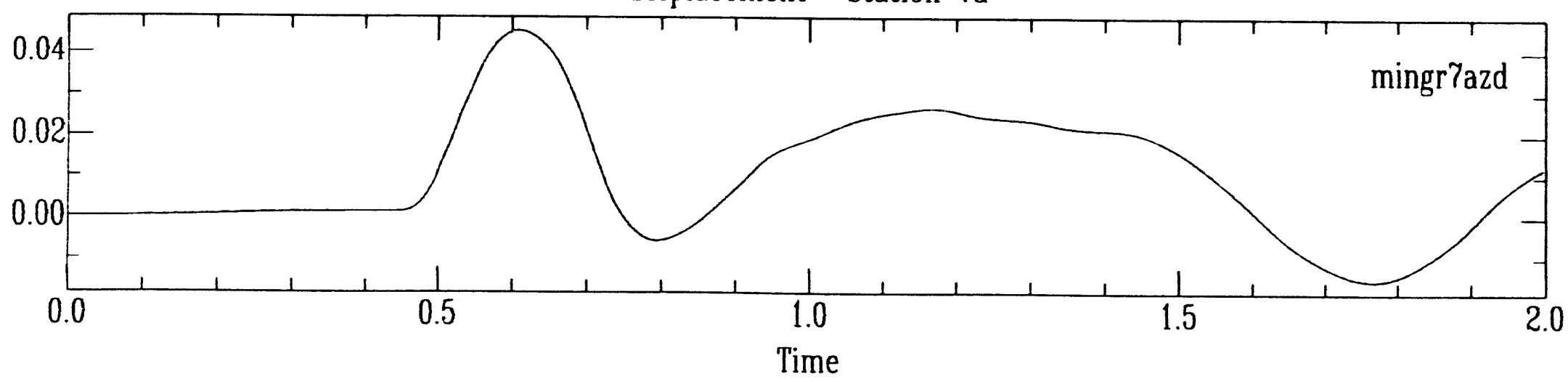




\section{Mineral Quarry Surface Ground Motion}

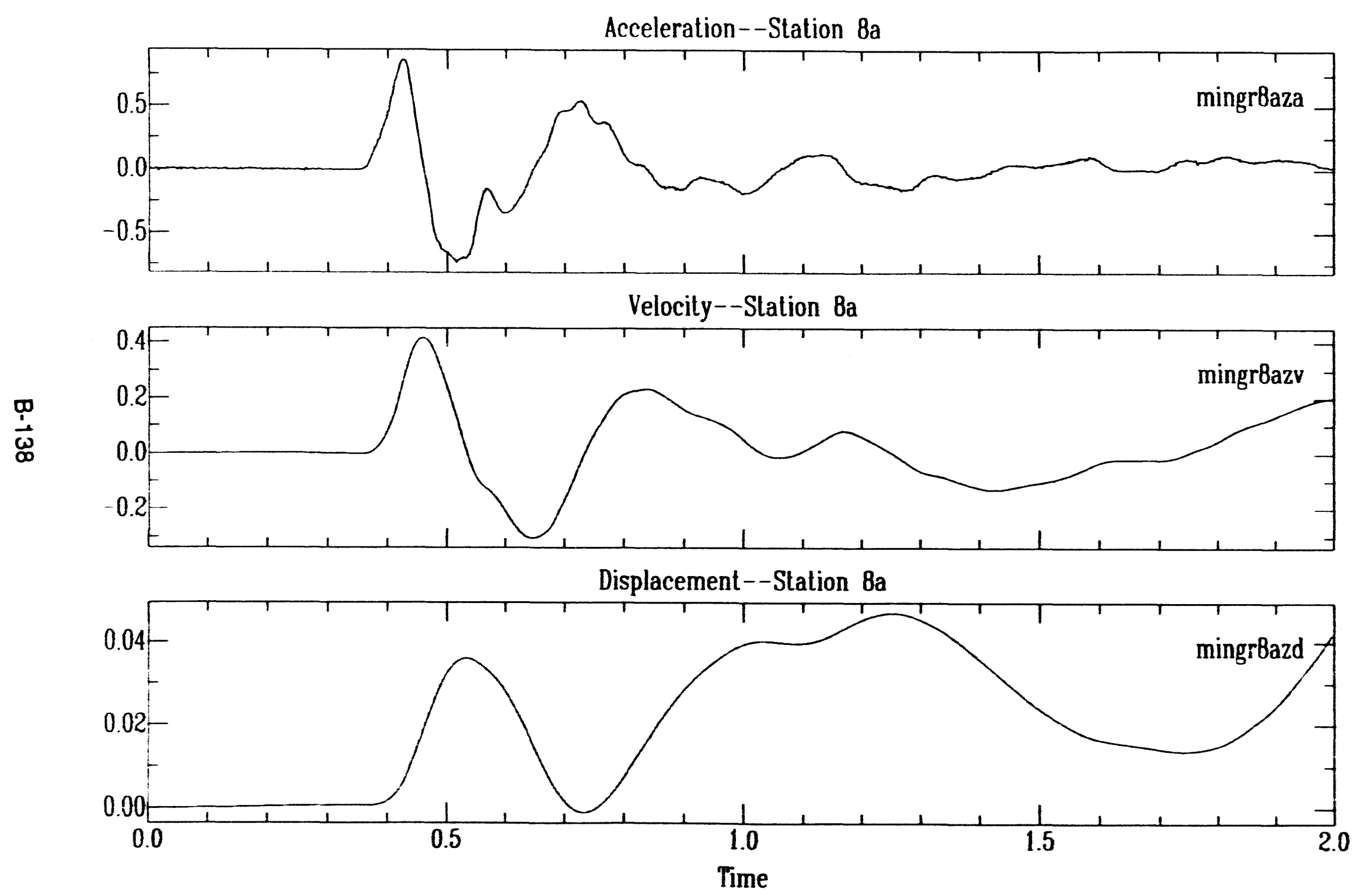




\section{Mineral Quarry Surface Ground Motion}

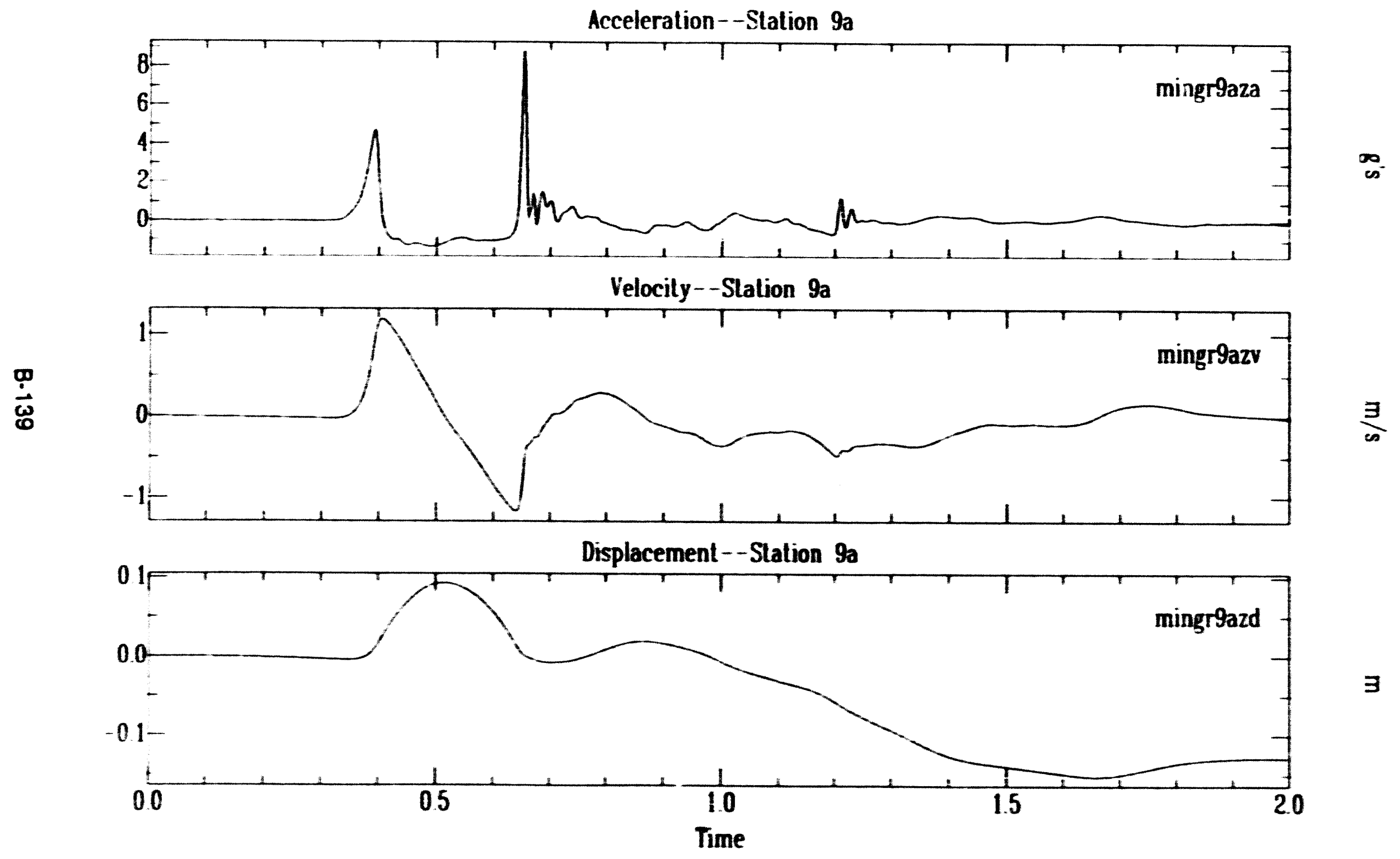



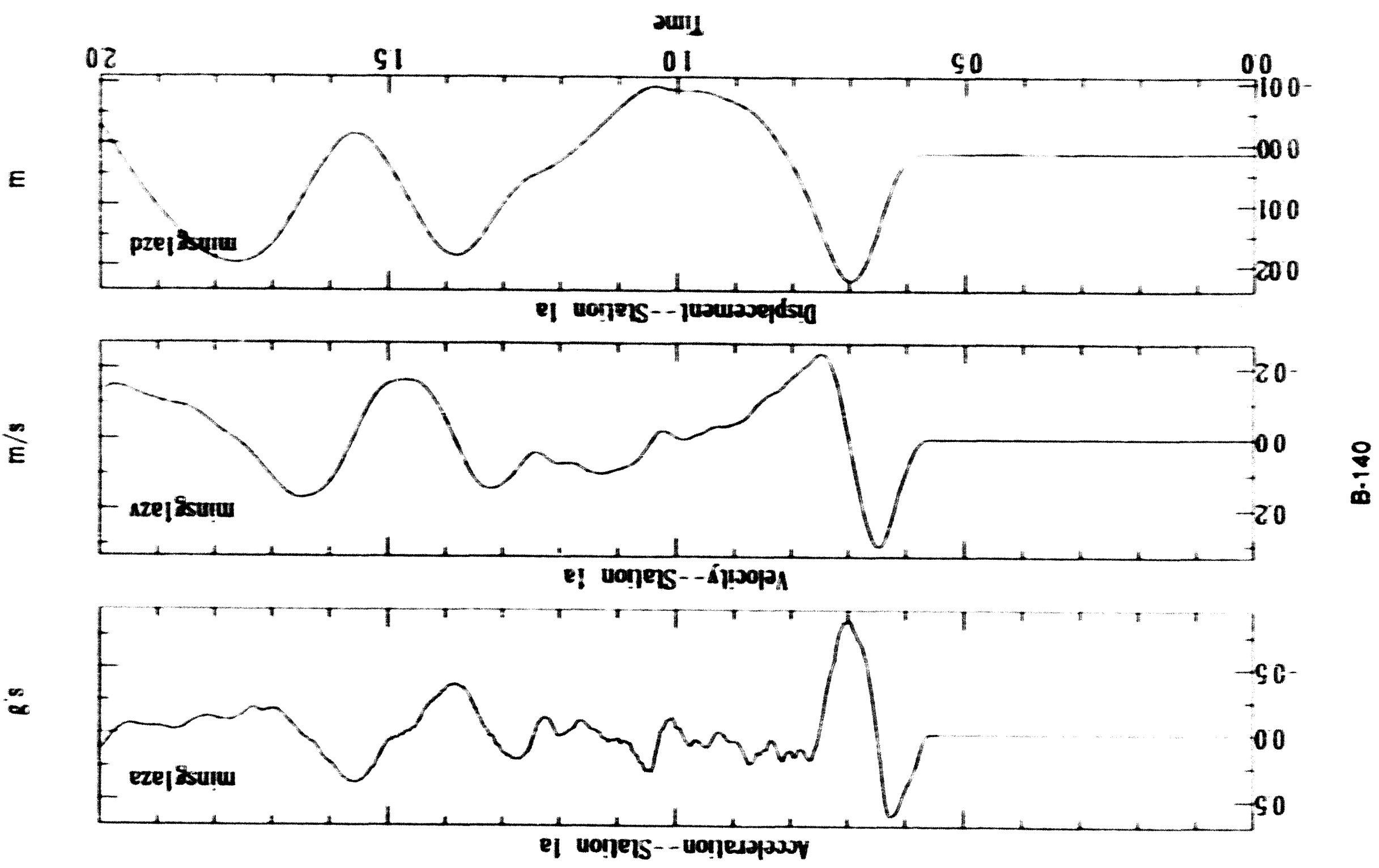

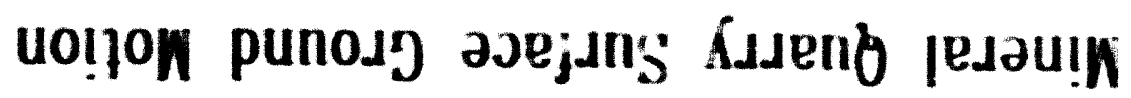




\section{Vineral Quarry Surface Ground Motion}

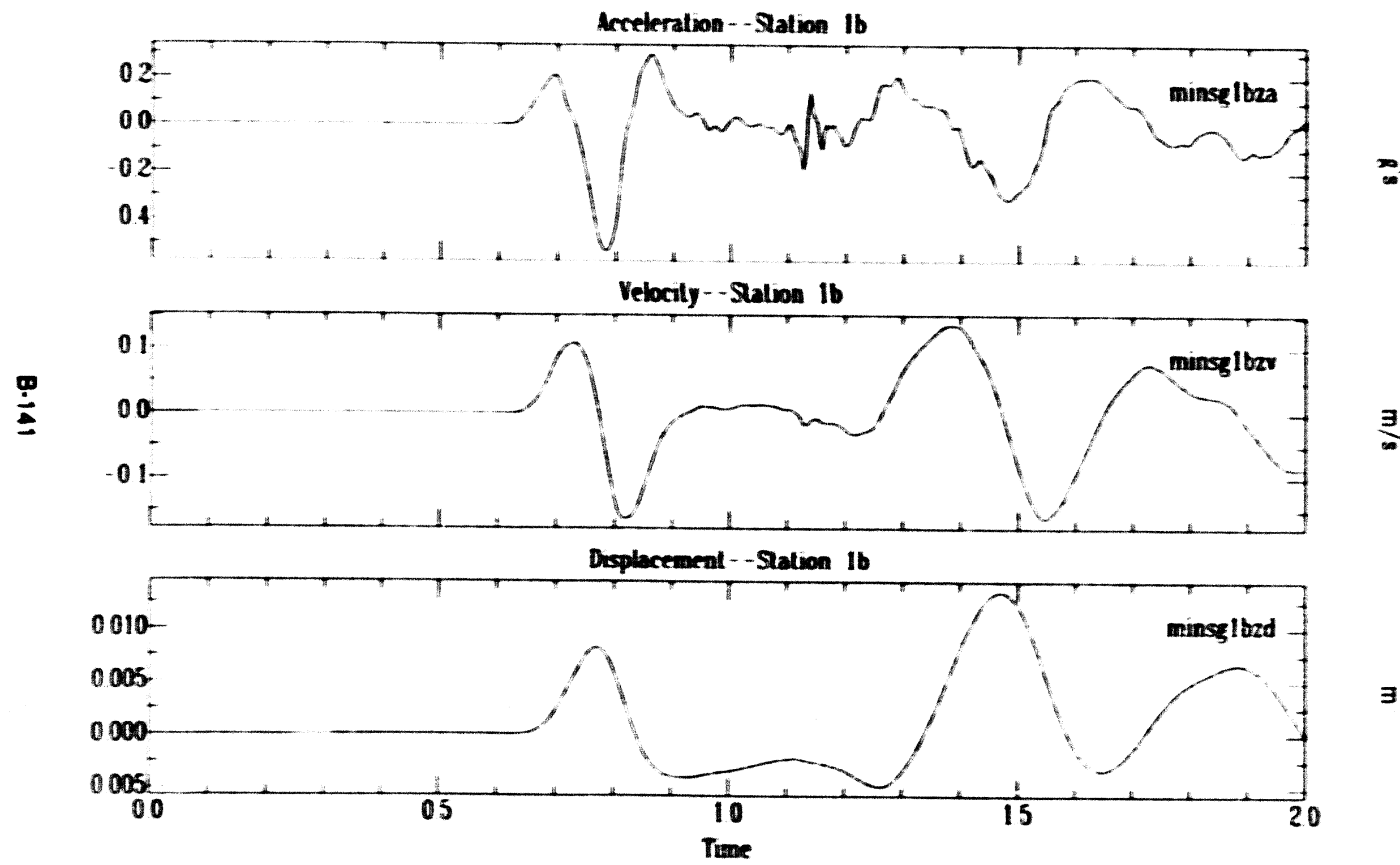




\section{Nineral Quarry Surface Ground Motion}

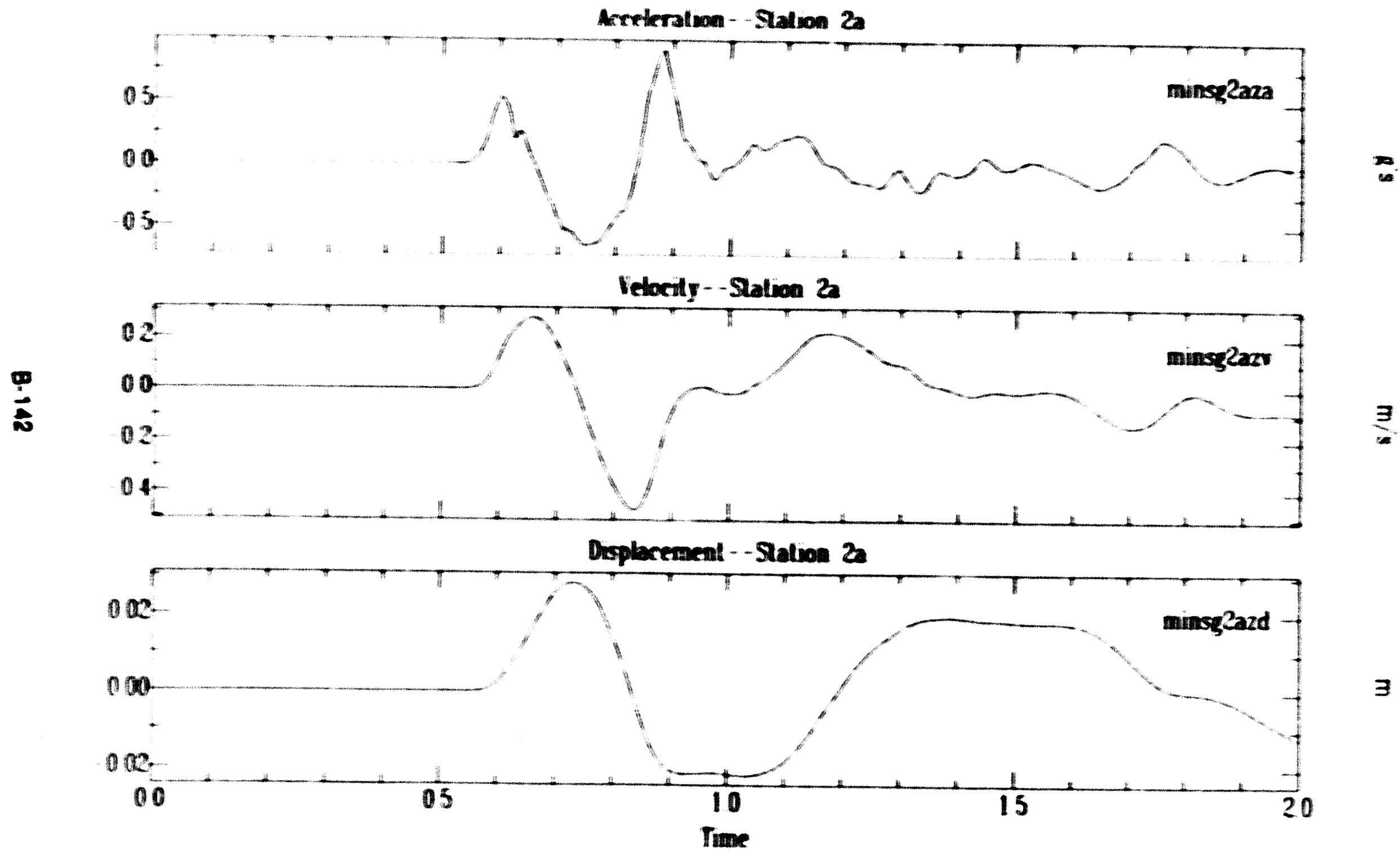




\section{Vineral Quarry Surface Ground Motion}

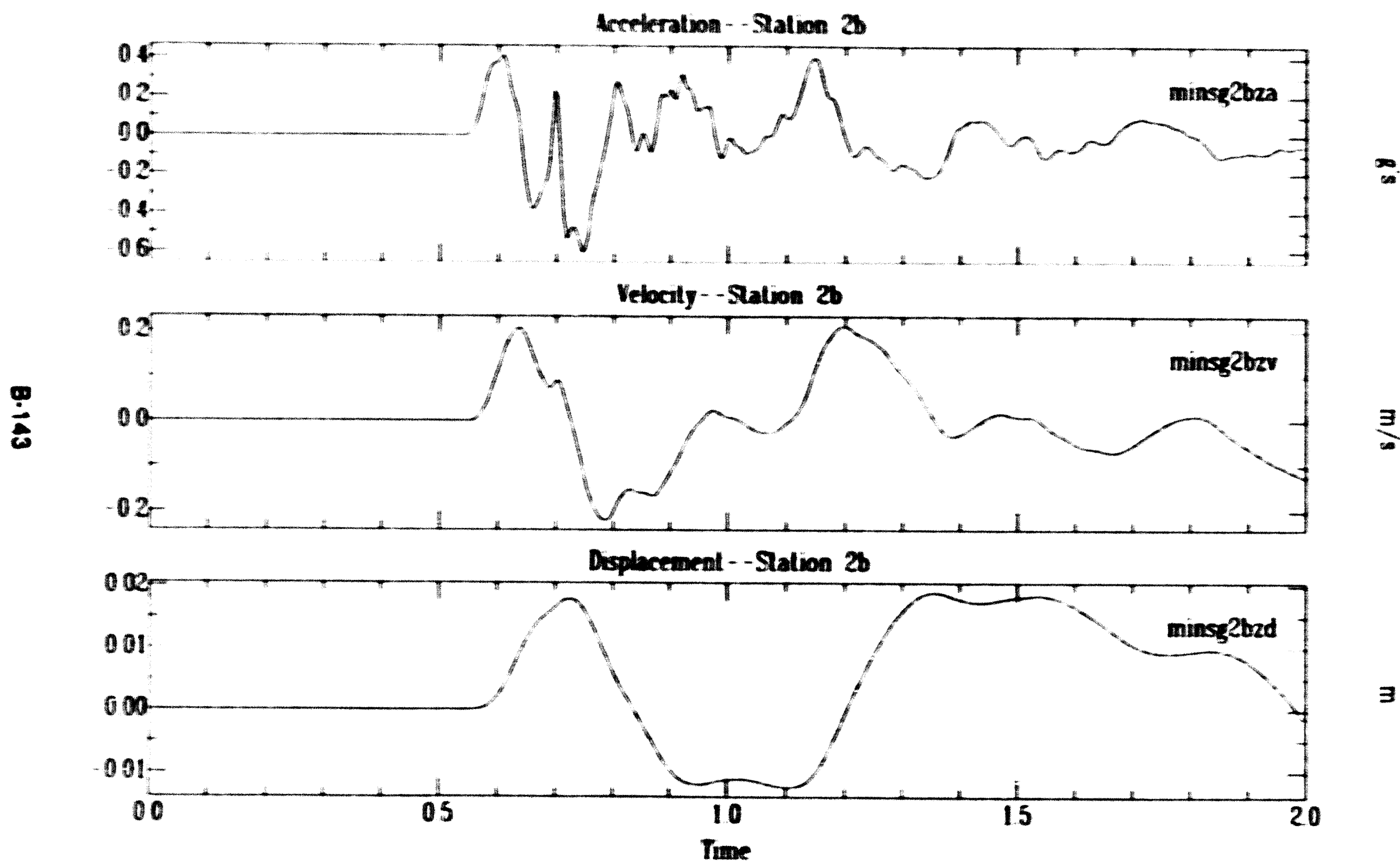




\section{Nineral Quarry Surface Ground Motion}

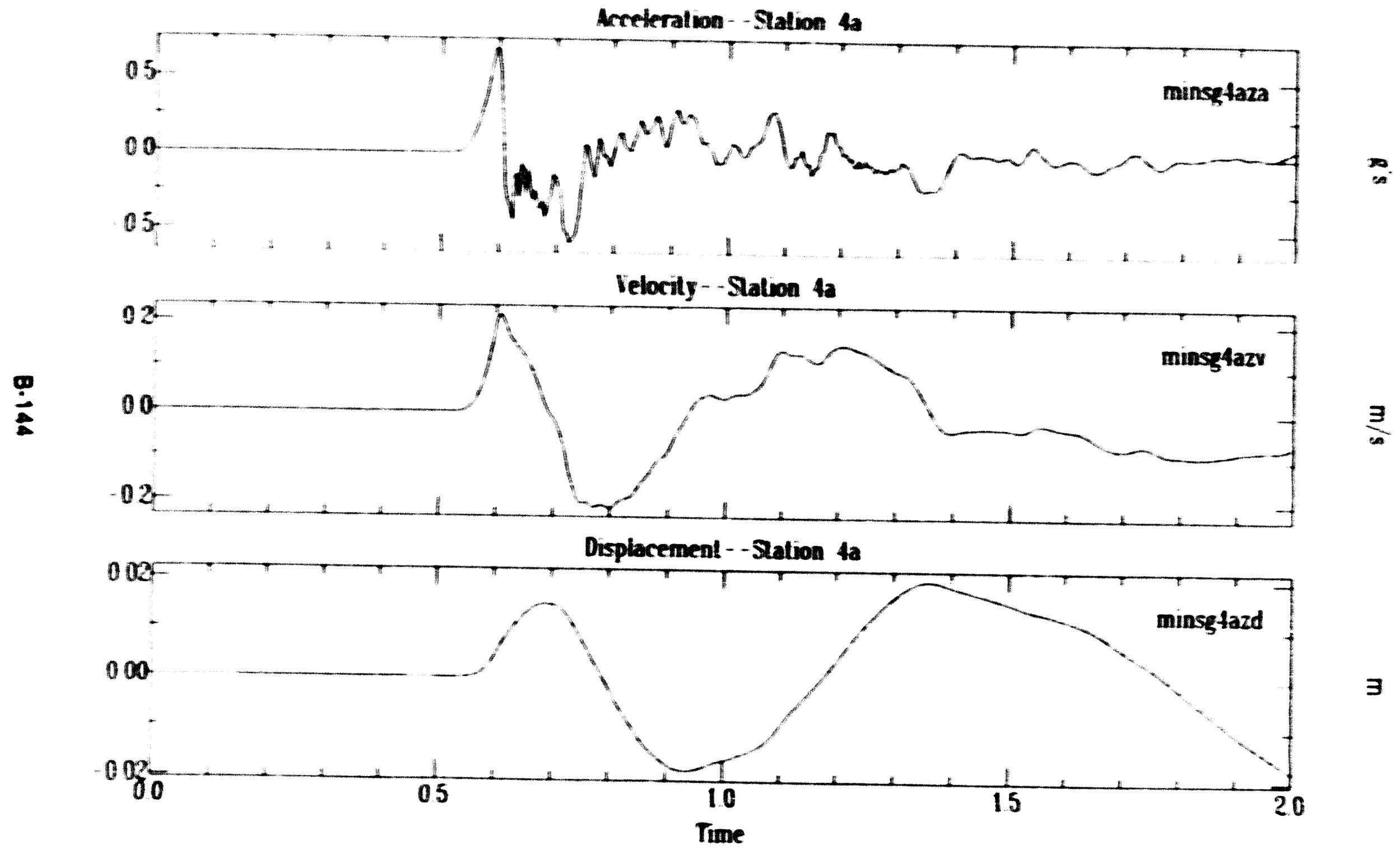




\section{Vineral Quarry Surface Ground Motion}

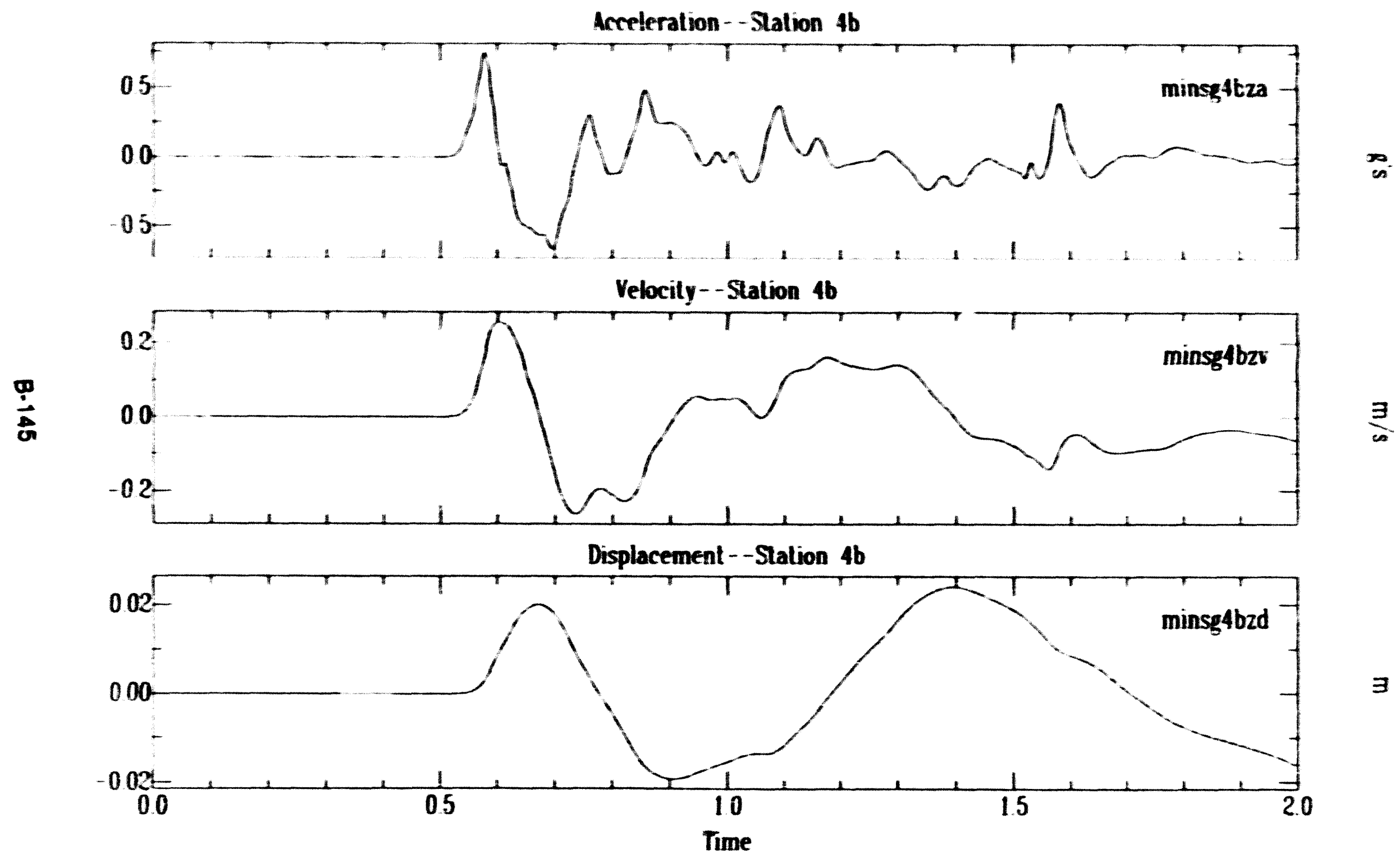




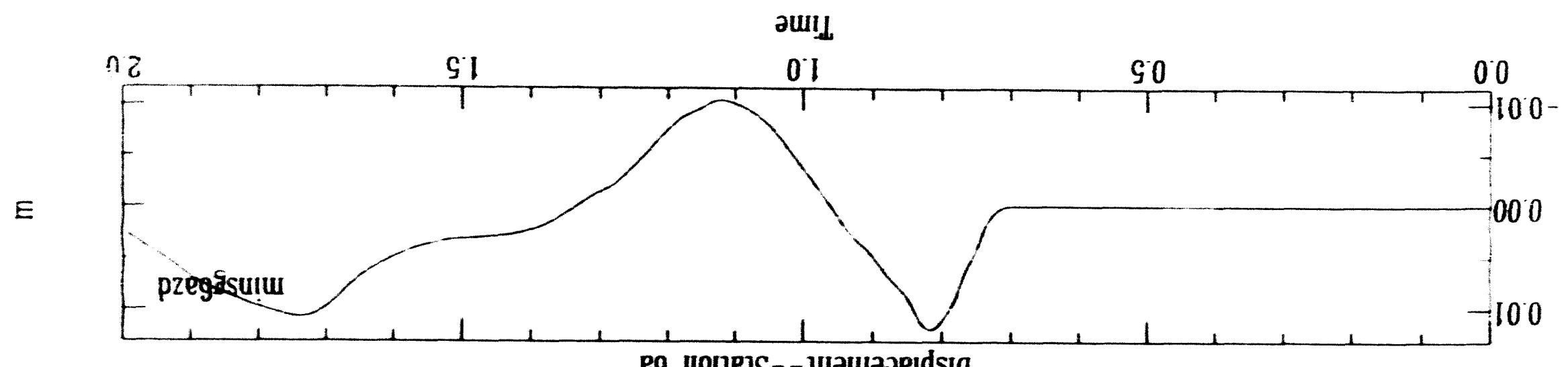

eg чоп̣еาS-- |иәшәวе|ds! 0
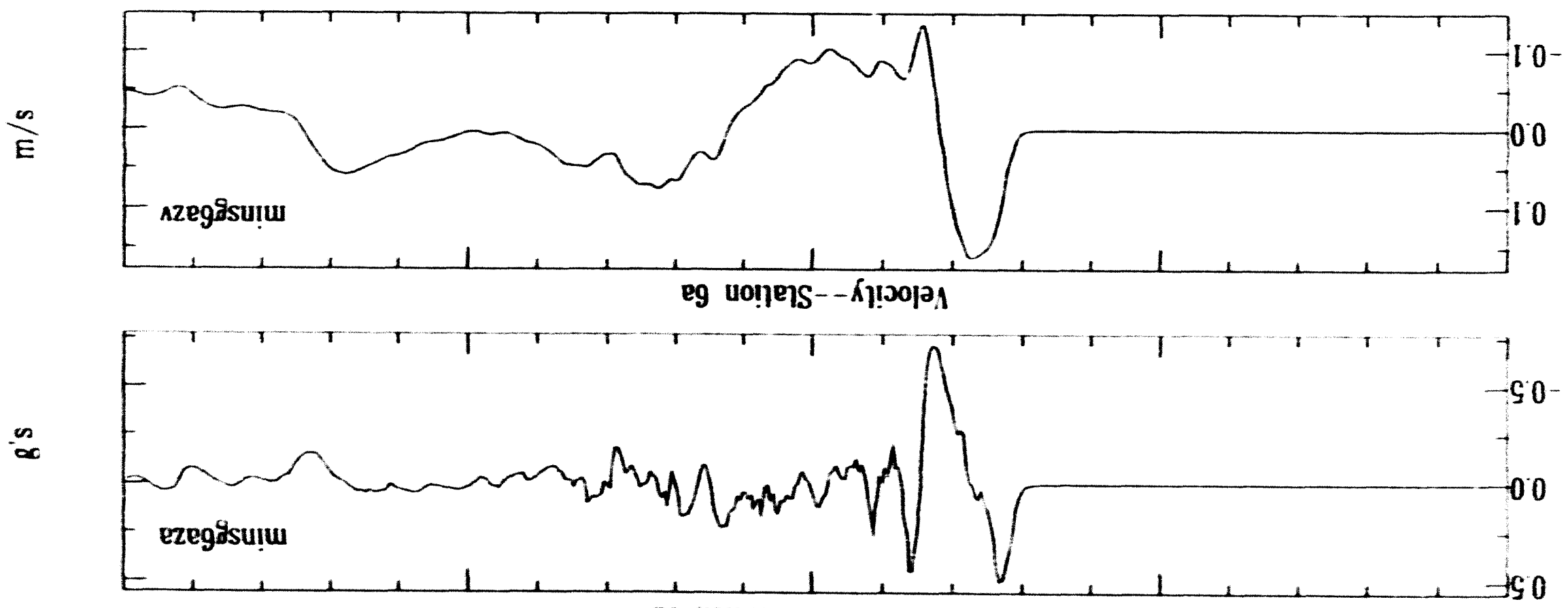

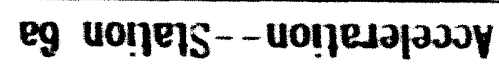

uo!̣on punodg ajejans Kddeni() ןedau!k 


\section{Mineral Quarry Surface Ground Motion}

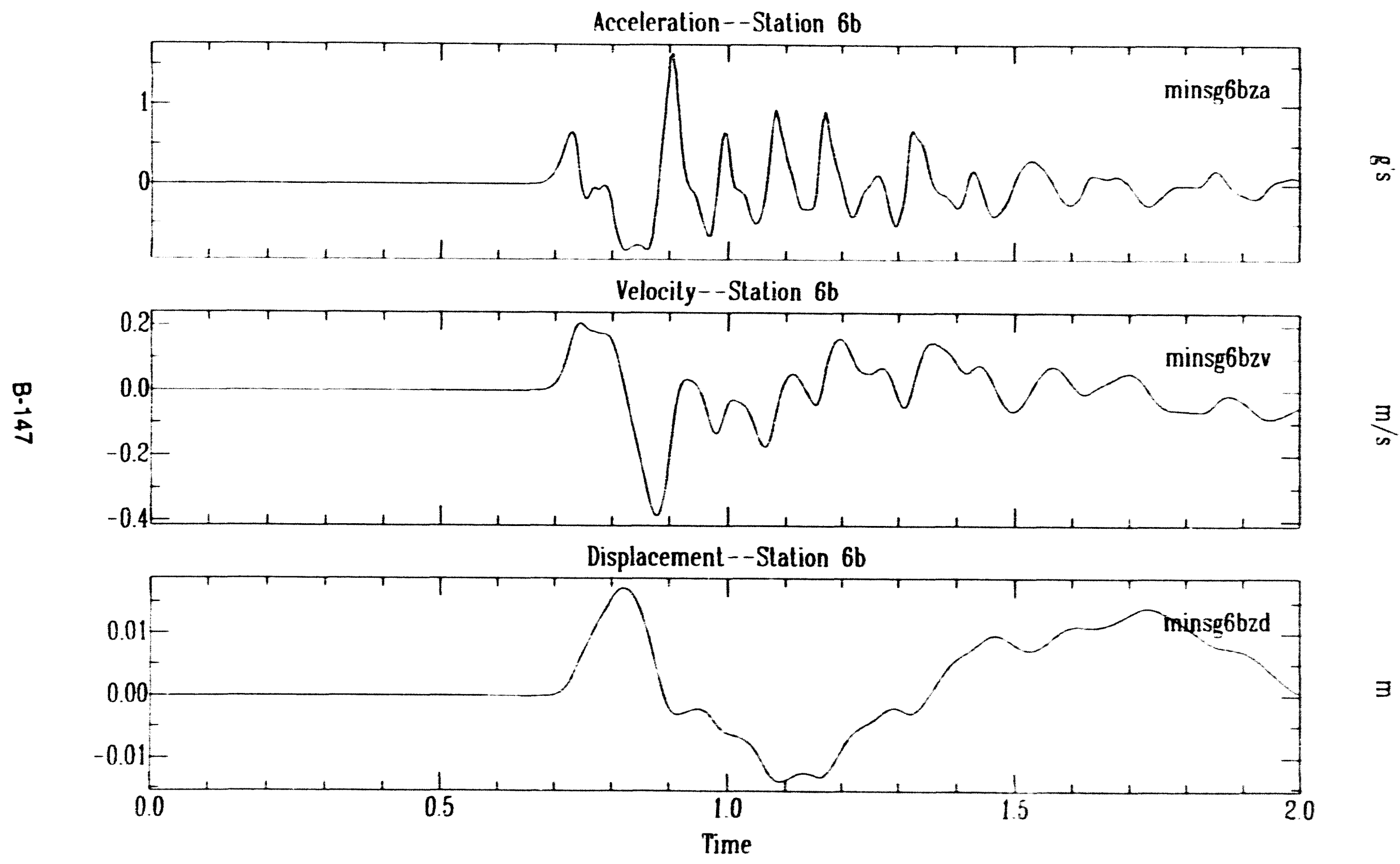



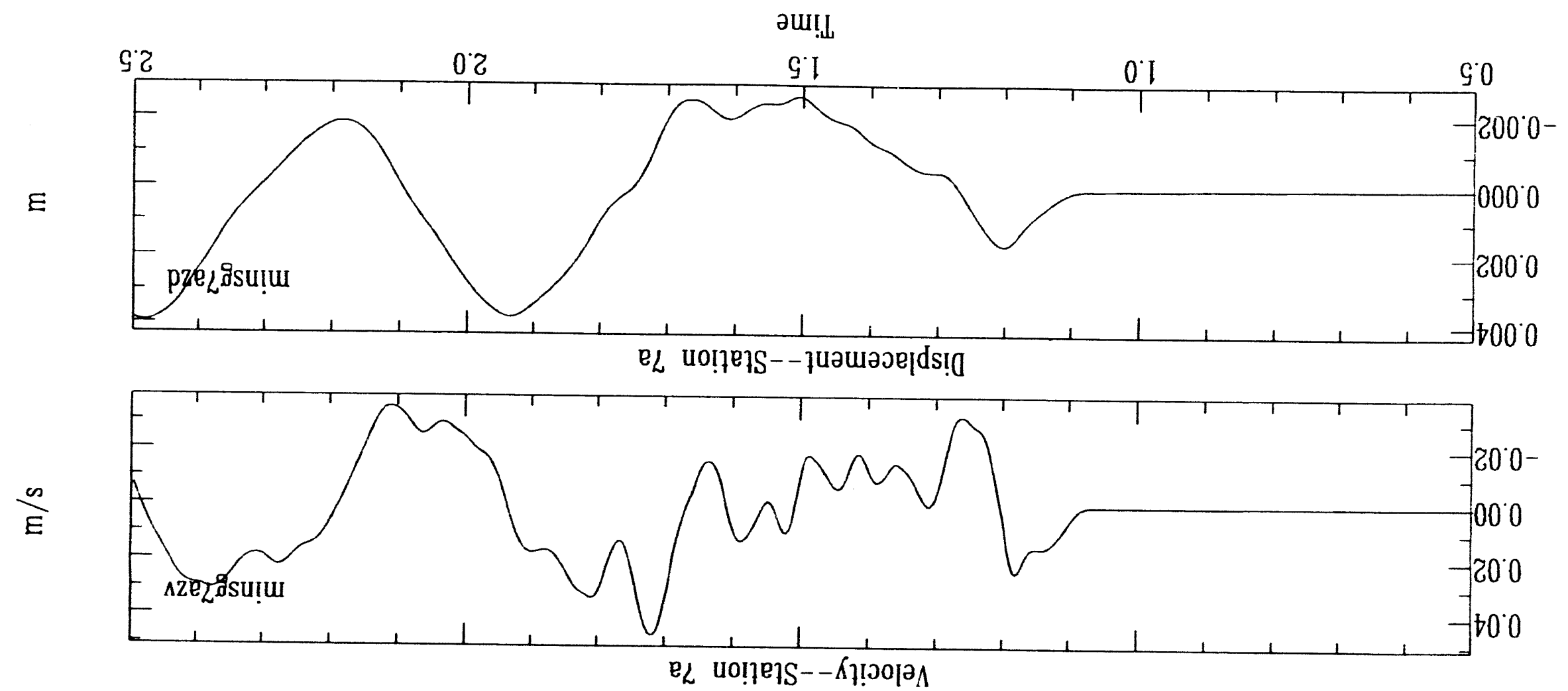

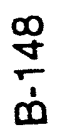

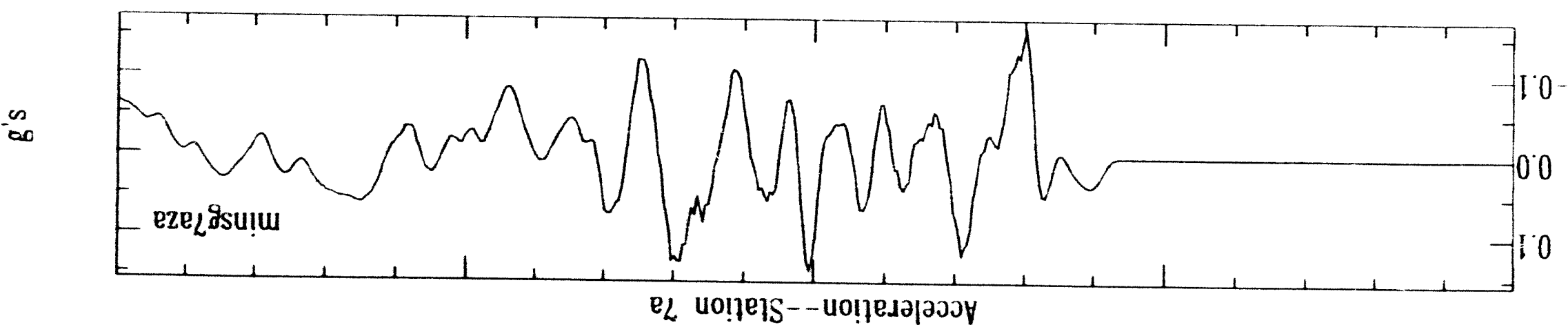

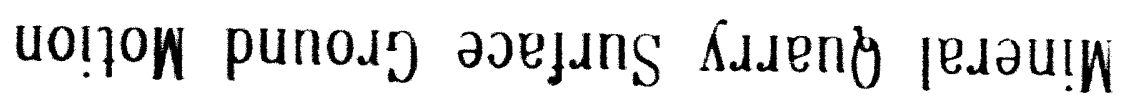




\section{Mineral Quarry Surface Ground Motion}

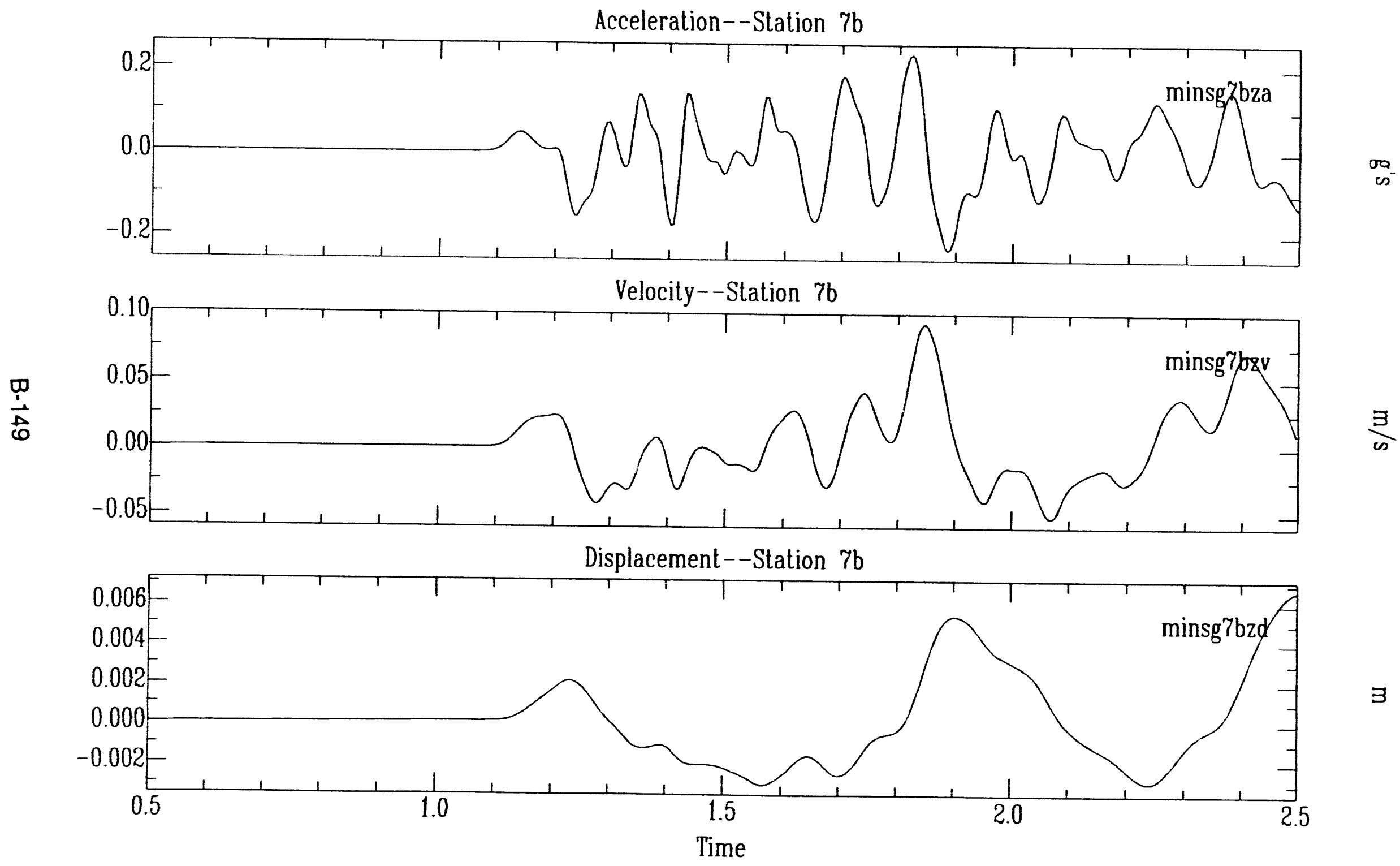




\section{Mineral Quarry Surface Ground Motion}

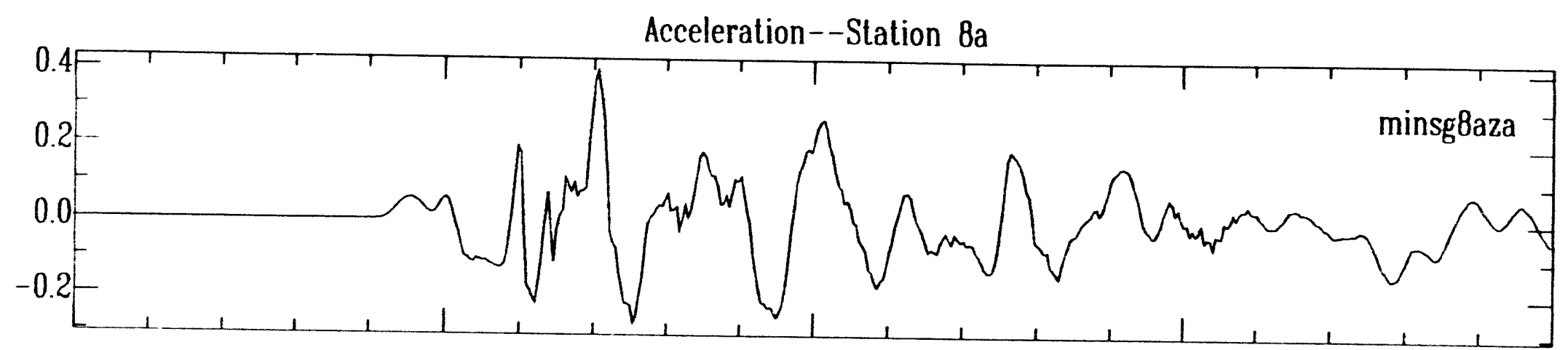

Velocity--Station $8 \mathrm{a}$

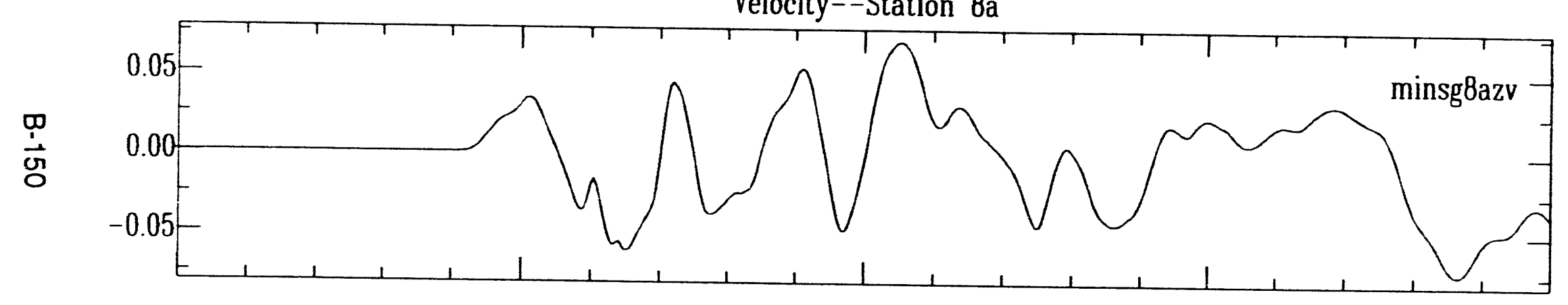

$\frac{3}{\infty}$

Displacement--Station 8a

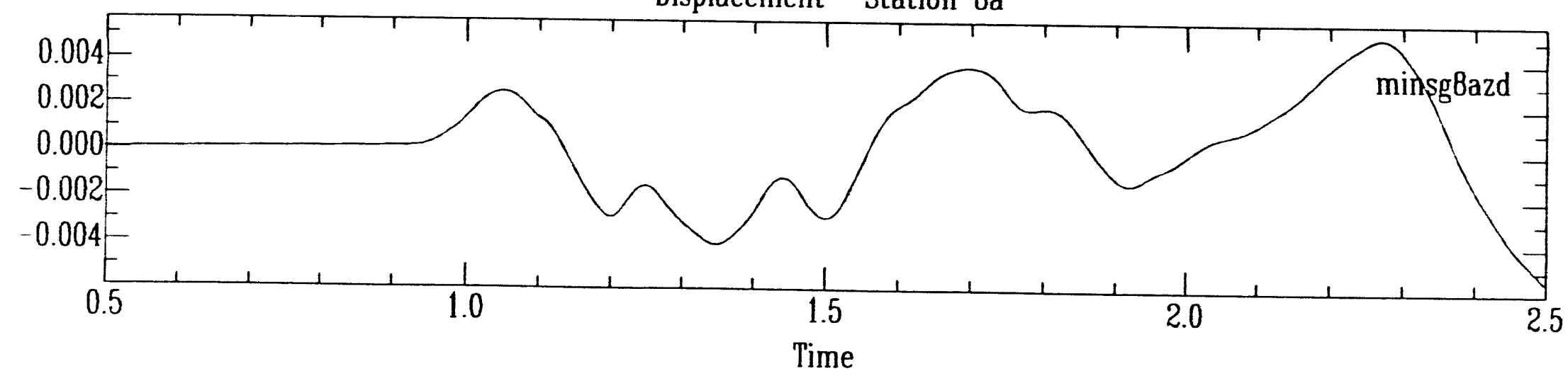




\section{Mineral Quarry Surface Ground Motion}

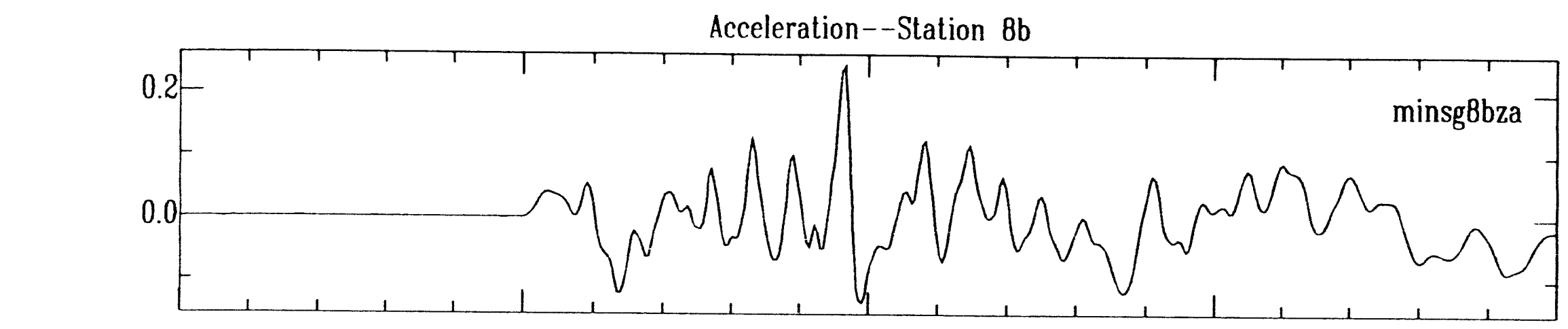

Velocity--Station $8 \mathrm{~b}$

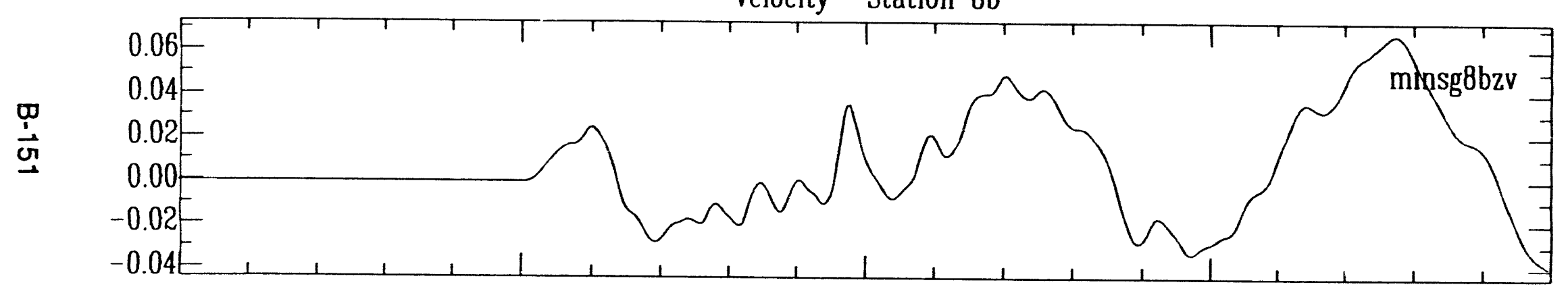

Displacement--Station 8b

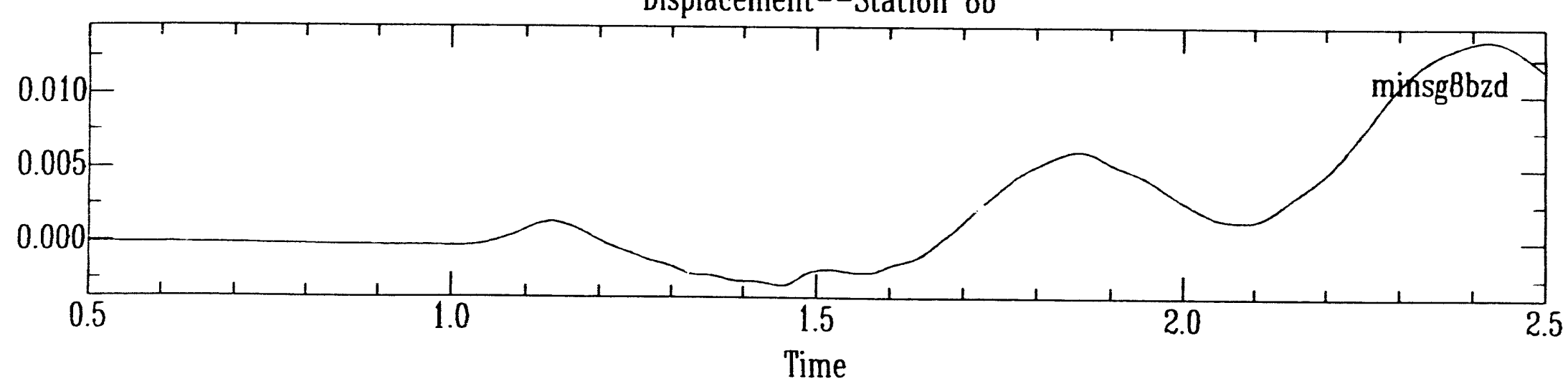




\section{Mineral Quarry Surface Ground Motion}

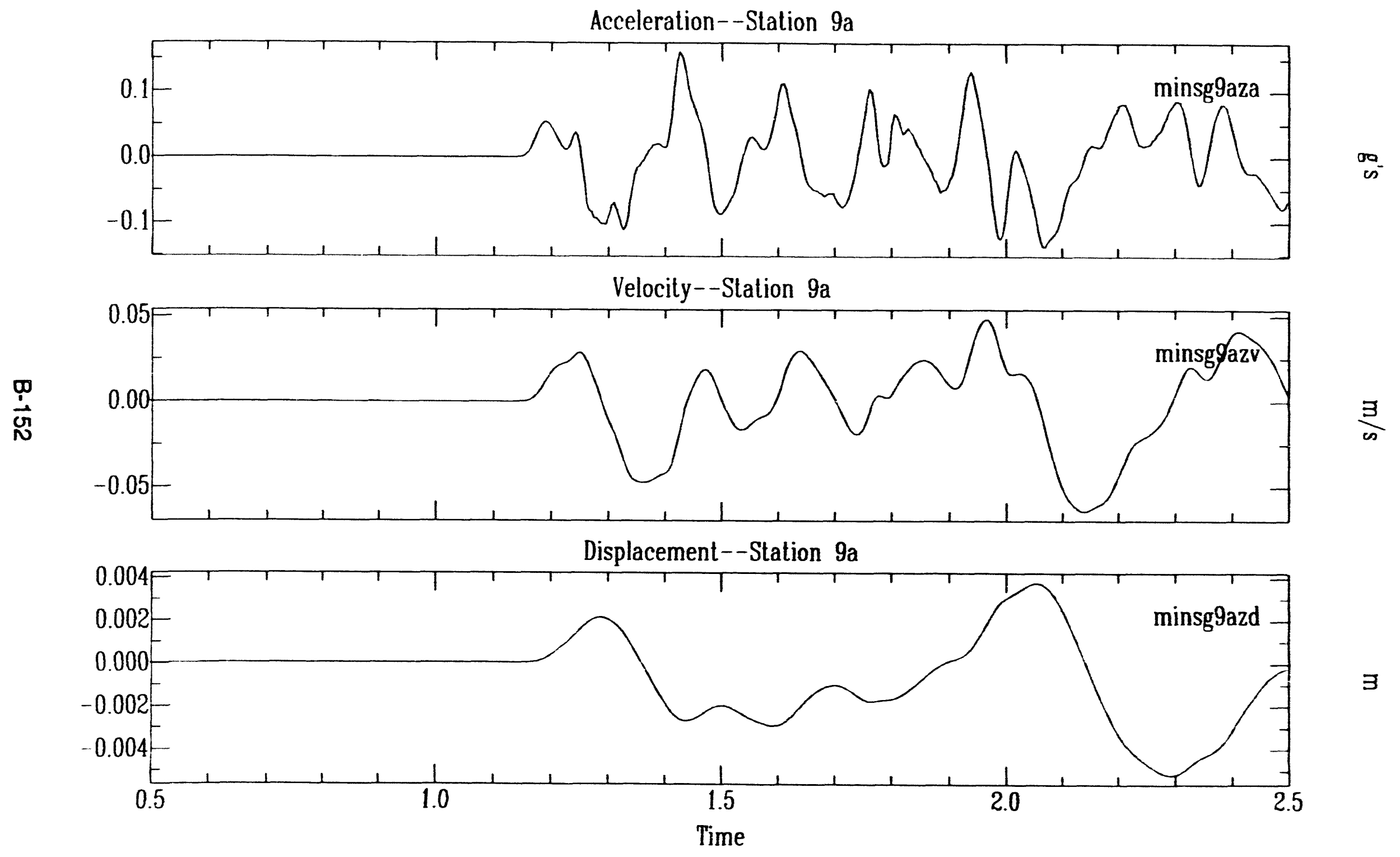




\section{Mineral Quarry Surface Ground Motion}

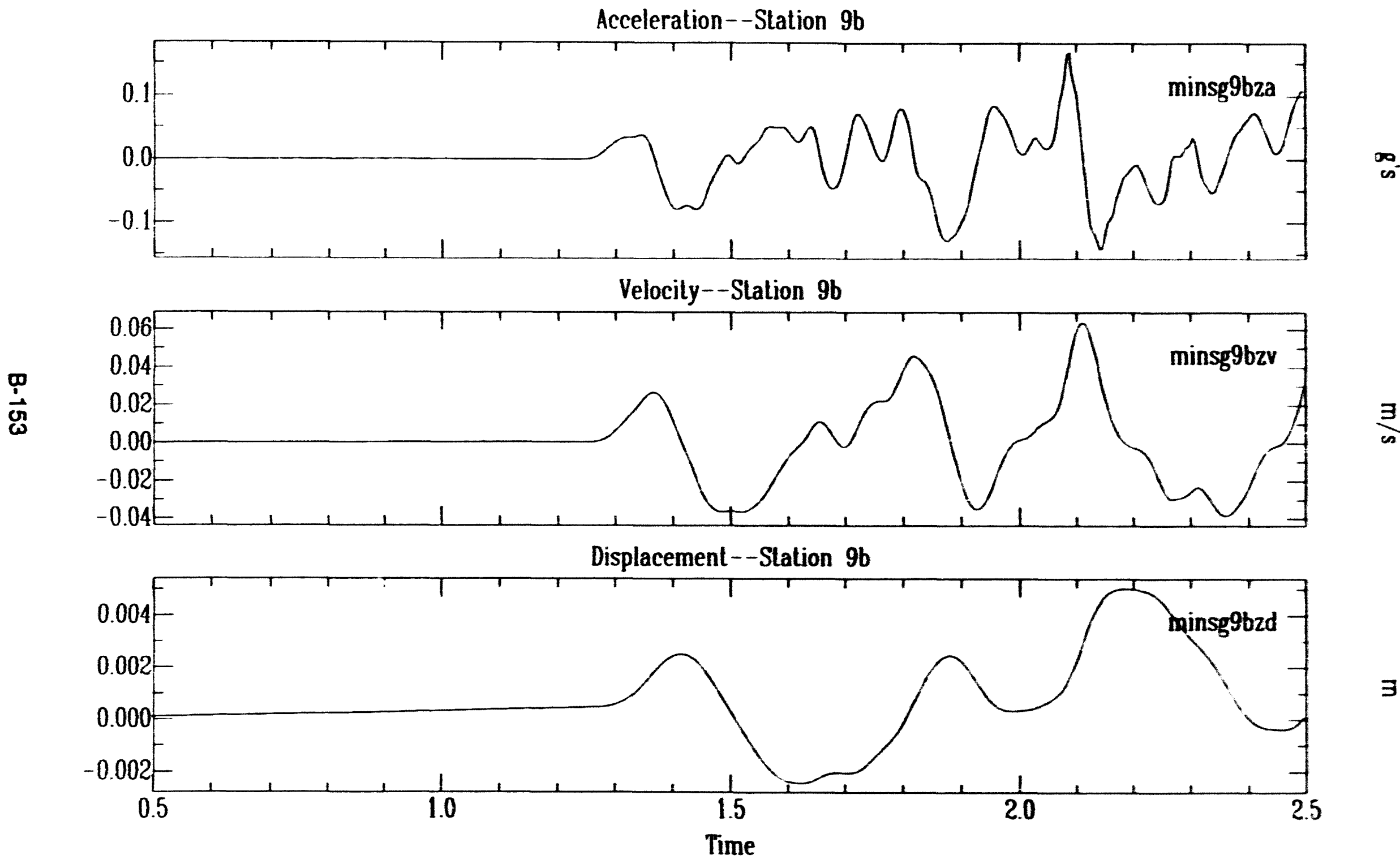




\section{Mineral Quarry Surface Ground Motion}

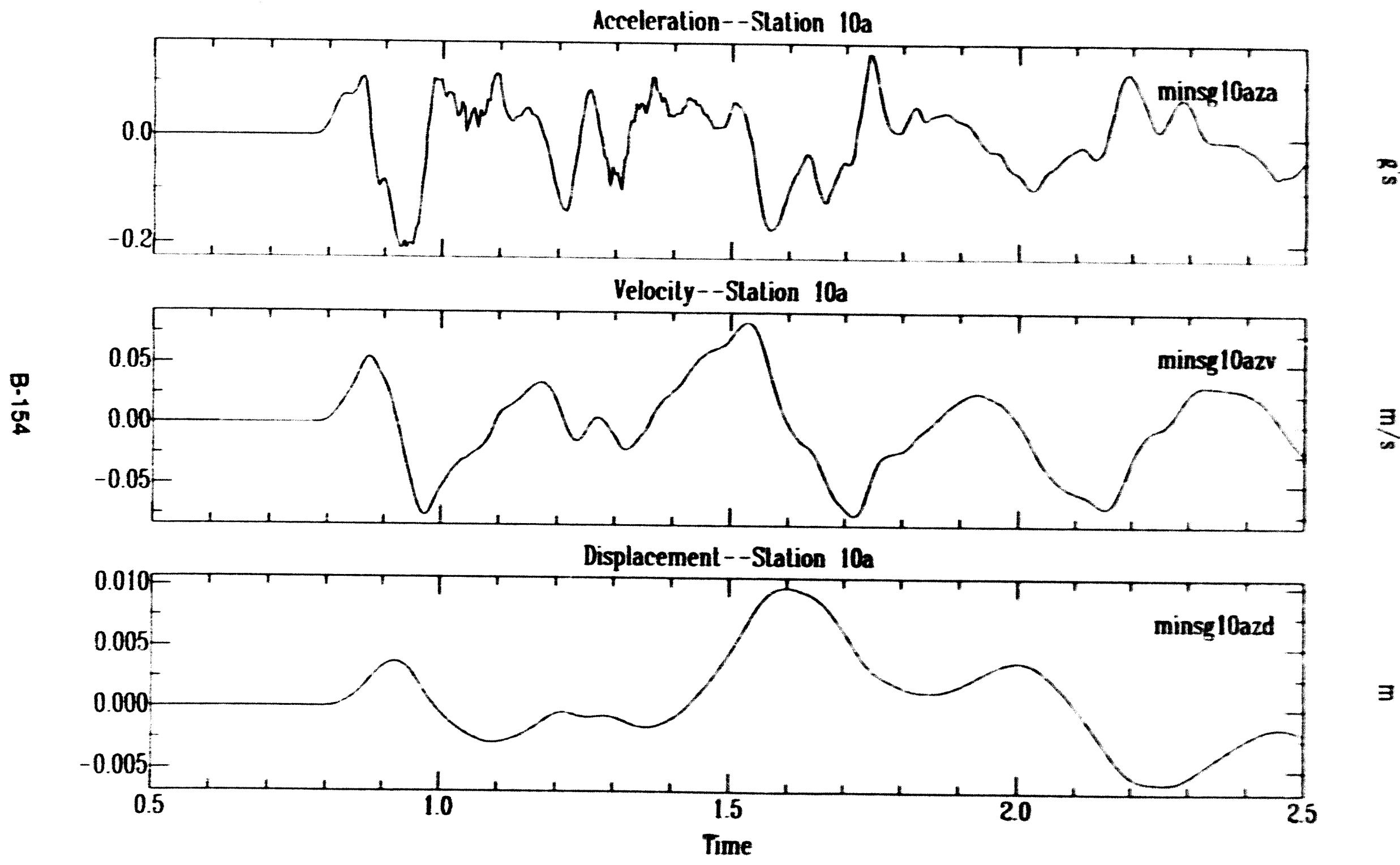




\section{Mineral Quarry Surface Ground Motion}

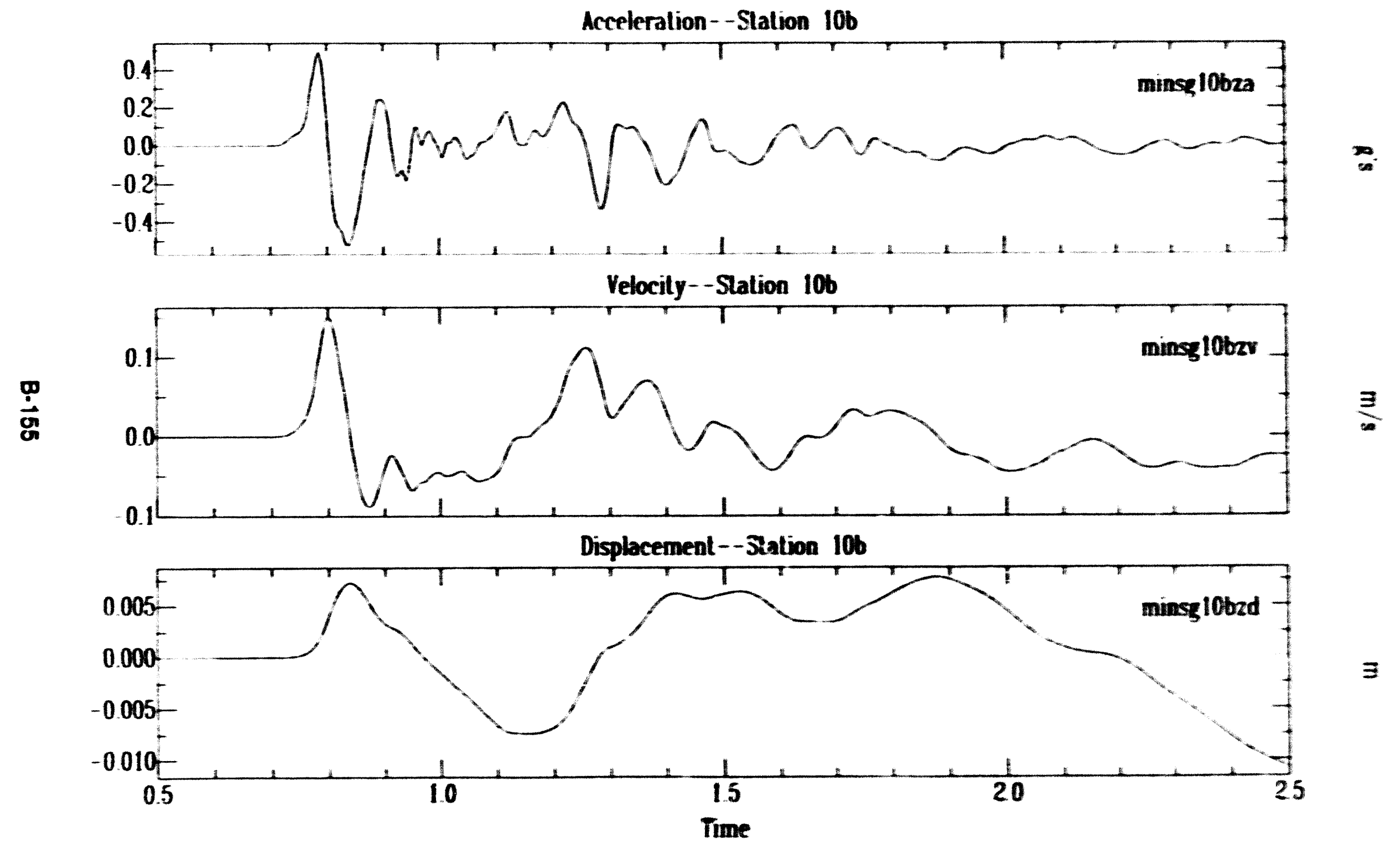




\section{Vineral Quarry Surface Ground Motion}

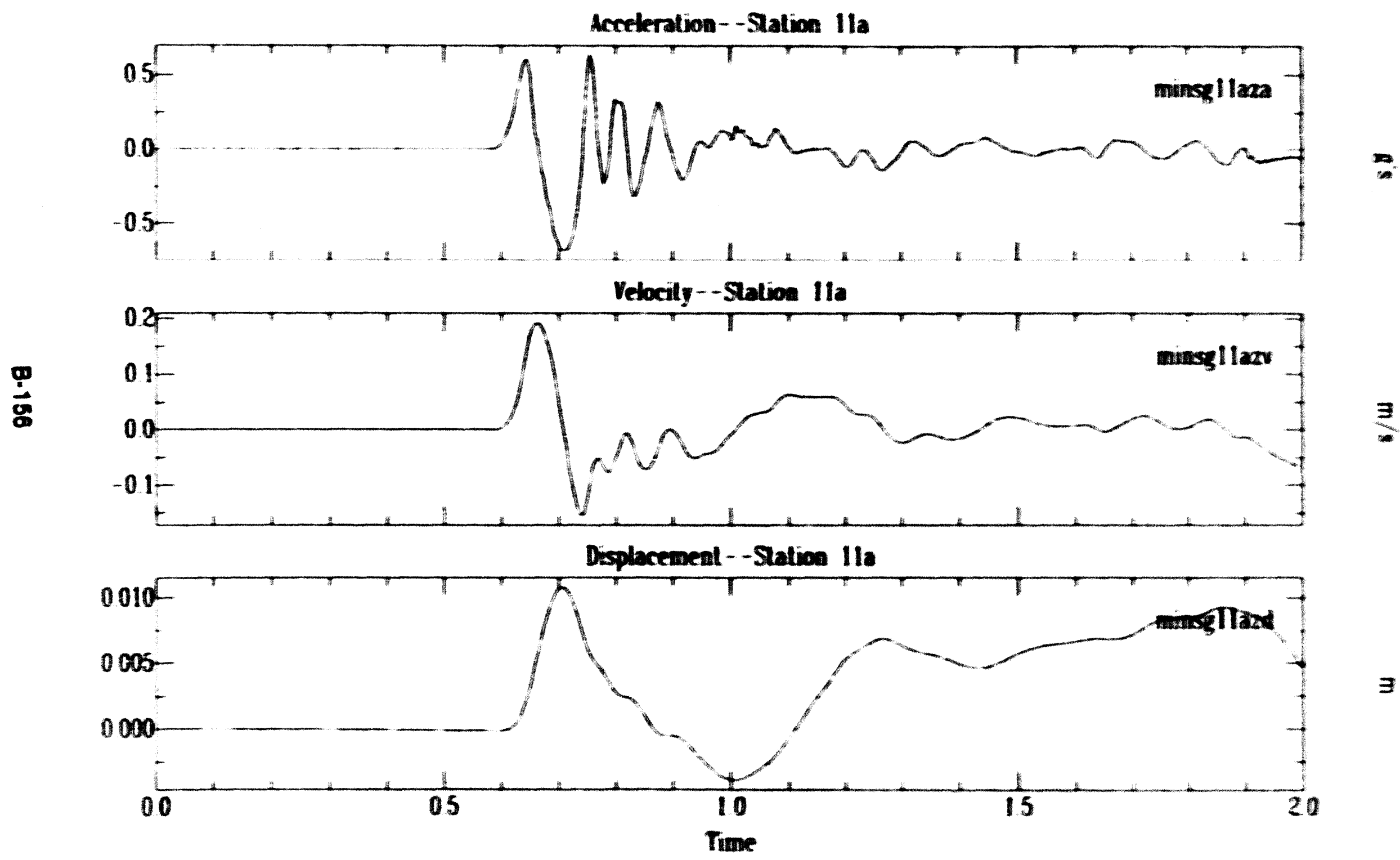




\section{Mineral Quarry Surface Ground Motion}

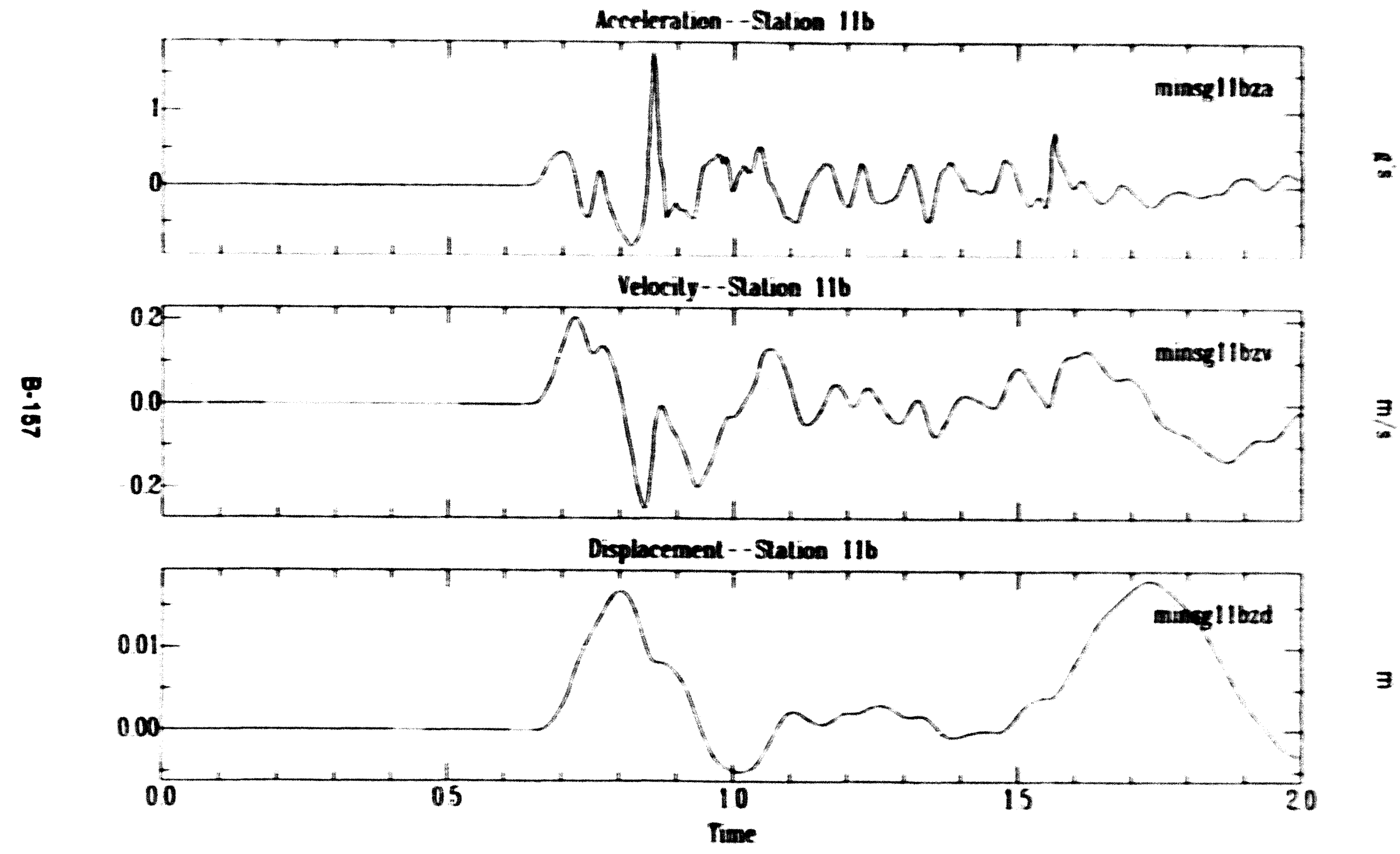



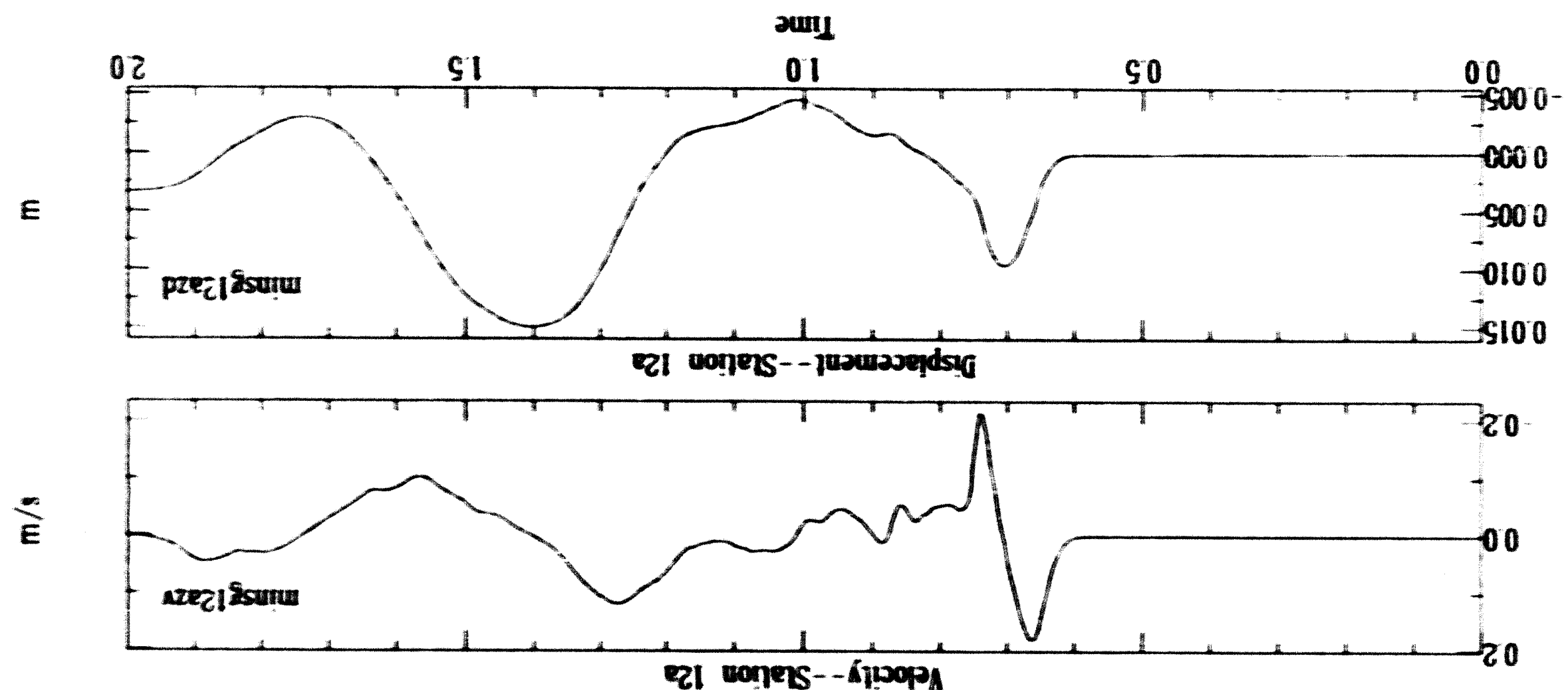

$\frac{8}{\dot{0}}$

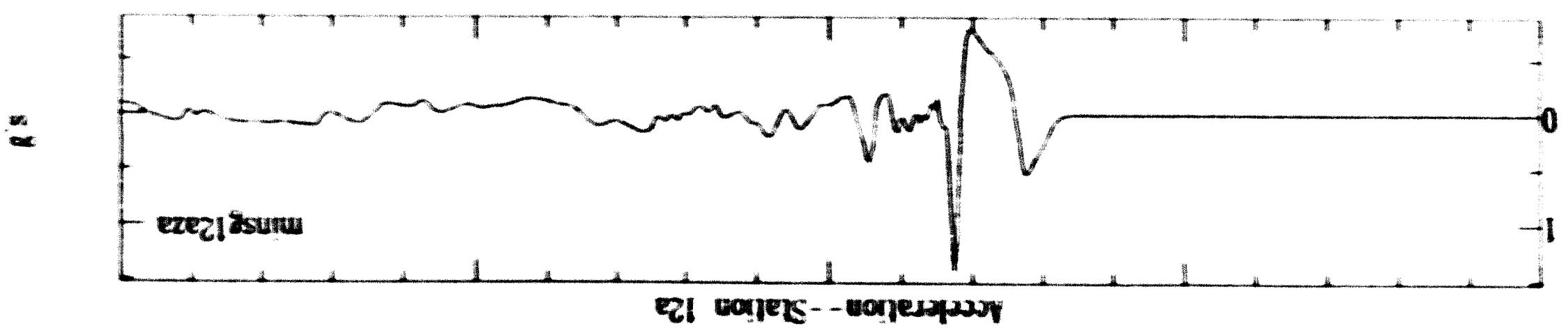

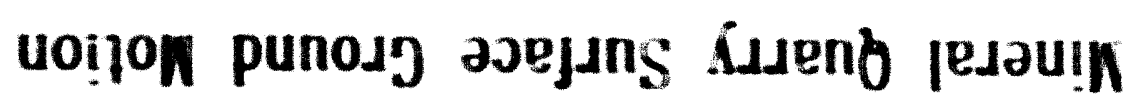




\section{Mineral Quarry Surface Ground Motion}

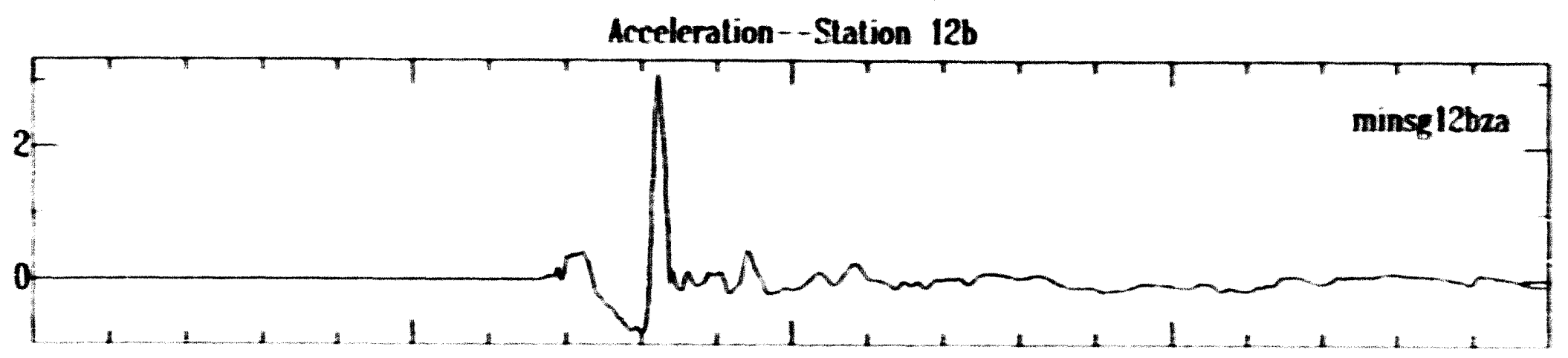

Velocily--Station $12 b$

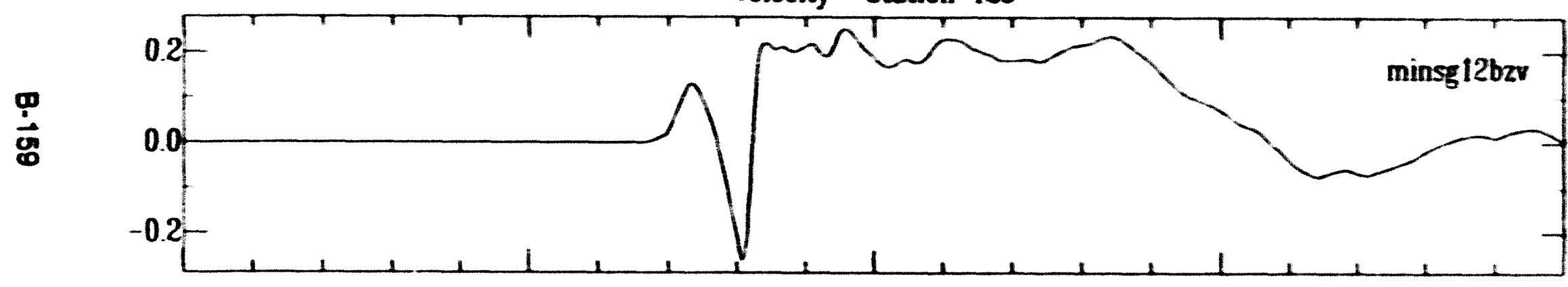

$\exists$

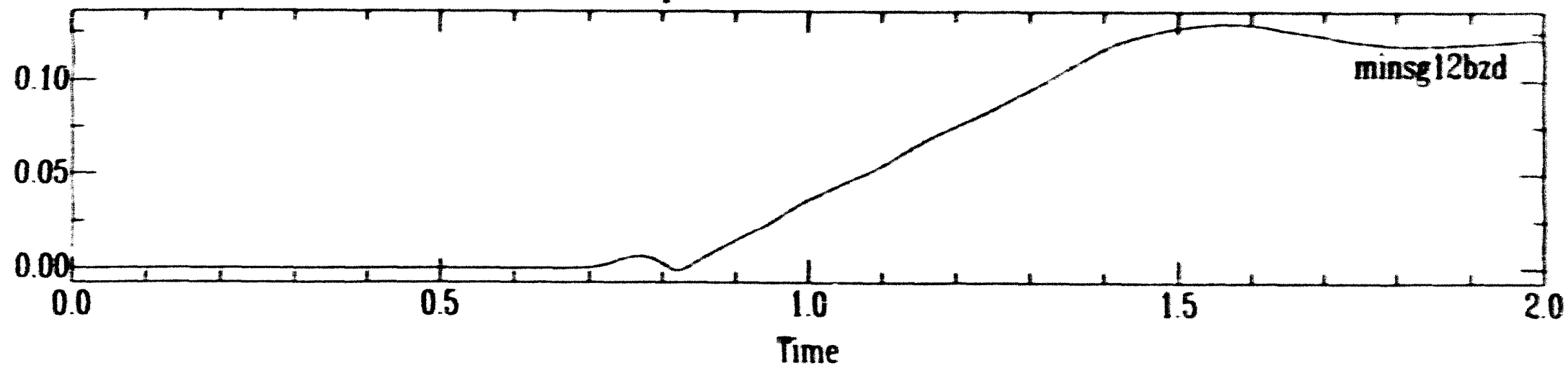




\subsection{Boxar (U19ba)}

Experiment design. To map the ground motions from a normally buried underground nuclear explosion outside of the spall zone, an extensive set of ground-motion sensors was fielded on Bexar (Table 13 and Fig. 17). In addition to the $15 \mathrm{~J}-8$ stations inside the spall zone (including three near SGZ), 39 ESA stations were fielded using portable dightal recorders. These last ranged in distance from just inside the edge of predicted spall (about $1.8 \mathrm{~km}$ from SGZ) out to more than $8 \mathrm{~km}$ from SGZ.

Resulte. The J.8 results were originally reported out in April 1991 (Memo J-8.91.073, R. S. Fitzhugh to F. N. App). The following note was included in this memo (italics added):

- Data users please note: Because of a RIDS problem, the acceleration data was digitized at a sample rate of only $53 \mathrm{~Hz}$, which probably affected the 'narrow' peak signal amplitudes and the integrated data. Also the baseline offset correction was not operating properly."

All of the J-8 stations indicated spall. Of the 39 ESA stations fielded, 31 triggered properly and recorded data. Only three of these functioning ESA stations recorded signals near the spall level (BEXSG12A, 29A, and 32A). These results are shown in the data traces in Fig. 18 (43 pages). 


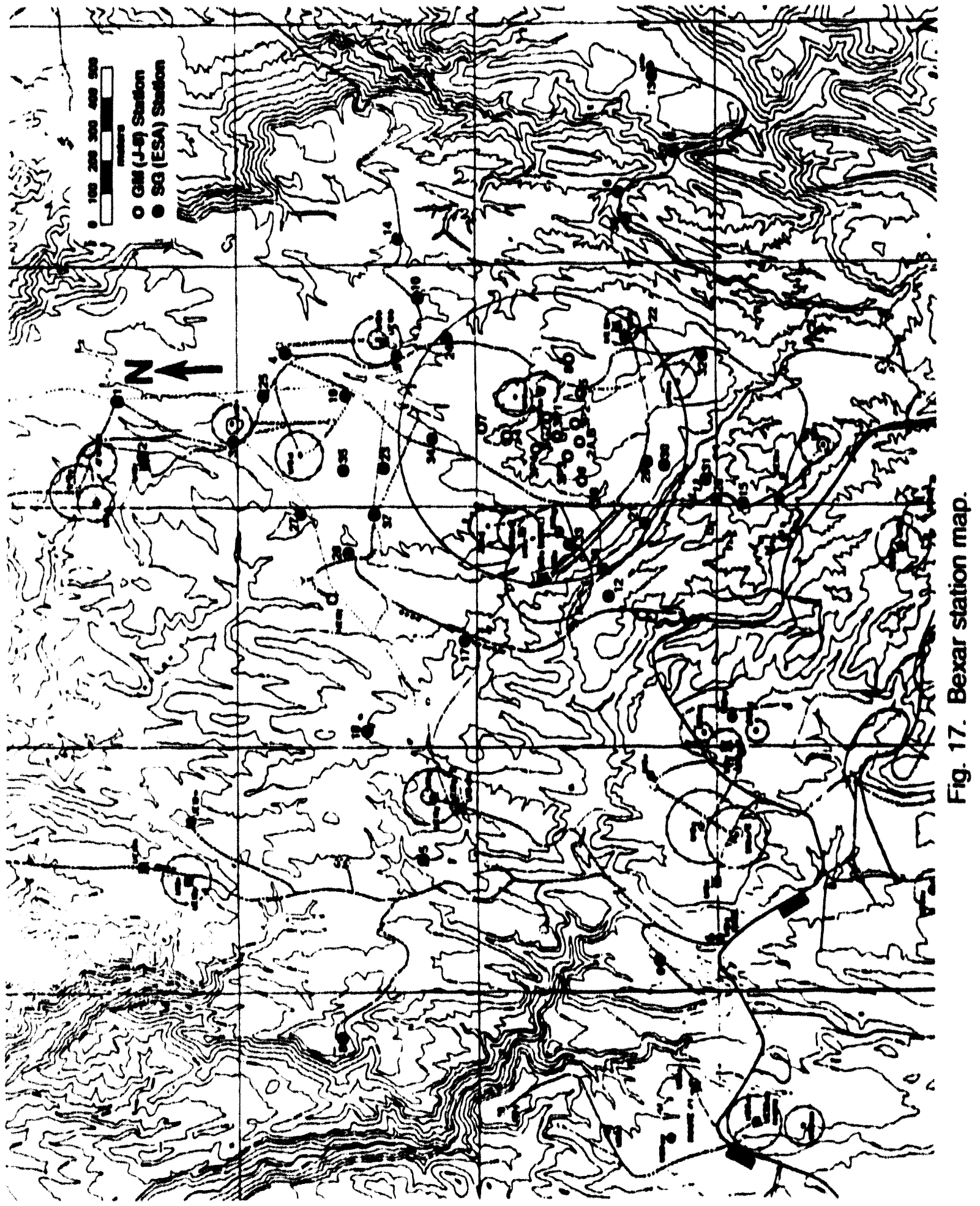


Table 13. Bexar Station Data

\begin{tabular}{|c|c|c|c|c|c|c|}
\hline $\begin{array}{l}\text { Station } \\
\text { Name }\end{array}$ & Location & Accelerometer & $\begin{array}{l}\text { Northing (m) } \\
282534\end{array}$ & $\begin{array}{l}\text { Easting(m) } \\
183764\end{array}$ & $\begin{array}{l}\text { Slant Range } \\
629(m) \text { DOB }\end{array}$ & Travel Time \\
\hline BEXGM1A & $15 \mathrm{~m} \mathrm{S08W}$ & Endevco $25 \mathrm{~g}$ & 282519 & 183762 & 629 & 0.29 \\
\hline BEXGM1B & $15 \mathrm{~m} \mathrm{~S} 08 \mathrm{~W}$ & Endevco $25 \mathrm{~g}$ & 282519 & 183762 & 629 & 0.29 \\
\hline BEXGM1C & $15 \mathrm{~m} \mathrm{~S} 08 \mathrm{~W}$ & Endevco $25 \mathrm{~g}$ & 282519 & 183762 & 629 & 0.29 \\
\hline BEXGM2A & $258 \mathrm{~m} \mathrm{~S} 30 \mathrm{~W}$ & Endevco $25 \mathrm{~g}$ & 282311 & 183635 & 680 & 0.315 \\
\hline BEXGM2B & $258 \mathrm{~m} \mathrm{~S} 30 \mathrm{~W}$ & Endevco $25 \mathrm{~g}$ & 282311 & 183635 & 680 & 0.315 \\
\hline BEXGM3P1 & $258 \mathrm{~m} \mathrm{~N} 60 \mathrm{E}$ & Endevco $25 \mathrm{~g}$ & 282663 & 183987 & 680 & 0.31 \\
\hline BEXGM3P2 & $258 \mathrm{~m} \mathrm{~S} 30 \mathrm{E}$ & Endevco $25 \mathrm{~g}$ & 282311 & 183893 & 680 & 0.31 \\
\hline BEXGMЗР3 & $258 \mathrm{~m} \mathrm{~S} 60 \mathrm{~W}$ & Endevco $25 \mathrm{~g}$ & 282405 & 183541 & 680 & 0.32 \\
\hline BEXGM3P4 & $258 \mathrm{~m} \mathrm{~N} 32 \mathrm{~W}$ & Endevco $25 \mathrm{~g}$ & 282753 & 183628 & 680 & 0.295 \\
\hline BEXGM4A & $640 \mathrm{~m} \mathrm{~N}$ & Endevco $20 \mathrm{~g}$ & 283174 & 183764 & 897 & 0.39 \\
\hline BEXGM5A & $640 \mathrm{~m} \mathrm{~S} 60 \mathrm{E}$ & Endevco $20 \mathrm{~g}$ & 282215 & 184318 & 897 & 0.42 \\
\hline BEXGM6A & $640 \mathrm{~m} \mathrm{~S} 60 \mathrm{~W}$ & Endevco $20 \mathrm{~g}$ & 282214 & 183210 & 897 & 0.42 \\
\hline BEXGM7A & $977 \mathrm{~m}$ N04E & Endevco $20 \mathrm{~g}$ & 283509 & 183831 & 1162 & 0.48 \\
\hline BEXGM8A & $968 \mathrm{~m} \mathrm{S79E}$ & Endevco $20 \mathrm{~g}$ & 282356 & 184716 & 1155 & 0.55 \\
\hline BEXGM9A & $915 \mathrm{~m} \mathrm{S60W}$ & Endevco $20 \mathrm{~g}$ & 282075 & 182974 & 1109 & 0.47 \\
\hline BEXSG1A & $5510 \mathrm{~m} \mathrm{NO4E}$ & Terra Tec $5.28 \mathrm{~g}$ & 288020 & 184126 & 5534 & 5.221 \\
\hline BEXSG2A & $2930 \mathrm{~m} \mathrm{~N} 35.1 \mathrm{~W}$ & Terra Tec $5.28 \mathrm{~g}$ & 284927 & 182072 & 2997 & 5.338 \\
\hline BEXSG3A & $4130 \mathrm{~m} \mathrm{N1.5W}$ & Terra Tec $5.28 \mathrm{~g}$ & 286630 & 183643 & 4146 & 5.248 \\
\hline BEXSG4A & $3440 \mathrm{~m}$ N16.5E & Terra Tec $5.28 \mathrm{~g}$ & 285847 & 184728 & 3507 & 5.133 \\
\hline BEXSG5A & 5640 m N73.2W & Terra Tec $5.28 \mathrm{~g}$ & 282929 & 178613 & 5204 & 5.286 \\
\hline BEXSG6A & $6810 \mathrm{~m} \mathrm{S78W}$ & Terra Tec $5.28 \mathrm{~g}$ & 281094 & 177112 & 6835 & 5.157 \\
\hline BEXSG7A & $2960 \mathrm{~m} \mathrm{~S} 15.6 \mathrm{~W}$ & Terra Tec $5.28 \mathrm{~g}$ & 279681 & 182978 & 3025 & 5.219 \\
\hline BEXSG9A & $2190 \mathrm{~m} \mathrm{N26.9E}$ & Terra Tec $5.28 \mathrm{~g}$ & 284504 & 184751 & 2291 & 5.097 \\
\hline BEXSG11A & $2520 \mathrm{~m} \mathrm{~S} 60.7 \mathrm{~W}$ & Terra Tec $3.7 \mathrm{~g}$ & 281296 & 181571 & 2596 & 5.246 \\
\hline BEXSG11B & 2700 m S57.5W & Terra Tec $3.7 \mathrm{~g}$ & 281075 & 181492 & 2772 & 5.243 \\
\hline BEXSG12A & $2210 \mathrm{~m} \mathrm{S68.5W}$ & Terra Tec $3.7 \mathrm{~g}$ & 281719 & 181712 & 2296 & 5.062 \\
\hline BEXSG13A & $4870 \mathrm{~m} \mathrm{S74.1E}$ & Terra Tec $3.7 \mathrm{~g}$ & 281223 & 188452 & 4908 & 5.139 \\
\hline BEXSG14A & $3100 \mathrm{~m} \mathrm{N51.8E}$ & Terra Tec $3.7 \mathrm{~g}$ & 284466 & 186196 & 3169 & 5.214 \\
\hline BEXSG15A & $2650 \mathrm{~m} \mathrm{~S} 22.7 \mathrm{~W}$ & Terra Tec $3.7 \mathrm{~g}$ & 280091 & 182754 & 2717 & 5.074 \\
\hline BEXSG16A & $3180 \mathrm{~m} \mathrm{S72.1E}$ & Terra Tec $3.7 \mathrm{~g}$ & 281572 & 186792 & 3239 & 5.334 \\
\hline BEXSG17A & $2900 \mathrm{~m} \mathrm{N68.2W}$ & Terra Tec $3.7 \mathrm{~g}$ & 283603 & 181067 & 2969 & 5.209 \\
\hline BEXSG18A & $4460 \mathrm{~m} \mathrm{~N} 57.8 \mathrm{~W}$ & Terra Tec $3.7 \mathrm{~g}$ & 284898 & 179981 & 4505 & 5.259 \\
\hline BEXSG19A & 2690 m N9.1E & Terra Tec $3.7 \mathrm{~g}$ & 285190 & 184182 & 2761 & 5.052 \\
\hline
\end{tabular}


Table 13. Bexar Station Data (Concluded)

\begin{tabular}{|c|c|c|c|c|c|c|}
\hline $\begin{array}{l}\text { Station } \\
\text { Name }\end{array}$ & Location & Accelerometer & $\begin{array}{l}\text { Northing (m) } \\
282534\end{array}$ & $\begin{array}{l}\text { Easting(m) } \\
183764\end{array}$ & $\begin{array}{l}\text { Slant Range } \\
629(m) \text { DOB }\end{array}$ & Travel \\
\hline BEXSG20A & $3840 \mathrm{~m}$ S65.2E & Terra Tec $3.7 \mathrm{~g}$ & 280941 & 187256 & 3889 & 4.697 \\
\hline BEXSG21A & $1710 \mathrm{~m} \mathrm{~S} 40.7 \mathrm{~W}$ & Endevco & 281234 & 182653 & 1822 & 3.305 \\
\hline BEXSG22A & $1570 \mathrm{mS51.1E}$ & Endevco & 281626 & 185040 & 1688 & 6.166 \\
\hline BEXSG23A & $2180 \mathrm{~m} \mathrm{~N} 12.7 \mathrm{~W}$ & Endevco & 284665 & 183279 & 2274 & 3.483 \\
\hline BEXSG24A & $1670 \mathrm{~m} \mathrm{~N} 43.1 \mathrm{E}$ & Endevco & 283739 & 184904 & 1774 & 8.514 \\
\hline BEXSG25A & $3640 \mathrm{~m}$ N6.3E & Endevco & 286145 & 184178 & 3689 & 8.953 \\
\hline BEXSG26A & $2930 \mathrm{~m} \mathrm{N30.6W}$ & TerraTec $\mathbf{3 . 7 g}$ & 285050 & 182268 & 2994 & 3.699 \\
\hline BEXSG26B & $2930 \mathrm{~m}$ N30.6W & Endevco & 285050 & 182268 & 2994 & 3.675 \\
\hline BEXSG27A & $3330 \mathrm{~m} \mathrm{N17.8W}$ & Endevco & 85562 & 182700 & 3271 & 8.721 \\
\hline BEXSG29A & $1290 \mathrm{~m} \mathrm{~S} 20.5 \mathrm{~W}$ & Endevco & 281314 & 183318 & 1443 & 9.922 \\
\hline BEXSG32A & $2060 \mathrm{~m} \mathrm{~S} 25.8 \mathrm{E}$ & Endevco & 280653 & 184659 & 2176 & 9.788 \\
\hline BEXSG37A & $2550 \mathrm{~m} \mathrm{N25.6W}$ & Endevco & 284829 & 182657 & 2625 & 9.242 \\
\hline
\end{tabular}




\section{Bexar Surface Ground Motion}

Acceleration--Station 1a

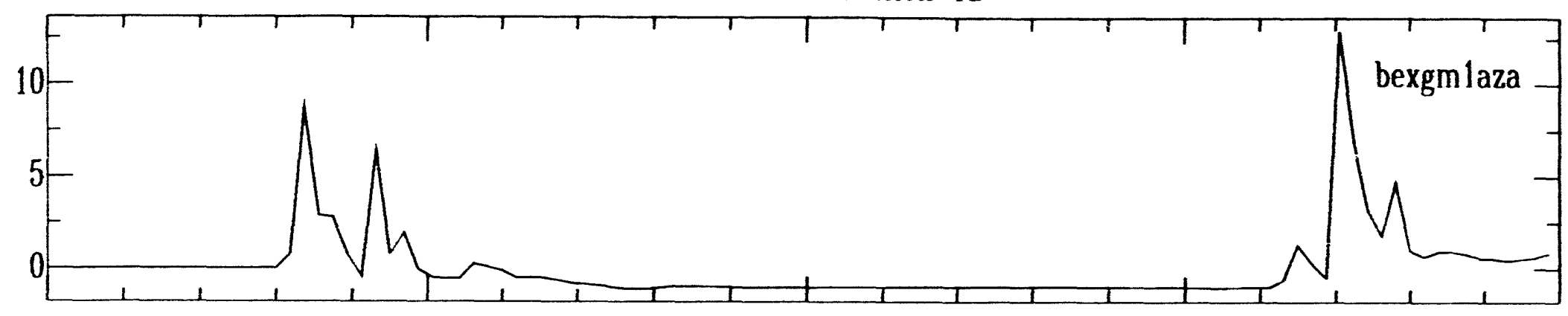

Velocity--Station la

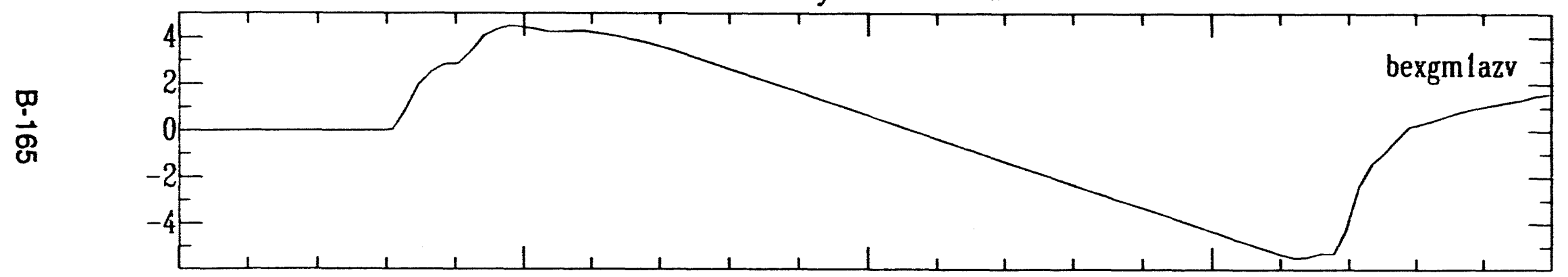

$\frac{3}{\infty}$

Displacement--Station la

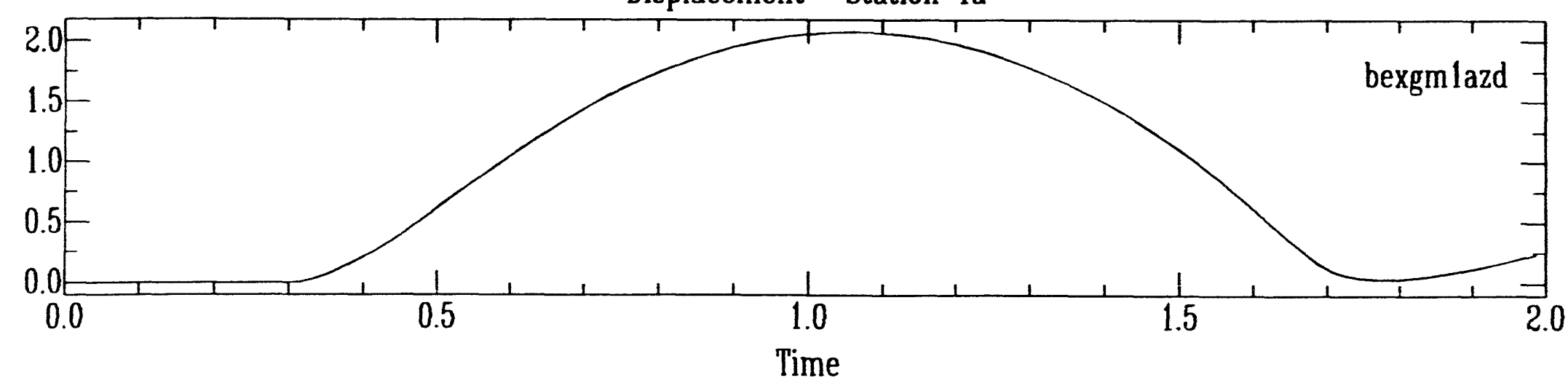




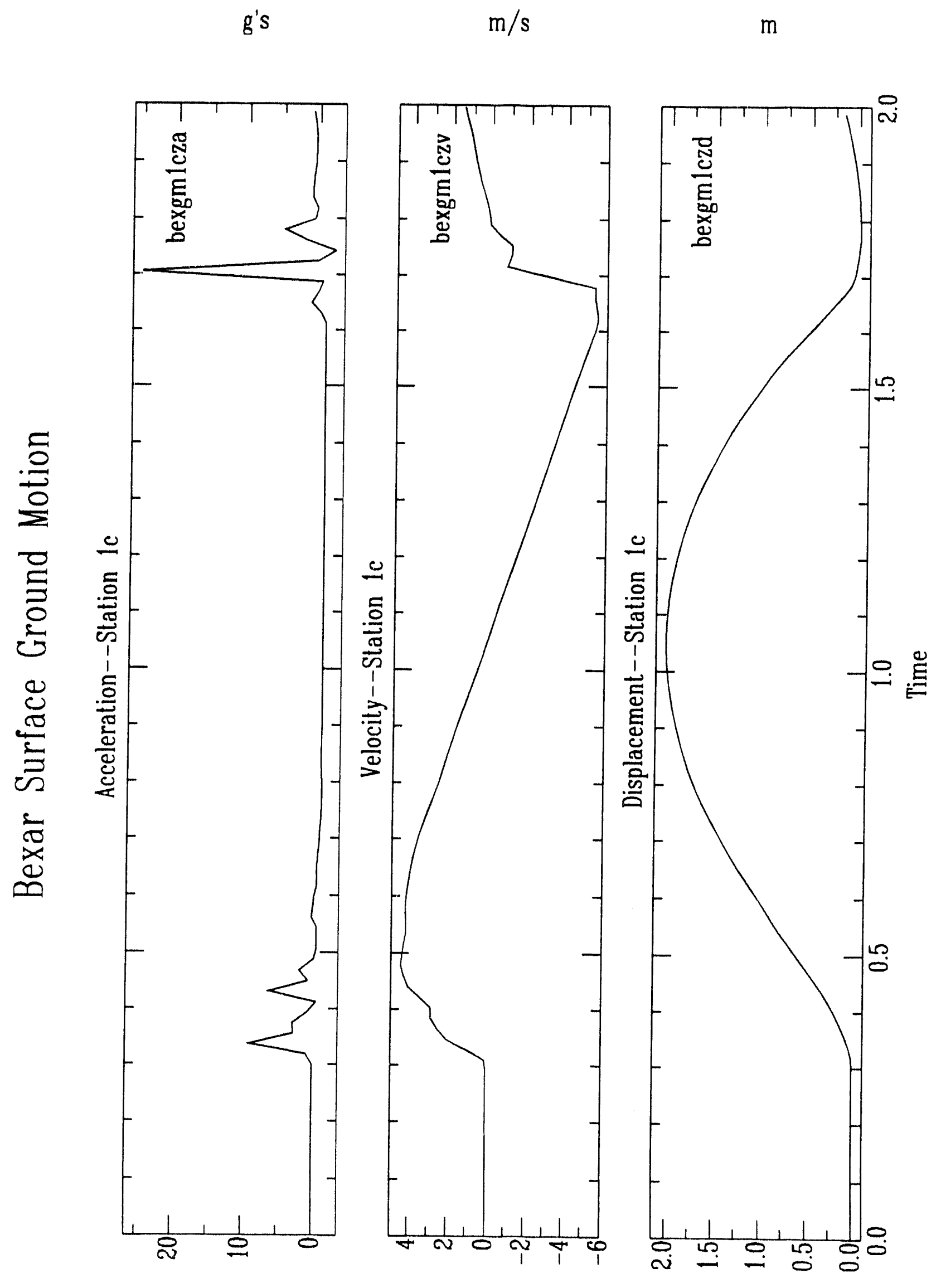




\section{Bexar Surface Ground Motion}

Acceleration--Station 2a

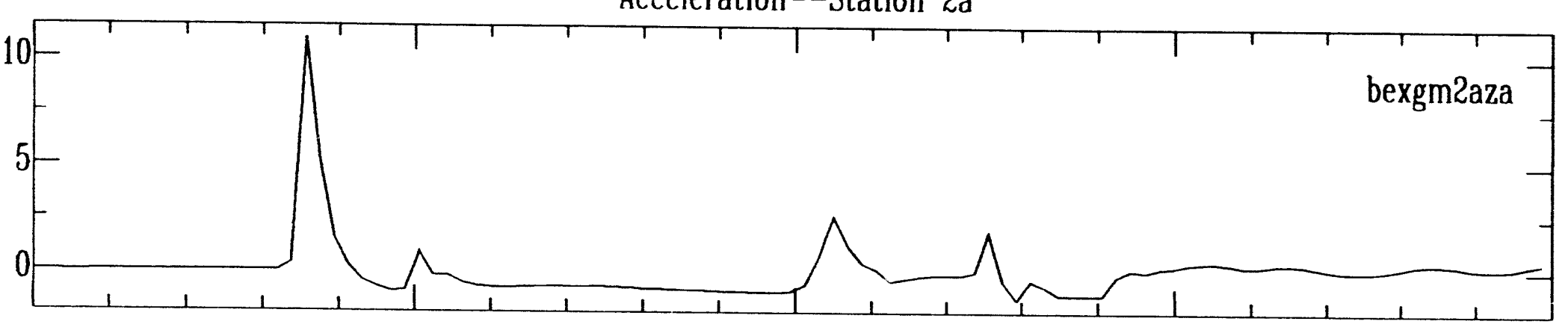

Velocity--Station 2a

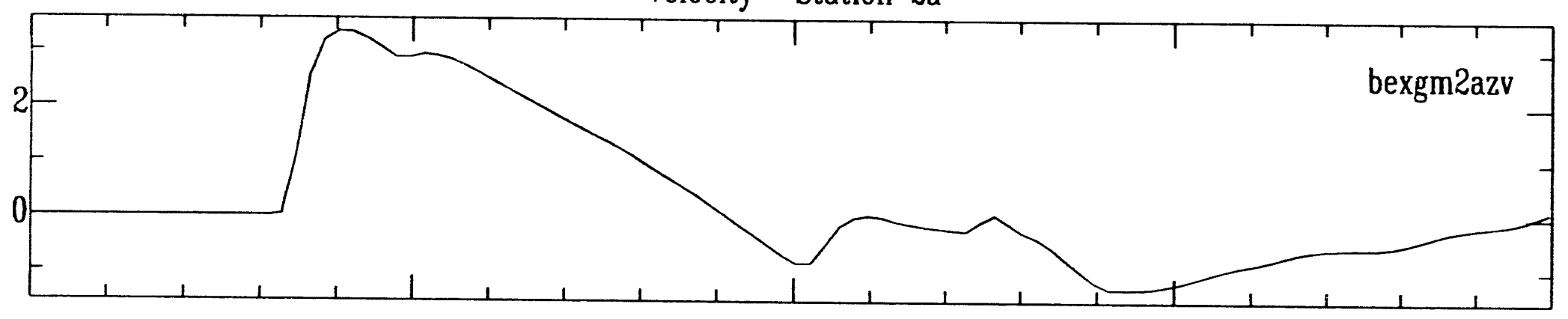

$\frac{3}{\infty}$

Displacement--Station 2a

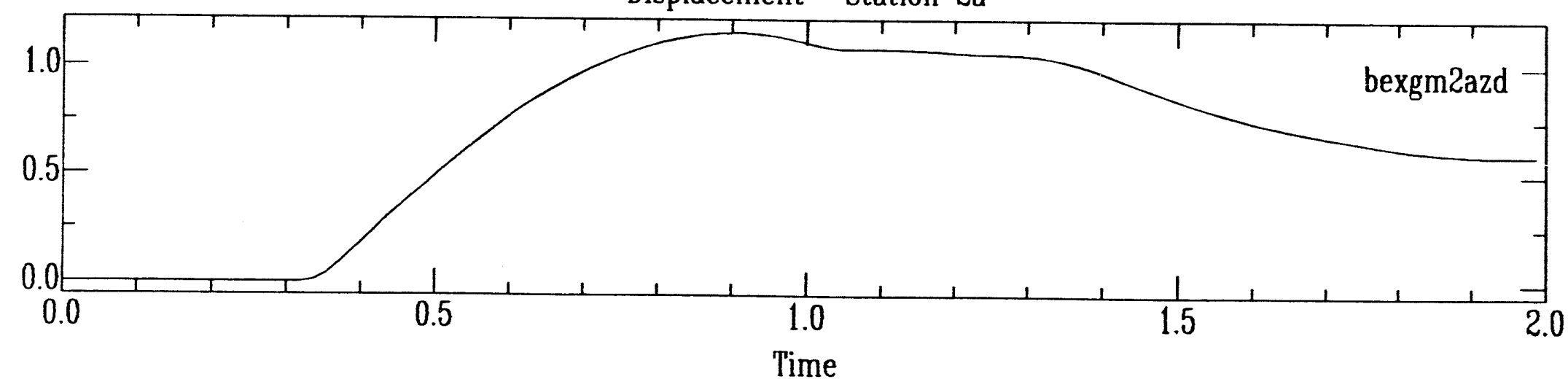




\section{Bexar Surface Ground Motion}

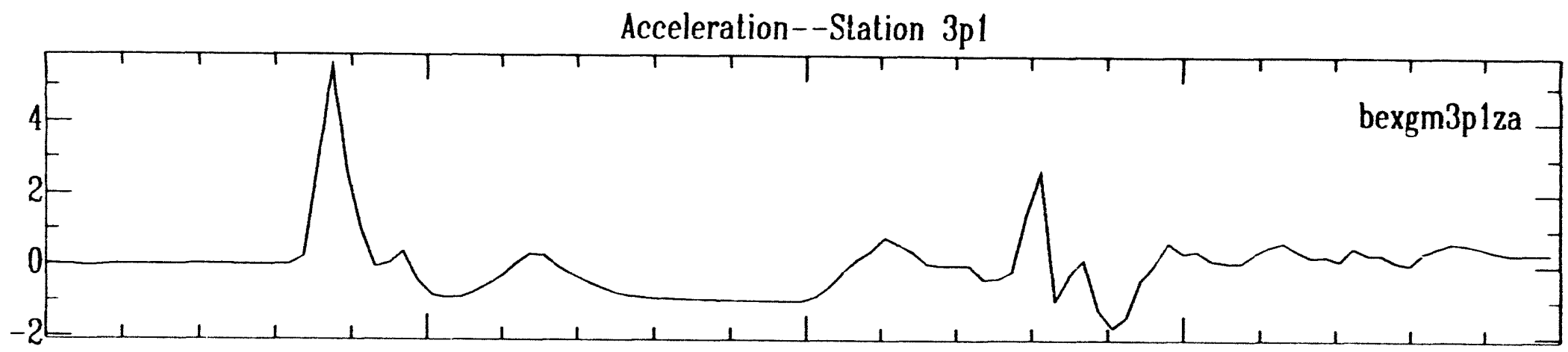

Velocity--Station 3p1

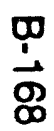

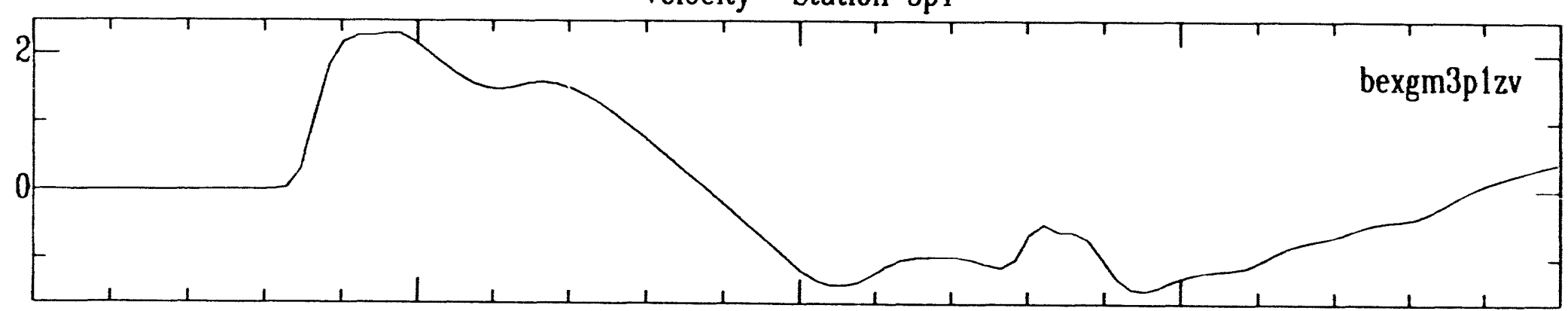

$\frac{3}{\infty}$

Displacement--Station 3p1

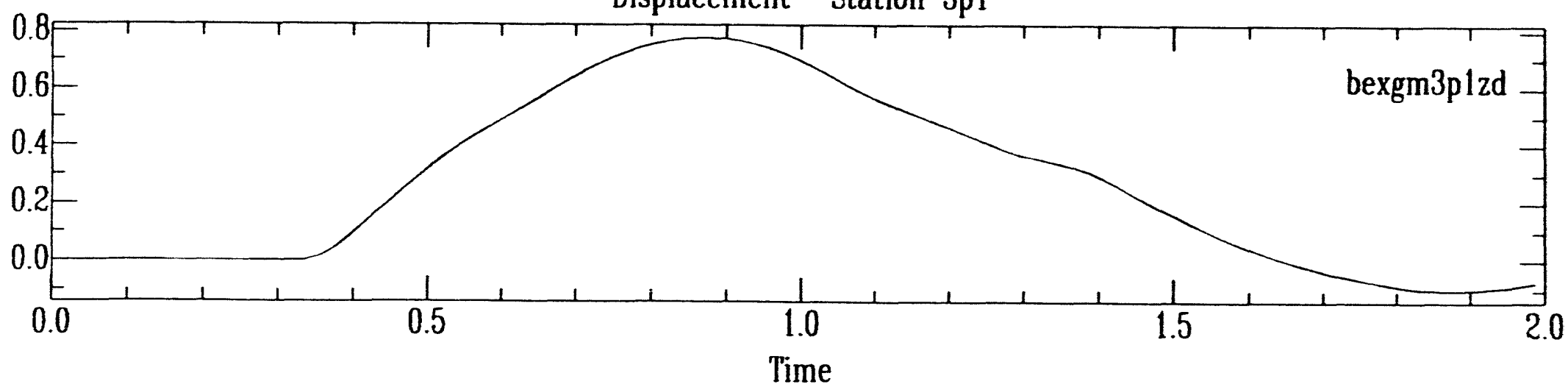



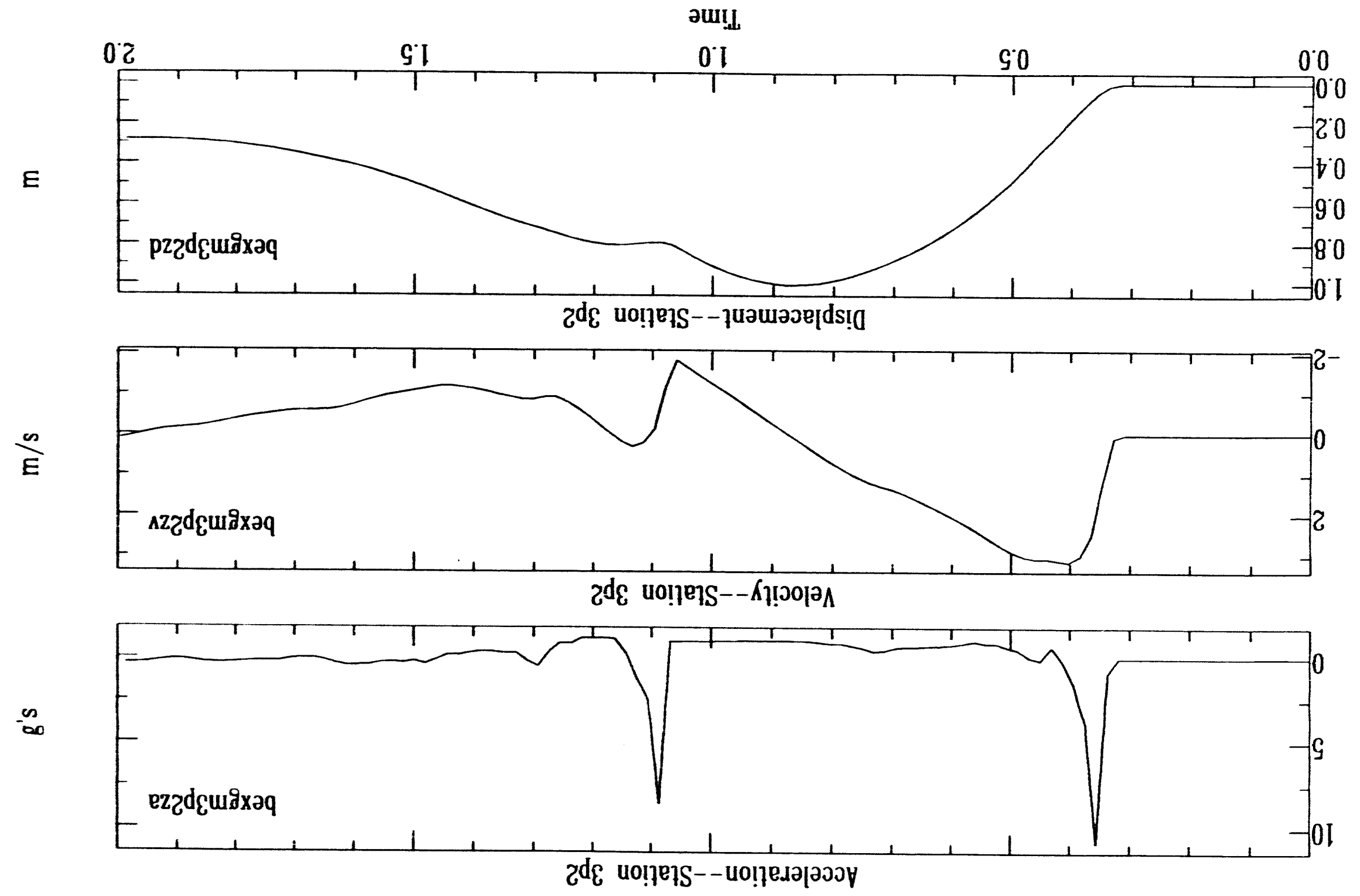

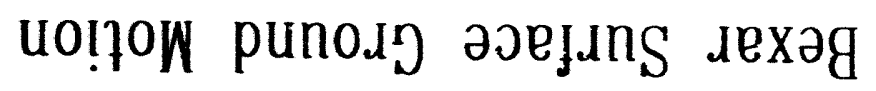




\section{Bexar Surface Ground Motion}
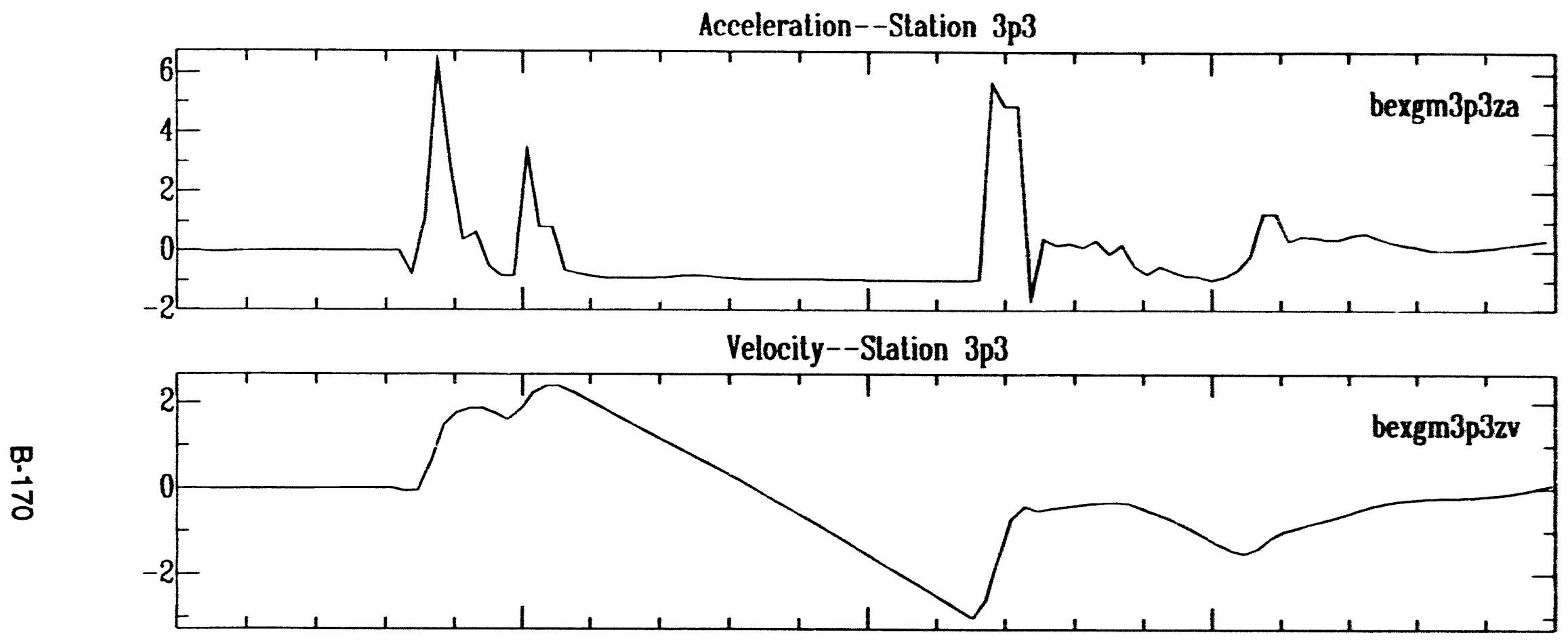

Displacement--Station 3p3

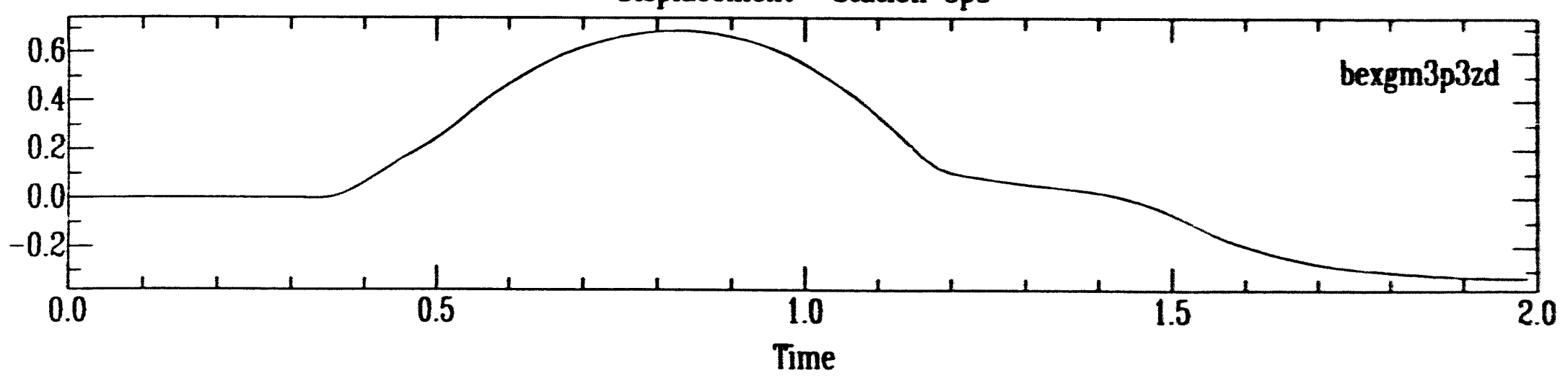



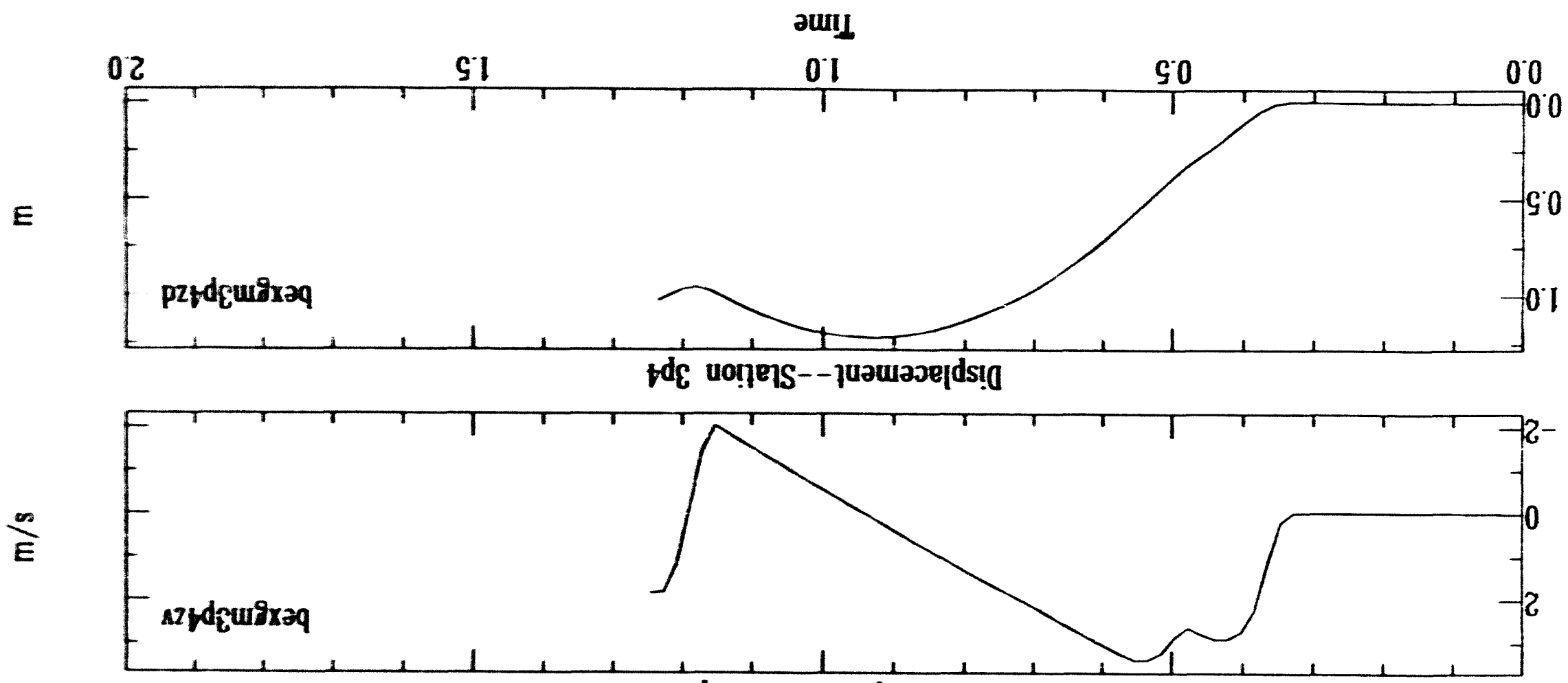

$\frac{\bar{T}}{\text { ต่ }}$

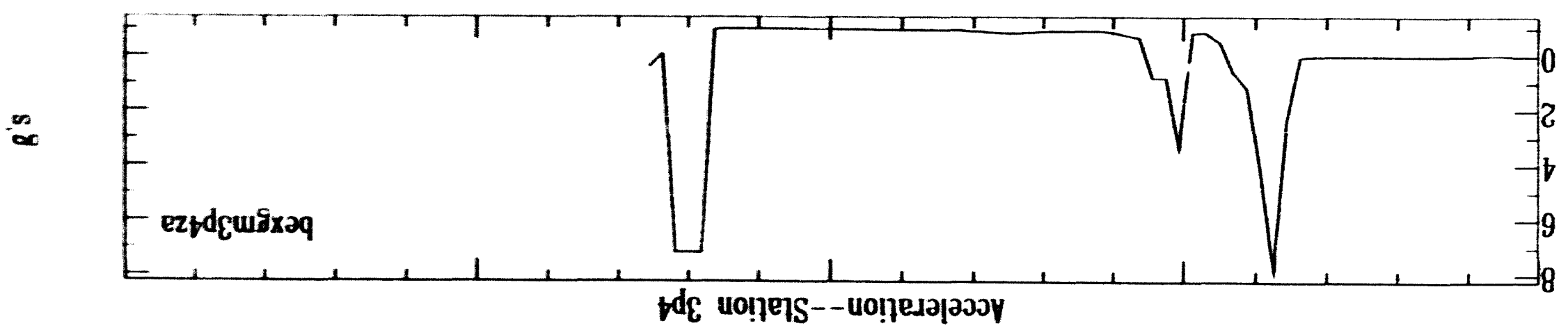

uo!ฺo punodg วэejans dexәg 

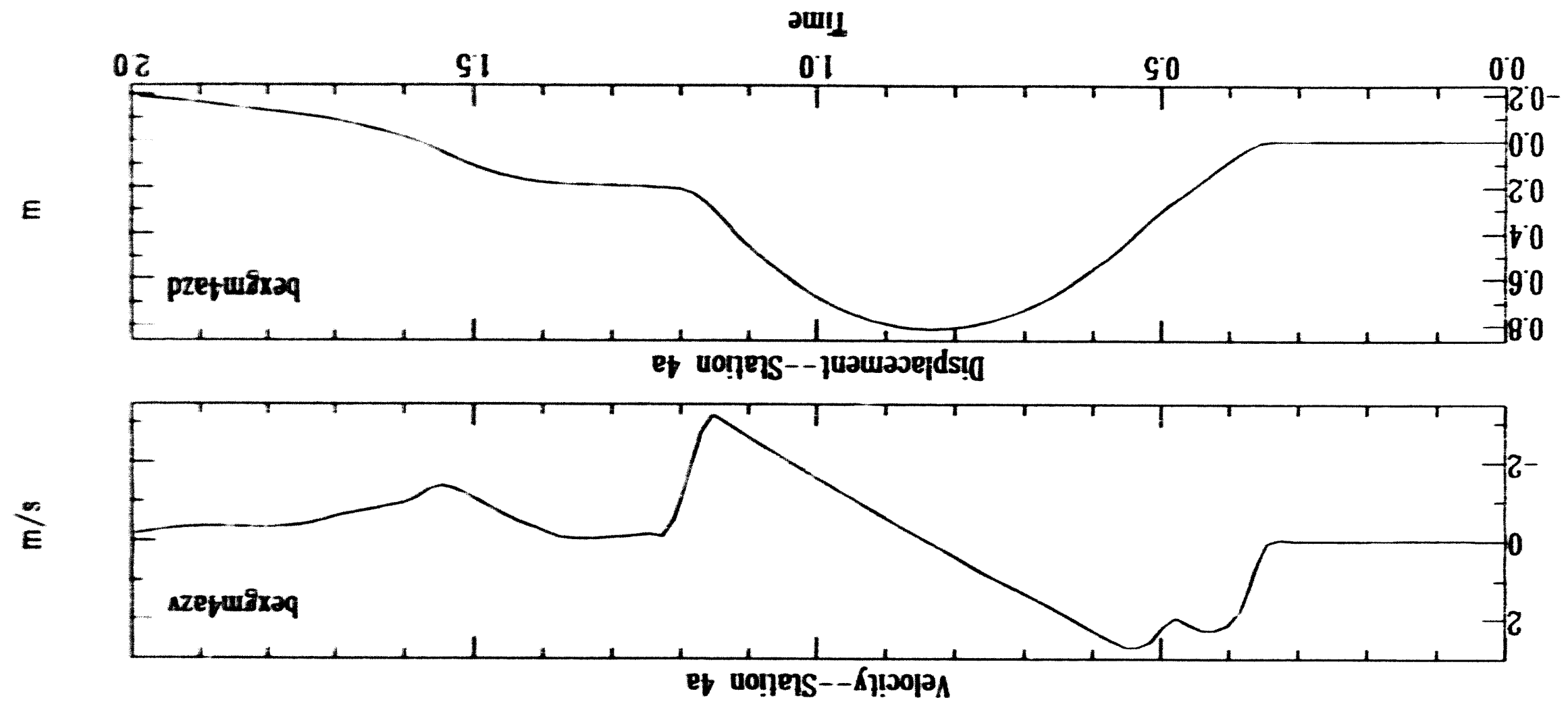

$\frac{N}{\infty}$

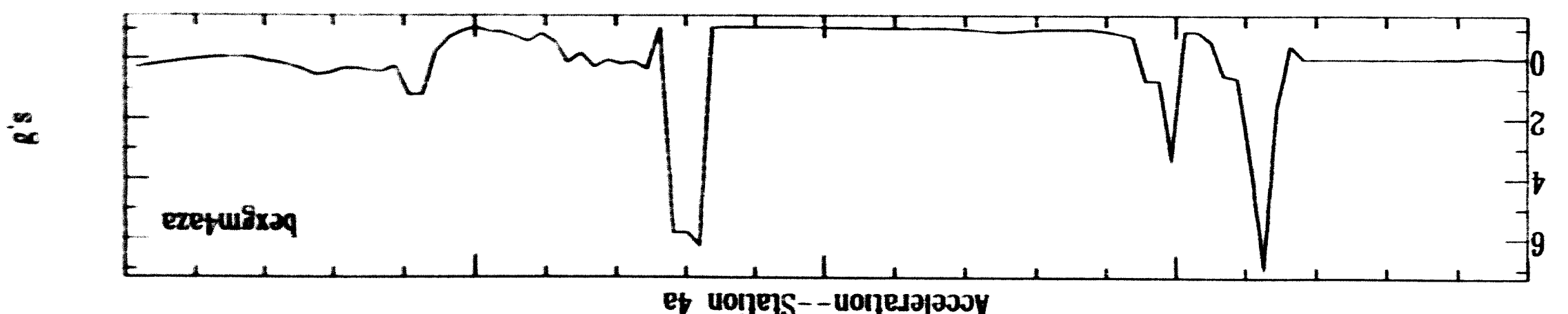

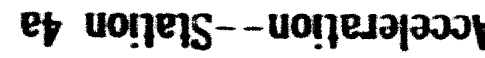

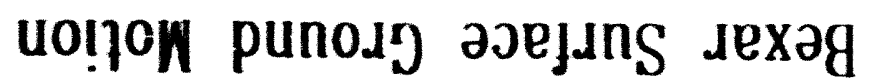


Bexar Surface Ground Motion

Acceleration--Station $5 a$

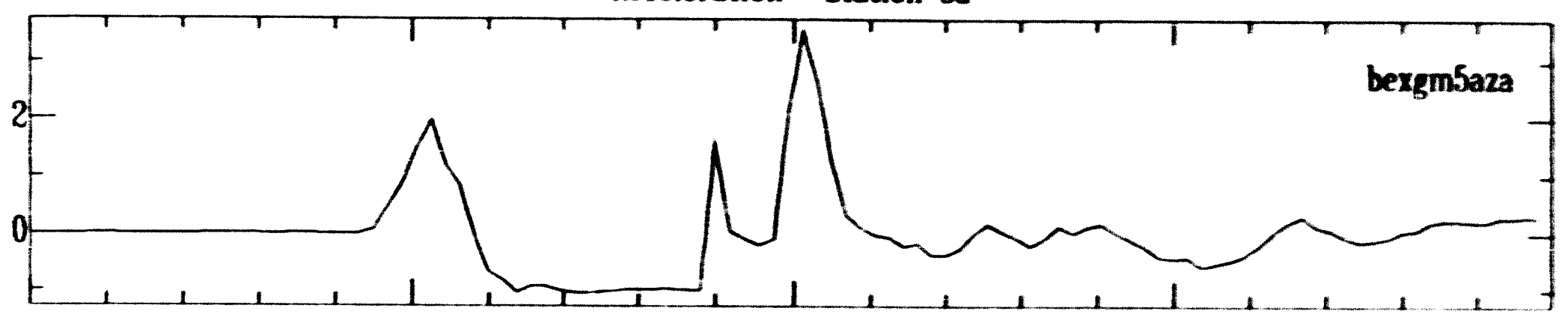

Velocily--Station $5 a$

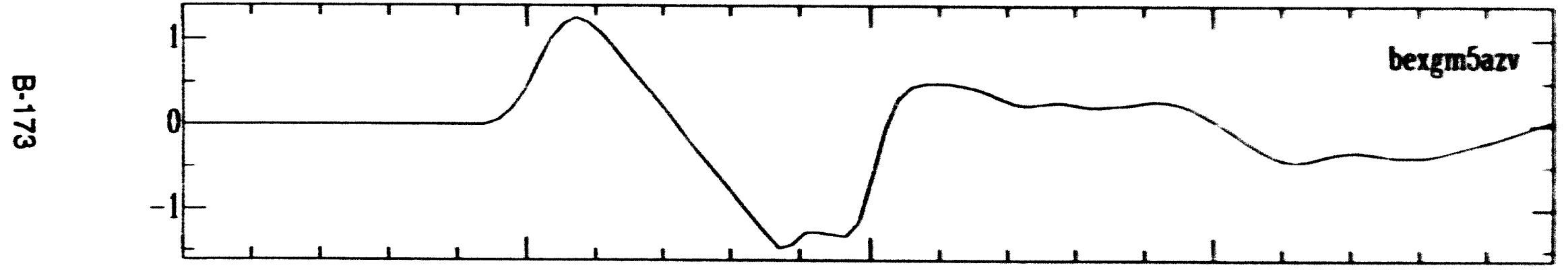

Displacement--Station 5 a

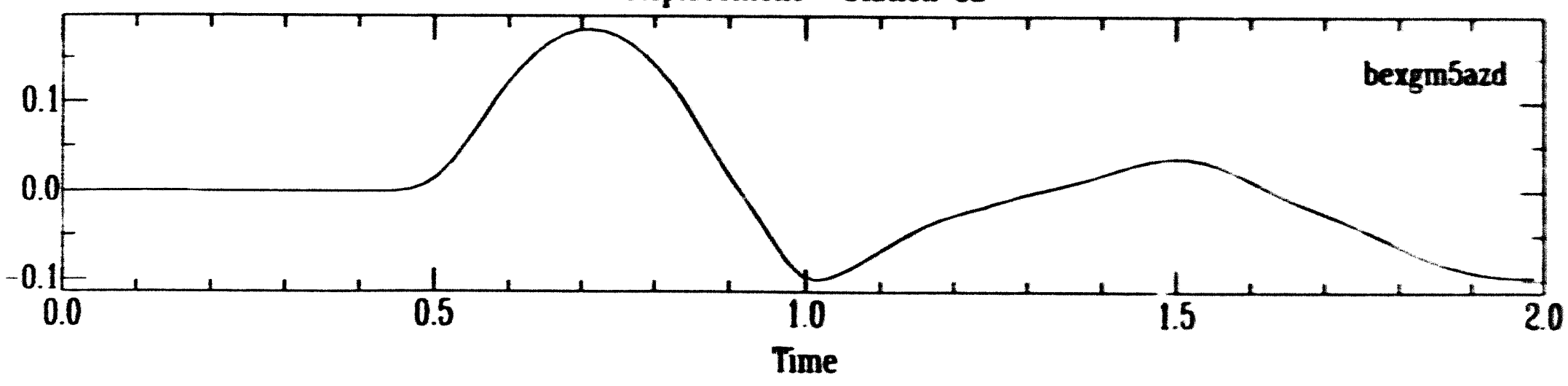




\section{Bexar Surface Ground Motion}

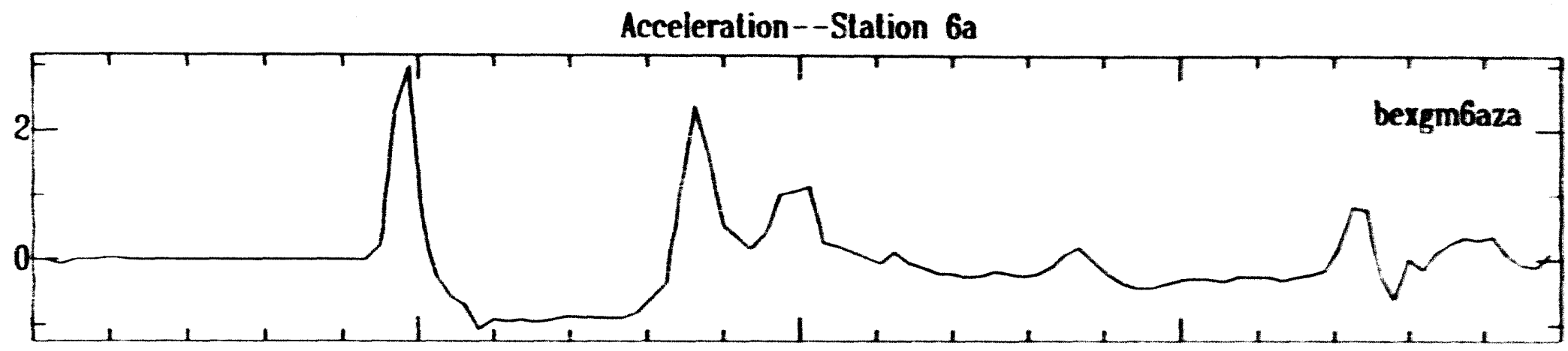

Velocily--Station $6 a$

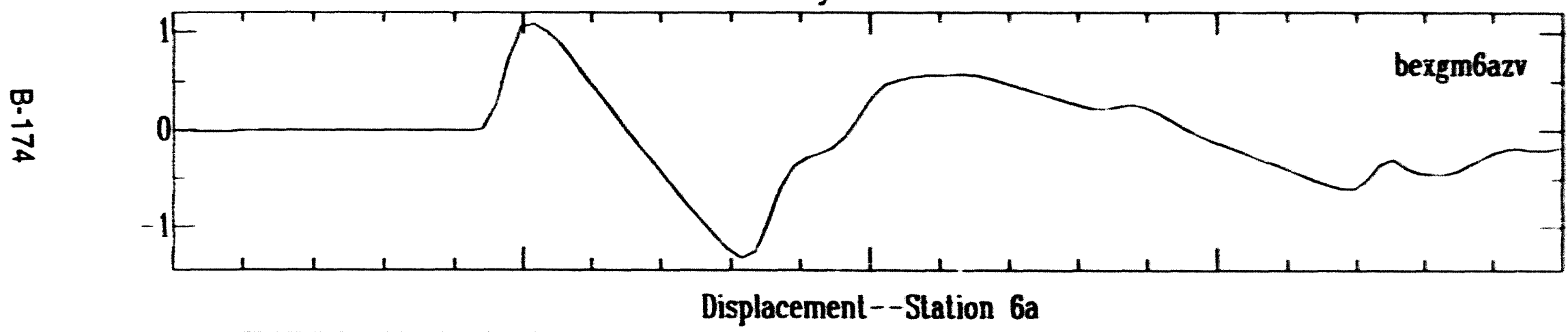

$\underset{\infty}{\exists}$

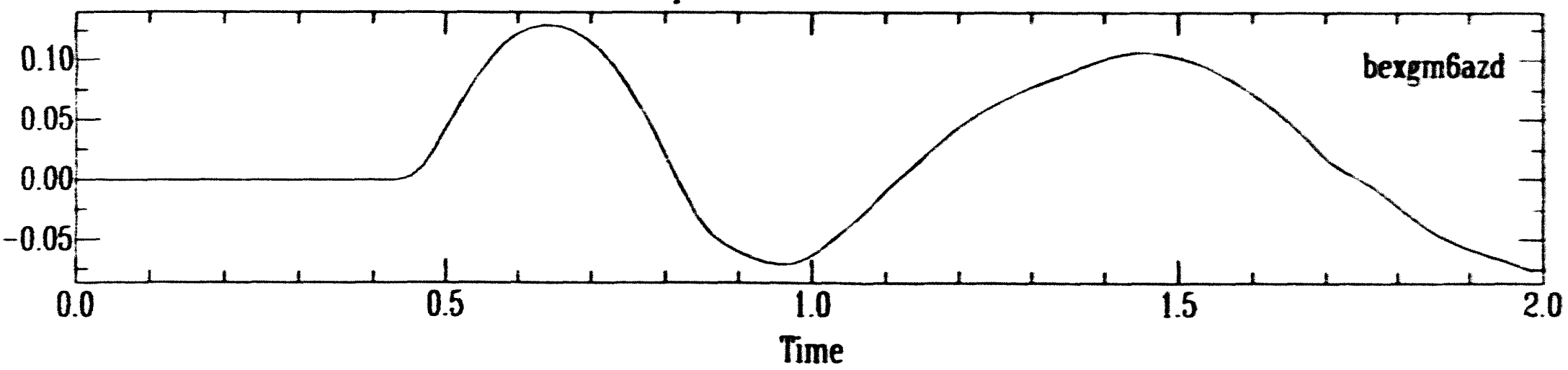




\section{Bexar Surface Ground Motion}

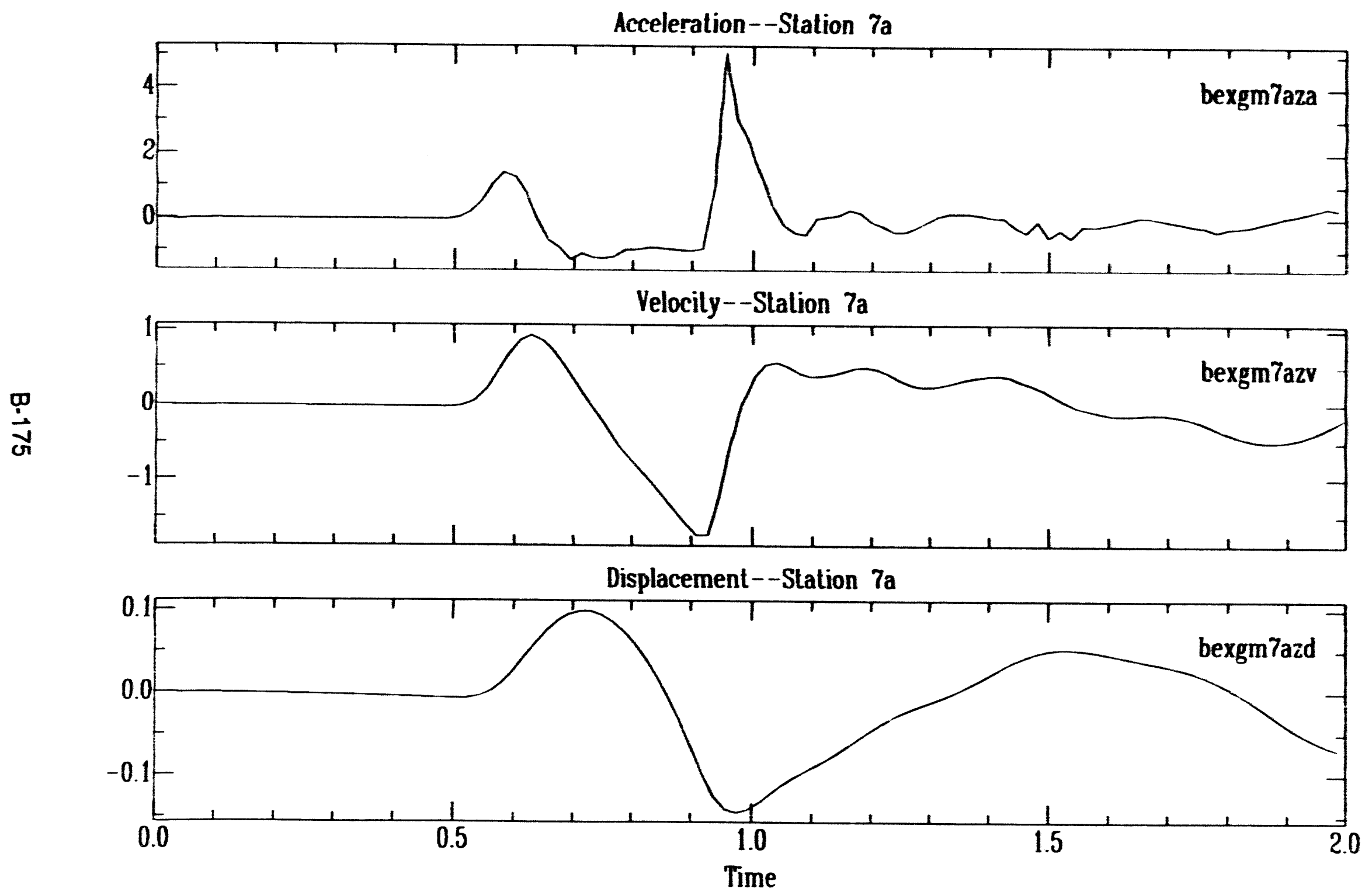




\section{Bexar Surface Ground Motion}

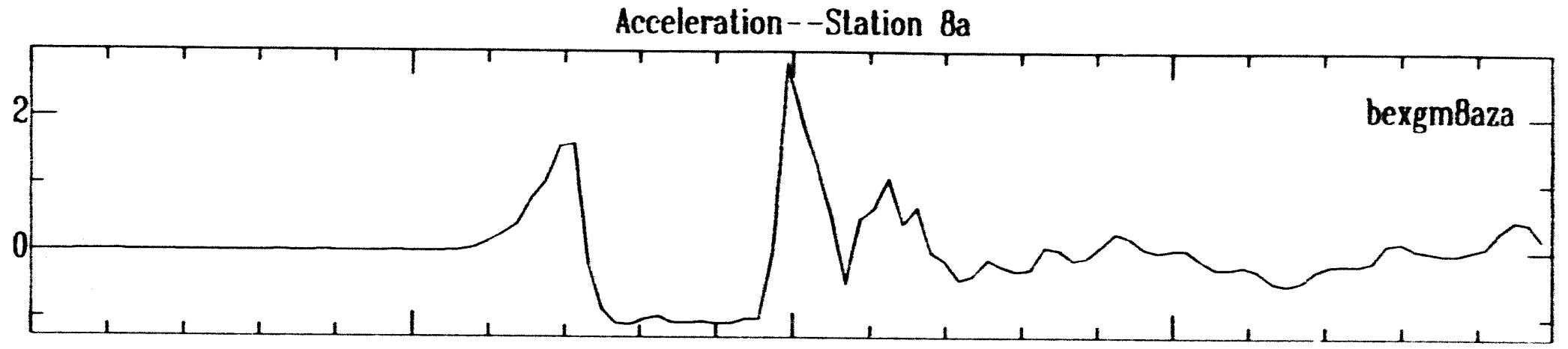

Velocily--Station Ba

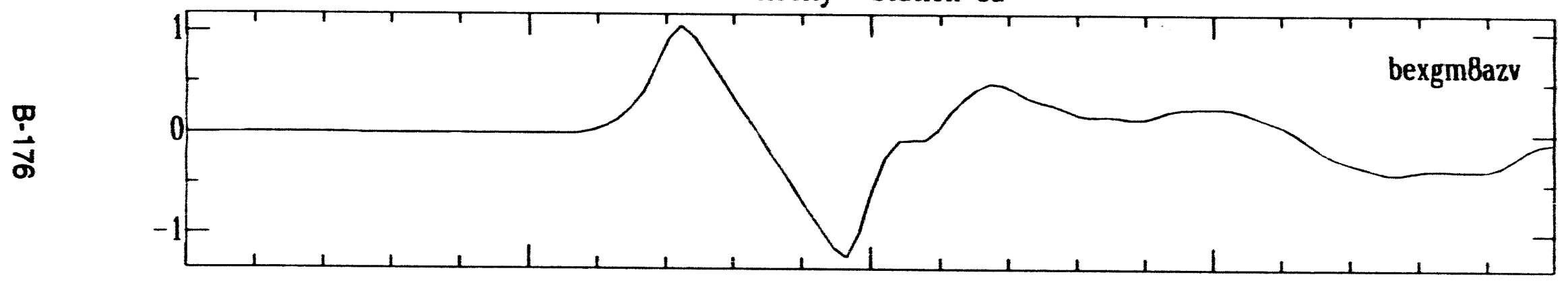

Displacement--Station 8a

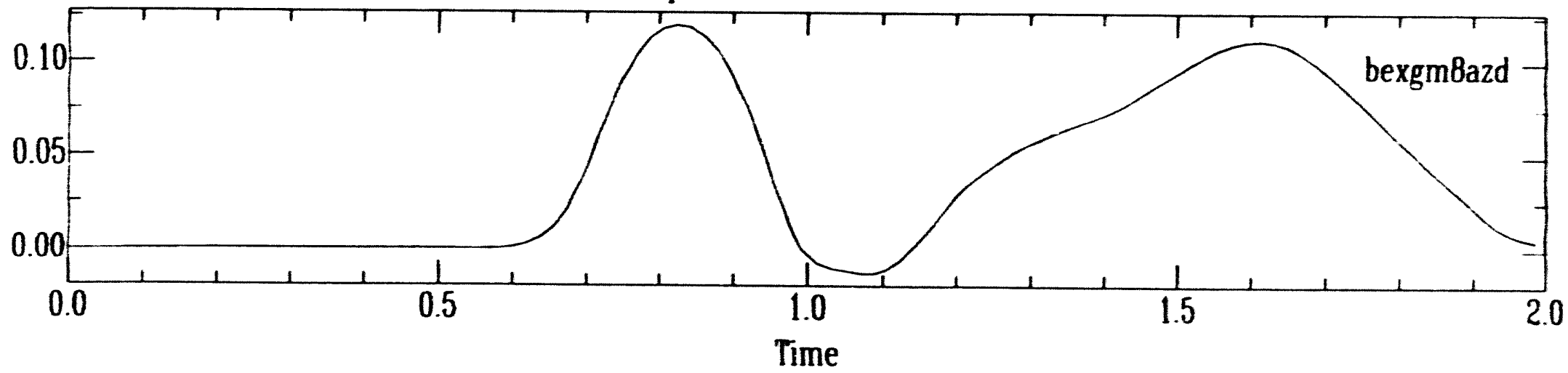


Bexar Surface Ground Motion

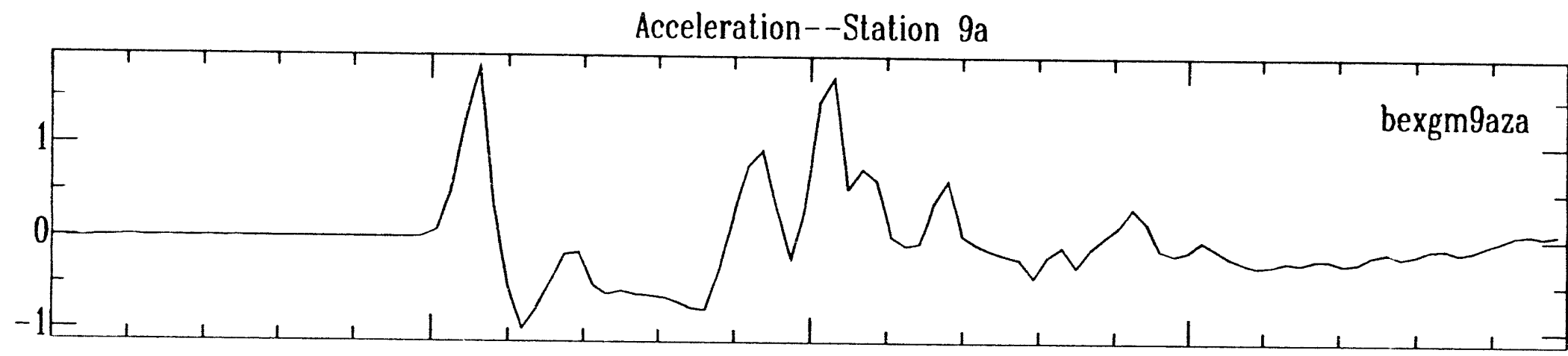

Velocity--Station 9a

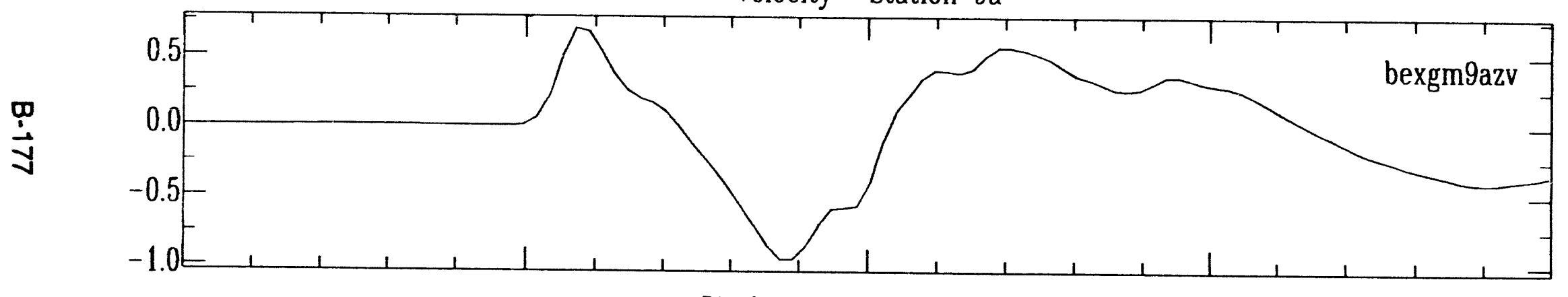

Displacement--Station 9a

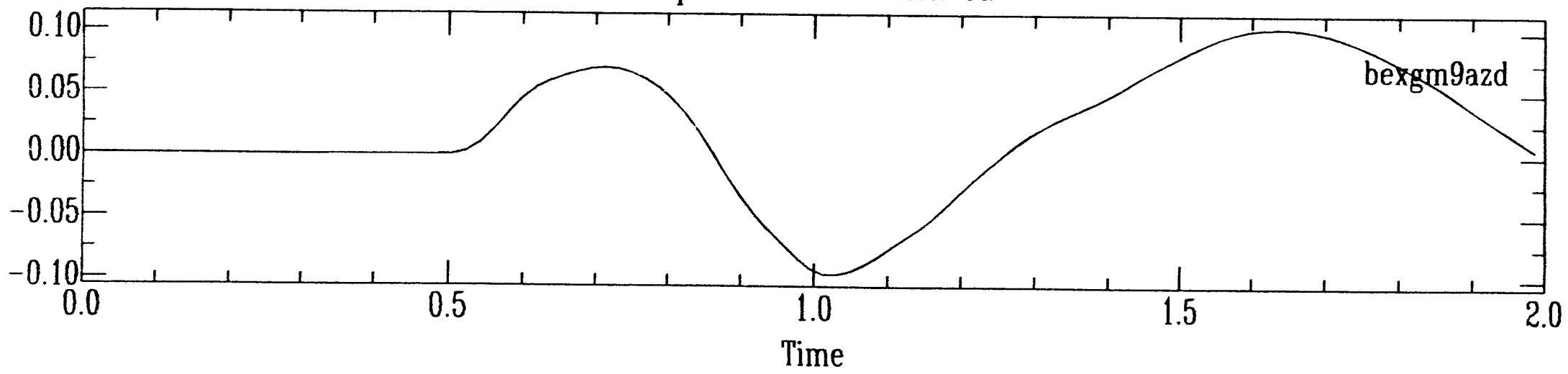




\section{Bexar Surface Ground Motion}

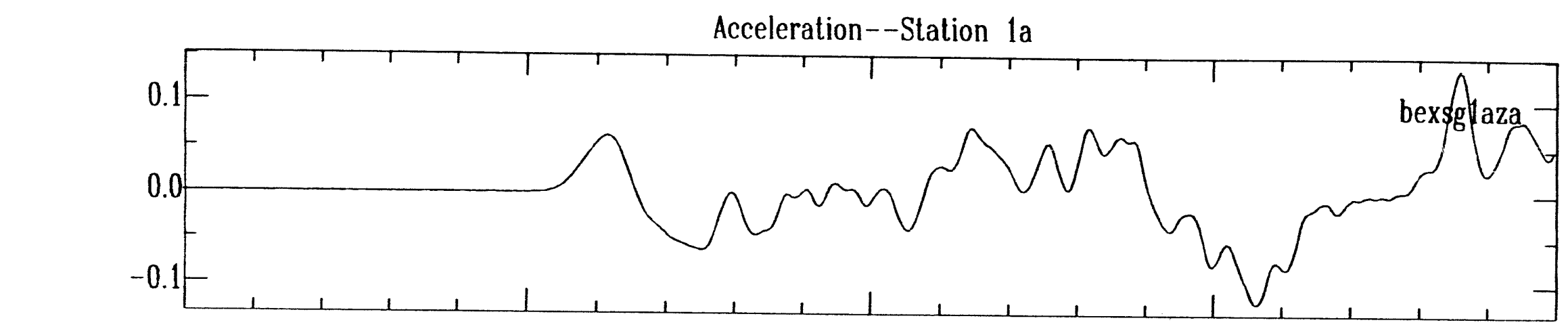

Velocity--Station 1a

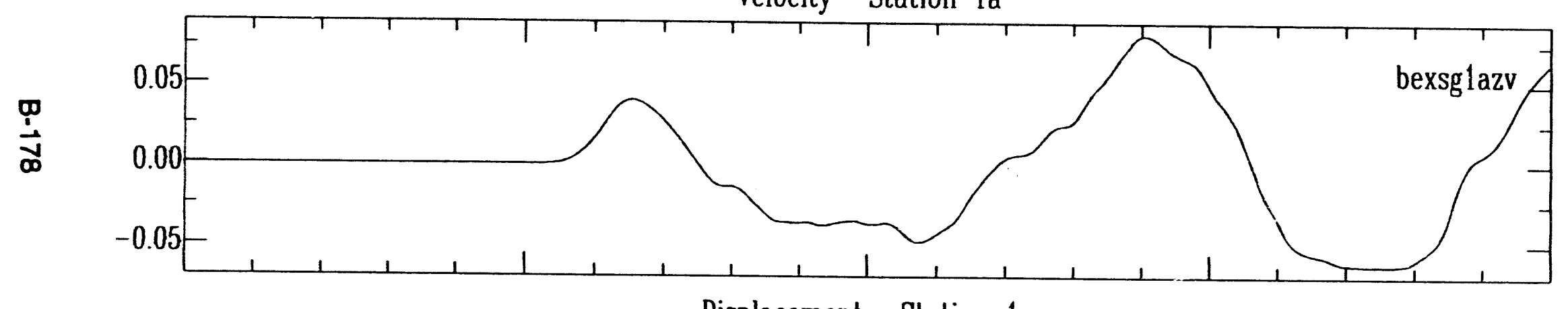

$\frac{3}{\infty}$

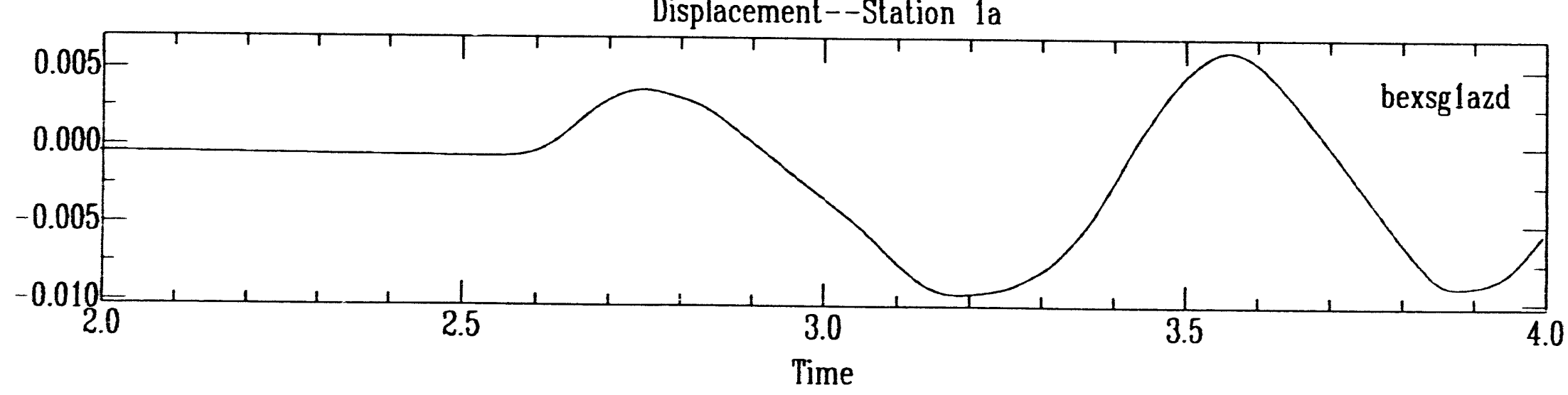


Bexar Surface Ground Motion

Acceleration--Station 2a

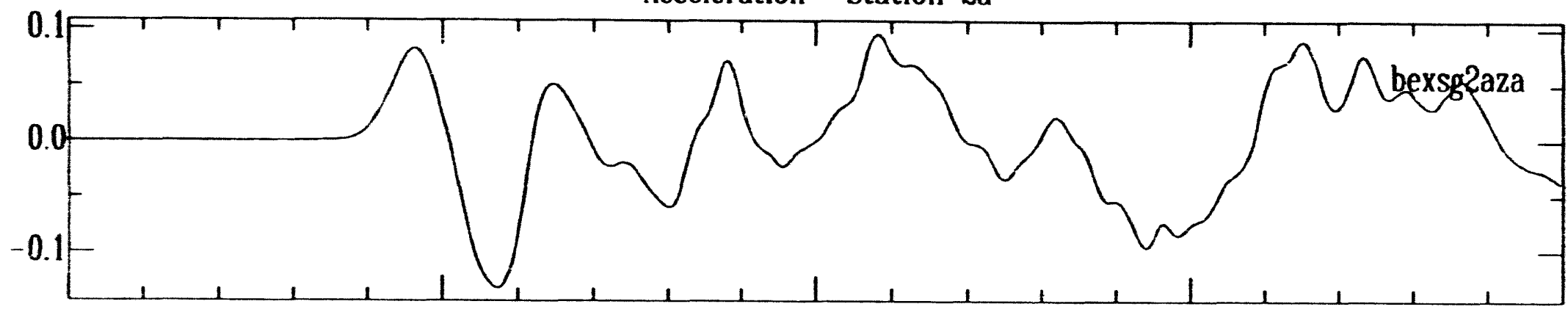

$\infty$

$\frac{3}{\infty}$

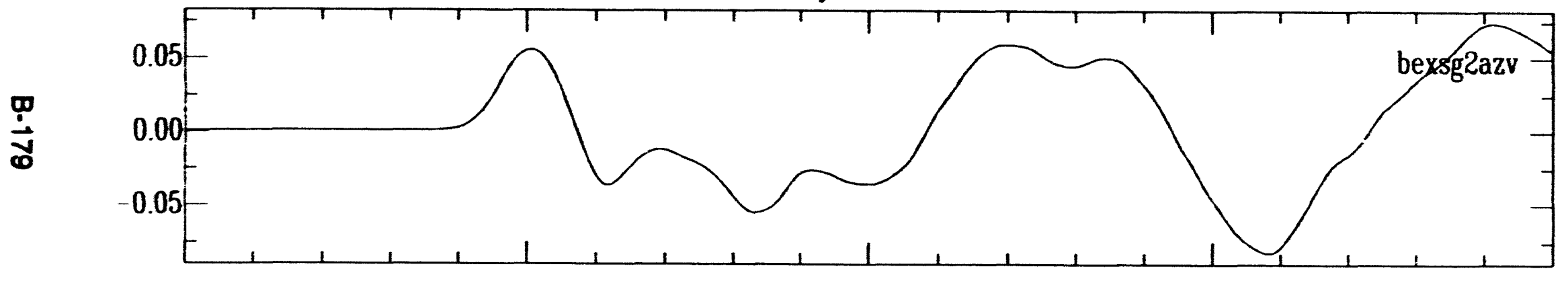

Displacement--Station 2a

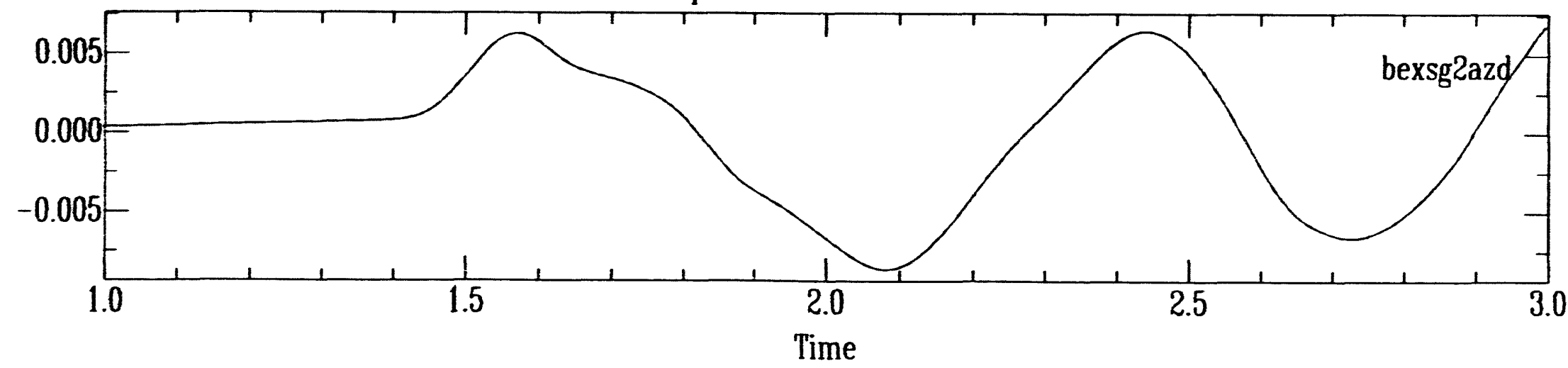




\section{Bexar Surface Ground Motion}

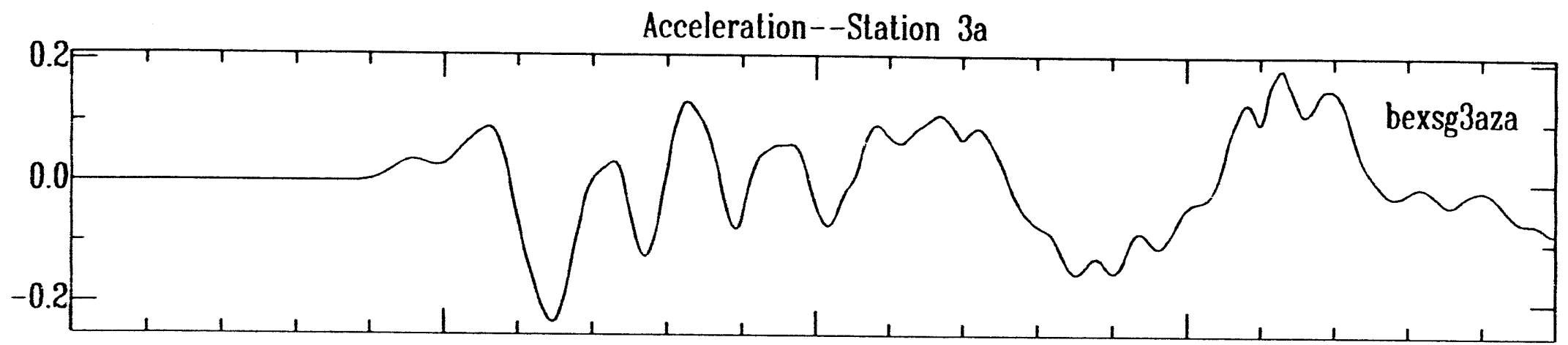

os

Velocity--Station 3a

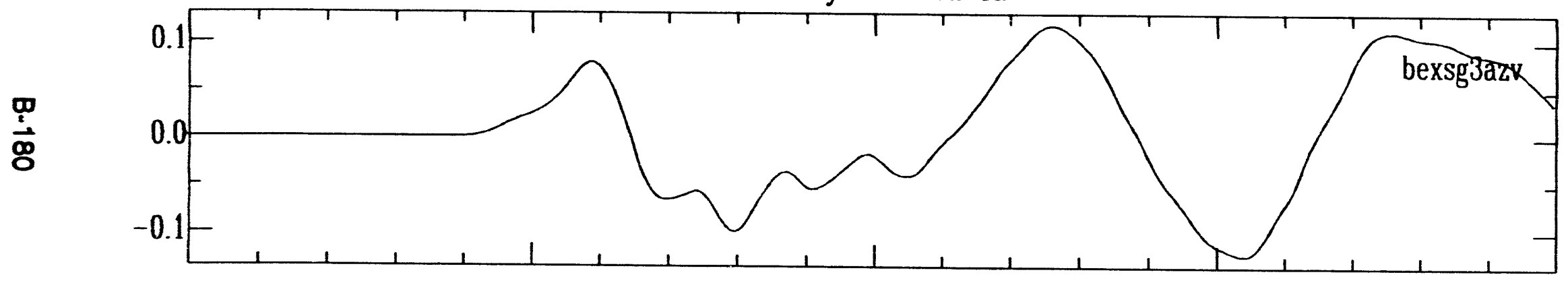

$\frac{3}{\infty}$

Displacement--Station 3a

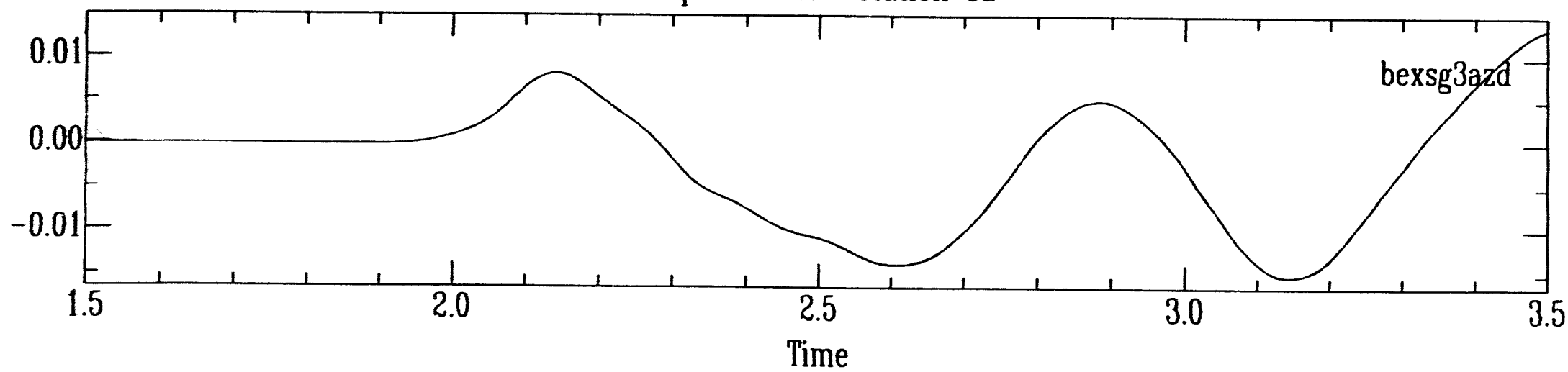




\section{Bexar Surface Ground Motion}

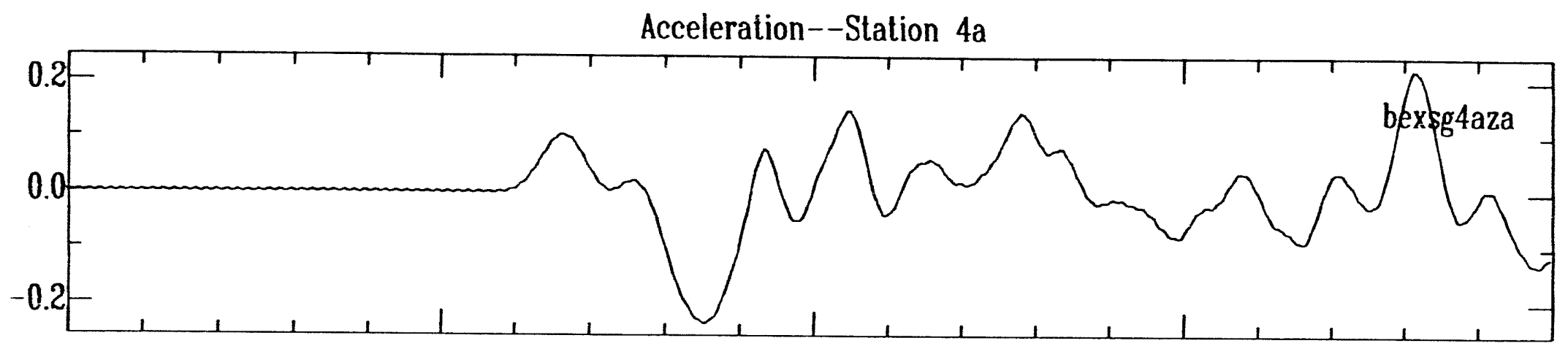

Velocity--Station 4a

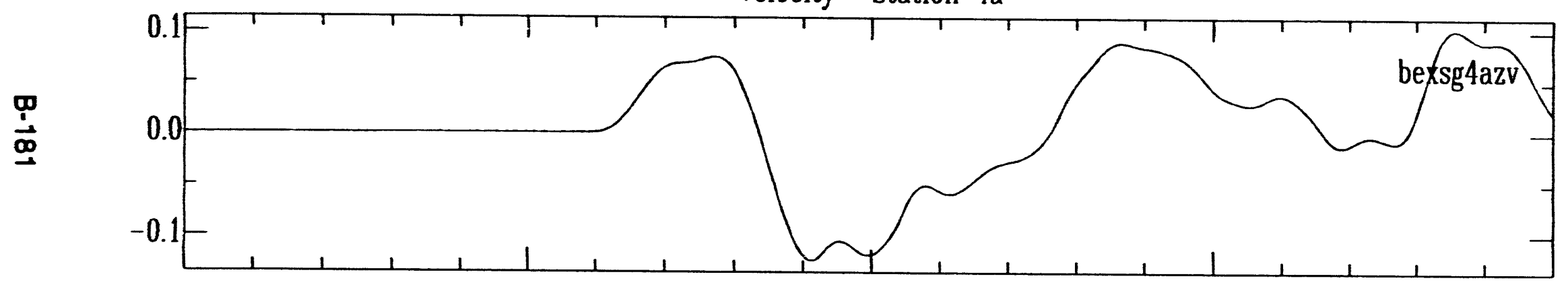

Displacement--Station 4a

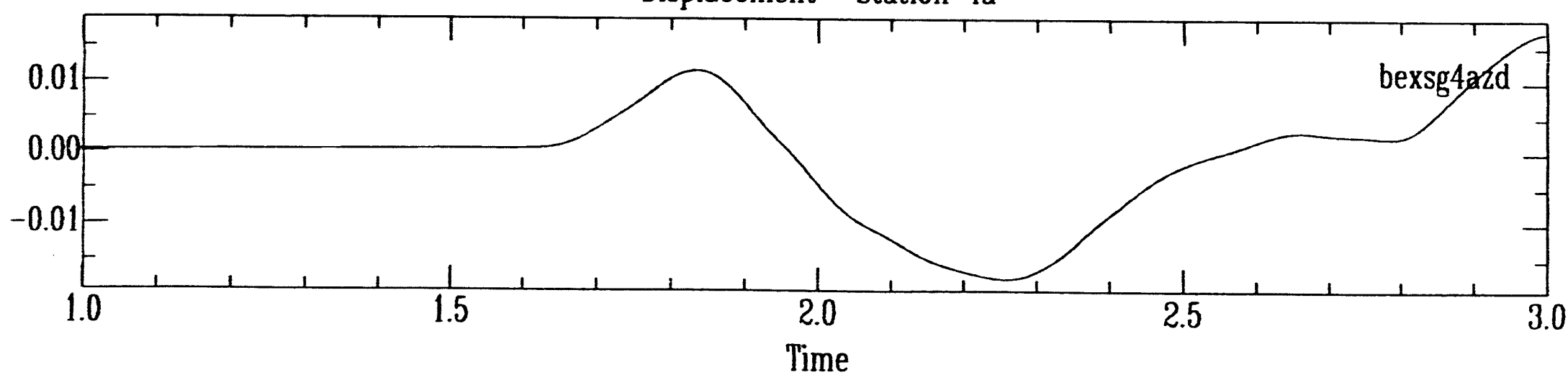




\section{Bexar Surface Ground Motion}
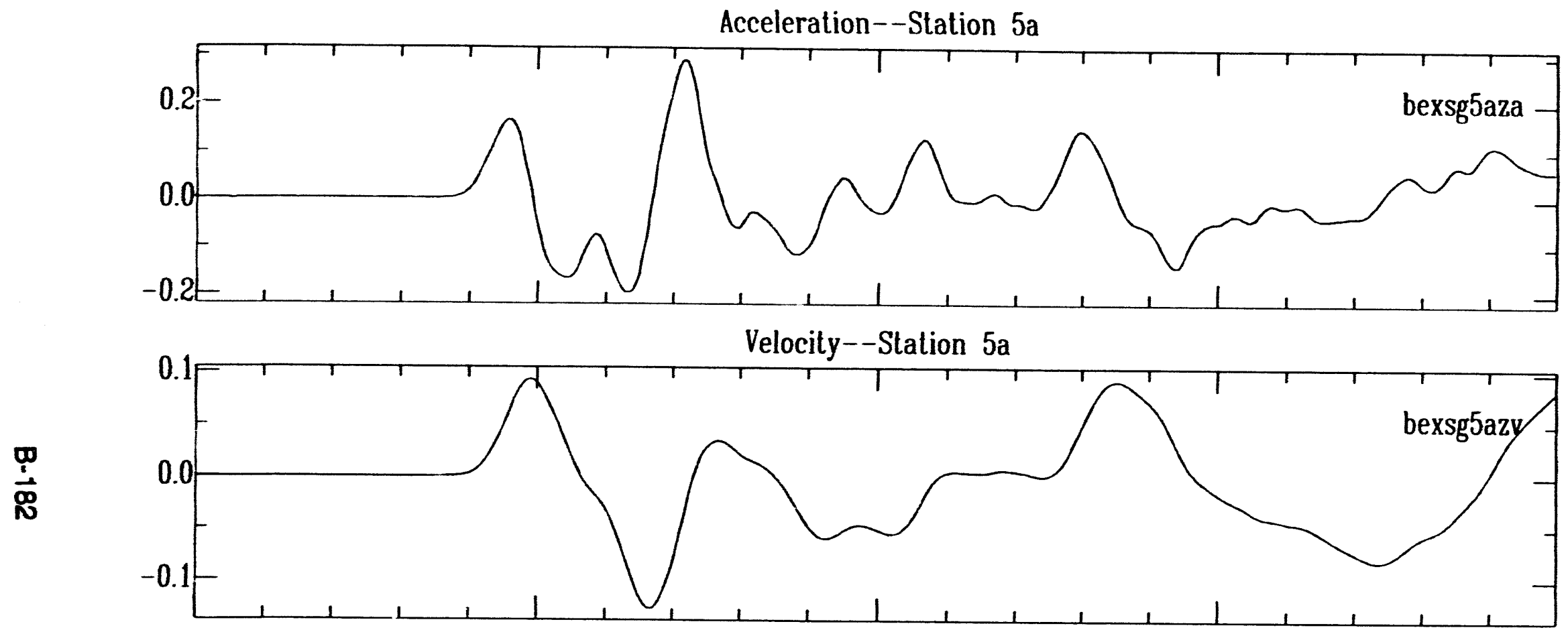

Displacement--Station 5a

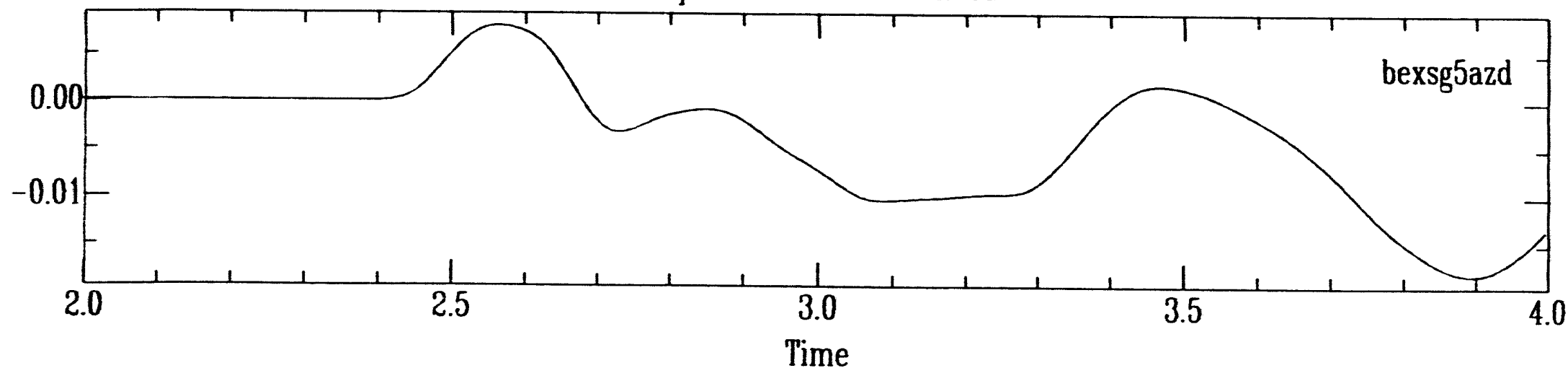




\section{Bexar Surface Ground Motion}

Acceleration--Station 6a

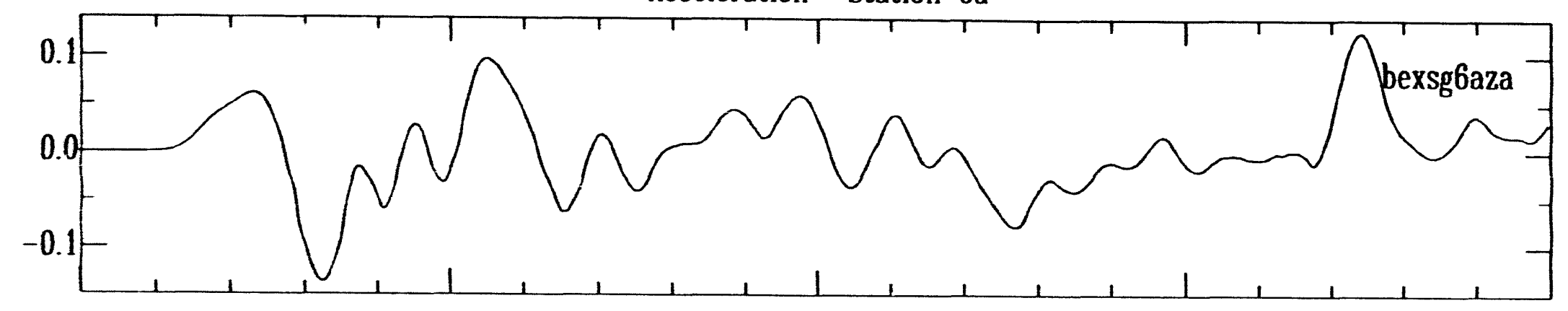

Velocity--Station 6a

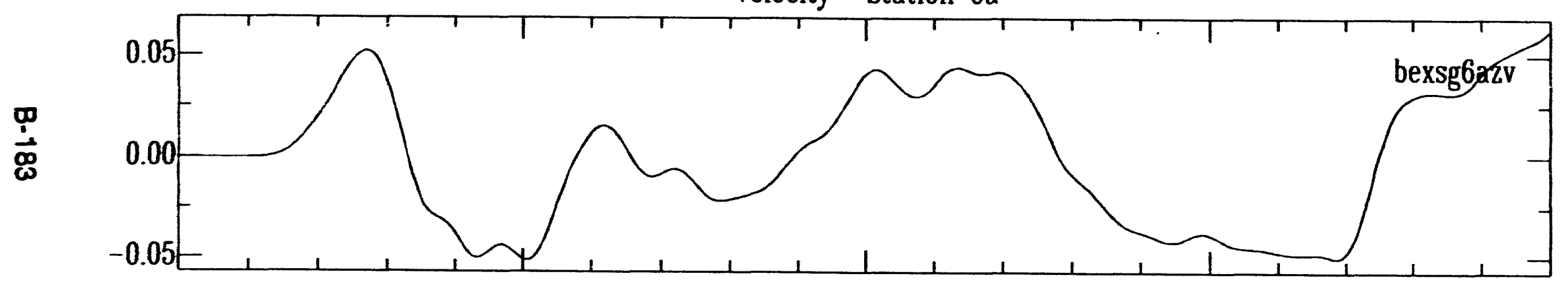

Displacement--Station 6a

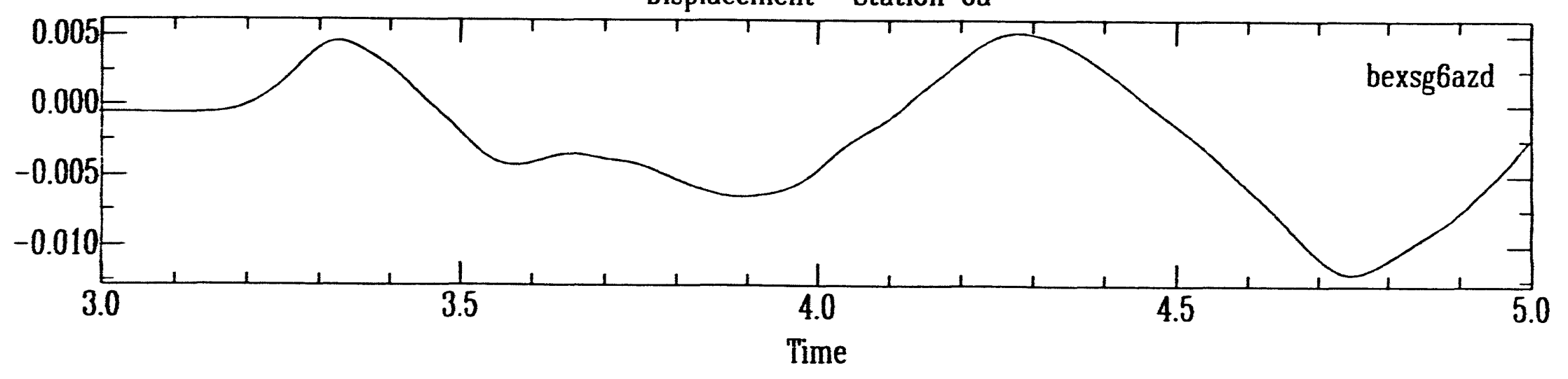




\section{Bexar Surface Ground Motion}

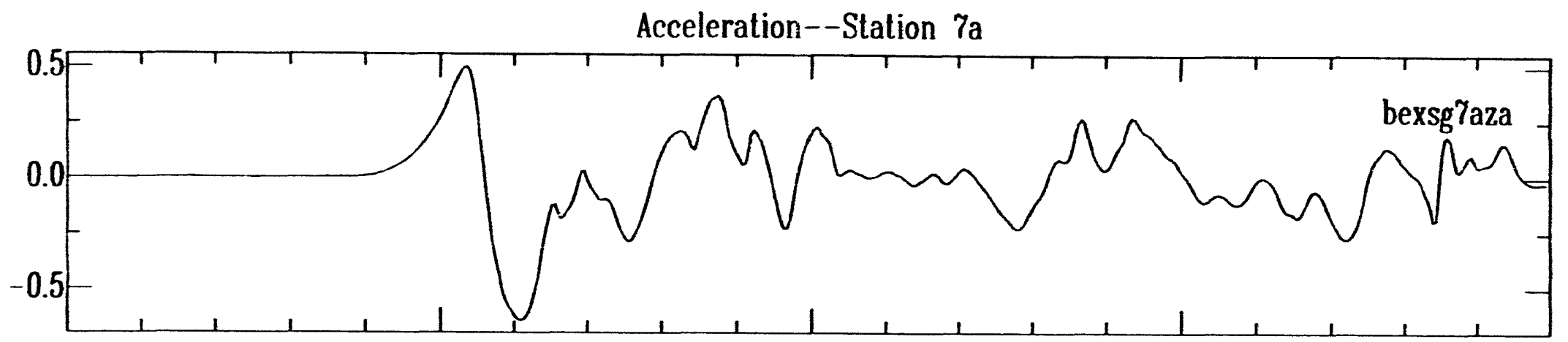

Velocity--Station 7a

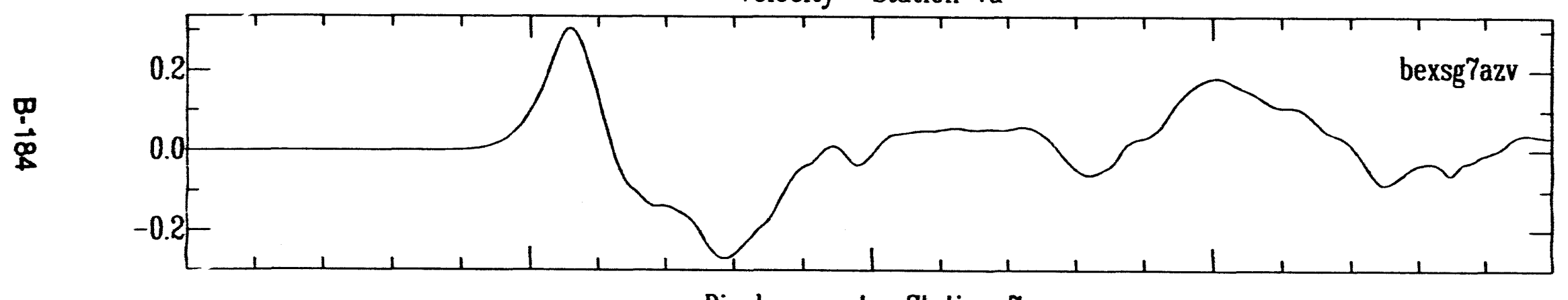

Displacement--Station 7a

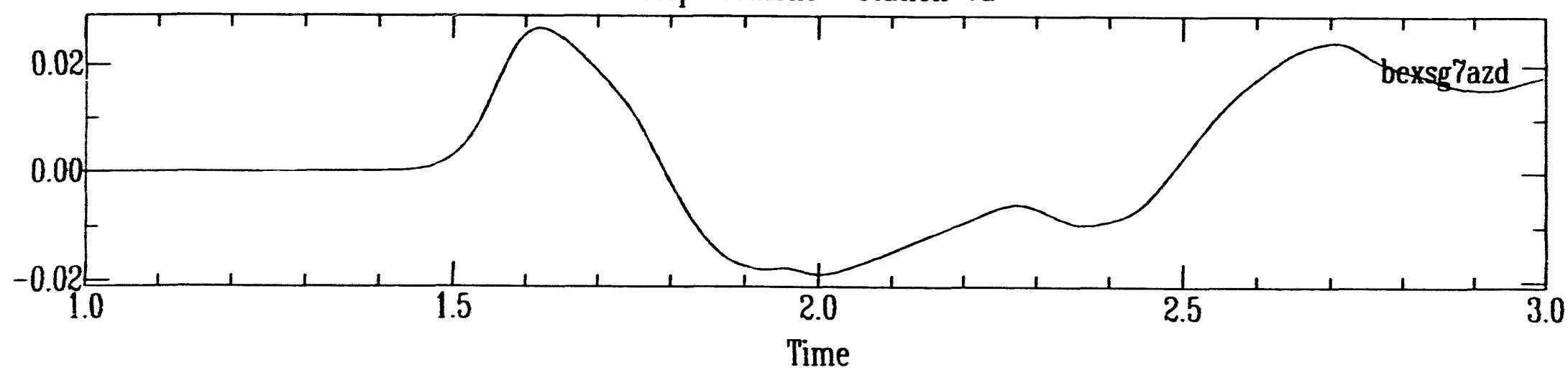




\section{Bexar Surface Ground Motion}

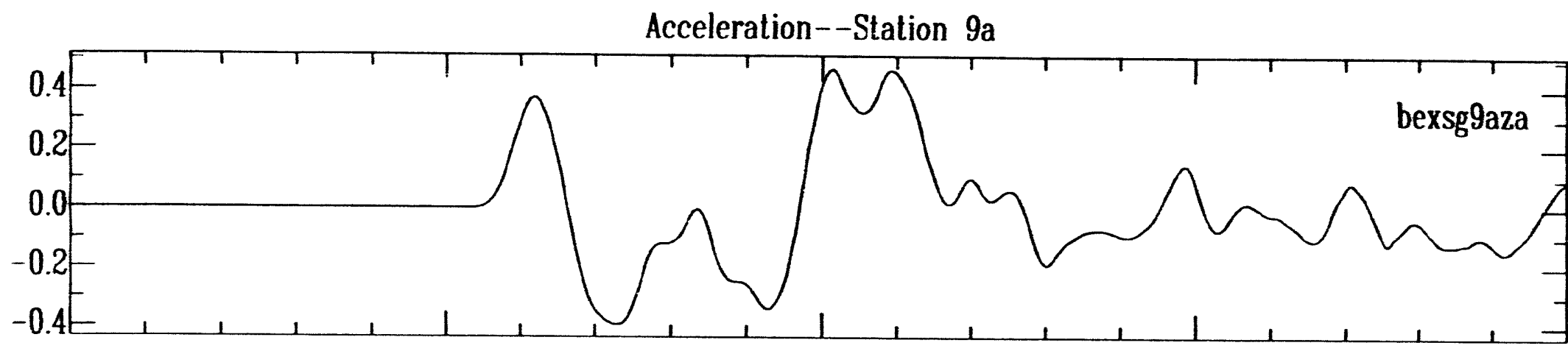

Velocity--Station 9a

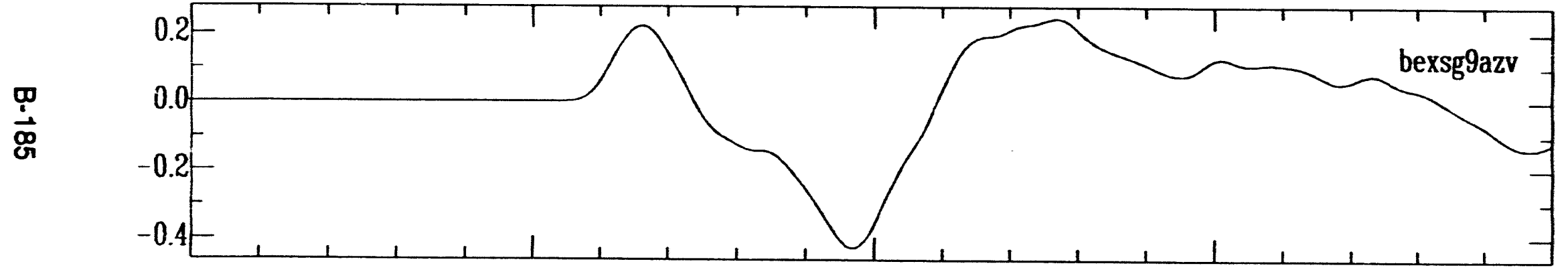

Displacement--Station 9a

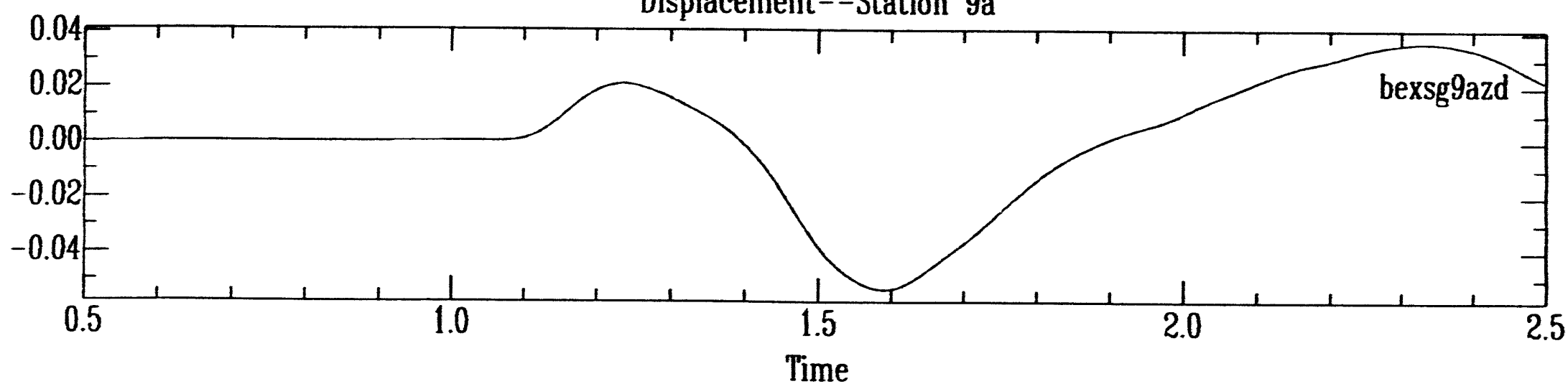




\section{Bexar Surface Ground Motion}

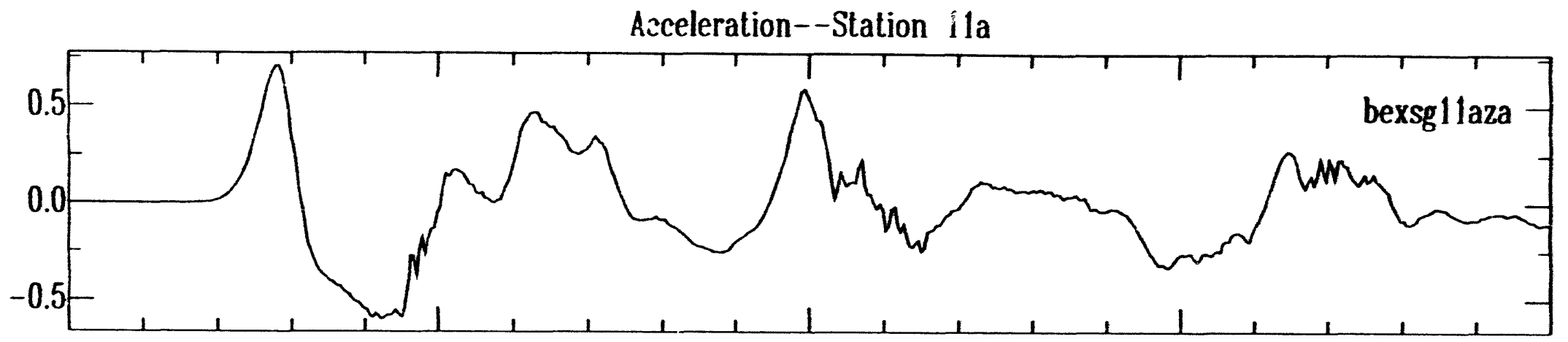

Velocity--Station 11a

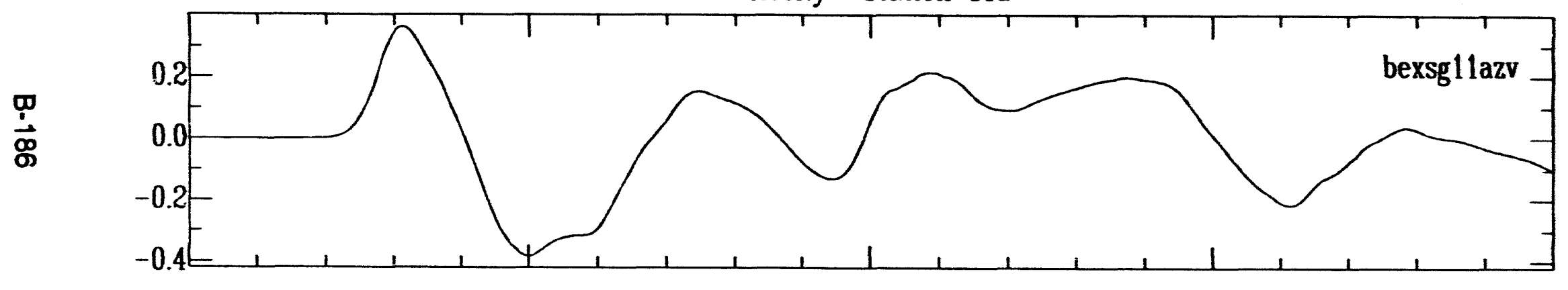

Displacement--Station 11a

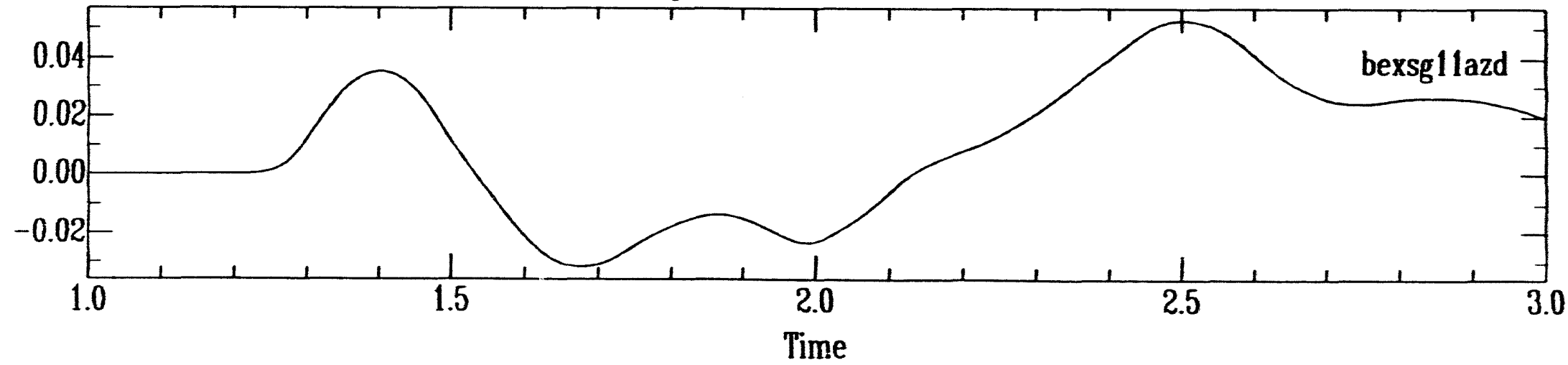




\section{Bexar Surface Ground Motion}

Acceleration--Station 1/b

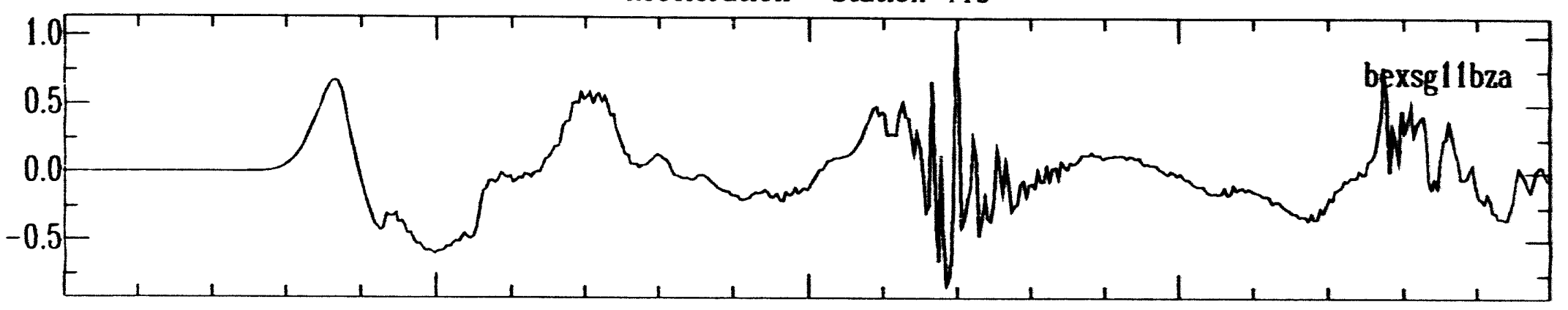

Velocity--Slation $11 \mathrm{~b}$

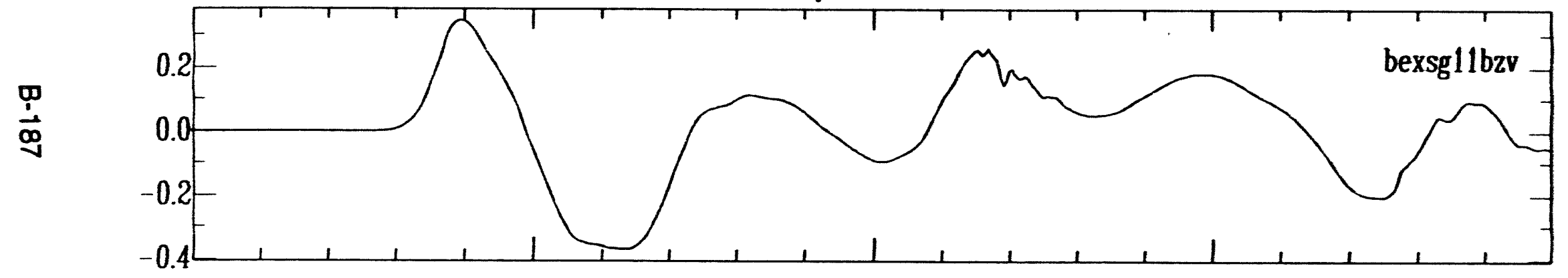

Displacement--Station 11b

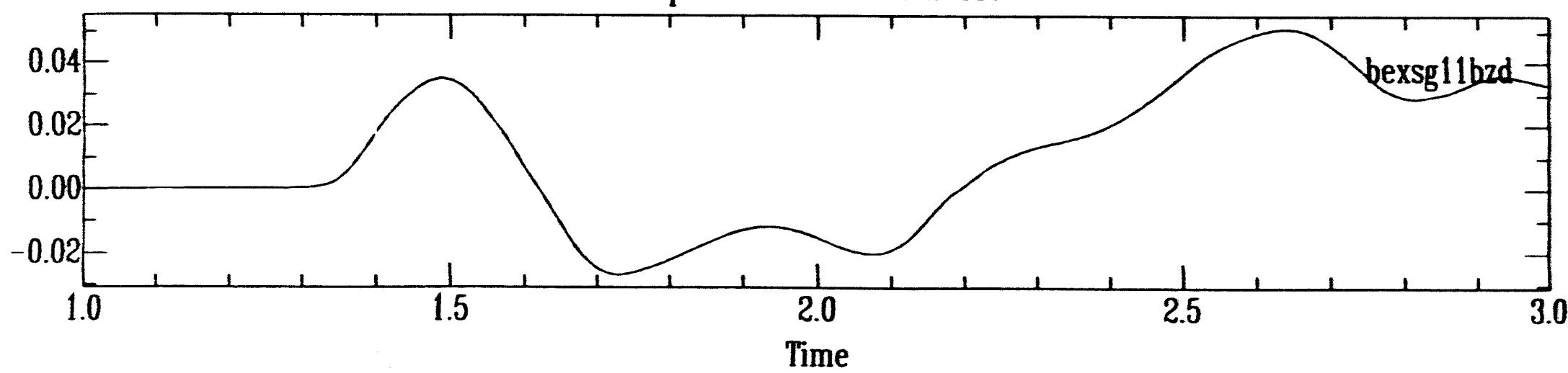




\section{Bexar Surface Ground Motion}

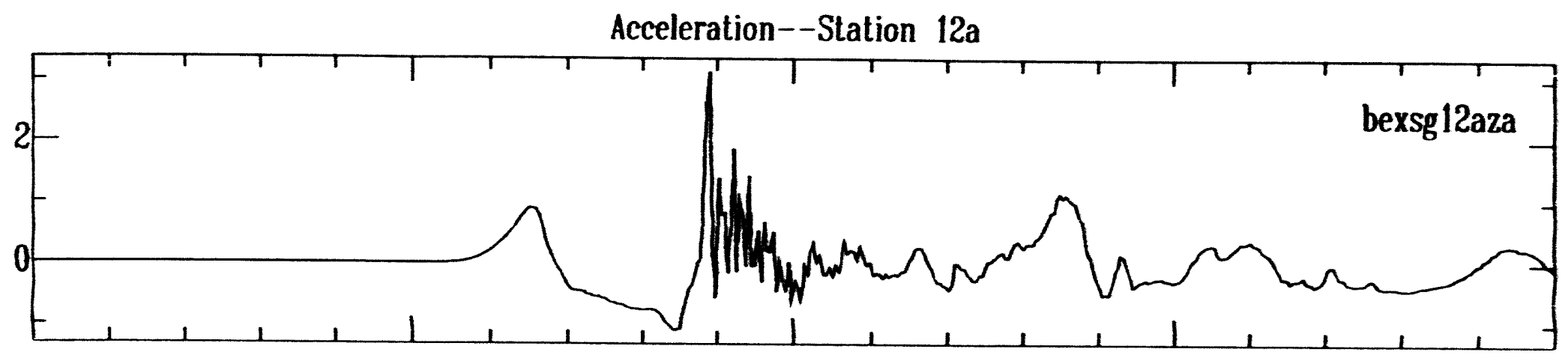

Velocity--Station 12a

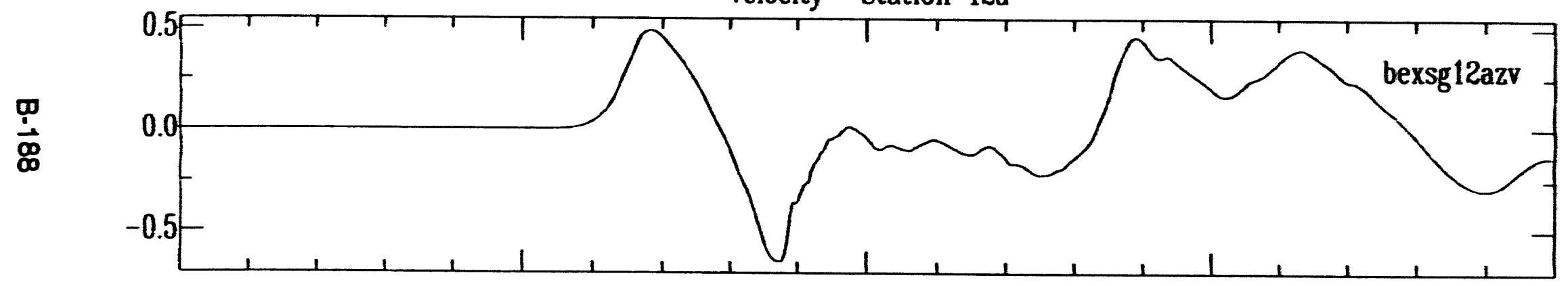

Displacement--Station 12a

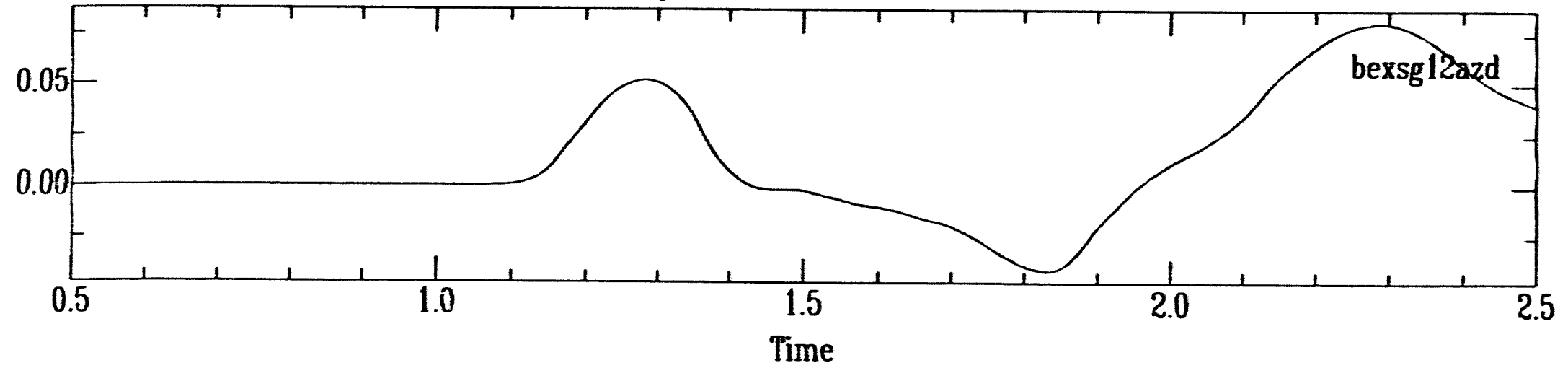




\section{Bexar Surface Ground Motion}

Acceleration--Station 13a
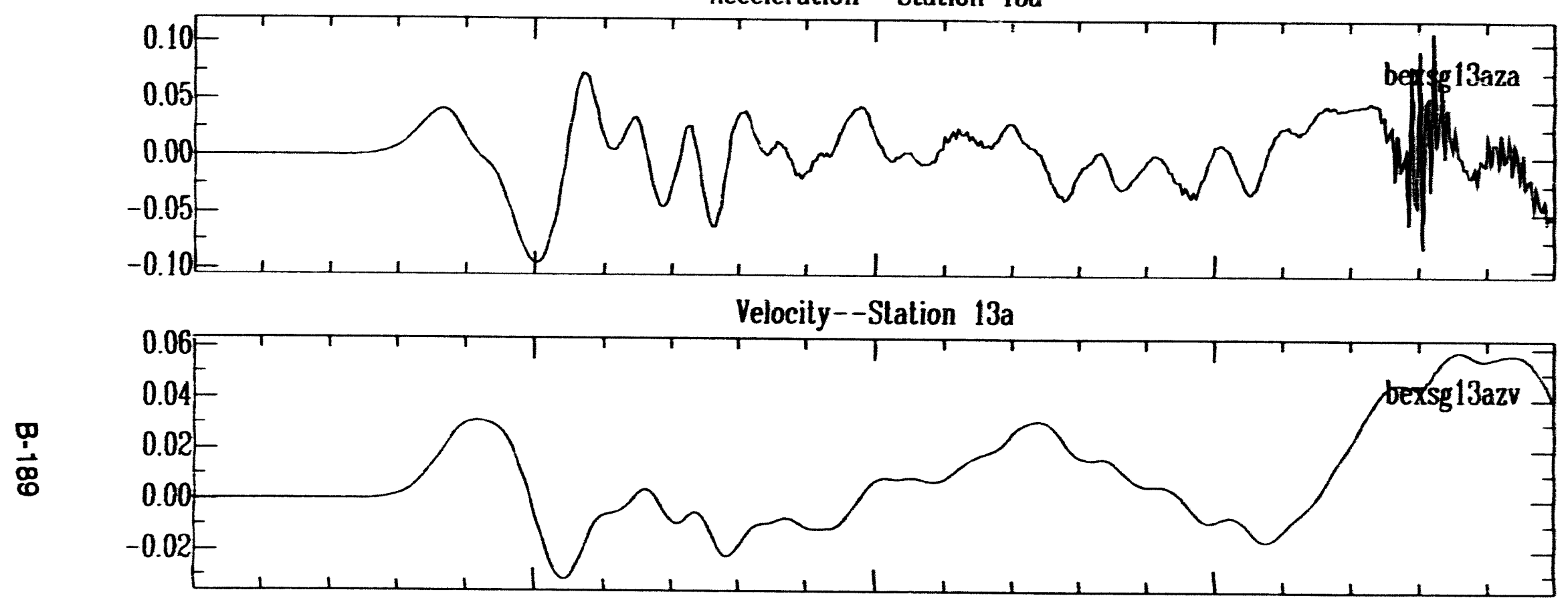

Displacement--Station 13a

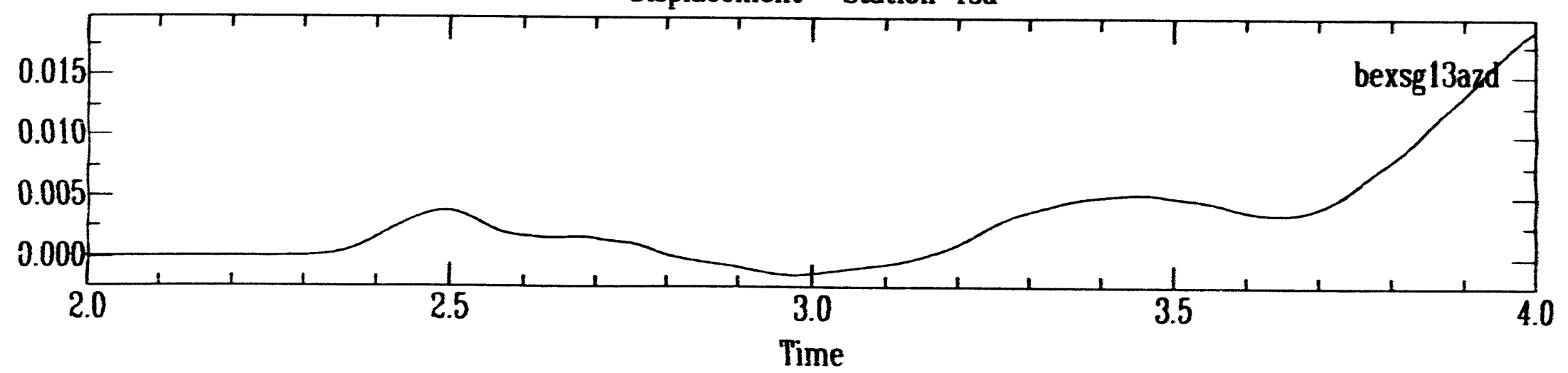




\section{Bexar Surface Ground Motion}

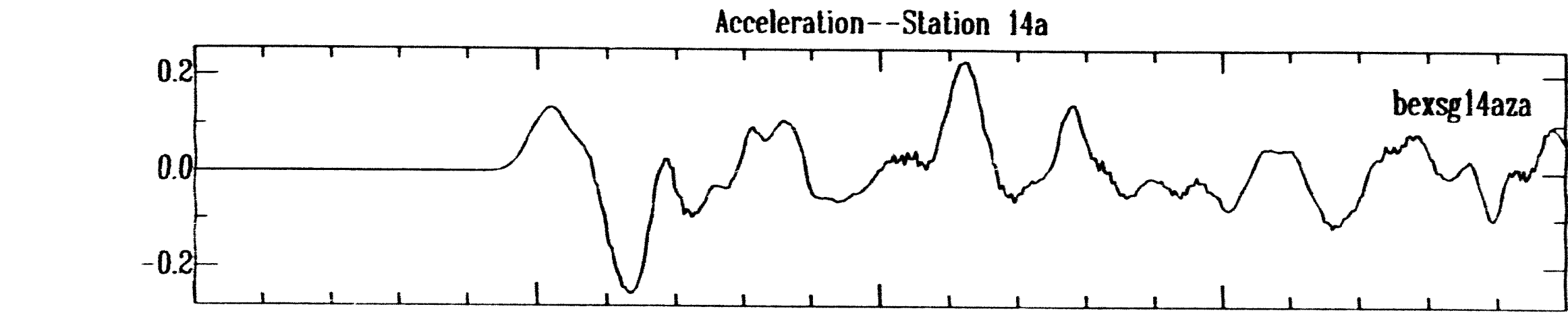

Velocity--Station 14a

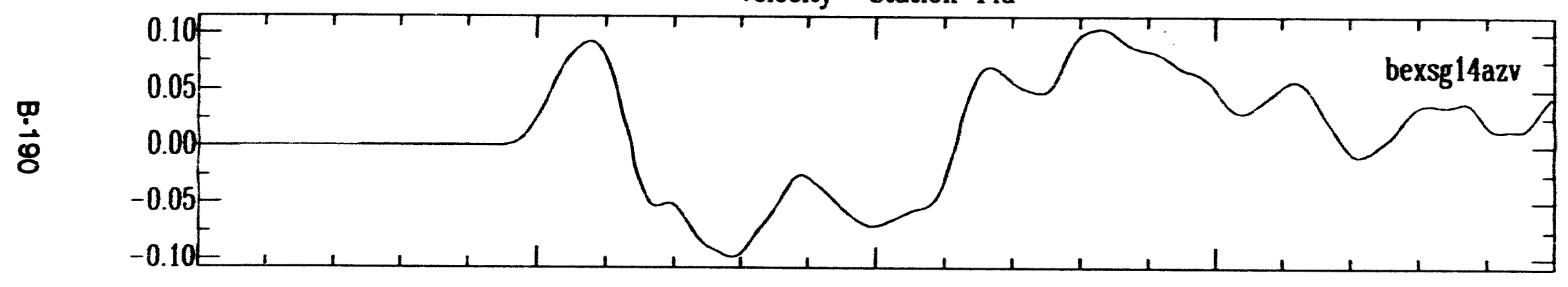

Displacement--Station 14a

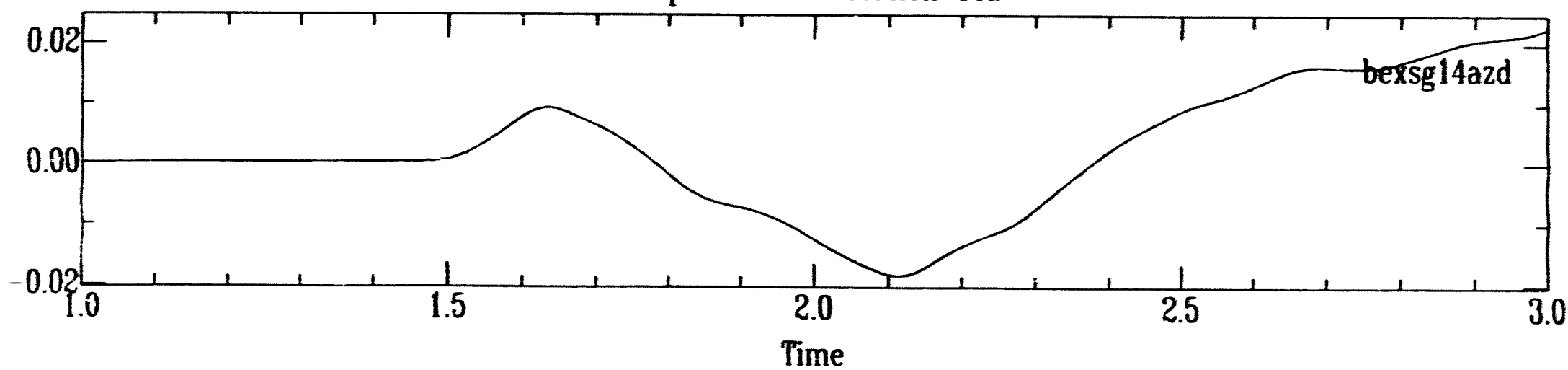




\section{Bexar Surface Ground Motion}

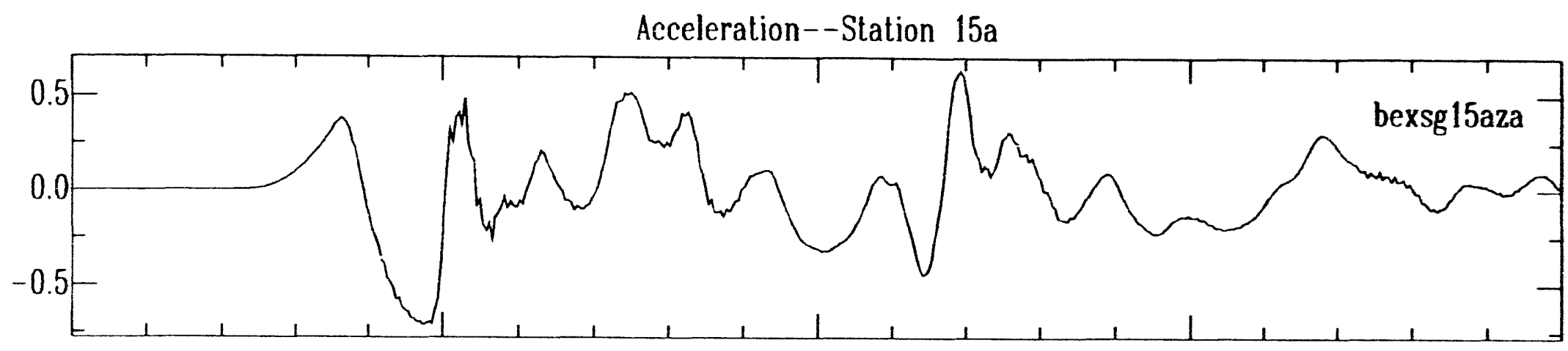

Velocity--Station $15 \mathrm{a}$

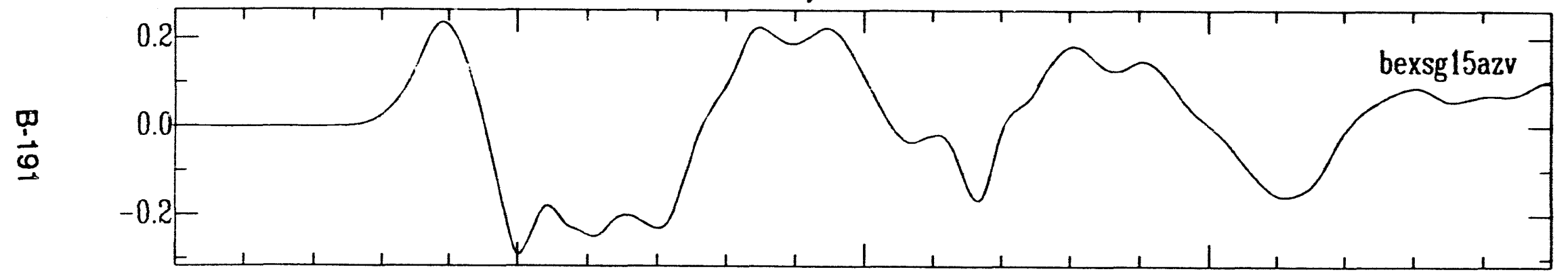

$\frac{3}{\infty}$

Displacement--Station $15 \mathrm{a}$

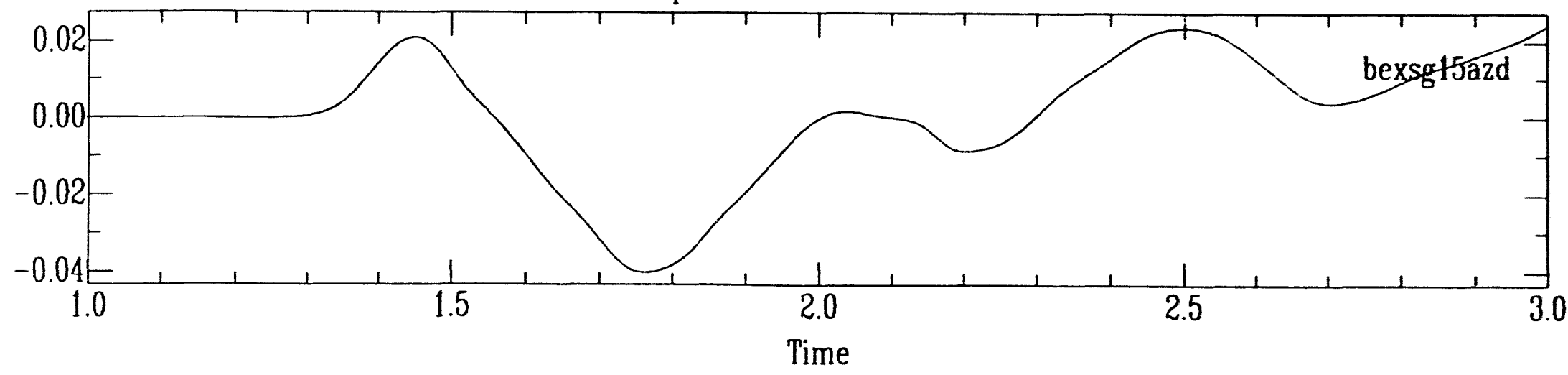




\section{Bexar Surface Ground Motion}

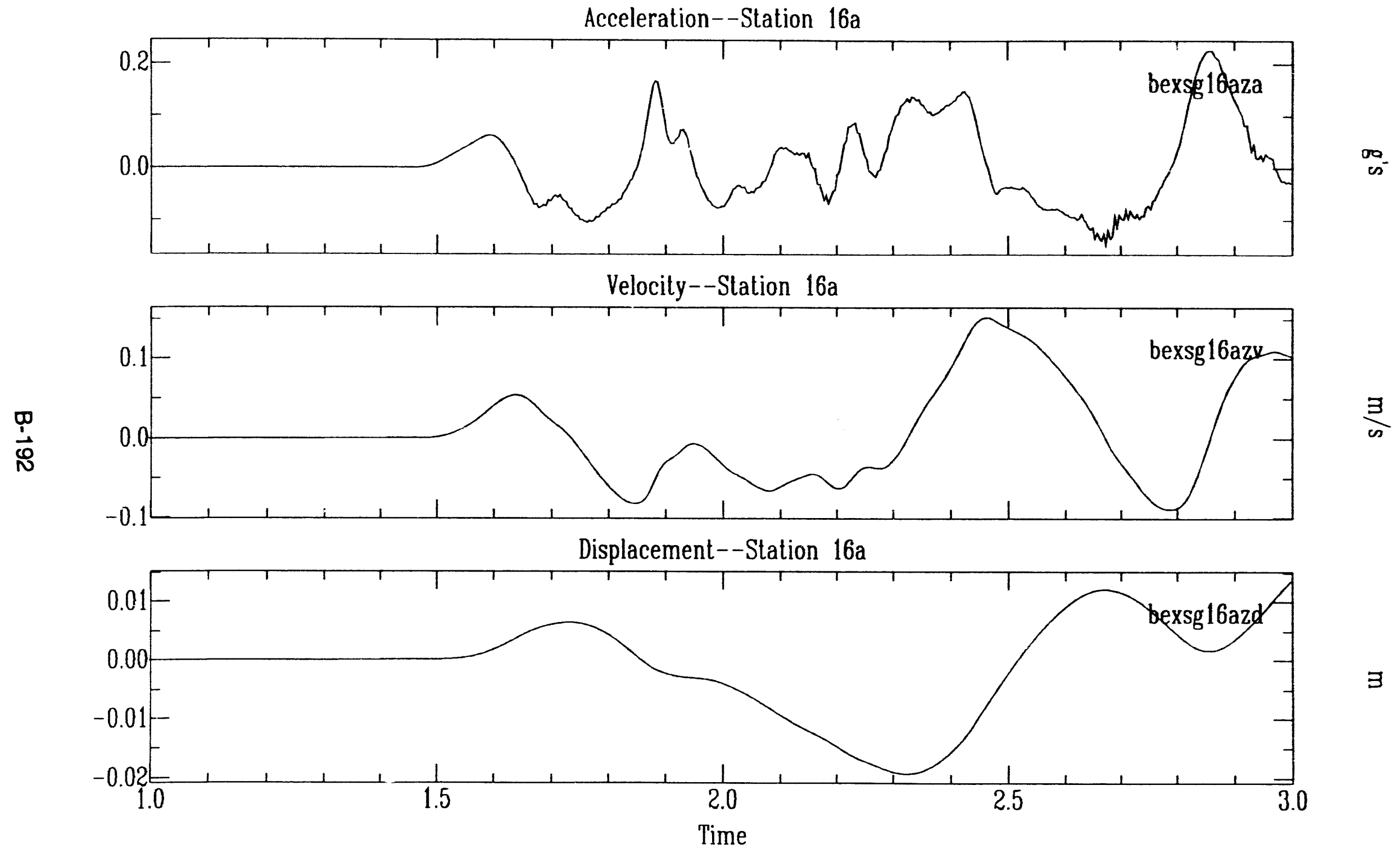




\section{Bexar Surface Ground Motion}

Acceleration--Station 17a

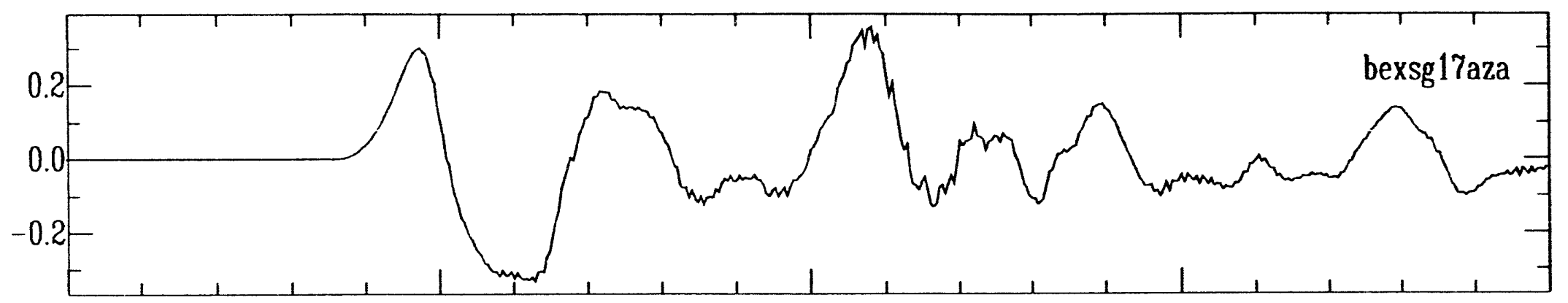

is

Velocity--Station $17 a$

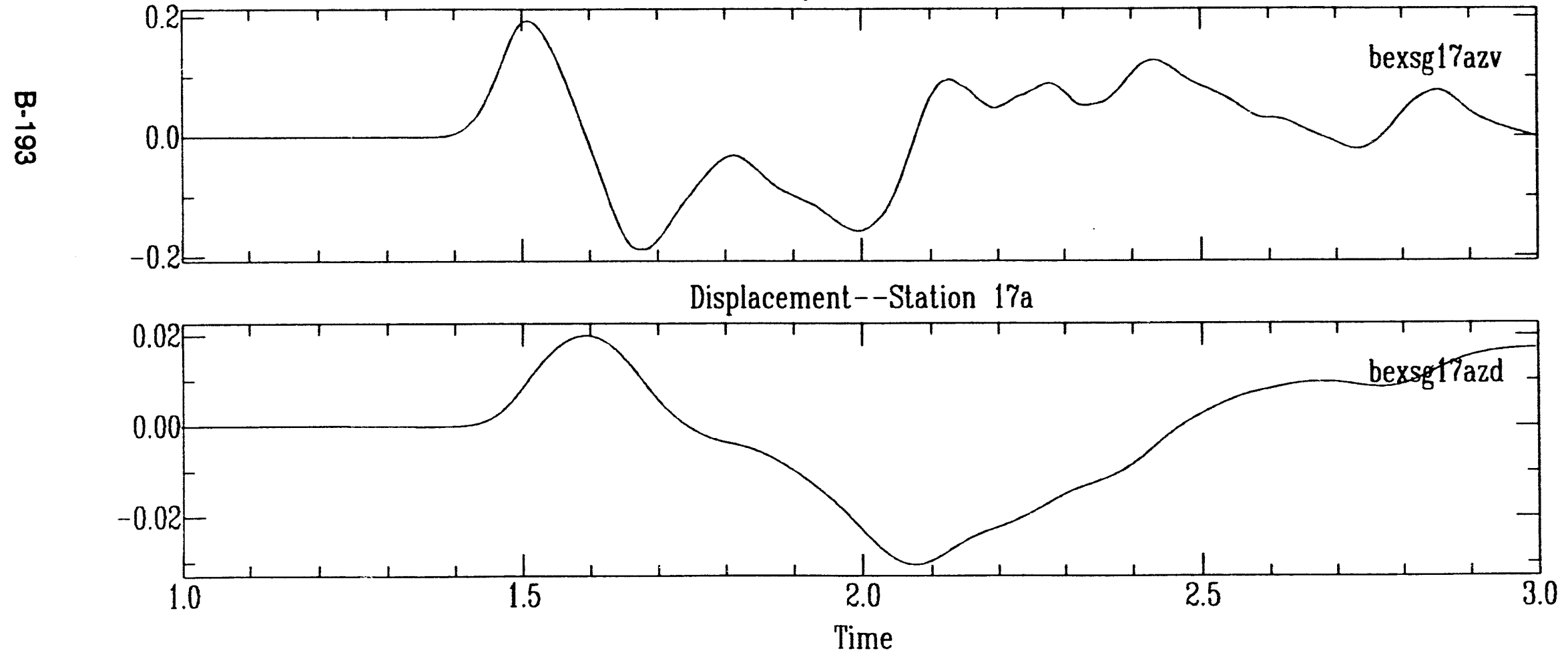




\section{Bexar Surface Ground Motion}

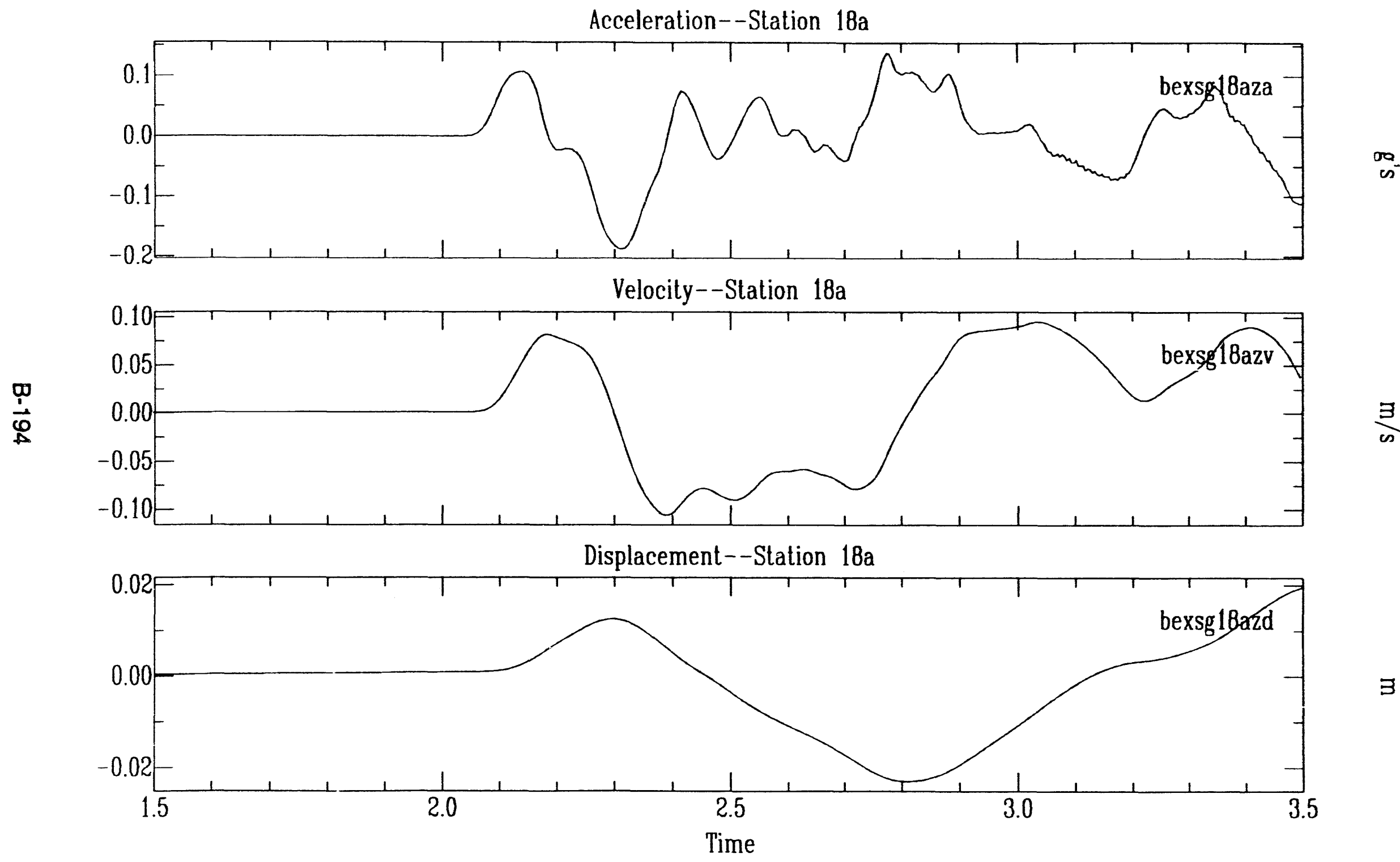




\section{Bexar Surface Ground Motion}

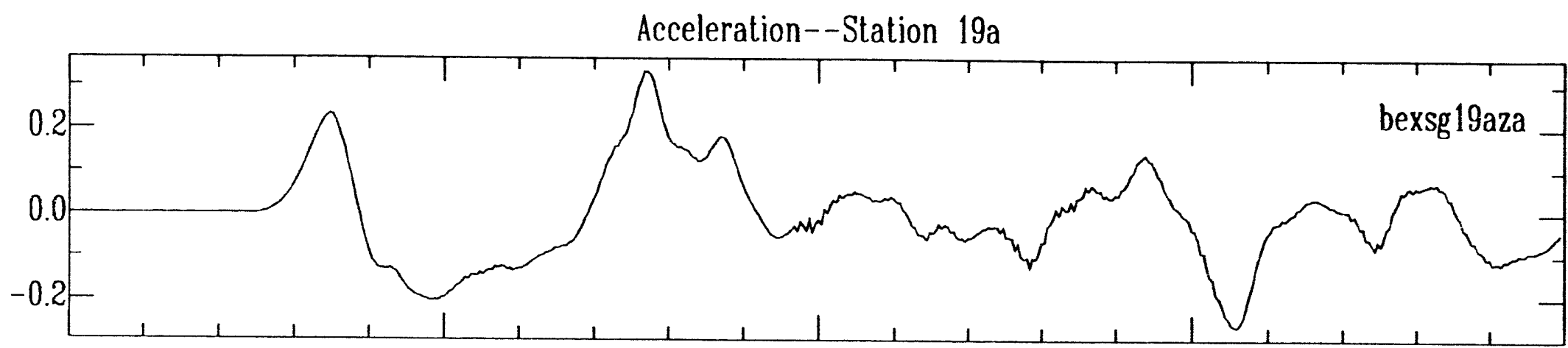

Velocily--Station 19a

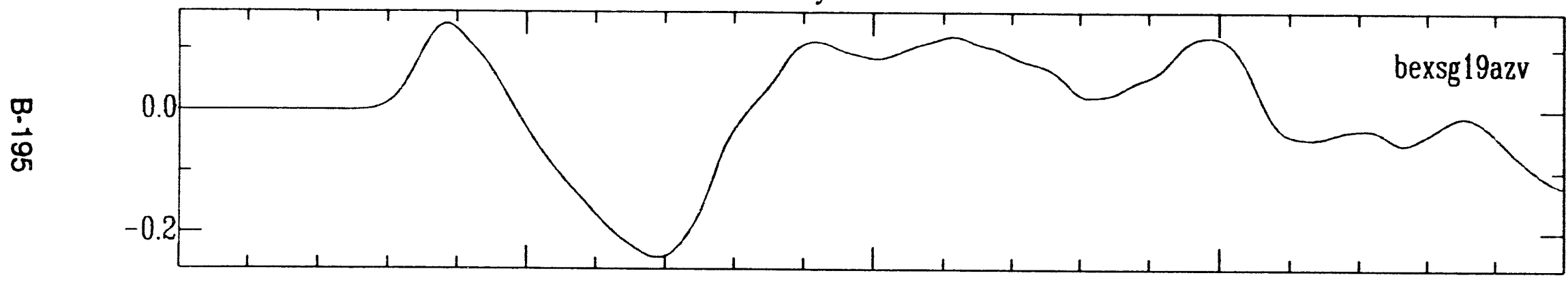

$\frac{3}{\infty}$

Displacement--Station $19 a$

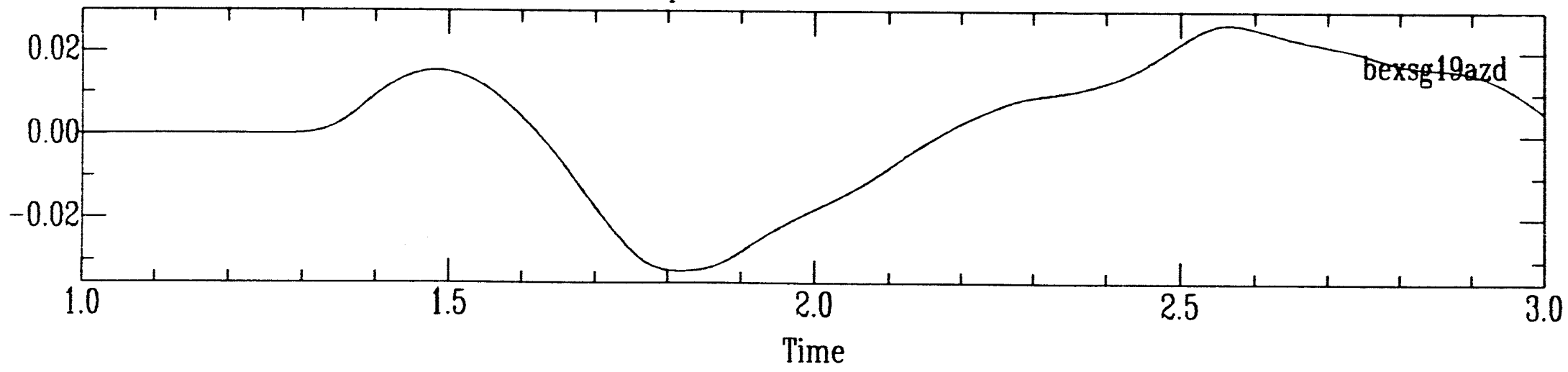




\section{Bexar Surface Ground Motion}

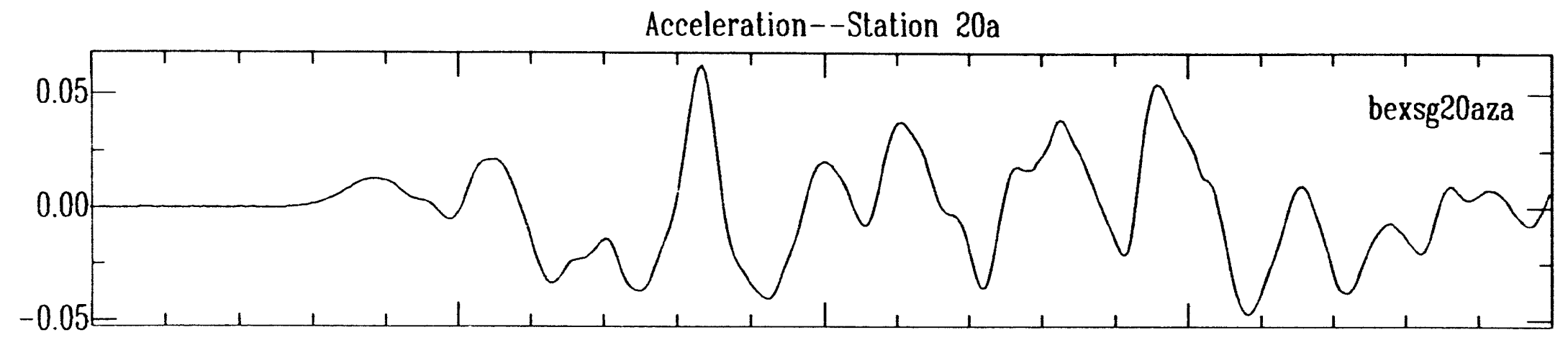

Velocity--Station 20a

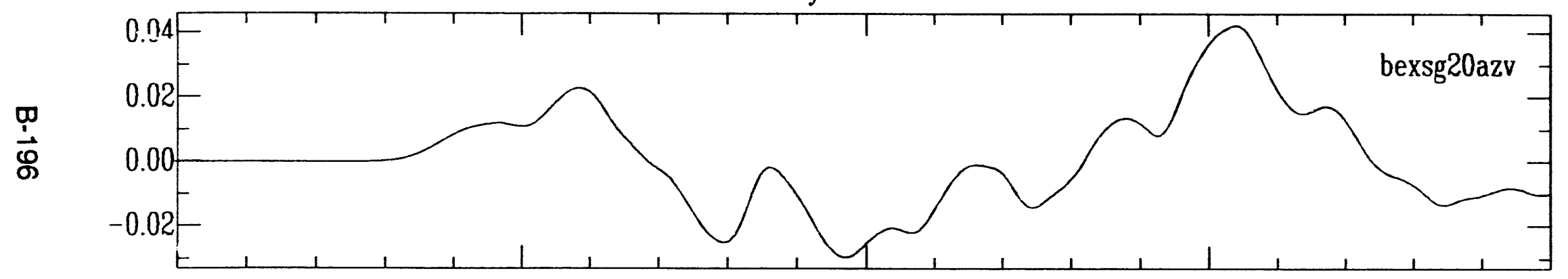

$\frac{3}{\infty}$

Displacement--Station 20a

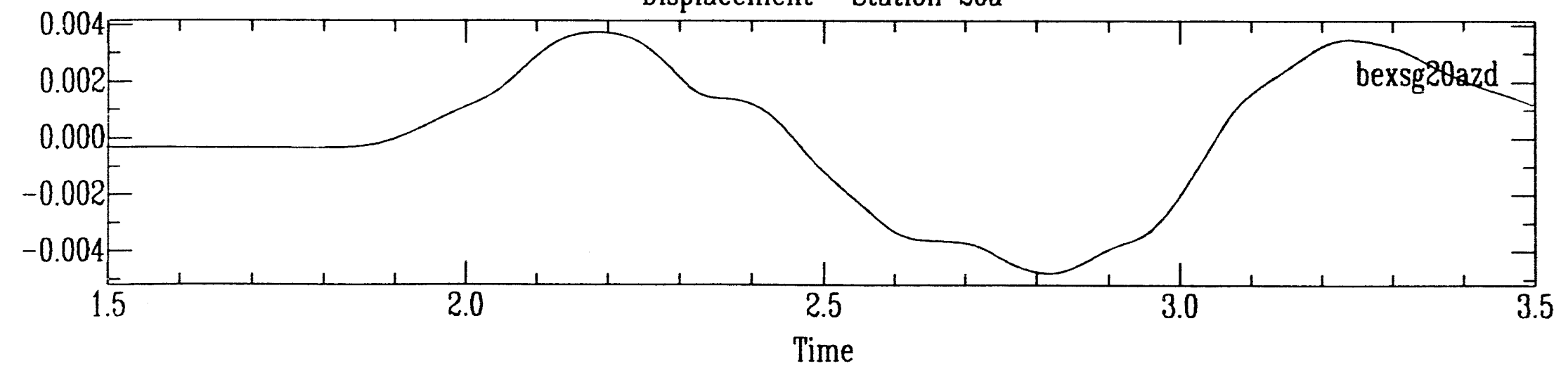




\section{Bexar Surface Ground Motion}

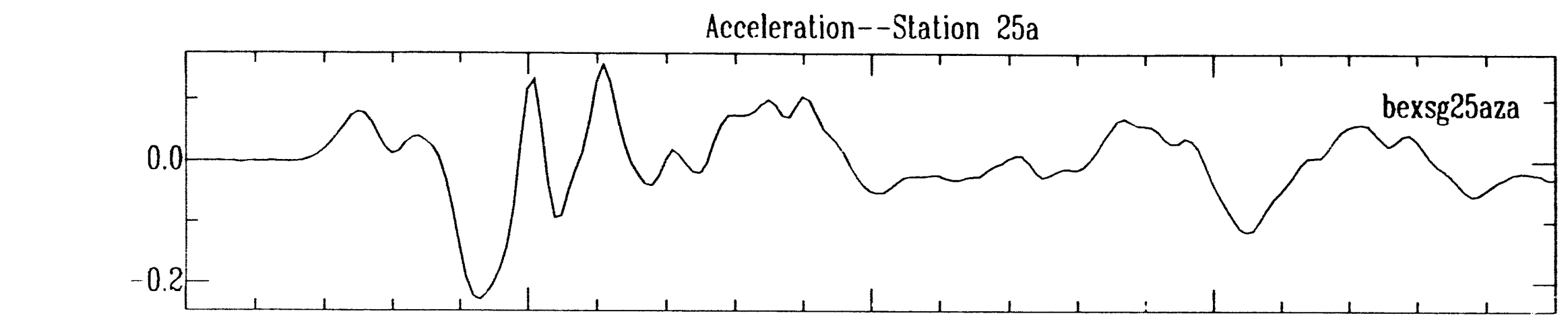

Velocity--Station 25a

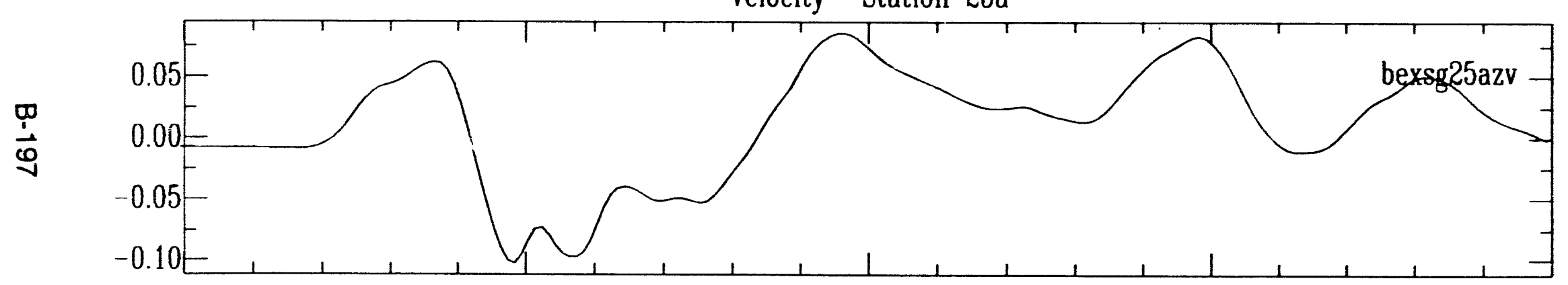

$\frac{3}{\infty}$

Displacement--Station 25a

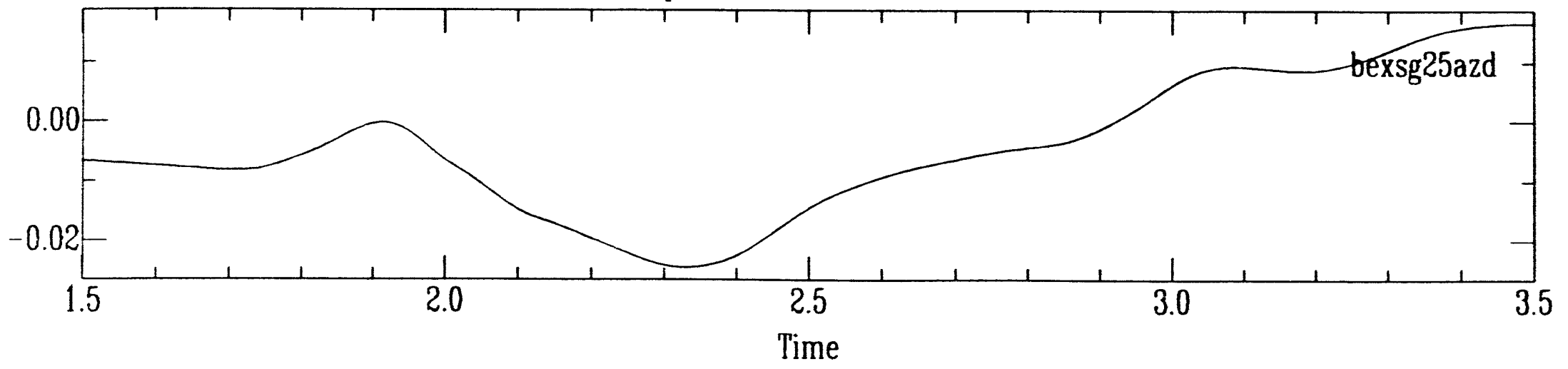




\section{Bexar Surface Ground Motion}

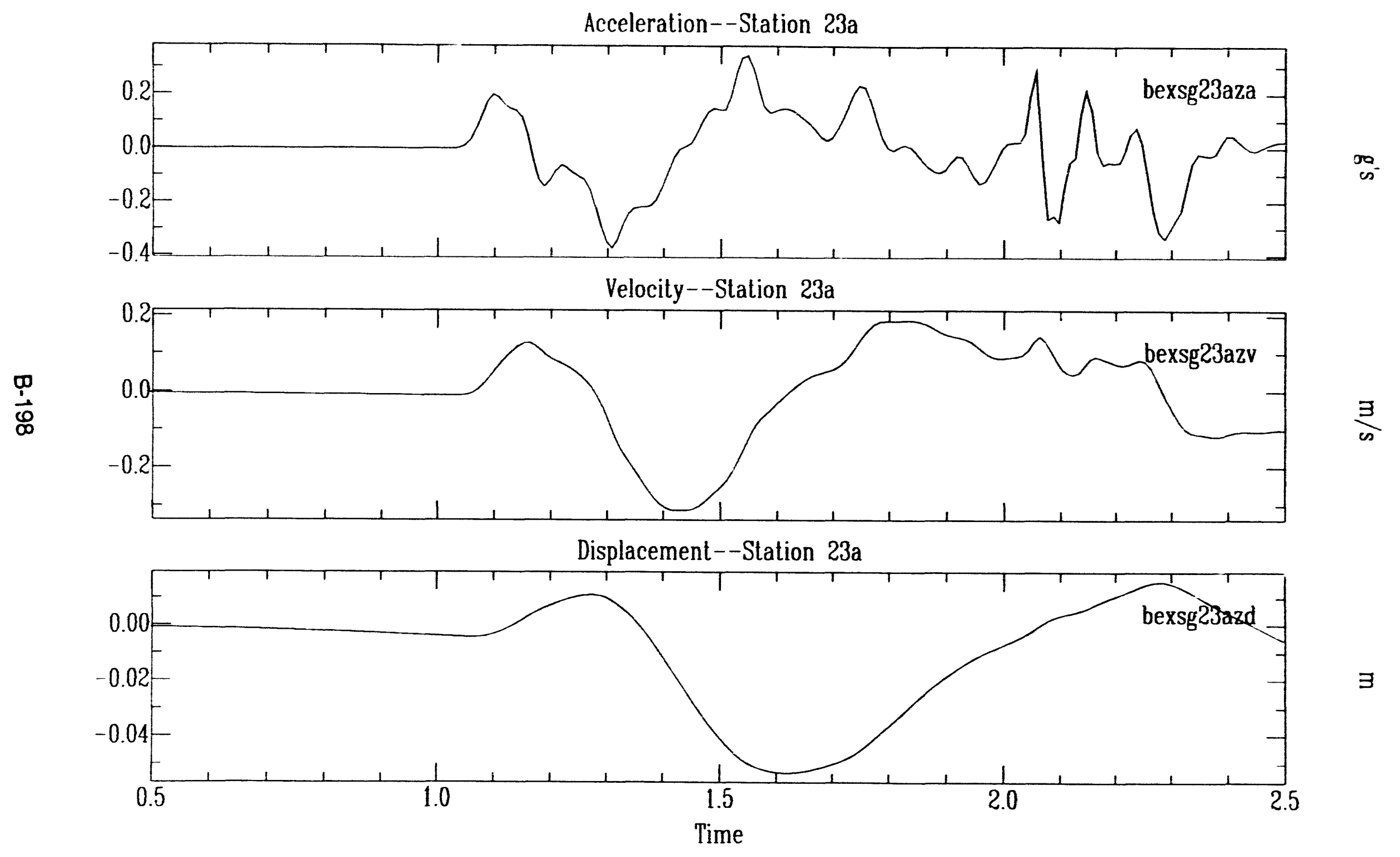




\section{Bexar Surface Ground Motion}

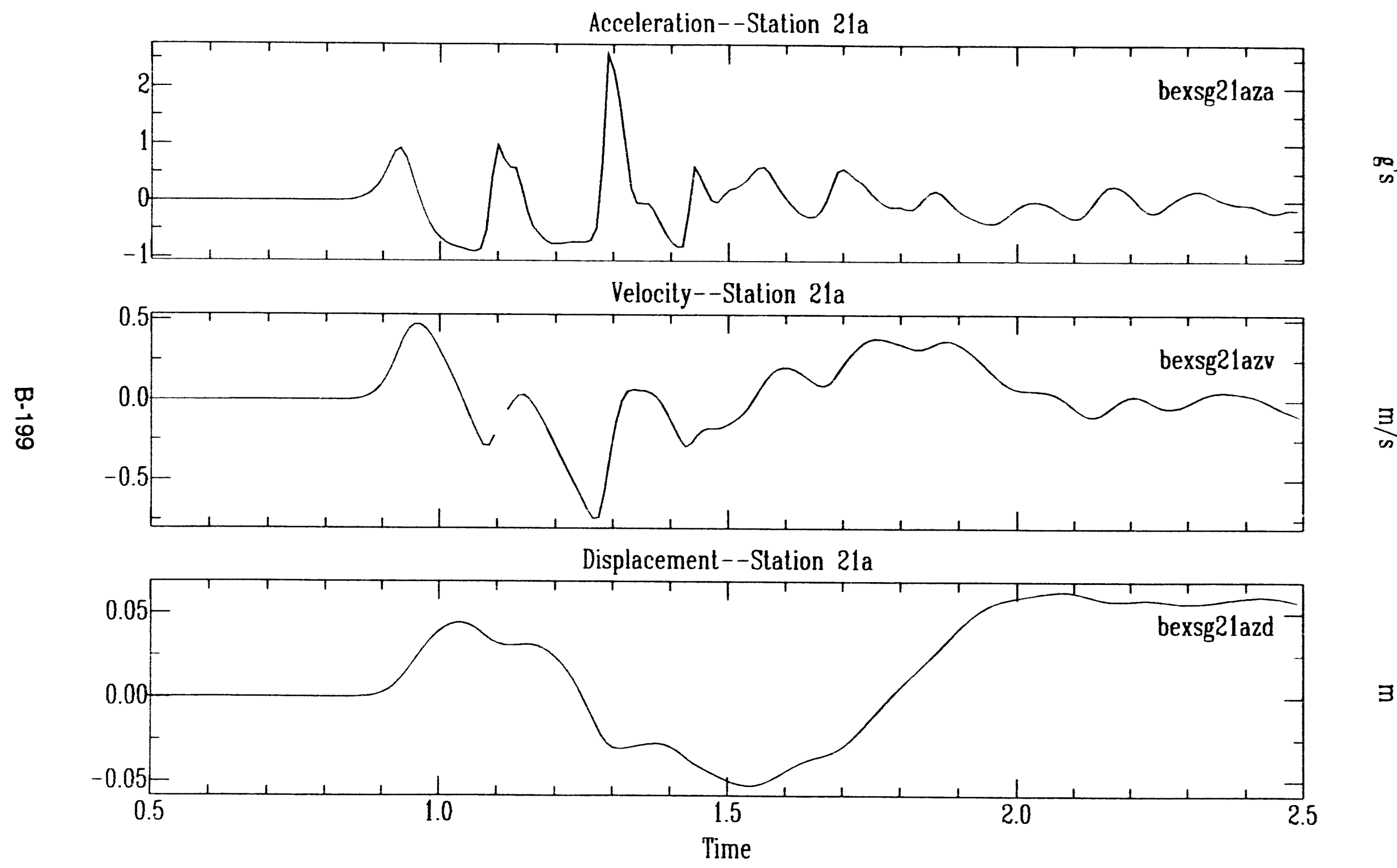




\section{Bexar Surface Ground Motion}

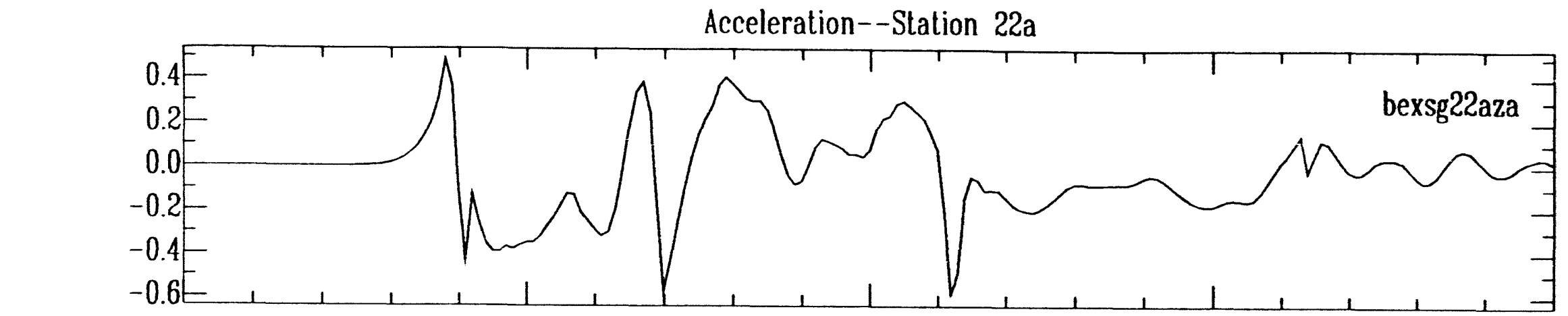

Velocity--Station 22a

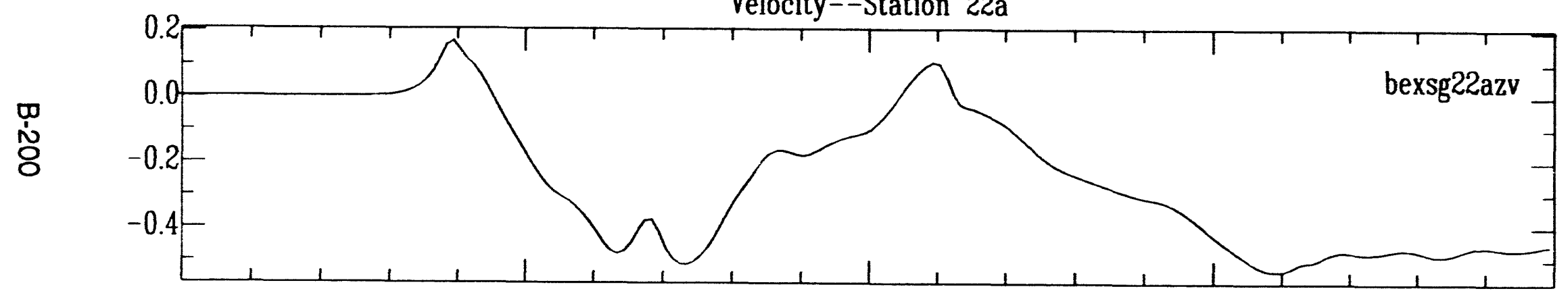

$\frac{3}{6}$

Displacement--Station 22a

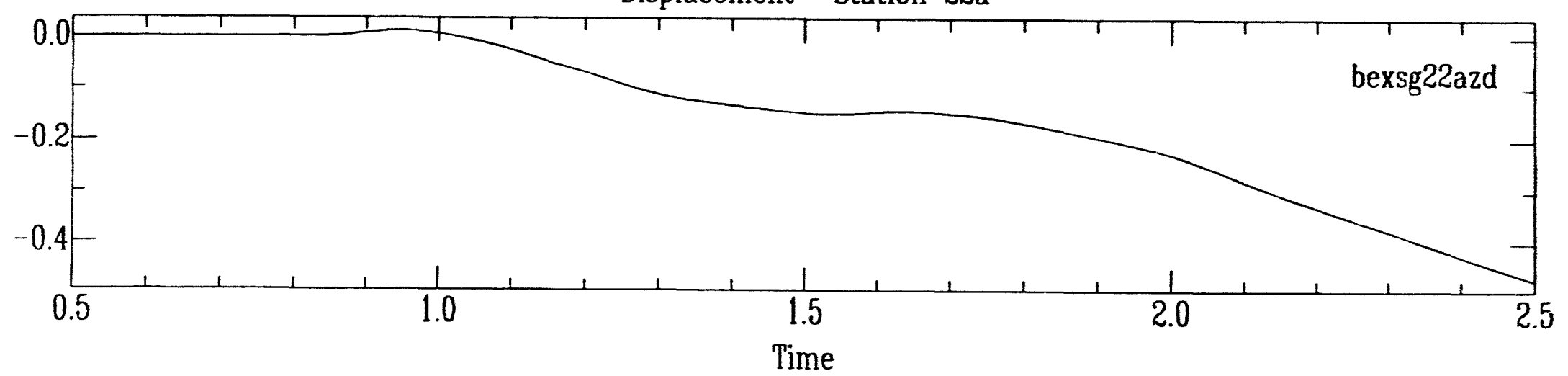




\section{Bexar Surface Ground Motion}

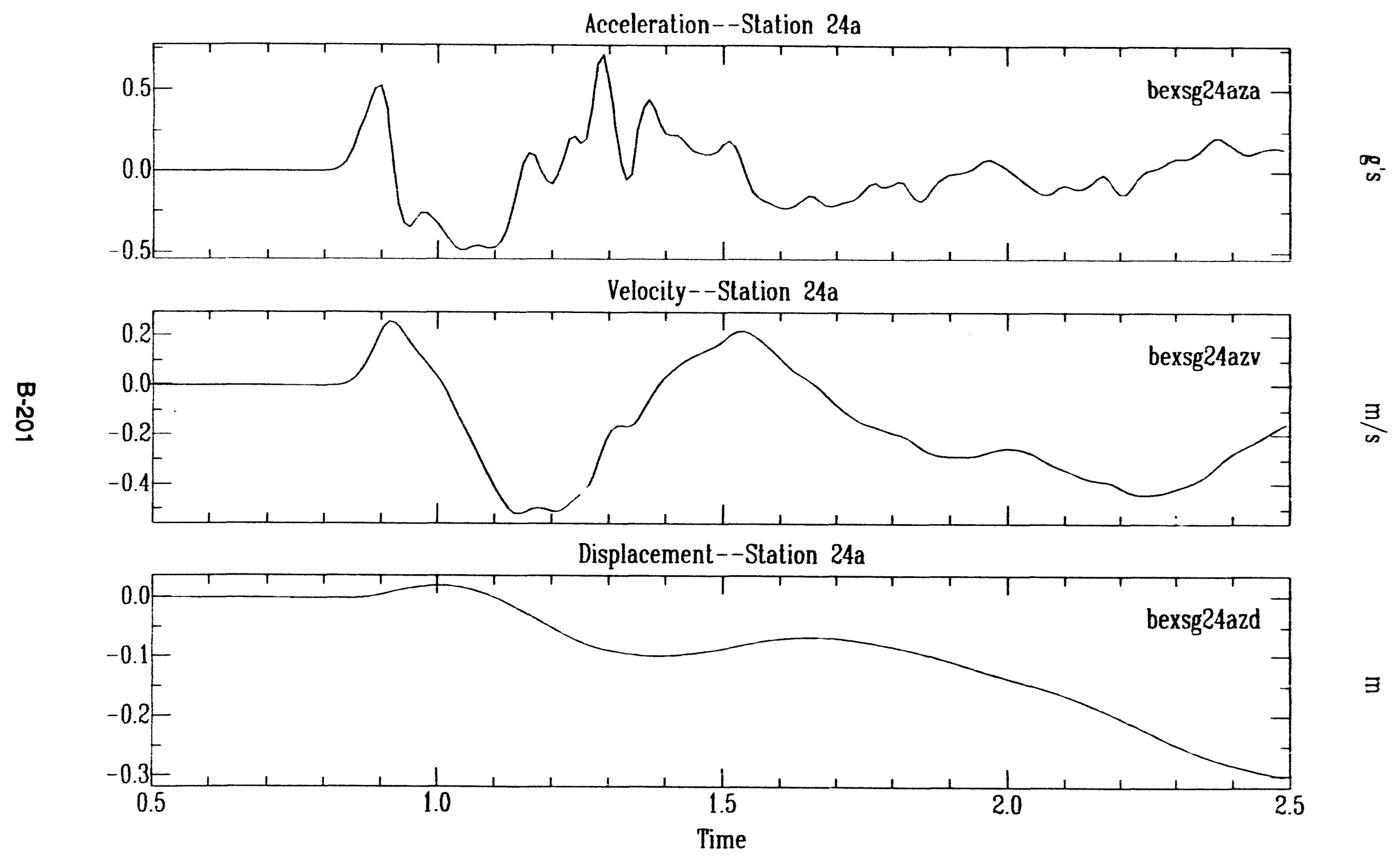




\section{Bexar Surface Ground Motion}

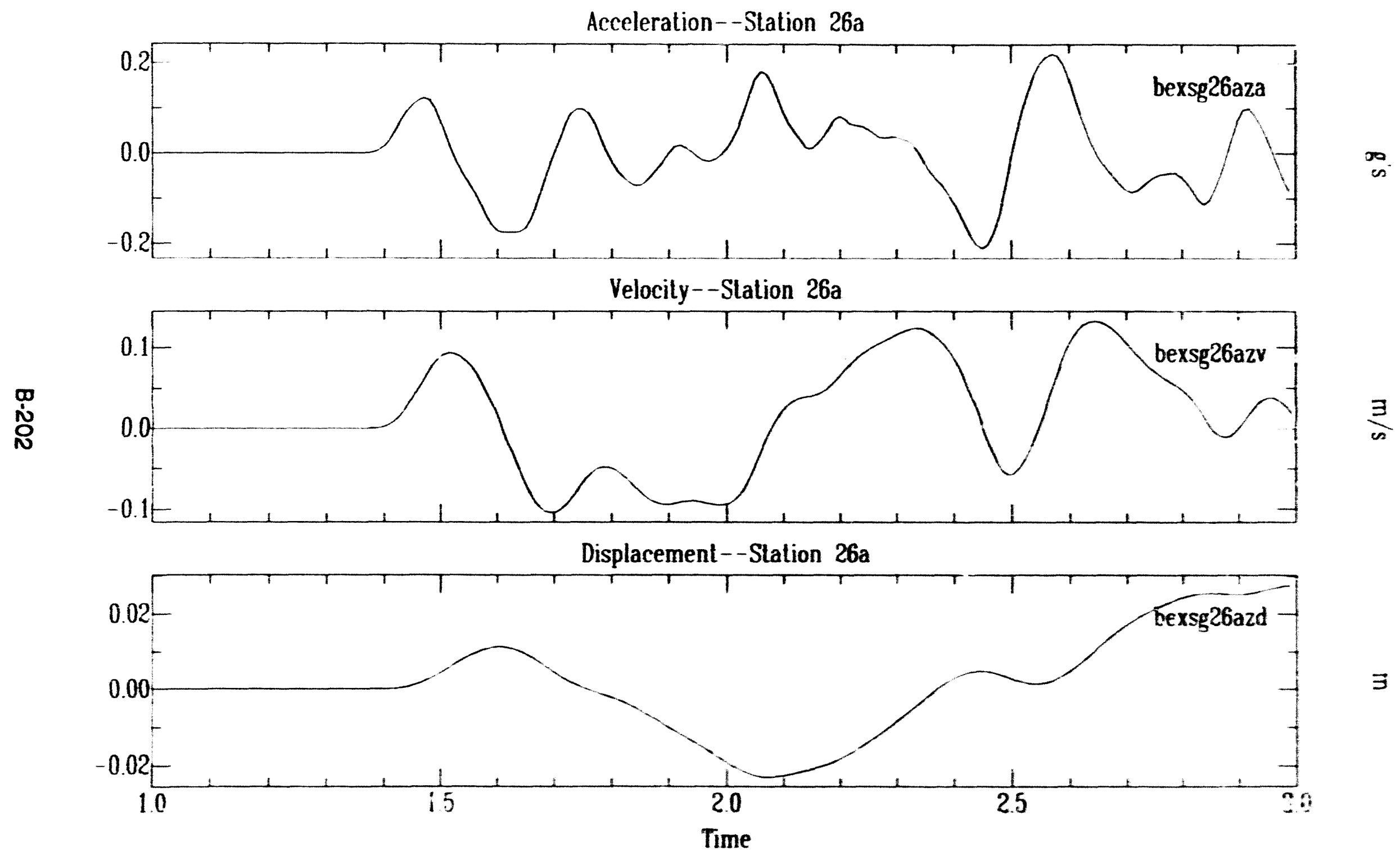




\section{Bexar Surface Ground Motion}

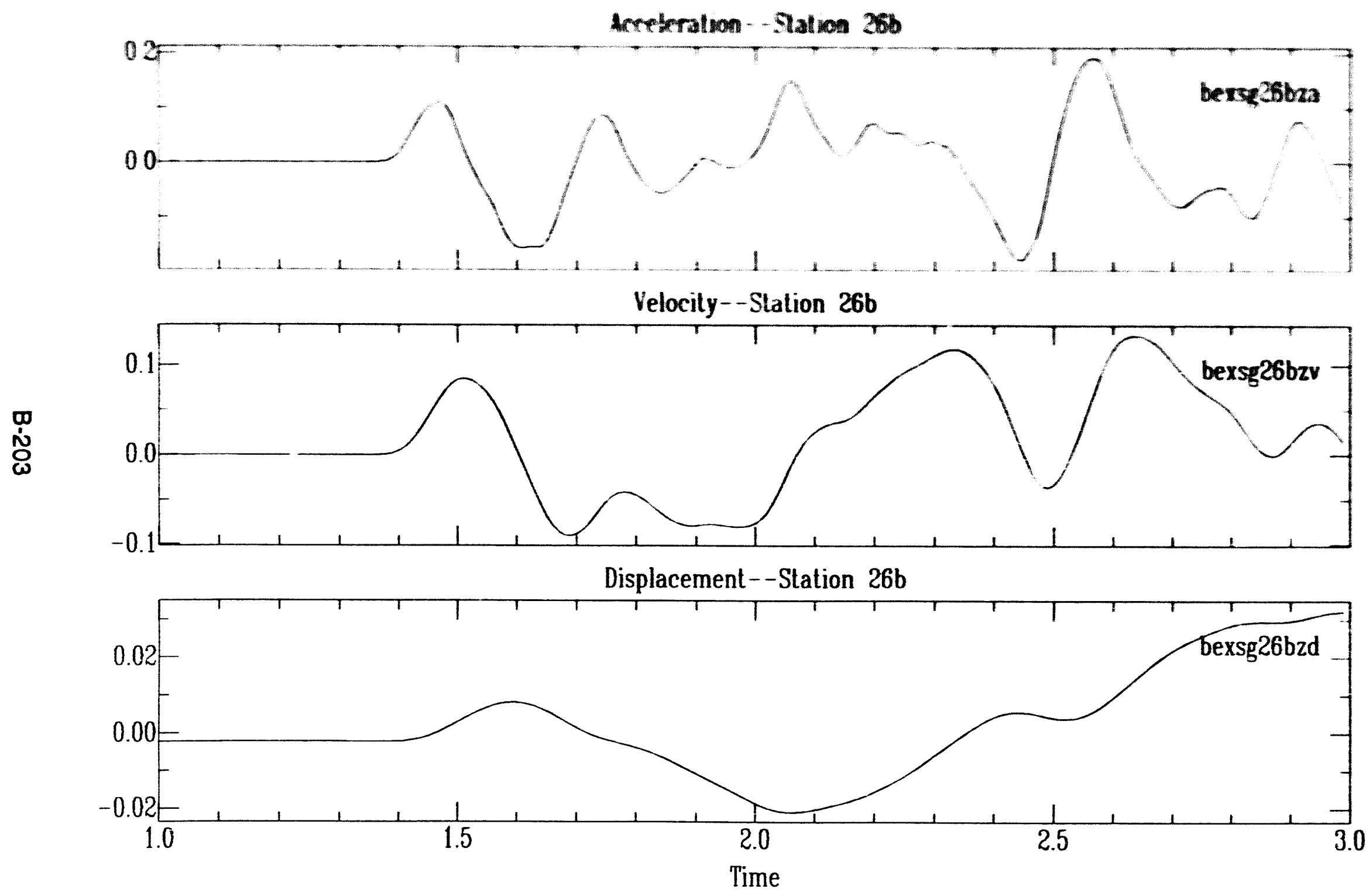




\section{Bexar Surface Ground Motion}
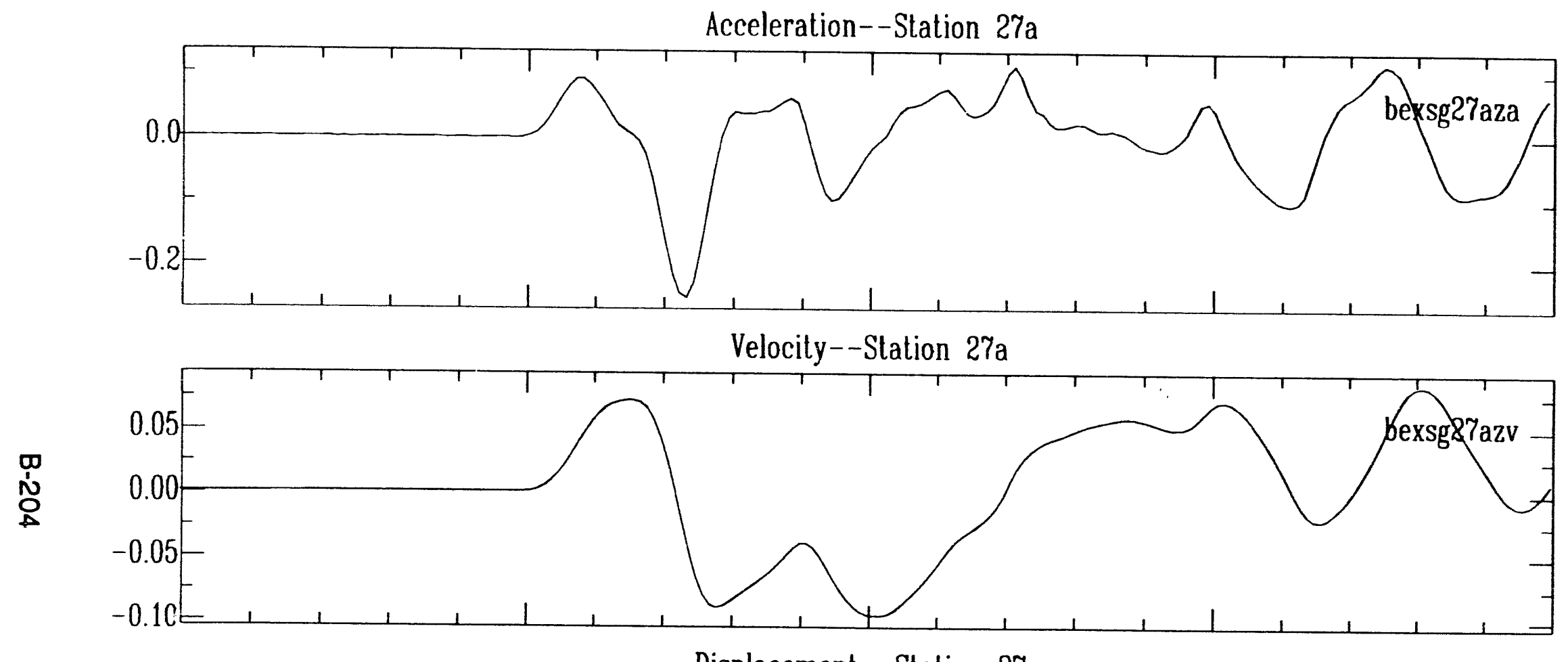

$\frac{3}{\infty}$

Displacement--Station 27a

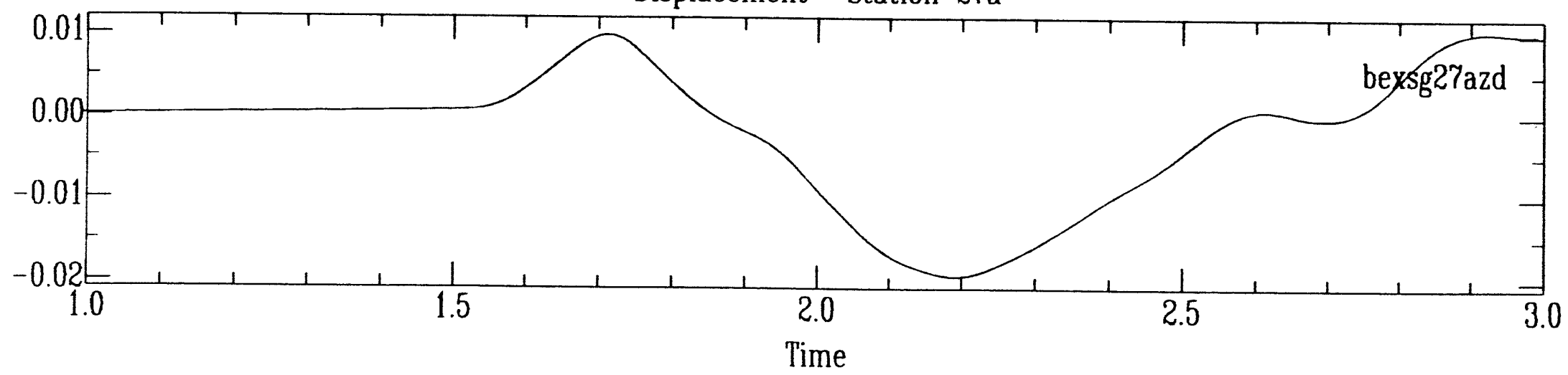




\section{Bexar Surface Ground Motion}

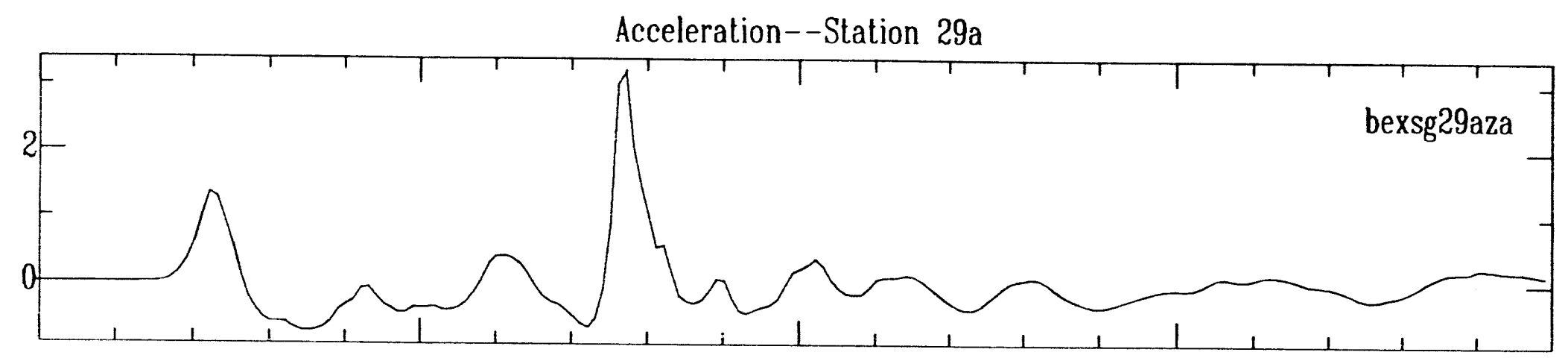

Velocity--Station 29a

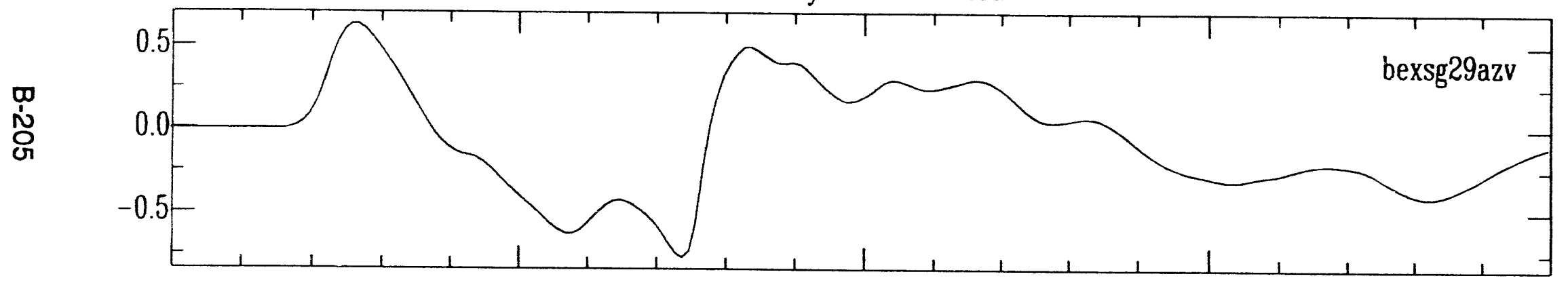

Displacement--Station 29a

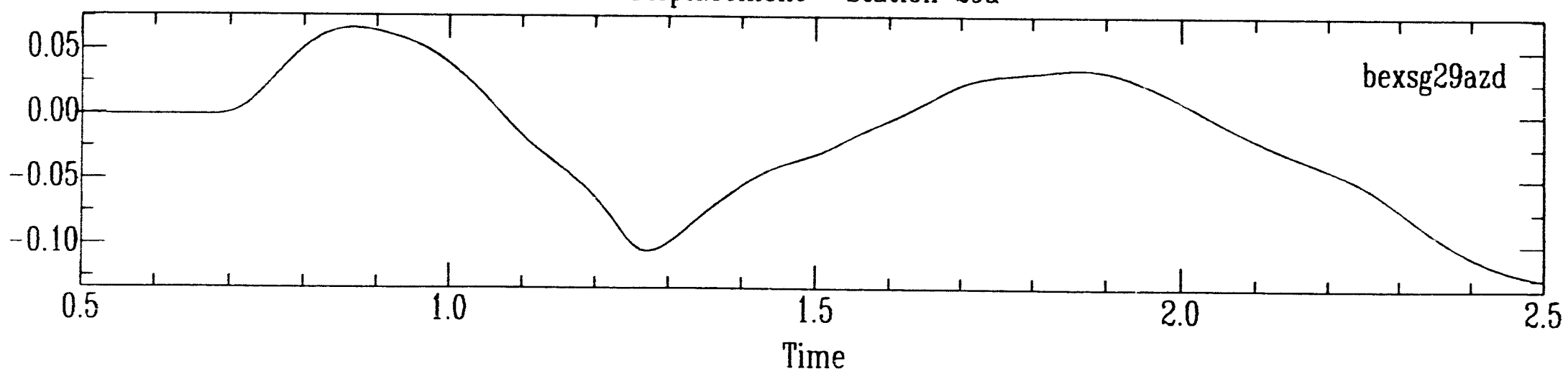




\section{Bexar Surface Ground Motion}

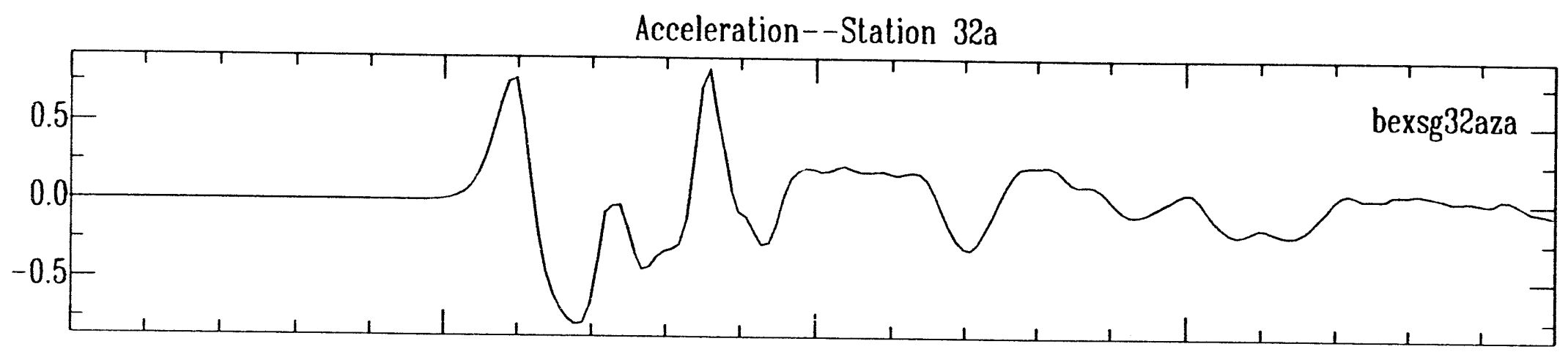

Velocity--Station 32a

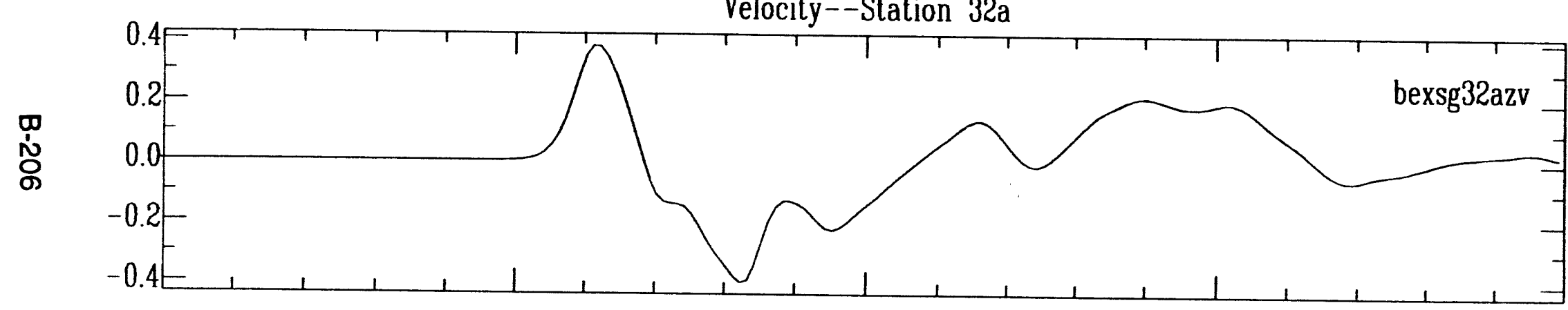

Displacement--Station 32a

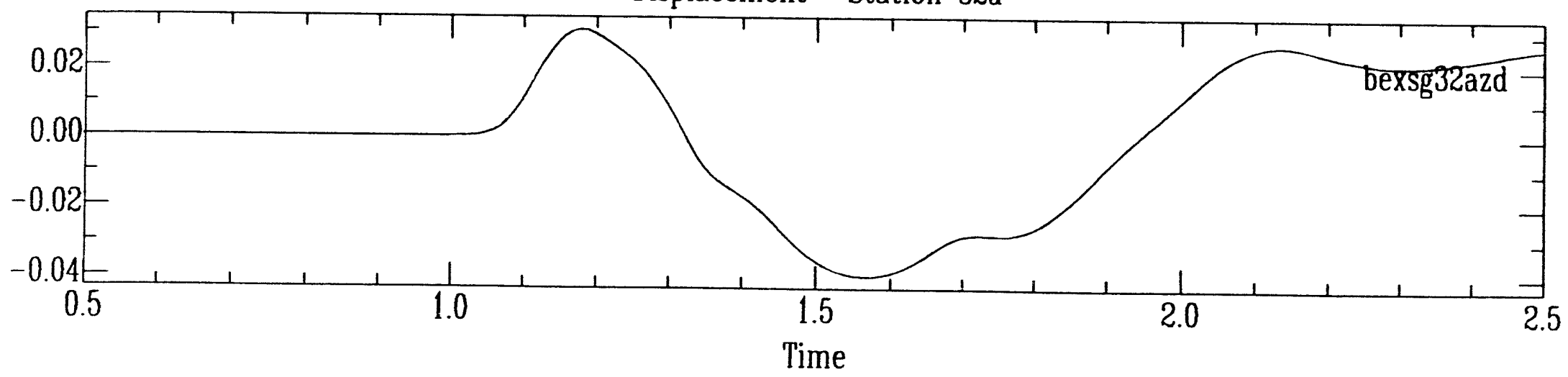




\section{Bexar Surface Ground Motion}

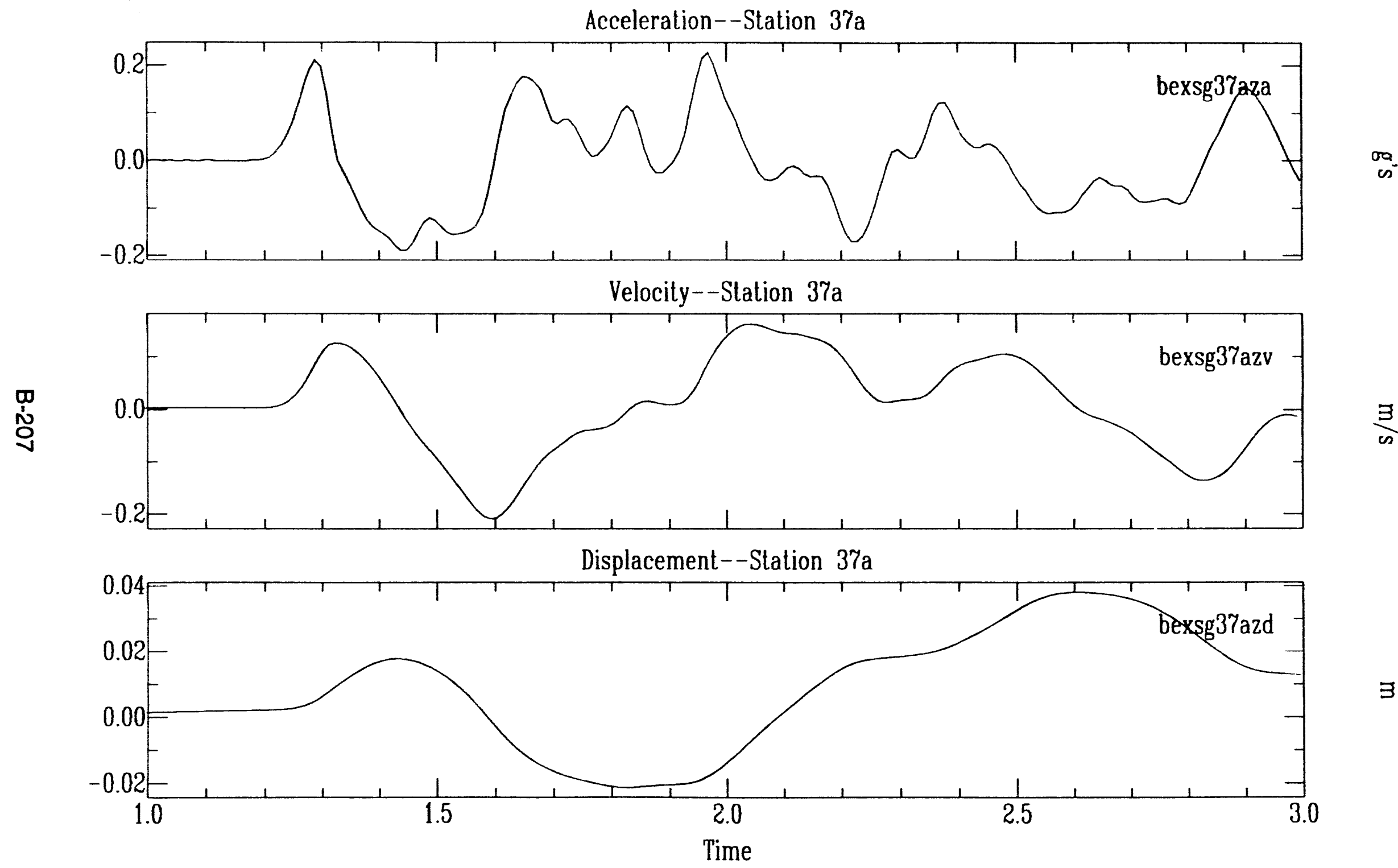




\subsection{Junction (U19bg)}

Experiment design. For the Los Alamos event Junction, a SIG ground-motion experiment (Table 14 and Fig. 20) and a standard J-8 accelerometer array of 12 stations were deployed (Table 15 and Fig. 19). Junction was fired 26 March 1992. Hole U19bg is located in the central part of Area 19, in close proximity to U19ad (Chancellor), U19ar (Cybar), and 19ay (Amarillo). Figure 19 shows the location of U19bg with the predicted spall radius indicated. The seven SIG arrays planned are also indicated.

Results. Weather conditions were a major factor in the SIG experiment. Rain and snow storms prevented fieldwork for most of the week preceding the event, which, together with poor road conditions, prevented the deployment of two of the planned SIG arrays (arrays \#1 and \#3). SIGs were deployed at 56 sites at five of the planned arrays. The azimuths and approximate distances of each array are listed in Table 14. The SIG deployment used some of the same locations used for the Contact and Amarillo events (though the array numbers were changed for Junction).

For this experiment, two SIGs were deployed at each location: a golf ball and a steel ball. Each ball was placed directly on the ground. A nail with a piece of flagging was pushed into the ground and the ball placed on top. Sracing between the golf ball and steel ball locations at a site was approximately $2 \mathrm{~m}$ on arrays \#2 and \#7; spacing between the two balls on the other arrays was approximately $10 \mathrm{~m}$. Spacing between sites was 50 paces, or approximately $40-50 \mathrm{~m}$.

The J-8 accelerometer data were originally reported out in April 1992 (Memo J-8-92. 100, R. S. Fitzhugh to F. N. App). All of the J-8 stations recorded spall signals, which are shown in Fig. 21 (12 pages).

Table 14. Junction SIG Arrays

$\begin{array}{ccc}\text { Array } & \begin{array}{c}\text { Distance } \\ \text { from SGZ }\end{array} & \begin{array}{c}\text { Azimuth } \\ \text { from SGZ }\end{array} \\ 1 & 2500 \mathrm{~m} & 50 \\ 2 & 2000 \mathrm{~m} & 100 \\ 3 & 750 \mathrm{~m} & 170 \\ 4 & 1500 \mathrm{~m} & 335 \\ 5 & 2500 \mathrm{~m} & 320 \\ 6 & 2250 \mathrm{~m} & 290 \\ 7 & 2000 \mathrm{~m} & 240\end{array}$




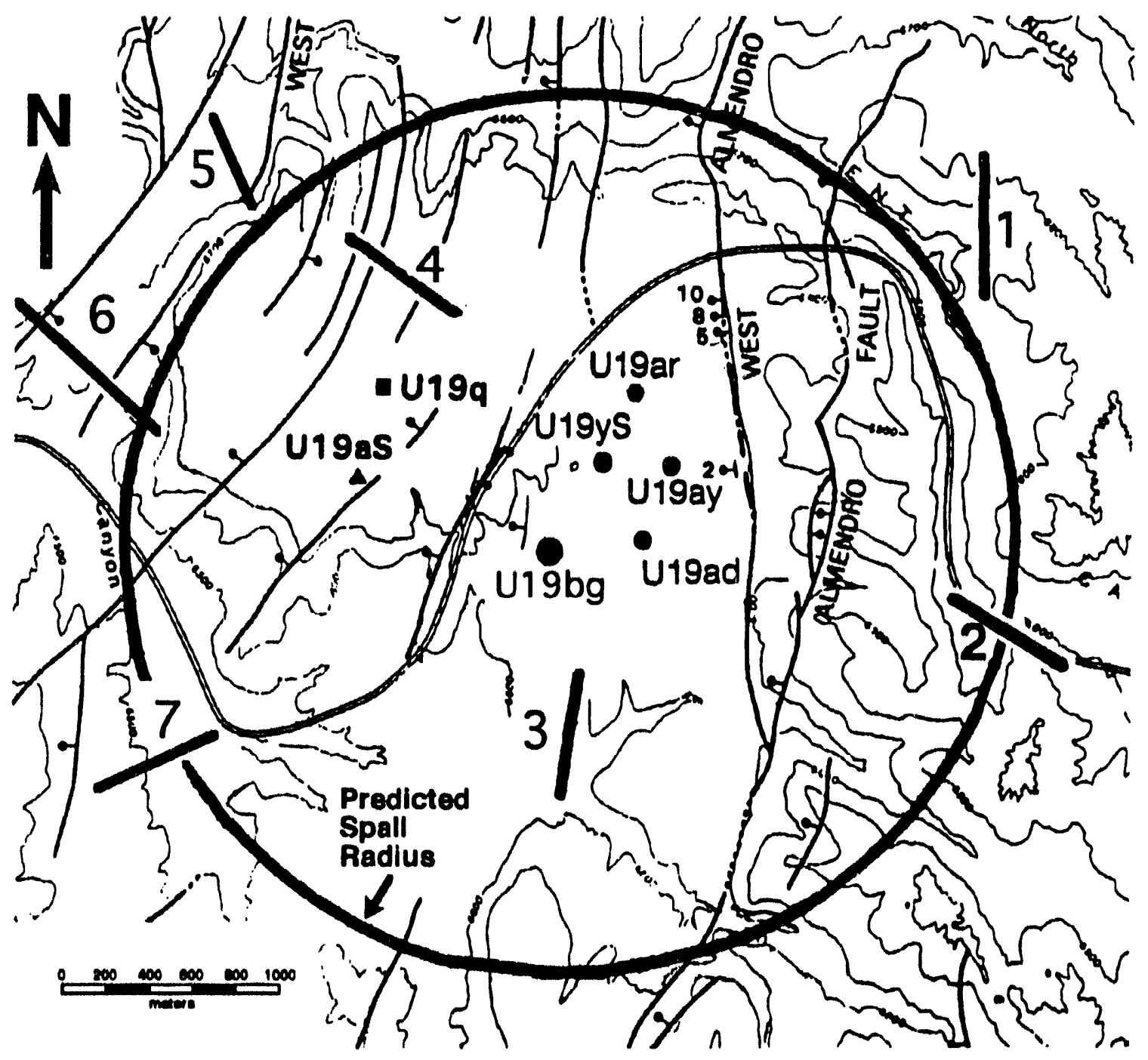

Fig. 19. Junction SIG experiment plan. 


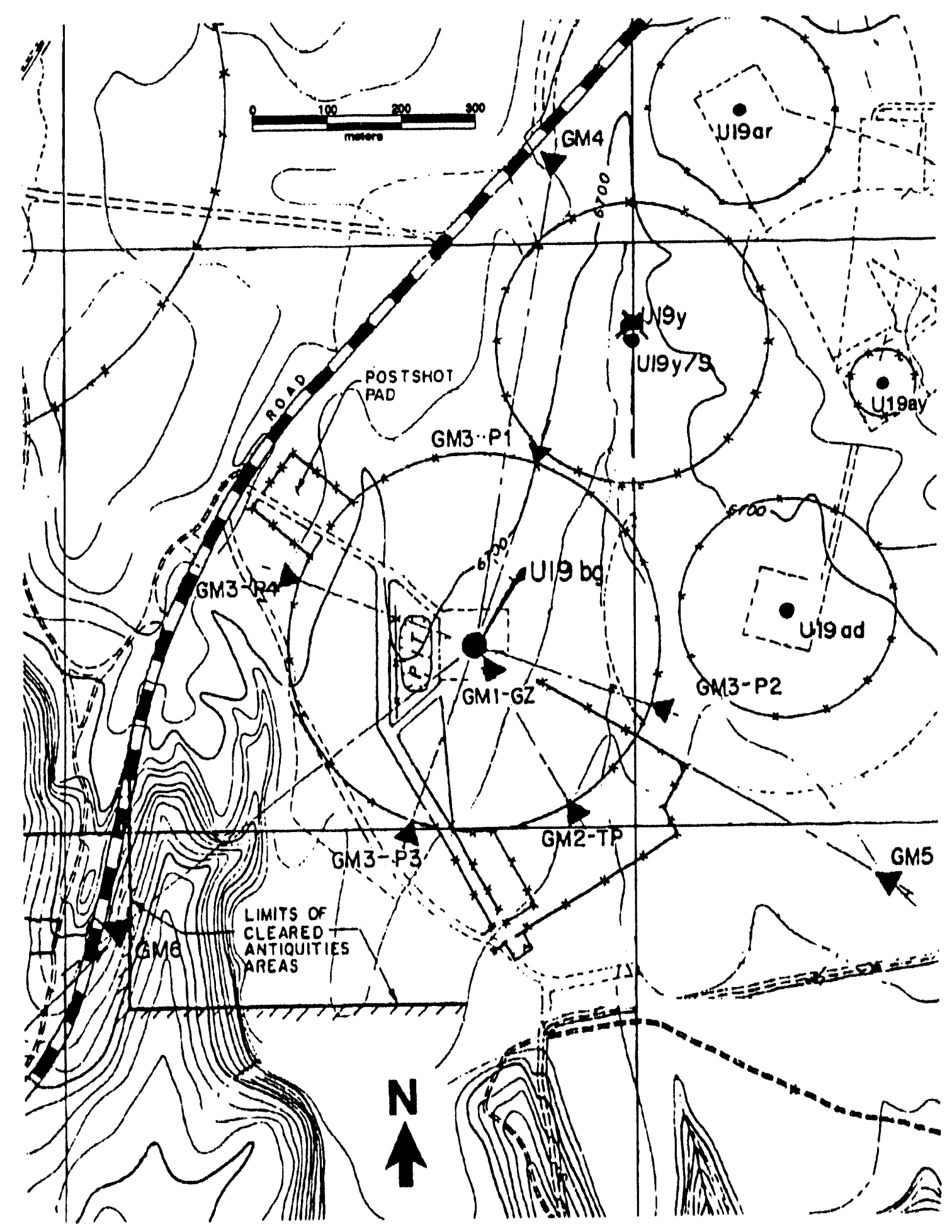

Fig. 20. Junction station map. 
Table 15. Junction Ground-Motion Station Data

\begin{tabular}{|c|c|c|c|c|c|c|}
\hline $\begin{array}{l}\text { Station } \\
\text { Name }\end{array}$ & $\begin{array}{l}\text { Station Loc } \\
\text { u19'sg }\end{array}$ & Accelerometer & $\begin{array}{l}\text { Northing (m) } \\
279898\end{array}$ & $\begin{array}{l}\text { Easting (m) } \\
179617\end{array}$ & $\begin{array}{l}\text { Slant Range } \\
622(m) \text { DOB }\end{array}$ & Travel Time \\
\hline JUNGM1A & $15 \mathrm{~m} \mathrm{S3OE}$ & Endevco $10 \mathrm{~g}$ & 279884 & 179625 & 622 & 0.297 \\
\hline JUNGM1B & $15 \mathrm{~m}$ S30E & Endevco $25 \mathrm{~g}$ & 279884 & 179625 & 622 & 0.3 \\
\hline JUNGM1C & $15 \mathrm{~m}$ S30E & Endevco $25 \mathrm{~g}$ & 279884 & 179625 & 622 & 0.298 \\
\hline JUNGM2A & $250 \mathrm{~m}$ S20E & Endevco $25 \mathrm{~g}$ & 279663 & 179702 & 670 & 0.313 \\
\hline JUNGM2B & $250 \mathrm{~m}$ S20E & Endevco $25 \mathrm{~g}$ & 279663 & 179702 & 670 & 0.313 \\
\hline JUNGM3P1 & $250 \mathrm{~m}$ N2OE & Endevco $25 \mathrm{~g}$ & 280132 & 179702 & 670 & 0.303 \\
\hline JUNGM3P2 & $250 \mathrm{~m}$ S70E & Endevco $25 \mathrm{~g}$ & 279812 & 179852 & 670 & 0.306 \\
\hline JUNGM3P3 & $250 \mathrm{~m}$ S20W & Endevco $25 \mathrm{~g}$ & 279663 & 179531 & 670 & 0.307 \\
\hline JUNGM3P4 & $250 \mathrm{~m}$ N70W & Endevco $25 \mathrm{~g}$ & 279983 & 179382 & 670 & 0.327 \\
\hline JUNGM4A & $622 \mathrm{~m}$ N10E & Endevco $20 \mathrm{~g}$ & 280509 & 179725 & 879 & 0.39 \\
\hline JUNGMSA & $622 \mathrm{~m} \mathrm{S60E}$ & Endevco $20 \mathrm{~g}$ & 279586 & 180155 & 880 & 0.42 \\
\hline JUNGM6A & $622 \mathrm{~m}$ S53W & Endevco $20 \mathrm{~g}$ & 279526 & 179118 & 880 & 0.375 \\
\hline
\end{tabular}


Table 16. Junction SIG Results

Loca-

tion

Golf Ball

Dist Azd

(cm)

Steol Ball

Dist Azi

(cm)

$\begin{array}{ll}8.9 & 125\end{array}$

11.485

$0.0 \quad 0$

....

$0.0 \quad 0$

11.4225

5.1305

$\begin{array}{ll}7.6 & 105\end{array}$

$35.6 \quad 265$

$22.9 \quad 190$

17.8265

$5.1 \quad 255$

$7.6 \quad 135$

ง $4-3$

4-5

4-6

4-7

4-8

4-9

4-10

4-11

4-12

5-1

5-2

$5-3$

5-4

5-5

5-6
19.1295

6.3275

8.9240

6.3215

$24.1 \quad 175$

8.9290

$10.2 \quad 225$

$15.2 \quad 310$

175.3255

$15.2 \quad 310$

14.0265

$0.0 \quad 0$

10.2320

0.00

\section{Comment}

Fracture zone through ball bcations. Disruption zone between 4-4 and 4-5.

Fracture zone through ball locations, heading $210^{\circ}$.

Small scarp between 4-9 and 4-10, rocks overturned.

Some cracking, disruption zone between 4-10 and 4-11; some cracking.

Reactivation of major fracture just west of SIGs, heading $350^{\circ}$ $0^{\circ}$; second fracture $10 \mathrm{~m}$ west.

Balls moved downstope.

Balls moved downstope.

Balls moved downstope. 
Table 16. Junction SIG Results (Concluded)

\begin{tabular}{|c|c|c|c|c|}
\hline \multirow{2}{*}{$\begin{array}{l}\text { Loca- } \\
\text { tion }\end{array}$} & \multicolumn{2}{|c|}{ Golf Ball } & \multicolumn{2}{|c|}{ Steal Ball } \\
\hline & $\begin{array}{l}\text { Dist } \\
\text { (cm) }\end{array}$ & Axd & $\begin{array}{l}\text { Dist } \\
\text { (cm) }\end{array}$ & Ad \\
\hline $5-7$ & 0.0 & 0 & 0.0 & 0 \\
\hline $5-8$ & 7.6 & 110 & 6.3 & 270 \\
\hline $6-1$ & 27.9 & 285 & 31.8 & 235 \\
\hline $6-2$ & 27.9 & 270 & 24.1 & 260 \\
\hline $6-3$ & 12.7 & 180 & 24.1 & 90 \\
\hline $6-4$ & 12.7 & 180 & 24.1 & 275 \\
\hline $6-5$ & 21.6 & 165 & 17.8 & 125 \\
\hline $6-6$ & 5.1 & 105 & 6.3 & 35 \\
\hline $6-7$ & 8.9 & 220 & 5.1 & 265 \\
\hline $6-8$ & 14.0 & 140 & 10.2 & 95 \\
\hline $6-9$ & $\ldots$ & $\cdots$ & 0.0 & 0 \\
\hline क 6-10 & 7.6 & 120 & 3.8 & 275 \\
\hline ก́ 6-11 & 0.0 & 0 & 0.0 & 0 \\
\hline$\vec{A}$ 6-12 & 7.6 & 105 & 0.0 & 0 \\
\hline 6-13 & 12.7 & 310 & 7.6 & 345 \\
\hline $6-14$ & 2.5 & 300 & 6.3 & 145 \\
\hline $6-15$ & 7.6 & 40 & 5.1 & 115 \\
\hline $6-16$ & 20.3 & 15 & 6.3 & 115 \\
\hline $7-1$ & 14.0 & 275 & 8.9 & 140 \\
\hline $7-2$ & 14.0 & 285 & 0.0 & 0 \\
\hline $7-3$ & 0.0 & 0 & 0.0 & 0 \\
\hline $7-4$ & 8.9 & 165 & 2.5 & 70 \\
\hline $7-5$ & 6.3 & 255 & 0.0 & 0 \\
\hline $7-6$ & 2.5 & 290 & 7.6 & 305 \\
\hline $7-7$ & 7.6 & 65 & 0.0 & 0 \\
\hline $7-8$ & 5.1 & 345 & 0.0 & 0 \\
\hline $7-9$ & 11.4 & 130 & 0.0 & 0 \\
\hline $7-10$ & 0.0 & 0 & 0.0 & 0 \\
\hline
\end{tabular}

Comment

Balls moved downslope.

Balls moved dowmslope.

Steel ball moved upslope, stopped in a depression.

Fracture zone between 6-3 and 6-4.

Fracture zone between 6-4 and 6-5, 20m west of 6-4.

Golf ball moved upslope. 


\section{Junction Surface Ground Motion}

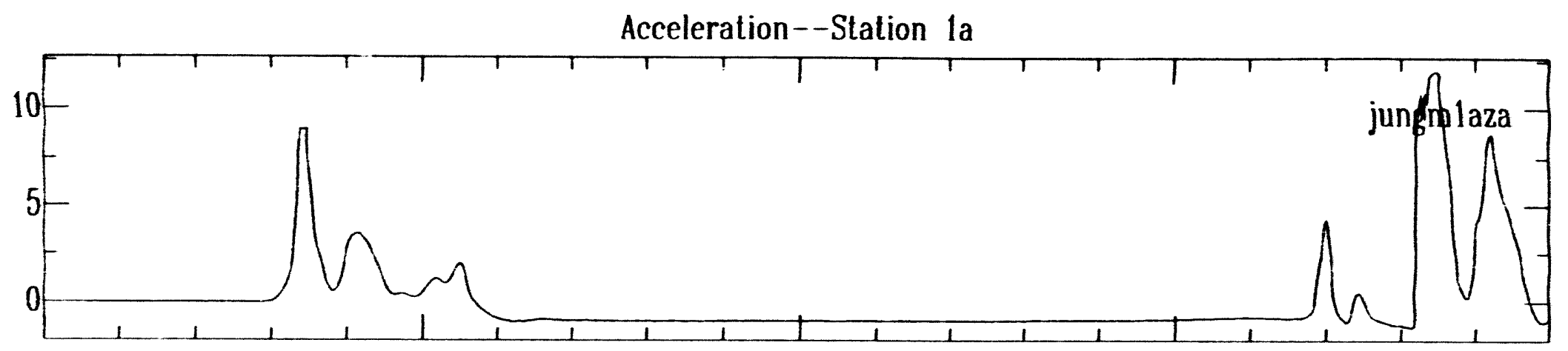

Velocity--Station 1a

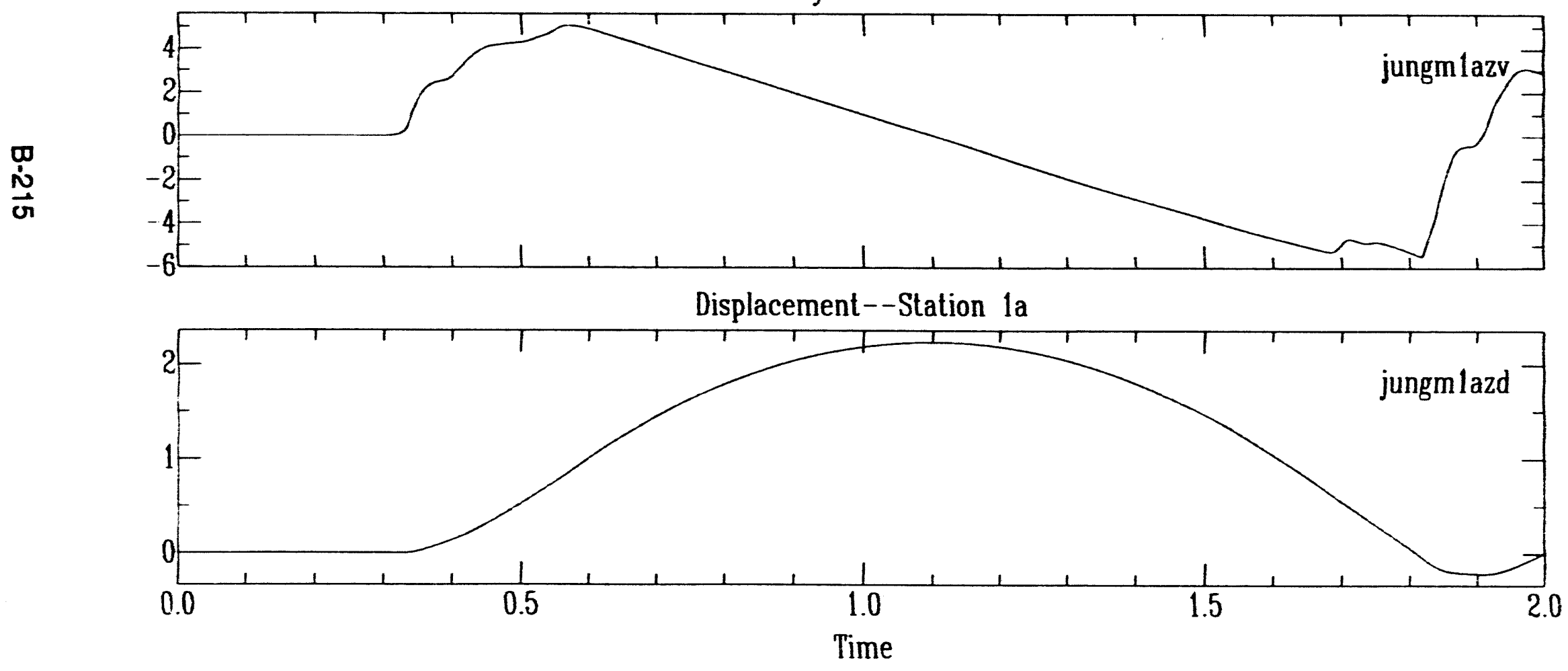




\section{Junction Surface Ground Motion}

Acceleration--Station 1b

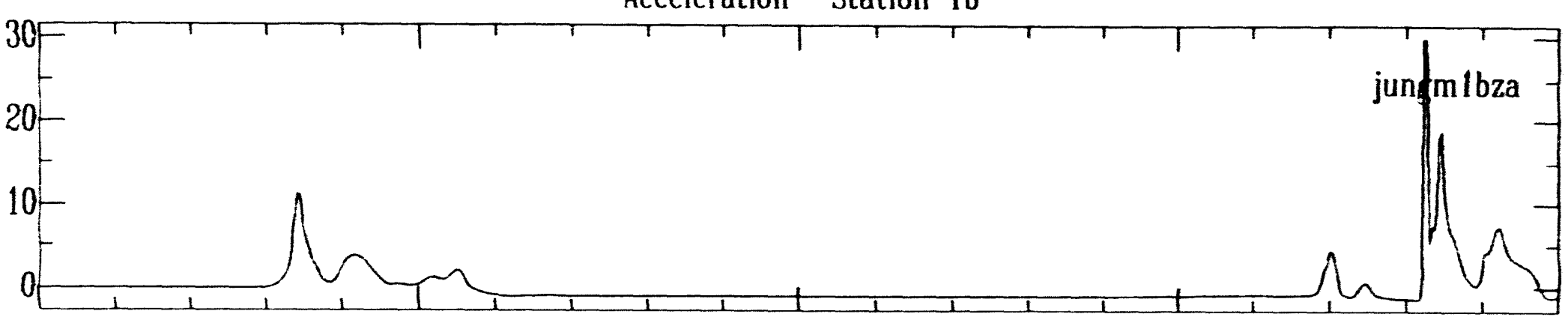

Velocity--Station 16

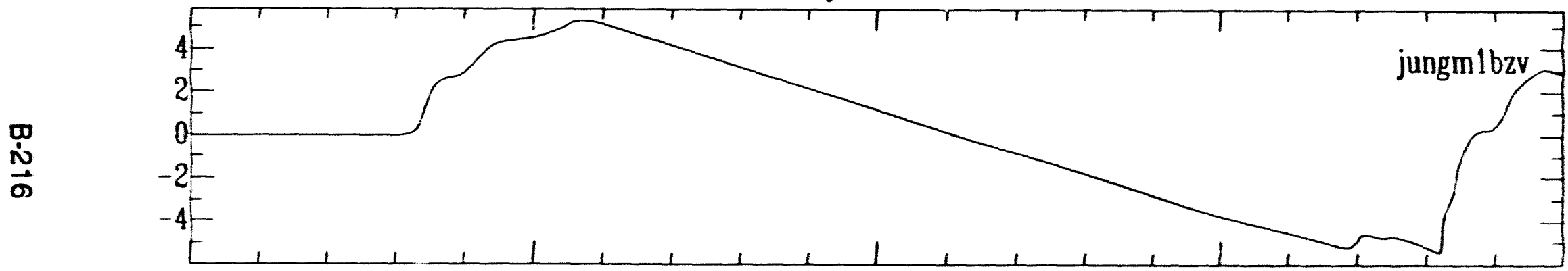

Displacement--Station 1b

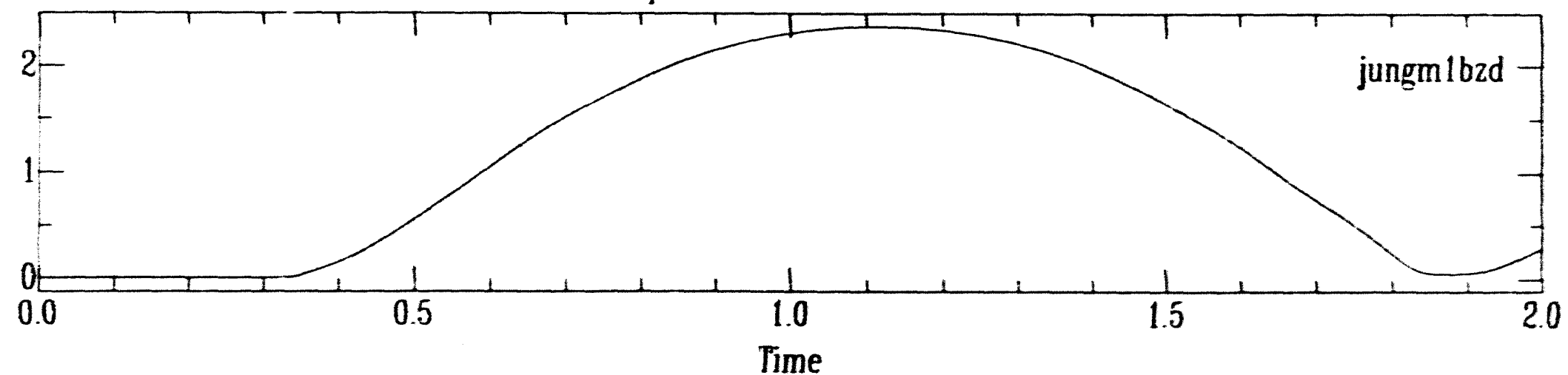




\section{Junction Surface Ground Motion}

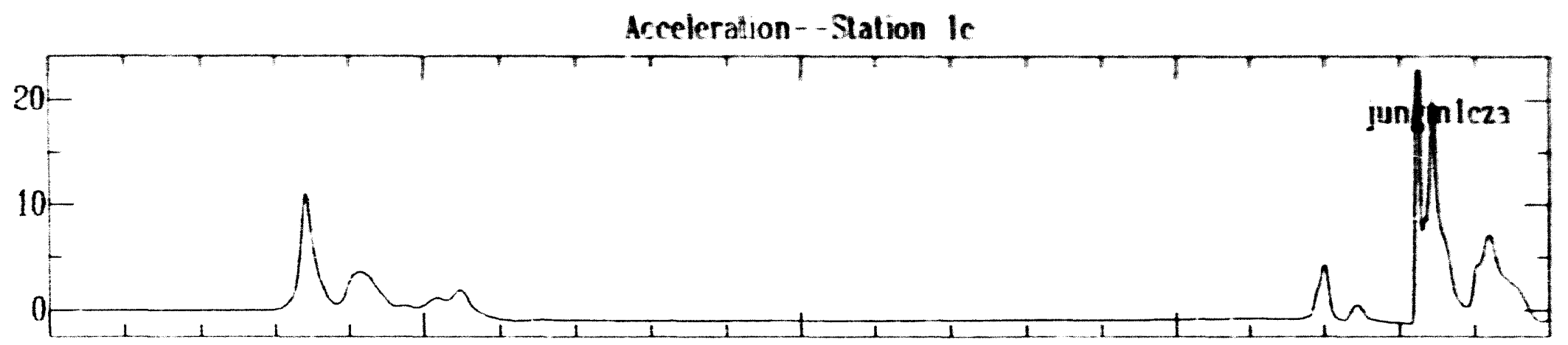

Velocity--Station Ic

ֻึ

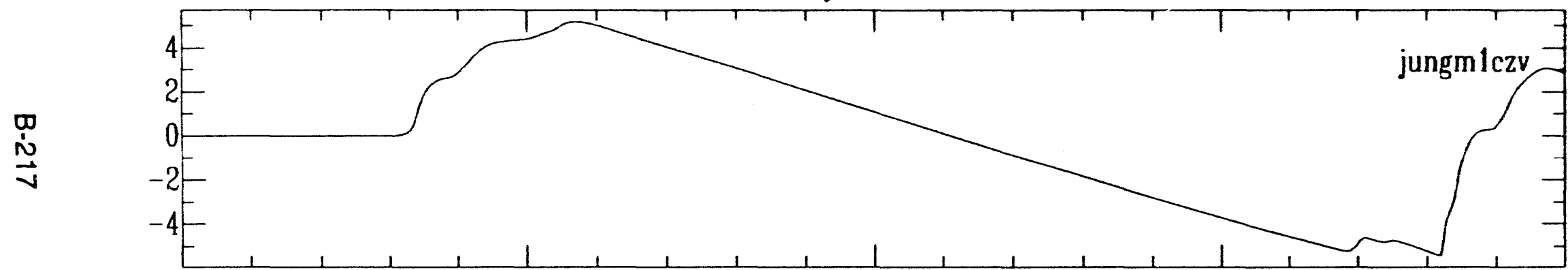

Displacement--Station Ic

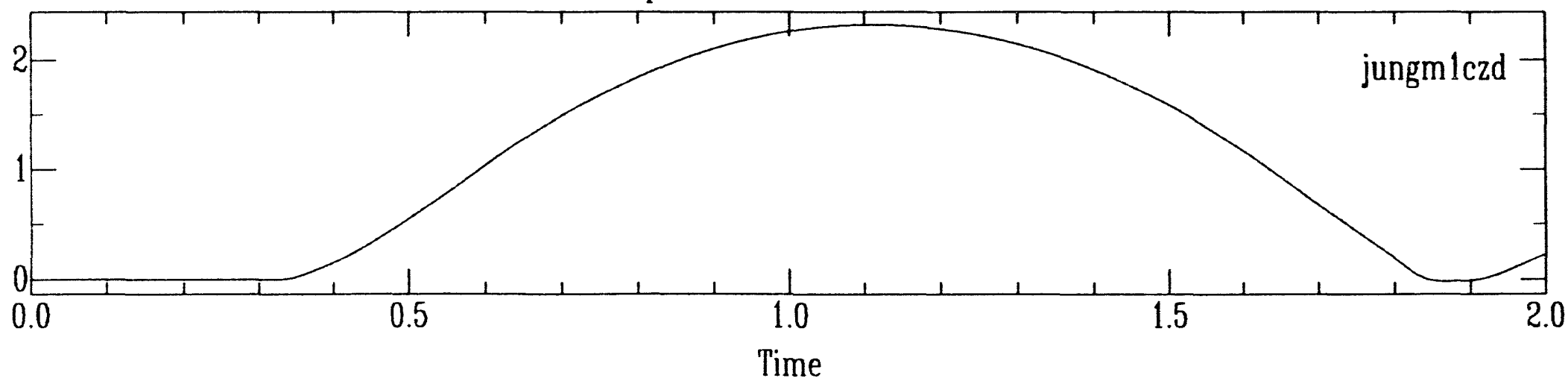



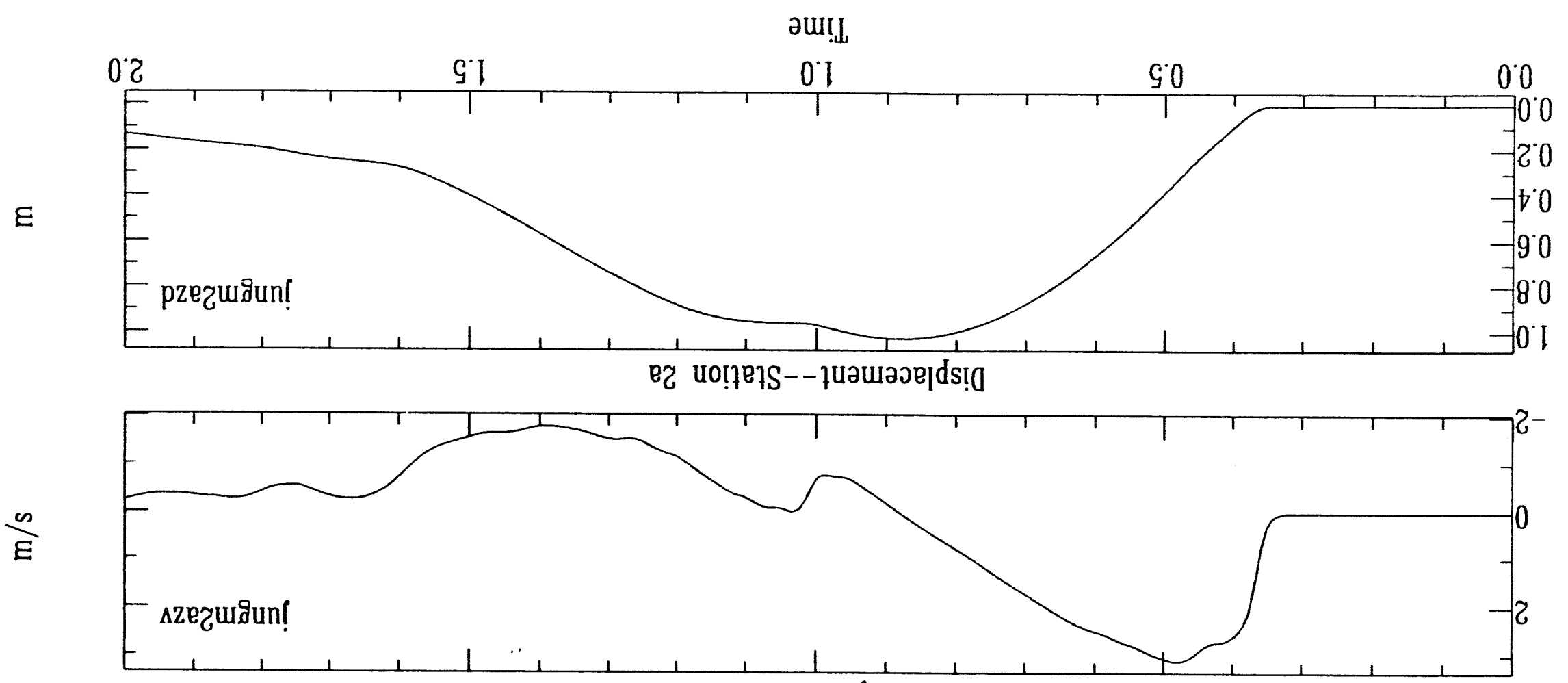

$\stackrel{\infty}{\text { ஸे }}$

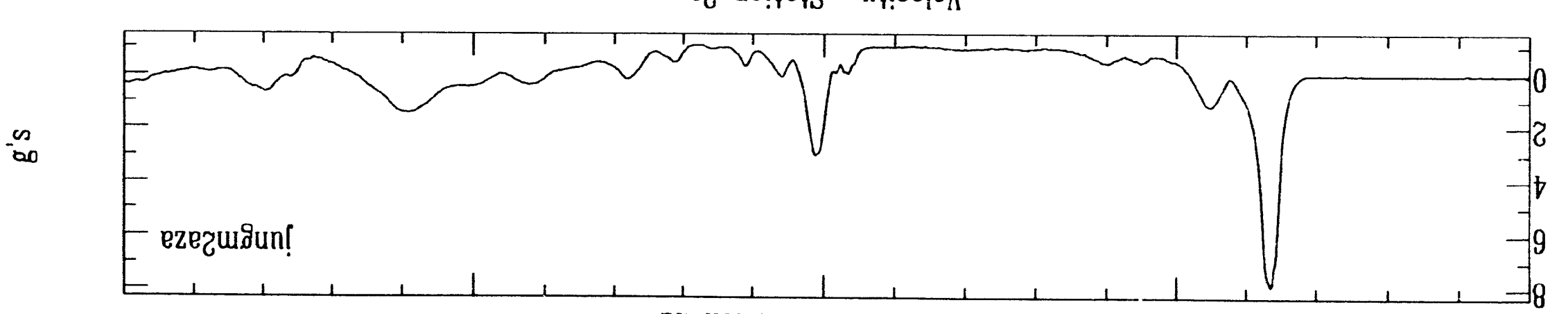

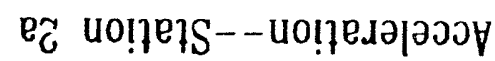

uo!noN punodn zoejuns uo!̣oun 


\section{Junction Surface Ground Motion}

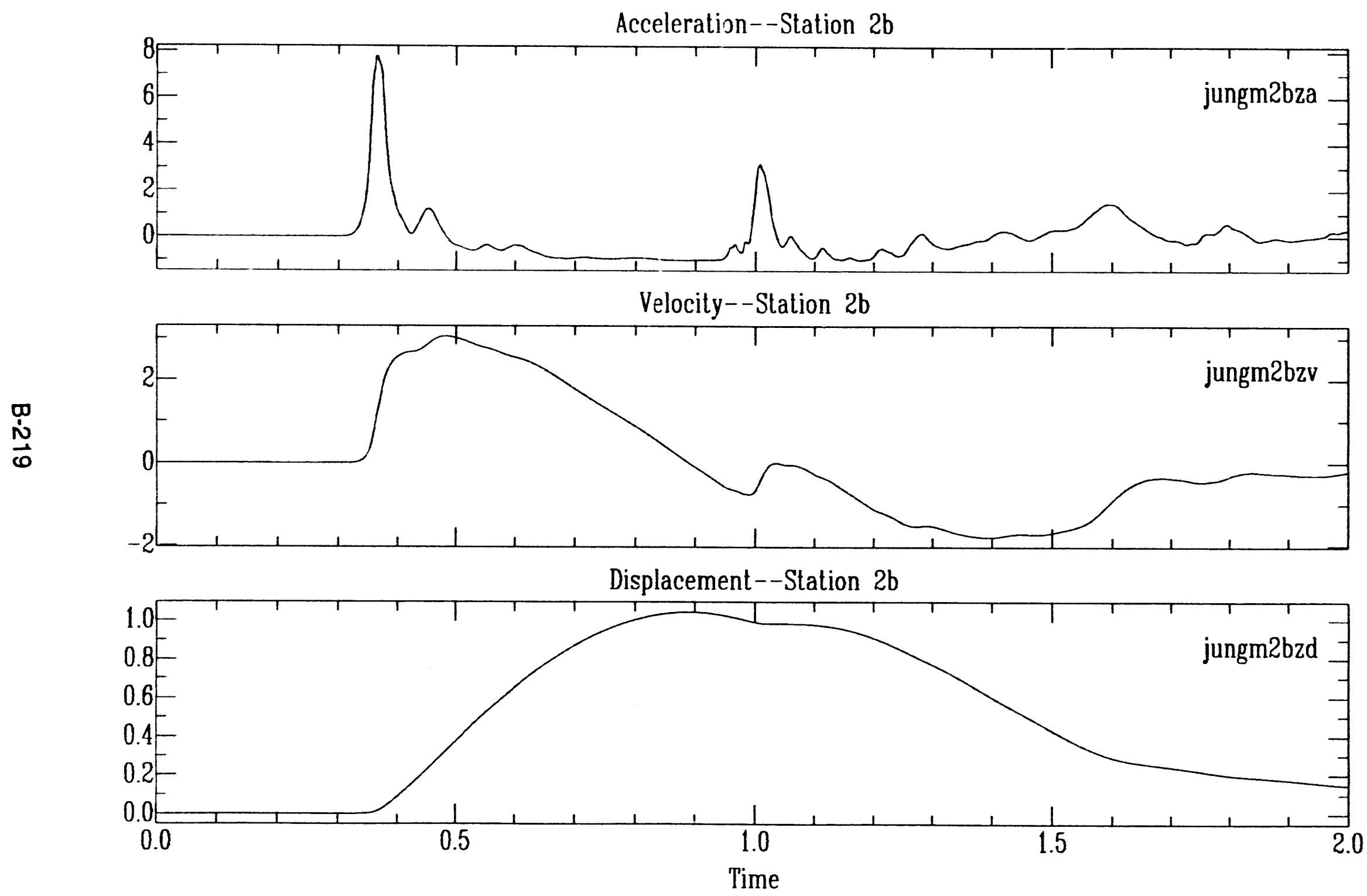

$\infty$ 

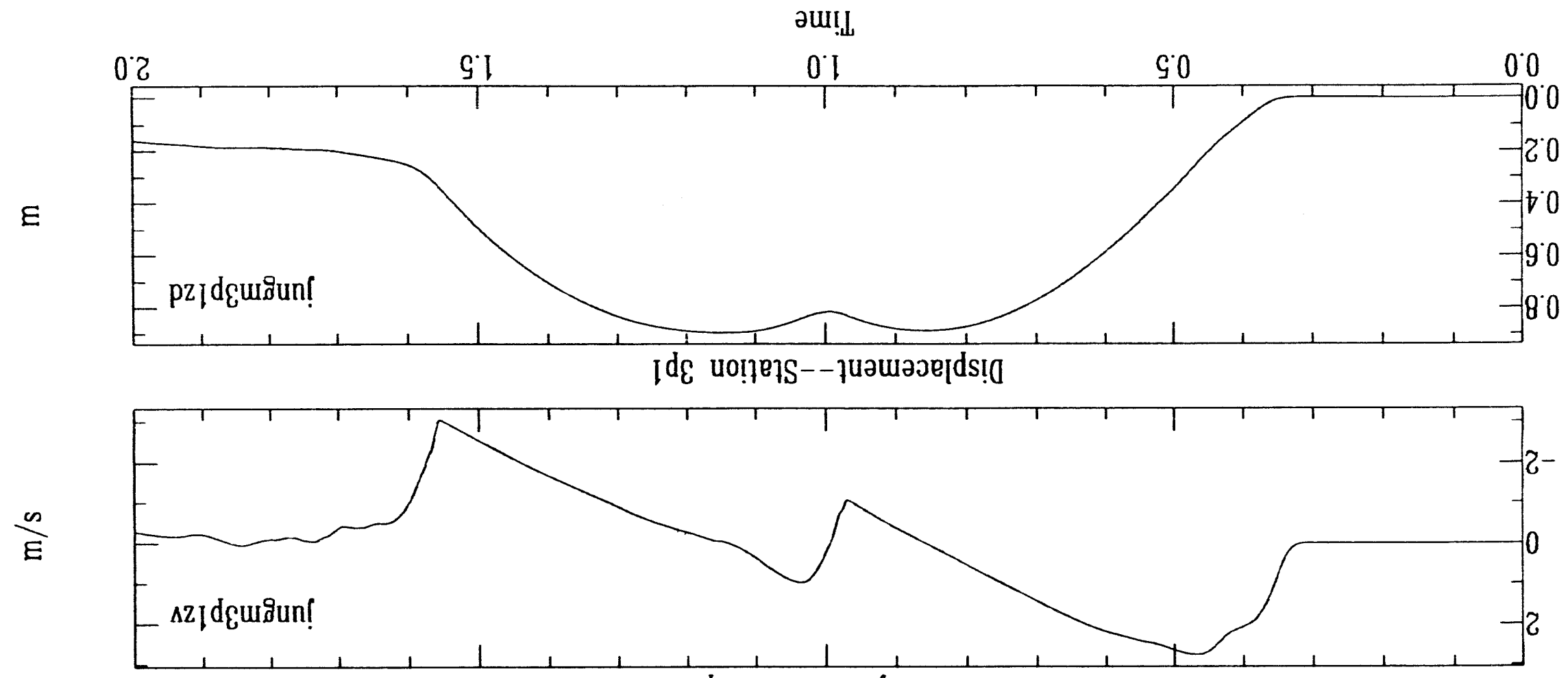

尽

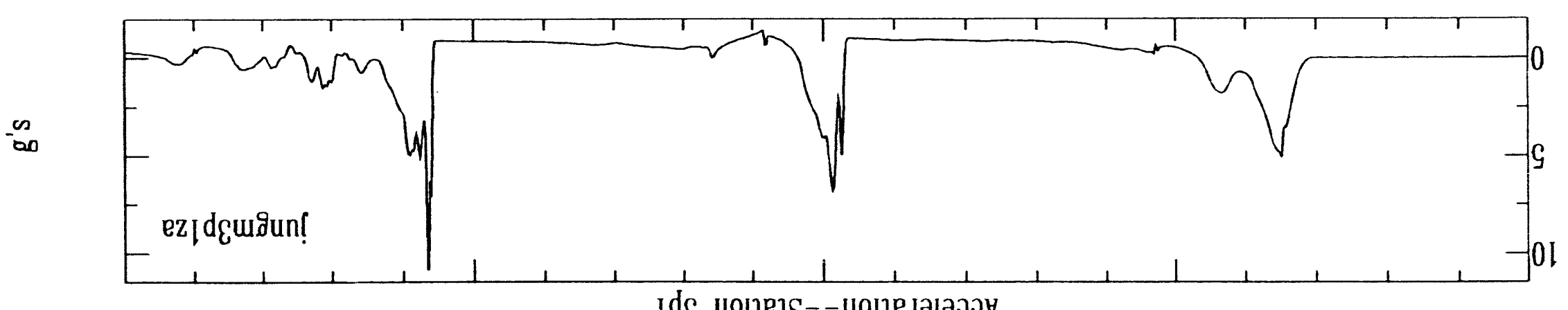

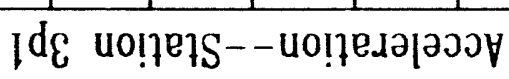

uolfow punodn ajejans uolpoun 


\section{Junction Surface Ground Motion}

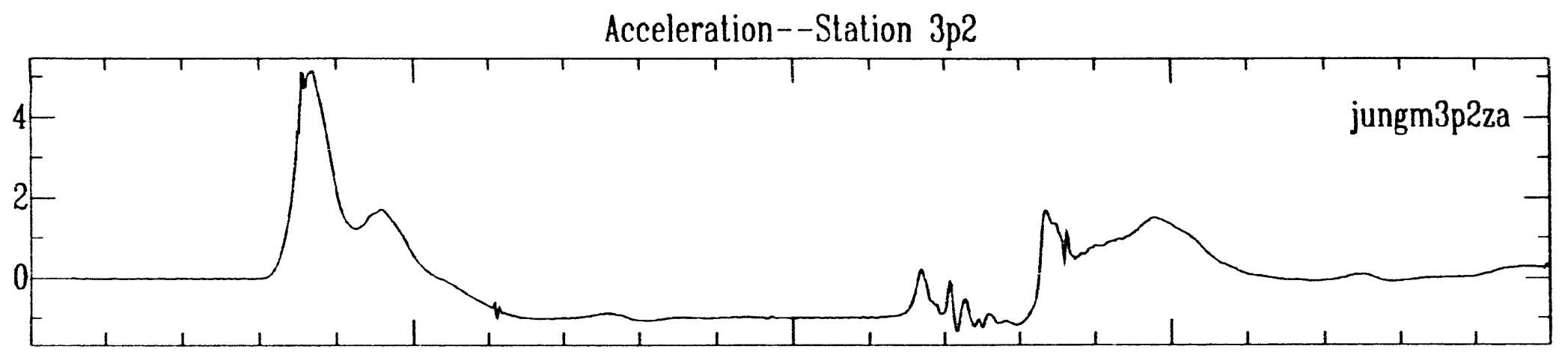

Velocity--Station 3p2

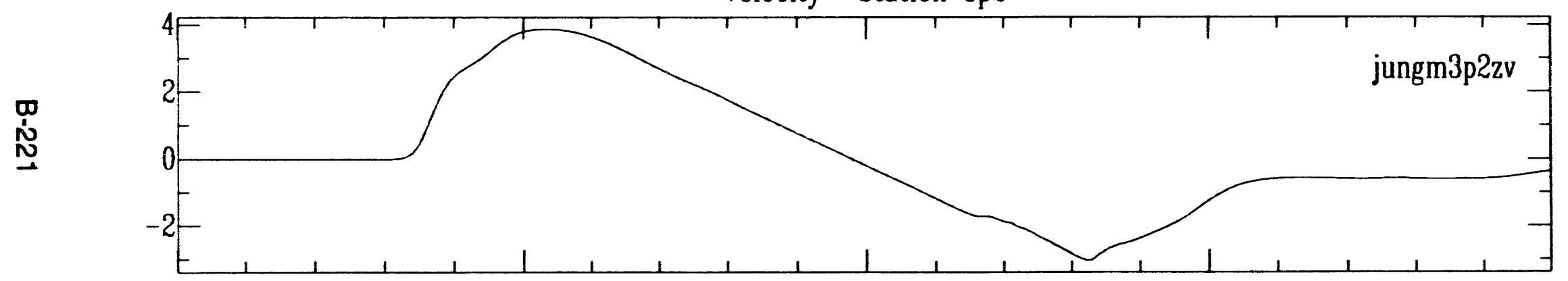

$\frac{3}{\infty}$

Displacement--Station 3p2

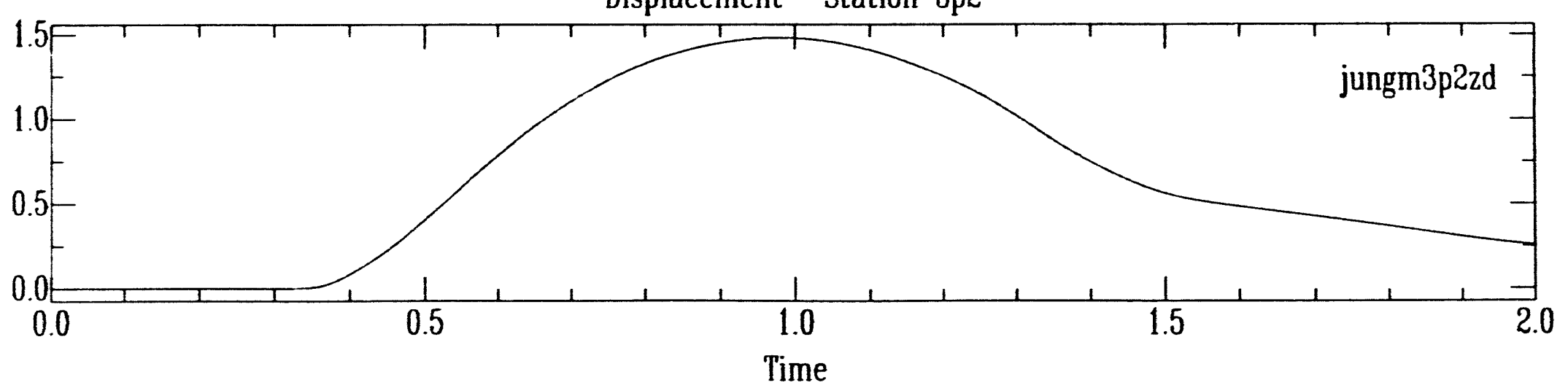




\section{Junction Surface Ground Motion}

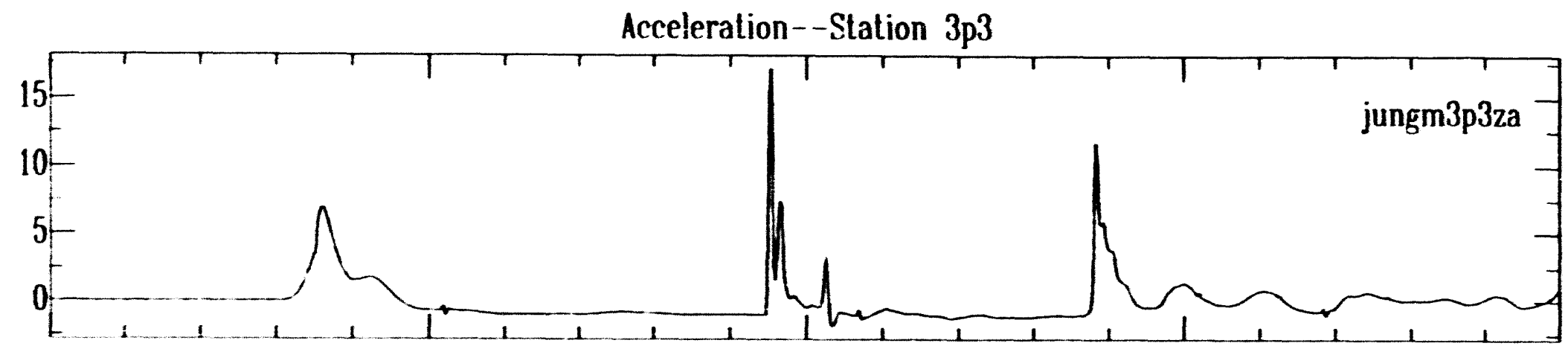

Velocity--Station 3p3

$\boldsymbol{\infty}$
$\mathbf{N}$
$\mathbf{N}$

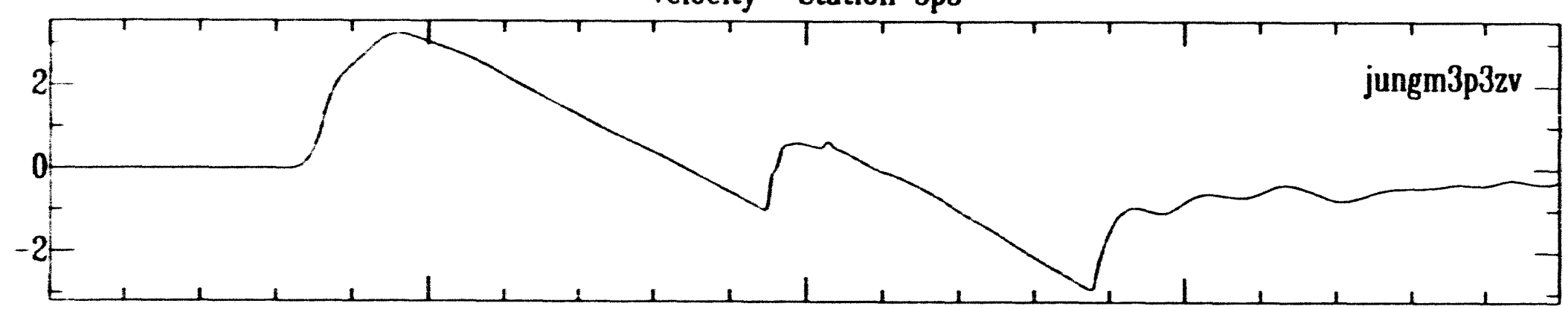

3

Displacement--Station 3p3

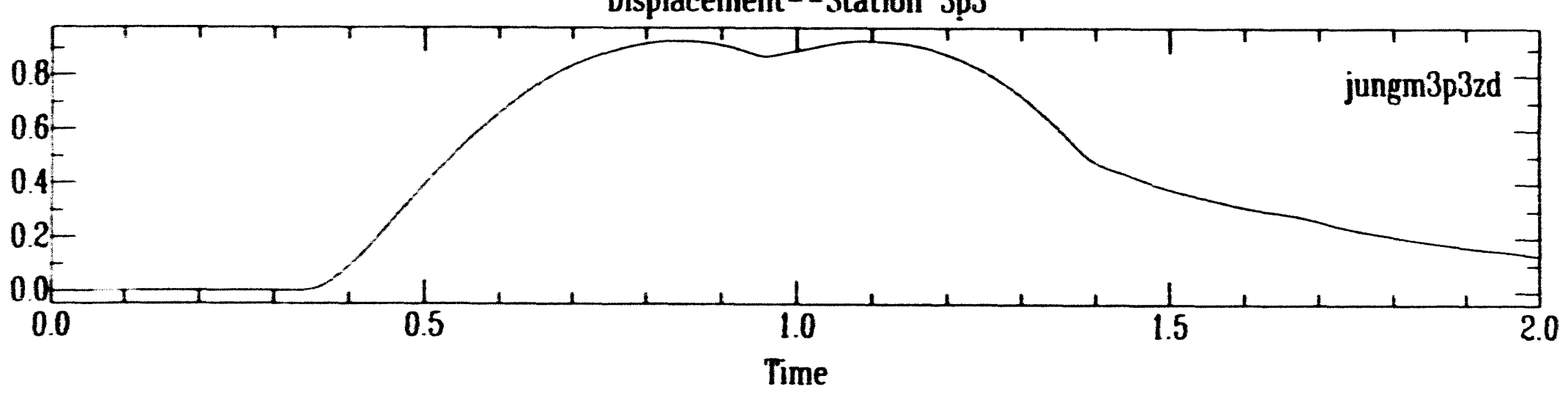




\section{Junction Surface Ground Motion}

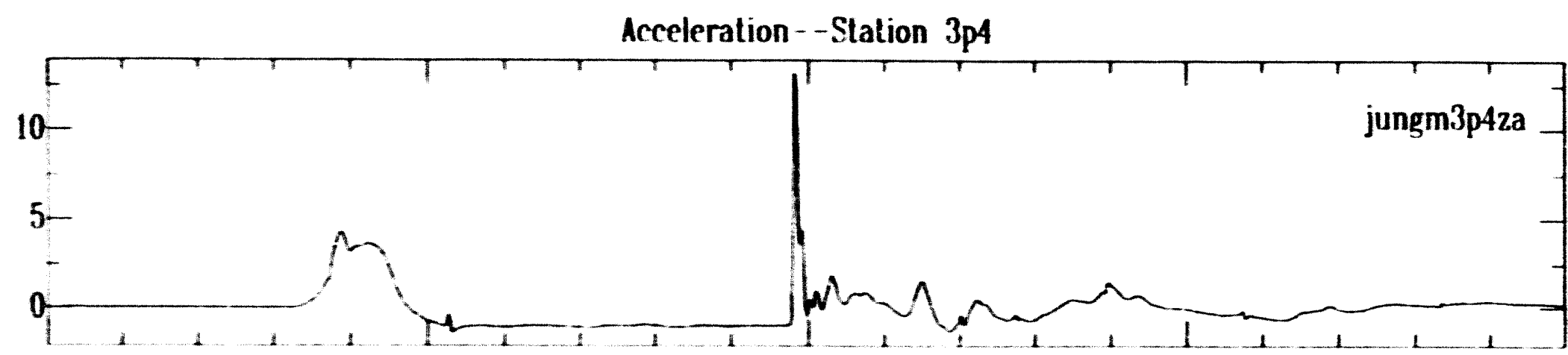

Velocity--Station 3p4

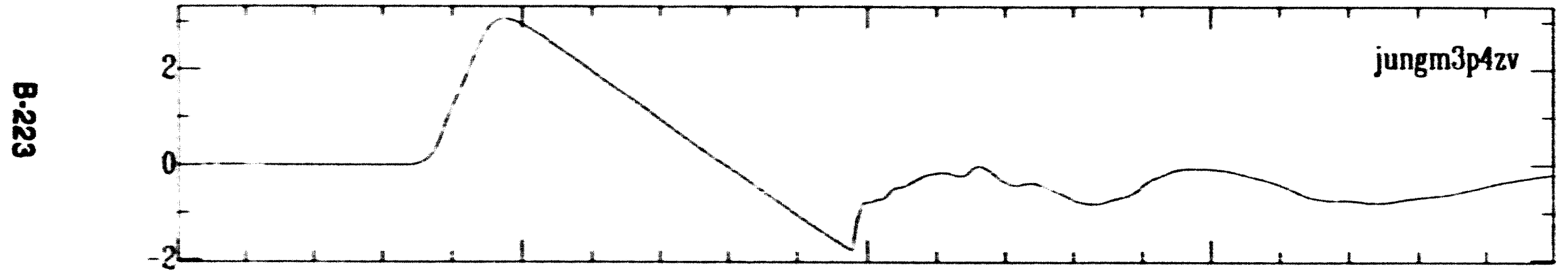

Displacement--Station 3p4

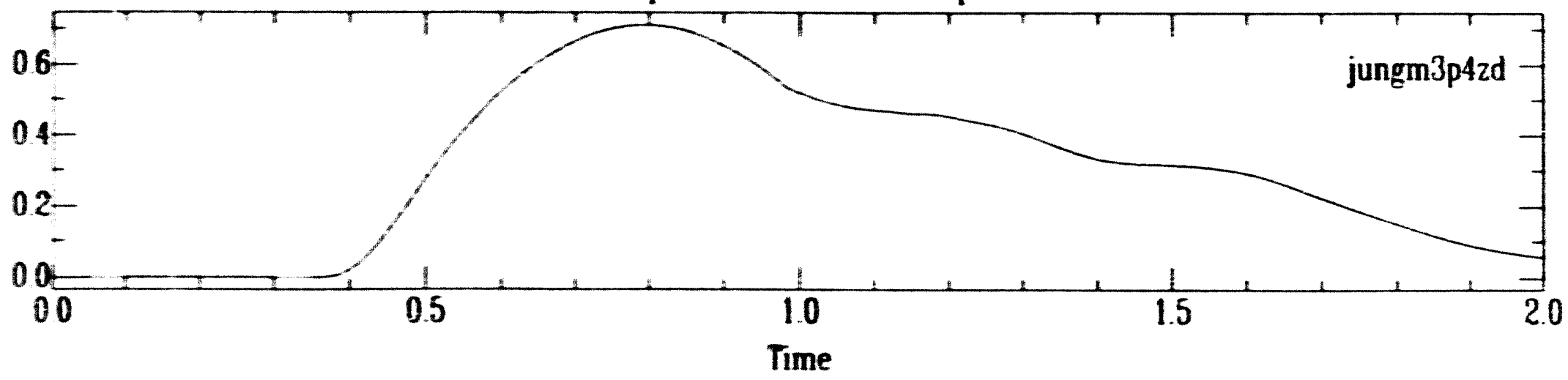



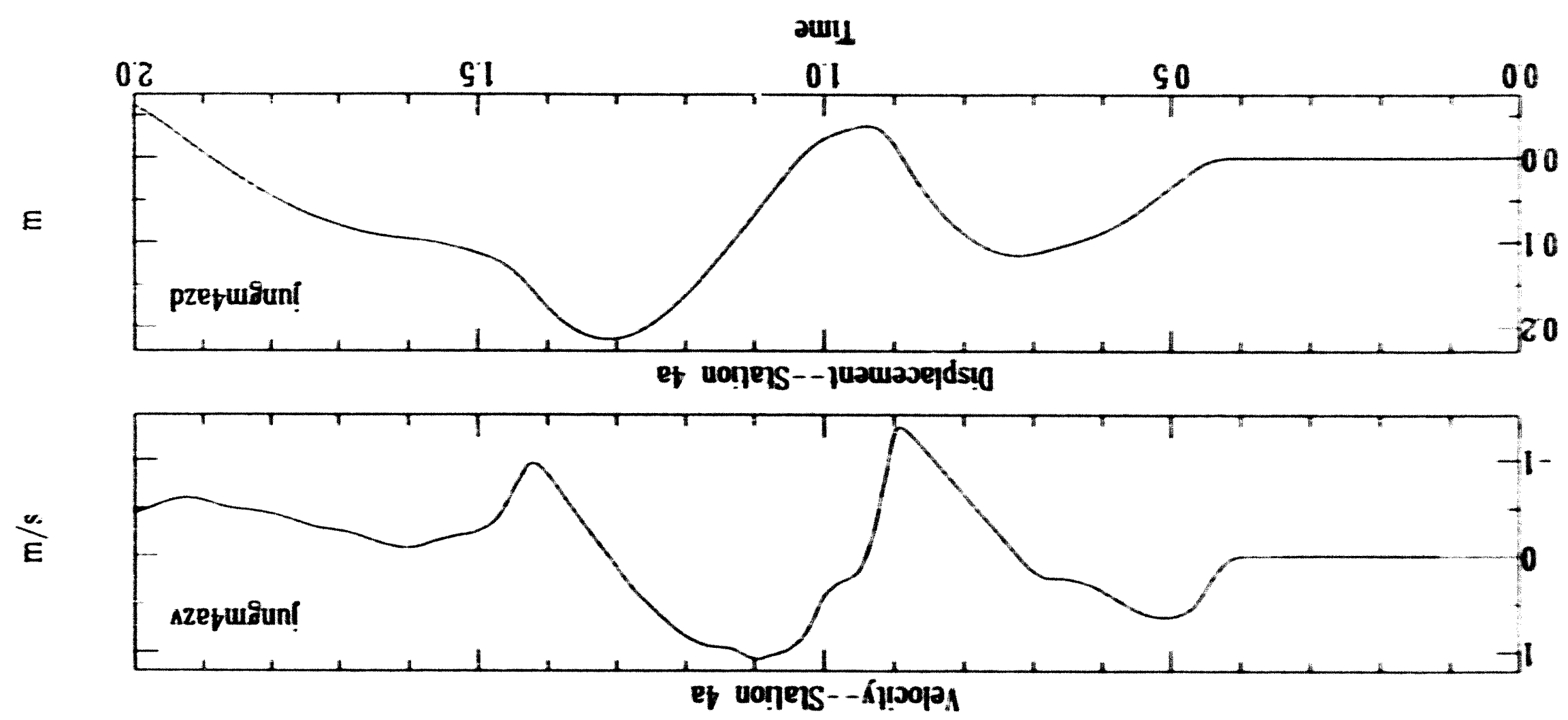

$\frac{8}{4}$

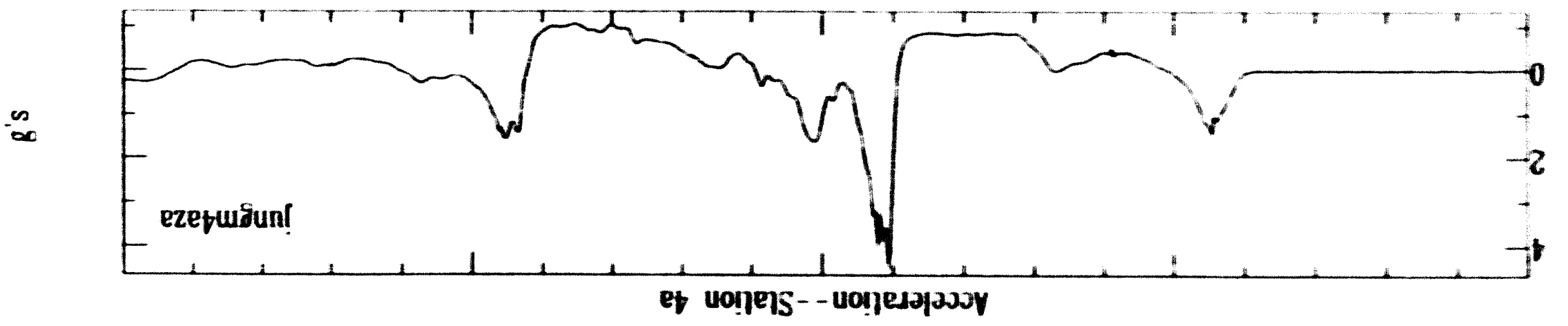

uo!now punodJ วJejans uo!̣unf 
Junction Surface Ground Motion

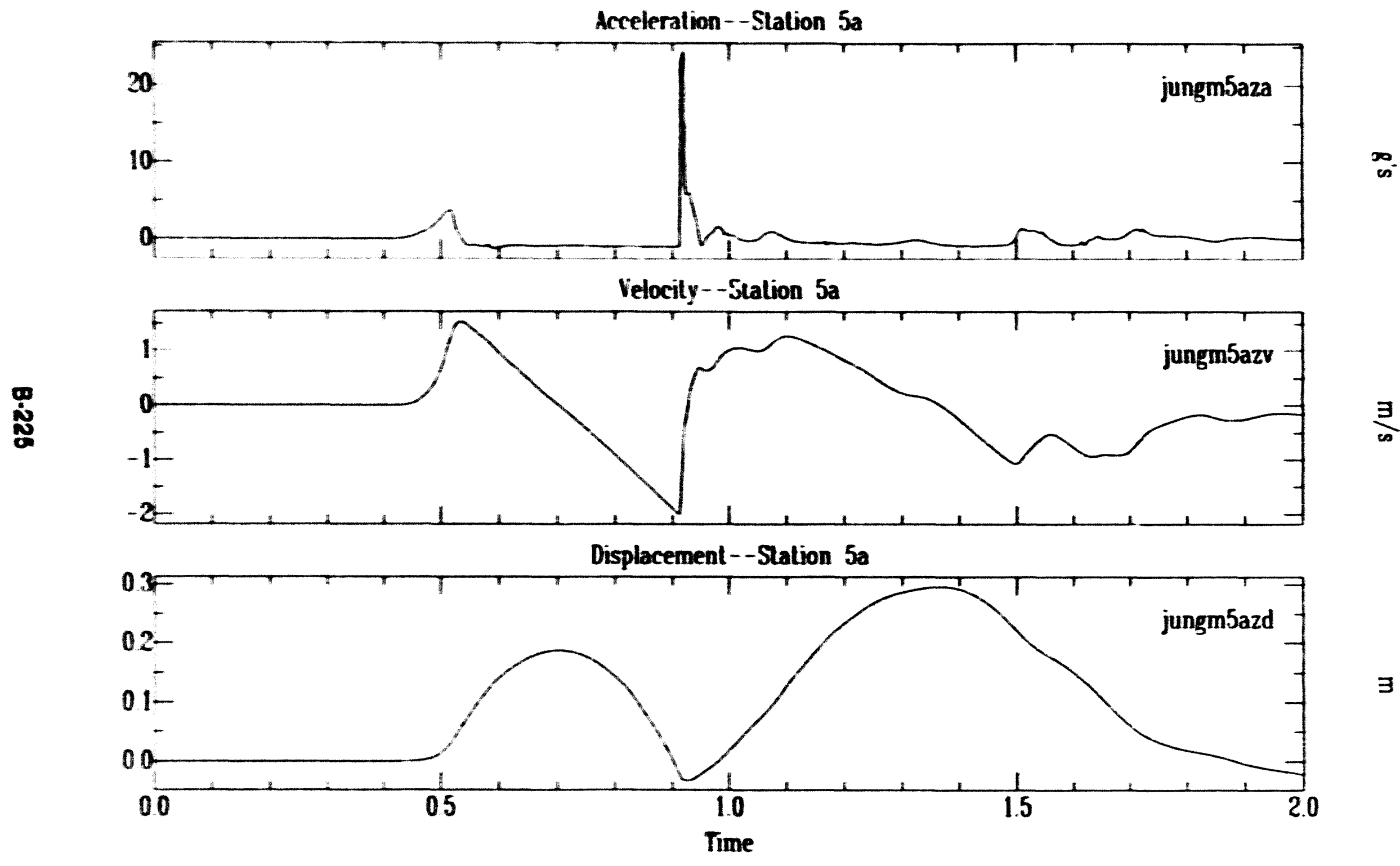



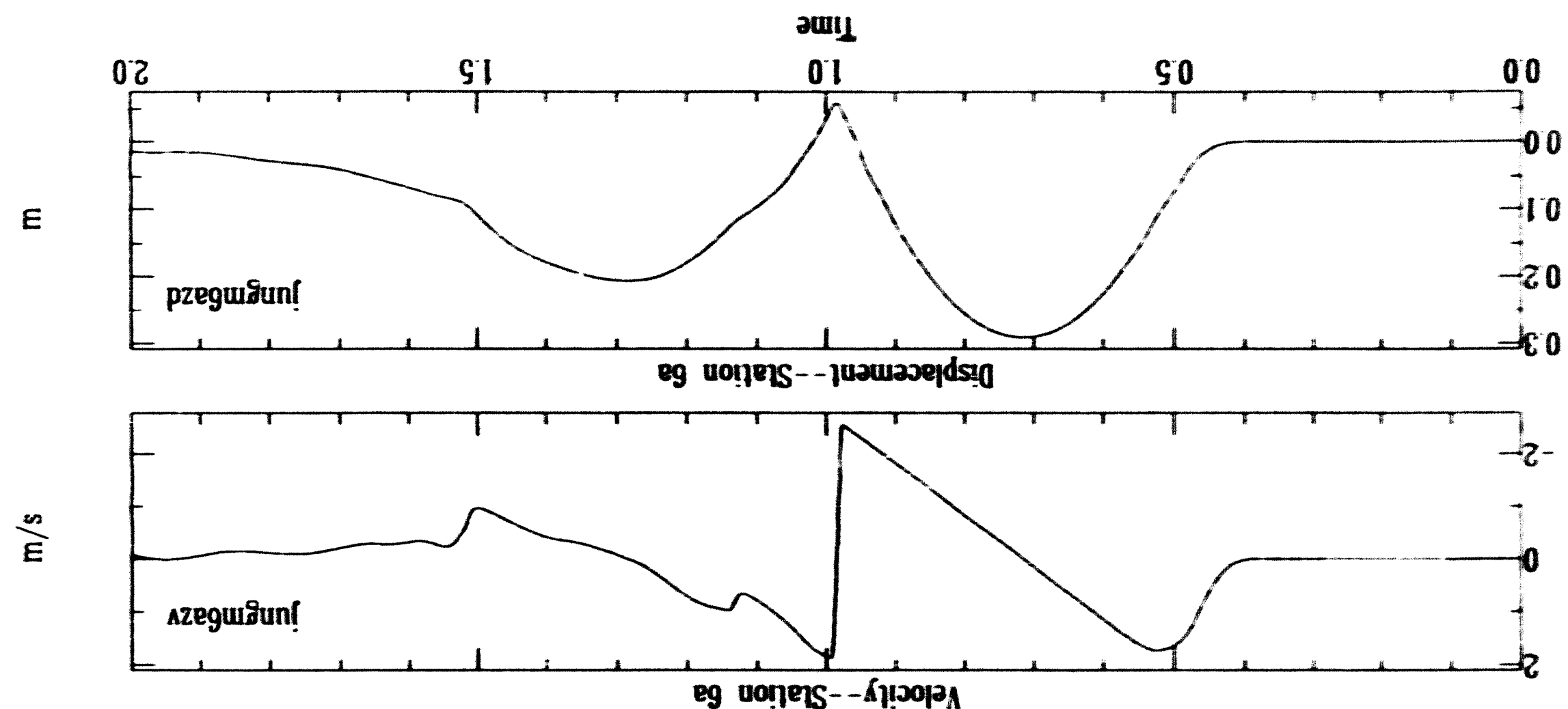

$\frac{8}{\alpha}$

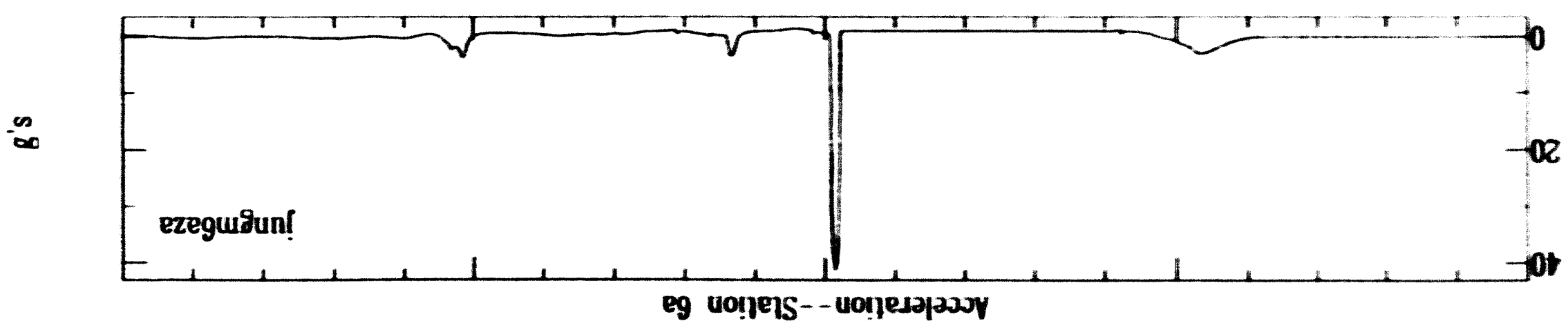

uo!̣ok punodg วכejans uo!̣une 


\section{Additional information}

These data plus the horizontal and tangential components reside as digital time-serles files in the Ground-Motion Database, a GEODES DBMS database on a VAX VMS minicomputer. The vertical data presenter herein also reside as Seismic Analysis Code (SAC) from Lawrence Livermore National Laboratory files on a Sun workstation. For additional information regarding these data sets you may contact:

For general information:

Thomas A. Weaver

Los Alamos National Laboratory

EES-3, MS C335

Los Alamos. Now Mexico 87545

Telephone: 505 667.8464

Fax: 505667.4739
For SAC file information:

Dlane F. Baker

Los Alamos National Laboratory

EES-3, MS C335

Los Alamos, New Mexico 87545

Telephone: 505 687.3776

Fax: $505667-4739$

For VAX GM database information:

Fred App

Los Alamos National Laboratory

EES-3, MS F659

Los Alamos. New Mexico 87544

Telephone: 505 667.6872

Fax: 505 685.3681

\section{Acknowlodgoments}

This work is sponsored by the Department of Energy, Oflice of Arms Control and Nonprollieration (DOE/AN). The ettorts of Brian Slump. Craig Pearson, and Karl Thomasen of SMU, and Bob Reinke. DNAA, in the collection and analysis of the data for several of the events, are gratelully acknowledged. The cooperation of Fred ApD (LANL) on the Ground.Motion Database and Tom Tunnell of EG\&G in data repair is also gratelully acknowledged. Finally, the authors appreciate the persistent prodding and advice of Bob Jetries and Eric Jones (LANL) and Marv Denny (DOE/AN) 10 prepare and release these data for the use of others. 

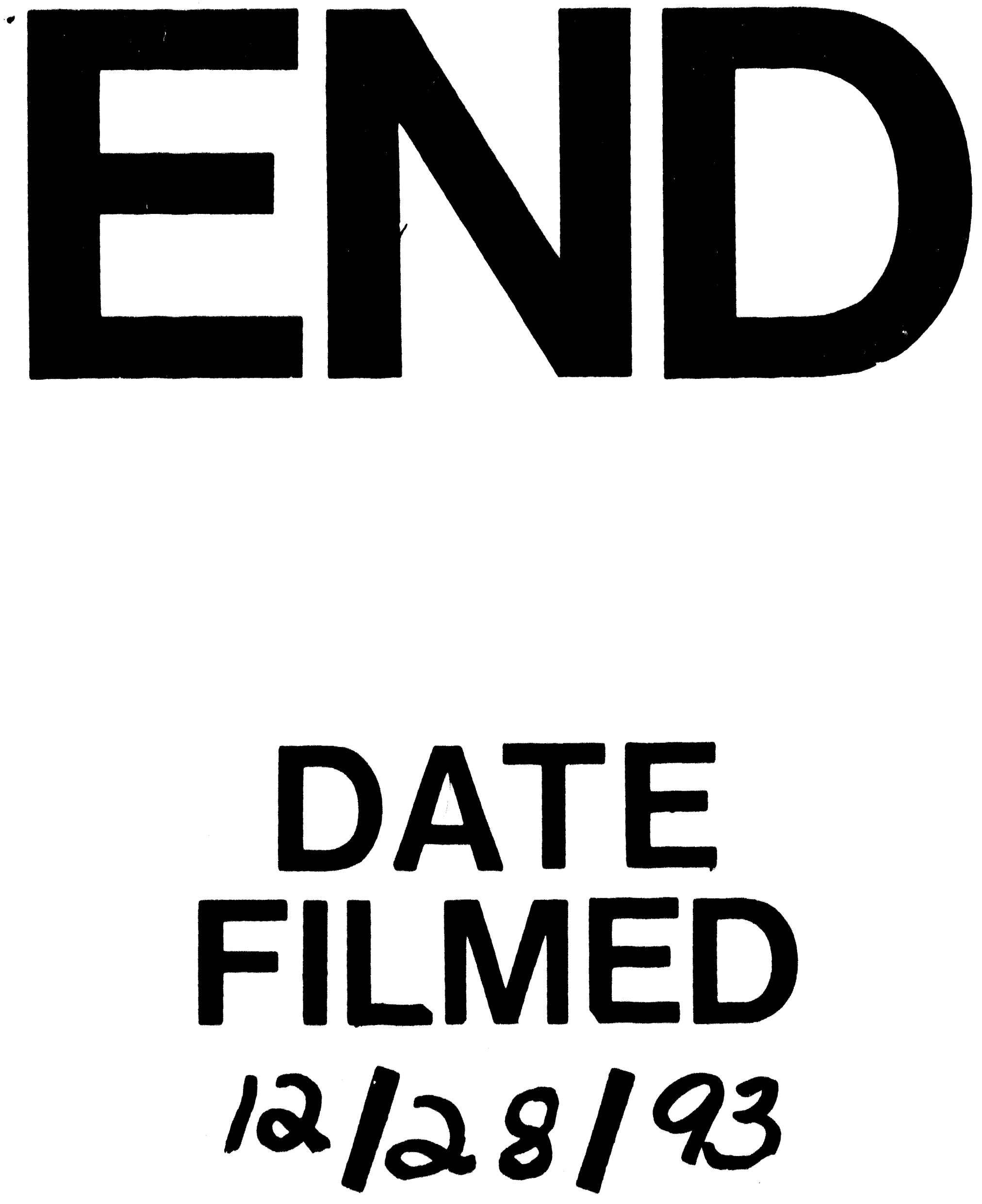
